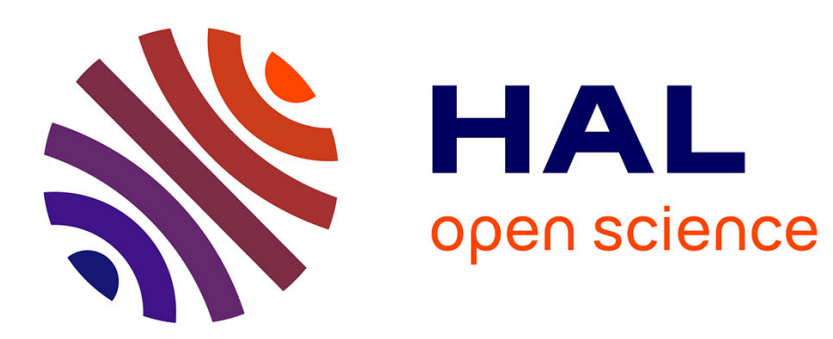

\title{
Development of tools for quantum engineering using individual atoms : optical nanofibers and controlled Rydberg interactions
}

Sylvain Ravets

\section{- To cite this version:}

Sylvain Ravets. Development of tools for quantum engineering using individual atoms: optical nanofibers and controlled Rydberg interactions. Optics [physics.optics]. Institut d'Optique Graduate School, 2014. English. NNT : 2014IOTA0019 . tel-01132435

HAL Id: tel-01132435

https://pastel.archives-ouvertes.fr/tel-01132435

Submitted on 17 Mar 2015

HAL is a multi-disciplinary open access archive for the deposit and dissemination of scientific research documents, whether they are published or not. The documents may come from teaching and research institutions in France or abroad, or from public or private research centers.
L'archive ouverte pluridisciplinaire HAL, est destinée au dépôt et à la diffusion de documents scientifiques de niveau recherche, publiés ou non, émanant des établissements d'enseignement et de recherche français ou étrangers, des laboratoires publics ou privés. 


\title{
INSTITUT
d'OPTIQUE

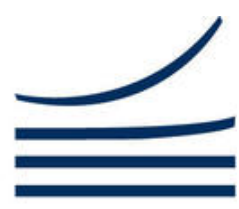

G R A D U A T E S C H O O L

ParisTech

\section{INSTITUT D'OPTIQUE GRADUATE SCHOOL ÉCOLE DOCTORALE ONDES ET MATIERE}

\author{
PHYSIQUE
}

THĖSE

pour l'obtention du grade de Docteur en science de l'Institut d'Optique Graduate School préparée au Laboratoire Charles Fabry

soutenue le 18/12/2014

par

Sylvain RAVETS

\section{Development of tools for quantum engineering using individual atoms:}

optical nanofibers and controlled Rydberg interactions

Directeur de thèse :

Co-directeur de thèse :

Antoine BrowaEys

Luis Orozco

Composition du jury :

Président du jury :

Rapporteurs :

Examinateurs :
Pierre Pillet

Michel Brune

Arno Rauschenbeutel

Antoine BrowaEys

József FORTÁGH

Thierry LAHAYE

Luis Orozco
Laboratoire Charles Fabry

Joint Quantum Institute, University of Maryland

Laboratoire Aimé Cotton

Laboratoire Kastler Brossel

- Vienna Center for Quantum Science and Technology

- Laboratoire Charles Fabry

- Physikalisches Institut, Universität Tübingen

- Laboratoire Chales Fabry

- Joint Quantum Institute, University of Maryland 



\section{Remerciements}

Ce manuscrit présente les travaux réalisés de Septembre 2011 à Décembre 2014 au cours de mon doctorat à l'Institut d'Optique. J'ai eu la chance d'y bénéficier d'un formidable environnement scientifique et de conditions de travail idéales au sein du Laboratoire Charles Fabry, et je tiens tout d'abord à remercier Christian Chardonnet, Pierre Chavel puis Patrick Georges de m'y avoir accueilli durant ces trois années.

Je remercie également les rapporteurs de ma thèse, Michel Brune et Arno Rauschenbeutel, pour leur lecture attentive de mon manuscrit et pour leurs commentaires. Merci en particulier à Michel Brune d'avoir passé du temps à m'aider à corriger une petite erreur qui s'était glissée dans la version initiale du Chapitre 9. Pierre Pillet et József Fortágh m'ont aussi fait l'honneur d'accepter de prendre part au jury de ma thèse, et je les remercie des discussions très intéressantes que nous avons pu avoir à cette occasion.

J'aimerais maintenant exprimer toute ma gratitude envers mon directeur de thèse, Antoine Browaeys, pour m'avoir donné la chance de travailler dans son groupe sur un dispositif expérimental exceptionnel, et pour m'avoir ouvert les portes du Joint Quantum Institute. Antoine a eu une importance toute particulière dès le début de ma formation de physicien. Après une première rencontre lors d'une discussion informelle sur les interférences de plasmons uniques en 2008, j'ai aussi eu l'occasion de suivre son cours d'introduction à l'optique quantique en 2009. C'est sans aucun doute ce cours qui m'a donné l'envie de poursuivre dans le domaine de la physique quantique. C'est pourquoi je suis revenu vers Antoine Browaeys en 2011, dans l'espoir de pouvoir travailler avec lui pour ma thèse. Merci donc de m'avoir accordé ta confiance, me permettant de connaître, au-delà du Professeur Antoine Browaeys, le chercheur et l'ami Antoine avec qui je partage, en plus du style vestimentaire, les goûts culinaires et musicaux. 
Luis Orozco a su faire preuve à mon égard d'un sens de l'hospitalité absolument remarquable dès mon arrivée aux Etats-Unis, et m'a permis de me sentir chez lui comme chez moi. Je le remercie donc vivement de m'avoir laissé les clés de son appartement, de sa voiture, de m'avoir fait découvrir les concerts de la bibliothèque du congrès et plein d'autres choses encore. Luis, je me suis vraiment senti chez toi comme en famille. Tu as transformé ma manière de voir les choses, et je tâcherai de suivre cet exemple dans le futur. Au laboratoire, j'ai pu profiter d'une liberté totale dans un environnement exceptionnel. Tes conseils, tes intuitions et ton goût du travail bien fait m'ont marqué et continueront à m'influencer dans le futur.

Travailler dans deux endroits différents m'a aussi permis de bénéficier d'interactions avec nombre de physiciens de talent, dont les conseils et les discussions ont contribué de manière essentielle à l'avancement de mon travail de thèse. Un grand merci à Thierry Lahaye pour sa disponibilité, son habileté expérimentale et son sens de la formule. Ce fut un plaisir de partager un bureau avec toi pendant deux ans. Charles Adams nous a fait le plaisir de venir passer un mois en notre compagnie, l'occasion rêvée pour étudier les transitions microndes entre états de Rydberg. Merci également à Steve Rolston, Fred Wellstood, Chris Lobb, Trey Porto et Gretchen Campbell pour nos discussions hebdomadaires. Je remercie enfin Bill Philips qui n'a pas hésité, malgré le décalage horaire, à se lever à l'aube le jour de ma soutenance de thèse pour pouvoir participer à la traditionnelle séance de questions qu'il affectionne tant.

Je ne compte plus le nombre d'heures passées en compagnie de Henning Labuhn et Daniel Barredo ces deux dernières années. Par notre complémentarité, nous avons su former une équipe soudée et efficace. L'informatique n'a apparemment aucun secret pour Henning, et je reste très impressionné par sa capacité à appréhender tout nouvel outil avec une facilité et une rapidité déconcertantes. Je suis heureux de partager avec Daniel l'amour du détail et de la maniaquerie, et le remercie d'avoir systématiquement pris le temps de me raccompagner en voiture après les nombreuses soirées passées ensemble à prendre des données. Je tiens à vous exprimer toute mon amitié en souvenir des bons moments passés au laboratoire, mais aussi à l'extérieur du laboratoire. Florence Nogrette a permis l'avancement rapide du projet à un moment important de ma thèse et je l'en remercie. Merci également à Lucas Béguin et Aline Vernier d'avoir mis en place une expérience d'une stabilité sans faille, et d'avoir pris soin de me former à son utilisation à mon retour des Etats-Unis. Je tiens enfin à souligner le rôle de Chales Evellin qui, sur une ancienne version de l'expérience, 
à initié ma formation en faisant preuve d'une rigueur expérimentale exemplaire et inspirante. Je remercie enfin tous les membres du groupe d'optique quantique de l'Institut d'Optique avec lesquels j'ai eu plaisir à interagir pendant ma thèse, avec une mention particulière pour Yvan Sortais, Radu Chicireanu, Andreas Fuhrmaneck, Ronan Bourgain, Joseph Pellegrino et Stephan Jennewein.

$\mathrm{Au}$ Joint Quantum Institute, j'ai fait équipe avec Jon Hoffman, qui est probablement celui qui a le plus facilité mon intégration aux Etats-Unis. J'ai beaucoup apprécié travailler avec lui, et garde le souvenir de nos nombreux fous rires. Merci pour tout, Jon. Jon et moi avons aussi eu la chance de collaborer avec Fredrik Fatemi et Guy Beadie, sans qui nous ne nous serions jamais lancés dans l'étude de la propagation de modes d'ordre supérieur dans nos fibres optiques nanométriques. Je ne saurais oublier Jeff Grover et Kristen Voigt, que je remercie infiniment pour leur amitié, ainsi que tous les membres de l'aventure Atoms and Squids, Pablo Solano, Zaeill Kim, Jongmin Lee, Jared Hertzberg, Peter Kordell, Austin Wood et David Wong-Campos.

J'ai eu la chance de pouvoir exercer une mission d'enseignement au sein de l'Institut d'Optique dont je remercie toute l'équipe pédagogique, administrative et technique. Merci en particulier à Lionel Jacubowiez, Fabienne Bernard, Thierry Avignon et Cédric Lejeune du service des travaux pratiques, ainsi qu'à Nathalie Westbrook et François Goudail avec qui j'ai eu plaisir à enseigner les travaux dirigés de polarisation.

Mon séjour américain n'aurait pu se réaliser sans l'aide financière des fondations Fulbright et Monahan. Les travaux de recherche présentés dans ce manuscrit ont également bénéficié du soutien financier de la NSF via le Physics Frontier Center du Joint Quantum Institute, du soutien financier de l'Union Européenne, via les projets ARENA, AQUTE, HAIRS et RYSQ ainsi que du soutien financier de la Région Ile-de France via les projets LUMAT et LAGON.

Je tiens enfin adresser quelques mots à ceux qui contribuent, en dehors du laboratoire, à mon bien être et mon épanouissement personnel, et en premier lieu mes parents ainsi que mes frères Séverin et Savinien. Je ne saurais oublier mes colocataires Emily, Lucy, Bethany, Florent et Guilhem, les amis et amies du lycée, de l'ENS Cachan ainsi que de l'orchestre des Planches à Musique, que je remercie pour 
tous ces moments agréables, passés, présents et futurs. 


\section{Contents}

$\begin{array}{ll}\text { Introduction } & 1\end{array}$

I Optical nanofiber fabrication and characterization for a hybrid quantum system

1 Optical trapping of atoms around nanofibers $\quad 9$

1.1 Experimental constraints imposed by the system . . . . . . . . . . . . 10

1.2 Trapping atoms around nanofibers . . . . . . . . . . . . . . . . 12

1.3 Outline of the first part . . . . . . . . . . . 16

2 Heat-and-pull apparatus $\quad 17$

2.1 Heat-and-pull apparatus . . . . . . . . . . . . . . . . . . 18

2.2 Pulling algorithm . . . . . . . . . . . . . . . . . . . . 19

2.3 Important pre-pull steps . . . . . . . . . . . . . . . . . . . . 21

2.4 Characterization of our tapered optical fibers . . . . . . . . . . . . . 22

3 Intermodal energy transfer in a tapered optical fiber 39

3.1 Fiber used . . . . . . . . . . . . . . . . . . . . 40

3.2 Modes in an optical fiber . . . . . . . . . . . . . . . . 40

3.3 Mode evolution in a tapered fiber . . . . . . . . . . . . . . . . . 42

3.4 Adiabatic tapers . . . . . . . . . . . . . . . . . . . 43

3.5 Intermodal energy transfer in a 2 mrad tapered fiber . . . . . . . . . 44

3.6 Quality of our fibers . . . . . . . . . . . . . . . 48

3.7 Nanofibers for a hybrid quantum system . . . . . . . . . . . . . 48

4 Propagation of higher-order modes in nanofibers $\quad 61$

4.1 Motivations . . . . . . . . . . . . . . . . . . 62

4.2 First family of excited modes . . . . . . . . . . . . . 63 
4.3 Transmission signals . . . . . . . . . . . . . . . . . 63

4.4 Reduced-cladding fibers . . . . . . . . . . . . . . . 65

4.5 Increasing adiabaticity . . . . . . . . . . . . . . . 66

\section{Interactions between two and three Rydberg atoms in arrays of optical tweezers}

5 Properties of Rydberg atoms $\quad 81$

5.1 Energy spectrum of alkali Rydberg atoms . . . . . . . . . . . . . . 82

5.2 Exaggerated properties of Rydberg atoms . . . . . . . . . . . . 84

5.3 Rydberg atoms in magnetic and electric fields . . . . . . . . . . . 85

5.4 Interactions between Rydberg atoms: a brief overview . . . . . . . . . 89

5.5 Rydberg blockade . . . . . . . . . . . . . . . . . . . . . . 91

5.6 Outline of the second part . . . . . . . . . . . . . . 93

$6 \quad$ Experimental apparatus $\quad 95$

6.1 Trapping and imaging single atoms . . . . . . . . . . . . . . . 96

6.2 Creating arrays of traps . . . . . . . . . . . . . . . . . 101

6.3 Coherent excitation to a Rydberg state . . . . . . . . . . . . . 105

6.4 Modeling our system . . . . . . . . . . . . . . . . . . . . . . . . . . 114

6.5 Control of electric fields . . . . . . . . . . . . . . . . . . . 118

6.6 Single-site addressability . . . . . . . . . . . . . . . . . . . . 121

6.7 Conclusion . . . . . . . . . . . . . . . . . . . . . 124

7 Van der Waals interactions between Rydberg atoms $\mathbf{1 2 5}$

7.1 Van der Waals interactions . . . . . . . . . . . . . . . . . . . . 126

7.2 Rydberg blockade in three-atom systems . . . . . . . . . . . . 135

7.3 Measurement of the van der Waals interaction . . . . . . . . . . . . . 141

7.4 Angular dependence of the van der Waals interaction . . . . . . . . . 144

7.5 Conclusion . . . . . . . . . . . . . . . . . . . . . 151

8 Resonant energy transfer between Rydberg atoms 153

8.1 Resonant interaction between two Rydberg atoms . . . . . . . . . . . 157

8.2 Choice of the Rydberg states . . . . . . . . . . . . . . . . . . 160

8.3 Coherent manipulation of Rydberg states . . . . . . . . . . . . . . . . 164

8.4 Coherent resonant energy transfer between two Rydberg atoms . . . . 167

8.5 Coherent resonant energy transfer in a chain of Rydberg atoms . . . . 179 
8.6 Full numerical simulation of the three-atom system . . . . . . . . . . 184

8.7 Conclusion . . . . . . . . . . . . . . . . . . . . . . . . . . 194

9 Coherent dipole-dipole coupling at a "Förster" resonance 197

9.1 Finding a Förster resonance between two Rydberg atoms . . . . . . . 200

9.2 Interaction between two atoms at a Förster resonance . . . . . . . . . 203

9.3 Spectroscopy of two interacting atoms at a Förster resonance . . . . . 207

9.4 Observation of Förster oscillations . . . . . . . . . . . . . . . . . . 217

9.5 Angular dependence of the interaction . . . . . . . . . . . . 225

9.6 Förster resonance in a magnetic field . . . . . . . . . . . . . . . . 233

9.7 Conclusion . . . . . . . . . . . . . . . . . . . . 236

$\begin{array}{ll}\text { Outlook } & 239\end{array}$

$\begin{array}{ll}\text { Bibliography } & 246\end{array}$ 


\section{Introduction}

Comprehending the nature of the transition from the microscopic quantum world to the classical macroscopic world is still a great challenge in physics. At a singleparticle level, quantum mechanics offers a successful description of the microscopic world [Dehmelt (1990); Haroche and Raimond (2006)]. At the macroscopic level, one can accurately describe phenomena using global variables while disregarding the underlying microscopic structure [Reif (1965); Landau and Lifshitz (1976)]. At the intermediate, so-called mesoscopic scale, a transition occurs where the situation is more complex, since the system cannot be considered macroscopic while the particle number starts to be large (typically a few tens to hundreds of particles), rendering the microscopic treatment hardly tractable in the most general case. The main difficulty resides in solving the equations of quantum mechanics for large numbers of interacting particles, due to the exponential growth with the particle number of the needs in computational resources. As an example, no classical computer can do $a b$ initio predictions for the dynamics of systems containing more than $\simeq 30$ two-level particles subject to long-range interactions [Aspuru-Guzik and Walther (2012)]. One envisioned solution is it to engineer a mesoscopic quantum system that we can control and manipulate in the laboratory, thus producing a quantum simulator [Cirac and Zoller (2012)]. This foreseeing idea of R. Feynman [Feynman (1982)] was the starting point of the field of quantum engineering, which is now also motivated by the perspectives offered by the quantum processing of information [Nielsen and Chuang (2000)] and by the usefulness of some quantum states for precision measurements (quantum metrology) [Wineland et al. (1992)], for instance.

Today, an exceptional level of mastery has been reached for the manipulation and control of two-level systems (called "qubits" in the language of quantum information) encoded on individual quantum objects, which led to the awarding of the 2012 Nobel Prize in physics to D. Wineland and S. Haroche [Wineland (2013); Haroche (2013)]. Currently, different types of qubits are being actively studied as shown in Figure 1. Some of them, such as atoms, ions, photons [Bloch, Dalibard, and Nascimbene (2012); 
a

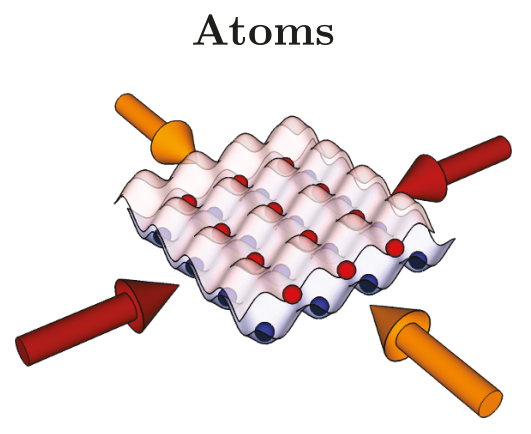

b
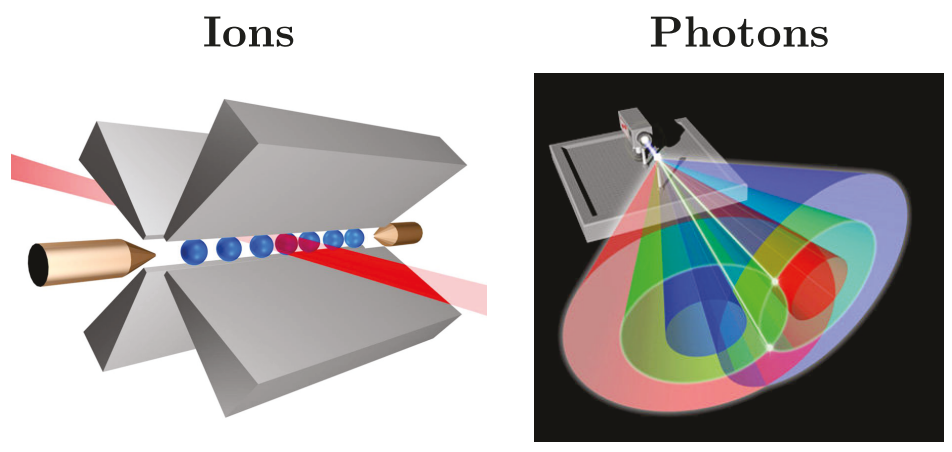

Nitrogen-vacancy centers

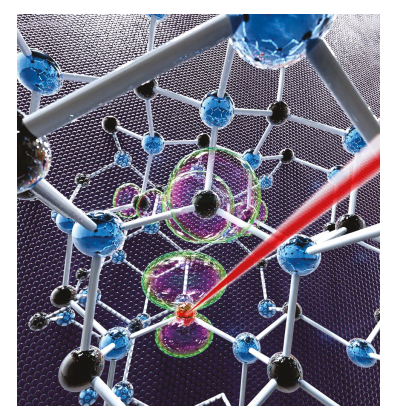

\section{Superconducting} qubits

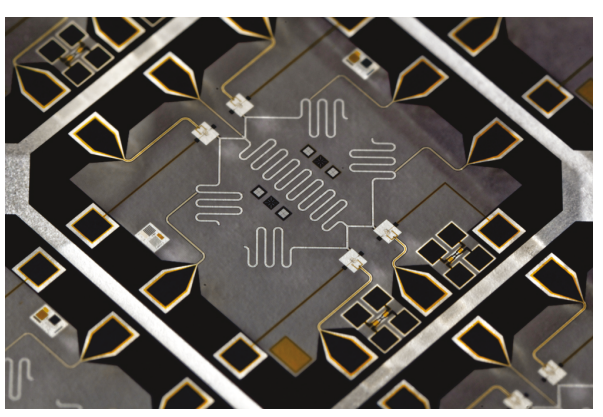

Figure 1: Examples of artificial quantum systems. a, In atomic and molecular physics, qubits can be encoded on neutral atoms (from the group of F. Gerbier at lkb, Paris), ions (from the group of P. Zoller at uibk, Innsbruck) or photons (from the ESA website) for instance. $\mathbf{b}$, In condensed matter physics, one can manufacture two-level systems such as NV-centers (from the group of C. van de Walle at UCSB, Santa Barbara), quantum dots [Reitzenstein and Forchel (2010)] or superconducting qubits (from the group of J. M. Martinis at UCSB, Santa Barbara). Due to their quantum properties, those objects are sometimes called "artificial atoms".

Blatt and Roos (2012); Aspuru-Guzik and Walther (2012)] are used in the field of atomic and molecular physics. Others are being manufactured, such as quantum dots, superconducting qubits and NV centers [Houck, Tureci, and Koch (2012)], which are essentially two-level systems ("artificial atoms") that show quantum properties. All those platforms show great promise for quantum simulation and quantum information applications [Georgescu, Ashhab, and Nori (2014)], and scientists are currently working on increasing the number of interacting qubits in their systems [Ladd et al. (2010)]. To date, with the successful creation of 14-qubit entangled states [Monz et al. (2011)], the trapped ions community is often considered as the most advanced towards this goal. That number begins to be interesting in testing quantum simulations that can barely be done with classical computers [Jurcevic et al. (2014); 
Richerme et al. (2014)]. However, every community is facing technical challenges in scaling up the number of qubits due to the limitations inherent to every system.

Fortunately, the different available systems are subject to different limitations, so that a complementary approach is possible. The wide variety of existing platforms leads to a broad range of fundamental properties and experimental implementations. Hybrid approaches [Xiang et al. (2013)] take advantage of the broad diversity of available systems, by combining their advantages while hopefully compensating for their weaknesses in order to create a robust and scalable quantum system [Sørensen et al. (2004); Andre et al. (2006); Rabl et al. (2006); Imamoğlu (2009); Marcos et al. (2010); Jessen et al. (2014)]. In atomic and molecular physics for example, photons or neutral atoms can be well isolated from their environment, thus showing low decoherence over long durations. However, their weak interactions render their manipulation and control slow and experimentally demanding. On the other hand, nanofabricated systems (superconducting qubits or quantum dots for instance) benefit from relatively easy and fast manipulation [Devoret and Martinis (2004)]. One possible architecture for a quantum simulator could combine an ensemble of nanofabricated qubits used as a fast quantum processor with an ensemble of atomic qubits serving as a good quantum memory.

This thesis describes my work on two different systems that are being developed towards these goals, one located at the Joint Quantum Institute (JQI) in Maryland, and the other located at the Institut d'Optique (IOGS) in Palaiseau.

- Figure 2a shows the current envisioning of the system under construction at JQI [Hoffman et al. (2011)], on which I worked from September 2011 to February 2013. The project aims at building a hybrid system of neutral atom spins coupled to superconducting qubits. Laser-cooled rubidium atoms will be trapped a few microns away from a superconducting circuit that resonates at the hyperfine frequency of the rubidium atoms, allowing magnetic coupling between the atoms and the superconductor. As this will be done in a dilution refrigerator environment, there are major technical constraints, in particular on the heating induced by light losses. The rubidium atoms will therefore be trapped and transported in the evanescent field of an ultrathin tapered optical fiber, a technique recently demonstrated in the group of A. Rauschenbeutel [Vetsch et al. (2010)].

- Figure $2 \mathrm{~b}$ shows the system at the Institut d'Optique, where I worked starting from February 2013. This project was specially designed for applications in 
a

b
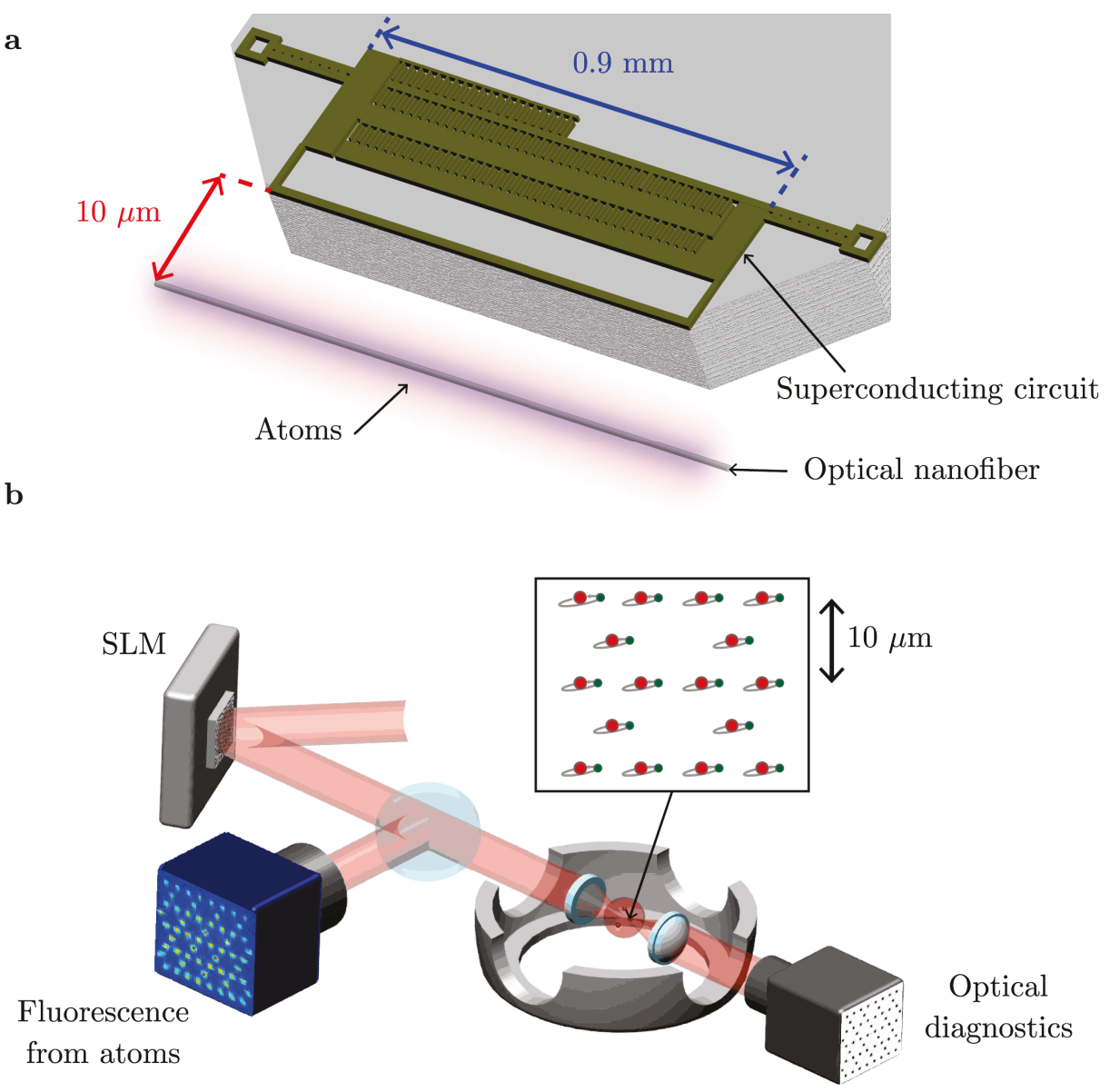

Figure 2: The two systems at JQI and IOGS. a, Current envisioning of the hybrid system in construction at JQI. Neutral rubidium atoms trapped around an ultrathin optical fiber are placed a few microns away from a superconducting qubit, allowing magnetic coupling between the two quantum objects. b. Single rubidium atoms are trapped in different sites of an array of dipole traps. The atoms are excited to Rydberg states, to allow large couplings between them.

quantum information and quantum simulation with Rydberg atoms [Saffman, Walker, and Mølmer (2010)]. Here, we use neutral atoms trapped in arrays of dipole traps, that we excite to highly interacting Rydberg states, so as to achieve strong interactions and thus fast operation times between neutral atoms. While this system is not, strictly speaking, a hybrid system, the approach is similar as one combines the large coherence times of neutral ground state atoms, with the large interactions between Rydberg atoms. This field has excited an increasing interest from the theoretical [Jaksch et al. (2000); Lukin et al. (2001)] and experimental [Comparat and Pillet (2010)] points of view.

The first part of this thesis describes the work I did at the JQI. When I joined the group, there was no expertise on fiber tapering. My work, jointly with J. E. Hoffman, 
consisted in the construction of a fiber puller apparatus, and the production and characterization of our tapered optical fibers. After an introductory Chapter presenting the necessary background on fibers (see Chapter 1), I will detail in Chapter 2 the construction of our fiber puller apparatus. The characterization of our nanofibers, showing transmissions as high as 99.95\%, is discussed in Chapter 3. Finally, we studied the propagation of higher-order modes in the fiber, where we obtain high levels of transmission, that offer new degrees of freedom and potentialities for atom trapping [Sagué, Baade, and Rauschenbeutel (2008)]. Those results are presented in Chapter 4 .

The second part of my thesis focuses on the work I did at the IOGS, jointly with H. Labuhn and D. Barredo. During my thesis, we performed experiments on small arrays of two and three interacting atoms allowing full characterization of the interatomic Rydberg interactions present in our system. This part starts by an introduction to the necessary background on Rydberg atoms (see Chapter 5). Following the work by L. Béguin [Béguin (2013)], we briefly recall the principles of our setup in Chapter 6, and we detail our recent additions to this setup (improvement of the laser stability, addition of a SLM to increase the number of atoms, implementation of addressability in our arrays). We then study the effect of anisotropic van der Waals interactions on the excitation dynamics of two-atom and three-atom systems (see Chapter 7). In Chapter 8, we use the resonant dipole-dipole interactions to simulate spin dynamics in a two-atom and three-atom chain. We finally demonstrate our ability to control resonant dipole-dipole interactions by electrically tuning a two-atom system to a Förster resonance (see Chapter 9).

The following articles have been published in the context of this thesis:

- Hoffman, J. E., Ravets, S., Grover, J. A., Solano, P., Kordell, P. R., WongCampos, J. D., Orozco, L. A., and Rolston, S. L., "Ultrahigh transmission optical nanofibers," AIP Advances 4, 067124 (2014).

- Ravets, S., Hoffman, J. E., Kordell, P. R., Wong-Campos, J. D., Rolston, S. L., and Orozco, L. A., "Intermodal energy transfer in a tapered optical fiber: optimizing transmission," J. Opt. Soc. Am. A 30, 2361 (2013).

- Ravets, S., Hoffman, J. E., Orozco, L. A., Rolston, S. L., Beadie, G., and Fatemi, F. K., "A low-loss photonic silica nanofiber for higher-order modes," Opt. Express 21, 18325 (2013).

- Nogrette, F., Labuhn, H., Ravets, S., Barredo, D., Béguin L., Vernier, A., Lahaye, T., and Browaeys, A., "Single-Atom Trapping in Holographic 2D Arrays 
of Microtraps with Arbitrary Geometries," Phys. Rev. X 4, 021034 (2014).

- Labuhn, H., Ravets. S., Barredo, D., Béguin L., Nogrette, F., Lahaye, T. and Browaeys, A., "Single-atom addressing in microtraps for quantum-state engineering using Rydberg atoms," Phys. Rev. A 90, 023415 (2014).

- Barredo, D., Ravets. S., Labuhn, H., Béguin L., Vernier, A., Nogrette, F., Lahaye, T., and Browaeys, A. "Demonstration of a Strong Rydberg Blockade in Three-Atom Systems with Anisotropic Interactions," Phys. Rev. Lett. 112, 183002 (2014).

- Barredo, D., Labuhn, H., Ravets. S., Lahaye, T., Browaeys, A. and Adams, C. S., "Coherent Excitation Transfer in a "Spin Chain" of Three Rydberg Atoms," arXiv:1408.1055 (2014).

- Ravets. S., Labuhn, H., Barredo, D., Béguin, L., Lahaye, T., and Browaeys, A. "Coherent dipole-dipole coupling between two single Rydberg atoms at an electrically-tuned Förster resonance," Nat. Phys. 10, 914 (2014). 


\section{Part I}

Optical nanofiber fabrication and

characterization for a hybrid quantum system 



\section{Chapter 1}

\section{Optical trapping of atoms around nanofibers}

\section{Contents}

1.1 Experimental constraints imposed by the system ... 10

1.2 Trapping atoms around nanofibers .......... 12

1.2 .1 Optical nanofibers . . . . . . . . . . . . . . . . . . . . . . . . 13

1.2 .2 Intensity profile . . . . . . . . . . . . . . . . . . . . . . . . . . . . 13

1.2 .3 Trapping potential . . . . . . . . . . . . . 15

1.2.4 Nanofiber trapping for a quantum hybrid system . . . . . 15

1.3 Outline of the first part ............ 16

The first part of this thesis concentrates on the experiments I performed at the university of Maryland in the context of the development of a particular quantum hybrid system [Hoffman et al. (2011)]. Hybrid systems have attracted a lot of interest over the last few years, and several approaches for achieving this goal of combining two different types of quantum systems are under study [Wallquist et al. (2009)]. One promising avenue is to interface a quantum memory from atomic, molecular and optical physics with a fast quantum processor from solid-state physics [Tian et al. (2004); Sørensen et al. (2004); Rabl et al. (2006)].

Along these lines, our approach focuses on interfacing a few individual neutral atoms with superconducting qubits by means of a direct magnetic coupling between the two systems [Sørensen et al. (2004); Verdú et al. (2009); Hoffman et al. (2011); Bernon et al. (2013)]. Trapping a few individual atoms a few micrometers away from 


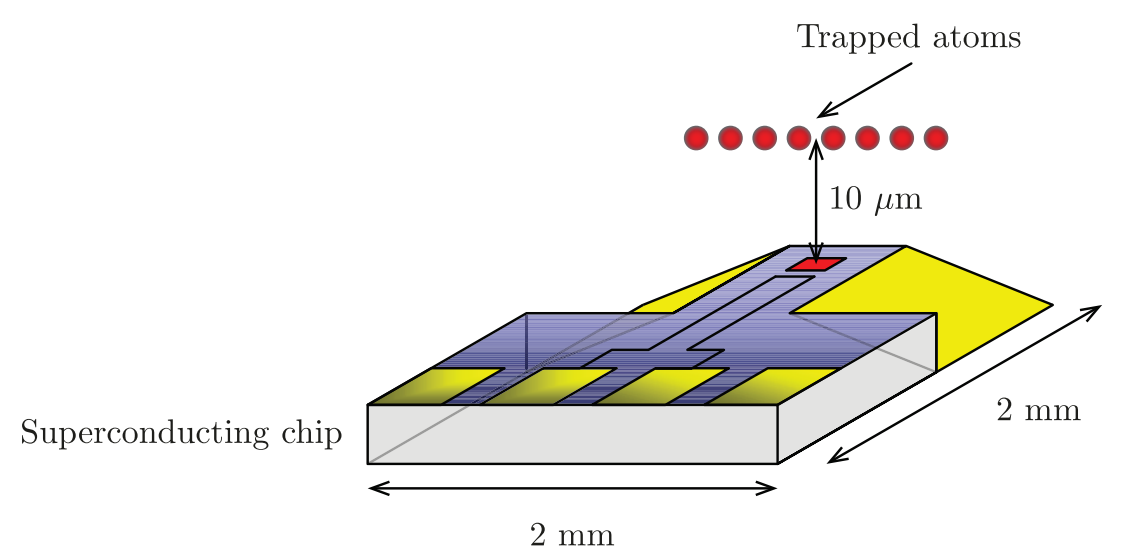

Figure 1.1: Original envisioning of the quantum hybrid system (adapted from [Hoffman et al. (2011)]). A few individual atoms are placed a few micrometers away from a superconducting qubit. The setup requires a trapping technique that allow a tight confinement of the atoms and does not illuminate the superconductor.

the superconducting surface, thus requires a good control over the atomic positions with respect to the superconducting qubit (see the original envisioning of the hybrid system in Figure 1.1). This can be accomplished using tight optical dipole traps of volume $\sim 1 \mu \mathrm{m}^{3}$ with high oscillation frequencies $\sim 100 \mathrm{kHz}$ [Grimm, Weidemüller, and Ovchinnikov (2000)], a feature hardly achievable with magnetic traps. Moreover, because we want to use the magnetic coupling between the atoms and the superconducting qubit (which is sensitive to magnetic fields due to its superconductivity), we prefer to avoid the presence of any extra sources of magnetic fields near the superconducting surface. As a consequence, we have chosen to optically trap the atoms above the superconducting surface, using the optical dipole force created by shining far-detuned lasers on the atoms. Optical dipole forces have been used in diverse experimental systems to trap and manipulate particles [Grimm, Weidemüller, and Ovchinnikov (2000)]. However for our purpose, the presence of a $10 \mathrm{mK}$-temperature surface close to the atoms imposes stringent experimental constraints.

\subsection{Experimental constraints imposed by the system}

The typical temperature needed to operate a superconducting qubit is $\sim 10 \mathrm{mK}$. In practice, we use a Triton 200 Cryofree dilution refrigerator from Oxford Instruments at $12 \mathrm{mK}$. This raises the challenge of trapping the atoms a few microns away from the superconducting surface placed inside the dilution refrigerator, in a mK-temperature 
a

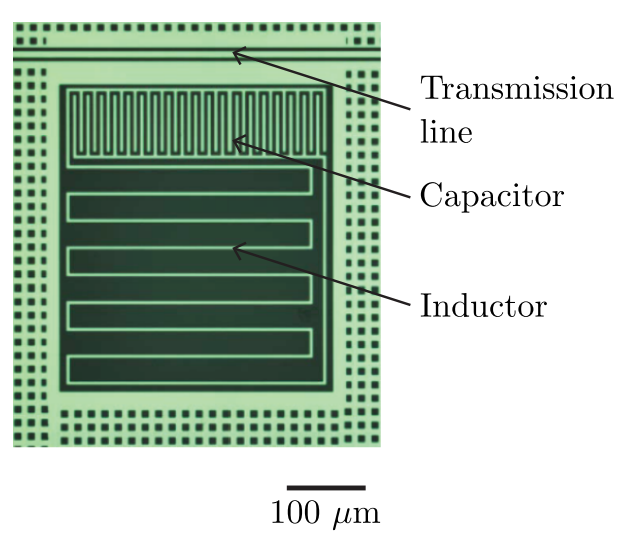

b

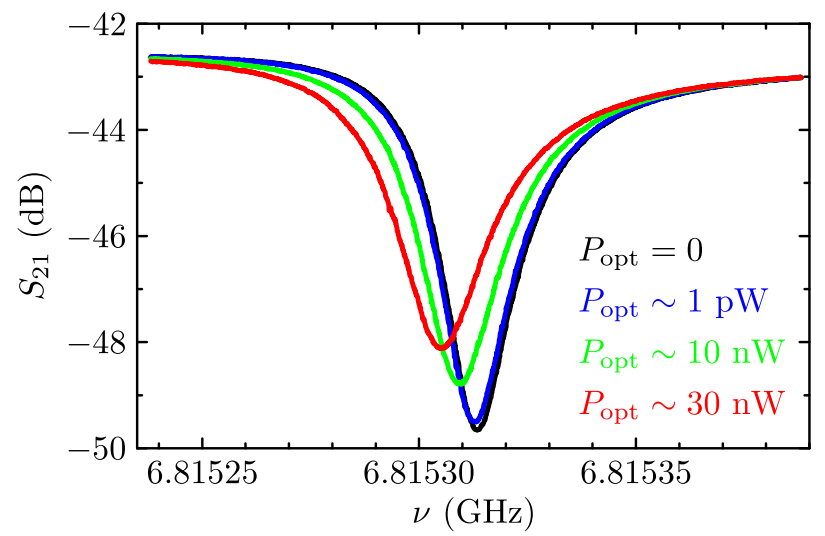

Figure 1.2: Testing the sensitivity of a superconducting resonator to light. a, Image of the microwave resonator used in this experiment. The niobium resonator is made of an inductor and a capacitor that are coupled to a microwave transmission line [Kim et al. (2011)]. Dark regions show the sapphire substrate. b, Response of the circuit to light. When shining light of wavelength $780 \mathrm{~nm}$ on the resonator, its resonance frequency, as well as its quality factor, are significantly modified for laser powers as low as a few $\mathrm{nW}$.

environment [Jessen et al. (2014)]. The two main constraints are:

- The cooling power in the final stage of the dilution refrigerator (where we want to trap the atoms) is $\sim 200 \mu \mathrm{W}$. However, laser cooling and laser trapping of atoms typically require tens to hundreds of milliwatts of laser power. Any photon hitting the cold surfaces due to clipping or scattering will generate heat in the dilution fridge, on top of the intrinsic damage to the superconductivity. As a consequence, one has to ensure that the heat generated by those photons is small enough to still be able to operate the cryostat at $12 \mathrm{mK}$.

- Superconducting circuits are extremely sensitive to light [Barends et al. (2011); Córcoles et al. (2011)]. As a matter of fact, superconducting nanowires are now used as single-photon detectors [Eisaman et al. (2011)], where the absorption of a single-photon causes a small region of the nanowire to go normal, allowing its detection. On our project, K. Voigt (a graduate student in the group) and J. Hertzberg (a postdoctoral researcher in the group), illustrated the extreme sensitivity of a superconducting resonator (see Figure 1.2a) by shining light at a wavelength $780 \mathrm{~nm}$ directly into it with a beam that covers its entire surface. The black curve in Figure $1.2 \mathrm{~b}$ plots the transmission of a microwave field through the superconducting LC circuit as a function of the microwave frequency, showing the resonance of the superconducting circuit. When increasing the laser power, the resonance frequency shifts and the quality factor of the res- 
onator decreases. They measured that a laser power as low as a few $\mathrm{nW}$ is enough to significantly change the resonance frequency (by a few tens of $\mathrm{kHz}$ ) and the quality factor of the resonator.

As a consequence, we need to use an atom trapping technique that minimizes the diffusion and losses of photons. For that purpose, the JQI group decided to implement a technique demonstrated in 2010 in the group of A. Rauschenbeutel [Vetsch et al. (2010)], that uses the evanescent field guided around an optical nanofiber to trap the atoms [Balykin et al. (2004); Le Kien, Balykin, and Hakuta (2004); Vetsch et al. (2010); Goban et al. (2012)]. This technique allows trapping the atoms with precise positioning, while maintaining the light strongly confined, minimizing the scattering.

\subsection{Trapping atoms around nanofibers}

The trapping technique is based on the optical dipole force created by shining fardetuned light on the atoms [Grimm, Weidemüller, and Ovchinnikov (2000)]. The dipole potential generated by the light for an ideal two-level atom is given, in the rotating wave approximation, by:

$$
U(\boldsymbol{r})=\frac{3 \pi c^{2}}{2 \omega_{0}^{3}} \frac{\Gamma}{\Delta} I(\boldsymbol{r})
$$

where $\Delta$ is the frequency detuning from the atomic transition of frequency $\omega_{0}, \Gamma$ is the spontaneous decay rate of the excited state and $I(\boldsymbol{r})$ is the light intensity. For red-detuned light $(\Delta<0)$, the potential minimum is found at the beam intensity maximum. On the other hand, blue-detuned light $(\Delta>0)$ repels the atoms toward the light intensity minima. These properties are widely used to create optical dipole traps for atoms. It has been shown that strongly focused lasers allow trapping individual atoms [Schlosser et al. (2001); Sortais et al. (2007)]. Strong focusing leads to high intensities, limiting the laser power needed to trap individual atoms. As a consequence, trapping atoms in microscopic traps, that have a size comparable to the wavelength of the light used for trapping, allows producing traps with a few millikelvin depth using laser powers as low as a few tens of milliwatts (see Section 1.2.3). This is a major advantage in view of trapping atoms in the $10 \mathrm{mK}$ stage of a dilution fridge. 


\subsubsection{Optical nanofibers}

Optical nanofibers are optical fibers having a radius that is smaller than the wavelength of the propagating light. Like standard optical fibers, nanofibers are waveguides where the light is guided by total internal refraction at the interface between two media of different indices. Because the diameter of a nanofiber is smaller than the wavelength, part of the light propagates outside the waveguide, in the form of an evanescent wave. Nanofibers offer an interesting platform to interface atoms with light, allowing trapping of atoms in the vicinity of the fiber surface. The standard trapping scheme uses a two-color trap [Le Kien, Balykin, and Hakuta (2004); Vetsch et al. (2010)]. For ${ }^{87} \mathrm{Rb}$ for example, we combine a $730 \mathrm{~nm}$ beam (blue detuned from the $\mathrm{D}$ lines of ${ }^{87} \mathrm{Rb}$ ) with a $1064 \mathrm{~nm}$ beam (red-detuned from the $\mathrm{D}$ lines of ${ }^{87} \mathrm{Rb}$ ) to create a trapping potential for the atoms.

\subsubsection{Intensity profile}

The calculated intensity profile inside and outside a nanofiber with a $180 \mathrm{~nm}$ radius is shown in Figure 1.3a, where the surface of the fiber is represented by the black circle. The trapping fields are linearly polarized, as represented by the red and blue arrows in Figure 1.3a. Outside the fiber, we observe the evanescent field formed by the trapping beams. Using a linear polarization for the trapping beam breaks the cylindrical symmetry in the system, and the evanescent field shows two maxima at the positions where the transverse part of the electric field is orthogonal to the fiber surface. The evanescent field is maximum at the fiber surface, and then decays exponentially for larger distances. One can show [Kien et al. (2004)] that the characteristic decay length for the intensity outside the fiber is:

$$
\Lambda=\frac{1}{\sqrt{\beta^{2}-k_{0}^{2}}}
$$

Here, $k_{0}$ is the free-space wavevector and $\beta$ is the propagation constant ${ }^{1}$ of the field along the fiber axis $z$ :

$$
\left[\begin{array}{l}
\boldsymbol{E}(\boldsymbol{r}, t) \\
\boldsymbol{H}(\boldsymbol{r}, t)
\end{array}\right]=\left[\begin{array}{c}
\boldsymbol{E}(r, \phi) \\
\boldsymbol{H}(r, \phi)
\end{array}\right] \exp [i(\beta z-\omega t)],
$$

where $\boldsymbol{E}$ and $\boldsymbol{H}$ denote the electric and magnetic fields for a cylindrically symmetric waveguide. We observe that $1064 \mathrm{~nm}$ and $730 \mathrm{~nm}$ beams have different decay lengths,

\footnotetext{
${ }^{1}$ In general, the propagation constant can be written $\beta=n_{\text {eff }} k_{0}$, where $n_{\text {eff }}$ is the effective refractive index seen by the light as it is guided by the fiber.
} 

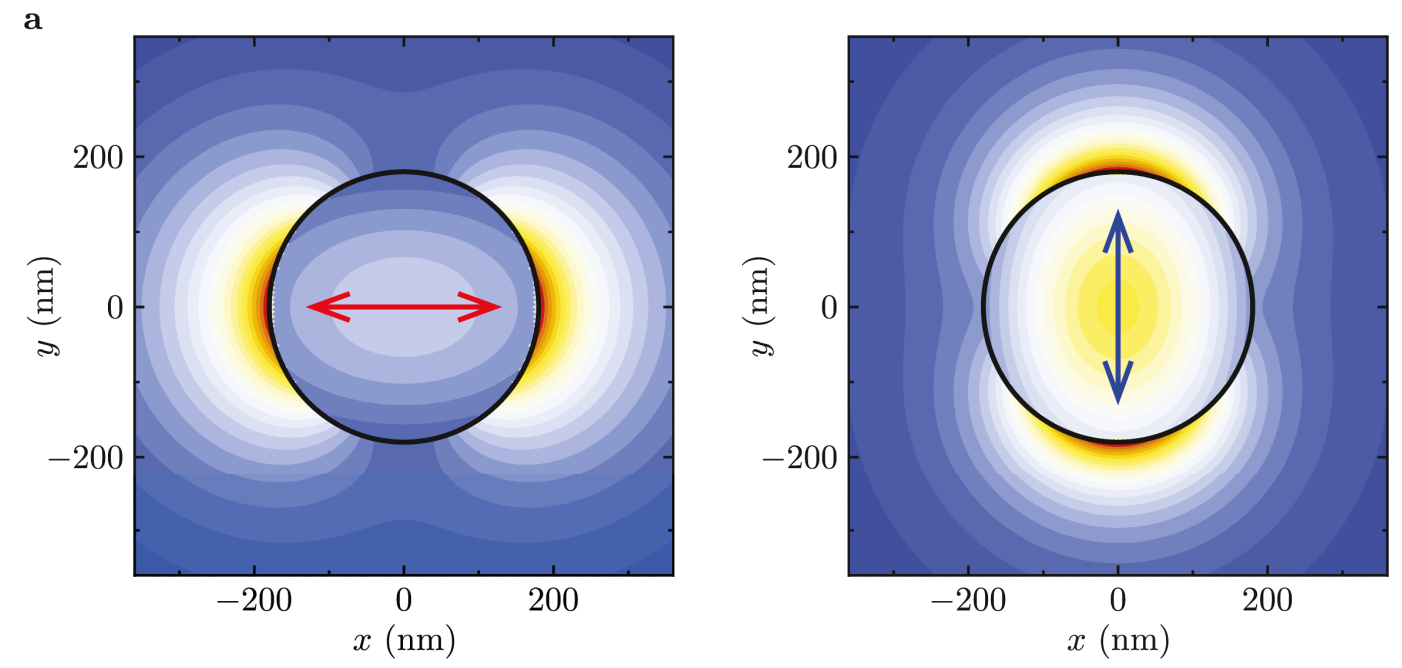

b

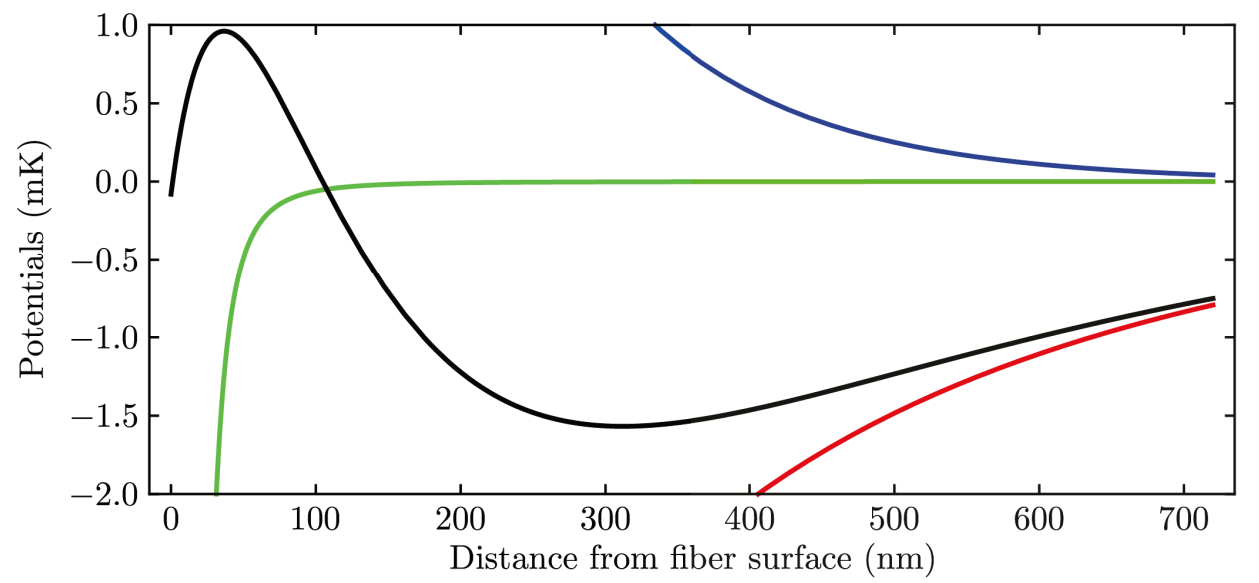

c

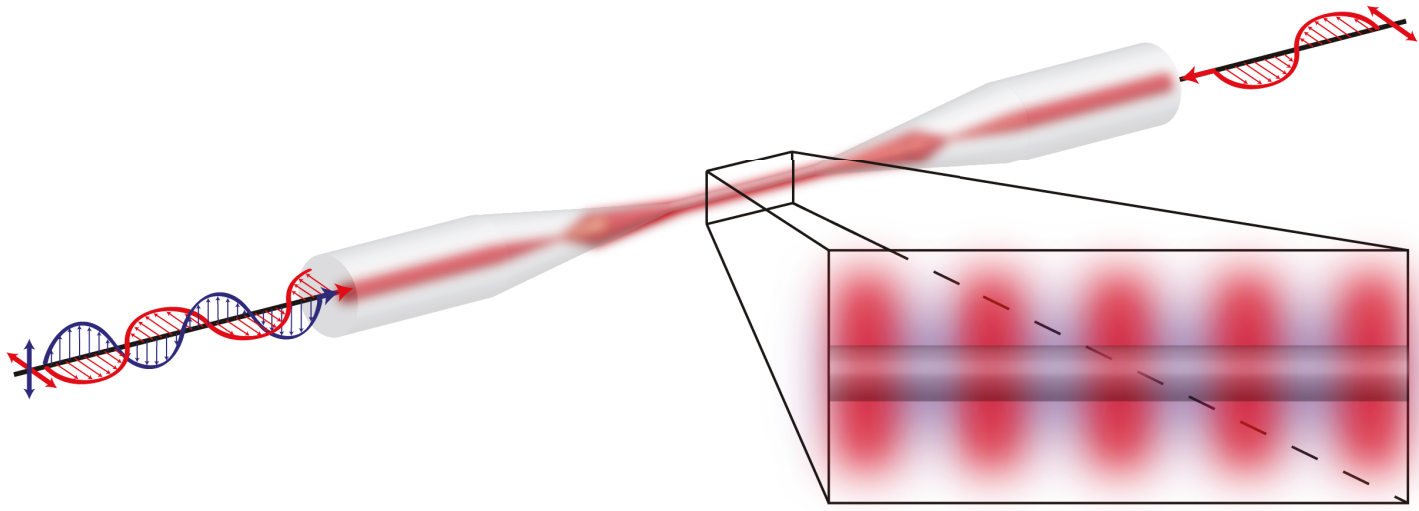

Figure 1.3: Trapping atoms around nanofibers (adapted from [Hoffman (2014)]). a, Calculated intensity profile around a $360 \mathrm{~nm}$ nanofiber for horizontally polarized light of wavelength $1064 \mathrm{~nm}$ (left), and vertically polarized light of wavelength $730 \mathrm{~nm}$ (right). b, Trapping potentials obtained for $7.5 \mathrm{~mW}$ of blue-detuned light and $4.5 \mathrm{~mW}$ of red-detuned light. The red curve shows the attractive potential created by the red-detuned light. The blue curve is the repulsive potential created by the blue-detuned light. The green curve represents the attractive van der Waals forces created by the dielectric surface of the fiber on an atom. The resulting trapping potential is shown in black. c, Two-color trapping scheme using a nanofiber. The inset shows the red-detuned and blue-detuned light around the nanofiber. 
which will allow creating a potential minimum for the atoms, as we now explain.

\subsubsection{Trapping potential}

By summing the attractive potential created by the red-detuned beam, the repulsive potential created by the blue-detuned beam and the attractive van der Waals potential created by the nanofiber itself ${ }^{2}$, we obtain the trapping potential shown in Figure 1.3b (black curve). In total, we observe that using $12 \mathrm{~mW}$ of trapping power, we create a trap with a depth of a few $\mathrm{mK}$ and located a few hundreds of nanometers away from the fiber surface.

Figure. 1.3c shows the experimental setup for trapping atoms around nanofibers used by E. Vetsch et al. in [Vetsch et al. (2010)]. The linearly polarized reddetuned and blue-detuned beams are launched into the nanofiber, thus creating an attractive and a repulsive potential for the atoms. The group of A. Rauschenbeutel has shown that the use of orthogonal linear polarizations provides two positions on either side of the fiber where the red-detuned light intensity and the blue-detuned light are simultaneously maximum and minimum respectively. This allows confining azimuthally the atoms around the fiber. Finally, by retro-reflecting the red-detuned laser beam inside the fiber, one creates a red-detuned standing wave around the nanofiber. This allows confining the atoms along the fiber axis in two different 1D lattices located on either side of the fiber (see inset in Figure 1.3c).

\subsubsection{Nanofiber trapping for a quantum hybrid system}

Adapting this trapping technique to our system presents four main advantages:

- The trapping light is tightly confined around the nanofiber, in the form of an evanescent wave. This tight confinement leads to large intensities so that a moderate laser power $(\sim 10 \mathrm{~mW})$ is necessary to trap the atoms.

- The nanofiber can also be used to send resonant probe light on the atoms (light of wavelength $780 \mathrm{~nm}$ in the case of rubidium), with no need for an extra optical path in the cryostat.

- The nanofiber acts as a waveguide which allows the photons to remain tightly confined over the full length of the nanofiber, that can reach a few centimeters

\footnotetext{
${ }^{2}$ For the van der Waals potential, we consider the potential created by an infinite dielectric on a ground state atom [Courtois, Courty, and Mertz (1996); Landragin et al. (1996)].
} 
(see Chapter 2). This contrasts with Gaussian beams that diverge as they propagate in free space.

- Nanofibers can show extremely low losses, as we will demonstrate in Chapter 3. This leads to low light scattering, allowing for a low heat load in the cryostat and very little light shining onto the superconducting circuit.

All those advantages make nanofiber traps an ideal platform for trapping atoms in a dilution fridge, a few micrometers away from the superconducting surface.

\subsection{Outline of the first part}

The next three Chapters describe the fabrication and characterization of the nanofibers. Chapter 2 focuses on the construction of the pulling apparatus I built with J. E. Hoffman to manufacture our own nanofibers. Particular attention is paid to the procedures to follow in order to obtain ultra-high transmission nanofibers. Chapter 3 shows our work to identify the measured residual sources of losses. A modal analysis of our transmission signals using spectrograms allows us to find experimental conditions where the residual losses are only attributed to slight non-adiabaticities in the chosen fiber profile, and not to other technical limitations of our apparatus. The obtained transmissions, in excess of $0.9995 \pm 0.0002$, agree well with calculations. In Chapter 4 , we measure the transmission of higher-order modes through our nanofibers. Using a special type of fibers (reduced-cladding fibers), we show that we can achieve more than $97 \%$ transmission of the first family of modes.

Note: The results I obtained during my stay at the Joint Quantum Institute are described at length in the three papers [Hoffman et al. (2014); Ravets et al. (2013a,b)]. As a consequence, in this part, each Chapter consists of a short introduction to the results detailed in the attached papers. 


\section{Chapter 2}

\section{Heat-and-pull apparatus for manufacturing ultrahigh transmission optical nanofibers}

\section{Contents}

2.1 Heat-and-pull apparatus . . . . . . . . . . 18

2.2 Pulling algorithm . . . . . . . . . . . . . 19

2.2.1 Fixed flame and constant pulling velocity . . . . . . . . 19

2.2 .2 Sweeping the flame . . . . . . . . . . . . . . 20

2.3 Important pre-pull steps . . . . . . . . . . . . . 21

2.4 Characterization of our tapered optical fibers $\ldots \ldots 22$

Over the last twenty years, the light-guiding properties of optical nanofibers have excited a lot of interest in different fields of physics and engineering [Brambilla (2010); Morrissey et al. (2013)]. The tight confinement of light inside nanofibers as well as its guidance in the form of an evanescent wave have been used for various applications. For example, the high-intensities obtained in nanofibers have led to the observation of non-linear effects like supercontinuum generation [Leon-Saval et al. (2004)]. Nanofibers can also be interfaced with their surrounding environment by means of evanescent coupling, allowing their use as optical sensors [Chen et al. (2013); Morrissey et al. (2013)] and their integration to existing fiber network for applications in quantum information science [Kimble (2008)]. All those applications benefit from high transmission nanofibers, through a reduction of scattered light and laser power 


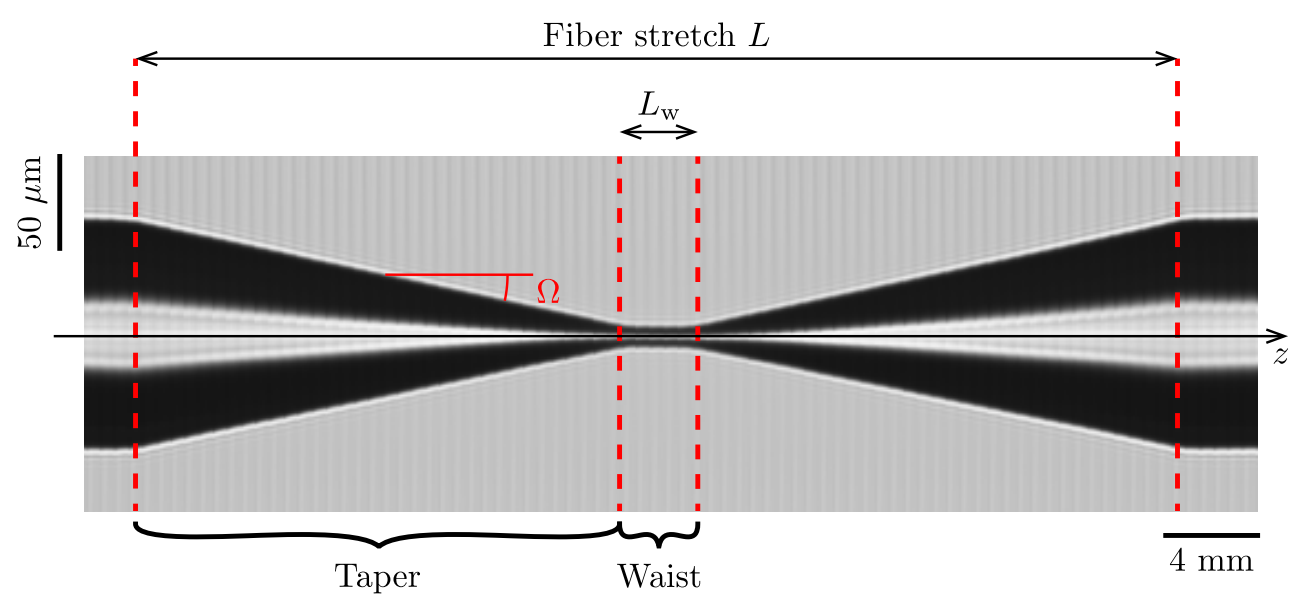

Figure 2.1: Image of a tapered fiber. The Figure consists of an horizontally compressed stack of 100 optical microscope images (see the difference between the horizontal and vertical scales). Note that the optical fiber is a glass cylinder that acts as a cylindrical lens. The observed dark and bright regions are due to this lensing effect. In order to be able to resolve the fiber over its entire length using an optical microscope, we have pulled it down to a waist of $5 \mu \mathrm{m}$, with a few mm length. The taper geometry is linear, with a tapering angle $\Omega=2 \mathrm{mrad}$.

requirements [Tong et al. (2003)]. To obtain high transmissions, some care has to be taken in the manufacturing process of the nanofibers. This is precisely what I focused on during my stay at the Joint Quantum Institute. In this Chapter, we concentrate on the construction of the pulling apparatus I built with J. E. Hoffman to manufacture our own nanofibers. We summarize the procedure detailed in [Hoffman et al. (2014)]. Particular attention is paid to the important cleaning and alignment steps to follow in order to obtain ultra-high transmission nanofibers.

\section{$2.1 \quad$ Heat-and-pull apparatus}

We produce optical nanofibers by heating a standard, $125 \mu \mathrm{m}$ diameter, optical fiber while pulling it on both extremities [Bilodeau et al. (1987); Birks and Li (1992); Bures and Ghosh (1999); Brambilla, Finazzi, and Richardson (2004)]. Following the work of the group of A. Rauschenbeutel [Warken (2007)], we use an oxyhydrogen flame at stoichiometric combination to bring a $\sim 0.75 \mathrm{~mm}$ fiber portion to a temperature that exceeds its softening point $(\simeq 1860 \mathrm{~K})$. Two high-precision computer-controlled motors pull on the fiber ends at a typical velocity of $0.1 \mathrm{~mm} / \mathrm{s}$. To produce a fiber of chosen geometry, we calculate the trajectories of the motors using an algorithm that relies on conservation of volume. Using this so-called "heat-and-pull" method, we produce tapered optical fibers that are composed of two conical tapers that connect 
the standard optical fiber to a uniform waist (see microscope image in Figure 2.1). The uniform waist can reach diameters of a few hundreds of nanometers, producing a nanofiber with a length that can vary between a few $\mathrm{mm}$ to $10 \mathrm{~cm}$. This method for manufacturing nanofibers has several advantages:

- We have full control over the shape of the nanofiber, and obtain nanofibers that are uniform over their entire length, which is not necessarily the case for nanofibers produced with other methods using fusion splicers or chemical etchants [Brambilla (2010)].

- The tapered sections connect the nanofiber to a standard optical fiber, which eases its manipulation since we benefit from all the technologies developed for optical fibers in telecommunication.

- The tapers act as mode couplers for the nanofiber, which transfer the light form the $125 \mu \mathrm{m}$ diameter fiber to the nanofiber and reciprocally. By careful design of adiabatic tapers, one can reach ultrahigh transmissions as we demonstrated experimentally (see transmission signal in Figure 3.3b).

\subsection{Pulling algorithm}

This Section summarizes the main ingredients of the pulling model and the pulling algorithm.

\subsubsection{Fixed flame and constant pulling velocity}

We first suppose that the flame is fixed and that we pull the fiber on both extremities at a constant velocity $v_{\mathrm{p}}$. At $t=0$, the fiber radius is $R$. The flame uniformly softens a cylindrical fiber portion, with a height equal to $L_{0}$. After a time $d t$, we assume that the softened portion of glass has been elongated and forms a new cylinder, of radius $R+d R$ and height $L_{0}+d z=L_{0}+v_{\mathrm{p}} d t$. Conservation of volume gives [Birks and Li (1992)]:

$$
\pi R^{2} L_{0}=\pi(R+d R)^{2}\left(L_{0}+d z\right)
$$

Solving the differential equation leads to:

$$
R(z)=R_{0} \exp \left(-\frac{z}{2 L_{0}}\right)
$$


For a fixed flame and a constant pulling velocity $v_{\mathrm{p}}$, we obtain a tapered fiber with exponential taper profiles and a waist of length $L_{0}$. However, for our application we need more control on the taper profile:

- We need control over the length of the waist (in the current envisioning of the setup, $L_{\mathrm{w}} \simeq 10 \mathrm{~cm}$ ). Reaching the desired size of the waist with a fixed flame would require to use a flame that is more than $10 \mathrm{~cm}$ long, leading to instabilities.

- The tapers have to be short enough in order to fit in the cryostat. To produce tapered fibers with a nanofiber waist, we need to reduce the fiber radius by a factor of $\simeq 250$, leading to exponential tapers that are too long. As a consequence, we need to have some control on the taper geometry in order to create shorter tapers.

\subsubsection{Sweeping the flame}

We control the taper geometry by moving the flame while pulling the fiber, as described by F. Warken in [Warken (2007)]. We divide the pull into different steps $n$, during which the flame moves at a velocity $v_{\mathrm{f}, n}$ over a distance $L_{n}$, while the fiber is being pulled at both extremities with velocities $\pm v_{\mathrm{p}, n} / 2$ (where $v_{\mathrm{p}, n} / 2<v_{\mathrm{f}, n}$ to ensure that the flame can sweep a portion of the fiber). At each step, the points of the fiber that are fully swept by the flame are evenly heated, which effectively varies the size of flame and allows us to get some control over the taper geometry [Birks and Li (1992)]. Assuming a linear profile of the velocities inside the flame, the velocity $v(z)$ of a point of the fiber of coordinate $z$, which is located inside the flame at time $t$ reads:

$$
v(z)=\frac{d z}{d t}=\frac{v_{\mathrm{p}, n}}{L_{0}}\left(z-\frac{L_{0}}{2}-v_{\mathrm{f}, n} t\right),
$$

where $v_{\mathrm{p}, n}$ is the pulling velocity at step $n$. Solving this equation of motion, we obtain the time needed for the flame to fully sweep a point:

$$
t_{0, n}=\frac{L_{0}}{v_{\mathrm{p}, n}} \ln \left(\frac{2 v_{\mathrm{f}, n}+v_{\mathrm{p}, n}}{2 v_{\mathrm{f}, n}-v_{\mathrm{p}, n}}\right) .
$$

Equation 2.2 gives the relation between the waist radius at step $n, R_{\mathrm{w}, n}$, and the waist radius at the previous step, $R_{\mathrm{w}, n-1}$ after a heating time $t_{0, n}$ :

$$
R_{\mathrm{w}, n}=R_{\mathrm{w}, n-1} \exp \left(-\frac{t_{0, n} v_{\mathrm{f}, n}}{2 L_{0}}\right)
$$




\section{Pulling algorithm}

We calculate the pulling parameters $v_{\mathrm{f}, n}, v_{\mathrm{p}, n}$ and $L_{n}$ iteratively, starting from the target fiber waist radius $R_{\mathrm{w}}$ until reaching the initial radius $R_{0}$. In practice, we fix the flame velocity to $v_{\mathrm{f}, n} \equiv v_{\mathrm{f}}=2.1 \mathrm{~mm} / \mathrm{s}$ for the entirety of the pull. At step $n$, the waist radius $R_{\mathrm{w}, n+1}$ is imposed by the chosen geometry. Using Equation 2.4 and Equation 2.5, we obtain the pulling velocity $v_{\mathrm{p}, n}$. The remaining parameter we need to calculate is the sweeping distance of the flame $L_{n}$. Using conservation of volume, we relate the length of the waist at step $n-1, L_{\mathrm{w}, n-1}$, to the length of the waist at step $n, L_{\mathrm{w}, n}$ :

$$
R_{\mathrm{w}, n-1}^{2} L_{\mathrm{w}, n-1}=R_{\mathrm{w}, n}^{2} L_{\mathrm{w}, n}+R_{\mathrm{w}, n-1}^{2}\left(v_{\mathrm{f}}-\frac{v_{\mathrm{p}, n}}{2}\right) t_{0, n} .
$$

Finally, producing a waist of size $L_{\mathrm{w}, n}$ at step $n$ requires a sweeping distance $L_{n}$ that is given by:

$$
\left(L_{n}-v_{\mathrm{f}} t_{0, n}\right)\left(v_{\mathrm{f}}+\frac{v_{\mathrm{p}, n}}{2}\right)=v_{\mathrm{f}} L_{\mathrm{w}, n} .
$$

Once those parameters are calculated, we can pull tapered fibers with a chosen taper geometry and a chosen size of waist. Note that we do not move the flame in practice, but we encode its motion into the one of the motors.

\subsection{Important pre-pull steps}

The procedure to obtain ultrahigh transmission tapered nanofibers involves a series of cleaning and alignment steps:

- We pay particular attention to the pre-pull cleanliness of the fiber. Indeed, any remaining particulate from buffer removal, solvent evaporation or any dust on the surface of the fiber will burn during the pull and greatly diminish the transmission. We use our in situ optical microscope to image the fiber prior to the pull and ensure that there is not any visible particulate on the surface within the resolution of the optical microscope.

- Once the fiber is cleaned, we position it on the pulling motors. Using our in situ optical microscope, we verify that the fiber is properly tensioned, and we carefully align it with respect to the center of the flame. The alignment is precise to a few microns over the full length of the fiber $(\mathrm{a} f \mathrm{few} \mathrm{cm})$. 


\subsection{Characterization of our tapered optical fibers}

We characterize our nanofibers using different diagnostics:

- We first validate the accuracy of our simulation of the expected fiber profile using an in situ optical microscope. We image an optical microfiber over its entire length ${ }^{1}$, and compare the obtained profile to the calculated one. The root mean square of the relative difference between the two profiles is smaller than $2 \%$, showing the accuracy of our algorithm and pulling apparatus for large radii.

- We use a scanning electron microscope to measure the nanofiber profile below a micrometer and verify that our nanofibers truly achieve the desired diameter. For an expected diameter of $500 \mathrm{~nm}$, we measured a diameter of $536 \pm 12 \mathrm{~nm}$, where the error is systematic.

- We measure the transmission of light of wavelength $780 \mathrm{~nm}$ through the fiber during the pulling process (see transmission signal in Figure 3.3b). We obtain single-mode transmissions of $99.95 \pm 0.02 \%$, which represents a loss from tapering of $2.6 \times 10^{-5} \mathrm{~dB} / \mathrm{mm}$ when normalized to the entire stretch. The measured losses present an improvement of two orders of magnitude when compared to work reported elsewhere [Brambilla (2010)].

- Transferred to a high vacuum environment, our nanofibers of typical radius equal to $250 \mathrm{~nm}$, can withstand the application of $400 \mathrm{~mW}$ of laser power at a wavelength of $760 \mathrm{~nm}$. This level of power is well above the one needed for our experiments.

Applying our pulling procedure, we have shown that we can produce tapered optical nanofibers with a 0.9995 transmission. For a typical trapping power of $30 \mathrm{~mW}$, this represents a loss of $15 \mu \mathrm{W}$ inside the dilution refrigerator. The next Chapter shows our work to understand the origin of the residual sources of losses.

\footnotetext{
${ }^{1}$ Here, we pulled the fiber to a final radius of a few micrometers, so as to remain above the resolution of our optical microscope.
} 


\title{
Ultrahigh transmission optical nanofibers
}

\author{
J. E. Hoffman, ${ }^{1}$ S. Ravets, ${ }^{1,2}$ J. A. Grover, ${ }^{1}$ P. Solano, ${ }^{1}$ P. R. Kordell, ${ }^{1}$ \\ J. D. Wong-Campos, ${ }^{1}$ L. A. Orozco, ${ }^{1}$ and S. L. Rolston ${ }^{1, a}$ \\ ${ }^{1}$ Joint Quantum Institute, Department of Physics, University of Maryland, and \\ National Institute of Standards and Technology, College Park, MD 20742, U.S.A. \\ ${ }^{2}$ Laboratoire Charles Fabry, Institut d'Optique, CNRS Univ Paris-Sud, \\ Campus Polytechnique, RD 128, 91127 Palaiseau cedex, France
}

(Received 26 March 2014; accepted 13 May 2014; published online 17 June 2014)

\begin{abstract}
We present a procedure for reproducibly fabricating ultrahigh transmission optical nanofibers (530 nm diameter and $84 \mathrm{~mm}$ stretch) with single-mode transmissions of $99.95 \pm 0.02 \%$, which represents a loss from tapering of $2.6 \times 10^{-5} \mathrm{~dB} / \mathrm{mm}$ when normalized to the entire stretch. When controllably launching the next family of higher-order modes on a fiber with $195 \mathrm{~mm}$ stretch, we achieve a transmission of $97.8 \pm 2.8 \%$, which has a loss from tapering of $5.0 \times 10^{-4} \mathrm{~dB} / \mathrm{mm}$ when normalized to the entire stretch. Our pulling and transfer procedures allow us to fabricate optical nanofibers that transmit more than $400 \mathrm{~mW}$ in high vacuum conditions. These results, published as parameters in our previous work, present an improvement of two orders of magnitude less loss for the fundamental mode and an increase in transmission of more than $300 \%$ for higher-order modes, when following the protocols detailed in this paper. We extract from the transmission during the pull, the only reported spectrogram of a fundamental mode launch that does not include excitation to asymmetric modes; in stark contrast to a pull in which our cleaning protocol is not followed. These results depend critically on the pre-pull cleanliness and when properly following our pulling protocols are in excellent agreement with simulations. (c) 2014 Author(s). All article content, except where otherwise noted, is licensed under a Creative Commons Attribution 3.0 Unported License. [http://dx.doi.org/10.1063/1.4879799]
\end{abstract}

\section{INTRODUCTION}

Optical nanofibers have seen widespread use in science and engineering applications in the last thirty years. ${ }^{1,2}$ The tight confinement of the evanescent field around the optical nanofiber, ${ }^{3}$ unique light geometries provided by the fiber modes, ${ }^{4-6}$ low loss, and promise of improved atomlight interaction ${ }^{7-11}$ have led to increased interest in the physics community. Optical micro- or nanofibers are used for sensing and detection, ${ }^{12,13}$ and coupling light to resonators, ${ }^{12,14-18} \mathrm{NV}$ centers, ${ }^{19}$ or photonic crystals. ${ }^{20-22}$ Optical nanofiber fabricated systems can be connected to an existing fiber network to provide applications in quantum information science. ${ }^{23}$ The development of atom traps around optical nanofibers affords new avenues of research ${ }^{4,8,10}$ including loss-limited hybrid quantum systems. These applications can benefit from high transmission nanofibers through a reduction in unwanted stray light fields produced from non adiabatic mode excitation and reduced laser power requirements. Here we present the tools and procedures necessary to create ultrahigh transmission nanofibers.

Previous work has focused on the post-pull environmental controls in humidity and air purity; ${ }^{24}$ here we focus on the critical pre-pull steps necessary to achieve an ultrahigh transmission before handling the known environmental effects. Following the protocols and procedures we have produced fibers with $99.95 \%$ transmission when launching the fundamental mode. We have also launched

\footnotetext{
${ }^{\mathrm{a} C}$ Corresponding author: rolston@umd.edu
} 
higher-order modes, ${ }^{25,26}$ where we achieve transmissions of greater than $97 \%$ for the first family of excited modes. This level of transmission has required a thorough optimization of the pulling algorithm and of the cleaning procedure. Here, we explicitly explain our technique and our apparatus that produces fibers that we will use in a series of experiments in quantum hybrid systems. ${ }^{27,28}$

Our pulling technique, using a well-known methodology, ${ }_{1}^{1}$ requires two pulling motors and a stationary oxyhydrogen heat source. The flame brushing method allows us to reliably produce optical nanofibers with controllable taper geometries and a uniform waist. The waist can vary in length from 1 to $100 \mathrm{~mm}$, and we can achieve radii as small as hundreds of nanometers. ${ }^{29-33}$ Rather than sweep the flame back and forth over the fiber, we keep the flame stationary; this action prevents the creation of small air currents, which could lead to nonuniformities on the fiber waist and is equivalent to transforming to the rest frame of the flame. This transformation is applicable to other pulling techniques as well, so there would be no need to scan a heat source.

Other common techniques for optical nanofiber production include micro-furnaces, fusion splicers, chemical etchants, and $\mathrm{CO}_{2}$ lasers. ${ }^{34-39}$ Chemical etching generally produces lower transmission than other heat and pull methods and offers less control over the shape of the taper and the length of the waist. $\mathrm{A} \mathrm{CO}_{2}$ laser produces high-transmission optical nanofibers but the final diameter is limited by the power and focus of the laser. Here we present, to best of our knowledge, the highest recorded transmission optical nanofibers, with a loss of $2.6 \times 10^{-5} \mathrm{~dB} / \mathrm{mm}$ on the fundamental mode, ${ }^{1,40}$ with controllable taper geometries and long fiber waists which are suitable for the space and optical constraints of cryogenic environments. ${ }^{28}$

The paper is organized as follows: Section II discusses the experimental setup. In Sec. III we describe the necessary steps for a typical pull, detailing the cleaning and alignment process. Sec. IV examines the quality of the produced fibers by measuring their transmission and comparing our results to other reported measurements. In Sec. V we report the transmission of more than $400 \mathrm{~mW}$ through the nanofiber under high vacuum conditions. Sec. VI presents our conclusions. Appendices A and B detail procedures and controls.

\section{EXPERIMENTAL SETUP}

This sections describes the details of the experimental setup. Our work follows the originally Mainz and currently Vienna group. ${ }^{32}$ A more detailed discussion of the algorithm and experimental verification can be found in Appendix A.

\section{A. The fiber-pulling apparatus}

The fiber-puller apparatus (see. Fig. 1 and Table I) consists of a heat source that brings the glass to a temperature greater than its softening point $\left(1585^{\circ} \mathrm{C}\right.$ for fused silica $\left.{ }^{41}\right)$ and two motors that pull the fiber from both ends. We use two computer-controlled motors, Newport XML 210 (fiber motors), mounted to a precision-ground granite slab with dimensions $12^{\prime \prime} \times 48^{\prime \prime} \times 4^{\prime \prime}$, flat to $3.81 \mu \mathrm{m}$ on average. The granite slab serves two purposes: it is a damping weight and a flat surface. The weight of the granite slab, exceeding $100 \mathrm{~kg}$, damps the recoil from the fiber motors as they change direction at the end of every pull step. Without a flat surface the motors will not work to specification, leading to parasitic effects on the nanofiber: the pitch or yaw of the motor can vary the distance between the fiber and the flame, changing the effective size of the flame and pulling the fiber in various directions (negating any pre-pull alignment). The motors are mounted to the granite by L-bracket adapters designed to not deform the motors from the the granite surface. We then mount the granite to an optical breadboard on three points so that surface imperfections of the optical table do not distort the granite slab.

The fiber motors, have $210 \mathrm{~mm}$ of motion, with a minimum incremental motion of $0.01 \mu \mathrm{m}$ and an on-axis accuracy of $3 \pm 1.5 \mu \mathrm{m}$ ( 1 in Fig. 1). The resolution of the motors is much smaller than any other length scale in our system, making the motors suitable for pulling. The fiber motors are controlled with a Newport XPS controller, which allows us to implement trajectories with a constant acceleration, resulting in jerkless motion during each step of the pull. 
a)

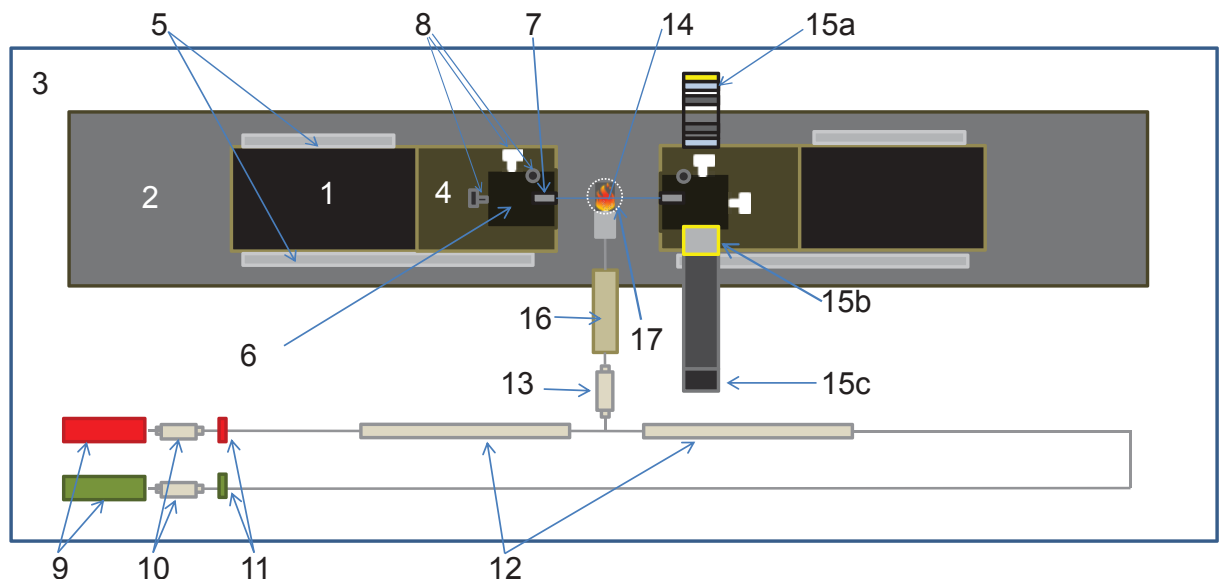

b)

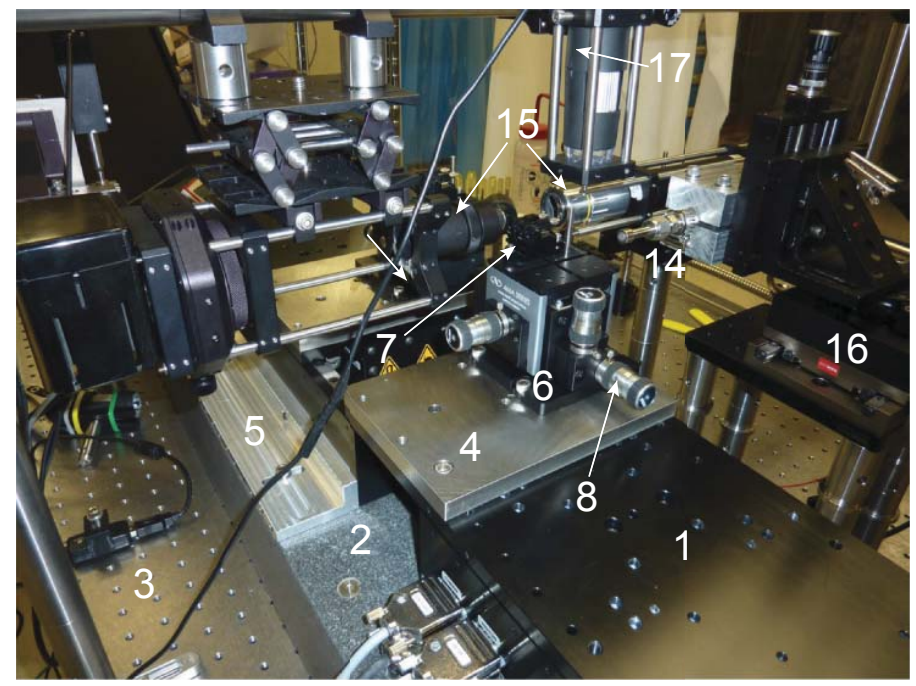

FIG. 1. a) A schematic of the fiber pulling setup (view from above). b) A photograph of the pulling apparatus with prominent pieces numbered as in Table I. 1) Fiber motors. 2) Granite slab. 3) Optical breadboard. 4) Adapter plates. 5) L brackets. 6) XYZ fiber alignment flexure stages. 7) Fiber holders. 8) Adjustment screws. 9) Flow meters. 10) Filters. 11) Valves. 12) Pipes. 13) Filter. 14) Nozzle. 15) (a) Illumination system, (b) Optical microscope, and (c) CCD. 16) Flame positioning stepper motor. 17) 2 MP USB microscope positioned orthogonally to the fiber. The entire apparatus is inside a cleanroom rated to ISO Class 100

Attached to each XML 210 (1 in Fig. 1) are Newport 466A flexure stages (6 in Fig. 1), each with a Newport 466A-710 fiber clamp on top (7 in Fig. 1). We position the v-grooves of the fiber clamp on each stage at the minimum separation allowed by the parameters of a given pull, which is typically $3 \mathrm{~cm}$. Separating the fiber clamps at the minimum distance minimizes the fiber sag during the pull. The $\mathrm{v}$-grooves of the fiber clamps on the left and right fiber motors must be aligned within micrometers to achieve a high transmission. We align the v-grooves using Newport DS-4F ( 8 in Fig. 1) and AJS100-0.5 (8 in Fig. 1) micrometers, attached to the flexure stages to allow for three axis translation. To perform the alignment we use an in situ optical microscope (15(a)-(c) in Fig. 1).

The optical microscope includes a Mitutoyo M Plan APO 10X infinity-corrected objective and a Point Grey Flea2G CCD camera (15(a)-(c) in Fig. 1). The Flea2G has $2448 \times 2048,3.45 \times 3.45 \mu \mathrm{m}^{2}$ pixels. With the inclusion of the long working distance microscope objective each pixel corresponds to $0.345 \times 0.345 \mu \mathrm{m}^{2}$ in the image. The microscope is illuminated by a Köhler illumination system composed of a thermal light source, two condenser lenses, and two apertures. 
TABLE I. List of equipment parts for the pulling apparatus with numbers corresponding to Fig. 1.

\begin{tabular}{|c|c|c|}
\hline Item & Part & Description \\
\hline 1 & Newport XML 210 & Computer-controlled high precision motor \\
\hline 2 & Granite slab & $12^{\prime \prime} \times 48^{\prime \prime} \times 4^{\prime \prime}$, Flat to $3.81 \mu \mathrm{m}$ \\
\hline 3 & Newport VH3660W & $3^{\prime} \times 5^{\prime}$ workstation \\
\hline 4 & Adapter Plate & Adapts metric XML 210 to $466 \mathrm{~A}$ \\
\hline 5 & L brackets & Adapts XML 210 to granite \\
\hline 6 & Newport 466A & Compact XYZ fiber alignment flexure stages \\
\hline 7 & Newport 466A-710 & Double arm bare fiber holder double V-grooves \\
\hline 8 & Newport DS-4F & High precision adjuster, $8.0 \mathrm{~mm}$ coarse, $0.3 \mathrm{~mm}$ fine travel \\
\hline 8 & Newport AJS100-0.5 & High precision small knob adjustment screw, $12.7 \mathrm{~mm}$ travel \\
\hline 9 & Omega FMA 5400/5500 & Flow meters \\
\hline 10 & Swagelok SS-4F-7 & Particulate filter, 7 micron pore size \\
\hline 11 & Swagelok SS-4P4T & Valve to close the flow of gas \\
\hline 12 & Swagelok SS-FM4SL4SL4-12 & Stainless steel flexible tubing \\
\hline 13 & GLFPF3000VMM4 & "Mini Gaskleen filter" from Pall, removal rating: $\geq 0.003 \mu \mathrm{m}$ \\
\hline 14 & Stainless Steel Custom flame nozzle & $29,228 \mu \mathrm{m}$ holes in a $1 \times 2 \mathrm{~mm}^{2}$ array \\
\hline 15 & Optical microscope & Microscope objective, CCD, and illumination system \\
\hline $15 \mathrm{a}$ & Illumintation System & Kohler illumination system \\
\hline $15 b$ & Mitutoyo M Plan APO 10X & Microscope objective, $0.28 \mathrm{NA}$, working distance $33.5 \mathrm{~mm}$ \\
\hline $15 \mathrm{c}$ & Flea2G CCD camera & $2448 \times 2048$ pixels, $3.45 \times 3.45 \mu \mathrm{m}^{2}$ pixels \\
\hline 16 & Thorlabs DRV014 & $50 \mathrm{~mm}$ Trapezoidal Stepper Motor Drive \\
\hline 17 & USB microscope & 200x, 2 MP USB microscope \\
\hline 18 & Platinum wire & Platinum catalyst to ignite flame \\
\hline 19 & Clean room & ISO class 100 cleanroom \\
\hline
\end{tabular}

We use an oxyhydrogen flame as a heat source to thin the fibers, in a stoichiometric mixture of hydrogen and oxygen to ensure that water vapor is the only byproduct. Stainless steel gas lines introduce the hydrogen (red) and oxygen (green) to two Omega FMA 5400/5500 flow meters (9 in Fig. 1). The flow rates are set to $30 \mathrm{~mL} / \mathrm{min}$ and $60 \mathrm{~mL} / \mathrm{min}$ for oxygen and hydrogen respectively.

Directly after the flow meters is a coarse particle filter (10 in Fig. 1), followed by a valve for safety (11 in Fig. 1). We mix the gases in a tee after a flexible stainless steel tube (12 in Fig. 1). The gas mixture is finely filtered with a high quality $3 \mathrm{~nm}$ filter (13 in Fig. 1). Finally the hydrogenoxygen mixture exits through a custom-made nozzle (14 in Fig. 1). The nozzle is composed of two parts and is constructed out of stainless steel. The first part is a $6.5 \mathrm{~mm}$ diameter plate with a $3.175 \mathrm{~mm}$ thickness. The plate has a $1 \times 2 \mathrm{~mm}^{2}$ array of $29,228 \mu \mathrm{m}$ holes. The long axis of the holes is perpendicular to the fiber axis. The second part of the nozzle is an adapter in which the first plate is pressure fit into the gas line. The outer diameter is $9.5 \mathrm{~mm}$ and is counter sunk $3.4 \mathrm{~mm}$ with an inner diameter of $6.35 \mathrm{~mm}$. This piece connects to $6.35 \mathrm{~mm}$ outer diameter tubing that then connects to stainless steel gas line with a Swagelok connector. The adapter was heated, allowing it to expand, so that the plate would slide into the countersunk inner diameter. The design diameter serves as a flame arrestor, while still allowing for the gas flow to be in the laminar regime.

We ignite the flame using a resistively-heated platinum wire as a catalyst. This process is clean and prevents the deposition of any particulate on the fiber from the ignition process. The nozzle is clamped to a Thorlabs DRV014 motor (16 in Fig. 1), the flame motor, that translates the flame in front of the fiber for the duration of the pulling process. The flame motor introduces and removes the heat source and during the pull we fix the horizontal distance between the nozzle and the front edge of the fiber at $0.5 \mathrm{~mm}$, as depicted in Fig. 1. We have found experimentally that a distance of 0.4-0.6 mm provides the proper heat distribution from our flame. Working outside this range for our flow rates significantly reduces the reproducibility of the fabrication process. If the flow rates were to change, the optimal working distance between the nozzle and fiber would need to be modified and it would be necessary to remeasure the effective width of the flame (see Sec. A 2).

The entire pulling apparatus is inside a cleanroom initially specified as ISO Class 100. If any fiber buffer remains or dust lands on the fiber at any time the transmission will degrade (see Appendix B). 


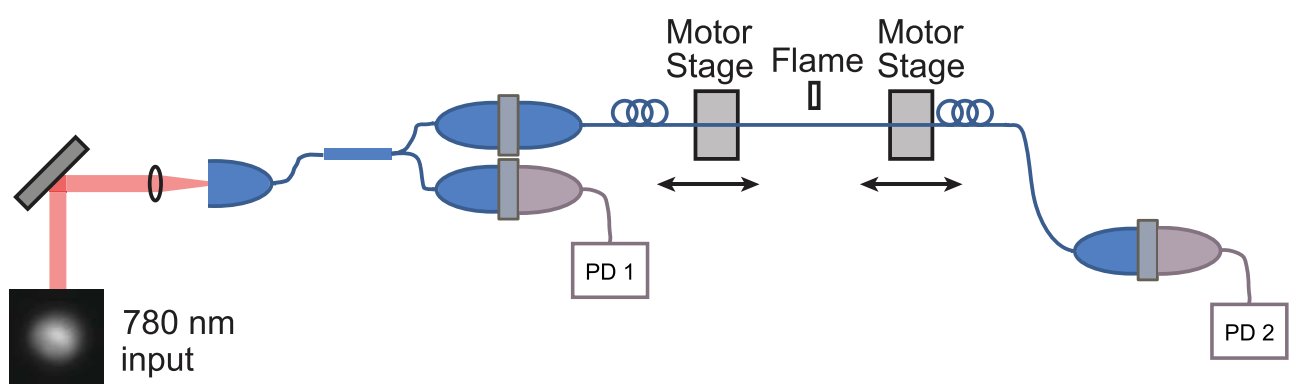

FIG. 2. The experimental setup to monitor the transmission when launching the fundamental mode.

\section{B. Transmission monitoring setup}

Figure 2 shows the transmission monitoring setup for the fundamental mode. Using a $780 \mathrm{~nm}$ Vortex laser, we launch light into a fiber and split the light with a 50/50 in-fiber beam splitter. In one output of the beam splitter we record the laser power using a Thorlabs DET36A photodetector. The other output is connected to a FC connectorized fiber that we fusion splice to Fibercore SM800 fiber. We then place the SM800 fiber in the fiber puller and record the intensity of light through the fiber at the output of the fiber puller using another DET36A for the duration of the pull. We record data on a DPO7054 Tektroniks oscilloscope in high resolution mode set to collect $10^{7}$ samples. We normalize the signal through the fiber puller to the laser drift throughout the pull.

When launching higher-order modes, a superposition of the $L P_{11}$ family of modes, we do not use the in-fiber beam splitter. Instead, we use a pick-off to track the laser drift and then free-space couple light into an SM1500 fiber from Fibercore with an initial cladding diameter of $50 \mu \mathrm{m}$.

We generate the $L P_{11}$ family superposition by launching a Gaussian beam from a New Focus Vortex laser through a phase plate. ${ }^{42,43}$ One side of the phase plate writes a $\pi$ phase shift on half of the beam. This generates a two-lobed mode that approximates the $T E M_{01}$ free space optical mode. The $T E M_{01}$ free space optical mode is then coupled into an SM1500 fiber and excites a superposition of the $T E_{01}, T M_{01}$, and $H E_{21}$ modes. There is less than one percent fundamental mode corruption, which we take into consideration when calculating the transmission.

\section{THE PULLING PROCESS}

The setup for an ultrahigh transmission pull involves a series of cleaning and alignment steps. We outline this procedure in this section.

\section{A. Cleaning procedure}

Obtaining a high transmission through an optical nanofiber requires a detailed analysis of the pre-pull cleanliness of the fiber. If any particulate remains from the fiber buffer or if dust arrives on the fiber before being introduced to the flame, the particulate will burn and greatly diminish the final transmission, see Appendix B. Furthermore, evaporate from solvents can decrease transmission.

Our cleaning procedure starts by mechanically removing the protective plastic buffer to expose the glass of the fiber to the flame. Then we use isopropyl alcohol on lens tissue to remove larger particulate. A few wipes of acetone ${ }^{44}$ are then applied with class $10 \mathrm{a}$ cleanroom wipes from Ted Pella, in order to dissolve smaller remnants of the buffer. A final cleaning with methanol using class 10a cleanroom wipes removes any evaporate left from the previous solvents. After, we carefully lay the fiber into the grooves of the fiber clamps on the pulling apparatus and image the entire length of cleaned fiber using the optical microscope. If there is any visible dust, particulate, or evaporate, within the $2 \mu \mathrm{m}$ resolution of the optical microscope, we start the cleaning procedure over. If the fiber is clean, we proceed to align it. 

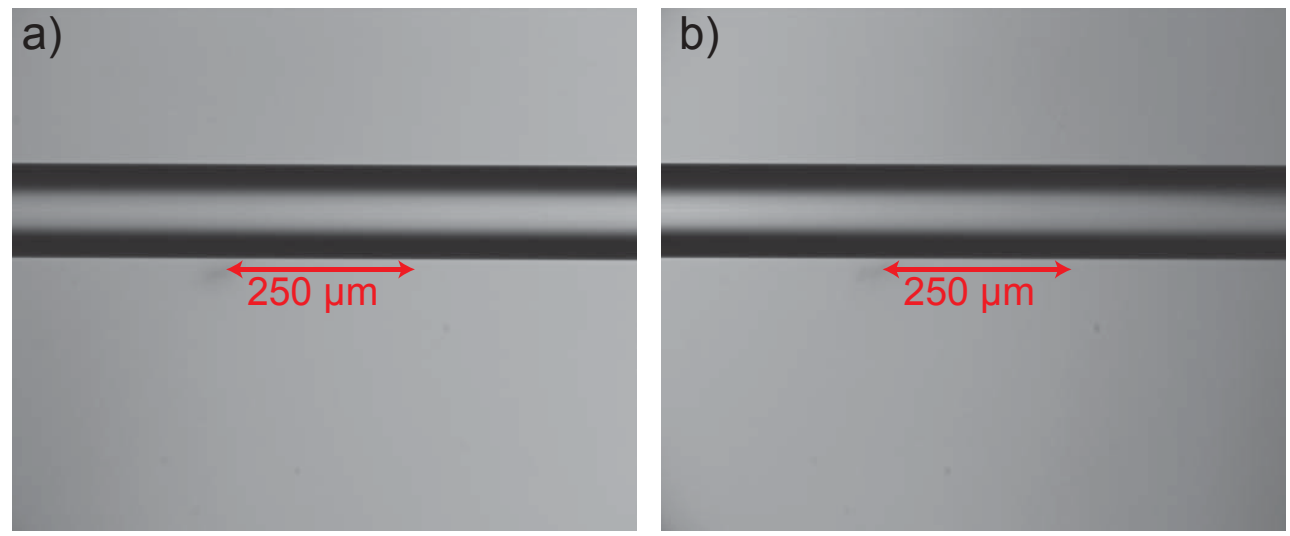

FIG. 3. Images of the section of fiber held directly next to the left (a) and right (b) fiber clamps respectively. The two images are separated by $3 \mathrm{~cm}$.

\section{B. Alignment procedure}

The alignment procedure begins by properly tensioning the fiber. We tension the fiber by moving the fiber motors apart in $200 \mu \mathrm{m}$ increments until the fiber slides through the fiber clamps, which typically takes $800 \mu \mathrm{m}$ of total displacement. This allows the fiber to reach a uniform tension. However, early measurements showed the fiber to be overtensioned: introducing the fiber to the flame will yield immediate thinning, even if the motors are stationary. As a result, we then untension the fiber in $20 \mu \mathrm{m}$ increments until the fiber buckles. We observe the buckling process (the fiber bending inwards and then straightening under the inward force from the motors) with a 2 MP USB digital microscope mounted orthogonally to the flame above the center of the fiber, see 17 in Fig. 1.

The buckling process results in no loss in transmission and no thinning visible through the optical microscope upon introducing the fiber to the flame within the microscope's resolution of \pm 2 $\mu \mathrm{m}$.

Once the fiber is properly tensioned, we align the fiber such that the sections of fiber directly next to the left fiber clamp and right fiber clamp are equidistant from the optical microscope and at the same height. We translate each section of fiber in front of the optical microscope using the fiber motors, see Fig. 1, and align the height and focus of each fiber section using the micrometers attached to the flexure stage until both images overlap. If we see a sag in the fiber caused by the buckling we carefully retention the fiber in $5 \mu \mathrm{m}$ steps until the fiber is straight as in Fig. 3 . The microscope objective has a $3 \mu \mathrm{m}$ depth of field, so by matching the diameter of the lensed light from the cladding and core we ensure the v-grooves of the fiber clamps on each motor are equidistant from the camera, and therefore the nozzle of the flame. This alignment is on the order of micrometers over a length of centimeters. Once the images overlap, see Fig. 3, the fiber is ready to be pulled.

\section{RESULTS}

Here we discuss the results obtained from following the procedures outlined in the previous sections. We present details on the transmissions achieved by following cleaning and alignment procedures. Finally, we detail methods to aid in understanding the entire modal evolution during the fiber pull as a final check on the quality of the nanofibers we produce.

\section{A. Transmission}

Figure 4(a) shows the transmission as a function of time during the pull for an optical nanofiber with a $2 \mathrm{mrad}$ angle taper to a radius of $6 \mu \mathrm{m}$ and exponential profile to reach a final waist radius of $250 \mathrm{~nm}$, with a fiber waist length of $5 \mathrm{~mm}$. We achieve a transmission of $99.95 \pm 0.02 \%$, or a loss of $2.6 \times 10^{-5} \mathrm{~dB} / \mathrm{mm}$. The error listed in the transmission is the standard deviation. We see from 
a)

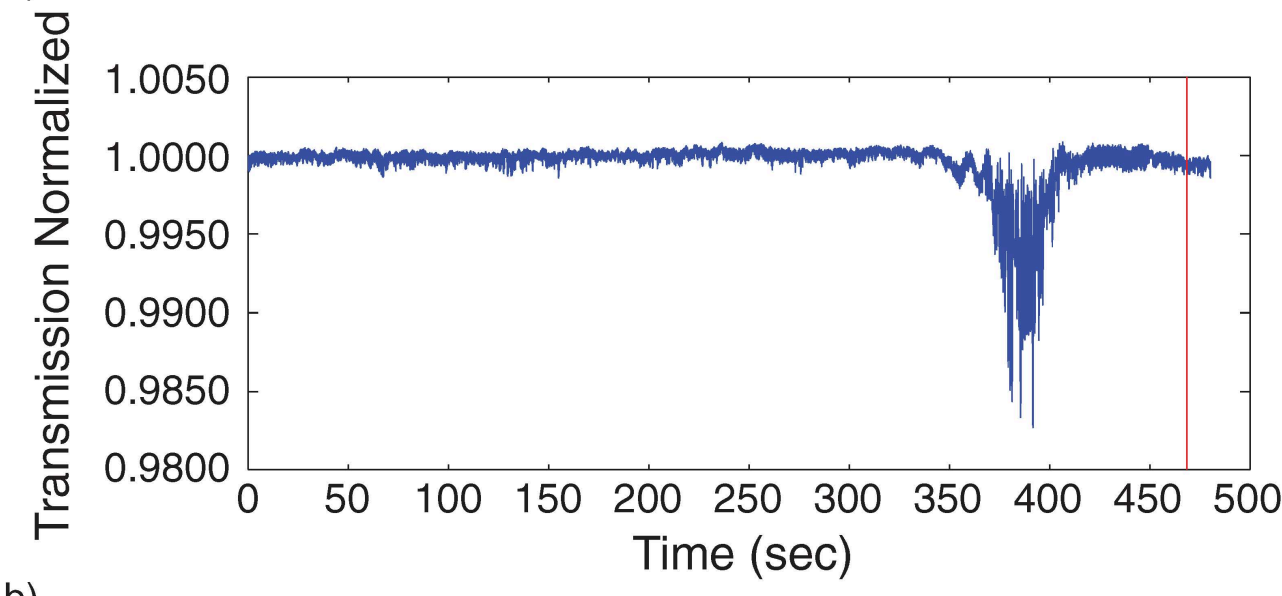

b)

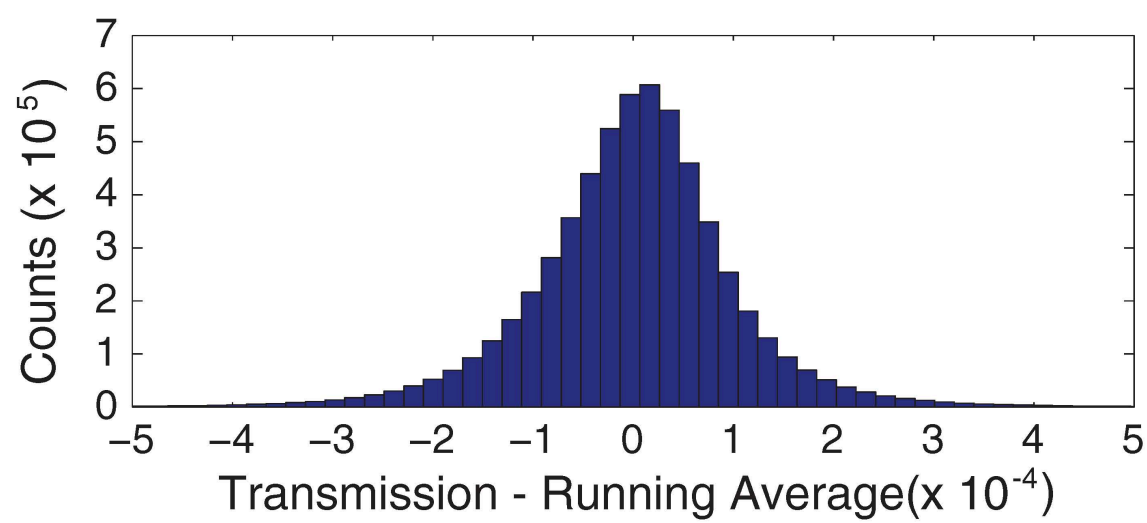

c)

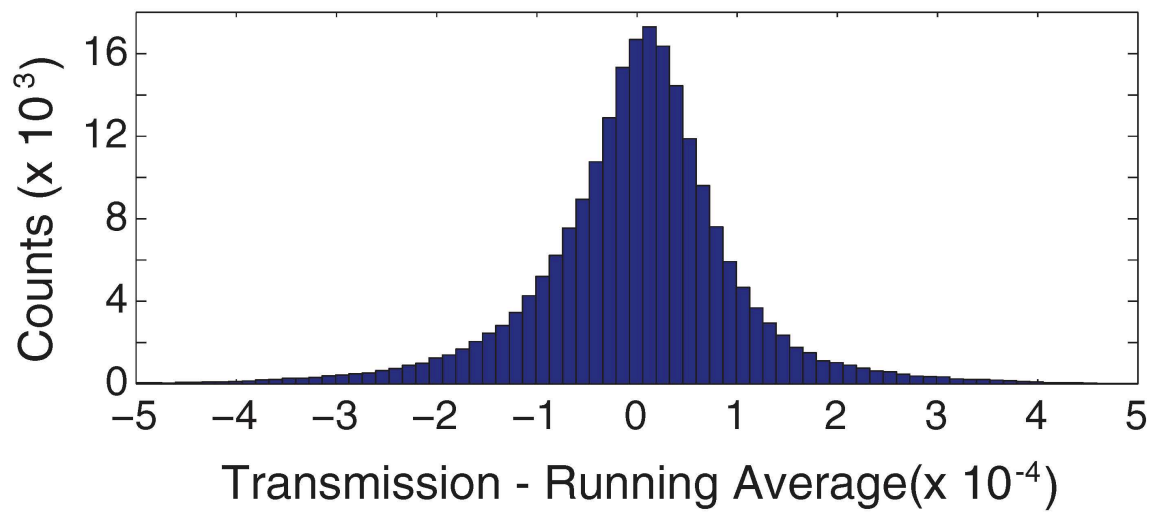

FIG. 4. a) The normalized transmission as a function of time during the pull through a nanofiber with an angle of 2 mrad to a radius of $6 \mu \mathrm{m}$ and exponential profile to a final waist radius of $250 \mathrm{~nm}$. The length of the waist is $5 \mathrm{~mm}$. The fiber has a transmission of $99.95 \pm 0.02 \%$ or equivalently a loss of $2.6 \times 10^{-5} \mathrm{~dB} / \mathrm{mm}$. b) Histogram of data taken from the beginning of the pull before higher order mode excitation. c) Histogram of transmission data taken after the pull ended.

Fig. 4(b) and 4(c) a histogram of the data ranges we use to take the mean for the average value at the beginning of the pull and the average value at the end of the pull. We obtain a standard deviation of $1.0 \times 10^{-4}$ for the data in Fig. 4(b) and $1.0 \times 10^{-4}$ for the data in 4(c). This leads to a standard deviation of the final transmission of $2.3 \times 10^{-4}$. There are possible systematic errors such as drifts in the amplifier gain, but we expect them to be of the same order or smaller than the quoted standard deviation. 


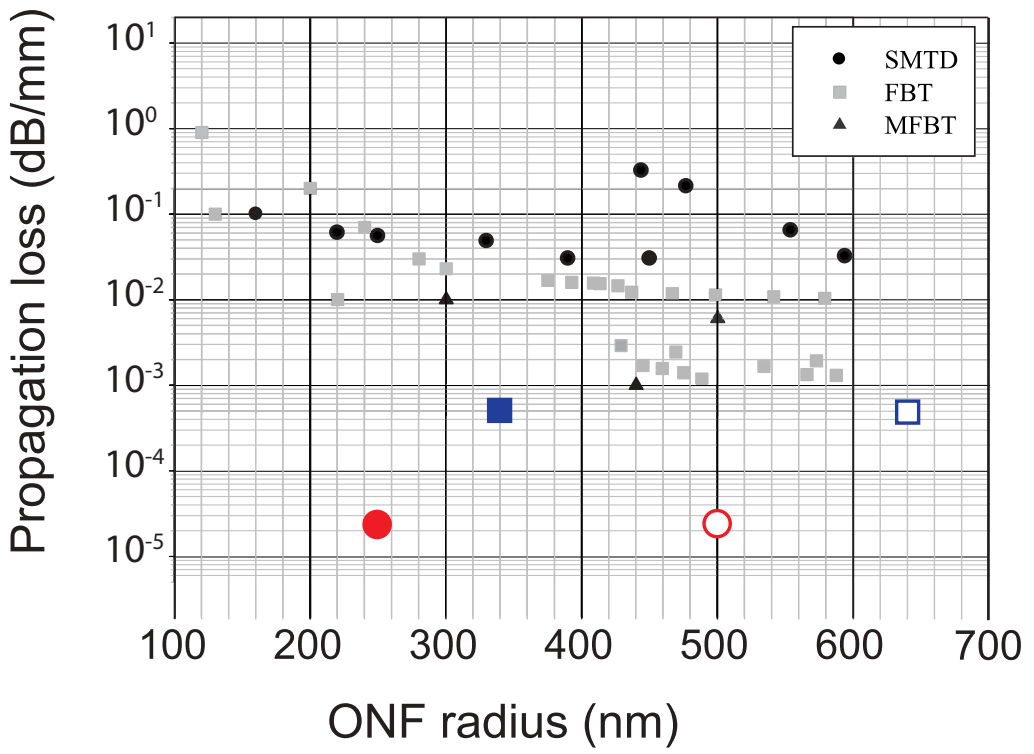

FIG. 5. Propagation loss as a function of optical nanofiber radius as compiled in Ref. 1 edited to include our results for optical nanofiber loss in $\mathrm{dB} / \mathrm{mm}$. The smaller, solid gray and black squares, circles, and triangles, represent previous results. The solid red circle represents our result for loss launching the fundamental mode. The open red circle scales the fundamental mode result to the effective radius to compare results with equivalent V numbers at $1550 \mathrm{~nm}$. The solid blue square represents our loss when launching the higher-order modes. Similarly the open blue square scales the result to the effective radius.

The final transmission is determined by taking the mean of the data after the pull ends, delineated by the red line in Fig. 4, and dividing by the value of the normalized signal at the beginning of the pull, which we must determine. We take a cumulative average of the transmission from the beginning of the pull until just before any higher order modes are excited, see Sec. IV B. Using this we see that the transmission steadies at $99.95 \%$. We find this a fair method because there is no detectable loss over this range and no beating between modes (Appendix B) in the signal since we have yet to excite any higher order modes, and by checking the cumulative average we show that the transmission listed is steady and a lower bound.

Previous work has focused on telecom light at $1550 \mathrm{~nm}$. In Fig. 5 we plot the propagation loss as a function of optical nanofiber radius for different pulling techniques as compiled in Ref. 1 and references therein. We extend the axes to overlay our results. Figure 5 shows that the lowest loss for previous work on the fundamental mode is on the order of $10^{-3} \mathrm{~dB} / \mathrm{mm}$ at $1550 \mathrm{~nm}$, with final radii of between $440-600 \mathrm{~nm}$. Our result for the fundamental mode has a loss of $2.6 \times 10^{-5} \mathrm{~dB} / \mathrm{mm}$ when the loss is taken over the entire $84 \mathrm{~mm}$ stretch. If the loss is only attributed to the $5 \mathrm{~mm}$ waist this becomes $4.34 \times 10^{-4} \mathrm{~dB} / \mathrm{mm}$. These results mark an improvement of two orders of magnitude over previous work. ${ }^{1,45,46}$ For higher-order mode pulls using SM1500 fiber with an initial diameter of $50 \mu \mathrm{m}$, the taper angle was $0.4 \mathrm{mrad}$ until a radius of $6 \mu \mathrm{m}$ and then had an exponential profile until reaching a uniform waist radius of $280 \mathrm{~nm}$. Here we achieved a loss of $5 \times 10^{-4} \mathrm{~dB} / \mathrm{mm}$, when taken over the entire stretch, which represents less loss than the previous results for fundamental mode launches.

Since the $\mathrm{V}$ number is proportional to the fiber radius divided by the input wavelength we find it fair to compare our results at a wavelength of $780 \mathrm{~nm}$ to the results in Fig. 5 at $1550 \mathrm{~nm}$ by scaling our final radius by a factor of 2. The solid red circle and blue square in Fig. 5 represent the actual radius of the pull while the open red circle and blue square are designed to scale our results to equivalent $\mathrm{V}$ numbers for inputs at $1550 \mathrm{~nm}$ and represent an effective radius. This means our effective final radius for the fundamental mode is $500 \mathrm{~nm}$ and for the higher modes $640 \mathrm{~nm}$. This ultra-high transmission is reproducible to better than $1 \%$ over time with the same fiber, when following the cleaning and alignment procedure outlined in Sec. III. 


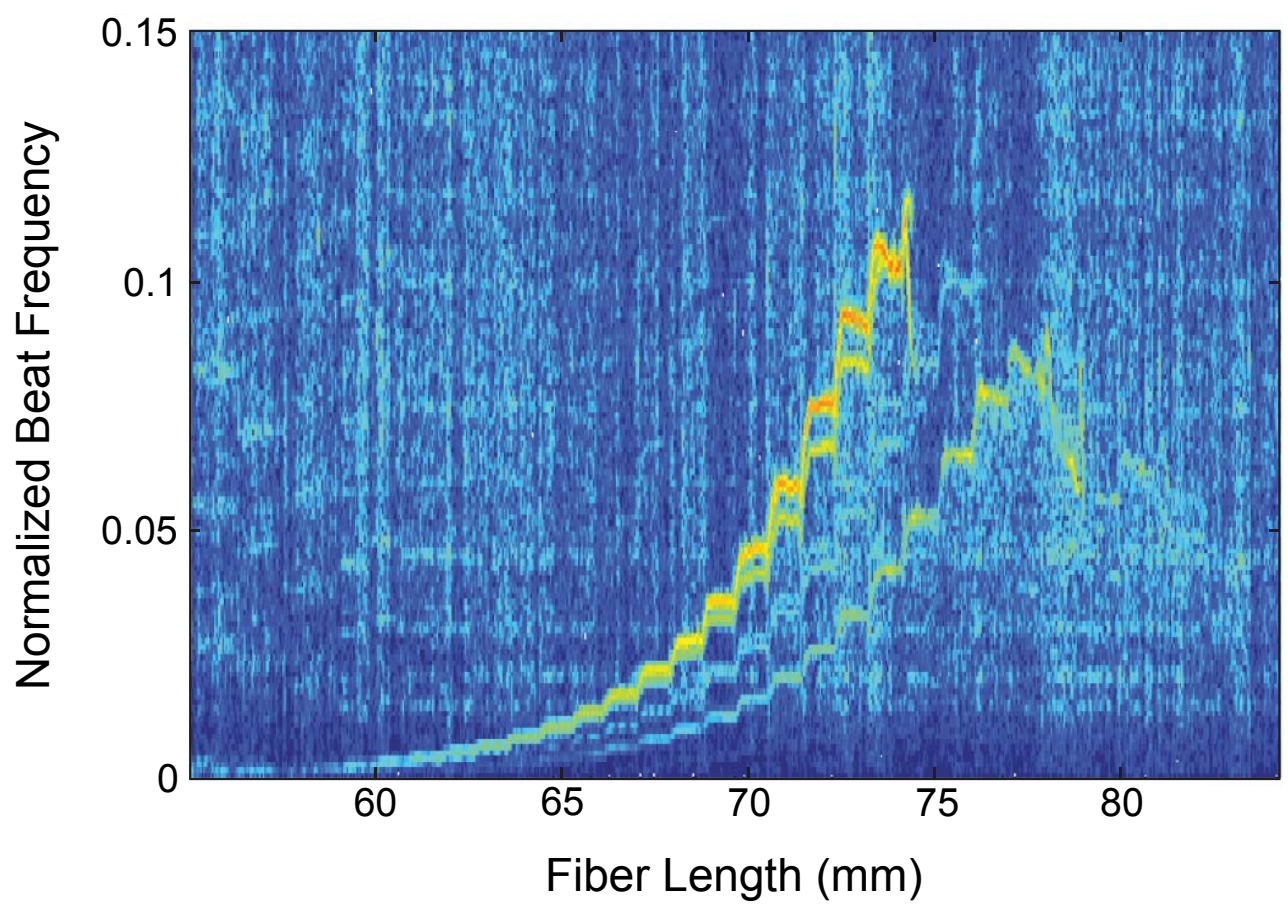

FIG. 6. Spectrogram of the transmission data from Fig. 4. The excited curves correspond to higher-order modes of the same symmetry as the fundamental mode: $E H_{11}, H E_{12}$, and $H E_{13}$.

Using a numerical Maxwell's equations solver, FIMMPROP, ${ }^{47}$ we simulate the expected transmission through a fiber with the same profile as in our pulls. We find the expected transmission to be $99.97 \%,{ }^{25}$ through a one - sided taper profile matching the $2 \mathrm{mrad}$ pull depicted in Fig. 4 , which is consistent with our experimental result that measures the transmission through the entire nanofiber. Furthermore, when launching the next family of modes through the fiber the FIMMPROP simulations were well-matched to the achieved transmissions. ${ }^{26}$ This implies that we are not limited by the pulling apparatus.

\section{B. Spectrogram Analysis}

We analyze the quality of the nanofiber using a spectrogram, a short-time Fourier transform of the transmission data, also sometimes referred to as the Gabor Transform. The spectrogram allows us to extract the entire modal evolution in the nanofiber during the pull. Each curve corresponds to the evolving spatial beat frequency between the fundamental mode and excited modes propagating in the fiber, while the contrast corresponds to the energy transferred from the fundamental mode. Following this we can use theory and simulation to identify all modes that are excited during the pull. A detailed description, with full theoretical background, can be found in Ref. 25.

Figure 6 is a spectrogram of the transmission data from Fig. 4. We see that for a successful 2 mrad pull with SM800 fiber we expect to observe a few higher order mode excitations. If modes are excited that are asymmetric to the fundamental mode, we know the cylindrical symmetry of the fiber was broken during the pulling process ${ }^{25}$ which can aid in identifying and fixing the error in the pulling apparatus.

It is worth noting that the modal excitation remains in the family of the same symmetry as the fundamental mode. To the best of our knowledge, this is the first report of modal excitation that remained purely in the symmetric family of modes. Previous work has seen asymmetric excitations to the $T E_{01}, T M_{01}$, and $H E_{21}$ modes. ${ }^{35,48}$ In Appendix B, we demonstrate this is not the case for an uncleaned fiber. 


\section{POWER MEASUREMENTS}

Once the pull is complete the fiber is transferred to a HV chamber beneath a HEPA filter. Without keeping the fiber in a clean environment the transmission will degrade as dust accumulates on the surface of the nanofiber waist and taper, which will cause the fiber to break under high powers in vacuum due to heating. ${ }^{49}$

To prevent the leakage current from impinging on the nanofiber, we avoided direct line of sight between the ion pump and the nanofiber by placing the ion pump on an elbow. Then between the elbow and the ion pump we placed a grounding mesh to prevent the electric field from penetrating past the mesh. With this arrangement a $250 \mathrm{~nm}$ radius nanofiber has withstood the application of more than $400 \pm 12 \mathrm{~mW}$ from a Ti:Sapphire laser at $760 \mathrm{~nm}$ in $\mathrm{HV}$ conditions.

\section{CONCLUSION}

We provide the necessary procedures to clean, prepare, and pull an ultrahigh transmission nanofiber in a reproducible way. The work is validated through microscopy, and we present the transmission results of a standard 2 mrad pull yielding a transmission of $99.95 \pm 0.02 \%$ or loss of $2.6 \times 10^{-6} \mathrm{~dB} / \mathrm{mm}$, an improvement of two orders of magnitude for the fundamental mode. When launching higher-order modes we have losses of $5 \times 10^{-4} \mathrm{~dB} / \mathrm{mm}$. The transmission results are in excellent agreement with transmission simulations, implying the limiting factor in transmission comes from a lack of pre-pull cleanliness. We provide a detailed cleaning protocol, which greatly improves the reproducibility for ultrahigh transmission fibers and produces the first recorded tapers without asymmetric modal excitation. We provide evidence that the pre-pull cleanliness is critical to achieving ultrahigh transmission nanofibers. These fibers can achieve efficient guidance with short, controllable taper lengths and are usable for various atomic physics applications. During the manuscript writing process we became aware of similar independent work..$^{50}$

\section{ACKNOWLEDGMENTS}

Work supported by National Science Foundation of the USA through the Physics Frontier Center at the Joint Quantum Institute, Army Research Office Atomtronics MURI, and S. R. thanks the Fulbright Foundation for support. We acknowledge the support of the Maryland NanoCenter and its NispLab. We would like to thank Fredrik Fatemi and Guy Beadie for their major contributions to the higher-order mode studies and Prof. A. Rauschenbeutel for his support and interest in this project.

\section{APPENDIX A}

We pull our fibers using a flame brushing technique. ${ }^{30-33}$ A section of fiber, less than a millimeter in length, is brought to its softening point using a clean oxyhydrogen flame and then pulled by two high-precision motors.

Our algorithm, ${ }^{51}$ based on the work of the originally Mainz and currently Vienna group, ${ }^{32}$ calculates the trajectories of the motors needed to produce a fiber with the desired final radius, length of uniform waist, and taper geometry. The tapers are formed by a series of small sections that are well approximated by lines, allowing us to form a linear taper with a given angle down to a radius of $6 \mu \mathrm{m}$, which connects to an exponential that smoothly reduces to a submicron radius, typically $250 \mathrm{~nm}$. The slope of the linear section generally varies between 0.3 and $5 \mathrm{mrad}$. Our algorithm divides the pull into steps defined by their pulling velocity and the traveling length of the flame. We recursively calculate the parameters, starting from the desired final radius, $r_{w}$, until reaching the initial radius, $r_{0}$. The full details and code can be found at Ref. 44 .

\section{Motor Control}

The model produces a velocity profile that is a square wave in time. Experimentally, we approximate the square wave in three parts: 
1. A ramp up to $v_{b, n} \pm v_{f, n} / 2$

2. A constant pull velocity equal to $v_{b, n} \pm v_{f, n} / 2$

3. A ramp down in velocity to zero.

Where $v_{b, n}$ is the velocity of the flame in step $\mathrm{n}$ and $v_{f, n}$ is the velocity that the fiber motors move apart. The addition of $v_{b, n}$ arises from the transformation to the rest frame of the flame. Typically, $v_{b, n}$ is an order of magnitude greater than $v_{f, n}$. When transforming to the rest frame of the flame, both motors move in the direction the flame would have swept in that step. The motor whose pull velocity is in the same direction as the flame motion will lead while the other motor will lag. We have verified this sequence using the encoder of the motor that allows us to record the trajectory of the motors and by looking at the output of a Michelson interferometer with one arm spanning the two motorized stages.

\section{Measurement of the flame width}

One experimental parameter fundamental to the algorithm is the effective size of the flame, $L_{0}$, which corresponds to the zone of the fiber inside the flame that melts and thins during the pulling process. The softening point for the fused silica used by Fibercore for the SM800 fiber occurs at $1585^{\circ} \mathrm{C}$. The best way to evaluate this is to measure the impact of the flame on the fiber, since our flame cannot be observed by eye.

Working with reproducible conditions requires that we fix the working distance between the fiber and the nozzle. As a consequence, the fiber is always at the same spot inside the flame and always sees the same distribution of temperature. We check the distance with a microscope before each pull and fix it to $400 \pm 50 \mu \mathrm{m}$.

We measure $L_{0}$ by fixing the flame and letting both motors move apart at a constant velocity. Conservation of volume leads to an exponential profile with a waist of length $L_{0}$, and the radius profile is given by :

$$
r_{w}=r_{0} \exp \left(-\frac{t_{h} v_{f}}{2 L_{0}}\right)
$$

where $t_{h}$ is the heating time and $r_{0}$ the unmodified radius of the fiber.

We use our imaging system (15 in Fig. 1) to measure the radius of the waist of the fiber for different values of $v_{f} t_{h}$, and fit $\ln \left(r_{0} / r_{w}\right)$ to extract $L_{0}$.

The measurement consists of fixing the pulling velocity at $0.05 \mathrm{~mm} / \mathrm{s}$, varying the heating time from 2 to $32 \mathrm{~s}$, and then measuring the final radius of the waist. We limit ourselves to times less than 40 seconds to stay within the $2 \mu \mathrm{m}$ resolution of our imaging system.

We characterize the size of the flame by plotting $\ln \left(r_{0} / r_{w}\right)$ as a function of $v_{f} t_{h}$ we obtain a fit with a reduced $\chi^{2}$ of 1.07 that yields $L_{0}=0.753 \pm 0.014 \mathrm{~mm}$. This parameter should be checked from time to time as the pulling apparatus is used since it can vary by a small amount.

Measuring the length of the waist or fitting the profiles of the taper to an exponential are less accurate methods than the above procedure because Eq. (A1) assumes a uniform hot zone, $L_{0}$. In this measurement we keep the flame fixed, which means that our hot zone is not uniform. During the actual pulling procedure we sweep, which creates an effective uniform hot zone. Here, the section of fiber located at the central point of the flame is thinned the most, as a result it is more accurate to measure the profile of the fiber after tapering and find the smallest radius to extract the value of $L_{0}$.

\section{Microscopy validation}

We validate the accuracy of our simulation of the expected fiber profile using both an in situ optical microscope and a scanning electron microscope (SEM). Figure 7(a) shows the measured (blue markers) and simulated profiles (red lines) of a fiber taper imaged optically. The taper profile is designed to have three angles, 5, 2, and $3 \mathrm{mrad}$, that taper down to radii of 50, 35 and $25 \mu \mathrm{m}$, respectively. An exponential profile smoothly links the radius of $25 \mu \mathrm{m}$ down to the the final radius 


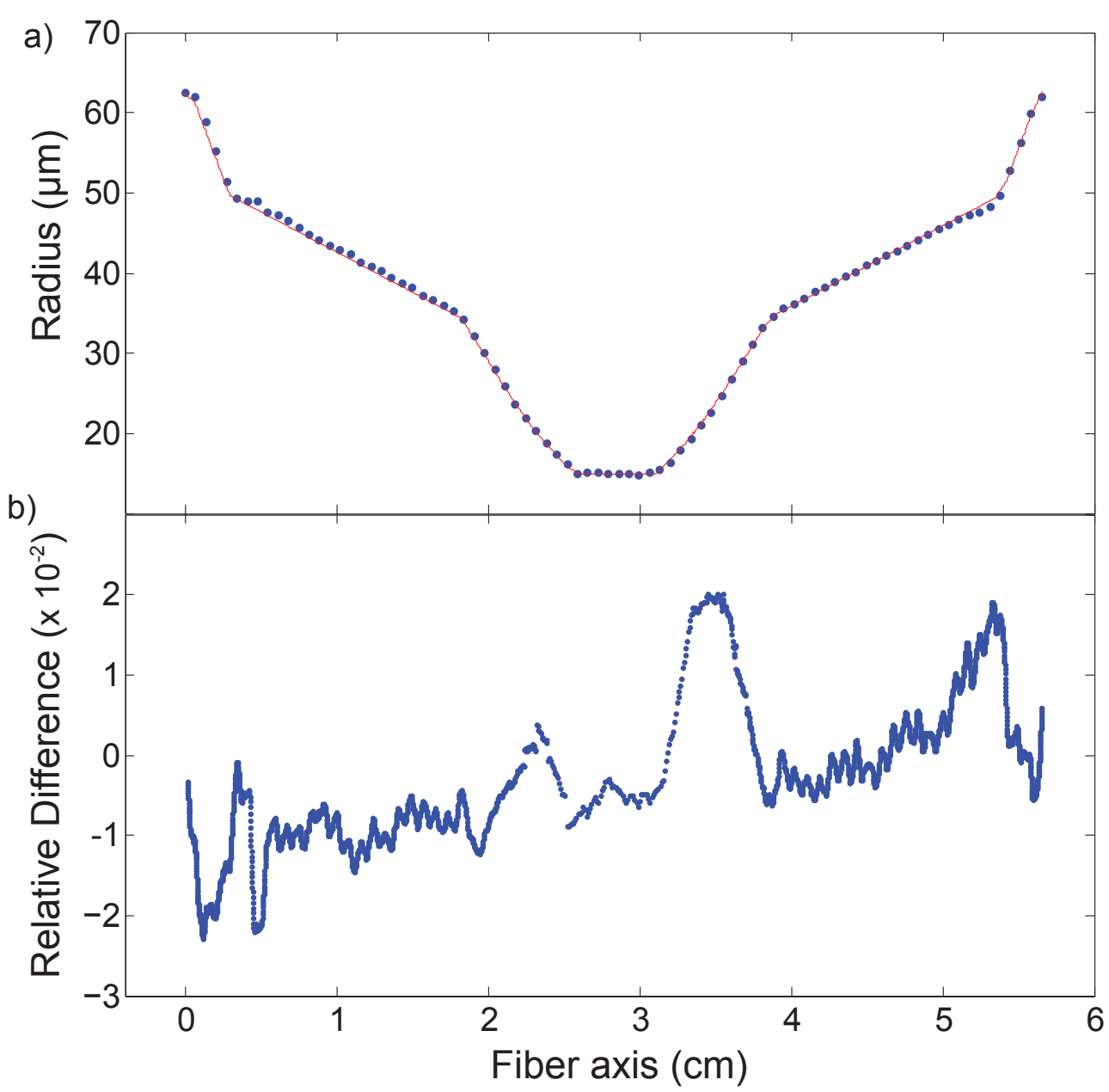

FIG. 7. Profile of a multiple-angled, linearly tapered fiber. (a) The blue dots are measurements taken using the optical microscope and the red line represents the profile shape from the fiber tapering simulation. The pull was for a final radius of $15 \mu \mathrm{m}$. The taper profile was designed to have three angles, 5, 2, $3 \mathrm{mrad}$, that taper down to radii of 50, 35 and $25 \mu \mathrm{m}$, respectively. The error in each measurement is dominated by a systematic error of $\pm 2.5 \mu \mathrm{m}$. (b) The relative difference between the expected profile and the measured profile with an RMS value of 0.0187 .

of $15 \mu \mathrm{m}$. The final radius is chosen to be well above the resolution of our optical microscope. The length of the uniform waist is chosen to be $5 \mathrm{~mm}$ long. Figure 7(a) is a compilation of optical microscope images taken of the entirety of the tapered fiber. An edge finding technique then measures the profile of the fiber at different cuts. The error in the measured radius is dominated by a systematic error of $\pm 2.5 \mu \mathrm{m}$ due to the finite resolution of the imaging system. We first use an image of the unmodified fiber, which has a diameter of $125.1 \mu \mathrm{m}$, to determine the pixel to micron conversion. The number of pixels measured for an unmodified fiber has an error of a few pixels as a result of the resolution of the optical microscope. We then binarize the gray levels of the pixels and choose a threshold such that the diameter of the unmodified fiber matches the pixel count from the previous measurement. The edge finding technique itself has an error of about 0.5 pixels for a flat length of fiber resulting from the binarization process. Figure 7(b) displays the relative difference between the measured image radius and the simulated radius normalized to the expected radius. The largest deviation is slightly larger than $2 \%$, while the RMS value is 0.0187 . This verifies the accuracy of our algorithm and pulling apparatus for larger radius tapers.

We use a SEM to measure the nanofiber profile below a micrometer to verify that our nanofibers truly achieve the desired diameter. Figure 8, shows a SEM image of a nanofiber, coated with 

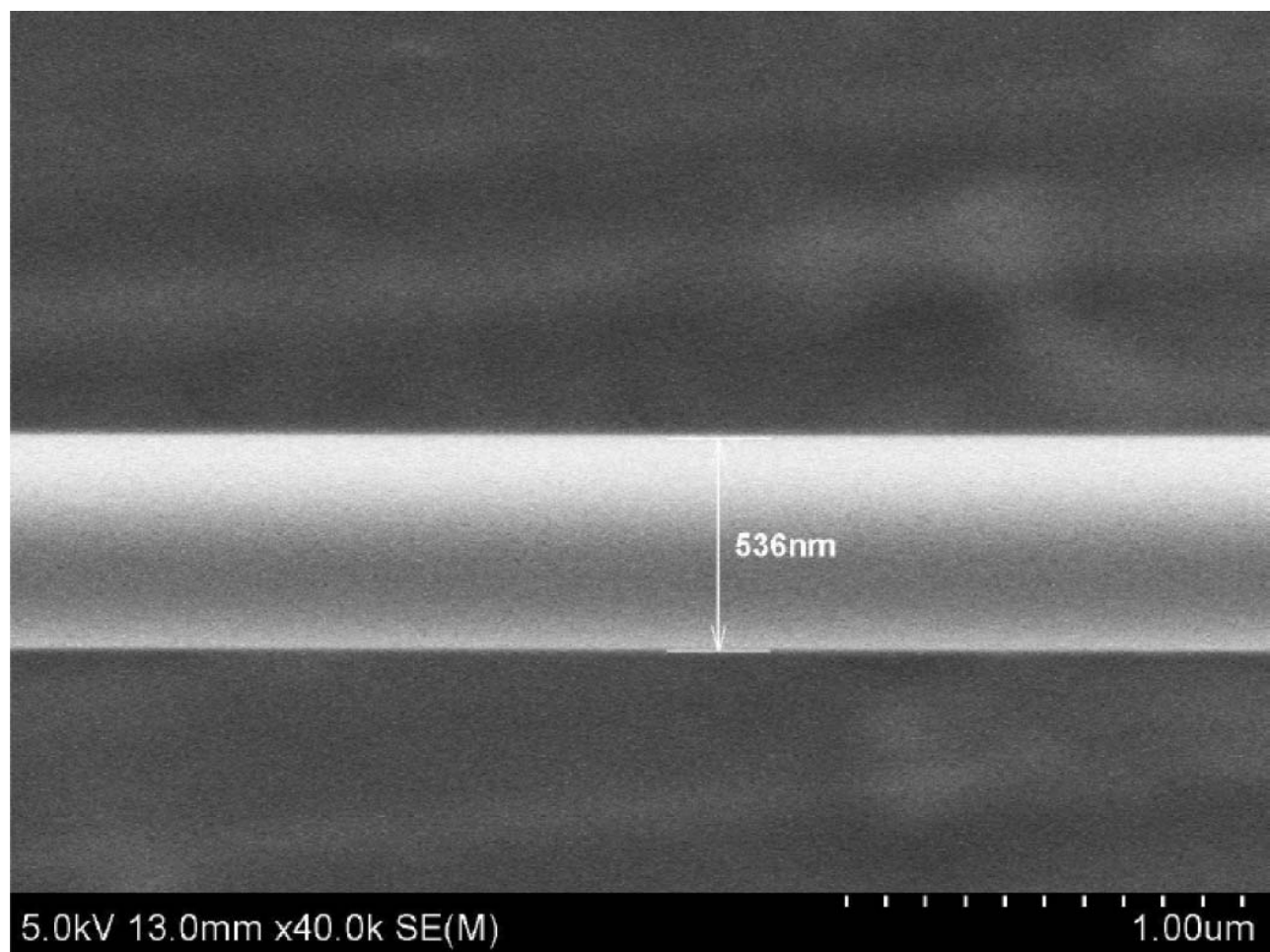

FIG. 8. A SEM image taken in the NISP lab at UMD. We measure a radius of $536 \pm 12 \mathrm{~nm}$, the expected diameter of the waist is $500 \mathrm{~nm}$.

graphite, with an expected diameter of $500 \mathrm{~nm}$, and a measured diameter of $536 \pm 12 \mathrm{~nm}$. The error is systematic, coming from the scaling factor associated with the SEM calibration. We attribute this small disagreement to thermal forces that push the fiber away from the nozzle at the end of the pull when the fiber is thin. We could compensate for this in the algorithm by adjusting the effective hot zone as the fiber tapers, but we have not found it necessary to do so.

\section{APPENDIX B}

Any particulate accumulation on the optical fiber before the pull begins will compromise the quality of the optical nanofiber: it will degrade the transmission, excite higher order modes, change the modal evolution, and scatter light. If any particulate accumulates on the fiber before the pull, the maximum possible transmission for a given taper geometry will not be achieved. Using FIMMPROP, as described in IV A, we have a sense of what this ideal transmission is for a given geometry, and if our transmission deviates, it can generally be attributed to a lack of proper cleaning. If the nanofiber environment is not clean or has a high humidity the transmission will decrease after a pull is finished. ${ }^{24}$ Furthermore, if any dust accumulates on the nanofiber surface, it will not withstand high powers under vacuum.

If the fiber is not properly cleaned before pulling, the final transmission can vary by a few percent. Figure 9 displays the extreme case of mechanically stripping the buffer and not cleaning the fiber at all before pulling. Here, the transmission is only $80.5 \%$ for a $2 \mathrm{mrad}$ taper down to $r_{w}=250 \mathrm{~nm}$, leading to more than a $19 \%$ loss in transmission when compared to a properly cleaned fiber. The spectrogram in Fig. 9(b) shows excitation to excited asymmetric mode: $T E_{01}, T M_{01}$, and $H E_{21}$, identified by arrows, that were not present when the fiber was properly cleaned. It is further interesting that there is more energy transferred to these asymmetric modes than any other modes.

Before every pull, we follow the cleaning procedure described in Sec. III A. After imaging the fiber, we decide whether or not we should start the pull or restart the cleaning process. We restart 
a)

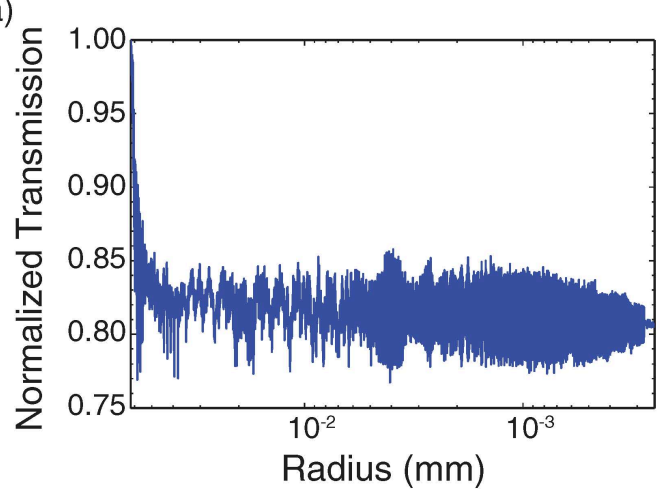

b)

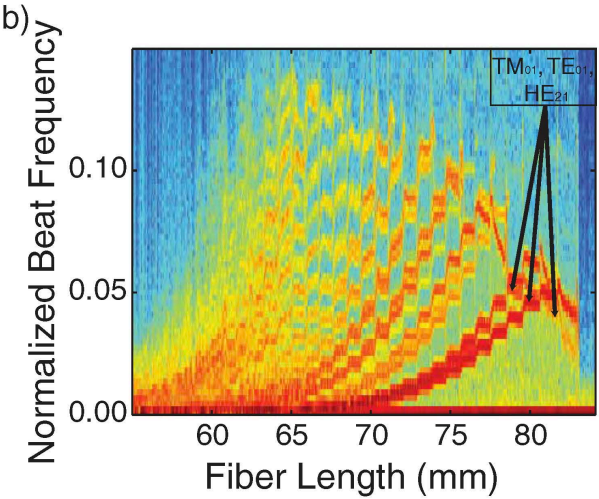

FIG. 9. (a) Transmission signal for an uncleaned 2 mrad tapered fiber. The transmission of this fiber is $80.50 \%$. (b) Spectrogram of the transmission data. We can distinguish the excitation of many higher order modes. Of special interest are the curves identified, the asymmetric $T E_{01}, T M_{01}$, and $H E_{21}$ modes.

if anything is obstructing the light traveling through the fiber reaching the CCD. When there is particulate attached on top of or below the fiber, we use a wipe with methanol and remove it. If there is nothing observable within the resolution of the optical microscope we proceed with the pull. When we do not follow these criteria the reproducibility in the transmission will change by a few percent. When we apply this cleaning method, the variability between runs is better than $1 \%$.

The origin of the particulate can come in various forms: remnants of plastic buffer, solvent evaporate, or any small particulate floating in the air. We believe the most common source to be the buffer. Since we use a mechanical fiber stripper to remove the buffer, micro or macroscopic pieces of buffer remain on the fiber after stripping. We apply wipes to remove the buffer remnants. This removal process can be imperfect because mechanical strippers are not designed to make contact with the actual glass of the fiber. Buffer remnants are a particularly insidious form of particulate. The plastic is generally designed with a higher index of refraction than the cladding to help remove cladding modes. If the buffer remains, we believe it may burn into the fiber. This higher index irregularity can lead to an excitation of higher order modes.

During the pull there may be signatures that the fiber was not properly cleaned. These take the form of large losses in transmission and the excitation of higher order modes. If there is initially loss or beating in the transmission this is a sign that the fiber was not properly cleaned; this is displayed in Fig. 9(a). The fiber starts single mode in the core and therefore there should be no beating between modes and negligible losses in the initial pulling process, before the fundamental mode becomes a cladding mode, in which the tapering process reduces the effective index of refraction to and then below the index of refraction of the cladding.

As the fiber continues to taper and the effective index of refraction of the fundamental mode approaches the index of refraction of the cladding, the mode begins to leak from the core. This is when higher order mode excitation can occur. For the SM800 fiber used in this study, the transition occurs at a radius of $19.4 \mu \mathrm{m}$. This transition is captured by Media 1 in Fig. 10. Here, we see that as the radius reduces, the effective index of refraction approaches the index of refraction of the cladding and the mode begins to leak from the core. If the beating between higher order modes occurs before this point, this is evidence that the fiber was damaged. A nanofiber with a 2 mrad geometry, if handled properly, typically excites only three higher order modes: $E H_{11}, H E_{12}$, and $H E_{13} .{ }^{25}$ In Fig. 9(b), each curve corresponds to beating between different modes and we can identify more than twenty excited modes as a result of the buffer remnants.

Furthermore, any dust on the nanofiber will cause it to break under high power in vacuum. The cleanroom environment and the cleanliness of our pulling and transfer procedures allow us to achieve nanofibers that transmit more than $400 \pm 12 \mathrm{~mW}$ in an $\mathrm{HV}$ environment.

We believe that chemically removing the buffer could be beneficial to the fiber transmission. Chemical removal can lead to less mechanical damage to the fiber and properly remove all of the 


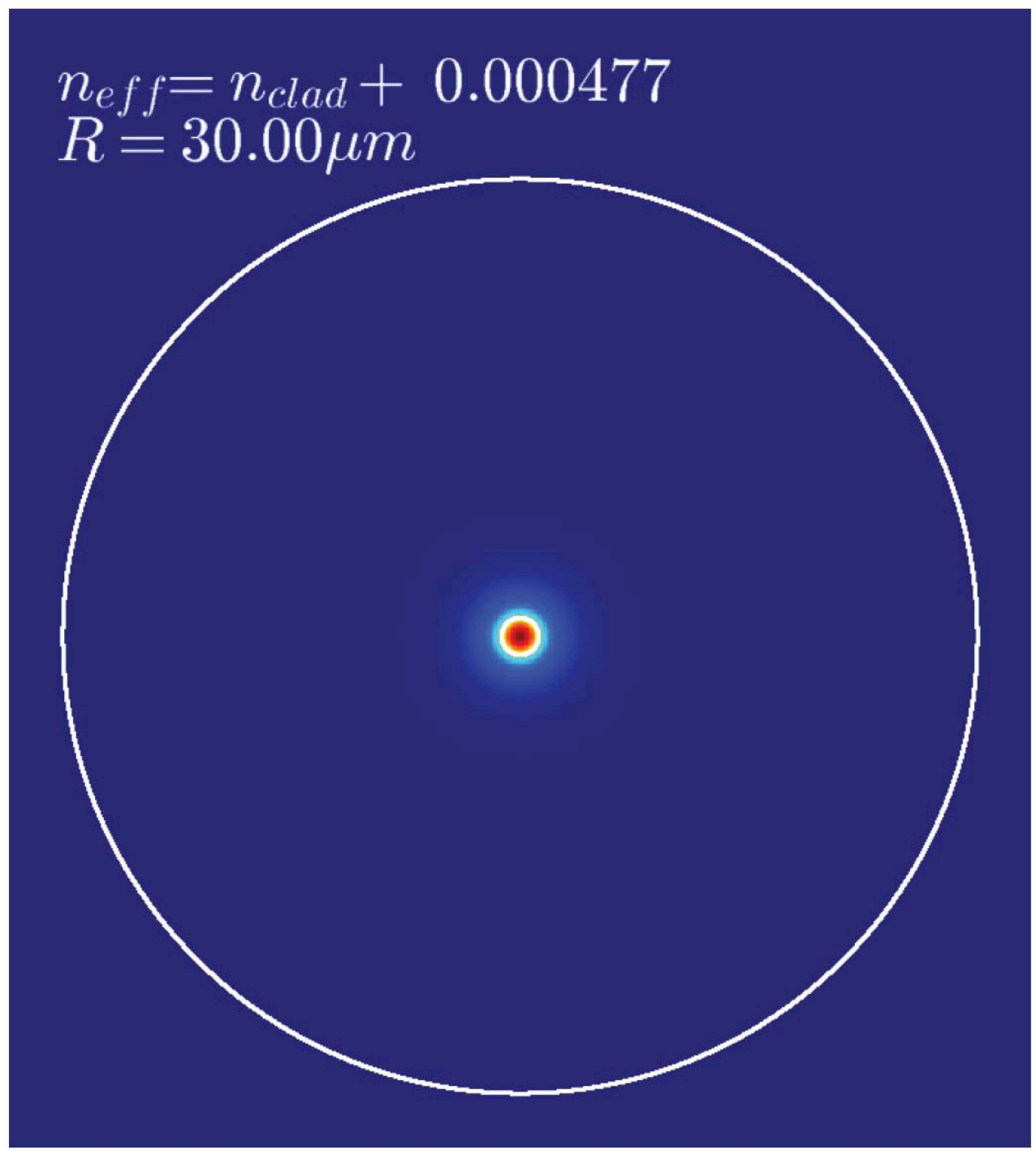

FIG. 10. The transition of the fundamental mode from a core to a cladding mode. The intensity of each frame is renormalized for visualization. The modes are obtained using the numerical solver FIMMPROP (multimedia view). [URL: http://dx.doi.org/10.1063/1.4879799.1]

buffer. This is not a critical issue, since our transmission is in good agreement with simulations from FIMMPROP, but it could improve reproducibility and ease the cleaning process.

${ }^{1}$ G. Brambilla, Journal of Optics 12, 043001 (2010)

${ }^{2}$ M. J. Morrissey, K. Deasy, M. Frawley, R. Kumar, E. Prel, L. Russell, V. G. Truong, and S. Nic Chormaic, Sensors 13, 10449 (2013).

${ }^{3}$ F. Le Kien, J. Q. Liang, K. Hakuta, and V. I. Balykin, Optics Communications 242, 445 (2004).

${ }^{4}$ F. Le Kien, V. I. Balykin, and K. Hakuta, Phys. Rev. A 70, 063403 (2004).

${ }^{5}$ D. Reitz, and A. Rauschenbeutel, Optics Communications 2854705 (2012).

${ }^{6}$ G. Sagué, A. Baade, and A. Rauschenbeutel, New Journal of Physics 10, 113008 (2008).

${ }^{7}$ D. J. Alton, N. P. Stern, T. Aoki, H. Lee, E. Ostby, K. J. Vahala, and H. J. Kimble, Nat. Phys. 7, 159 (2011).

${ }^{8}$ A. Goban, K. S. Choi, D. J. Alton, D. Ding, C. Lacroûte, M. Pototschnig, T. Thiele, N. P. Stern, and H. J. Kimble, Phys. Rev. Lett. 109, 033603 (2012).

${ }^{9}$ F. Le Kien, S. Dutta Gupta, V. I. Balykin, and K. Hakuta, Phys. Rev. A 72, 032509 (2005).

${ }^{10}$ E. Vetsch, D. Reitz, G. Sagué, R. Schmidt, S. T. Dawkins, and A. Rauschenbeutel, Phys. Rev. Lett. 104, 203603 (2010).

${ }^{11}$ C. Wuttke, M. Becker, S. Brückner, M. Rothhardt, and A. Rauschenbeutel, Opt. Lett. 37, 1949 (2012)

${ }^{12}$ J. C. Knight, G. Cheung, F. Jacques, and T. A. Birks, Opt. Lett. 22, 1129 (1997). 
${ }^{13}$ K. P. Nayak, P. N. Melentiev, M. Morinaga, F. L. Kien, V. I. Balykin, and K. Hakuta, Opt. Express 15, 5431 (2007).

${ }^{14}$ G. Kakarantzas, T. A. Birks, and P. St. J. Russell, Opt. Lett. 27, 1013 (2002).

${ }^{15}$ Y. Louyer, D. Meschede, and A. Rauschenbeutel, Phys. Rev. A 72, 031801 (2005).

${ }^{16}$ M. J. Morrissey, K. Deasy, Y. Wu, S. Chakrabarti, and S. N. Chormaic, Review of Scientific Instruments 80, 053102 (2009).

${ }^{17}$ S. M. Spillane, T. J. Kippenberg, O. J. Painter, and K. J. Vahala, Phys. Rev. Lett. 91, 043902 (2003).

${ }^{18}$ M. Fujiwara, T. Noda, A. Tanaka, K. Toubaru, H.-Q. Zhao, and S. Takeuchi, Opt. Express 20, 19545 (2012).

${ }^{19}$ T. Schröder, M. Fujiwara, T. Noda, H.-Q. Zhao, O. Benson, and S. Takeuchi, Opt. Express 20, 10490 (2012).

${ }^{20}$ K. P. Nayak and K. Hakuta, Opt. Express 21, 2480 (2013).

${ }^{21}$ M. Sadgrove, R. Yalla, K. P. Nayak, and K. Hakuta, Opt. Lett. 38, 2542 (2013).

22 J. D. Thompson, T. G. Tiecke, N. P. de Leon, J. Feist, A. V. Akimov, M. Gullans, A. S. Zibrov, V. Vuletić, and M. D. Lukin, Science 340, 1202 (2013).

${ }^{23}$ H. J. Kimble, Nature 453, 1023 (2008).

${ }^{24}$ M. Fujiwara, K. Toubaru, and S. Takeuchi, Opt. Express 19, 8596 (2011).

${ }^{25}$ S. Ravets, J. E. Hoffman, P. Kordell, J. D. Wong-Campos, S. L. Rolston, and L. A. Orozco, J. Opt. Soc. Am. A 30, 2361 (2013).

${ }^{26}$ S. Ravets, J. E. Hoffman, L. A. Orozco, S. L. Rolston, G. Beadie, and F. K. Fatemi, Opt. Express 21, 18325 (2013).

${ }^{27}$ M. Hafezi, Z. Kim, S. L. Rolston, L. A. Orozco, B. L. Lev, and J. M. Taylor, Phys. Rev. A 85, 020302 (2012).

${ }^{28}$ J. E. Hoffman, J. A. Grover, Z. Kim, A. K. Wood, J. R. Anderson, A. J. Dragt, M. Hafezi, C. J. Lobb, L. A. Orozco, S. L. Rolston, J. M. Taylor, C. P. Vlahacos, and F. C. Wellstood, Revista Mexicana De Fisica 57, 1 (2011).

${ }^{29}$ R. Garcia-Fernandez, W. Alt, F. Bruse, C. Dan, K. Karapetyan, O. Rehband, A. Stiebeiner, U. Wiedemann, D. Meschede, and A. Rauschenbeutel, Applied Physics B 105, 3 (2011).

${ }^{30}$ F. Warken, E. Vetsch, D. Meschede, M. Sokolowski, and A. Rauschenbeutel, Opt. Express 15, 11952 (2007)

${ }^{31}$ F. Bilodeau, K. O. Hill, S. Faucher, and D. C. Johnson, Lightwave Technology, Journal of 6, 1476 (1988).

${ }^{32}$ F. Warken, "Ultra thin glass fibers as a tool for coupling light and matter," Ph.D. thesis, Rheinische Friedrich-Wilhelms Universitat (2007).

${ }^{33}$ T. A. Birks and Y. W. Li, Lightwave Technology, Journal of 10, 432 (1992).

${ }^{34}$ T. E. Dimmick, G. Kakarantzas, T. A. Birks, and P. St. J. Russell, Appl. Opt. 38, 6845 (1999).

${ }^{35}$ L. Ding, C. Belacel, S. Ducci, G. Leo, and I. Favero, Appl. Opt. 49, 2441 (2010).

${ }^{36}$ H. J. Kbashi, Journal of Materials Science \& Technology 28, 308 (2012).

${ }^{37}$ P. Lambelet, A. Sayah, M. Pfeffer, C. Philipona, and F. Marquis-Weible, Appl. Opt. 37, 7289 (1998).

${ }^{38}$ J. M. Ward, D. G. O'Shea, B. J. Shortt, M. J. Morrissey, K. Deasy, and S. G. Nic Chormaic, Review of Scientific Instruments 77, 083105 (2006).

${ }^{39}$ H. Yokota, E. Sugai, and Y. Sasaki, Optical Review 4, A104 (1997).

${ }^{40}$ A. Stiebeiner, R. Garcia-Fernandez, and A. Rauschenbeutel, Opt. Express 18, 22677 (2010).

${ }^{41}$ Incorporated, C., Spec sheet.

${ }^{42}$ F. K. Fatemi, Opt. Express 19, 25143 (2011)

${ }^{43}$ J. A. Pechkis and F. K. Fatemi, Opt. Express 20, 13409 (2012).

${ }^{44}$ The programs are available at the Digital Repository of the University of Maryland DRUM at http://hdl.handle.net/1903/15069.

${ }^{45}$ G. Brambilla, V. Finazzi, and D. J. Richardson, Opt. Express 12, 2258 (2004).

${ }^{46}$ S. Leon-Saval, T. A. Birks, W. J. Wadsworth, P. St. J. Russell, and M. W. Mason, Opt. Express 12, 2864 (2004).

${ }^{47}$ Ltd, P. D., "FIMMWAVE/FIMMPROP," http://www.photond.com.

${ }^{48}$ F. Orucevic, V. Lefèvre-Seguin, and J. Hare, Opt. Express 15, 13624 (2007).

${ }^{49}$ C. Wuttke and A. Rauschenbeutel, Phys. Rev. Lett. 111, 024301 (2013).

${ }^{50}$ J. M. Ward, A. Maimaiti, Vu H. Le, and S. G. Nic Chormaic, ArXiv e-prints (2014), arXiv:1402.6396.

${ }^{51}$ We used acetone for the data shown in this paper; however, we do not recommend its use because it can prolong the cleaning process. SM800 fibers have a buffer made of dual acrylate, which dissolves in acetone. This is fine for chemical removal of the buffer when heated or paired with other chemicals, but when cleaning with a wipe, the acetone can spread small buffer particulate along the stripped portion of fiber, which can burn when introduced to the flame. 


\section{Chapter 3}

\section{Intermodal energy transfer in a tapered optical fiber: optimizing transmission}

\section{Contents}

3.1 Fiber used . . . . . . . . . . . . . . 40

3.2 Modes in an optical fiber . . . . . . . . . . . . 40

3.3 Mode evolution in a tapered fiber . . . . . . . . 42

3.4 Adiabatic tapers . . . . . . . . . . . . . 43

3.5 Intermodal energy transfer in a $2 \mathrm{mrad}$ tapered fiber . . 44

3.5.1 Transmission signal . . . . . . . . . . . . . . . 46

3.5.2 Explanation of the observed beating . . . . . . . . . 46

3.5.3 Spectrogram of the transmission signal . . . . . . . . 47

3.6 Quality of our fibers . . . . . . . . . . . . 48

3.7 Nanofibers for a hybrid quantum system . . . . . . 48

We have seen in Chapter 1 that the total internal reflexion at the interface between two media with different indices of refraction allows light to be guided in optical fibers. A more detailed analysis of light propagation in optical fibers is given in terms of electromagnetic modes that are obtained by solving Maxwell's equations in a cylindrical dielectric waveguide [Yariv (1990)]. In this Chapter, we analyze our transmission signals in terms of modes so as to get more insight into light propagation in our tapered optical fibers. 


\section{$3.1 \quad$ Fiber used}

For the experiments presented in this Chapter, we have used a $125 \mu \mathrm{m}$ diameter SM800 fiber from Fibercore. The fiber is composed of a core of index of refraction $n_{\text {core }}=1.45861$, and a cladding of index of refraction $n_{\text {cladding }}=1.45367$ that is surrounded by air. The manufacturer indicates that the fiber is single-mode for wavelengths that are greater than $792 \mathrm{~nm}$. The numerical aperture (N.A.) of the fiber is given by:

$$
\text { N.A. }=\sqrt{n_{\text {core }}^{2}-n_{\text {clad }}^{2}}=0.12 \text {. }
$$

The fiber is surrounded by air, and we model it by the following step-index structure:

$$
\begin{cases}n=n_{\text {core }} & \text { for } \quad 0 \leq r<a, \\ n=n_{\text {clad }} & \text { for } \quad a \leq r \leq R, \\ n=1.00029 & \text { for } \quad r>R,\end{cases}
$$

where $a$ is the core radius, $R$ is the fiber radius and $r$ is the radius in cylindrical coordinates.

\subsection{Modes in an optical fiber}

We review in this Section the basic properties of optical fibers without demonstration (for the full derivation, see [Yariv (1990)]). Because of the cylindrical symmetry around the $z$-axis, it is convenient to work in the cylindrical coordinate system. The fields propagate along the $z$-axis, and we thus assume:

$$
\left[\begin{array}{l}
\boldsymbol{E}(\boldsymbol{r}, t) \\
\boldsymbol{H}(\boldsymbol{r}, t)
\end{array}\right]=\left[\begin{array}{c}
\boldsymbol{E}(r, \phi) \\
\boldsymbol{H}(r, \phi)
\end{array}\right] \exp [i(\beta z-\omega t)],
$$

Solving Maxwell's equations, one obtains a discrete set of solutions describing the modes that are guided inside the core of the waveguide. The guided modes are labeled by their mode family number $l$ and their mode order $m$ [Yariv (1990)]. The amplitude of their electromagnetic field oscillates as a function of $r$ inside the core (where $m$ is the number of field zeros), and decays exponentially outside. In general, for $l \neq 0$, we observe that the fields show non-zero longitudinal components $E_{z}$ and $H_{z}$, which distinguishes them from transverse modes ${ }^{1}$. In this case, the guided modes

\footnotetext{
${ }^{1}$ Satisfying all the boundary conditions in a cylindrical waveguide introduces non-zero longitudinal components for $\boldsymbol{E}$ and $\boldsymbol{H}$.
} 
are called "hybrid modes", and labeled $\mathrm{HE}_{l m}$ and $\mathrm{EH}_{l m}$. For $l=0$, we obtain a family of $\mathrm{TE}_{0 m}$ modes that are transverse electric $\left(E_{z}=0\right)$ and a family of $\mathrm{TM}_{0 m}$ modes that are transverse magnetic $\left(H_{z}=0\right)$. One observes that for modes that are guided inside the core, the propagation constants must satisfy:

$$
n_{\text {clad }} k_{0}<\beta_{l m} \leq n_{\text {core }} k_{0},
$$

where $k_{0}$ is free-space wavevector. We define the effective index of refraction $n_{\text {eff }}$ :

$$
\beta_{l m}=n_{\text {eff }} k_{0}
$$

where $n_{\text {eff }}$ depends on $l$ and $m$. The effective index $n_{\text {eff }}$ can be interpreted as the index of refraction experienced by the mode as it propagates through the medium. As a consequence, depending on the value of $n_{\text {eff }}$, we can identify several types of modes:

- Core modes have most of their energy confined in the core, while a small portion of their energy leaks into the cladding. Thus, as we have seen, $n_{\text {clad }}<n_{\text {eff }} \leq n_{\text {core }}$.

- Solutions of Maxwell's equation verifying $1<n_{\text {eff }} \leq n_{\text {clad }}$ correspond to "cladding modes", that are guided by the cladding-to-air interface.

- Finally, $n_{\mathrm{eff}}=1$ corresponds to radiation modes that are radiated into the air and that are thus not guided by the fiber.

The number of modes that are accepted inside the core depends on the size of the core $a$, the wavelength of the light $\lambda$ and the numerical aperture of the fiber. We define the dimensionless parameter $V_{\text {core }}$ :

$$
V_{\text {core }}=\frac{2 \pi}{\lambda} a \text { N.A. }=\frac{2 \pi}{\lambda} a \sqrt{n_{\text {core }}^{2}-n_{\text {clad }}^{2}} .
$$

In the approximation $V_{\text {core }} \gg 1$, one can show that the number of bound modes in the core is $\propto V_{\text {core }}^{2} / 2$. When $V_{\text {core }} \leq 2.405$, the only bound mode that can propagate inside the core is the $\mathrm{HE}_{11}$ mode: in this case, the fiber is single-mode, and the $\mathrm{HE}_{11}$ mode is called the fundamental mode. We also define the dimensionless parameter $V_{\text {clad: }}$

$$
V_{\text {clad }}=\frac{2 \pi}{\lambda} R \sqrt{n_{\text {clad }}^{2}-n_{\text {air }}^{2}},
$$

where $n_{\text {air }}$ is the air index of refraction. For $V_{\text {clad }} \gg 1$, the number of modes that are guided by the cladding-to-air interface is $\propto V_{\text {clad }}^{2} / 2$. In this Chapter, we are interested 

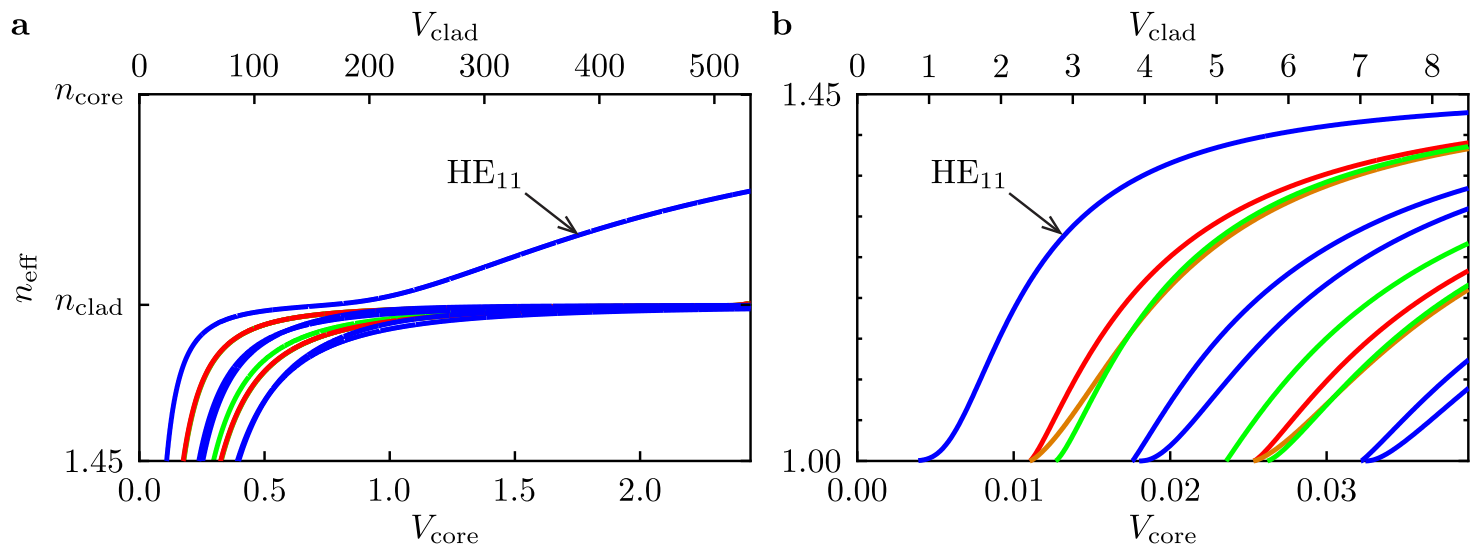

Figure 3.1: Modal evolution in a tapered fiber. a, Dispersion relations for the first few excited modes of the families $l=0$ (TE modes are in red and TM modes in orange), $l=1$ (blue curves) and $l=2$ (green curves). The curves were calculated using the fully vectorial finite difference mode solver from commercial software FIMMWAVE ${ }^{2}$. For the largest values of $V_{\text {core }}$ and $V_{\text {clad }}$, the fiber is initially single mode. When $V_{\text {core }} \simeq 0.76$, the fundamental mode can not be guided as a core mode anymore, and escapes into the cladding. b, Zoom of the dispersion relations for low fiber radii. As we decrease $V_{\text {core }}$ and $V_{\text {clad }}$, higher-order modes get cutoff progressively. Below $V_{\text {clad }}=2.405$, the nanofiber is single mode.

in the propagation, through a tapered fiber, of the fundamental, $\mathrm{HE}_{11}$ mode.

\subsection{Mode evolution in a tapered fiber}

We initially launch the fundamental mode $\mathrm{HE}_{11}$ in the core of the unpulled fiber. In order to efficiently launch the fundamental mode into the nanofiber, the light has to remain confined to the $\mathrm{HE}_{11}$ everywhere inside the tapered fiber. As the light propagates through the fiber, it first encounters a taper where the radii $a$ and $R$ (and thus $V_{\text {core }}$ and $V_{\text {clad }}$ ) decrease ${ }^{3}$. The plots in Figure 3.1a and Figure 3.1b show the evolution of the effective indices $n_{\text {eff }}$ as a function of $V_{\text {core }}$ and $V_{\text {clad }}$ of the first few modes of families with $l=0,1$ and 2 for light of wavelength $\lambda=780.24 \mathrm{~nm}$. There are three important steps in the propagation of the $\mathrm{HE}_{11}$ mode through the taper:

- Initially, $V_{\text {core }}=2.4412$, and the effective index of the fundamental mode verifies $n_{\text {clad }}<n_{\text {eff,HE }} \leq n_{\text {core }}$. The light is guided by the core-to-cladding interface, and propagates as a core mode. As $V_{\text {core }}$ decreases, $n_{\text {eff,HE }}$ decreases and approaches $n_{\text {clad }}$.

\footnotetext{
${ }^{2}$ FIMMWAVE/FIMMPROP by Photon Design Ltd., http://www.photond.com.

${ }^{3}$ We assume that the core and the cladding radii decrease at the same rate along the taper, which implies that there is no diffusion of the core into the cladding during the tapering process.
} 
- When $V_{\text {core }} \simeq 0.76$, we observe that $n_{\text {eff, } \mathrm{HE}_{11}}=n_{\text {clad }}$, showing that the core radius has become too small to support the fundamental mode $(a \simeq 0.78 \mu \mathrm{m})$. The light escapes into the cladding and is guided by the cladding-to-air interface. At this point, the size of the waveguide $(R \simeq 19.4 \mu \mathrm{m})$ is large compared to the wavelength, and therefore: $V_{\text {clad }} \simeq 165 \gg 1$. As a consequence, the number of modes that are accepted inside the cladding, together with the fundamental mode, is of the order of $10^{4}$. Moreover, we observe in Figure 3.1a that the loworder cladding modes all have similar indices of refraction $n_{\text {eff }} \simeq n_{\text {clad }}$, showing that energy can be easily transferred from the $\mathrm{HE}_{11}$ mode to higher-order modes. As a consequence, the moment when the radius of the fiber waist reaches $R \simeq$ $19.4 \mu \mathrm{m}$ is a critical step in the pulling process.

- Further decreasing $R$ (or $V_{\text {clad }}$ in Figure 3.1b), we observe that the effective indices decrease and approach $n_{\text {air }}$. The effective indices of the higher-order modes successively reach their cutoff condition $n_{\text {eff }}=n_{\text {air }}$, where they stop being guided by the fiber. When $V_{\text {clad }}=2.405(R \simeq 300 \mathrm{~nm})$, the fiber is single mode again: only the fundamental mode $\mathrm{HE}_{11}$ is transmitted through the fiber, whereas light that has been transferred to higher-order modes during the propagation radiates in the air.

As it propagates through the tapered fiber, the fundamental mode experiences drastic changes. Energy transfers between modes can occur, especially at the moment when the mode escapes from the core to the cladding, and where the splitting between adjacent propagation constants is the smallest. For a perfectly cylindrical fiber, energy transfers only occur between modes of same $l$. Energy transfers between modes of different $l$ are a consequence of the presence of asymmetries.

\subsection{Adiabatic tapers}

With a typical radius of $\simeq 250 \mathrm{~nm}$, the nanofibers used for trapping atoms are single mode. Higher-order modes that are excited in the tapers subsequently reach their cutoffs and radiate into the air. Any higher-order excitation in the taper leads to losses inside the dilution fridge. As a consequence, we want the fundamental mode to evolve adiabatically through the taper [Snyder and Love (1983)] so as not to excite higher-order modes. Adiabaticity can be thought in a simple optical sense, as making sure that there is always total internal reflection as the ray propagates. As a 


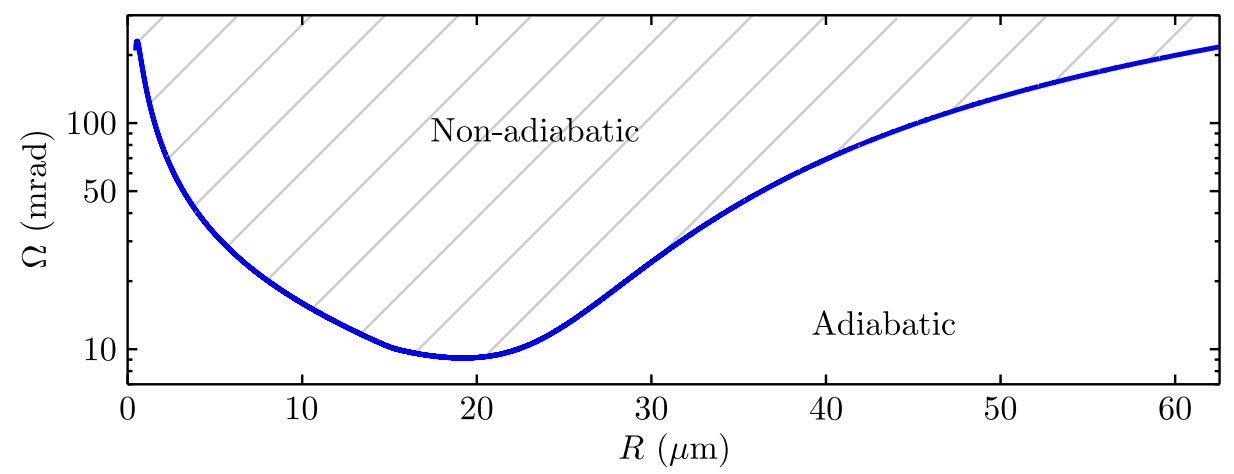

Figure 3.2: Adiabaticity criteria. For taper angles that are small enough, the fundamental mode evolves adiabatically in the taper. The blue curve gives an upper limit (obtained when $z_{t}=z_{b}$ ) on the taper angles one has to use top reach adiabaticity.

consequence, adiabaticity is reached for tapers that are long enough, more precisely when the characteristic length of the taper:

$$
z_{t}=\frac{R}{\tan \Omega}
$$

is large compared to the characteristic beat length (the spatial frequency of the beating) between the fundamental mode and the first excited mode of the family $l=1$ (the $\mathrm{EH}_{11}$ mode):

$$
z_{b}=\frac{2 \pi}{\beta_{\mathrm{HE}_{11}}-\beta_{\mathrm{EH}_{11}}}=\frac{\lambda}{n_{\mathrm{eff}, \mathrm{HE}_{11}}-n_{\mathrm{eff}, \mathrm{EH}_{11}}} .
$$

Solving the equation $z_{b}=z_{t}$, we obtain an upper limit on the taper angle $\Omega$ as a function of $R$. In order to obtain an adiabatic taper $\left(z_{t} \gg z_{b}\right)$ the taper angles must be small compared to the ones indicated by the blue curve in Figure 3.2. As expected, we observe that the adiabaticity condition is the most stringent for $R \simeq 20 \mu \mathrm{m}$, where the light escapes from the core to the cladding. Our $2 \mathrm{mrad}$ linear tapers show angles that are well below this adiabaticity threshold everywhere along the taper, and are consequently adiabatic according to this criteria.

\subsection{Intermodal energy transfer in a $2 \mathrm{mrad}$ tapered fiber}

We analyze the propagation of the $\mathrm{HE}_{11}$ mode through a $2 \mathrm{mrad}$ tapered fiber by monitoring the transmission of a few milliwatts of light of wavelength $780.24 \mathrm{~nm}$ during the tapering process. Figure 3.3a shows the transmission monitoring setup for 


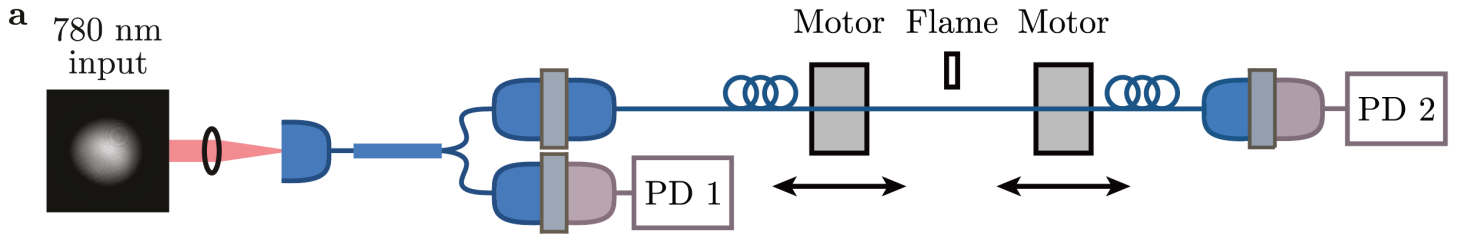

b

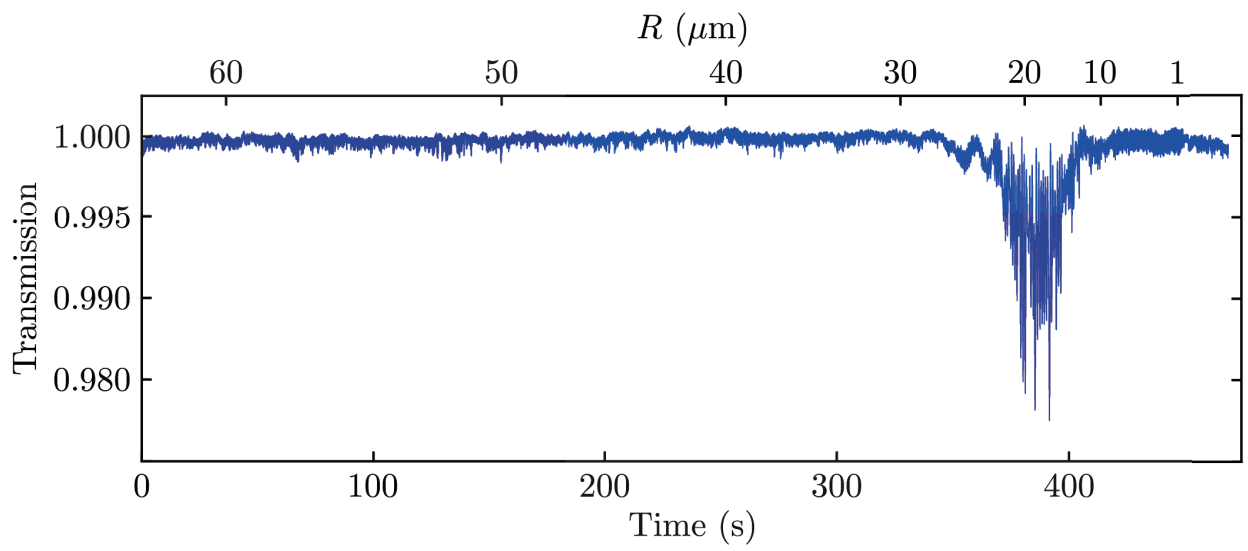

c

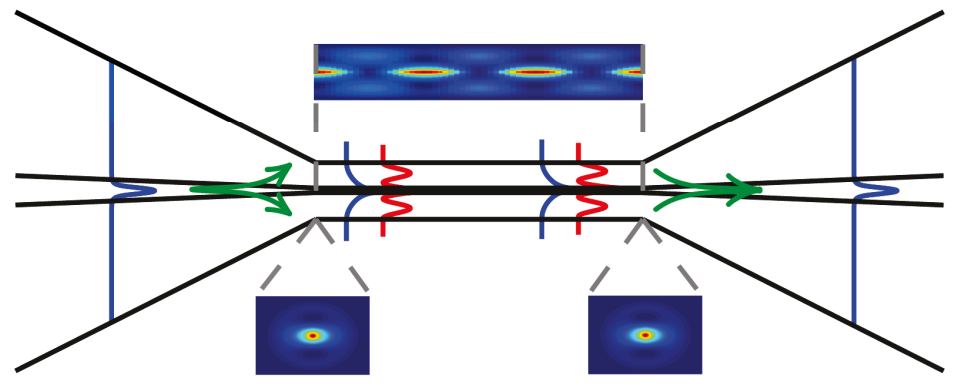

d

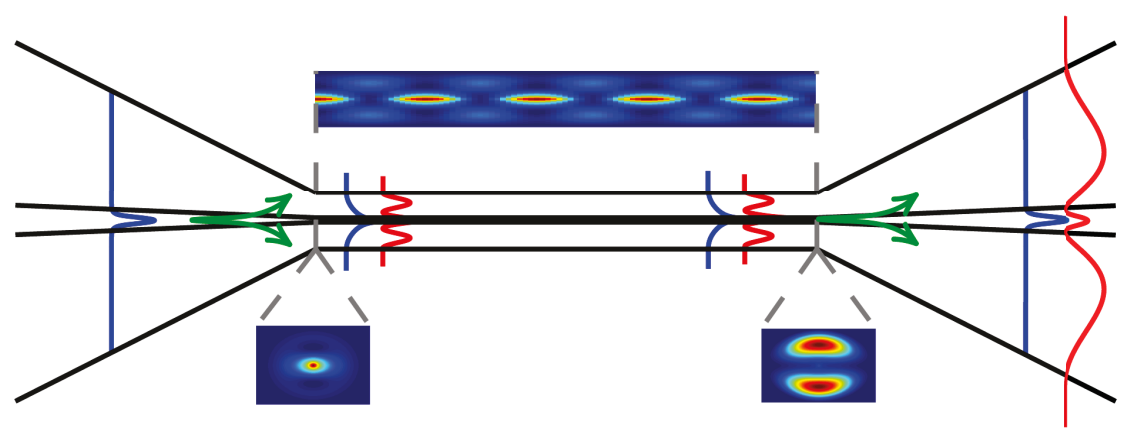

Figure 3.3: Transmission of the fundamental mode through a tapered fiber. a, Experimental setup to monitor the transmission when launching the fundamental mode. $\mathbf{b}$, Measured transmission of the $\mathrm{HE}_{11}$ mode while tapering the fiber. The top axis shows the time evolution of the radius of the fiber waist, as calculated using our pulling algorithm. Around $R=20 \mu \mathrm{m}$, we observe the presence of beating. c, Schematic of the modal evolution in the transition region. All the power is initially contained in the fundamental mode (blue profile). When the core of the fiber becomes too small compared to the wavelength, the light escapes into the cladding (green arrows) and some higher-order modes can be excited (red profile). As they propagate through the fiber, the modes show some beating. d, The quantity of light that couples back to the core in the second taper depends on the fiber length, which leads to the observation of some beating in the transmission signals. 
the fundamental mode. We launch the input beam into a fiber and split the light using an in-fiber beam splitter. One output is connected to a photodetector to measure the laser power while the other output is fusion spliced to the fiber placed in the fiber puller. Using another photodetector, we record the light intensity transmitted through the fiber during the pull.

\subsubsection{Transmission signal}

Figure 3.3b shows the obtained transmission signal as a function of time and fiber radius. We clearly observe in the transmission signal the different steps of the modal evolution described earlier:

- The mode is initially launched in the core of the single-mode fiber. We observe a constant transmission during the first $100 \mathrm{~s}$ of the pull, showing that the fiber is completely adiabatic for radii that are larger than $30 \mu \mathrm{m}$.

- Around $R=20 \mu \mathrm{m}$, we observe some oscillations in the transmission signal. This corresponds to the place where the fundamental mode escapes from the core to the cladding. The oscillations show that some energy has been transferred to some higher-order modes as we will demonstrate later.

- As we continue to thin the fibers, the higher-order modes that have been excited cut off and couple to radiation modes in the air. Below $R=300 \mathrm{~nm}$, the nanofiber is single mode, and the transmission signal is steady again. We measure a transmission of $99.95 \pm 0.02 \%$.

\subsubsection{Explanation of the observed beating}

Energy transfers to higher-order modes occur during the core-to-cladding transition because of non-adiabaticities. When this is the case, several modes co-propagate in the fiber with different propagation constants and thus accumulate the phase differences [Orucevic, Lefèvre-Seguin, and Hare (2007)]:

$$
\Phi_{i, j}(L)=\int_{0}^{L}\left[\beta_{i}(R(z))-\beta_{j}(R(z))\right] \mathrm{d} z
$$

where $L$ is the fiber stretch and $\beta_{i}$ (resp. $\beta_{j}$ ) is the propagation constant of mode $i$ (resp. mode $j$ ). Depending on the fiber length, the phase accumulation between the modes leads to a different field distribution when the light re-enters the fiber core in the second taper at $R \simeq 20 \mu \mathrm{m}$. The fraction of energy that couples back into the core 


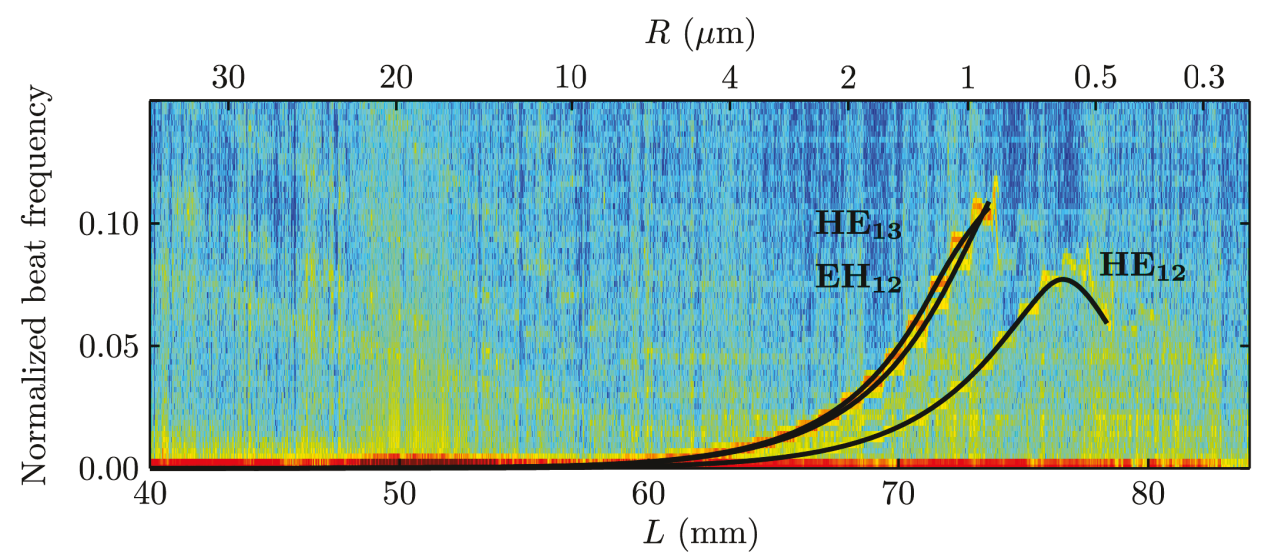

Figure 3.4: Spectrogram of the transmission signal obtained for the $2 \mathrm{mrad}$ tapered fiber. The spectrogram shows the power spectral density (PSD) of the transmission signal of Figure 3.3b as a function of the fiber stretch $L$ and the spatial angular frequency. The top axis shows the time evolution of the waist radius as calculated using our pulling algorithm. The spectrogram shows the beating between the fundamental mode and three higher-order modes after the fundamental mode escapes from the core to the cladding $(R \simeq 20 \mu \mathrm{m})$.

depends on the field distribution at this point. If the modes travel through an integer number of beat lengths (see Figure 3.3c), the field distribution returns to its initial input and all the energy couples back into the core. If the modes experience a noninteger number of beat lengths (see Figure 3.3d), the field distribution is different from what it was initially and only a fraction of energy can couple back into the core. At the fiber output, we only measure on the detector the quantity of light that coupled back into the fiber core and as a consequence, we observe some beating in the transmission signal.

\subsubsection{Spectrogram of the transmission signal}

As we pull the fiber, the phase does not accumulate at a constant rate, and as a consequence, the spatial frequency of the beating $K$ is a function of the total stretch L [Orucevic, Lefèvre-Seguin, and Hare (2007)]:

$$
K_{i, j}(L)=\frac{1}{2 \pi} \frac{d \Phi_{i, j}}{d L} .
$$

We extract the evolution of the frequencies contributing to the beating using spectrograms, which plot local, windowed Fourier transforms of the transmission signal as a function of time. Figure 3.4 displays the spectrogram of the transmission signal of Figure 3.3b. We observe that several lines appear around $R=20 \mu \mathrm{m}$, at the moment where the fundamental mode escapes from the core. Each curve shows the beating 
between the fundamental mode and a higher-order mode that has been excited due to the presence of non-adiabaticities in the fiber profile. The curves terminate before the end of the pull, when the excited mode cuts off. Using the calculated dispersion relations for the fiber (see Figure 3.1) as well as our pulling algorithm that simulates the evolution of the fiber profile after each step of the pull (see Chapter 2) we calculate the expected spatial frequencies for $\simeq 1000$ pairs of modes of different families $(l=1$ to 6$)$ and order $(m=1$ to 20$)$. Without any adjustable parameter, we observe that three of the calculated curves (see black lines in Figure 3.4) show a perfect matching with the three ones observed experimentally. Using the spectrograms, we are thus able to identify the higher-order modes that are excited during the pull. We determine that the excited modes are $\mathrm{HE}_{12}, \mathrm{EH}_{12}$ and $\mathrm{HE}_{13}$. All those modes belong to the same family of modes as $\operatorname{HE}_{11}(l=1)$, which shows that the cylindrical symmetry of our linear tapers is conserved during the pull.

\subsection{Quality of our fibers}

We have measured a $99.95 \pm 0.02 \%$ transmission for a 2 mrad tapered fiber. Using the commercial software FIMMWAVE, we calculate an expected ${ }^{4}$ fundamental mode transmission of $99.94 \%$. The measured value is in agreement with the expected one, which confirms that for that pull, the main source of losses come from non-adiabaticities in the fiber profile and that we are not subject to other technical limitations of our pulling apparatus. For other fiber profiles with a varying slope, a spectrogram analysis has shown that modes of different $l$ get excited, revealing the presence of asymmetries in the fiber profile. We conclude that linear tapers with a constant slope are optimal for our fiber puller, since they do not introduce any asymmetries that could couple modes with different $l$.

\subsection{Nanofibers for a hybrid quantum system}

In view of introducing our nanofibers close to a superconducting surface in a cryogenic environment, our study of the losses in our tapered optical fibers allowed us to draw the following conclusions:

- For the linear $2 \mathrm{mrad}$ fiber used in this Chapter, the tapers are $\simeq 4 \mathrm{~cm}$ long,

\footnotetext{
${ }^{4}$ We simulate the propagation of the fundamental mode through a single taper only. We obtain a transmission of $99.97 \%$, which allows estimating the total transmission as the square of the obtained value.
} 
which is compact enough given the geometrical constraints we have in the cryostat. Decreasing $\Omega$ would lead to even more adiabatic fibers, but would also produce longer tapers.

- For $\simeq 30 \mathrm{~mW}$ of trapping power, a $99.95 \%$ transmission means that $15 \mu \mathrm{W}$ of power are scattered in the dilution refrigerator, which is well below the $200 \mu \mathrm{W}$ cooling power of the cryostat. The observed losses in our $2 \mathrm{mrad}$ linear tapered fiber are thus low enough in terms of generated heat.

- We have demonstrated that the observed losses are mainly due to non-adiabaticities in the taper profile. In this case, the photons escape from the fiber tapers, at the position where the higher-order modes cutoff. This part of the fiber is not facing directly the superconducting chip, which thus leaves some latitude to protect the resonator using beam blocks .

- Finally, another source of losses that is present in optical fibers and that we have not mentioned yet is Rayleigh scattering. Rayleigh scattering is due to the presence of density fluctuations and impurities in fused silica, and leads to $\simeq 3 \mathrm{~dB} / \mathrm{km}$ of losses for light of wavelength $\simeq 780 \mathrm{~nm}$ [Snyder and Love (1983)]. For $\simeq 30 \mathrm{~mW}$ of input power, the $\simeq 100 \mu \mathrm{m}$ portion of nanofiber that is directly facing the superconducting chip thus scatters $\simeq 1.5 \mathrm{nW}$ of power $^{5}$. When the fiber is placed directly above the chip, we estimate that about half of these scattered photons hit the superconducting chip, which corresponds to $\simeq 750 \mathrm{pW}$ of absorbed power.

As a conclusion, our measurements show great promise for the integration of our nanofibers into a $10 \mathrm{mK}$ environment. The obtained losses are compatible with the available cooling power of the dilution fridge. However, due to the extreme sensitivity to light of the original resonator, the estimated amount of power scattered directly on the superconducting chip $\lesssim 1 \mathrm{nW}$ remained too high. As a consequence, our measurements have led to the fabrication of another superconducting resonator. This resonator incorporates in a new design of the system that minimizes the illumination of the resonator, as we will see in Outlook.

\footnotetext{
${ }^{5}$ For the red-detuned light used for trapping, the quantity of losses due to Rayleigh scattering is even smaller. Here, we give a slight overestimate of the quantity of scattered light.
} 


\title{
Intermodal energy transfer in a tapered optical fiber: optimizing transmission
}

\author{
S. Ravets, ${ }^{1,2}$ J. E. Hoffman, ${ }^{1}$ P. R. Kordell, ${ }^{1}$ J. D. Wong-Campos, ${ }^{1}$ S. L. Rolston, ${ }^{1}$ and L. A. Orozco ${ }^{1, *}$ \\ ${ }^{1}$ Joint Quantum Institute, Department of Physics, University of Maryland, and National Institute of Standards and \\ Technology, College Park, Maryland 20742, USA \\ ${ }^{2}$ Laboratoire Charles Fabry, Institut d'Optique, CNRS, Univ Paris Sud, 2 avenue Augustin Fresnel, \\ 91127 Palaiseau cedex, France \\ *Corresponding author: lorozco@umd.edu
}

Received July 26, 2013; revised September 27, 2013; accepted September 30, 2013; posted October 3, 2013 (Doc. ID 194689); published October 25, 2013

\begin{abstract}
We present an experimental and theoretical study of the energy transfer between modes during the tapering process of an optical nanofiber through spectrogram analysis. The results allow optimization of the tapering process, and we measure transmission in excess of $99.95 \%$ for the fundamental mode. We quantify the adiabaticity condition through calculations and place an upper bound on the amount of energy transferred to other modes at each step of the tapering, giving practical limits to the tapering angle. ( 2013 Optical Society of America

OCIS codes: $\quad$ (060.2310) Fiber optics; (060.2280) Fiber design and fabrication; (060.2270) Fiber characteri-

zation; (060.2400) Fiber properties; (350.5500) Propagation; (030.4070) Modes.

http://dx.doi.org/10.1364/JOSAA.30.002361
\end{abstract}

\section{INTRODUCTION}

The study of mode coupling in an optical waveguide [1] is fundamentally important for good control of connectorization and transmission. This is especially true for tapered optical fibers with subwavelength waists, where light propagates in a mode that exhibits a large evanescent component propagating outside the waveguide. Nanofibers are ideal for probing nonlinear physics, atomic physics, and other sensing applications [2-5]. As the light propagates through the taper, it successively encounters regimes where the fiber is single mode, multimode, and then single mode again. Careful design of adiabatic tapers leads to ultralow loss fibers []. Adiabatic criteria give an upper limit on how steep a taper can be, but are too vague for optimization of transmission. Here we are interested in giving quantitative bounds and constraints on the taper geometry.

Using a spectrogram analysis of the transmission signal through the fiber [7], we are able to identify the modes excited during the tapering process and extract the amount of energy transferred to each of these modes. Using this analysis, we show the importance of geometry control and fiber cleanliness in reaching transmissions as high as $99.95 \%$ in commercial fibers at $780 \mathrm{~nm}$. Our nanofibers can handle more than $400 \mathrm{~mW}$ of optical power in ultrahigh vacuum. After reaching the cut-off radius, the excited modes couple to radiative modes [8] and diffract outside of the fiber.

Our analysis provides a path to fully model the electromagnetic field evolution in a nanofiber. This is crucial for a complete modeling of the coupling between light and matter $[\underline{9}, \underline{10}]$. In the example of atoms trapped on the evanescent field around a nanofiber waist, it is necessary to know the coupling coefficients between the modes of the field and the atoms. This work details the modal evolution in the fiber, opening perspectives for the design of even more adiabatic fibers, making them usable in extreme conditions [11].

This paper presents our protocols, diagnostics, and characterization tools for fabricating nanofibers. It is structured as follows: we first overview our experimental goals and conditions in Section 2. Section 3 presents the modal evolution in tapered fibers. We then study in Section 4 adiabaticity in tapered fibers. Section $\underline{5}$ analyzes in more detail the transmission signal. We introduce the spectrogram to analyze the transmission [7] in Section 6 . By modeling and diagnosing the fiber pull, we identify in Section $\underline{7}$ crucial elements to improve the transmission. Section $\underline{8}$ looks into the other losses present in the fiber. Section $\underline{\underline{9}}$ is the conclusion of the paper.

\section{MOTIVATION AND CONSTRUCTION OVERVIEW}

Controlling neutral atoms with dipole traps is a successful and promising avenue for the implementation of a growing number of scientific and technical applications [12]. The offresonant interaction between light and atoms in the presence of an intensity gradient produces a dipole force that can generate traps: detuning below atomic resonance attracts atoms to go to the most intense region, creating an optical tweezer $[13,14]$, and above-resonance detuning keeps the atom in the intensity minima, requiring more complicated geometries [15-18]. One drawback of optical tweezers obtained by tightly focusing a laser beam comes from diffraction, which limits the trapping volume extension in the axial direction. One solution to this limitation is the use of optical nanofibers $[\underline{4}, \underline{9}, \underline{19}]$. These devices offer enough light confinement and guidance to trap atoms over a few centimeters in the axial direction and present the advantage of being integrable to other devices $[\underline{10}, \underline{20}-22]$. We are interested in introducing this device into 
a $12 \mathrm{mK}$ cryogenic environment to probe interactions between a trapped neutral atom and a superconducting circuit [11].

Following the work of Warken in [23], we produce our fibers using a heat-and-pull technique, summarized below (see Ref. [24] for details on the algorithm and the hardware). An oxyhydrogen flame at stoichiometric combination brings a $0.75 \mathrm{~mm}$ long fiber portion to a temperature that exceeds its softening point. Two high-precision computer controlled motors pull on the fiber ends at a typical velocity of $0.1 \mathrm{~mm} / \mathrm{s}$. We use an algorithm that relies on conservation of volume, which calculates the trajectories of the motors to produce a fiber of chosen geometry. The code is available at the DRUM Digital Repository of the University of Maryland [25].

We pull a SM800 fiber from Fibercore that has a numerical aperture of 0.12 and a cutoff wavelength of $794 \mathrm{~nm}$. Using the Sellmeier coefficients provided by Fibercore, we determine the core $\left(n_{\text {core }}=1.45861\right)$ and the cladding $\left(n_{\text {clad }}=\right.$ 1.45367) indices of refraction. The pull is divided into approximately 100 steps, such that the taper is composed of a series of sections small enough to be considered linear. Our tapers are generally composed of a section with a constant few mrad taper angle that reduces the fiber to a radius of $6 \mu \mathrm{m}$, and then connects to an exponential section that gently reaches submicrometer radii (on the order of $250 \mathrm{~nm}$ ). The central waist is uniform, and its length can be between $5 \mathrm{~mm}$ and $10 \mathrm{~cm}$. A pull generally lasts for a few hundreds of seconds.

\section{MODAL EVOLUTION}

\section{A. Modes in a Cylindrical Waveguide}

The description of modes in a cylindrical waveguide using Maxwell equations can be found in several references, e.g., $[8,26]$. The modal fields vary as $\exp \left[i\left(\beta_{l m} z-\omega t\right)\right]$, where $\beta_{l m}$ is the propagation constant of the mode of symmetry and order $(l, m)$. The propagation of light inside a two-layered step index fiber depends on the $V$ parameter of the fiber,

$$
V=\frac{2 \pi}{\lambda} a \sqrt{n_{1}^{2}-n_{2}^{2}}
$$

where $a$ is the core radius, $n_{1}$ is the core index of refraction, $n_{2}$ is the surrounding medium index of refraction, and $\lambda$ is the free space wavelength. The relation between $\beta_{l m}$ and the $V$ parameter is called the dispersion relation of mode $(l, m)$. In our tapers, we can approximate the fiber as a two-layer step index cylindrical waveguide in two regions: at the beginning of the taper, the light is confined to the core and guided through the core-to-cladding interface. We assume that the core and the cladding radii decrease at the same rate along the taper, which implies that there is no diffusion of the core into the cladding during the tapering process. In the waist, what was initially the core in the center of the fiber is now negligible $\left(a_{\text {core }} \approx 10 \mathrm{~nm} \ll \lambda\right)$. The light is then guided through the cladding-to-air interface.

\section{B. Three-Layer Fiber}

Since we continuously decrease the fiber radius during the pull, the fundamental mode leaks from the core to the cladding. In that region, the presence of the core, the cladding, (a)

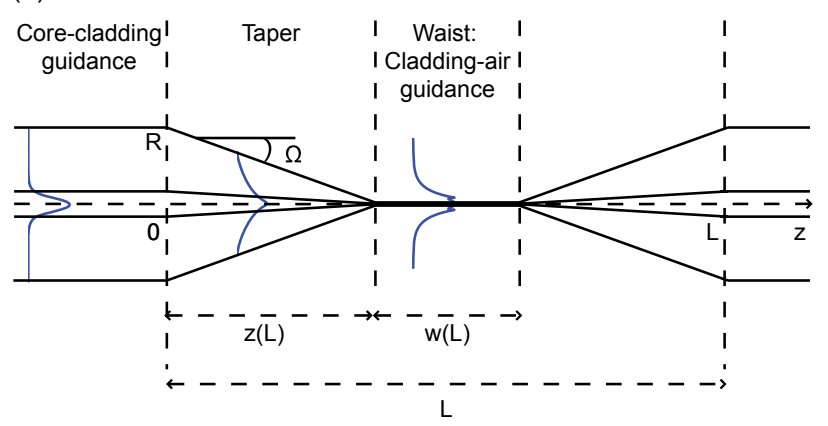

(b)

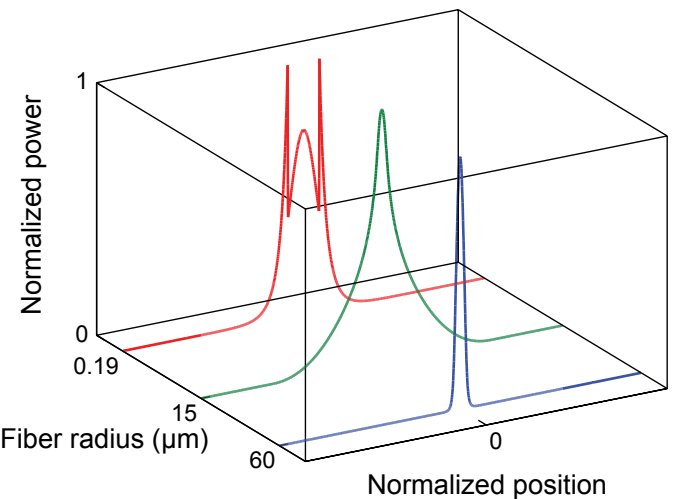

Fig. 1. (a) Schematic of the stretched fiber. At a given time, the fiber is composed of two tapers and a uniform waist of radius $r$ and length $w$. The total stretch is equal to $L$. (b) Calculated intensity profile of the mode for a radius of fiber equal to $60 \mu \mathrm{m}, 15 \mu \mathrm{m}$, and $190 \mathrm{~nm}$. Note that the position axes are not quantitative, and have been scaled to make the plots visible. The profiles are normalized to their maximum power.

and the air influence the mode (see Fig. 1). A proper treatment has to take into account all of those interfaces. We model our fibers by a three-layered structure, and we calculate the dispersion relations for a series of modes using the fully vectorial finite difference mode solver from commercial software FIMMWAVE [27]. Figure $\underline{2}$ shows a plot of $n_{\text {eff }}=\beta / k_{0}$ as a function of the radius of the SM800 fiber described in Section 2.

We are interested in modes that are initially launched into the core, thus guided by the core-to-cladding interface. Core modes have most of their energy contained in the core, and their effective indices of refraction satisfy $n_{\text {clad }}<n_{\text {eff }}<n_{\text {core }}$. Figure 2 shows that the $\mathrm{HE}_{11}$ mode effective index is initially greater than $n_{\text {clad }}=1.45367$ (green curve indicated by an arrow). Some higher-order modes from the LP11 family may be accepted in the core, close to their cutoff condition. (The fiber cutoff wavelength is $792 \mathrm{~nm}>780.24 \mathrm{~nm}$, so strictly speaking, we are not working in the fiber single-mode regime.) Experimentally, we filter higher-order modes that have been launched or excited with a $1.27 \mathrm{~cm}$ diameter mandrel, effectively placing us into the single-mode regime.

When the fiber radius decreases, $n_{\text {eff }}^{\mathrm{HE} 11}$ approaches $n_{\text {clad }}$. Since we model the fiber by a three-slab cylindrical waveguide, the cladding area is finite: the core becomes too small to support the fundamental mode around the point where $n_{\text {eff }}^{\text {HE11 }}$ reaches $n_{\text {clad }}(R=19.43 \mu \mathrm{m}$ in Fig. 2$)$. The mode progressively leaks into the cladding to be guided by the cladding-to-air interface. The characteristic length-scale of the waveguide is $R \gg \lambda$, and many modes can be guided by the 


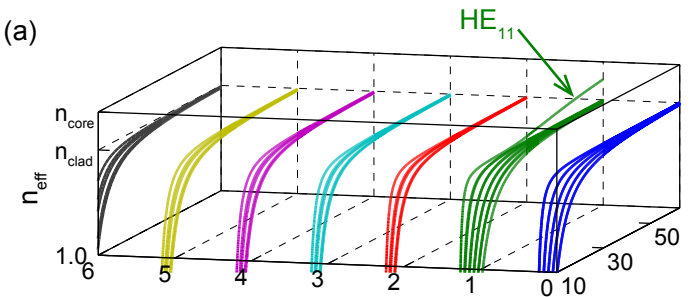

(b)

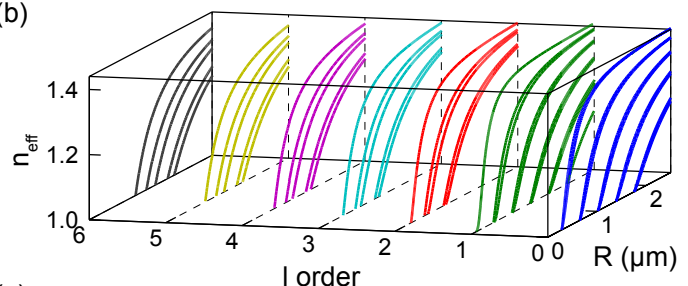

(c)

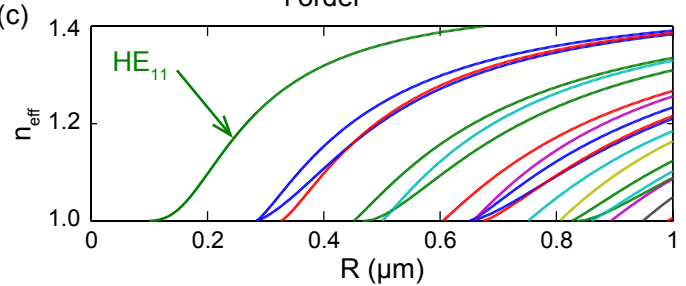

Fig. 2. Dispersion relations for various modes $\mathrm{TE}_{0 m}, \mathrm{TM}_{0 m}, \mathrm{HE}_{l m}$, and $\mathrm{EH}_{l m}(l=1$ to 5$)$ as a function of radius calculated for a three-layer model using FIMMPROP. Here, $n_{\text {air }}=n_{\text {vacuum }}=1$. We show the first few modes of each family. (a) and (b) Threedimensional representation of the dispersions for different mode families. (c) Projection for the smaller values of $R$.

cladding-to-air interface ( $n_{\text {air }}<n_{\text {eff }}<n_{\text {clad }}$ ), together with the fundamental mode. As long as $R \gg \lambda$, the air has little influence on the effective index of many of the accepted modes ( $n_{\text {eff }} \approx n_{\text {clad }}$ for all the modes shown in Fig. 2). The indices are so close to each other that the modes interact and exchange energy easily. For that reason, this is the critical region of the taper, where the adiabaticity condition is the most stringent. By symmetry, for a fully cylindrical fiber, intermodal energy transfer will only happen between modes of the same family (one color in Fig. 2). Energy transfers between modes from different families are a consequence of the presence of asymmetries.

Further decreasing $R$, we observe that the modes' effective indices approach $n_{\text {air }}=1$. The dispersion curves separate, and adiabaticity can again be easily achieved. When the index of refraction of a mode reaches $n_{\text {air }}$, the mode is not guided by the fiber anymore and radiates into the air. This radius, specific to each mode, is called its cutoff. The highly excited modes leave the fiber first, and the number of modes allowed in the waveguide decreases progressively [see Fig. 2(c)]. Under $0.3 \mu \mathrm{m}$, the only mode that can propagate is the $\mathrm{HE}_{11}$ mode, whose index asymptotically approaches 1 . The fiber is once again single mode.

\section{ADIABATICITY IN FIBERS}

Achieving high transmission in nanofibers requires precise control of the taper geometry, where the mode adiabatically escapes from the core to the cladding before coupling back to the core $[8,28]$. High transmission through tapered nanofibers is indicative of their quality $[\underline{6}, \underline{29}]$.

\section{A. Adiabaticity Criterion}

The mode conversion in a taper is strongly related to the taper geometry. If a taper is too short (taper angle too steep), the mode evolution is nonadiabatic, and we observe losses. Inversely, as the taper is lengthened, the mode conversion is more adiabatic. In the limit of a very shallow angle, we intuitively understand that the transmission can reach $100 \%$, since all the energy remains in the fundamental mode throughout the evolution. Following this idea, an adiabaticity criterion has been derived [8] relating the characteristic taper length, $z_{t}$, to the characteristic beating length between two modes, $z_{b}$.

$z_{t}$ is the length associated with the tapering angle $\Omega$ at radius $R$, defined by

$$
z_{t}=\frac{R}{\tan (\Omega)}
$$

$z_{b}$ is the beat length between two modes (the spatial frequency of the beating)

$$
z_{b}=\frac{2 \pi}{\beta_{1}-\beta_{2}}=\frac{\lambda}{n_{\mathrm{eff}, 1}-n_{\mathrm{eff}, 2}}
$$

where $\beta_{1}$ is the fundamental mode propagation constant at radius $R$ and $\beta_{2}$ is the propagation constant at radius $R$ of the first excited mode with the same symmetry as the fundamental mode $\left(\mathrm{EH}_{11}\right)$. Equation (3) relates the beat length to the inverse of the distance between two curves in Fig. 2. Mode conversion in a taper is adiabatic when the fiber is long enough: $z_{t} \gg z_{b}$ [8]. If the two modes are close, $z_{b}$ is large, making the adiabaticity condition more difficult to satisfy. The choice of $\mathrm{EH}_{11}$ gives the most stringent condition on the fiber length, as it produces the shortest beat length between the fundamental mode and any mode with symmetry $l=1$. Nevertheless, this condition remains too vague when one wants to optimize the taper geometry for a given transmission.

Using the dispersion relations from FIMMPROP, we can solve the equation $z_{t}=z_{b}$, when the beat length equals the taper length. The blue curve in Fig. 3 separates the plane into

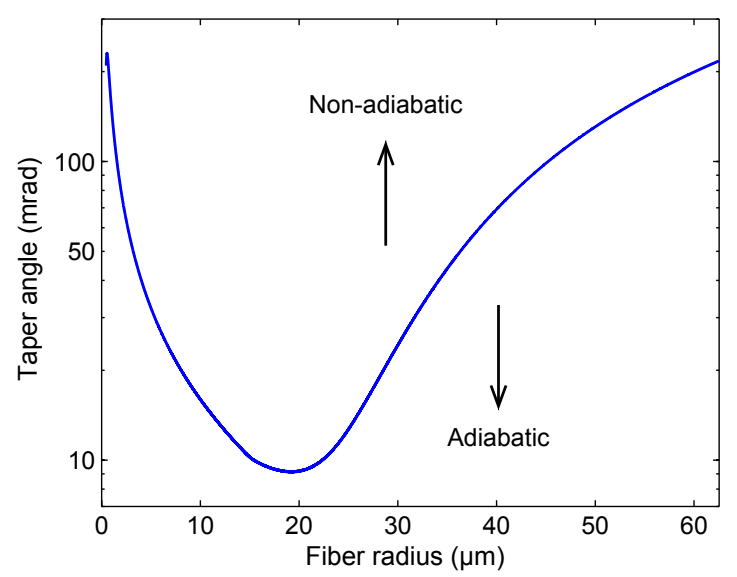

Fig. 3. Upper boundary for the taper angle $\Omega$ as a function of the radius of the fiber set by $z_{b}=z_{t}$. Note the logarithmic scale for the vertical axis. The core-to-cladding diameter ratio for this fiber is $2.535 / 62.55$ and is fixed for the entirety of the pull. $n_{\text {core }}=$ 1.45861 and $n_{\text {clad }}=1.45367$. 
two regions: in order to be adiabatic, taper angles need to be much smaller than the ones indicated on the curve. Above the curve, the angles correspond to nonadiabatic tapers.

Figure 3 gives an upper limit on the taper angle at a specific radius using the condition $z_{b}=z_{t}$ from Eqs. (2) and (3). It does not provide any quantitative information on the intermodal energy transfer for a given taper: calculations in Section 4.D show that the angles in Fig. 3 lead to large energy transfers. We are interested in producing fibers with high transmissions, greater than $99.90 \%$, and we need to find the optimal geometry necessary to reach a specific transmission.

\section{B. Transmission of a Tapered Fiber Section}

We perform numerical simulations with FIMMPROP to explore the parameter space and find the optimal adiabatic profile for a given transmission. The fiber tapers from $62.55 \mu \mathrm{m}$ radius down to $250 \mathrm{~nm}$ radius. Using the indices of refraction for our SM800 fiber (see Section 2), we divide the taper into a discrete series of linear sections (32 sections in this work). At the end of each section we project the output field into the first family of modes (here we use the 15 first modes of family 1) to obtain specific amplitude and phase information in terms of the excited modes. The $S$ matrix, relating input and output, contains all the mode phases and amplitudes necessary to relate the input and output fields of that particular section.

Figure $\underline{4}$ shows the modal evolution in a tapered section when the input is in the fundamental mode. When $\Omega$ is small (or the length $L$ is large), the modal evolution is adiabatic and the transmission approaches unity as seen in the plot for the
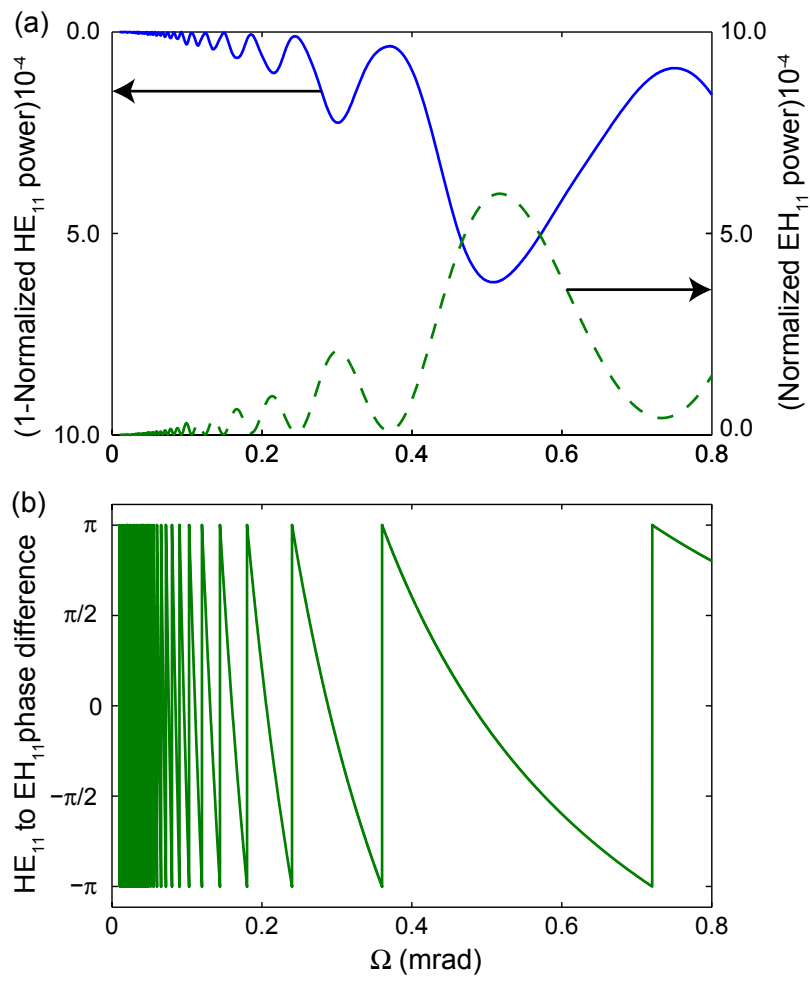

Fig. 4. Transmission of one section (tapering from 25.5 to $23.5 \mu \mathrm{m}$ ) as a function of angle when the input is the fundamental mode. (a) Amplitude of the fundamental $\mathrm{HE}_{11}$ (continuous blue) and the first higher-order mode $\mathrm{EH}_{11}$ (dashed green). (b) Phase difference between the fundamental and the first higher-order mode. normalized power in the $\mathrm{HE}_{11}$ mode in Fig. 4 . When $\Omega$ increases, some energy couples to higher-order modes, and the fundamental mode transmission decreases. For the small angles considered here, Fig. 4(a) shows energy transfer to one mode only ( $\mathrm{EH}_{11}$ mode-dashed green curve). Energy transfer to other modes ( $\mathrm{HE}_{12}$ mode and higher) is negligible within the resolution of the plot. The oscillations in the transmission are due to modal dispersion in the fiber, which leads to spatial beating: two modes see different indices of refraction and accumulate a phase difference as they propagate through the fiber (see Section 3.A). The phase accumulation increases and can become large for small angles (or increased fiber length). In the particular situation of Fig. $\underline{4(\mathrm{~b})}$ where only two modes beat together, the $\mathrm{EH}_{11}$ power reaches local maxima for zero phase differences and local minima for $\pi$ phase differences. The situation can become complex when more than two modes are excited. Consequently, there exist some situations in which large intermode energy transfer during propagation still results in good fundamental mode transmission. Thanks to mode spatial interferences, most of the energy can couple back to the fundamental mode during the propagation. In this case, one relies on interference in the nonadiabatic effects.

\section{Genetic Algorithm}

We obtain the total transmission $T$ after calculating the projection on the fundamental mode of the full $S$ matrix, given by the product of all $S$ matrices for each section. We want to find the shortest tapered fiber given a target transmission. For this task, we use the genetic algorithm function from MATLAB to find an optimal solution. This approach is efficient with large problems and allows the use of information of previous runs to improve the computing time in contrast with Monte Carlo methods and other optimization techniques that use deterministic approaches. Typical parameters for the algorithm are a population size of 500 , a crossover probability of 0.7 , a mutation probability of 0.025 , and a number of generations of 500 . The genetic algorithm can probe a large parameter space: for each section, we have calculated $1500 S$ matrices, for angles that can vary between 10 and $1.57 \mathrm{rad}$. We run the algorithm more than 1000 times with different sets of parameters to approach the global minimum.

\section{Fully Adiabatic Fiber}

We will define total transmissions greater than $T=0.9990$ as a fully adiabatic fiber. In this section, we investigate fibers with limited intermode energy transfers during the pull. This means that the power contained in the fundamental mode cannot deviate too much from $T$ everywhere in the taper. In this case, the interference between higher-order modes plays a minimal role in the final transmission. We benefit from the robustness with respect to variation in parameters that is associated with an adiabatic process. We obtain the most strict condition on the angles that can be used to reach a specific transmission. We run the algorithm with the added condition that the transmission of each small taper section is greater than $T$. That way, we make sure that the fundamental mode power is greater than $T$ at 32 points in the taper. Between those points the fundamental mode power can oscillate, but remains constrained around $T$, ensuring the limitation of intermode energy transfer everywhere in the taper. 


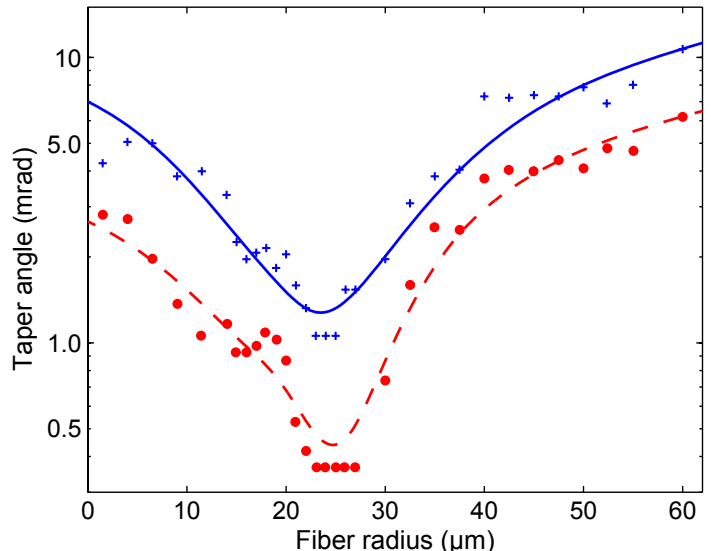

Fig. 5. Optimal adiabatic tapers calculated with the genetic algorithm for $T=99.90 \%$ (blue crosses and continuous line) and $T=$ 99.99\% (red-dotted and -dashed line), where the intermode energy transfers are limited. Each marker corresponds to the optimum angle for a section. The lines are guides for the eye.

Figure 5 shows results from the genetic algorithm for optimized adiabatic fiber tapers using target transmissions of $99.90 \%$ and $99.99 \%$. We plot the taper angle as a function of the fiber radius as in Fig. 3. We observe similar behavior: large taper angles are allowed for large fiber radii, and then reach a minimum around the transition region at $20 \mu \mathrm{m}$, before increasing again at smaller radii. For $T=0.9999$, the optimal taper in Fig. 5 (red-dashed curve) shows angles as low as $0.4 \mathrm{mrad}, 30$ times smaller than the $z_{b}=z_{t}$ criteria. The results in Fig. $\underline{5}$ give precise bounds on adiabaticity, with minimum power transmitted to higher-order modes. This last point ensures that this algorithm is insensitive to phase effects: the final transmission is not a consequence of constructive interference between several modes and will be independent of perturbation to the fiber geometry.

Figure 6 shows the optimized taper profiles corresponding to $T=0 . \overline{9} 990$ (blue continuous line) and $T=0.9999$ (reddashed line). Strikingly, for $T=0.9999$ the optimized adiabatic taper is only $4.5 \mathrm{~cm}$ long, on the order of typical nonadiabatic taper lengths produced with a heat-and-pull method [24] (the 2 mrad taper presented Section 5 is $\approx 6 \mathrm{~cm}$ long and still presents nonadiabaticities). Note, however, that in Fig. $\underline{6}, \Omega$ varies continuously as a function of

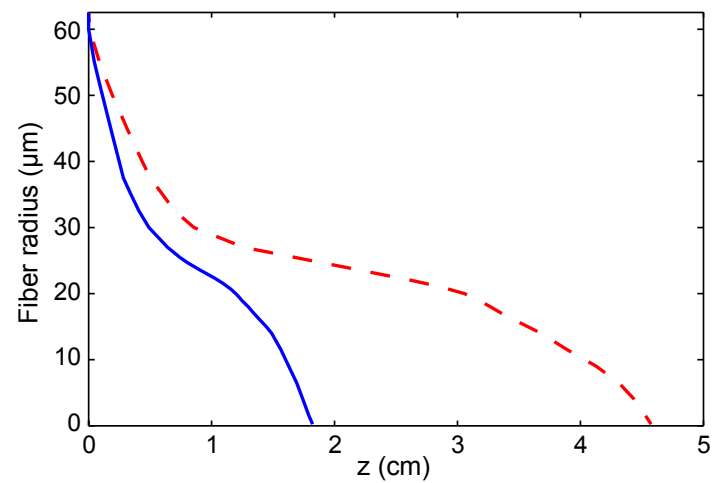

Fig. 6. Optimal taper profiles for $T=99.90 \%$ (continuous blue line) and $T=99.99 \%$ (dashed red line). The profiles are only based on the dots from Fig. 5 and not on the continuous lines. Note that the horizontal axis scale is in centimeters, whereas the vertical axis scale is in micrometers. $z$, and can be large at the beginning of the pull. Experimentally, we show below (see Section 7) that abrupt variations of $\Omega$ during the pull can induce detrimental asymmetries in the taper. With our apparatus, we have precise control of the taper geometry for linear and exponential profiles [24]. Reaching adiabaticity that way would require a linear taper angle $\Omega \approx 0.5 \mathrm{mrad}$, and a substantially increased length. One could chose to use smaller clad fibers [30] or to chemically pre-etch fibers, allowing shorter adiabatic tapers with improved handling.

\section{E. Utilizing Nonadiabaticity}

Limiting intermodal energy transfer in a taper to arbitrarily small values is possible, but can be impractical due to large taper lengths. An alternative approach consists of allowing large energy transfers, yet reaching high transmissions by careful design and phase control in the fiber. Section 3.A shows that different modes interfere together as they propagate in the taper. Taking advantage of this spatial beating, we can design fibers with particular phase combinations that allow high transmission, despite the presence of nonadiabaticities. In this section, we run the genetic algorithm with only a condition on the final transmission $(T \geq 0.9999)$ : intermodal energy transfer in each section is no longer limited. Using this nonadiabaticity, it is possible to produce short hightransmission tapers.

We calculate the shortest fiber length that has $99.99 \%$ total transmission in the fundamental mode using the genetic algorithm. Figure 7 (a) shows that the taper angles allowed here are much larger than the ones presented above in the adiabatic case (Fig. 5). At large fiber radii, the taper angle reaches $\approx 100 \mathrm{mrad}$. Closer to the transition region, the minimal taper angle can still be as large as 2 mrad. From the taper angles used here, we know that the fundamental mode is not (a)

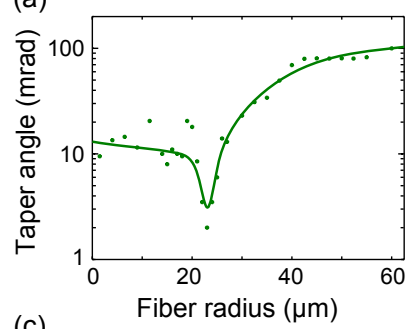

(b)
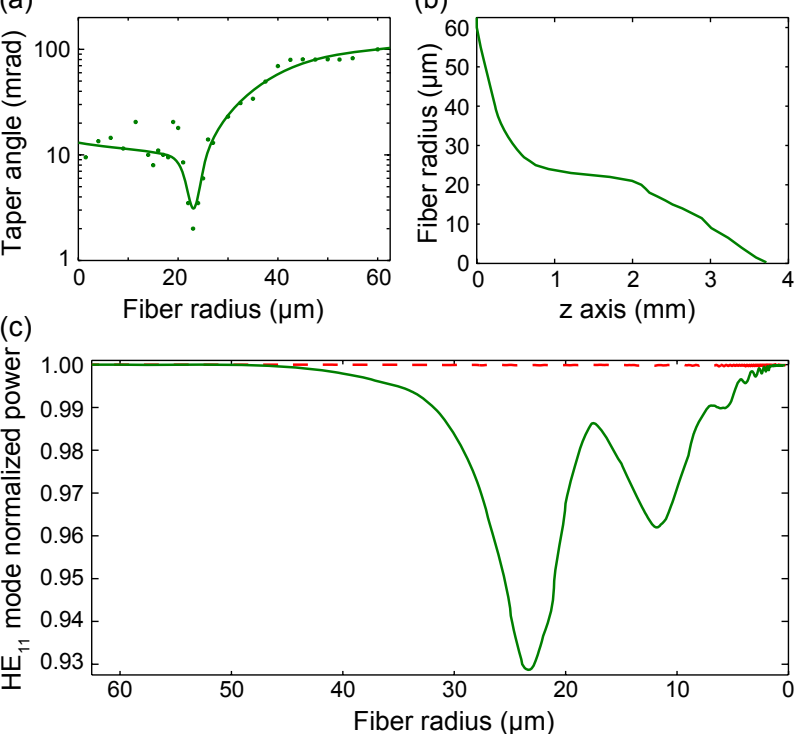

Fig. 7. Study of fiber profile for $99.99 \%$ transmission with optimized length given by the genetic algorithm. (a) Taper angles and fiber radius, squares with the continuous line to guide the eye. (b) Fiber radius and fiber length with a final length of $3.7 \mathrm{~mm}$ using the results in (a). (c) Fundamental mode transmission as a function of fiber radius for the optimized adiabatic fiber (red-dashed line) and the optimized nonadiabatic fiber (green continuous line). 
propagating adiabatically in this taper. Figure 7(b) shows the corresponding profile. Figure 7(c) shows a $99.99 \%$ transmission (green continuous line) fiber with a $3.7 \mathrm{~mm}$ length, a factor of 12 shorter than in the adiabatic case (red-dashed line) calculated using FIMMPROP. This greatly reduces the length requirements for high-transmission fibers, which is particularly relevant for our application.

Using FIMMPROP, we model the tapered structures presented in Figs. $\underline{6}$ and $\underline{7}$ by putting together a succession of 32 linear tapered sections obtained with the genetic algorithm. The input is set to $100 \%$ in the $\mathrm{HE}_{11}$ fundamental mode, and the simulation includes 15 modes of family $l=1$. We calculate the modal evolution (phases and amplitudes) along the taper. In the adiabatic optimized case [red-dashed curve Fig. 7(b)], we confirm that the power contained in the fundamental mode is close to $99.99 \%$ throughout the taper. Higherorder mode excitations are negligible, and the evolution is adiabatic. Using nonadiabaticity [green curve Fig. 7(b)], we observe large energy transfer to higher-order modes. Around $R=23 \mu \mathrm{m}$, more than $7 \%$ of the energy has been transferred to higher-order modes. However, using this particular geometry, the resulting phase combinations lead to high transmission in the fundamental mode.

Nonadiabaticity can lead to high transmission with shorter tapers, which is particularly useful for taper design. In the rest of the paper, we experimentally study fibers that exhibit this behavior. Exploiting nonadiabaticity requires particular attention because of their sensitivity to mode phases: deviations from the calculated profile might lead to situations in which mode interference causes large losses, with less energy ending in the fundamental mode than initially expected. One needs to reproduce the calculated geometry as accurately as possible. As discussed above, producing the taper in Fig. $\underline{7}$ with a continuously varying angle is not the best option for us, due to the presence of large angles and possible experimental asymmetries. Moreover, this particular taper length $(3.7 \mathrm{~mm})$ is too small in comparison to the heating-zone size $(0.75 \mathrm{~mm}$ in our experiment) to accurately produce such a profile. Our typical profiles start with a linear section ( $\Omega$ of a few mrad) down to $6 \mu \mathrm{m}$ radius, followed by an exponential section down to $250 \mathrm{~nm}$ radius. We calculate with FIMMPROP the $\mathrm{HE}_{11}$ mode evolution through such a taper $(\Omega=2 \mathrm{mrad})$ and show that it benefits from nonadiabatic effects, leading to high transmission.

We start by investigating geometries we can produce with good accuracy using our fiber puller. Figure 8 shows the transmission of the first few modes of family $l=\overline{1}$ through a $2 \mathrm{mrad}$ taper. We create a taper with FIMMPROP that reproduces the experimental profile, which has been validated with microscopy measurements [24]. Initially, all the power is contained in the fundamental mode. Around $R=23 \mu \mathrm{m}, \approx 0.4 \%$ of the energy is transferred to higher-order modes because of nonadiabaticities (up to $\mathrm{HE}_{13}$, the fifth mode of family $l=1$ ). This illustrates that non-negligible higher-order mode excitations can be observed below the $z_{b}=z_{t}$ limit (the taper angle $\Omega=$ $2 \mathrm{mrad}$ is at least a factor of five below the $z_{b}=z_{t}$ limit everywhere in the taper). Those modes beat together, and by the end of the taper, $99.97 \%$ of the energy is transmitted through the fundamental mode. For different taper angles, we observe that our typical tapers benefit from nonadiabaticity (see Section 5). If there is still room for optimization, the simplicity

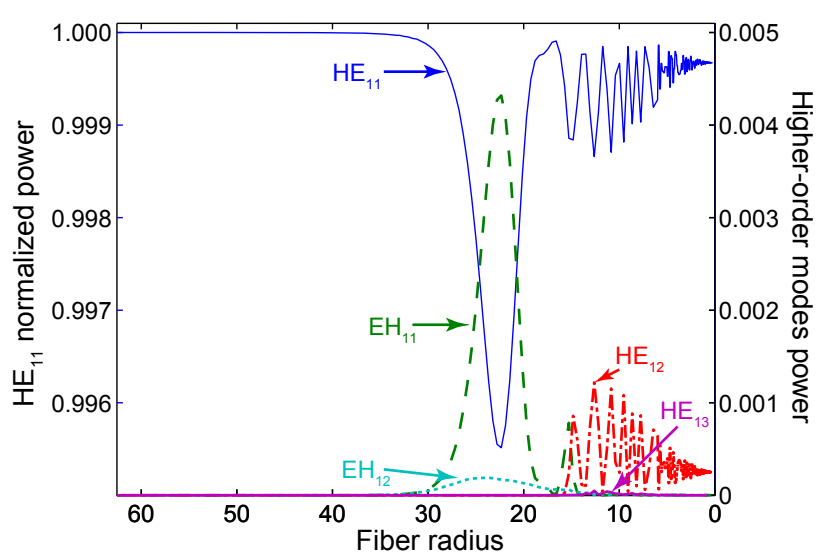

Fig. 8. Mode evolution for a 2 mrad linear fiber down to $250 \mathrm{~nm}$ radius. During the propagation through the taper, some energy is transferred from the fundamental $\mathrm{HE}_{11}$ (blue thin continuous line) to four higher-order modes $\mathrm{EH}_{11}$ (green long-dashed line), $\mathrm{EH}_{12}$ (light bluedotted line), $\mathrm{HE}_{12}$ (red-dashed-dotted line), and $\mathrm{EH}_{13}$ (purple thick continuous line). The final transmission through one taper is $99.97 \%$ on the $\mathrm{HE}_{11}$ fundamental mode.

of the linear geometry makes it the ideal candidate for our application.

\section{ANALYSIS OF THE TRANSMISSION SIGNAL}

We evaluate the quality of a pull by monitoring the transmission of a few milliwatts from a $780.24 \mathrm{~nm}$ laser through the fiber during the process. We normalize the signal to remove fluctuations of the laser intensity. Figure 9(a) shows a typical transmission as a function of time for a successful $2 \mathrm{mrad}$ pull. The transmission and normalization fiber outputs are connected to two Thorlabs DET10A photodetectors, which deliver a signal to a SR570 low-noise differential preamplifier from Stanford Research Systems. A Tektronix DPO7054 digital oscilloscope set on high-resolution mode and sample rate of 10-20 ksample/s records the data. The fiber is thinned during the pull, and as its radius decreases, we observe different notable features in the transmission signal. Figure 9(b) shows the relation between time and radius for the particular pull of Fig. 9(a) calculated using the algorithm for fiber pulling that was validated in [24], with a deviation from the experimental measurements lower than $8 \%$ at all diameters.

\section{A. Single-Mode Section}

The fiber is initially close to being single mode $(V \approx 2.45)$ at the light wavelength we use to measure the transmission. We carefully launch the fundamental mode with a $1.27 \mathrm{~cm}$ radius of curvature mandrel wrap, to filter higher-order modes from the initial launch. During the first $100 \mathrm{~s}$ (down to $25 \mu \mathrm{m}$ radius), we observe a constant transmission. A 2 mrad taper is completely adiabatic in this region (see Fig. 3). The fundamental mode is confined to the core and does not interact with any other mode.

\section{B. From Single Mode to Multimode}

As the fiber radius decreases, the fundamental mode effective index approaches the cladding index of refraction (see Fig. 2). The fiber core becomes too small to support the fundamental mode, which progressively leaks into the cladding to become guided by the cladding-to-air interface. The point at which the 

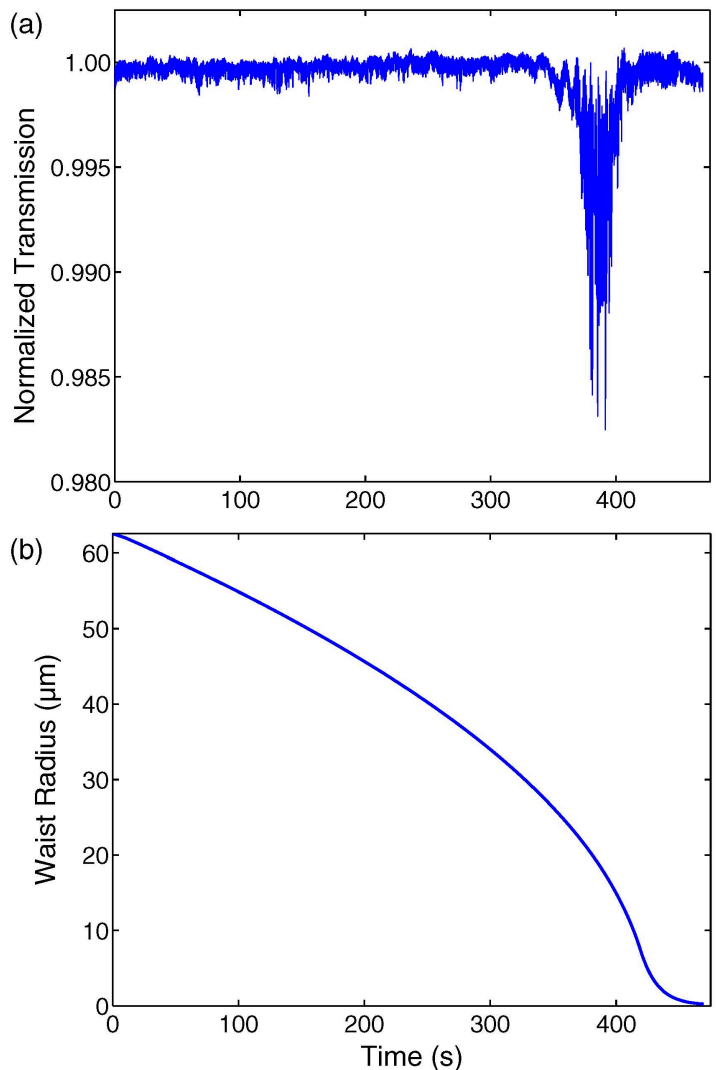

Fig. 9. (a) Transmission through a 2 mrad fiber as a function of time during the manufacturing process. (b) Evolution of the waist radius during the pull, calculated from the algorithm described in [24]. The final radius is $250 \mathrm{~nm}$.

fundamental mode leaks into the cladding is $n_{\text {eff }}^{\mathrm{HE} 11}=n_{\text {clad }}$, at $R=19.43 \mu \mathrm{m}$. At that point, the waveguide is so large in comparison to the wavelength of the light that the fiber is multimode $(V \approx 170)$. The dispersion relation curves of all the modes are close to each other [see Fig. 2(a)], and the modes can easily interact. Figure 5 shows that the tapering angle has to be smaller than $0.3 \mathrm{mrad}$ in order to be adiabatic in that region. The transmission signal therefore shows mode beating (see Fig. 10).

Energy transfer to higher-order modes occurs during the transition from core to cladding because of this nonadiabaticity. For a cylindrically symmetric fiber, such a transfer of energy is only possible from the fundamental mode to other modes of order $l=1$ (by symmetry). Once they have been excited in the fiber, those modes coexist and propagate together with different propagation constants, given by the dispersion relation curves (green curves in Fig. 2). The optical path length inside the fiber is

$$
(L)_{n}=\int_{\text {fiber }} n_{\text {eff }}^{n}(z) \mathrm{d} z .
$$

Equation (4) shows that different modes accumulate a phase difference. The modulation observed in the transmission signal around radius $20 \mu \mathrm{m}$ is a signature of the presence of higher-order modes beating together.

The beating signal is related to the relative phases when light couples back into the fiber core as $R$ increases in the

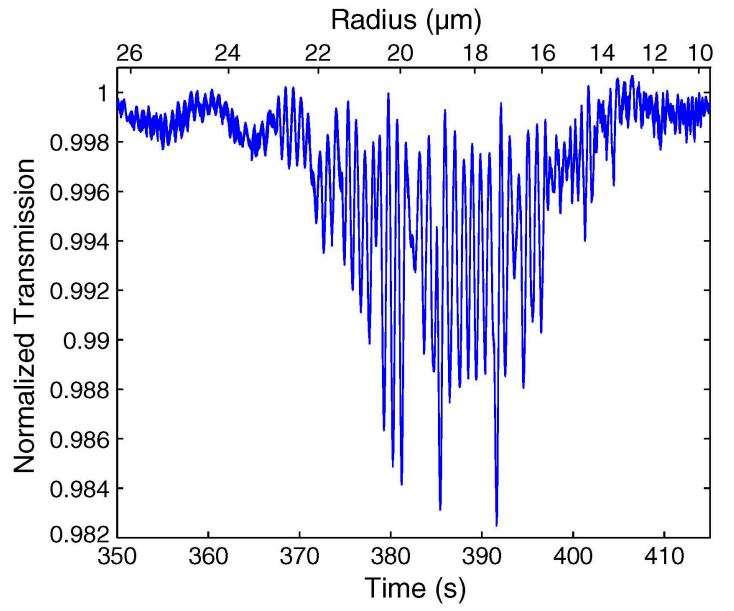

Fig. 10. Zoom of the transmission of the 2 mrad pull shown in Fig. 9 when the radius of the waist is near $20 \mu \mathrm{m}$. We see oscillations in the transmission signal, due to the beating between the fundamental mode and higher-order modes excited at a radius of $20 \mu \mathrm{m}$. The top vertical scale is the fiber radius at the waist.

(a)

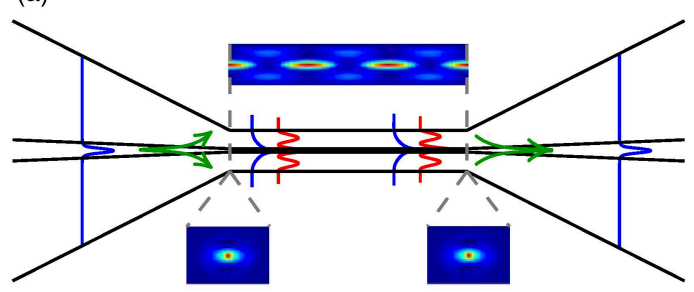

(b)

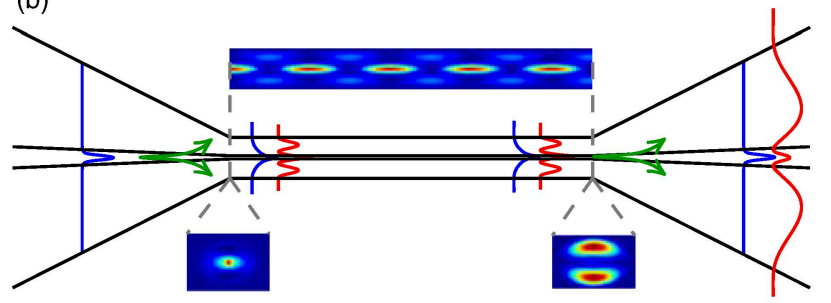

Fig. 11. Schematic of the modal evolution in the transition region. All the power is initially contained in the fundamental mode (blue profile). When the core of the fiber becomes too small compared to the wavelength, the light escapes into the cladding (green arrows) and some higher-order modes can be excited (red profile). The radius of the waist is equal to $20 \mu \mathrm{m}$, so that the excited modes do not experience any cutoff as they propagate through the waveguide. (a) The length of the fiber is an integer number of beating lengths. (b) Length of the fiber, not an integer of beating lengths. The mode profiles were calculated with FIMMPROP.

second taper. When $R$ reaches $19.43 \mu \mathrm{m}$ in the second taper, energy couples back into the core. Although the two tapers are identical, the presence of beating between modes breaks the symmetry (see Fig. 11). Depending on the fiber length, the phase accumulation between modes leads to a different field distribution entering the core at $19.43 \mu \mathrm{m}$. The fraction of energy that can couple back into the core depends on the field distribution at this point. If the modes travel through an integer number of beat lengths, the field distribution returns to its initial input. The reciprocity theorem implies that all the energy couples back into the core. If the modes experience a 
noninteger number of beat lengths, the field distribution is different from what it was initially and only a fraction of energy can couple back into the core: the rest of the energy couples to cladding modes. The cladding light is not detected since we filter the higher-order modes placing a mandrel wrap in front of the detector. At the fiber output, we therefore only observe on the detector the light that coupled back into the fiber core.

\section{Single Mode Again}

As we continue to thin the fiber, the modes' effective indices approach the air index of refraction. When $R$ (equivalently, the $V$ parameter) becomes small enough, the excited modes cut off and couple to radiation modes in air. Meanwhile, the fundamental mode's effective index asymptotically approaches $n_{\text {air }}$ without reaching a cutoff. A small enough fiber can consequently be single mode again after all the higher-order modes cutoff. For the SM800 fiber, the single-mode cutoff occurs at $300 \mathrm{~nm}$ radius. After this cutoff, we do not see any beating anymore, and the transmission is steady again: we measure a transmission of $99.950(23) \%$, where the dispersion of the distribution is $5.8 \times 10^{-3}$ and the dispersion on the mean is $1.2 \times 10^{-5}$. Possible systematic effects related to fiber cleanliness and the detectors' and amplifiers' long-term stability prevent us from giving a better bound than $0.023 \%$ to the measured uncertainty in the transmission, but $T$ is close to unity, both in the measurement and in the simulation. Note that the simulation Section 4.E looks at the propagation through a single taper. In the present case, light goes through two tapers, explaining why the measured transmission is slightly smaller than the simulated one.

\section{SPECTROGRAMS}

We extract the evolution of the frequencies contributing to the beating process as a function of pull time using spectrograms, which plot local, windowed Fourier transforms of the transmission signal as a function of time. We use the spectrogram function in MATLAB with a window of 8192 points and an

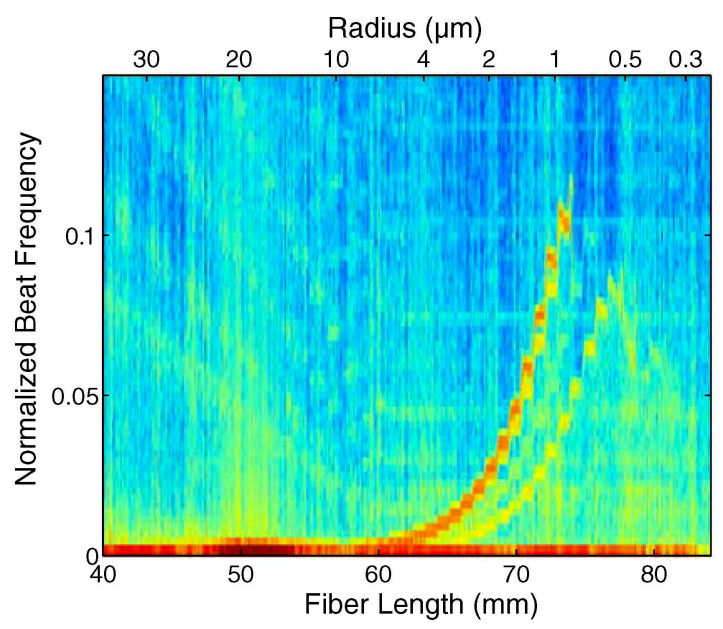

Fig. 12. Transmission spectrogram of a 2 mrad pull (see the time evolution in Fig. 9) as a function of the stretch $L$ of the fiber, showing the chirp of the beating frequency and the abrupt end of the beating. The top vertical scale shows the waist radius calculated from the algorithm. The colormap corresponds to the power spectral density (PSD). overlap of 7000 points. [See Fig. 12 for an example of a spectrogram of the transmission from Fig. 9(a)]. The modulation in the transmission does not have a single frequency. The frequency is chirped for various reasons. First, the stretch of the fiber is not a linear function of time. Its form depends on the chosen pulling parameters, and can be calculated using our algorithm. Second, the propagation constants of the modes are not only radius-dependent, but the way they evolve also depends on the mode. The difference between two curves varies as a function of $R$, which means that the phase does not accumulate at a constant rate.

Each curve in the spectrogram signals the interaction between two modes beating at a given frequency. They all appear when the fiber enters the multimode regime $(R \approx 19.43 \mu \mathrm{m})$. The presence of these curves indicates nonadiabaticities in the pull. The curves terminate before the end of the pull, at a point that corresponds to the cutoff of one of the two beating modes. We now have the task to identify which modes are excited, how they are excited, and whether there is a way to suppress their excitation. Given the specificity of the phase accumulation for a couple of modes, it is possible to label the modes excited during the pull and use the spectrogram as a diagnostic to evaluate the adiabaticity and symmetry of the fibers.

\section{A. Modeling the Pull}

The phase accumulation between two modes is a function of their optical path length, which depends on the geometry of the fiber at a time $t$ [see Eq. (4) above]. When the stretch at that time is equal to $L$, the phase accumulation between two modes is

$$
\Phi_{i, j}(L)=\int_{0}^{L}\left[\beta_{i}(r(z))-\beta_{j}(r(z))\right] \mathrm{d} z,
$$

with spatial frequency $K[\underline{7}]$

$$
K_{i, j}(L)=\frac{1}{2 \pi} \frac{d \Phi_{i j}}{d L} .
$$

\section{B. Identifying the Modes}

We know the profile at the end of a step from the pulling algorithm described in [24]. We use the dispersion relations obtained with FIMMPROP to calculate the differences, $\Delta \beta_{i, j}$, in propagation constants for mode $i$ and mode $j$. By integrating numerically $\Delta \beta_{i, j}(z)$ at each step, we obtain $\Phi_{i, j}(L)$. A numerical differentiation of $\Phi_{i, j}$ with respect to $L$ gives us the evolution of the spatial frequency as a function of step (see Fig. 13). From our simulation of the pull, we know the stretch as a function of time, and we can plot the evolution of the spatial frequency as a function of time.

We calculate the spatial frequency for a thousand pairs of modes with different radial symmetry ( $l=1$ to 6$)$ and azimuthal order ( $m=1$ to 20 ), and we map them on the spectrogram. We can then identify and label the curves observed on the spectrogram by looking for their overlap with the experimental curves. We get an excellent matching without any scale adjustments.

We can identify in the spectrogram (2 mrad tapered fiber) the signature of beating between four modes (see Fig. 14). All 

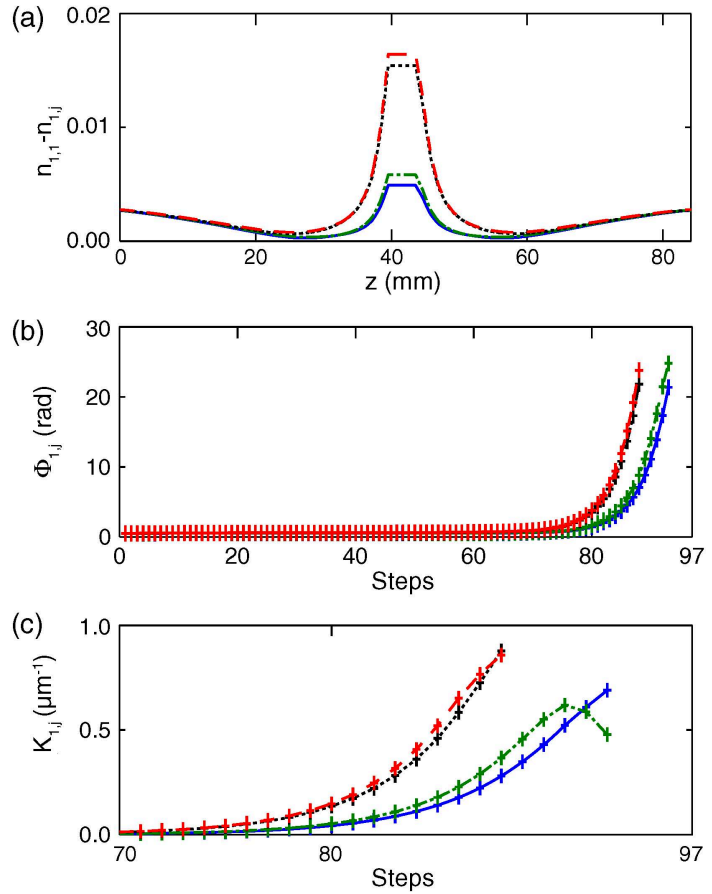

Fig. 13. Study of the differences between the fundamental mode and the first four excited modes of family 1 . (a) $\Delta \beta_{1, j}$ as a function of length along the fiber axis (difference between the indices of refraction at step 75). (b) Phase accumulation $\Phi_{1, j}$ as a function of step. (c) Spatial frequency $K_{1, j}$ of the beating as a function of step. The lines (long-dashed red, continuous blue, short-dashed black, and longshort dashed green) join the calculated points.

those modes have the symmetry $l=1$ (see Fig. 2 ) as expected for a cylindrically symmetric fiber. We observe higher-order mode excitation up to the fifth mode of family $l=1$, which

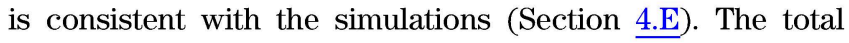
$\mathrm{HE}_{11}$ transmission is 0.9995 , meaning that $0.05 \%$ of the energy has been transferred to the other modes. We suppose here that all the losses come from the transfer of power to other modes. This power is radiated in air when those modes reach cutoff. The contribution of other losses such as Rayleigh scattering is expected to be much smaller. The power spectral density (PSD), which defines the colormap in a spectrogram, gives a representation of how the remaining power is distributed between the higher-order modes as a function of time. By plotting the PSD at different times, we evaluate the power contained in each branch contributing to the beating. Below $R=4 \mu \mathrm{m}$, those contributions are almost constant, and the higher-order mode relative power is distributed as follows: $5.5 \pm 0.5 \%$ in $\mathrm{HE}_{12}, 9 \pm 0.5 \%$ in $\mathrm{EH}_{12}$, and $85.5 \pm 0.5 \%$ in $\mathrm{HE}_{13}$. Note that we only resolve the beating between the fundamental mode and one excited mode. The beating between excited modes exists, but this second-order effect is too weak to be visible in the spectrogram.

\section{APPLICATION: QUALITY OF THE PULL}

We can use the spectrogram analysis to design and diagnose its quality while pulling a fiber. The number of modes excited and which modes are excited give us information about the adiabaticity, asymmetries, and quality of the fiber after the pull.

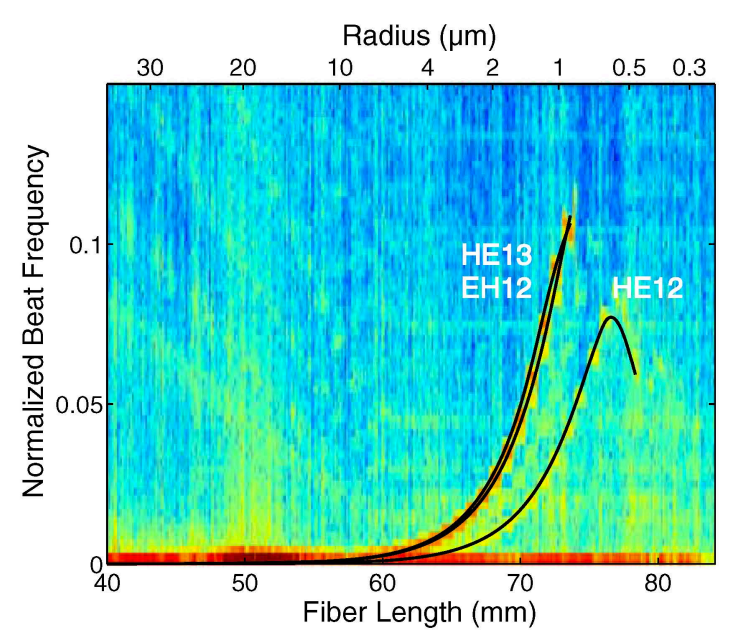

Fig. 14. Identification of the modes beating for the 2 mrad tapered fiber spectrogram of Fig. 12 . Only modes from family 1 are beating.

\section{A. Multiangle Taper}

The beating amplitude and higher-order modes excitations seen in Figs. $\underline{9}$ and 12 show that the angle of tapering near the critical region at $19.43 \mu \mathrm{m}$ is nonadiabatic. A shallower taper angle around that region could lead to a more adiabatic transition. Following this idea, we study a fiber with a $2 \mathrm{mrad}$ angle until a radius of $20 \mu \mathrm{m}$, and then decrease the angle to $0.75 \mathrm{mrad}$. After $R=6 \mu \mathrm{m}$, the pull is exponential down to $R=250 \mathrm{~nm}$.

We see that the transmission at the end of the pull is only $97.850 \%$ from Fig. 15. This corresponds to a transfer of energy to the higher-order modes larger than $3 \%$, a factor of 60 worse than in the linear $2 \mathrm{mrad}$ pull (Fig. 9). The beating amplitude is much larger than in the $2 \mathrm{mrad}$ case. This is surprising since this pull is designed to be more adiabatic, and simulations with FIMMPROP confirm that we still expect a transmission $T \geq 99.90 \%$.

\section{B. Tracking Asymmetries}

The spectrogram analysis in Fig. 16 shows excitation to the $\mathrm{TE}_{01}, \mathrm{TM}_{01}$, and $\mathrm{HE}_{21}$ modes, which do not belong to the family of the fundamental mode. The largest transfer is still to the same family, with a different distribution. Coupling to other families should not be observed for a fiber with cylindrical symmetry. This suggests that our multiple angle tapers introduce some asymmetries in the fiber. We imaged the fiber using an optical microscope near the angle change regions (see Fig. 17) to further investigate the decrease of transmission.

Figure 17 shows that the bottom angle of the fiber exceeds the top angle. Although the measured diameters are as expected, superimposed plots of the top and bottom edges show that there are imperfections around the transition. We observe a peak at the transition radius $(R \approx 20 \mu \mathrm{m})$ in the distance between the edges. We believe that the excitation of higherorder modes at this radius is a consequence of this asymmetry. We do not observe the same imperfection around the transition region for a $2 \mathrm{mrad}$ flat fiber. The abrupt change in angle exacerbates imperfections in the pulling process by introducing some asymmetries. These results further support the idea that single-angle linear tapers are good candidates for our application. Further increasing adiabaticity would require us to decrease $\Omega$, leading to large taper lengths. 

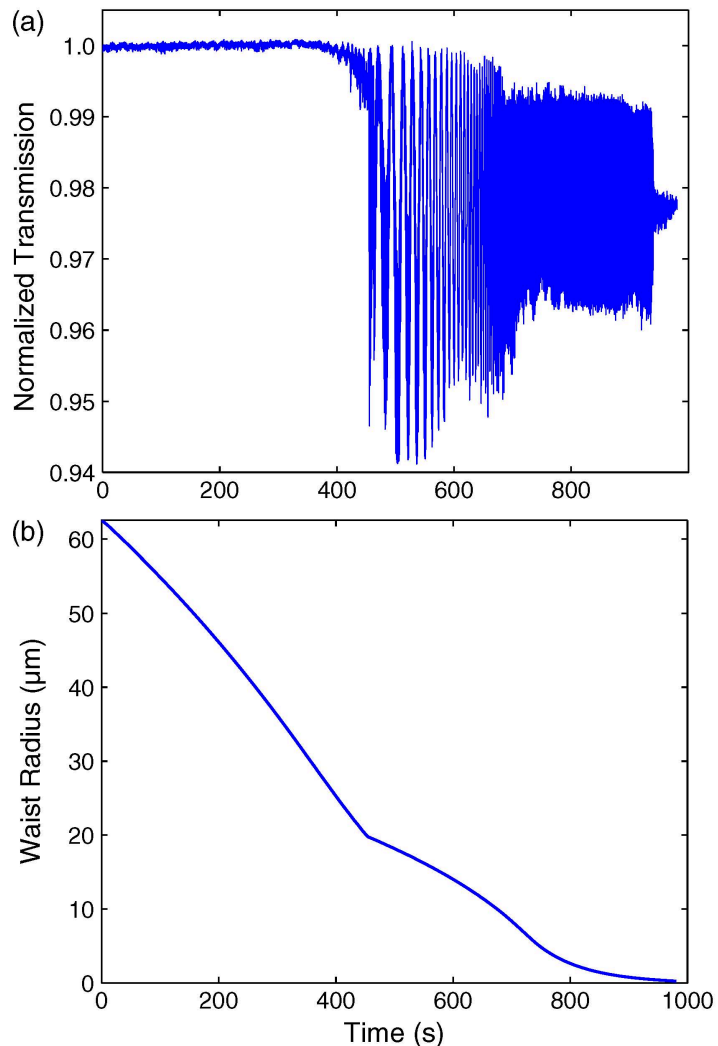

Fig. 15. (a) Normalized transmission through the fiber as a function of time during the manufacturing process. (b) Evolution of the radius of the waist during the pull. Based on the algorithm, we initially taper the fiber with a $2 \mathrm{mrad}$ angle until a radius of $20 \mu \mathrm{m}$; the angle changes to $0.75 \mathrm{mrad}$ until the radius of the fiber is equal to $6 \mu \mathrm{m}$, where the radius exponentially decreases down to $250 \mathrm{~nm}$.

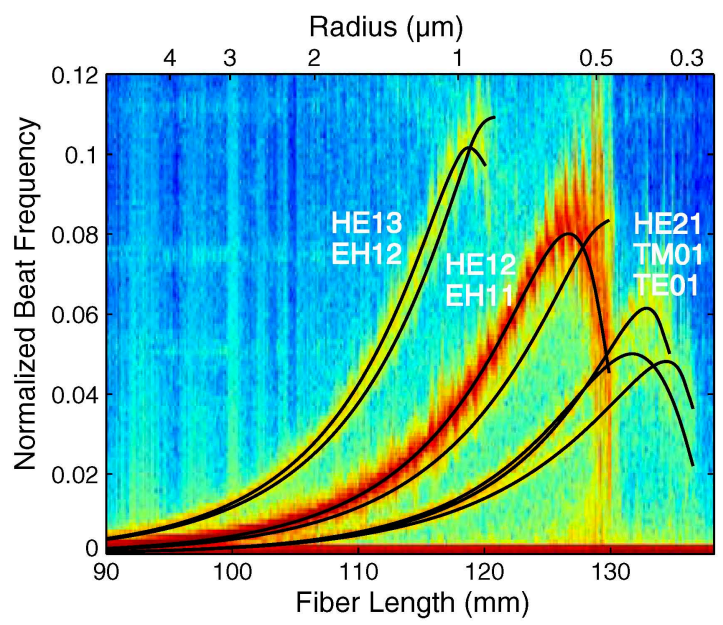

Fig. 16. Spectrogram of the transmission data shown in Fig. 15. The solid black curves are the ones given by the simulation. The modes are labeled in the figure. The total transmission in the fundamental is 0.97850 . For $R \leq 2 \mu \mathrm{m}$ we calculate from the PSD that the remaining energy is distributed between seven higher-order modes as follows: $\mathrm{TE}_{01}(0.08 \%), \mathrm{TM}_{01}(0.05 \%), \mathrm{EH}_{11}(0.35 \%), \mathrm{EH}_{12}(0.05 \%), \mathrm{HE}_{12}$ (98.4\%), $\mathrm{HE}_{13}(0.2 \%)$, and $\mathrm{HE}_{21}(0.87 \%)$.

Because of geometrical and handling constraints, we find it ideal to work with 2 mrad tapers. To work with steep and multiple angles might require a smaller flame or a more symmetric heating. (a)<smiles></smiles>

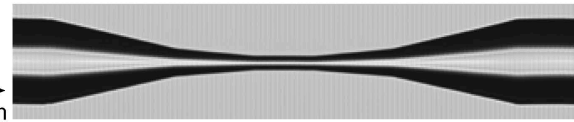

(b)

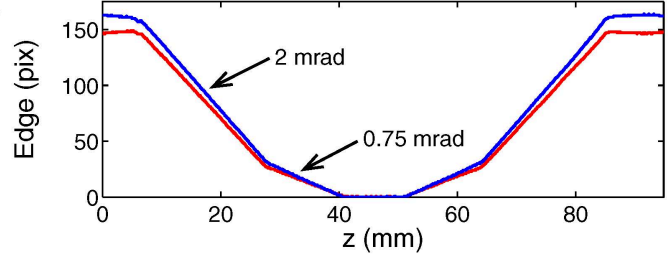

(c)

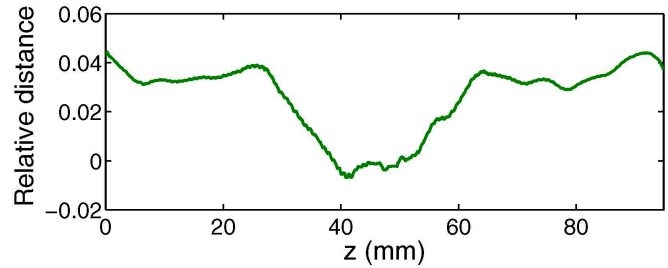

Fig. 17. Study of the asymmetry of a pulled fiber. The fiber has a $10 \mu \mathrm{m}$ radius with an angle change from 2 to $0.75 \mathrm{mrad}$ at $20 \mu \mathrm{m}$. (a) 100 images taken with an optical microscope stacked and horizontally compressed to enhance any asymmetries. (b) Profile of the bottom edge (blue curve) and top edge (red curve) of the fiber. The abrupt change in angle at $20 \mu \mathrm{m}$ introduces an asymmetry at this radius. (c) Relative difference between the two edges (normalized by the diameter of the fiber) as a function of $z$.

\section{UNDERSTANDING THE LOSSES}

\section{A. Losses}

Understanding the losses in nanofibers is important for our future applications [11,24], which require knowledge of such photon loss. We identify two main loss mechanisms that contribute to the final losses: coupling to higher-order modes through nonadiabaticities and scattering around the waist of the nanofiber [8]. Systematic effects such as the presence of impurities on the fiber surface, or asymmetries in the pull, enhance the losses through those mechanisms.

\section{B. Coupling to Higher-Order Modes}

We have observed and characterized in this paper the effect of nonadiabaticities in the taper. Their presence induces energy transfer to higher-order modes. As we reach the single-mode regime, those higher-order modes cutoff. They cannot be guided by the fiber anymore, and they diffract out as radiative modes. In a plane transverse to the fiber, one can observe a characteristic diffraction pattern further supporting the fact that this effect is most the important for the pulls considered in this study.

\section{Rayleigh Scattering}

Rayleigh scattering is present in any glass, leading to scattering of light and attenuation in the transmitted signal []. The attenuation coefficient for fused silica is small at a wavelength of $780.24 \mathrm{~nm}$. By imaging the fiber, it is possible to directly observe the scattering. Experimentally, it is particularly visible on the fiber waist, but remains of the order of $3 \mathrm{~dB} / \mathrm{km}$, justifying the fact that we neglected it in this paper.

\section{Systematic Effects}

Transmission varies drastically with the surface state of the fiber. When the fiber is initially dirty, the spectrogram analysis shows the excitation of more modes corresponding to more 
losses. We attribute this to the presence of impurities on the surface of the fiber at the beginning or during the pull. A dust particle on the fiber waist leads to losses through coupling to higher-order modes or scattering. The cleanliness of the fiber is critical before and during the pull. Such imperfections are avoidable by properly cleaning the fiber and imaging the fiber prior to a pull as explained in [24]. All the pulls presented in this paper were performed after applying the cleaning procedure described in [24].

\section{CONCLUSION}

We have demonstrated our ability to produce ultralow loss optical nanofibers. Reaching high transmissions is important for many nanofiber applications. We have described an algorithm that calculates the optimum taper length for a given transmission, or equivalently the optimum transmission for a given taper length. This new approach concerning adiabaticity in tapered fibers gives more precise bounds than the traditional adiabaticity condition, which helps in the design of a suitable taper geometry. We show that in our experiments, the transition from the single-mode regime to the multimode regime is nonadiabatic, inducing excitations of higher-order modes during the tapering. Having good control of the taper geometry is crucial for limiting losses due to those excitations.

The propagation of different modes during the pull leads to a characteristic beating pattern in the transmission. Plotting the spectrogram of the transmission signal and using a model of fiber pulling, we are able to identify the modes excited during the pull. This gives information for the analysis of the quality of a fiber and the understanding of loss factors, which will help in the manufacture of even more adiabatic fibers.

\section{ACKNOWLEDGMENTS}

We thank Prof. A. Rauschenbeutel for his interest and support on this project. This work was funded by the National Science Foundation through the Physics Frontier Center at the Joint Quantum Institute, and the Army Research Office Atomtronics MURI. S. R. acknowledges support from the Fulbright Foundation.

\section{REFERENCES}

1. A. Yariv, "Coupled-mode theory for guided-wave optics," IEEE J. Quantum Electron. 9, 919-933 (1973).

2. F. L. Kien, J. Liang, K. Hakuta, and V. Balykin, "Field intensity distributions and polarization orientations in a vacuum-clad subwavelength-diameter optical fiber," Opt. Commun. 242, 445-455 (2004).

3. S. Leon-Saval, T. Birks, W. Wadsworth, P. S. J. Russell, and M. Mason, "Supercontinuum generation in submicron fibre waveguides," Opt. Express 12, 2864-2869 (2004).

4. E. Vetsch, D. Reitz, G. Sagué, R. Schmidt, S. T. Dawkins, and A. Rauschenbeutel, "Optical interface created by laser-cooled atoms trapped in the evanescent field surrounding an optical nanofiber," Phys. Rev. Lett. 104, 203603 (2010).

5. J. Bures and R. Ghosh, "Power density of the evanescent field in the vicinity of a tapered fiber," J. Opt. Soc. Am. A 16, 1992-1996 (1999).

6. L. Tong, R. R. Gattass, J. B. Ashcom, S. He, J. Lou, M. Shen, I. Maxwell, and E. Mazur, "Subwavelength-diameter silica wires for low-loss optical wave guiding," Nature 426, 816-819 (2003).
7. F. Orucevic, V. Lefèvre-Seguin, and J. Hare, "Transmittance and near-field characterization of sub-wavelength tapered optical fibers," Opt. Express 15, 13624-13629 (2007).

8. A. W. Snyder and J. D. Love, Optical Waveguide Theory (Chapman \& Hall, 1983).

9. V. I. Balykin, K. Hakuta, F. L. Kien, J. Q. Liang, and M. Morinaga, "Atom trapping and guiding with a subwavelength-diameter optical fiber," Phys. Rev. A 70, 011401 (2004).

10. S. M. Spillane, T. J. Kippenberg, O. J. Painter, and K. J. Vahala, "Ideality in a fiber-taper-coupled microresonator system for application to cavity quantum electrodynamics," Phys. Rev. Lett. 91, 043902 (2003).

11. J. E. Hoffman, J. A. Grover, Z. Kim, A. K. Wood, J. R. Anderson, A. J. Dragt, M. Hafezi, C. J. Lobb, L. A. Orozco, S. L. Rolston, J. M. Taylor, C. P. Vlahacos, and F. C. Wellstood, "Atoms talking to squids," Rev. Mex. Fis. 57, 1-5 (2011)

12. R. Grimm, M. Weidemuller, and Y. B. Ovchinnikov, "Optical dipole traps for neutral atoms," Adv. At. Mol. Opt. Phys. 42, 95-170 (2000).

13. A. Ashkin, "Trapping of atoms by resonance radiation pressure," Phys. Rev. Lett. 40, 729-732 (1978).

14. S. Chu, J. E. Bjorkholm, A. Ashkin, and A. Cable, "Experimental observation of optically trapped atoms," Phys. Rev. Lett. 57, 314-317 (1986).

15. R. J. Cook and R. K. Hill, "An electromagnetic mirror for neutral atoms," Opt. Commun. 43, 258-260 (1982).

16. K.-H. Yang, W. C. Stwalley, S. P. Heneghan, J. T. Bahns, K.-K. Wang, and T. R. Hess, "Examination of effects of TEM $\mathrm{TH}_{01}^{*}$-mode laser radiation in the trapping of neutral potassium atoms," Phys. Rev. A 34, 2962-2967 (1986).

17. N. Davidson, H. Jin Lee, C. S. Adams, M. Kasevich, and S. Chu, "Long atomic coherence times in an optical dipole trap," Phys. Rev. Lett. 74, 1311-1314 (1995).

18. S. Kulin, S. Aubin, S. Christe, B. Peker, S. L. Rolston, and L. A. Orozco, "A single hollow-beam optical trap for cold atoms," J. Opt. B 3, 353-357 (2001).

19. A. Goban, K. Choi, D. Alton, D. Ding, C. Lacroûte, M. Pototschnig, T. Thiele, N. Stern, and H. Kimble, "Demonstration of a state-insensitive, compensated nanofiber trap," Phys. Rev. Lett. 109, 1-5 (2012).

20. X. Jiang, L. Tong, G. Vienne, X. Guo, A. Tsao, Q. Yang, and D. Yang, "Demonstration of optical microfiber knot resonators," Appl. Phys. Lett. 88, 223501 (2006).

21. K. P. Nayak, F. L. Kien, Y. Kawai, K. Hakuta, K. Nakajima, H. T. Miyazaki, and Y. Sugimoto, "Cavity formation on an optical nanofiber using focused ion beam milling technique," Opt. Express 19, 14040-14050 (2011).

22. C. Wuttke, M. Becker, S. Brückner, M. Rothhardt, and A. Rauschenbeutel, "Nanofiber Fabry-Perot microresonator for nonlinear optics and cavity quantum electrodynamics," Opt. Lett. 37, 1949-1951 (2012).

23. F. Warken, "Ultra thin glass fibers as a tool for coupling light and matter," Ph.D. thesis (Rheinische Friedrich-Wilhelms Universitat, 2007).

24. J. E. Hoffman, S. Ravets, J. Grover, P. Solano, P. R. Kordell, J. D. Wong-Campos, S. L. Rolston, and L. A. Orozco, "Heat and pull apparatus for ultrahigh transmission optical nanofibers," (in preparation).

25. http://drum.lib.umd.edu.

26. A. Yariv, Optical Electronics in Modern Communications (Oxford University, 1997).

27. Photon Design Ltd., "FIMMWAVE/FIMMPROP," http://www .photond.com

28. T. Birks and Y. Li, "The shape of fiber tapers," J. Lightwave Technol. 10, 432-438 (1992).

29. M. Fujiwara, K. Toubaru, and S. Takeuchi, "Optical transmittance degradation in tapered fibers," Opt. Express 19, 8596-8601 (2011).

30. S. Ravets, J. E. Hoffman, L. A. Orozco, S. L. Rolston, G. Beadie, and F. K. Fatemi, "A low-loss photonic silica nanofiber for higher-order modes,” Opt. Express 21, 18325-18335 (2013). 


\section{Chapter 4}

\section{Propagation of higher-order modes in tapered optical nanofibers}

\section{Contents}

4.1 Motivations .................... 62

4.2 First family of excited modes $\ldots \ldots \ldots 63$

4.3 Transmission signals . . . . . . . . . . . 63

4.4 Reduced-cladding fibers . . . . . . . . . . . 65

4.5 Increasing adiabaticity $\ldots \ldots \ldots 66$

We have shown that we are able to produce tapered optical nanofibers with unprecedented levels of transmission in the fundamental mode. Using the two-color trapping scheme described in Chapter 1, the JQI team has successfully trapped atoms around one of these nanofibers as we will show in Outlook. This trapping scheme uses the evanescent field created around the nanofiber by the fundamental, $\mathrm{HE}_{11}$ mode. Other trapping schemes that are based on the interference between different modes co-propagating in the fiber have also been proposed [Sagué, Baade, and Rauschenbeutel (2008)]. However, efficiently guiding higher-order modes in tapered optical fibers is experimentally challenging [Frawley et al. (2012)], and these schemes have not been implemented yet. In this Chapter, we investigate higher-order mode propagation in our tapered optical fibers. We achieve more than $97 \%$ transmission of the first family of excited modes, with less than $1 \%$ fundamental mode contamination. 
a

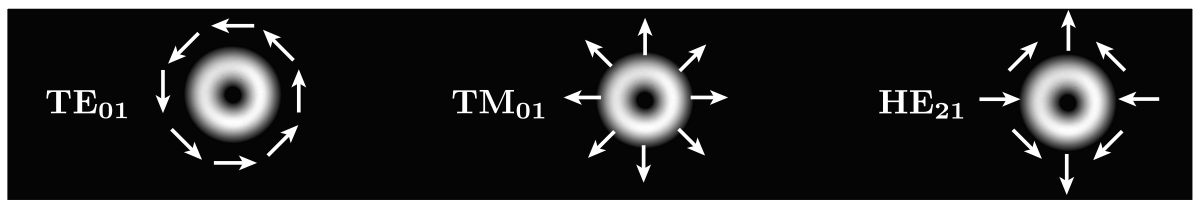

b

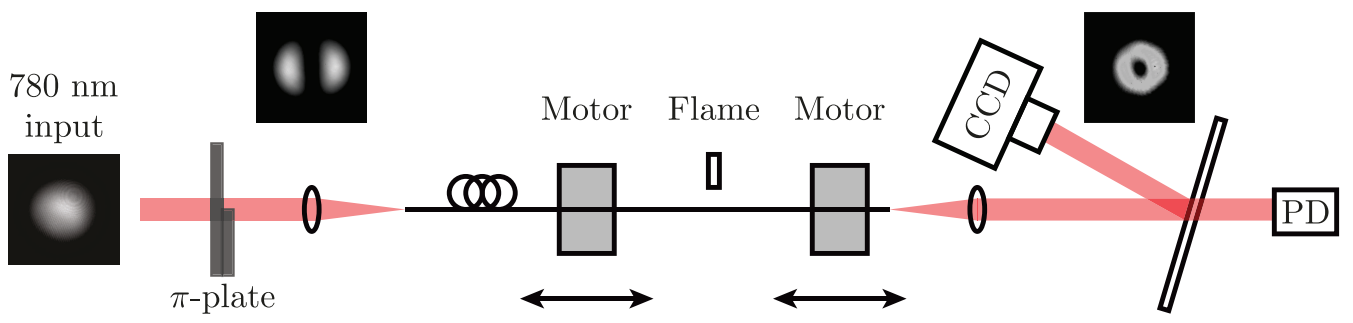

Figure 4.1: Launching higher-order modes. a, Higher-order modes of interest. Intensity and polarization profile of the first three higher-order modes $\mathrm{TE}_{01}, \mathrm{TM}_{01}$ and $\mathrm{HE}_{21}$. b. Experimental setup. A Gaussian beam passes through a $\pi$ phase plate and is coupled into the fiber. At the output of the fiber, we record the transmission of a few milliwatts of power on a photodetector (PD) and on a camera (CCD). The insets show typical beam images recorded on a CCD.

\subsection{Motivations}

The propagation of higher-order modes in optical fibers show some remarkable properties that are of interest for atom trapping:

- Higher-order modes experience a cutoff at a finite radius, unlike the $\mathrm{HE}_{11}$ mode. This allows controlling the extent of the evanescent field using larger fiber radii, leading to stronger fibers with improved handling.

- The energy distributions of higher-order modes are less confined into the fiber core than for the fundamental mode ${ }^{1}$. For a given input power, more energy propagates at the periphery of the fiber, which decreases the amount of laser power required for trapping atoms and thus the amount of Rayleigh scattering.

- When different modes propagate simultaneously inside the fiber, they beat. One can use the interference pattern created by this beating to axially confine the atoms, where the distance between the traps and the longitudinal trap size is determined by the spatial frequency of the beating.

\footnotetext{
${ }^{1}$ As a matter of fact, at a given fiber radius, the effective indices of higher-order modes are smaller than the one of the fundamental mode, showing that the higher-order modes are less confined into the fiber core.
} 


\subsection{First family of excited modes}

We are interested in the propagation in a tapered optical fiber of the three lowest higher-order modes $\mathrm{TE}_{01}, \mathrm{TM}_{01}$ and $\mathrm{HE}_{21}$ modes, that have the polarization profiles shown in Figure 4.1a. Initially, the fiber must be multimode so as to be able to launch these modes in the fiber core. The three lowest higher-order modes are allowed in the core when $V_{\text {core, } R_{0}}>2.8$, where $R_{0}$ is the initial fiber radius (see dispersion relations in Figure 3.1). We use commercial fibers that are designed to be single mode for light of wavelength $980 \mathrm{~nm}$ and $1500 \mathrm{~nm}$ (see Section 4.4), and that are thus multimode for the light of wavelength $780 \mathrm{~nm}$ we use in our experiments. To excite these modes, we send a Gaussian beam through a phase plate that imprints a $\pi$-phase shift on half of the beam (see Figure 4.1b), producing a two-lobed beam that approximates a $\mathrm{TEM}_{01}$ free-space mode. Because of the inversion of the polarization over half the incident beam, we excite a combination ${ }^{2}$ of the $\mathrm{TE}_{01}, \mathrm{TM}_{01}$ and $\mathrm{HE}_{21}$ modes when coupling the light into the fiber [Fatemi (2011); Pechkis and Fatemi (2012)]. We follow the mode evolution during a pull by monitoring the transmission of a few milliwatts of laser power. The transmission is monitored both by a CCD and by a photodetector.

\subsection{Transmission signals}

Figure 4.2 shows typical transmission signals obtained when exciting the fiber with a combination of the $\mathrm{TE}_{01}, \mathrm{TM}_{01}$ and $\mathrm{HE}_{21}$ modes. Four important steps labeled by $\mathrm{A}, \mathrm{B}, \mathrm{C}$ and $\mathrm{D}$ can be distinguished in the transmission signal in Figure $4.2 \mathrm{~b}$ :

A The modes are initially confined to the core, and the transmission is steady.

B The modes escape from the core to the cladding when the fiber radius reaches $R \equiv R_{\mathrm{c}}$. Because of non-adiabaticities in the taper profile, other higher-order modes (including, in particular the modes $\mathrm{TE}_{02}, \mathrm{TM}_{02}$ and $\mathrm{HE}_{22}$ ) get excited and we observe the beating between those modes.

C The fiber radius reaches $R \simeq 0.7 \mu \mathrm{m}\left(V_{\text {clad }} \simeq 6\right)$, which corresponds to the cutoff radius for the modes $\mathrm{TE}_{02}, \mathrm{TM}_{02}$ and $\mathrm{HE}_{22}$. Those modes radiate into the air, and as a consequence, we observe a decrease in the beating amplitude. For $R \leq 0.45 \mu \mathrm{m}\left(V_{\text {clad }} \leq 3.8\right)$, the only modes that are guided by the fiber are

\footnotetext{
${ }^{2}$ Stressed-induced birefringence can be use to selectively excite each of these modes, but we have not used this selectivity here. Initially the three modes are quasi-degenerate, and we couple to a combination of those three modes.
} 

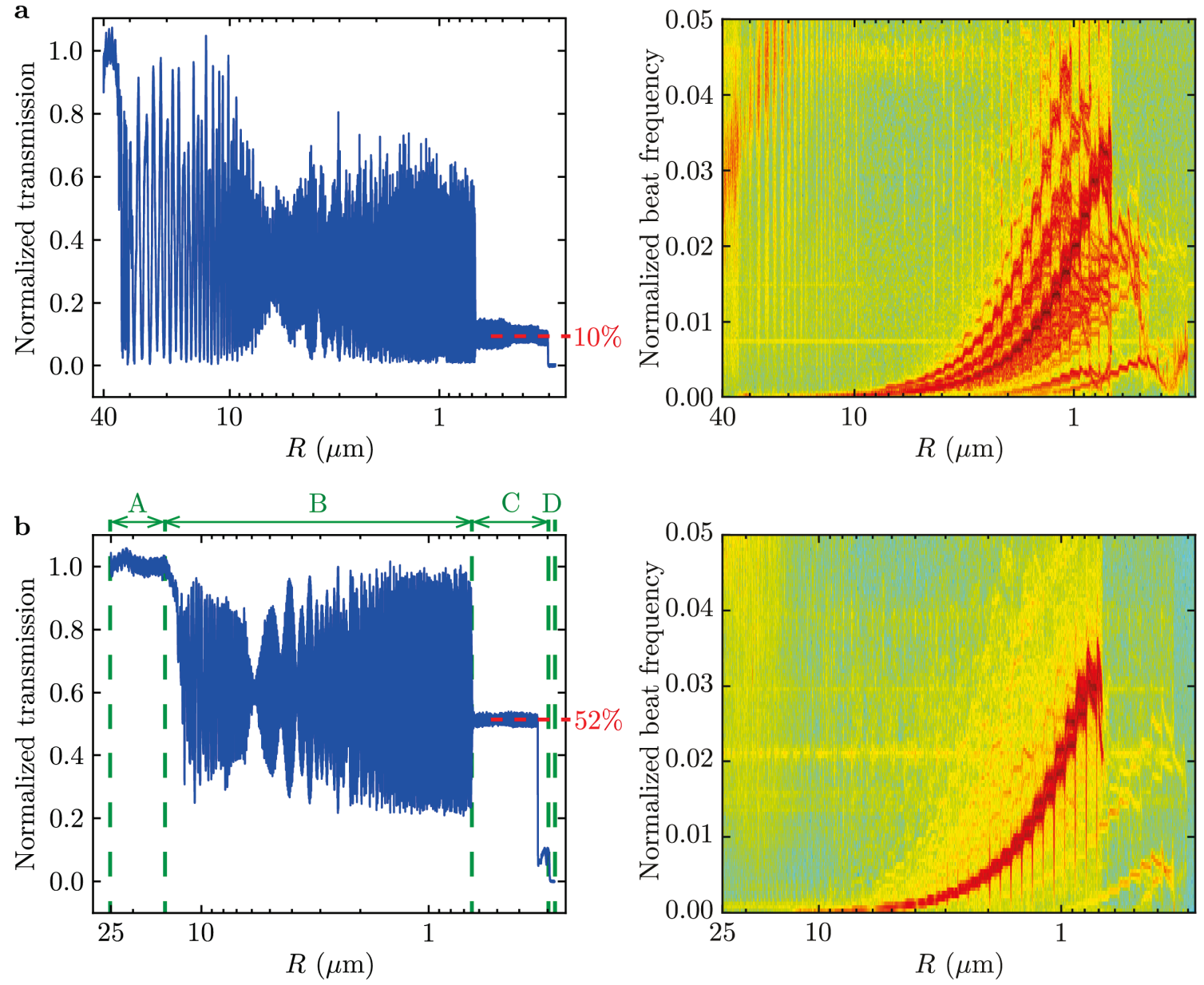

c
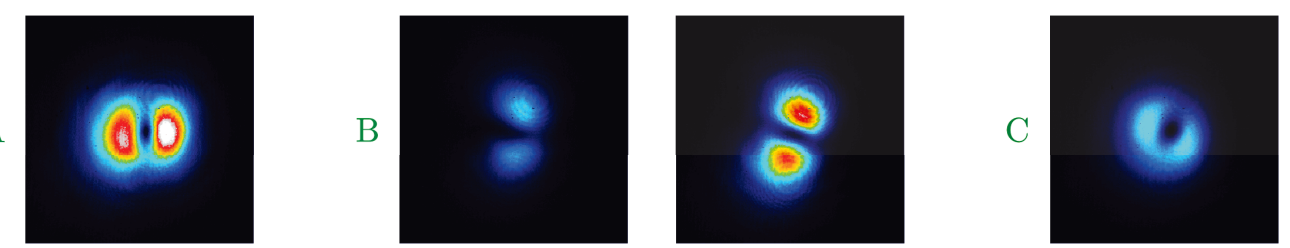

Figure 4.2: Comparison of the transmissions of higher-order modes obtained with different kinds of fibers. a, Fiber with an initial $80 \mu \mathrm{m}$ diameter. We obtain a $10 \%$ transmission of the higher-order modes through a $2 \mathrm{mrad}$, linearly tapered fiber. The spectrogram shows energy transfers to lots of modes. b. Fiber with an initial $50 \mu \mathrm{m}$ diameter. For this fiber, the spectrogram shows less energy transfers during the pull. c, CCD images of the output mode transmitted through the $50 \mu \mathrm{m}$ fiber at different steps of the pull, labeled with green capital letters A, B, C and D (see description in the text). 
Table 4.1: Properties of the different fibers pulled. We use a SM980G80 fiber from Thorlabs and a SM1500 fiber from fiber core. The SM1500 fiber shows a reduced cladding size and an increased N.A. (and thus an increased $V_{\text {core, }, R_{0}}$ ). Those properties are beneficial in order to obtain high transmissions for the higher-order modes.

\begin{tabular}{lllllll}
\hline & $\begin{array}{l}\boldsymbol{R}_{\mathbf{0}} \\
(\boldsymbol{\mu m})\end{array}$ & $\begin{array}{l}\boldsymbol{\lambda}_{\text {cutoff }} \\
(\mathbf{n m})\end{array}$ & N.A. & $\boldsymbol{V}_{\text {core, } \boldsymbol{R}_{\mathbf{0}}}$ & $\begin{array}{l}\boldsymbol{R}_{\mathrm{c}} \\
(\boldsymbol{\mu m})\end{array}$ & $\boldsymbol{V}_{\text {clad, } \boldsymbol{R}_{\mathrm{c}}}$ \\
\hline SM980G80 & 40 & 920 & 0.18 & 2.9 & 34 & 288 \\
SM1500 & 25 & 1396 & 0.3 & 4.3 & 13 & 119 \\
\hline
\end{tabular}

$\mathrm{TE}_{01}, \mathrm{TM}_{01}, \mathrm{HE}_{21}$ and $\mathrm{HE}_{11}$. We measure the transmission of the higher-order modes after this cutoff. For $R \simeq 330 \mathrm{~nm}$, the observed drop in transmission corresponds to the $\mathrm{HE}_{21}$ mode cutoff $\left(V_{\text {clad }}=2.8\right)$.

D For $R \simeq 290 \mathrm{~nm}$, the fiber reaches its single-mode cutoff $\left(V_{\text {clad }}=2.405\right)$ and only guides the fundamental, $\mathrm{HE}_{11}$ mode. For this fiber, losses in the fundamental mode are negligible. As a consequence, the small quantity of light that reaches the detector at the end of the pull (less than $2 \%$ ), indicates that we excite the first three higher-order modes with better than $98 \%$ purity.

\subsection{Reduced-cladding fibers}

We have compared the transmissions of the first few higher-order modes when tapering fibers with different numerical apertures and different initial radii $\left(R_{0}=40 \mu \mathrm{m}\right.$ and $\left.R_{0}=25 \mu \mathrm{m}\right)$. The parameters of those commercially available fibers are summarized in Table 4.1. We record the transmission signals obtained while tapering two different fibers with a $\Omega=2 \mathrm{mrad}$ tapering angle. We observe in Figure 4.2 the beneficial effects of fibers with a reduced cladding size and with a higher numerical aperture:

- The fiber with the initially smallest size and largest numerical aperture shows a $52 \%$ transmission while the other shows only a $10 \%$ transmission.

- The spectrograms in Figure 4.2 show less energy transfers when increasing N.A. and decreasing $R_{0}$. 


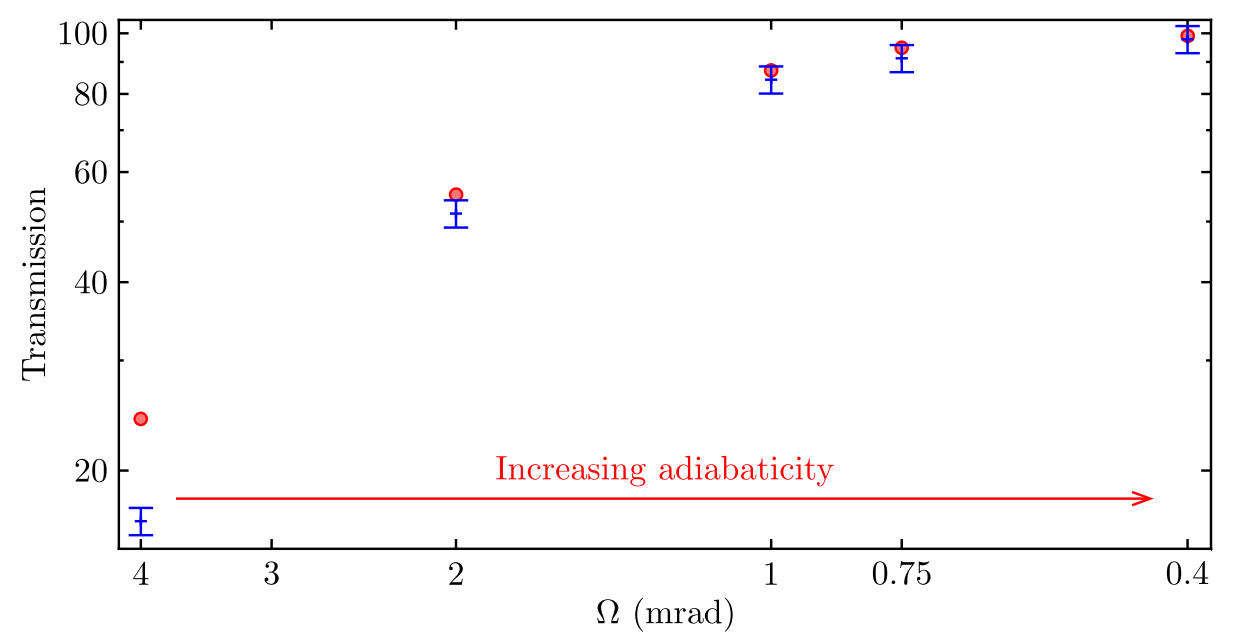

Figure 4.3: Reaching adiabaticity for higher-order modes. We also observe a good agreement between the experimental data (blue markers) and the transmission calculated using the commercial waveguide propagation software FIMMWAVE.

Indeed, we have seen that non-adiabaticities occur when the light escapes from the core to the cladding due to intermodal energy transfers. For the modes $\mathrm{TE}_{01}, \mathrm{TM}_{01}$ and $\mathrm{HE}_{21}$, this transition occurs when the fiber radius reaches $R_{\mathrm{c}}$ (see Table 4.1). One can show using Equation 3.6 and Equation 3.7 that when this transition occurs, the number $V_{\text {clad }}$ is equal to:

$$
V_{\text {clad }, R_{\mathrm{c}}}=\frac{2 \pi}{\lambda} R_{0} \frac{2.405}{V_{\text {core }, R_{0}}} \sqrt{n_{\text {clad }}^{2}-n_{\text {core }}^{2}} .
$$

Indeed, at the core-to-cladding transition, the number of modes that are accepted in the waveguide is $\propto V_{\text {clad, } R_{\mathrm{c}}}^{2} / 2$. As a consequence, reducing $R_{0}$ from $40 \mu \mathrm{m}$ to $25 \mu \mathrm{m}$ and increasing N.A. from 0.18 to 0.30 in Figure 4.2 leads to a significant decrease of the number of modes to which energy can be transferred during the pull due to non-adiabaticities ${ }^{3}$. The reduction in $R_{\mathrm{c}}$ also leads to an increase in the difference between adjacent propagation constants, leading to less mode interaction. As a consequence, fibers with a reduced cladding size and a high N.A. allow us to reach better transmissions.

\subsection{Increasing adiabaticity}

We have discussed in the previous Sections the transmission of higher-order modes in tapered fibers with a 2 mrad tapering angle. It is clear from Figure 4.2 that, for

\footnotetext{
${ }^{3} V_{\text {core }, R_{0}}$ and N.A. are proportional. As a consequence, increasing N.A. increases $V_{\text {core, } R_{0}}$ in Equation 4.1 .
} 
this value of $\Omega$, higher-order modes propagation is non-adiabatic. In this Section, we produce more adiabatic fibers by decreasing $\Omega$. Figure 4.3 shows the transmissions measured for tapering angles $\Omega$ that vary between $4 \mathrm{mrad}$ and $0.4 \mathrm{mrad}$. As expected, we observe that decreasing $\Omega$ (and thus producing more adiabatic tapers) increases the transmission of the first few higher-order modes. For $\Omega=0.4 \mathrm{mrad}$, we measure a transmission as high as $97.8 \pm 1.0 \%$. An advantage of working with fibers that have a reduced cladding size is that it allows to use tapering angles as low as $0.4 \mathrm{mrad}$ while keeping reasonable taper lengths. With the level of transmission reached here, our fibers become usable for implementing higher-order modes trapping schemes [Sagué, Baade, and Rauschenbeutel (2008)]. 


\title{
A low-loss photonic silica nanofiber for higher-order modes
}

\author{
S. Ravets, ${ }^{1,2}$ J. E. Hoffman, ${ }^{1}$ L. A. Orozco,,${ }^{1, *}$ S. L. Rolston, ${ }^{1}$ G. \\ Beadie, $^{3}$ and F. K. Fatemi ${ }^{3}$ \\ ${ }^{1}$ Joint Quantum Institute, Department of Physics, University of Maryland and National \\ Institute of Standards and Technology, College Park, Maryland 20742, USA \\ ${ }^{2}$ Laboratoire Charles Fabry, Institut d'Optique, CNRS, Univ Paris Sud, 2 Avenue Augustin \\ Fresnel, 91127 Palaiseau cedex, France \\ ${ }^{3}$ Optical Sciences Division, Naval Research Laboratory, Washington DC, 20375, USA \\ *lorozco@umd.edu
}

\begin{abstract}
Optical nanofibers confine light to subwavelength scales, and are of interest for the design, integration, and interconnection of nanophotonic devices. Here we demonstrate high transmission (>97\%) of the first family of excited modes through a $350 \mathrm{~nm}$ radius fiber, by appropriate choice of the fiber and precise control of the taper geometry. We can design the nanofibers so that these modes propagate with most of their energy outside the waist region. We also present an optical setup for selectively launching these modes with less than $1 \%$ fundamental mode contamination. Our experimental results are in good agreement with simulations of the propagation. Multimode optical nanofibers expand the photonic toolbox, and may aid in the realization of a fully integrated nanoscale device for communication science, laser science or other sensing applications.
\end{abstract}

(C) 2013 Optical Society of America

OCIS codes: (060.2270) Fiber characterization; (060.2280) Fiber design and fabrication; (350.5500) Propagation.

\section{References and links}

1. F. L. Kien, J. Liang, K. Hakuta, and V. Balykin, "Field intensity distributions and polarization orientations in a vacuum-clad subwavelength-diameter optical fiber," Opt. Commun. 242, 445-455 (2004).

2. S. Leon-Saval, T. Birks, W. Wadsworth, P. S. J. Russell, and M. Mason, "Supercontinuum generation in submicron fibre waveguides," Opt. Express 12, 2864-2869 (2004).

3. E. Vetsch, D. Reitz, G. Sagué, R. Schmidt, S. T. Dawkins, and A. Rauschenbeutel, "Optical interface created by laser-cooled atoms trapped in the evanescent field surrounding an optical nanofiber," Phys. Rev. Lett. 104, 203603 (2010).

4. L. Tong, R. R. Gattass, J. B. Ashcom, S. He, J. Lou, M. Shen, I. Maxwell, and E. Mazur, "Subwavelengthdiameter silica wires for low-loss optical wave guiding," Nature 426, 816-819 (2003).

5. T. Birks and Y. Li, “The shape of fiber tapers," J. Lightwave Technol. 10, 432-438 (1992).

6. X. Jiang, L. Tong, G. Vienne, X. Guo, A. Tsao, Q. Yang, and D. Yang, "Demonstration of optical microfiber knot resonators," Appl. Phys. Lett. 88, 223501 (2006).

7. K. P. Nayak, F. L. Kien, Y. Kawai, K. Hakuta, K. Nakajima, H. T. Miyazaki, and Y. Sugimoto, "Cavity formation on an optical nanofiber using focused ion beam milling technique," Opt. Express 19, 14040-14050 (2011).

8. C. Wuttke, M. Becker, S. Brückner, M. Rothhardt, and A. Rauschenbeutel, "Nanofiber fabry-perot microresonator for nonlinear optics and cavity quantum electrodynamics," Opt. Lett. 37, 1949-1951 (2012).

9. G. Brambilla, V. Finazzi, and D. Richardson, "Ultra-low-loss optical fiber nanotapers," Opt. Express 12, 22582263 (2004).

10. S. M. Spillane, T. J. Kippenberg, O. J. Painter, and K. J. Vahala, "Ideality in a fiber-taper-coupled microresonator system for application to cavity quantum electrodynamics,” Phys. Rev. Lett. 91, 043902 (2003). 
11. M. C. Frawley, A. Petcu-Colan, V. G. Truong, and S. N. Chormaic, "Higher order mode propagation in an optical nanofiber," Opt. Commun. 285, 4648-4654 (2012).

12. F. K. Fatemi, "Cylindrical vector beams for rapid polarization-dependent measurements in atomic systems," Opt Express 19, 25143-25150 (2011).

13. F. Warken, "Ultra thin glass fibers as a tool for coupling light and matter," Ph.D. thesis, Rheinische FriedrichWilhelms Universitat, Mainz, Germany (2007).

14. S. Ravets, J. E. Hoffman, P. Kordell, J. D. Wong, S. L. Rolston, and L. A. Orozco, "Intermodal energy transfer in a tapered optical fiber: Optimizing transmission," in preparation (2013).

15. G. Sagué, A. Baade, and A. Rauschenbeutel, "Blue-detuned evanescent field surface traps for neutral atoms based on mode interference in ultrathin optical fibres," New J. Phys. 10, 113008 (2008).

16. A. W. Snyder and J. D. Love, Optical Waveguide Theory (Chapman and Hall, London, 1983).

17. A. Yariv, Optical Electronics in Modern Communications (Oxford University, New York, 1997).

18. J. A. Pechkis and F. K. Fatemi, "Cold atom guidance in a capillary using blue-detuned, hollow optical modes," Opt. Express 20, 13409-13418 (2012).

19. T. E. Dimmick, G. Kakarantzas, T. A. Birks, and P. S. Russell, "Carbon dioxide laser fabrication of fused-fiber couplers and tapers," Appl. Opt. 38, 6845-6848 (1999).

20. J. E. Hoffman, S. Ravets, J. A. Grover, P. Solano, S. L. Rolston, and L. A. Orozco, "Manufacturing tapered optical fibers with a heat and pull method," in preparation (2013).

21. F. Orucevic, V. Lefèvre-Seguin, and J. Hare, "Transmittance and near-field characterization of sub-wavelength tapered optical fibers," Opt. Express 15, 13624-13629 (2007).

22. P. D. Ltd, "FIMMWAVE/FIMMPROP," http://www.photond.com.

\section{Introduction}

Optical nanofibers with a waist diameter smaller than the wavelength of the guided light are currently used for non-linear optics, atomic physics, sensing, and fiber coupling [1-3]. The intense evanescent field outside of an optical nanofiber is particularly interesting for atom trapping and strong atom-photon coupling. Tapering standard optical fiber down to submicron diameters has been a successful fabrication technique for a variety of applications [4-8], in which transmissions of the fundamental mode of the waveguide reaching more than $99 \%$ have been achieved [4,9]. Until now, the efficient guidance of higher-order modes has not been observed, due to the ease with which they couple to other modes, leading to large losses. This restricts most work with nanofibers to the single mode regime, where the diameter is small enough to only support the fundamental mode $H E_{11}[3,10]$.

This paper reports measurements with nanofibers using the first excited $T E_{01}, T M_{01}$, and $H E_{21}$ modes, which have azimuthal, radial, and hybrid polarization states, respectively. Prior work on higher-order mode propagation has shown $\approx 30 \%$ transmission of this $L P_{11}$ family of modes [11], with a mode purity at the fiber output of $\approx 70 \%$, which gives a total transmission of only $20 \%$. Here, by carefully controlling the taper geometry, and by choosing a commercially available 50 micron reduced-cladding-diameter fiber with increased numerical aperture, we demonstrate transmissions greater than $97 \%$ for light of $780 \mathrm{~nm}$ wavelength in the first excited $L P_{11}$ family of modes through fibers with a $350 \mathrm{~nm}$ waist radius. Furthermore, we present a setup that enables us to efficiently launch these three modes with exceedingly high purity at the output, where less than $1 \%$ of the light is coupled to the fundamental mode [12]. We follow the work of [13] to fabricate the nanofibers and use a series of diagnostics during the pull to monitor the quality of the fiber. We record the total transmission of the light and image the mode exiting the fiber for the duration of the pull. Analysis of the transmission as a function of time for different types of fibers allows us to estimate which modes are excited during the pull as well as their relative energies of excitation through the use of spectrograms [14].

These results open the way to efficiently use higher-order modes in optical nanofibers. Unlike the $H E_{11}$ mode, higher-order modes experience a cutoff at a finite radius. This allows improved control of the evanescent field extent at large radii, enabling stronger fibers and improved handling characteristics. Our work enables the usage of higher-order modes for atomic physics applications. In particular, the spatial interference between several of those modes can create 
unique evanescent field distributions on the waist, providing an easy and self-consistent way to break the symmetry along the propagation axis, suppressing the need to create a standing wave. This is particularly relevant to atomic physics applications for the realization of a one color, blue-detuned and state insensitive trapping potential for atoms [15].

\section{Mode propagation in an optical fiber}

References [16, 17] describe the modes in a cylindrical waveguide using Maxwell equations. The modal fields vary as $\exp [i(\beta z-\omega t)]$ where $\beta$ is the propagation constant of the mode. A mode propagates in the fiber with an effective index

$$
n_{\text {eff }}=\beta / k,
$$

where $k=2 \pi / \lambda$ is the free-space propagation constant and $\lambda$ is the free-space wavelength of the light. The propagation of light inside a two-layered step-index fiber, consisting of a core of radius $a$ and refractive index $n_{\text {core }}$ surrounded by a cladding of radius $R$ and refractive index $n_{\text {clad }}$, depends on $V$,

$$
V=\frac{2 \pi a}{\lambda} \sqrt{n_{\text {core }}^{2}-n_{\text {clad }}^{2}}
$$

$V$ plays an important role in our tapers, since the radius, $a$ in Eq. (2), varies enough that the interfaces seen by the modes change as they propagate through the taper. At the beginning of the taper, the light is confined in the core, and guided by the core-to-cladding interface with $V_{\text {core }}$ as in Eq. (2), and $n_{\text {clad }}<n_{\text {eff }}<n_{\text {core }}$. At the end of the taper, the core is negligible ( $a_{\text {core }} \approx 10 \mathrm{~nm} \ll \lambda$ ) and the light is guided by the cladding-to-air interface with $n_{\text {air }}<n_{\text {eff }}<$ $n_{\text {clad }}$. Between these two regimes, the light escapes the core to the cladding, and the relevant radius in Eq. (2) becomes $R$, which is much larger than $a$. Due to this radius increase, and the large index difference between $n_{\text {clad }}$ and $n_{\text {air }}, V>>1$. The fiber becomes highly multimode, as the number of bound modes is proportional to $V^{2} / 2$ [16]. Maintaining adiabaticity through this transition is critical.

\subsection{First family of excited modes}

Figure 1(a) shows the effective index of refraction against $V$ for several low-order modes in a nanofiber. When $V<2.405$, the fiber supports only the $H E_{11}$ mode. For a typical nanofiber, $n_{\text {core }} \approx 1.5$ and $n_{\text {clad }}=1$. The $T E_{01}$ and $T M_{01}$ modes are allowed for $V>2.405$, and the $H E_{21}$ mode is allowed for $V>2.8$. As long as $V$ remains lower than 3.8, only the four modes mentioned above are allowed. In the weakly guided regime, the modes of interest are known as the $L P_{11}$ family. We initially launch into the actual $L P_{11}$ family, because at the entrance, the fiber is weakly guided. The taper takes us into the strong guiding regime where the $L P_{11}$ splits into $T E_{01}, T M_{01}$, and $H E_{21}$. We will refer to the set of modes $\left(T E_{01}, T M_{01}\right.$, and $\left.H E_{21}\right)$ during the entirety of the pull as the $L P_{11}$ family. This simplifies discussions when referring to the full set of modes, especially in reference to excitations to modes or families with the same symmetry, i.e. $L P_{12}$ for $T E_{02}, T M_{02}$, and $H E_{22}$. Prior work has emphasized propagation of the $H E_{11}$ mode, where $V \leq 2.405[3,7,10]$. We are interested in selectively exciting and guiding the $\mathrm{LP}_{11}$ family through a nanofiber, in a regime where $2.405 \leq V \leq 3.8$. When expanded to free space, these modes have the intensity and polarization profiles shown in Fig. 1(b).

\subsection{Adiabaticity condition}

During the pull we continuously decrease $R$ and the light escapes the core to the cladding as it propagates through the taper. After light escapes the core, the presence of the core, the cladding, 
(a)

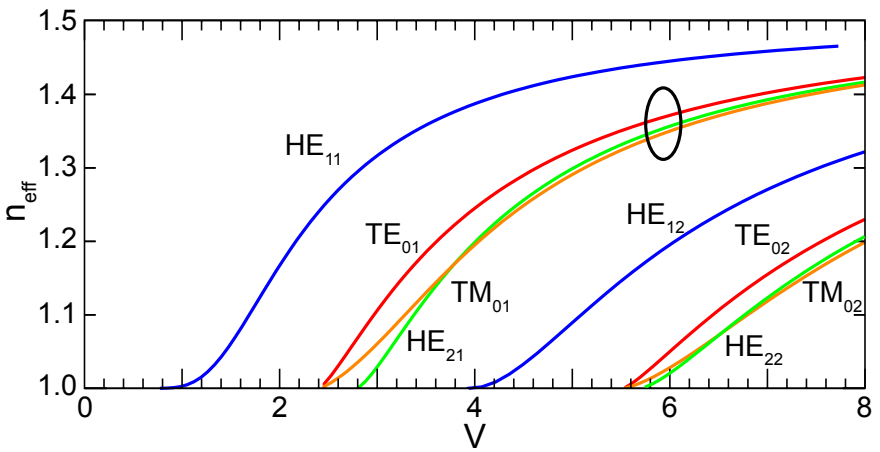

(b)

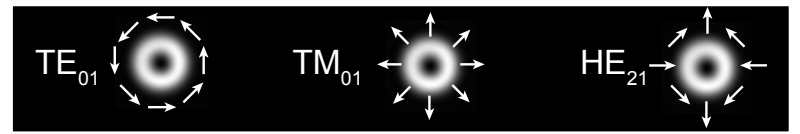

Fig. 1. (a) $n_{\text {eff }}$ indices of several low-order modes in a nanofiber with $n_{\text {clad }}=1.5$, surrounded by vacuum $\left(n_{\text {air }}=1\right)$. Below $V \approx 3.8$, two families of modes exist. In this work, we are emphasizing the $L P_{11}$ family (circled). By symmetry, modes in this family can interfere with the $T M_{02}, T E_{02}, H E_{22}$ family, which may be excited through non-adiabatic processes. (b) Intensity and polarization profiles of the $L P_{11}$ family of modes considered in this work.

and the air influence the mode. The fiber is highly multimode, and modes of the same symmetry can couple to each other. The mode evolution in a taper is strongly related to the shape of the taper. If a taper is too steep, the mode evolution is non-adiabatic resulting in low transmission. As the tapering angle $\Omega$ is reduced, the mode propagation becomes more adiabatic. Following this reasoning, an adiabaticity criterion has been derived [16] relating the characteristic length of the taper $z_{t}$, to the characteristic beat length between two modes $z_{b}$ where $z_{t}=R / \tan (\Omega)$, and $z_{b}=2 \pi /\left(\beta_{1}-\beta_{2}\right)=\lambda /\left(n_{\text {eff }}-n_{\text {eff }}\right)$.

The mode evolution in a taper is adiabatic when the fiber is long enough to satisfy $z_{t} \gg z_{b}$ [16]. For a typical silica optical fiber single mode at $\lambda=780 \mathrm{~nm}$, we calculate using this criterion a minimum $\Omega$ of a few milliradian for the limiting case of $z_{t}=z_{b}$. This implies that a taper requires sub-milliradian $\Omega$ to achieve adiabaticity in the region where light leaves the fiber core and becomes a cladding mode. Therefore, choosing an optical fiber with a small initial cladding radius (and thus a reduced $V_{\text {clad }}$ ) combined with a high numerical aperture is highly advantageous to maintaining adiabaticity [11]. Additionally, such a fiber reduces the overall drawing time, length requirements of the pulling apparatus, and the overall length of the taper.

\section{Experimental setup}

Figure 2 shows a diagram of our experimental setup. Here, we review the mode preparation, the pulling process, and the detection and analysis. We efficiently excite the $T M_{01}, T E_{01}$, and $H E_{21}$ modes using a fiber-based Cylindrical Vector Beam (CVB) generation method $[12,18]$. The fiber is drawn using a heat-and-pull method $[13,19,20]$. We measure the transmission while pulling, recording simultaneously the output of the photodiode to a digital storage oscilloscope and beam profiles on a Charge-Coupled Device (CCD) camera.

A New Focus Vortex laser delivers a $T E M_{00}$ Gaussian beam at $\lambda=780.24 \mathrm{~nm}$. This wavelength corresponds to the $D 2$ line of Rubidium, our atom of interest. We spatially filter this 


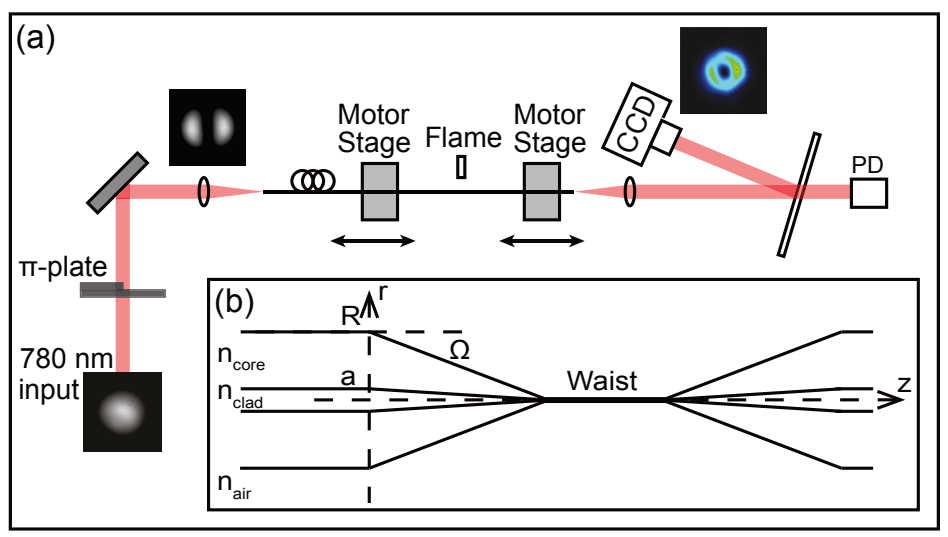

Fig. 2. (a) Simplified diagram of the experimental setup. A Gaussian beam passes through a $\pi$-phase plate and is coupled into the fiber to be drawn. On the output of the fiber, a photodetector (PD) and camera (CCD) monitor the transmission. Typical beam images are shown. (b) Schematic of tapered nanofiber.

beam using a polarization-maintaining optical fiber, and collimate with an asphere to a $1 / e^{2} \mathrm{di}-$ ameter of 630 microns. The Gaussian beam passes through a phase plate that imparts a $\pi$ phase shift on half of the beam, producing a two-lobed beam that approximates a $T E M_{01}$ free-space optical mode. The phase plate is on a translation stage, so that we can easily switch between the $L P_{11}$ and $L P_{01}$ families without adjusting alignment. Figure 2 shows profiles of the beam before and after the phase plate. We couple the beam into the fiber using a matched asphere. The coupling coefficients to the fiber modes are determined by the beam symmetry. The inversion of the polarization over half the incident beam allows us to selectively excite the $L P_{11}$ family with high efficiency. Stress-induced birefringence at the input end of the fiber can be used to selectvely excite the $T M_{01}, T E_{01}$, and $H E_{21}$ modes individually [12], but we have not used that selectivity here. The fundamental $H E_{11}$ mode is only excited through aberrations in the beam. To achieve efficient coupling into the $L P_{11}$ family, we wrap the fiber with two or three windings around a 4-mm diameter mandrel that attenuates any modes higher than the $L P_{11}$ family. Figure 2(a) shows typical input (black and white) and output (color) modes.

We detail our nanofiber pulling process in Ref. $[14,20]$. We start by clamping a fiber to two high-precision Newport XML 210 motor stages. We strip the buffer from the fiber and clean it thoroughly following the procedures in [20]. We image the fiber with an optical microscope and remove any undesired particle remaining on the fiber surface. This is important for the repeatability of our measurements. An oxyhydrogen flame with a stoichiometric combination brings a $0.75 \mathrm{~mm}$ long portion of the fiber to a temperature that exceeds the softening point of fused silica. We pull on the fiber ends at a typical relative velocity of $0.1 \mathrm{~mm} / \mathrm{s}$. We calculate the stage trajectories to produce a fiber of a chosen geometry using an algorithm that relies on conservation of volume [5]. We divide the pull into approximately 100 steps. Each step adds a small section to the taper that reduces the radius of the waist. The sections are small enough to be considered linear locally. The compilation of each small taper creates the final taper with a desired geometry, which is generally composed of a few mrad steep linear section that reduces to a radius of $6 \mu \mathrm{m}$, and then connects to an exponential section that gradually reaches a submicrometer radius. The central region forms a 7-mm uniform waist (see Fig. 2(b) for the geometry). To observe all possible mode cutoffs, the fibers are typically drawn to $R \approx 280$ $\mathrm{nm}$, at which point only the fundamental $H E_{11}$ mode propagates. We vary $\Omega$ from $0.4 \mathrm{mrad}$ to 4 
mrad, resulting in pull times lasting between 100 to 1000 seconds The output side of the fiber is held straight with no mode filtering. We follow the mode evolution for the duration of the pull by monitoring the transmission of a few $\mathrm{mW}$ of laser power through the fiber. The transmitted beam is monitored both by a CCD and by a photodetector (PD). The beamsplitter shown in the figure is tilted to as small an angle as possible to eliminate polarization-dependent reflections. Another PD, not shown in Fig. 2(a), measures the input laser power during the pull to normalize the transmission signal. We record PD signals with a Tektronix DPO7054 oscilloscope with 16bit resolution and a sample rate of 1-10 ksamples/s.

\section{Results}

In this section, we present our results on the transmission of the $L P_{11}$ mode family in the context of adiabaticity. We first analyze the improvements gained by choosing a fiber with a reduced cladding radius and increased numerical aperture. In [11], Frawley et al. looked at the improvement obtained by reducing the initial $R$ from $125 \mu \mathrm{m}$ to $80 \mu \mathrm{m}$. Moving from $80 \mu \mathrm{m}$ to $50 \mu \mathrm{m}$ fibers, we improve the transmission from $10 \%$ to $51 \%$ for a 2 -mrad taper. A spectrogram analysis depicts fewer and weaker excitations of higher-order modes. Second, we demonstrate an improvement in adiabaticity through the control of the taper geometry. Our ability to vary $\Omega$ allows for marked improvement in guidance efficiency through the nanofiber, increasing from $16 \%$ at $4 \mathrm{mrad}$ up to $97.8 \%$ at $0.4 \mathrm{mrad}$.

\subsection{Varying the fiber type}

\subsubsection{Transmission measurements}

Figure 3 shows typical transmissions obtained when exciting the fiber with a combination of $L P_{11}$ modes. We plot the normalized transmission during a single pull as a function of the fiber waist radius. We can identify four distinct regimes in Fig. 3. The modes are initially confined to the core (regime $A$ in Fig. 3). Adiabaticity can easily be achieved, and the transmission is steady. Because this fiber initially also supports the $L P_{02}$ and $L P_{21}$ modes and because our launch can weakly excite these modes, we observe a slight drop in transmitted power near $R=20 \mu \mathrm{m}$, when these higher-order modes become cladding modes. Regime $B$ occurs after the light has escaped from the core to the cladding. Because the cladding is typically much larger than the core $(R / a>10)$, and because $n_{\text {clad }}-n_{\text {air }} \gg n_{\text {core }}-n_{\text {clad }}, V$ increases by over two orders of magnitude $\left(V_{\text {clad }} \approx 200\right)$. If the core-cladding transition is not adiabatic, modes of similar symmetry are excited. In particular, we observe transfers of energy to the $L P_{12}$ family which contains the modes $T E_{02}, T M_{02}$, and $H E_{22}$. The interaction between those modes results in mode beating inside the fiber, and an oscillation in the amount of output light. The oscillations continue to $R \approx 0.7 \mu \mathrm{m}$ (regime $C$ ), where for a typical silica fiber, $V_{\text {clad }} \approx 6$. From this cutoff location, it is clear that much of the beating behavior is due to the $L P_{12}$ modes which have been excited through non-adiabatic transitions. The pull extends through the $T M_{01}, T E_{01}, H E_{21}$ cutoffs (regime $D$ ). After reaching the cutoff radius near $R=290 \mathrm{~nm}$, very little light reaches the photodetector - typically less than $1-2 \%$ - indicating low population of the fundamental mode. To determine the loss in the $H E_{11}$ mode, we measured the transmitted power before and after the pull by translating the phase step out of the way. The losses in this mode were negligible, meaning that we reach a mode purity of 98-99\%. Mode purity is of fundamental importance for nanofiber applications, and in particular for atomic physics where polarization control and stability are required.

We have compared the transmissions obtained using fibers of diameter $80 \mu \mathrm{m}$ and $50 \mu \mathrm{m}$, and observed the beneficial effects of smaller clad fibers and higher numerical aperture for $\Omega=2 \mathrm{mrad}$. This taper angle is chosen because it is non-adiabatic and highlights the effect of fiber diameter on adiabaticity. The improvement in adiabaticity is clear in Fig. 3, with a 

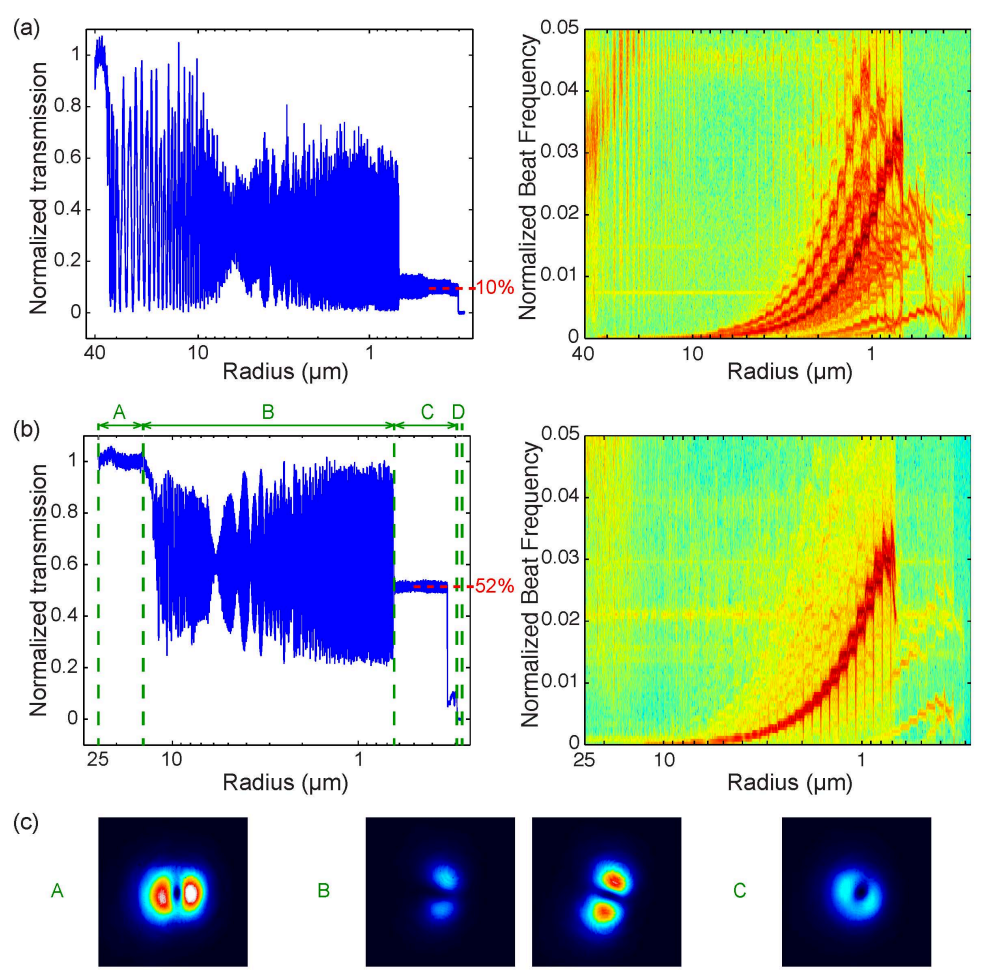

\begin{tabular}{lcccccc}
\hline & $\begin{array}{c}R_{0} \\
(\mu \mathrm{m})\end{array}$ & $\begin{array}{c}\lambda_{\text {cutoff }} \\
(\mathrm{nm})\end{array}$ & $N A$ & $V_{\text {core }, R_{0}}$ & $\begin{array}{c}R_{c}^{L P_{11}} \\
(\mu \mathrm{m})\end{array}$ & $V_{\text {clad }, R_{c}}$ \\
\hline Thorlabs SM980G80 & 40 & 920 & 0.18 & 2.9 & 33 & 288 \\
Fibercore SM1500 & 25 & 1396 & 0.3 & 4.3 & 14 & 119 \\
\hline
\end{tabular}

Fig. 3. Evolution while tapering of the transmission through fibers with a half angle of 2 mrad as a function of the radius of the waist. (a) Fiber with an initial diameter of $80 \mu \mathrm{m}$. (b) Fiber with an initial diameter of $50 \mu \mathrm{m}$. The spectrograms associated with those transmission curves give a clear picture of the power transfers during the pull. Note the logarithmic scales on the horizontal axis. (c) (1.0 MB) Movie of the evolution of the beam transmitted through the fiber measured on the CCD during a $2 \mathrm{mrad}$ pull of the $50 \mu \mathrm{m}$ fiber. We record one frame every second, and display them at a 7 frames per second speed. Sections A, B, $\mathrm{C}$, and $\mathrm{D}$ are described in the text. The properties of the fibers used are summarized in the table.

transmission of $52 \%$ obtained for the $50-\mu \mathrm{m}$ fiber, compared to the $10 \%$ transmission of the 80 $\mu \mathrm{m}$ fiber. The table in Fig. 3 compares the properties of the $80-\mu \mathrm{m}$ and $50-\mu \mathrm{m}$ fibers. Note that the SM1500 fiber initially supports the $L P_{01}, L P_{11}$ as well as the next families of excited modes $L P_{02}$ and $L P_{21}\left(V_{\text {core }, R_{0}}=4.3\right)$. These higher-order modes are substantially filtered, though not completely, by winding the fiber around a $4 \mathrm{~mm}$-diameter rod.

Using the numerical aperture (NA) and the cutoff wavelength provided by Thorlabs and Fibercore, we are able to derive using Eq. (2) the initial $V$ value $V_{c o r e, R_{0}}$, the radius at which the $L P_{11}$ family escapes from the core to the cladding $R_{c}^{L P_{11}}\left(V_{c o r e, R_{c}}=2.405\right.$ : the mode enters the 
cladding-to-air guidance regime) and the corresponding fiber $V$-number at that radius $V_{\text {clad }, R_{c}}$ for a wavelength of $780 \mathrm{~nm}$. Empirically, we obtain an estimate of $R_{c}^{L P_{11}}$ on the transmission plot when the oscillations start. The numbers agree quantitatively. We see that reducing the initial cladding radius and increasing the fiber numerical aperture allows the $L P_{11}$ family to leak from the core to the cladding at a smaller fiber radius. This results in a significantly reduced $V_{\text {clad }}$ when the modes escape from the core to the cladding: By reducing the cladding diameter from $80 \mu \mathrm{m}$ to $50 \mu \mathrm{m}$ and increasing the numerical aperture from 0.18 to 0.30 , the number of available modes decreases by more than an order of magnitude for these two commercially available fibers. Improvements could be achieved by pre-etching the fiber to a smaller diameter so that the initial core radius to cladding radius ratio $R / a$ is further reduced. In this case, the numerical aperture remains unchanged, and the number of available modes directly scales with the square of the ratio of initial cladding radii.

\subsubsection{Spectrograms}

In non-adiabatic propagation, the $L P_{11}$ modes couple to higher-order modes of the same symmetry, belonging to families $L P_{1 m}(m \geq 2)$. Because they propagate with different propagation constants during the pull, they accumulate a phase difference leading to interference in the amount of light that coupled back into the core. Since the photodetector only measures core light, this interference leads to oscillations in the transmission (Fig. 3). A useful way to examine these data is through spectrograms, which plot local, windowed Fourier transforms of the transmission signals as a function of waist radius. Plotting the spectrogram of the transmission signal $[14,21]$, we directly observe the contribution of various pairs of modes in the beating. Figure 3 shows the spectrograms for the $80-\mu \mathrm{m}$ and $50-\mu \mathrm{m}$ diameter fiber pulls. Each line in the spectrogram is specific to the beating between a $L P_{01}$ or $L P_{11}$ mode and another mode of similar symmetry excited during the pull. The curve ends when one of the modes reaches its cutoff: the energy is then lost via coupling to radiative modes.

The number of lines observed in a spectrogram is directly related to the excitation of higherorder modes through non-adiabatic processes from a single launched mode: the more lines present in the spectrogram, the less adiabatic the pull is. Moreover, the colormaps in Fig. 3 are normalized in such a way that the intensity of each red line gives the strength of the energy transferred. It is clear from Fig. 3 that more intense lines are present in the $80-\mu \mathrm{m}$ fiber than in the $50-\mu \mathrm{m}$, further supporting our observation of more stringent adiabaticity requirements for fibers with a large cladding radius and small numerical aperture.

\subsubsection{Imaging the fiber output}

We monitor the transmitted beam with a CCD, and obtain Media 1 showing both the core and cladding light as a function of time throughout the tapering process. Using a microscope objective, we first image the end of the fiber to observe the core-guided light (Media 1 in Fig. 3). The movie shows oscillations that result from mode competition, which modulates the amount of light that exits the fiber in the core.

Media 2 in Fig. 4(a) also depicts the transfer of energy from the core to the cladding. Because the cladding intensity is low compared with the core, we record two spatially separated images of the fiber simultaneously by using strong and weak reflections from a thick beamsplitter with AR-coated front face. Figure 4 shows the normalized fractions of energy exiting the fiber from the cladding and from the core. The two signals are out of phase. We note that the sum of the energy contained in the core and the energy contained in the cladding does not add up to the total energy input in the fiber. Outside the taper region, cladding light becomes highly scrambled and lost through the fiber buffer, resulting in both the speckle observed in Media 2 and the reduced total transmitted power. Residual cladding light is spatially filtered from hitting 


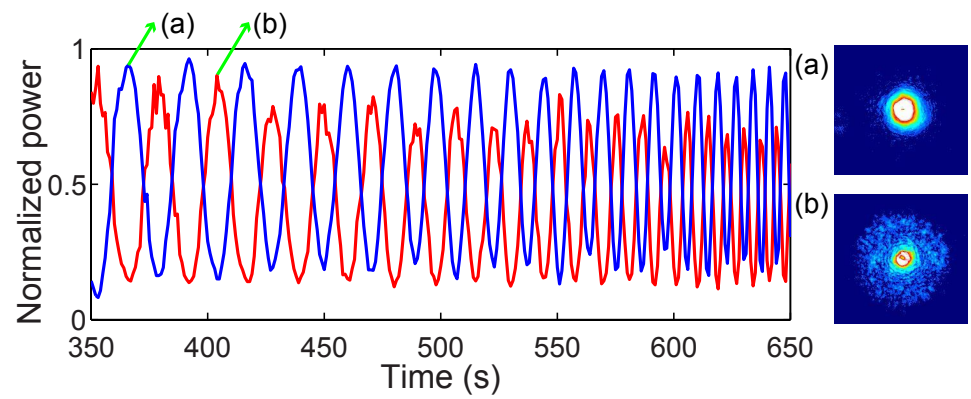

Fig. 4. Amount of light (normalized) exiting the fiber from the core (blue curve) and from the cladding (red curve). The signals are out of phase, confirming the transfer of energy between modes during the tapering. We observed the two simultaneously by using the two reflections from a thick beamsplitter. (a)-(b) (1.1 MB) Media 2 shows the evolution of the beam transmitted through a nanofiber during a portion of a pull, where the power is high enough to observe the cladding light.

the photodetector, so that the observed oscillations Fig. 3 are due only to core-guided light.

\subsection{Varying the angle}

The measurements in this section use the reduced-cladding Fibercore SM1500. For this fiber, the $L P_{11}$ modes transition to cladding modes near $R=13 \mu \mathrm{m}$. By using a reduced-diameter fiber, we observed a drastic improvement of the transmission of the $L P_{11}$ modes. To further improve the adiabaticity, it is necessary to look into more details of the tapering process itself. We have lowered $\Omega$ to improve the transmission over what is observed in the previous section. Figure 5 shows the results of draws using $\Omega=4,2,1,0.75$ and $0.4 \mathrm{mrad}$. Although each plot shows the same qualitative behavior as described in Fig. 3 for the 2-mrad pull, the strength of the features depends on $\Omega$.

The free-space mode at the fiber input has a spatial polarization that is an equal superposition of $H E_{21}$ and $T M_{01}$ (or $T E_{01}$ ). However, mode conversion occurs where the fiber is wound around the mode-filtering mandrel so that the distribution entering the nanofiber is unknown. Within the nanofiber waist, which is held fixed and straight, mode conversion is unlikely to occur so that the desired $L P_{11}$ mode can be achieved after the pull [12]. By $R=0.45 \mu \mathrm{m}$, only the $T M_{01}, T E_{01}$, and $H E_{21}$ modes are confined, with a small contribution in the fundamental $H E_{11}$. The $H E_{12}$ mode achieves cutoff at $V_{\text {clad }}=2.8(R \approx 330 \mathrm{~nm})$, earlier than the $T M_{01}$ and $T E_{01}$ modes, which reach cutoff at $V=2.4(R \approx 290 \mathrm{~nm})$. At $R \approx 330 \mathrm{~nm}$, the power is reduced by the $H E_{21}$ content, which is determined by the initial superposition of states entering the nanofiber.

The transmitted power in the $L P_{11}$ mode family is $16.6 \%$ for $\Omega=4 \mathrm{mrad}$. The transmitted power drops sharply near $R=13 \mu \mathrm{m}$, and undergoes strong oscillations between $10-80 \%$. For $\Omega=2 \mathrm{mrad}$, the oscillations below $R=13 \mu \mathrm{m}$ are reduced, with the transmission fluctuating between $30-90 \%$, and the transmitted power improves to $51.5 \%$. Further decreasing the angle to $\Omega=1,0.75$ and $0.4 \mathrm{mrad}$, improves the transmission to $84.3 \%, 91.2 \%$ and $97.8 \%$ respectively, where uncertainty is dominated by systematic effects that should be less than $1 \%$. Figure 5 also shows excellent agreement between the experimental results and those obtained using commercial waveguide propagation software [22]. For those calculations, the propagation was modeled using the targeted fiber geometry. In our transmission measurements, the scaling between time and radius is made using a separate algorithm that models the dynamics of the pull based on conservation of volume [20]. For $\Omega=0.4$ to $2 \mathrm{mrad}$, the mode cutoff positions we obtain using 
(a)

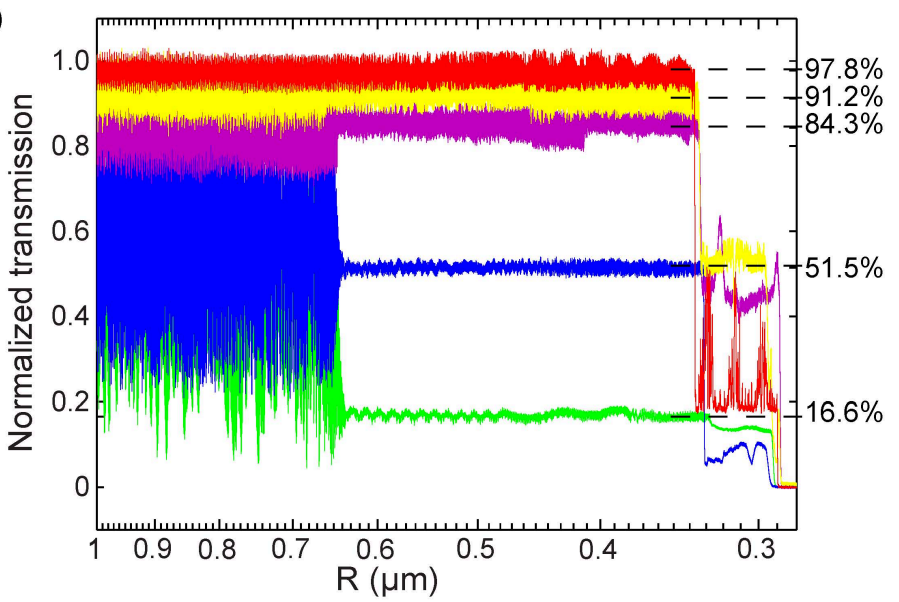

(b)

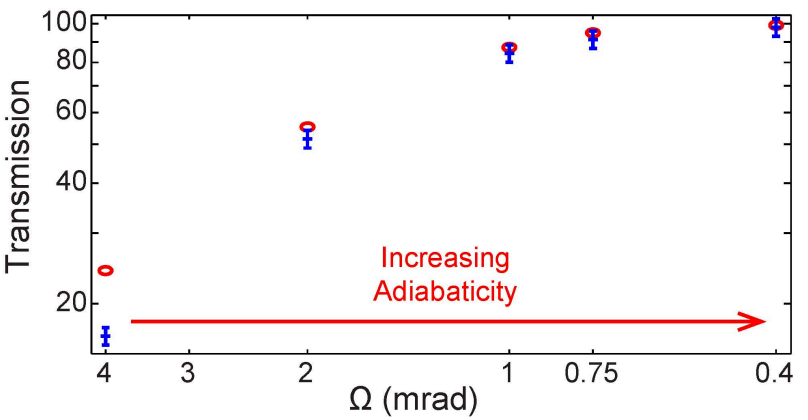

Fig. 5. (a) Transmission through a SM1500 nanofiber for $\Omega=4 \mathrm{mrad}$ (green), $2 \mathrm{mrad}$ (blue), $1 \mathrm{mrad}$ (purple), $0.75 \mathrm{mrad}$ (yellow), $0.4 \mathrm{mrad}$ (red). The transmission plots have the fundamental mode subtracted out (typically about $1 \%$ ) to accurately describe the total transmission of the $L P_{11}$ modes. The horizontal axis for the $4 \mathrm{mrad}$ pull was renormalized to take into account extra tension in the fiber due to the rapidity of the pull. (b) Simulated (red circles) and experimental (blue lines) final transmissions through the fiber as a function of angle. Decreasing $\Omega$ enables us to improve the transmission of the $L P_{11}$ family up to $97.8 \%$.

the scaling from the algorithm correspond directly to what is expected theoretically. We also observe excellent agreement between the experimental transmission measurements and the calculations, confirming the accuracy of our pulling procedure. We were not limited by systematic effects that might include accumulation of contaminants for longer pulls, and asymmetric profiles for faster ones. For the fastest pull (4 mrad), we observed a discrepancy due to systematic effects, and we must apply a different scaling to match the theoretical cutoffs.

For $\Omega=0.4 \mathrm{mrad}$, the most shallow angle studied, the amplitude of the oscillations, which is directly related to the energy transfer to undesired modes, is reduced to a few percent. The observed transmissions are due to losses into and out of the waist. Because the fiber is symmetric, the normalized transmitted power is the square of that in the waist. For $\Omega=0.4 \mathrm{mrad}$, this leads to $98.9 \%$ power in the waist. We believe that such a fiber is usable for various applications involving higher-order modes. We note that reaching adiabaticity for higher-order modes requires fibers that are substantially longer than for the fundamental mode. For the $H E_{11}$ mode, transmissions greater than $99 \%$ can easily be achieved for $\Omega$ up to $5 \mathrm{mrad}$ [14]. We also observed 
that when we remove the $\pi$-phase plate from the launch, the transmission in the fundamental mode is essentially equal to the transmission before pulling, as adiabaticity is strongly satisfied for this mode. During the tapering process, the higher-order modes escape from the core to the cladding earlier than the fundamental $H E_{11}$ mode. When the $H E_{11}$ mode finally transitions to a cladding mode, $R$ has decreased, so that $V$ and the number of available modes to couple to is smaller. The reduction in $R$ also leads to an increase in the difference between adjacent propagation constants, allowing less mode interaction and a steeper $\Omega$. Mode conversion also occurs throughout the fiber and not just at the core-cladding transition point, but this region has the most stringent adiabatic criterion.

\section{Conclusion}

We have demonstrated propagation of higher-order modes in nanofibers using the $T E_{01}, T M_{01}$, and $H E_{21}$ modes. By tapering the fiber with angles near $0.4 \mathrm{mrad}$ and using a commercial, offthe-shelf fiber with $50 \mu \mathrm{m}$ diameter, we have achieved transmission efficiency of $97.8 \%$ with excellent mode purity, a factor of four higher than previous work, and more than one order of magnitude improvement on mode purity. Critical to this work was a spectrogram analysis of the modes present during the pulling. Our experimental results agree with simulations of the propagation through the taper. High transmissions of $L P_{11}$ modes with high purity is a promising tool for atomic physics, expanding the possible intensity and polarization configurations of evanescent fields surrounding the nanofiber.

\section{Acknowledgments}

This work was funded by ONR, the ARO Atomtronics MURI, DARPA, and the NSF through the PFC at JQI. S. R. acknowledges support from the Fulbright Foundation. 


\section{Part II}

\section{Interactions between two and three Rydberg atoms in arrays of optical tweezers}





\section{Chapter 5}

\section{Properties of Rydberg atoms}

\section{Contents}

5.1 Energy spectrum of alkali Rydberg atoms . . . . . . . 82

5.2 Exaggerated properties of Rydberg atoms . . . . . 84

5.3 Rydberg atoms in magnetic and electric fields . . . . 85

5.3 .1 Zeeman effect.................. 86

5.3 .2 Stark effect . . . . . . . . . . . . . . . 87

5.4 Interactions between Rydberg atoms: a brief overview . $\quad 89$

5.5 Rydberg blockade ... . . . . . . . . . . . 91

5.6 Outline of the second part $\ldots \ldots \ldots . \ldots 93$

This second part concentrates on the experiments we performed at the Institut d'Optique on systems of neutral atoms trapped in arrays of optical tweezers. As we mentioned earlier (see Introduction), neutral atoms in their ground state are well isolated from their environment. However, they show weak interatomic interactions, leading to slow entanglement operation. To overcome this difficulty, the solution chosen in our setup is to use highly excited Rydberg atoms. Their remarkable properties allow coupling efficiently the different constituents of the system, a prerequisite for the creation of fast entanglement in the system.

A Rydberg atom is an atom in a highly excited state, where at least one electron has a large principal quantum number $n$ [Gallagher (2005)]. Rydberg states are named after the Swedish physicist Johannes Rydberg who proposed in 1888 an empirical formula describing the relation between the wavelengths of the spectral lines observed in alkali metal. This formula was later derived by N. Bohr within the 


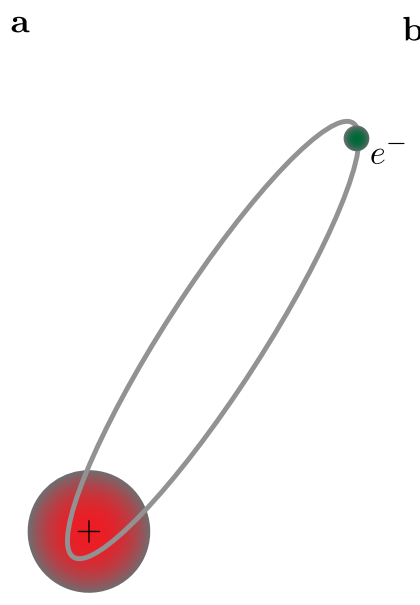

b $\quad E(\mathrm{THz})$

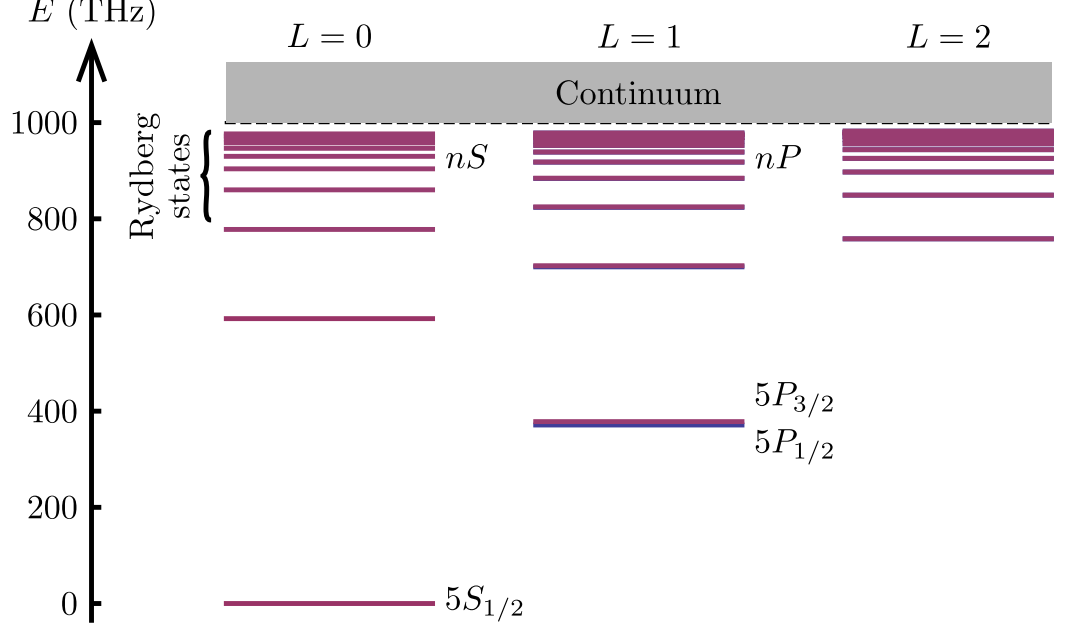

Figure 5.1: Classical and quantum pictures of a Rydberg atom. a, Classical picture of an alkali Rydberg atom. The valence electron spends most of its time far from the nucleus, experiencing essentially a charge $+e$. For low angular momentum states, the orbit can probe the inner core of the atom thus changing the effective charge seen by the electron. b, Quantum picture showing the spectrum of rubidium: the energy levels spacing decreases when $n$ increases. The Rydberg states are the ones close to the ionization threshold.

framework of his atomic model [Bohr (1913)], which provided the interpretation of the observed spectral lines as the result of transitions between high-lying states. Here, we concentrate on alkali atoms which possess only one valence electron. In a classical picture, the valence electron of an alkali Rydberg atom orbits far from the nucleus (see Figure 5.1a). Alkali Rydberg atoms can therefore be pictured classically as giant hydrogen atoms, which confers them interesting properties that are summarized in this introduction.

\subsection{Energy spectrum of alkali Rydberg atoms}

Classically, the valence electron of a Rydberg atom always remains far from the inner electronic core. In a first approximation, we can therefore consider that the valence electron experiences the Coulomb potential of a positive charge $+e$ created by the inner core. This places us exactly in the context of the description of the hydrogen atom, whose eigenergies are given as a function of $n$ by:

$$
E_{n}=-\frac{R_{y}}{n^{2}}
$$

where $R_{y} \simeq 13.6 \mathrm{eV}$. However, one main difference with the hydrogen atom is that, for Rydberg atoms, the inner core can not always be reduced to a simple charge 
Table 5.1: Quantum defects of ${ }^{87} \mathrm{Rb}$ for $\boldsymbol{n} \geq \mathbf{3 0}$. The quantum defects (obtained from [Li et al. (2003)] and [Han et al. (2006)]) decrease with increasing $L$ since the valence electron orbits further and further from the electronic core.

\begin{tabular}{lll}
\hline$L$ & $J$ & $\delta_{L, J}$ \\
\hline 0 & $1 / 2$ & 3.131 \\
1 & $1 / 2$ & 2.654 \\
& $3 / 2$ & 2.641 \\
2 & $3 / 2$ & 1.348 \\
& $5 / 2$ & 1.346 \\
3 & $5 / 2$ & 0.016 \\
& $7 / 2$ & 0.016 \\
\hline
\end{tabular}

$+e$. In particular, for low angular momenta $(L<3)$, the valence electron can probe the inner core (as shown in Figure 5.1a), which modifies the effective electric charge experienced by the electron and the position of the energy levels as a function of $L$. One describes this effect by applying small corrections to the values of $E_{n}$ using quantum defect theory [Gallagher (2005)], that expresses the energy of a state $|n, L\rangle$ by:

$$
E_{n, L}=-\frac{R_{y}}{n^{* 2}}
$$

where $n^{*}=n-\delta_{L}$ is the effective quantum number, that differs from $n$ by the quantity $\delta_{L}$ called the quantum defect. The energy spectrum of ${ }^{87} \mathrm{Rb}$ Rydberg states is represented in Figure 5.1b for $L \in\{0 ; 1 ; 2\}$. We observe indeed that the positions of the energy levels depend on $L$, in contrast with the case of the hydrogen atom.

Finally, the energy levels and thus the quantum defects are also affected, to a lesser extent, by the spin-orbit coupling between the magnetic moment of the electron and its spin. For Rydberg states, the coupling between the valence electron and the atom nucleus is so weak that the hyperfine structure (smaller than $1 \mathrm{MHz}$ for $n \geq 55$ ) can be neglected to a good approximation ${ }^{1}$. In the end, the quantum defects also depend slightly on the quantum number $J$, and the energies $E_{n, L, J}$ are given by Equation 5.2, where:

\footnotetext{
${ }^{1}$ In the rest of this thesis, we consequently express the Rydberg states in the fine basis $\left|n, L, J, M_{J}\right\rangle$.
} 


$$
n^{*}=n-\delta_{L, J}
$$

The quantum defects $\delta_{L, J}$ are measured experimentally, and depend on the atom considered. Table 5.1 lists the ${ }^{87} \mathrm{Rb}$ quantum defects measured, for $L \leq 3$, in [Li et al. (2003)] and [Han et al. (2006)]. For large angular momenta ( $L \geq 3)$, the valence electron always remains far from the inner electronic core, so that the energy spectrum approaches the one of hydrogen. As a consequence, we observe that $\delta_{L, J}$ abruptly decreases and approaches zero when $L$ increases $\left(\delta_{L, J} \simeq 0\right.$ for $\left.L \geq 4\right)$.

\subsection{Exaggerated properties of Rydberg atoms}

As "giant hydrogen atoms", alkali Rydberg atoms show exaggerated properties. Those properties can be derived using semi-classical models that lead to the simple scaling laws summarized below [Gallagher (2005)]:

- The mean radius of the atoms scales as $n^{* 2}$ :

$$
\langle r\rangle \simeq \frac{3}{2} n^{* 2} a_{0}
$$

As a result, the dipole coupling $\left\langle n, L, J, M_{J}\left|\hat{d_{ \pm}}\right| n^{\prime}, L \pm 1, J^{\prime}, M_{J^{\prime}}^{\prime}\right\rangle \simeq e\langle r\rangle$ between neighboring Rydberg states $\left|n, L, J, M_{J}\right\rangle$ and $\left|n^{\prime}, L^{\prime}=L \pm 1, J^{\prime}, M_{J^{\prime}}^{\prime}\right\rangle$ is large. As an example, in the case $n=59$ and $n \simeq n^{\prime}$ (see Chapter 9), typical transition dipole moments are $\simeq 3500 e a_{0}$, that is to say about 5000 times as big as the dipole of a water molecule. Throughout this thesis, we calculate the needed dipole matrix elements using a Matlab code developed by R. Chicireanu during his postdoctoral studies in the group ${ }^{2}$.

- As a consequence of this large dipole moment, Rydberg states are highly polarizable and therefore extremely sensitive to electric fields. For example, the polarizability of the state $59 D_{3 / 2}$ (see Chapter 9) is $\alpha \simeq 550 \mathrm{MHz} /(\mathrm{V} / \mathrm{cm})^{2}$, showing that a small electric field of $\simeq 100 \mathrm{mV} / \mathrm{cm}$ is enough to shift the energy of a state by as much as $\sim 3 \mathrm{MHz}$. The polarizability $\alpha$ scales as $n^{* 7}$ with the effective quantum number.

\footnotetext{
${ }^{2}$ The code is based on a numerical integration of the Rydberg radial wave functions while imposing for the energies of the states the ones given by Equation 5.2 (Numerov method [Zimmerman et al. (1979)]). For more details, see in [Béguin (2013)].
} 
- Rydberg states have a long radiative lifetime $\tau_{\text {tot }}$, of the order of $100 \mu$ s for the typical Rydberg states that we use. Rydberg states decay to other states due to spontaneous emission at a rate $\tau_{\text {rad }}^{-1}$ and also due to stimulated emission at a rate $\tau_{\mathrm{bb}}^{-1}$ induced by black-body radiation in an environment at temperature $T_{\text {Lab }} \simeq 300 \mathrm{~K}$. We have:

$$
\frac{1}{\tau_{\mathrm{tot}}}=\frac{1}{\tau_{\mathrm{rad}}}+\frac{1}{\tau_{\mathrm{bb}}} .
$$

A semi-empirical formula for $\tau_{\text {rad }}$ has been obtained from experimental and theoretical considerations [Gounand et al. (1976); Beterov et al. (2009)]:

$$
\tau_{\mathrm{rad}} \simeq \tau_{s} n^{* \delta},
$$

where $\tau_{s} \simeq 1.1 \mathrm{~ns}$ and $\delta \simeq 3.0$ for $\left|n D_{3 / 2}\right\rangle$ states. For $n=60$, this gives a radiative lifetime $\tau_{\text {rad }} \simeq 208 \mu \mathrm{s}$. Transitions between Rydberg states induced by blackbody radiation [Farley and Wing (1981)] occur at a rate:

$$
\tau_{\mathrm{bb}} \simeq \frac{3}{4}\left(\frac{\hbar c}{e^{2}}\right)^{3} \frac{\hbar}{k_{\mathrm{B}} T_{\mathrm{Lab}}} n^{* 2} \simeq(49 \mathrm{~ns}) \times n^{* 2},
$$

where $k_{\mathrm{B}}$ is the Boltzmann constant. For $n=60$ we have $\tau_{\mathrm{bb}} \simeq 169 \mu \mathrm{s}$. The two effects give a lifetime $\tau_{\text {tot }} \simeq 93 \mu$ s for the $\left|60 D_{3 / 2}\right\rangle$ state. This approximate value is reasonably close to the one given in [Beterov et al. $(2009)]\left(\tau_{\text {tot }} \simeq 108 \mu \mathrm{s}\right)$.

Rydberg atoms show both large dipole moments and long lifetimes. The large dipole moments lead to strong interactions and fast manipulation of the atoms, while the long timescales allow for long experimental sequences as well as manipulations within the Rydberg manifold as we will see in Chapter 8.

\subsection{Rydberg atoms in magnetic and electric fields}

In all our experiments, we apply a $3.3 \mathrm{G}$ magnetic field that is used to optical pump the atoms (see Chapter 6). Moreover, in Chapter 9, we use an electric field to control the interactions between the atoms. In this Section, we thus summarize the effects that electric and magnetic fields have on a Rydberg atom. 
Table 5.2: Landé factors for the Rydberg states of interest in this thesis. For the $3.3 \mathrm{G}$ magnetic field used in this experiment, the typical Zeeman shifts given in frequency units are of the order of a few MHz.

\begin{tabular}{ll}
\hline State & $g_{J}$ \\
\hline$\left|n S_{1 / 2}\right\rangle$ & 2 \\
$\left|n P_{1 / 2}\right\rangle$ & $2 / 3$ \\
$\left|n D_{3 / 2}\right\rangle$ & $4 / 5$ \\
$\left|n F_{5 / 2}\right\rangle$ & $6 / 7$ \\
$\left|n F_{7 / 2}\right\rangle$ & $8 / 7$ \\
\hline
\end{tabular}

\subsubsection{Zeeman effect}

Neglecting diamagnetism, there is nothing special about the influence of a magnetic field on Rydberg atoms as compared to ground state atoms, at least for low-lying $L$ states. A magnetic field $\boldsymbol{B}$ acts on an atom by means of its coupling to the magnetic dipole moment $\hat{\boldsymbol{\mu}}$ of the atom. This effect known as the Zeeman effect lifts the degeneracy of the magnetic sub-levels of the atom. For a magnetic field aligned along the $z$-axis, the interaction Hamiltonian reads:

$$
\hat{H}_{B}=-\hat{\boldsymbol{\mu}} \cdot \boldsymbol{B} \simeq \frac{\mu_{B}}{\hbar}\left(g_{S} \hat{S}_{z}+g_{L} \hat{L}_{z}\right) B_{z}
$$

where $\mu_{B}$ is the Bohr magneton $\left(\mu_{B} \simeq 1.4 \mathrm{MHz} / \mathrm{G}\right)$, and where the electron spin and electron orbital Landé factors are taken equal to $g_{S}=2$ and $g_{L}=1$ in Equation 5.8.

\section{Limit of a weak magnetic field}

In a weak magnetic field, the interaction Hamiltonian can be treated perturbatively. To lowest order, the unperturbed eigenstates are shifted by an energy

$$
\Delta E_{B}=\left\langle n, L, J, M_{J}\left|\hat{H}_{B}\right| n, L, J, M_{J}\right\rangle=g_{J} M_{J} \mu_{B} B
$$

where $g_{J}$ is the Landé factor:

$$
g_{J} \simeq \frac{3}{2}+\frac{S(S+1)-L(L+1)}{2 J(J+1)} .
$$


This perturbative approach is valid as long as the Zeeman shifts are small compared to energy splitting between states of different $J$, that are coupled by $\hat{H}_{B}$. The Landé factors for the states of interest in this Chapter are given in Table 5.2. For the magnetic field $B \simeq 3.3 \mathrm{G}$ used in our experiments, the Zeeman shifts are typically below $10 \mathrm{MHz}$. This is much smaller than the fine structure splitting for $P$-states (typically a few hundreds of $\mathrm{MHz}$ for the states used in this thesis) and $D$-states (typically a few tens of $\mathrm{MHz}$ for the states used in this thesis). However, for $F$ states, the fine structure splitting is smaller than $1 \mathrm{MHz}$ for the Rydberg states used in this thesis, which is typically smaller than the calculated Zeeman shift. In this case, one has to consider the full Hamiltonian $\hat{H}_{B}$.

\section{Full Hamiltonian $\hat{\boldsymbol{H}}_{B}$}

In large magnetic fields, the couplings between states $\left\{\left|n, L, J, M_{J}\right\rangle\right\}$ introduced by $\hat{H}_{B}$ modify the eigenstates of the system. After decomposition in the uncoupled basis $\left\{\left|n, L, M_{L}, S, M_{S}\right\rangle\right\}$, one can calculate the coupling due to the magnetic field between two states $\left|n^{\prime}, L^{\prime}, J^{\prime}, M_{J}^{\prime}\right\rangle$ and $\left|n, L, J, M_{J}\right\rangle$. The orthogonality of radial wavefunctions implies that couplings only occur when $\Delta n=0$. Moreover, the operators $\hat{L}_{z}$ and $\hat{S}_{z}$ conserve $L$, and therefore $\Delta L=0$. The relevant matrix elements of the Zeeman Hamiltonian are thus given by:

$$
\left\langle n, L, J^{\prime}, M_{J}^{\prime}\left|H_{B}\right| n, L, J, M_{J}\right\rangle=\mu_{B} B_{z} \sum_{M_{L}, M_{S}}\left(2 M_{S}+M_{L}\right) C_{L, M_{L} ; S, M_{S}}^{J, M_{J}} C_{L, M_{L} ; S, M_{S}}^{J^{\prime}, M_{J}^{\prime}}
$$

where $C_{L, M_{L} ; S, M_{S}}^{J, M_{J}}$ are the Clebsch-Gordan coefficients:

$$
C_{L, M_{L} ; S, M_{S}}^{J, M_{J}}=\left\langle L, M_{L} ; S, M_{S} \mid J, M_{J}\right\rangle
$$

Clebsch-Gordan coefficients are non-zero if and only if $M_{J}=M_{L}+M_{S}=M_{J}^{\prime}$. As a consequence, the magnetic field introduces couplings between states of different $J$. Note that the diagonal elements of $\hat{H}_{B}$ coincide with the Zeeman shifts obtained in the weak field limit in Equation 5.9.

\subsubsection{Stark effect}

An electric field $\boldsymbol{F}$ acts on an atom by means of its coupling to the electric dipole moment $\hat{\boldsymbol{d}}$ of the atom. Rydberg atoms have large dipole moments and are therefore particularly sensitive to electric fields due to the Stark effect. This property can be a drawback as one needs to pay attention to the electric environment, but it can also 
be used to control the interaction between two atoms as we will see in Chapter 9. In the presence of an electric field, the atomic states are perturbed under the influence of the Hamiltonian:

$$
\hat{H}_{F}=-\hat{\boldsymbol{d}} \cdot \boldsymbol{F} .
$$

\section{Second-order perturbation theory}

For low orbital momenta ( $L \leq 3$, the states of interest in this manuscript), Rydberg states are non-degenerate due to the electrostatic interactions between the ion core and the valence electron (see quantum defect theory in Section 5.1). Because the average value of the dipole moment vanishes in an atomic eigenstate, $\hat{H}_{F}$ has no effect to first order. In the presence of weak electric fields, we apply second-order perturbation theory. In this case, a state $\left|\phi_{0}\right\rangle=\left|n, L, J, M_{J}\right\rangle$ undergoes a quadratic energy shift, given by:

$$
\Delta E_{F}=\sum_{|\phi\rangle} \frac{\left|\left\langle\phi|\hat{\boldsymbol{d}} \cdot \boldsymbol{F}| \phi_{0}\right\rangle\right|^{2}}{E_{\phi}-E_{\phi_{0}}}=-\frac{1}{2} \alpha|\boldsymbol{F}|^{2},
$$

where the summation ranges over all states that are different from $\left|\phi_{0}\right\rangle$. In a first approximation, we will consider that the effect of the electric field is to shift the energy of the state $\left|n, L, J, M_{J}\right\rangle$ by the quantity $\Delta E_{F}$, while the state itself stays unperturbed. The coefficient $\alpha$ is called the polarizability of the state $\left|n, L, J, M_{J}\right\rangle$. Using the scaling laws given in Section 5.1, we can obtain an order of magnitude of the polarizability $\alpha$ :

- The numerator inside the sum in Equation 5.14 scales as the modulus squared of the product of a dipole matrix element times the electric field, $\left(n^{* 2} e a_{0}\right)^{2}|\boldsymbol{F}|^{2}$,

- The denominator inside the sum in Equation 5.14 scales as the difference in energy between two Rydberg states, $-2 R_{y} \times n_{r}^{*-3}$,

- As a consequence, we obtain an approximate expression for $\alpha$ :

$$
\alpha \simeq \frac{e^{2} a_{0}^{2} n^{* 7}}{R_{y}}
$$




\section{Full Stark Hamiltonian}

For larger angular momenta $(L \geq 4)$, the quantum defects vanish, which leads to some degeneracies between states. In this case, the electric field mixes the states, leading to new eigenstates for the system, with eigenenergies that vary linearly with $|\boldsymbol{F}|$. As a consequence, we determine the effect of $\boldsymbol{F}$ by diagonalizing numerically $\hat{H}_{F}$, where we calculate the dipole matrix elements numerically as described in Section 5.2. Note that $\hat{H}_{F}$ only couples states with $\left|\Delta M_{J}\right| \leq 1$ and $\Delta L= \pm 1$ which allows to significantly reduce the size of the basis. Moreover, the matrix elements decrease rapidly with $n$ and we only keep elements verifying $\Delta n \leq 4$. In this thesis, we only consider states with $L \leq 3$ in small electric fields $\leq 100 \mathrm{mV} / \mathrm{cm}$ (see Chapter 9 ). For those states we obtain the polarizabilities $\alpha$ by fitting the calculated Stark effect by a parabola for electric fields that are lower than $100 \mathrm{mV} / \mathrm{cm}$. We have verified that the polarizabilities obtained this way coincide with the ones obtained in Equation 5.14, showing that the perturbative approach is valid for the small fields used in this thesis (see Chapter 9). In this regime, the Stark Hamiltonian is a diagonal matrix, that shifts the eigenenergies by a quantity $\alpha|\boldsymbol{F}|^{2} / 2$ without disturbing the eigenstates ${ }^{3}$.

\subsection{Interactions between Rydberg atoms: a brief overview}

The presence of large dipole moments in our system of Rydberg atoms results in strong interatomic interactions. Indeed, two Rydberg atoms located at positions $\boldsymbol{R}_{A}$ and $\boldsymbol{R}_{B}$ interact predominantly through the dipole-dipole coupling described by the Hamiltonian:

$$
\hat{V}_{\mathrm{dip}}=\frac{1}{4 \pi \epsilon_{0}} \frac{\hat{\boldsymbol{d}}_{A} \cdot \hat{\boldsymbol{d}}_{B}-3\left(\hat{\boldsymbol{d}}_{A} \cdot \boldsymbol{n}\right)\left(\hat{\boldsymbol{d}}_{B} \cdot \boldsymbol{n}\right)}{R^{3}},
$$

where $\hat{\boldsymbol{d}}_{i}$ is the electric dipole moment of atom $i(i=A, B), \boldsymbol{R}=\boldsymbol{R}_{B}-\boldsymbol{R}_{A}$, and $\boldsymbol{n}=$ $\boldsymbol{R} / R$. As a consequence, we observe that the increased dipole moments immediately result in increased interaction energies. An estimate of the interaction can be obtained using the $n^{* 2}$ scaling of the transition dipoles moments:

$$
\left|V_{\text {dip }}\right| \sim \frac{1}{4 \pi \epsilon_{0}} \frac{e^{2} a_{0}^{2}}{R^{3}} n^{* 4}
$$

\footnotetext{
${ }^{3}$ One can show that the non-diagonal matrix elements are higher-order terms Cohen-Tannoudji, Dupont-Roc, and Grynberg (1988).
} 


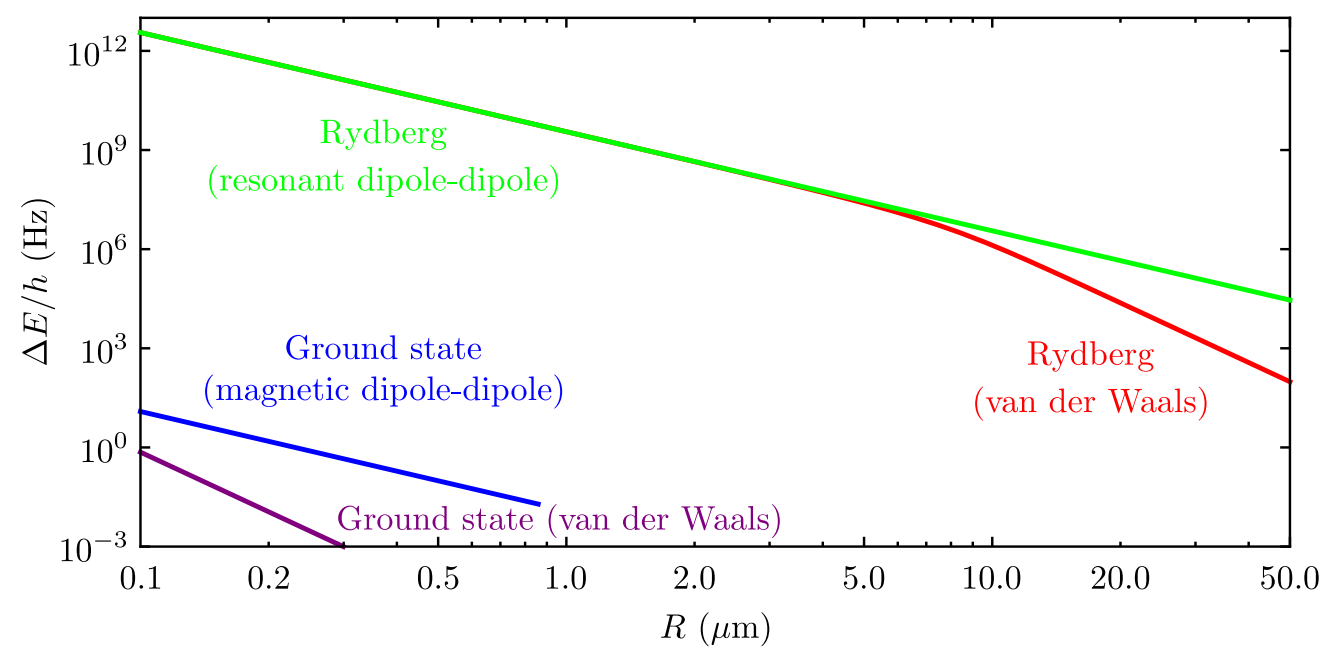

Figure 5.2: Two-body interaction strengths for rubidium atoms (adapted from [Saffman, Walker, and Mølmer (2010)]). The Figure shows the interaction strengths for ground state rubidium atoms (purple and blue curves) and Rydberg atoms excited to the $59 D_{3 / 2}$ level (green and red curves). The purple curve corresponds to the van der Waals interaction between ground state atoms $\left(\propto R^{-6}\right)$, and the blue curve represents the magnetic dipole-dipole interactions $\left(\propto \mu_{B}^{2} / R^{3}\right)$. The green and red curve correspond to electric dipoledipole interactions between Rydberg atoms. For the typical interatomic distances in our experiment, the interactions between Rydberg atoms are $\sim 10^{11}$ larger than between ground state atoms. For Rydberg states, we observe the existence of two regimes for the interaction. The green curve is an extrapolation of the resonant dipole-dipole interactions $\propto R^{-3}$ regime at large distances: this situation can be achieved by controlling the electric field, as we will demonstrate in Chapter 9.

For two atoms of principal quantum number $n=60$ and separated by a distance $R=10 \mu \mathrm{m}$, we obtain a typical interaction energy of $\sim h \times 10 \mathrm{MHz}$, which implies operation times of $\sim 100 \mathrm{~ns} \ll \tau_{\text {tot }}$ for our qubits.

Figure 5.2 compares the interaction strength for ground state rubidium atoms and Rydberg atoms excited to the $59 D_{3 / 2}$ state. At the typical interatomic distances in our experiment ( $\sim 1$ to $10 \mu \mathrm{m}$ ), ground state alkali atoms interact mainly via magnetic dipole-dipole coupling $\propto 1 / R^{3}$. At those distances, the interaction strength between two ground state atoms is lower than $10^{-2} \mathrm{~Hz}$. In our system the manipulation of ground state atoms is not possible on timescales that are smaller than the coherence time of the qubit. On the other hand, Rydberg atoms show electric dipole-dipole interactions that are approximately a factor $10^{11}$ larger than for ground state atoms (see red and green curves in Figure 5.2). At a few microns distance, Rydberg atoms show interactions of a few tens to a few hundreds of $\mathrm{MHz}$, in agreement with our estimations in Equation 5.17, which confirms the possibility to perform fast operations using Rydberg states. A closer look at the curves of the interactions for Rydberg 
atoms in Figure 5.2 shows the existence of two regimes. In the case of two atoms excited to the $59 D_{3 / 2}$ level, one observes that for $R \lesssim 8 \mu \mathrm{m}$, the interaction scales as $1 / R^{3}$ : this is the regime of resonant dipole-dipole interactions that we will discuss in Chapter 9. At larger distances, the interaction scales as $1 / R^{6}$ : this corresponds to the regime of van der Waals interactions that will be the subject of Chapter 7 .

\subsection{Rydberg blockade}

The large interactions between Rydberg atoms have inspired theorists who proposed to use them for the generation of entanglement and for applications in quantum information [Jaksch et al. (2000); Lukin et al. (2001)]. One extreme regime that allows fast creation of entanglement is the so-called "Rydberg blockade" that we describe in this paragraph in the case of two atoms $A$ and $B$ separated by a distance $R$. We consider each atom as a two-level system, with a ground state $|g\rangle$ and an excited Rydberg state $|r\rangle$ separated by an energy $E_{n}$ (see Figure 5.3). The two-atom system contains four pair-states: $|g g\rangle,|g r\rangle,|r g\rangle$ and $|r r\rangle$. In the van der Waals regime, the effect of the strong interaction between the two Rydberg atoms leads to a shift of the level $|r r\rangle$ by the interaction energy $\Delta E(R)$, while the other levels stay essentially unshifted. We assume that a laser is resonant with the excitation of a single atom to the Rydberg state. As a consequence of the dipole-dipole interaction, the laser is not resonant with the excitation of a pair of atoms to state $|r r\rangle$, provided that the linewidth of the excitation $\Omega$ is smaller than $\Delta E(R)$. The inhibition of the excitation of one of the two atoms by the dipole-dipole interaction occurs when $\Delta E(R) \gg \hbar \Omega$, which is a condition that is tolerant to experimental imperfections. This is one of the reasons why the Rydberg blockade triggered a huge interest in the field of quantum information.

When we illuminate the two atoms with the same laser field $\boldsymbol{E}_{L}$, we do not know which of them has been excited to the Rydberg state. This is the key to produce entanglement in the system: because of the Rydberg blockade we excite the system to a superposition state of having prepared one or the other atom to the Rydberg state

$$
\left|\Psi_{+}\right\rangle=\frac{1}{\sqrt{2}}\left(e^{i \boldsymbol{k} \cdot \boldsymbol{R}_{A}}|r g\rangle+e^{i \boldsymbol{k} \cdot \boldsymbol{R}_{B}}|g r\rangle\right),
$$

where $\boldsymbol{k}$ is the wavevector of the excitation laser and $\boldsymbol{R}_{A, B}$ are the positions of the atoms. For each atom, the laser couples the states $|g\rangle$ and $|r\rangle$ with the Rabi frequen- 


\section{One atom}

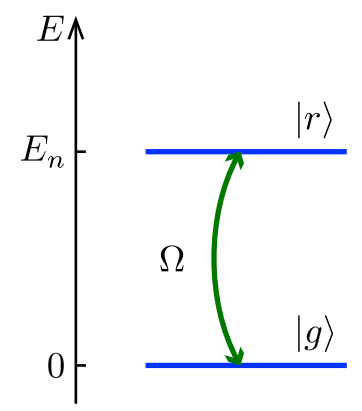

Two atoms

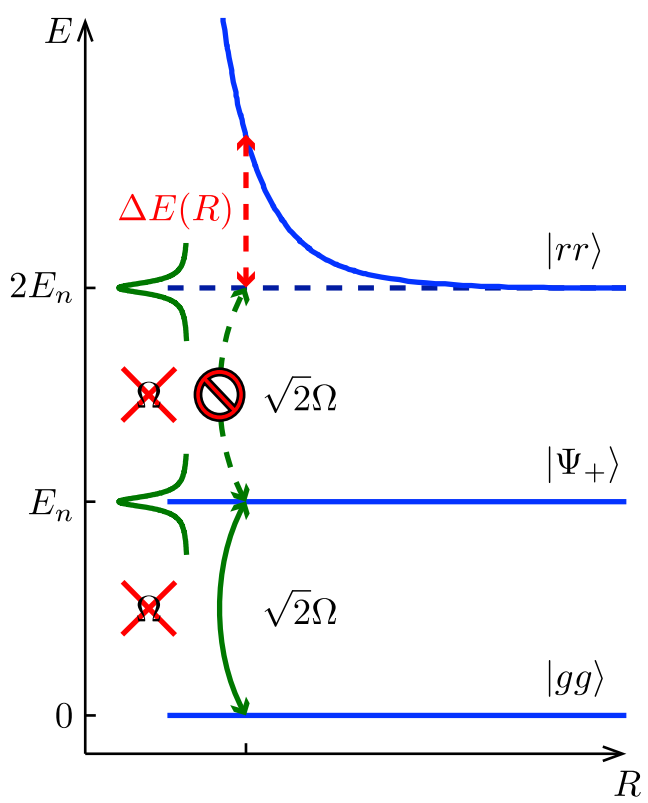

Figure 5.3: Principle of the Rydberg blockade between two atoms. The one-atom spectrum contains the ground state $|g\rangle$ and the Rydberg state $|r\rangle$. In the case of two atoms separated by a distance $R$, the van der Waals interaction shifts the doubly excited state $|r r\rangle$, preventing its excitation by a laser resonant with the one-atom transition. This results in the collective excitation of the state $\left|\Psi_{+}\right\rangle=(|g r\rangle+\exp (i \phi)|r g\rangle) / \sqrt{2}$.

$\operatorname{cies} \Omega_{A}$ and $\Omega_{B}$ :

$$
\hbar \Omega_{i}=-\left\langle r\left|\hat{\boldsymbol{d}}_{i} \cdot \boldsymbol{E}_{\mathrm{L}} e^{i \boldsymbol{k} \cdot \boldsymbol{R}_{i}}\right| g\right\rangle \equiv \hbar \Omega e^{i \boldsymbol{k} \cdot \boldsymbol{R}_{i}}
$$

As a consequence, one can calculate the laser coupling between $|g g\rangle$ and $\left|\Psi_{+}\right\rangle$:

$$
-\left\langle\Psi_{+}\left|\hat{\boldsymbol{d}}_{A} \cdot \boldsymbol{E}_{\mathrm{L}} e^{i \boldsymbol{k} \cdot \boldsymbol{R}_{A}}+\hat{\boldsymbol{d}}_{B} \cdot \boldsymbol{E}_{\mathrm{L}} e^{i \boldsymbol{k} \cdot \boldsymbol{R}_{B}}\right| g g\right\rangle=\sqrt{2} \hbar \Omega
$$

Note that the coupling from $|g g\rangle$ to the antisymmetric state

$$
\left|\Psi_{-}\right\rangle=\frac{1}{\sqrt{2}}\left(e^{i \boldsymbol{k} \cdot \boldsymbol{R}_{A}}|r g\rangle-e^{i \boldsymbol{k} \cdot \boldsymbol{R}_{B}}|g r\rangle\right)
$$

is zero: $\left|\Psi_{-}\right\rangle$is a dark state with respect to the laser excitation, that cannot be excited. In the Rydberg blockade regime, the two-atom system undergoes Rabi oscillations between $|g g\rangle$ and $\left|\Psi_{+}\right\rangle$at a frequency $\sqrt{2} \Omega$. Extended to the case of $N$ atoms where one atom only is excited while the excitation of the $N-1$ other atoms are blockaded, the system oscillates at a frequency $\sqrt{N} \Omega$ between the state $|g g \ldots g\rangle$ and 


$$
|\Psi\rangle=\frac{1}{\sqrt{N}} \sum_{j=1}^{N} e^{i \boldsymbol{k} \cdot \boldsymbol{R}_{j}}\left|g g \ldots r_{j} \ldots g\right\rangle
$$

The Rydberg blockade was first observed in disordered ensembles of cold atoms [Tong et al. (2004); Singer et al. (2004); Comparat and Pillet (2010)]. Today, it has been observed in systems as different as optical lattices [Viteau et al. (2011); Schauß et al. (2012)] or Bose-Einstein condensates [Heidemann et al. (2008); Löw et al. (2009); Balewski et al. (2013)], where it has proved to be a useful tool for the study of many-body physics [Anderson, Veale, and Gallagher (1998); Mourachko et al. (1998)], or photon-photon interactions [Dudin and Kuzmich (2012); Peyronel et al. (2012); Maxwell et al. (2013)]. With the level of control reached in an array of optical tweezers, new applications in quantum information and quantum simulation become possible. The demonstration of Rydberg blockade between two individual atoms at a controlled distance occurred in 2009 in our group [Gaëtan et al. (2009)] and simultaneously in the group of M. Saffman at the University of Wisconsin [Urban et al. (2009)]. This subsequently allowed the entanglement of two individual atoms using the Rydberg blockade at the Institut d'Optique [Wilk et al. (2010)] and the demonstration of a CNOT gate [Isenhower et al. (2010)] at the University of Wisconsin. These experiments set the ground for the experimental implementation of a Rydberg quantum simulator.

\subsection{Outline of the second part}

The large dipole moments in Rydberg atoms allow reaching the interaction energies required for fast manipulation and control of an ensemble of atomic qubits. A remarkable consequence of the large interactions between Rydberg atoms is the Rydberg blockade, as we will observe in Chapter 7 in the case of van der Waals interactions. One of the interests of the Rydberg blockade comes from the fact that it does not require any control over the interaction due to its robustness with respect to experimental fluctuations or imperfections. In Chapter 8 and Chapter 9, we work in a completely different regime where multiple Rydberg excitations are present in the system. In the regime of resonant dipole-dipole interaction, we show our ability to control and actively tune the interatomic interactions themselves, either using microwave or DC electric fields. 


\section{Chapter 6}

\section{Experimental apparatus}

\section{Contents}

6.1 Trapping and imaging single atoms . . . . . . . . . 96

6.1 .1 Our microscopic dipole traps . . . . . . . . . . . . . 96

6.1 .2 Single-atom trapping . . . . . . . . . . . . . . . . 98

6.1.3 Characteristics of the trap . . . . . . . . . . . . 100

6.2 Creating arrays of traps .............. 101

6.2.1 Spatial Light Modulator (SLM) . . . . . . . . . . . . . 101

6.2.2 The Gerchberg-Saxton algorithm . . . . . . . . . . . . 102

6.2.3 Improving the quality of our trapping arrays . . . . . . . . . 102

6.3 Coherent excitation to a Rydberg state . . . . . . . 105

6.3.1 Two-photon excitation . . . . . . . . . . . . . 105

6.3 .2 Laser system . . . . . . . . . . . . . . . . . . . 108

6.3.3 Rydberg detection and coherent excitation . . . . . . . . . 111

6.4 Modeling our system . . . . . . . . . . . . . . . 114

6.4.1 Discussion of the limitations . . . . . . . . . . . . . . . 114

6.4 .2 Modeling the system . . . . . . . . . . . . . . . . . . . . . . . . . . . . . .

6.4 .3 Detection errors . . . . . . . . . . . . . . . 117

6.4.4 Summary of the effects . . . . . . . . . . . . . . . . . . . . . . . . . . . . . .

6.5 Control of electric fields . . . . . . . . . . 118

6.5.1 Compensating residual electric fields . . . . . . . . . . 119

6.5.2 Calibration of the electrodes . . . . . . . . . . . . . . 119

6.5.3 Comparison with calculations . . . . . . . . . . 120

6.6 Single-site addressability . . . . . . . . . . 121

6.6 .1 The setup . . . . . . . . . . . . . . . . 121

6.6 .2 Illustration on a single atom . . . . . . . . . . . . . 122 
6.6.3 Addressing in arrays of atoms . . . . . . . . . . 123

6.7 Conclusion .................. 124

In this chapter, we describe our setup of neutral atoms trapped in arrays of optical dipole traps. This system has been designed to allow the engineering of quantum states in ordered ensembles of interacting atoms. The setup has been extensively described by L. Béguin in [Béguin (2013)], and we recall some of its principles in this chapter. In the first two sections, we concentrate on the techniques we use to produce ordered ensembles of single atoms. We first recall the principles of single atom trapping in an optical dipole trap. Secondly, we describe our addition of a Spatial Light Modulator (SLM) to the setup, which allows generating arrays of dipole traps with controllable geometries. We then concentrate in Section 6.3 on the techniques we use to excite an atoms to a Rydberg state. We detail in particular the addition of an ultra-stable cavity to the setup during my thesis, used to stabilize the frequencies of the Rydberg excitation lasers. The control of electric fields, which is fundamental when working with Rydberg atoms, is the subject of Section 6.5, where we describe in particular our recent calculations used to fully characterize the electric field inside the chamber. We finally describe our implementation of addressing of a single atom for Rydberg excitation.

\subsection{Trapping and imaging single atoms}

The core of our setup, shown schematically in Figure 6.1 is an ensemble of microscopic dipole traps, which allows to trap individual atoms [Schlosser et al. (2001); Schlosser, Reymond, and Grangier (2002)].

\subsubsection{Our microscopic dipole traps}

We use microscopic dipole traps ("optical tweezers") that are produced by tightly focusing a laser beam so as to generate a conservative dipole force on atoms that are illuminated by the laser [Ashkin (1970); Grimm, Weidemüller, and Ovchinnikov (2000)]. In particular, red-detuned dipole traps (with a detuning $\Delta$ ) produce an attractive dipole force that leads to trapping at the maximum intensity of the beam $I_{0}$. The trap depth $U_{0}$ is proportional to $I_{0}$ and inversely proportional to $\Delta$ :

$$
U_{0} \propto \frac{I_{0}}{\Delta}
$$




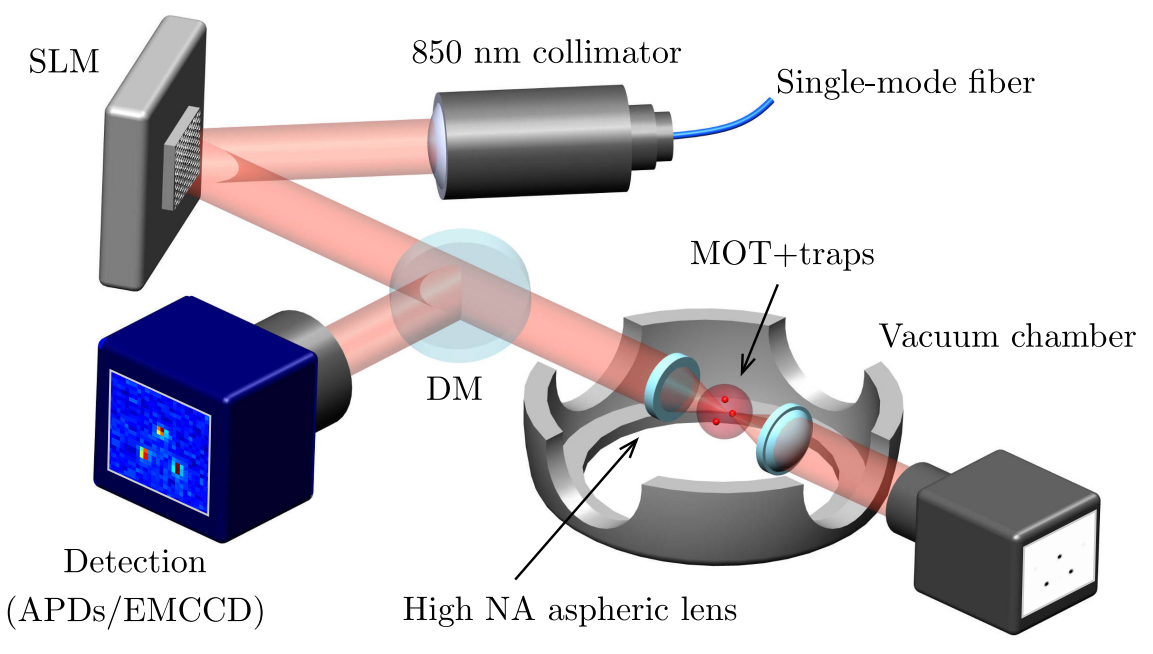

Diagnostics CCD

Figure 6.1: Generation of arrays of microtraps for single-atom trapping. The SLM imprints a calculated phase pattern on the $850 \mathrm{~nm}$ dipole trap beam. A high numerical aperture aspheric lens $\left(f_{\text {Asph }}=10 \mathrm{~mm}\right)$ under vacuum focuses it at the center of a MOT. The atomic fluorescence at $780 \mathrm{~nm}$ is reflected off a dichroic mirror (DM) and detected using Avalanche Photodiodes (APDs) or an EMCCD camera. A second aspheric lens identical to the first one collimates the $850 \mathrm{~nm}$ beam. The transmitted beam is used for trap diagnostics on a CCD.

In our experiment, we use light of wavelength $850 \mathrm{~nm}$, red-detuned from the $\mathrm{D}_{1}$ and $\mathrm{D}_{2}$ lines of rubidium, giving:

$$
\frac{1}{\Delta}=\frac{1}{3 \Delta_{1 / 2}}+\frac{2}{3 \Delta_{3 / 2}}
$$

where $\Delta_{1 / 2}$ is the detuning from the $\mathrm{D}_{1}$ line of ${ }^{87} \mathrm{Rb}$ and $\Delta_{3 / 2}$ is the detuning from the $\mathrm{D}_{2}$ line of ${ }^{87} \mathrm{Rb}$. One specificity of our setup, schematically shown in Figure 6.1, is the small size of our trap. We focus the trapping beam using a custom-made D-ZLaF52LA high-numerical aperture aspheric lens from LightPath technologies (N.A. =0.5). This creates a trap for ${ }^{87} \mathrm{Rb}$ atoms in the focal plane of the lens, with a radial dimension as small as $w_{0} \simeq 1 \mu \mathrm{m}$ [Sortais et al. (2007)]. Using $\simeq 3 \mathrm{~mW}$ of laser power, we obtain a typical trap depth of $U_{0} \simeq k_{B} \times 1 \mathrm{mK}$. We use, as a reservoir of atoms to load this trap, a cloud of cold atoms at temperature $\sim 100 \mu \mathrm{K}$, laser-cooled in a MOT. The optical tweezers are focused in the cold-atom cloud, allowing atoms to enter the trap at random times.

As long as they are illuminated by the MOT beams, the atoms absorb and spontaneously reemit resonant photons at a wavelength of $780 \mathrm{~nm}$ in all directions. We use 
this fluorescence to detect the trapped atoms. When an atom is in the trap, the scattered photons are collected by the aspheric lens used for trapping. We separate the fluorescence light from the trapping light using a dichroic mirror, shown in Figure 6.1 (DM). We measure the fluorescence signal using fiber-coupled avalanche photodiodes (APD) connected to photon counting modules. In this thesis, we performed experiments with arrays containing one, two and three atoms. In order to follow each atom individually, we spatially separate the fluorescence coming from different traps using a combination of two orthogonal edge mirrors separated by a distance of $\simeq 40 \mu \mathrm{m}$ (see Figure 6.2a). We image the plane of the atoms, with a magnification equal to 25 , on the intersection of the mirrors. One signal is transmitted between the two mirrors, while the two other ones are reflected in opposite directions. We couple the single atom signals to different single-mode optical fibers connected to independent detectors [Beugnon et al. (2006)]. This approach works for experiments with one, two or three atoms. To work with higher number of atoms, we have also used an iXon Ultra cooled 16-bit EMCCD camera from Andor. We conjugate the plane of the atoms with the plane of the camera (see Section 6.2) such that each fluorescence signal is imaged on a single pixel.

\subsubsection{Single-atom trapping}

Because our dipole trap has a small volume $\sim 1 \mu \mathrm{m}^{3}$, it is possible to isolate one single atom in the trap. Indeed, there exists a regime of densities of the cold-atom cloud where one atom at most is trapped at a time [Schlosser et al. (2001)]. In this single-atom regime, the entrance of more than one atom in the trapping region results in fast light-assisted collisions. Both atoms are expelled from the trap on timescales $\sim 10 \mu \mathrm{s}$, that are too fast to allow the collection of enough photons to detect twoatom events [Fuhrmanek et al. (2012)]. As a consequence, we only detect events with zero or one atom in the trap.

\section{Fluorescence signals}

Figure $6.2 \mathrm{~b}$ shows typical fluorescence signals observed in the single-atom regime, where we integrated the number of photon counts per 20 milliseconds time bins. We clearly distinguish two levels of fluorescence, that correspond to the absence or the presence of one and only one atom in the trap. We define a fluorescence threshold that makes the situation binary, with a low fluorescence level corresponding to no atom in the trap and a high fluorescence level corresponding to one and only one atom in the 


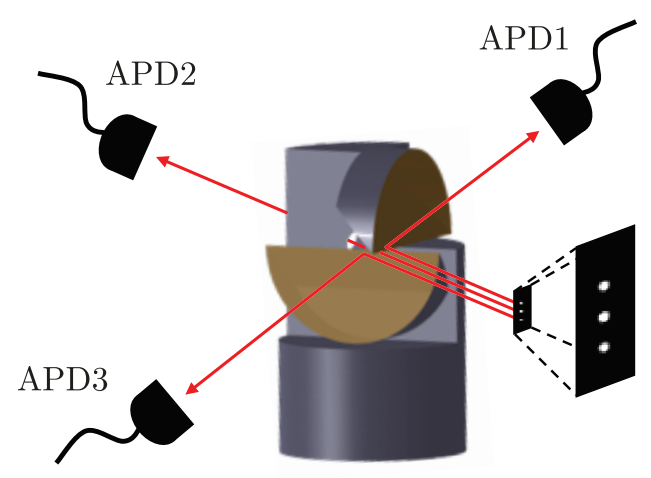

b

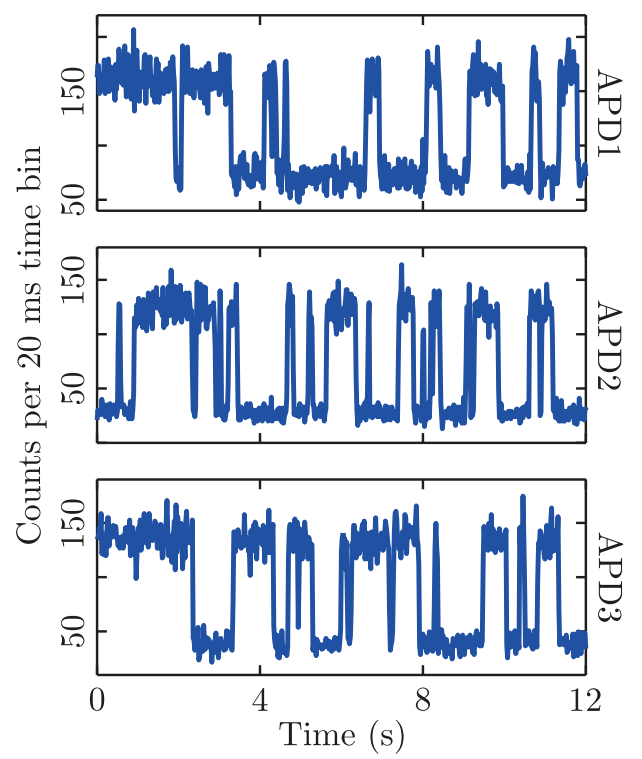

Figure 6.2: Observing single atoms with APDs. a, We spatially separate the three fluorescence signals using edge mirrors. The fluorescence light is coupled to APDs connected to photon counting modules. b, We plot the integrated number of counts per 20 milliseconds time bins. For each of the atoms, we observe the characteristic single atom signal consisting of two fluorescence levels corresponding to the presence or absence of one atom in the trap. We define a threshold to discriminate between the presence or the absence of one atom in the trap.

trap. Note that this method implies that the level of background has to be stable, especially for long runs. We were initially bothered by a fluctuating background coming from light from the MOT beams at wavelength $780 \mathrm{~nm}$, scattered off metallic vacuum parts, creating a speckle field that varies with time. To average out this speckle field, we added five piezoelectric crystals of maximal displacement of $\simeq 5 \mu \mathrm{m}$ on the mirrors of the MOT beams. The addition of those piezos did not lead to any noticeable change in the temperature of the atoms, but drastically improved the stability of the background level compared to the previous situation.

\section{Non-deterministic loading}

Since the atoms enter the trap at random times, the trap is loaded $\simeq 50 \%$ of the time, and our arrays are half-filled on average (non-deterministic loading). However, for some experiments (as it is the case in this thesis) it can be required to work with fully-loaded arrays of atoms. Working with fully-loaded arrays implies that we have to wait for the presence of one atom in each trap in order to trigger the experiment. The probability for this situation to occur is $1 / 2^{N}$ where $N$ is the number of traps. For the small arrays studied here $(N \leq 3)$, this leads to reasonable duty cycle times $\lesssim 1$ s. 
For future experiments with larger fully-loaded arrays of atoms, other deterministic schemes [Grunzweig et al. (2010); Beterov et al. (2011); Ebert et al. (2014)] will have to be implemented (see Outlook).

\subsubsection{Characteristics of the trap}

To characterize our traps, we performed single-atom measurements of the trap depth, the single atom temperature, and the trap frequency. We used standard sequences that have been detailed in [Darquié (2005); Beugnon (2007); Gaëtan (2009); Béguin (2013)], and that we briefly summarize below:

- We obtain the trap depth by measuring the lighshift introduced by the dipole trap beam on the ground state of a single atom. We shine the atom with a $\sigma^{+}$-polarized probe beam whose frequency is ramped around the resonance $\left|5 S_{1 / 2}, F=2, m_{F}=2\right\rangle \rightarrow\left|5 P_{3 / 2}, F=3, m_{F}=3\right\rangle$ [Tey et al. (2008); Shih and Chapman (2013)]. We record the number of fluorescence photons scattered by the atom as a function of the probe detuning. The measured light shift corresponds to the trap depth ${ }^{1}$. Using $\simeq 3 \mathrm{~mW}$ of laser power, we obtain a typical trap depth of $U_{0} \simeq k_{B} \times 1 \mathrm{mK}$.

- We measure the single atom temperature using a release and recapture method. We turn the trap off for a variable duration of time, and we measure the recapture probability at the end of the sequence. The data is compared with Monte-Carlo simulations of the single atom trajectories inside the trap, taking into account the energy distribution of an atom at a temperature $T$ [Tuchendler et al. (2008)]. We typically obtain a single atom temperature of $T \simeq 50 \mu \mathrm{K}$.

- To measure the radial trapping frequency, we first excite the monopole mode by turning the trap off for a few microseconds and turning it on again, as in [Sortais et al. (2007); Engler et al. (2000)]. When the trap is switched on again, the atoms oscillates in the trap. The confinement in the radial direction being much tighter than in the longitudinal direction, we mainly excite the radial motion of the atom at a frequency $\omega_{r}$. We then perform a release and recapture experiment, varying the intermediate holding time. The probability to recapture the atom at the end of the sequence depends on its kinetic energy

\footnotetext{
${ }^{1}$ Another method that we have used to obtain the trap depth consists in measuring, on a single atom, the position of a chosen Rydberg line. As mainly the ground state experiences a light shift, the frequency shift measured when leaving the trap on (as compared to when the trap is turned off during the measurement) corresponds to the trap depth.
} 
at the time we released the atom, and thus oscillates at the frequency $2 \omega_{r}$. Assuming the trapping beam to be a Gaussian, the measurement of the radial trapping frequency gives $\omega_{r} \simeq 2 \pi \times 90 \mathrm{kHz}$ for a trap depth of $\simeq 1 \mathrm{mK}$, which is consistent with a $1 / e^{2}$-radius of $1 \mu \mathrm{m}$ for the trapping beam. From this measurement, we infer the longitudinal trapping frequency $\omega_{x} \simeq 2 \pi \times 20 \mathrm{kHz}$.

\subsection{Creating arrays of traps}

Only two traps were used, in the early stage of the experiment, to characterize the setup and perform the first measurements [Béguin (2013)]. The two traps were created by two independent laser beams forming a small angle. One important task during my thesis was to increase the number of atoms in our system. A proof of principle of the possibility to do so by holographic generation of microtrap arrays had been performed at the Institut d'Optique on an older setup, with up to 5 traps [Bergamini et al. (2004)]. With the help of F. Nogrette, a research engineer at the Institut d'Optique who took care of the optical design, we implemented and pushed further this method on our setup: we demonstrated our ability to trap atoms in arrays with variable geometries containing up to 100 traps, and performed experiments in twoatom and three-atom systems (see Chapters 7, 8, and 9). Here, we briefly summarize our implementation of trap arrays. A more complete description can be found in [Nogrette et al. (2014)], and more details will be given in the thesis of H. Labuhn.

\subsubsection{Spatial Light Modulator (SLM)}

The holographic generation of dipole traps arrays uses a diffractive element in order to turn the collimated trapping beam into an array of traps after the aspheric lens. We use a X10468-02 reflective phase-modulating SLM from Hamamatsu (see Figure 6.1) to modulate the phase of the incoming trapping beam. The SLM has an active area of $12 \times 18 \mathrm{~mm}^{2}$ with a resolution of $600 \times 800$ pixels, and is illuminated by the trapping beam, which has been collimated to a $1 / e^{2}$ radius of $6.7 \mathrm{~mm}$ in order to cover the active area of the SLM as much as we can. Upon reflection on the SLM, we imprint a calculated phase pattern $\varphi(x, y)$ onto the trapping beam of initial Gaussian intensity $I_{0}(x, y)$ [Boyer et al. (2004); Bergamini et al. (2004); Gaunt and Hadzibabic (2012); Gaunt et al. (2013)]. An afocal telescope with a -0.8 magnification then conjugates the plane of the SLM with the plane of the aspheric lens to adapt the trapping beam to the active area of the aspheric lens aperture, while maintaining its collimation. The 
intensity distribution in the focal plane of the aspheric lens is given by the squared modulus of the 2D-Fourier transform of $\sqrt{I_{0}} \exp (i \varphi)$.

\subsubsection{Calculating the phase pattern: the Gerchberg-Saxton algorithm}

Starting with the known initial intensity of the beam $I_{0}$ we want to produce a desired trap array, with target intensity $I_{t}$ in the focal plane of the aspheric lens. The target intensity is a superposition of Gaussian peaks, with a $1 \mu \mathrm{m} 1 / e^{2}$ radius and centered at the location of the traps. Our goal is to calculate the phase $\varphi(x, y)$ we need to imprint on the beam to obtain the target intensity in the focal plane of the aspheric lens. We calculate $\varphi$ iteratively, using the Gerchberg-Saxton algorithm [Gerchberg and Saxton (1972)] that we have implemented in Matlab. We initialize $\varphi$ to a random phase $\varphi_{0}$, which is uniformly distributed over the range $[0,0.2 \times 2 \pi]$. Using fast Fourier transform, we propagate the electric field back and forth from the SLM plane to the plane of the atoms. At each step of the calculation, we impose that the amplitude of the field is $\sqrt{I_{0}}$ in the SLM plane and $\sqrt{I_{t}}$ in the focal plane:

- At step $n$, we obtain a phase $\varphi_{n}$ in the SLM plane. We then fix the electric field amplitude to $\sqrt{I_{0}}$ and, using fast Fourier transform, we propagate the electric field $\sqrt{I_{0}} e^{i \varphi_{n}}$ through the aspheric lens. We obtain the field $A_{n}^{f} e^{i \varphi_{n}^{f}}$ in the focal plane of the aspheric lens.

- In the plane of the atoms, we then fix the amplitude of the field to $\sqrt{I_{t}}$, and we propagate the field $\sqrt{I_{t}} e^{i \varphi_{n}^{f}}$ back to the SLM plane by inverse Fourier transform. We obtain the new phase $\varphi_{n+1}$.

- Convergence is reached when the difference between the calculated intensity $\left|A_{n}^{f}\right|^{2}$ and the target intensity $I_{t}$ is smaller than a chosen threshold.

After a few tens of iterations, the algorithm typically converges toward an approximate solution, where the intensity distribution in the focal plane of the lens is $I_{t}$ to a good approximation.

\subsubsection{Improving the quality of our trapping arrays}

We use several tools to characterize and optimize our trapping arrays. We first collect the trapping light after the vacuum chamber so as to image the intensity 

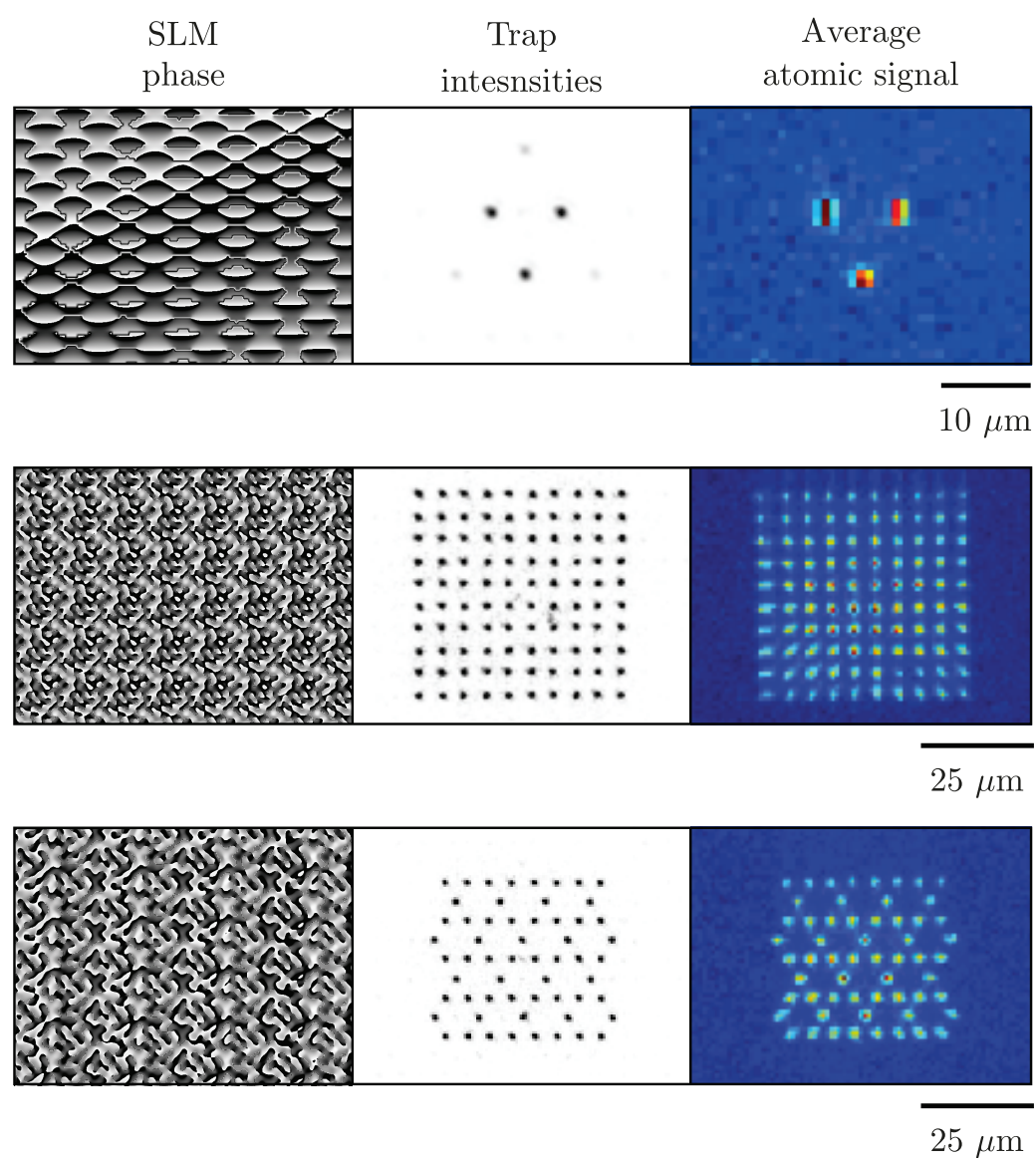

Figure 6.3: Examples of trapping arrays. For each panel, we show the calculated phase pattern used to create the array (left), an image of the resulting trap array taken with the diagnostics CCD (middle), and the average of 1000 fluorescence images of single atoms loaded into the traps (right). We have calibrated the distances between the traps, by measuring the displacement of a trap image on the CCD camera when we change the angle of incidence of the trapping beam by a known amount [Béguin (2013)]. We found a systematic uncertainty of $5 \%$, which will be the uncertainty given for the distances between atoms in the rest of this thesis.

pattern obtained in the focal plane of the aspheric lens. To do so, we re-collimate the trapping beam with the second aspheric lens placed in vacuum and facing the first one in a symmetrical configuration as shown in Figure 6.1. We then analyze the trapping light using either a CCD camera to measure its intensity distribution or a Shack-Hartmann sensor to measure the wavefront distortion at the output of the chamber. The second option is to look at the fluorescence signals of single atoms trapped in the produced arrays. To do so, we measure the fluorescence emitted by atoms in the trap using the EMCCD camera or the APDs (see Figure 6.1). These four approaches are complementary and allowed us to check and optimize the quality of our trapping arrays, as described below: 
- The CCD camera placed after the vacuum chamber allows us to image our trapping arrays (the plane of the CCD is conjugated with the plane of the atoms), as shown for several phase patterns (middle panels of Figure 6.3). We can use those images to improve the quality of our trapping arrays. Indeed, after we first calculate the phase needed for a given trap array, we generally notice on the CCD images some non-negligible dispersion in the trap intensities. For example, after we first calculated the phase for a $10 \times 10$ square lattice, the standard deviation of the trap intensities distribution was $\simeq 19 \%$. Such non-uniformities are detrimental to our experiment since the quality of singleatom trapping depends in the trapping power ${ }^{2}$. As a consequence, we use this diagnostic to retroact on the phase we imprint on the SLM: we modify the target intensity and continue to run the Gerchberg-Saxton with this updated target intensity so as to correct for the measured imperfections. In the new target image, we enhance the depths of the traps that show too low intensities on the CCD and we reduce the ones that showed too high intensities on the CCD. This leads to a new phase pattern, with improved distribution of trap intensities. Iterating this procedure a few times drastically improves the uniformity of our trapping arrays: for the $10 \times 10$ array showed in Figure 6.3, we have obtained a standard deviation in the distribution of trap intensities of $\simeq 1.4 \%$.

- After we installed the SLM, we also noticed on the CCD images the degradation of the quality of the traps when increasing the size of the array. This led us to analyze the wavefront of the trapping beam using a Shack-Hartmann wavefront sensor. Applying a flat phase on the SLM (i.e. one single trap centered on the field), we observed a significant deformation of the wavefront as the light traveled across the vacuum system ${ }^{3}$. This deformation is mainly due to imperfections of the optics (vacuum windows, aspheric lenses) and residual misalignments that introduce aberrations in the system. Following an approach commonly used in adaptive optics, we compensate for the optical aberrations of the system by applying on the SLM the conjugate of the measured distortion [López-Quesada, Andilla, and Martín-Badosa (2009)]. After this correction, we measured a root mean square deviation for a flat wavefront as low as $\simeq 0.014 \lambda$. This correction pattern is now systematically added to the holograms we display

\footnotetext{
${ }^{2}$ Too low powers lead to shallow traps, with short trapping times. Too high powers can place us outside of the single-atom regime.

${ }^{3}$ The measurement of the root mean square deviation from a flat wavefront gave $\simeq 0.05 \lambda$ before the vacuum chamber and $\simeq 0.15 \lambda$ after the vacuum chamber.
} 
on the SLM.

- Using the EMCCD camera, we have been able to demonstrate single-atom trapping in large lattices. The right panels in Figure 6.3 show the average of approximately 1000 EMCCD fluorescence images of single atoms in the traps. We demonstrated single-atom trapping in up to 100 traps separated by distances as small as $3 \mu \mathrm{m}$, in various geometrical configurations.

- Using the avalanche photodiodes, we repeated the temperature, trap depth and trap frequency measurements in an array of three traps. We obtained trap characteristics identical to the ones shown in Section 6.1.3, demonstrating the viability of the holographic approach for the production of our traps.

Our arrays are particularly adapted to Rydberg physics, where the large interatomic interactions allow working with interatomic distances as large as a few microns (in this thesis, interatomic distances range from $\simeq 4 \mu \mathrm{m}$ to $\simeq 50 \mu \mathrm{m}$ ). The versatility of this tool is also striking considering the ease with which it is possible to create various types of arrays, in comparison to more traditional techniques such as optical lattices [Dumke et al. (2002); Schlosser et al. (2011); Bloch (2005); Nelson, Li, and Weiss (2007)].

\subsection{Coherent excitation to a Rydberg state}

We have shown our ability to trap single atoms in arrays of optical dipole traps, with a typical interatomic separation of a few microns. In order to have strong interactions in our system, we use Rydberg states with principal quantum numbers $n \simeq 50-100$. In this section, we describe our Rydberg excitation scheme on a single atom. To realize our experiments, especially in view of quantum information applications [Jaksch et al. (1999)], one requirement is to coherently excite the atoms to Rydberg states. Here, we demonstrate our ability to perform Rabi oscillations on single atoms.

\subsubsection{Two-photon excitation}

Transition energies from the ground state to a Rydberg state typically lie in the UV domain, with wavelengths of $\sim 300 \mathrm{~nm}$. The coherent excitation of a single atom to a Rydberg state using a UV laser has been demonstrated recently [Hankin et al. (2014)]. However, it is generally preferred to use an intermediate state in a twophoton process [Singer et al. (2004); Afrousheh et al. (2004); Liebisch et al. (2005); 
a

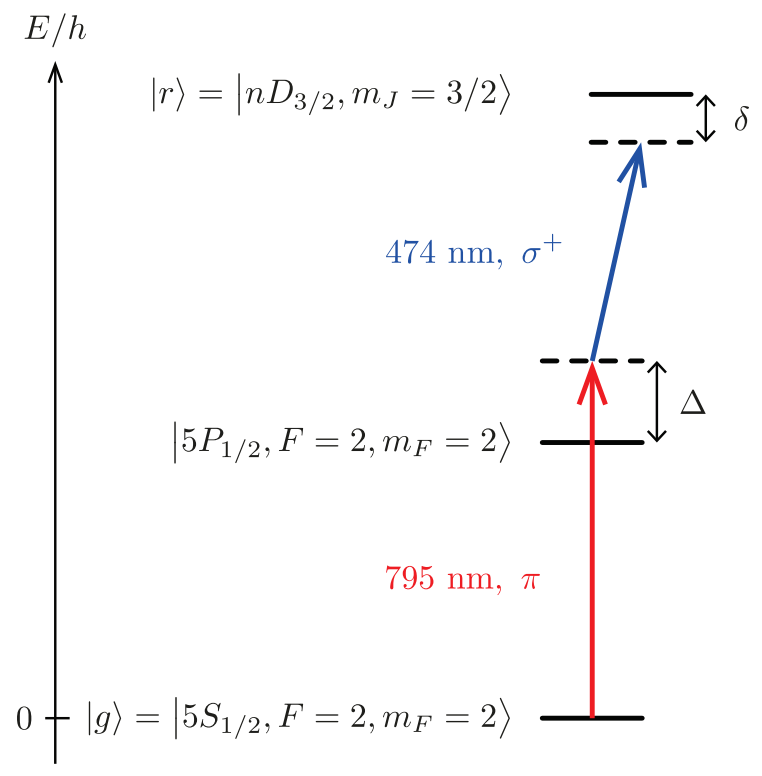

b

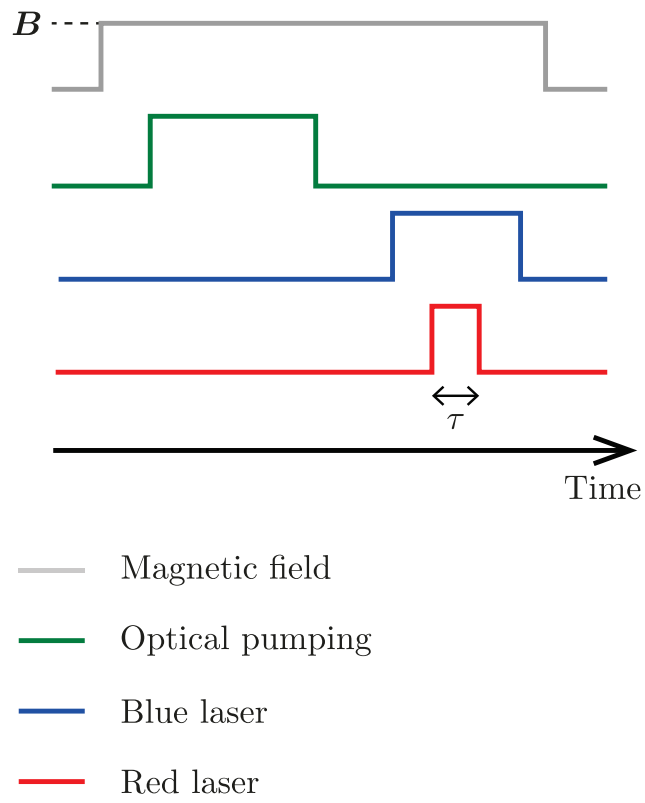

Figure 6.4: Excitation to Rydberg states. a, Level configuration for our two-photon excitation used to reach the Rydberg states. b, Typical excitation sequence. After optically pumping the atoms to the ground state, we shine the excitation beams on the atoms for a variable duration $\tau$.

van Ditzhuijzen et al. (2006); Johnson et al. (2008); Miroshnychenko et al. (2010)], due to the technical difficulties encountered when working with UV light ${ }^{4}$ [Tong et al. (2004); Hankin et al. (2014)].

\section{Laser configuration}

For two-photon excitation, we use a red laser of frequency $\omega_{R}$ that couples the ground state $|g\rangle=\left|5 S_{1 / 2}, F=2, M_{F}=2\right\rangle$ to the intermediate state $\left|5 P_{1 / 2}, F=2, M_{F}=2\right\rangle$ with a Rabi frequency $\Omega_{R}$, and a blue laser of frequency $\omega_{B}$ that couples the intermediate state to the chosen Rydberg state $|r\rangle$ with a Rabi frequency $\Omega_{B}$ (see Figure 6.4). The Rabi frequency $\Omega_{i}(i=\{R ; B\})$ is given by [Gaëtan (2009)]:

$$
\hbar \Omega_{i}=\left|d_{i}\right| \mathcal{E}_{i}
$$

where $\mathcal{E}_{i}$ is the amplitude of the field and $d_{i}$ is the dipole matrix element between the states that are coupled by the laser. We define the detunings $\Delta=\omega_{R}-E_{\left|5 P_{1 / 2}\right\rangle} / \hbar$ and

\footnotetext{
${ }^{4}$ Those difficulties include current technical limitations of UV lasers, and the accumulation of charges on surfaces hit by the UV laser due to the photoelectric effect.
} 
$\delta=\omega_{R}+\omega_{B}-E_{|r\rangle} / \hbar$. Under the assumption $\Omega_{R}, \Omega_{B} \ll \Delta$ and in the rotating wave approximation, it is possible to show that this situation is equivalent to coupling $|g\rangle$ to $|r\rangle$ with an effective Rabi frequency and an effective detuning:

$$
\begin{aligned}
& \Omega_{\mathrm{eff}}=\frac{\Omega_{R} \Omega_{B}}{2 \Delta}, \\
& \delta_{\mathrm{eff}}=\delta-\left(\frac{\left|\Omega_{R}\right|^{2}}{4 \Delta}-\frac{\left|\Omega_{B}\right|^{2}}{4 \Delta}\right) .
\end{aligned}
$$

\section{Typical Rabi frequencies}

The red laser is a DL100 commercial laser from Toptica, with a wavelength of $795 \mathrm{~nm}$. The detuning from the intermediate state is fixed ${ }^{5}$ to $\Delta=2 \pi \times 740 \mathrm{MHz}$ [Miroshnychenko et al. (2010)]. The laser beam has a measured $1 / e^{2}$ radius $w_{R} \simeq 105 \mu \mathrm{m}$ at the position of the atoms, which leads to the following typical order of magnitude for $\Omega_{R} / 2 \pi$ :

$$
\frac{\Omega_{R}}{2 \pi}=\frac{\left|d_{R}\right|}{h} \sqrt{\frac{4 P_{R}}{\pi \varepsilon_{0} c w_{R}^{2}}} \simeq 146 \sqrt{\frac{P_{R}}{1 \mathrm{~mW}}} \mathrm{MHz},
$$

where the value $d_{R} \simeq 1.73 e a_{0}$ was taken from [Steck (2010)]. We keep the possibility to vary the red laser power so as to tune $\Omega_{R}$ (the red laser power $P_{R}$ that is sent on the atoms varies between $100 \mu \mathrm{W}$ and $12 \mathrm{~mW}$ in the experiments presented in this thesis).

The $474 \mathrm{~nm}$ light comes from a TA-SHG-110 commercial laser from Toptica. A diode laser produces light with $\mathrm{a} \simeq 950 \mathrm{~nm}$ wavelength, that is frequency doubled to obtain blue light, with $\mathrm{a} \simeq 474 \mathrm{~nm}$ wavelength. Once the laser is locked (see Section 6.3.2), the detuning $\delta$ can be scanned by changing the frequency of an electrooptic modulator (see Section 6.3.2). The laser beam has a measured $1 / e^{2}$ radius $w_{B} \simeq 18 \mu \mathrm{m}$ at the position of the atoms. The Rabi frequency $\Omega_{B}$ depends on the principal quantum number $n$ of the chosen Rydberg state, and reads:

$$
\frac{\Omega_{B}}{2 \pi}=\frac{\left|d_{B}\right|}{h} \sqrt{\frac{4 P_{B}}{\pi \varepsilon_{0} c w_{B}^{2}}} \simeq 5.5\left(\frac{58^{*}}{n^{*}}\right)^{3 / 2} \sqrt{\frac{P_{B}}{1 \mathrm{~mW}}} \mathrm{MHz} .
$$

Here, the value $d_{B} \simeq 0.011 \times e a_{0}$ for the transition between the states $\left|5 P_{1 / 2}, M_{J}=1 / 2\right\rangle$ and $\left|58 D_{3 / 2}, M_{J}=3 / 2\right\rangle$ was taken from [Gaëtan (2009)], and we used the $\left(n^{*}\right)^{-3 / 2}$ scaling of the dipole coupling between low-lying states and Rydberg states. Since

\footnotetext{
${ }^{5} \mathrm{As}$ a consequence, the frequency of the $795 \mathrm{~nm}$ excitation laser is fixed in our experiments. The frequency measured with the wavemeter is $\nu_{R}=377105726 \pm 10 \mathrm{MHz}$.
} 
the dipole element $d_{B}$ is small compared to $d_{R}$, we generally use all the available power for the blue laser $\left(P_{B} \simeq 90 \mathrm{~mW}\right.$ on the atoms). In total, with the available power in the red and blue lasers, the largest effective Rabi frequency for the Rydberg transitions studied in this thesis is $\Omega_{\mathrm{eff}} \simeq 2 \pi \times 10 \mathrm{MHz}$.

\section{Polarization properties}

In our experiments the $795 \mathrm{~nm}$ light is $\pi$-polarized, and the $474 \mathrm{~nm}$ light can be either $\sigma^{+}$-polarized or $\sigma^{-}$-polarized, depending on the orientation of a quarter waveplate. The $\sigma^{+}$polarization allows coupling $|g\rangle$ to $|r\rangle=\left|n D_{3 / 2}, m_{j}=3 / 2\right\rangle$ states, whereas the $\sigma^{-}$polarization is used to excite $|r\rangle=\left|n S_{1 / 2}, m_{j}=1 / 2\right\rangle$ states. In most of the experiments performed here, we chose to work with $|r\rangle=\left|n D_{3 / 2}, m_{j}=3 / 2\right\rangle$ states $^{6}$, because they show a better coupling to $|g\rangle$. We chose the value of $n$ depending on the type of experiment we want to perform. In what follows, $n$ varies from 59 to 82 .

\subsubsection{Laser system}

The excitation lasers need to be on resonance with the studied transition to perform the two-photon excitation. Also, the relative phase drift (associated with the linewidths of the lasers) has to be stable and its rate of change has to be smaller than the Rabi frequency, of a few MHz typically.

\section{Our ultra-stable cavity}

One of our tasks during my thesis was to modify the previous laser system described in [Béguin (2013)] by installing an ultra-stable reference cavity in order to improve the laser stability. We stabilize the lasers using a commercial ultra-stable reference cavity from Stable Lasers Systems, as shown in Figure 6.5a. The ensemble is placed in a vacuum system at a pressure of $\simeq 10^{-7}$ mbar, and mounted on a breadboard which is enclosed in a wooden box lined internally with lead foam. The cavity, of length $L=$ $10 \mathrm{~cm}$, is made of a plane mirror and a spherical mirror with a radius of curvature $R_{c}=$ $50 \mathrm{~cm}$. The mirrors are mounted on an ultra-low expansion (ULE) glass spacer, which coefficient of thermal expansion cancels $\left(\alpha_{0}=0 \pm 30 \times 10^{-9} \mathrm{~K}^{-1}\right)$ at a temperature of $307.65 \pm 1.00 \mathrm{~K}$, as specified by the manufacturer. The cavity is placed under vacuum at a temperature of $307.65 \mathrm{~K}$, in a thermally regulated environment with $\Delta T=2 \mathrm{mK}$ precision. The zero-crossing temperature is known to a precision of $1 \mathrm{~K}$. A linear estimate around this point of the expansion coefficient change with temperature gives

\footnotetext{
${ }^{6}$ see one exception where we use S-states in Chapter 7.
} 

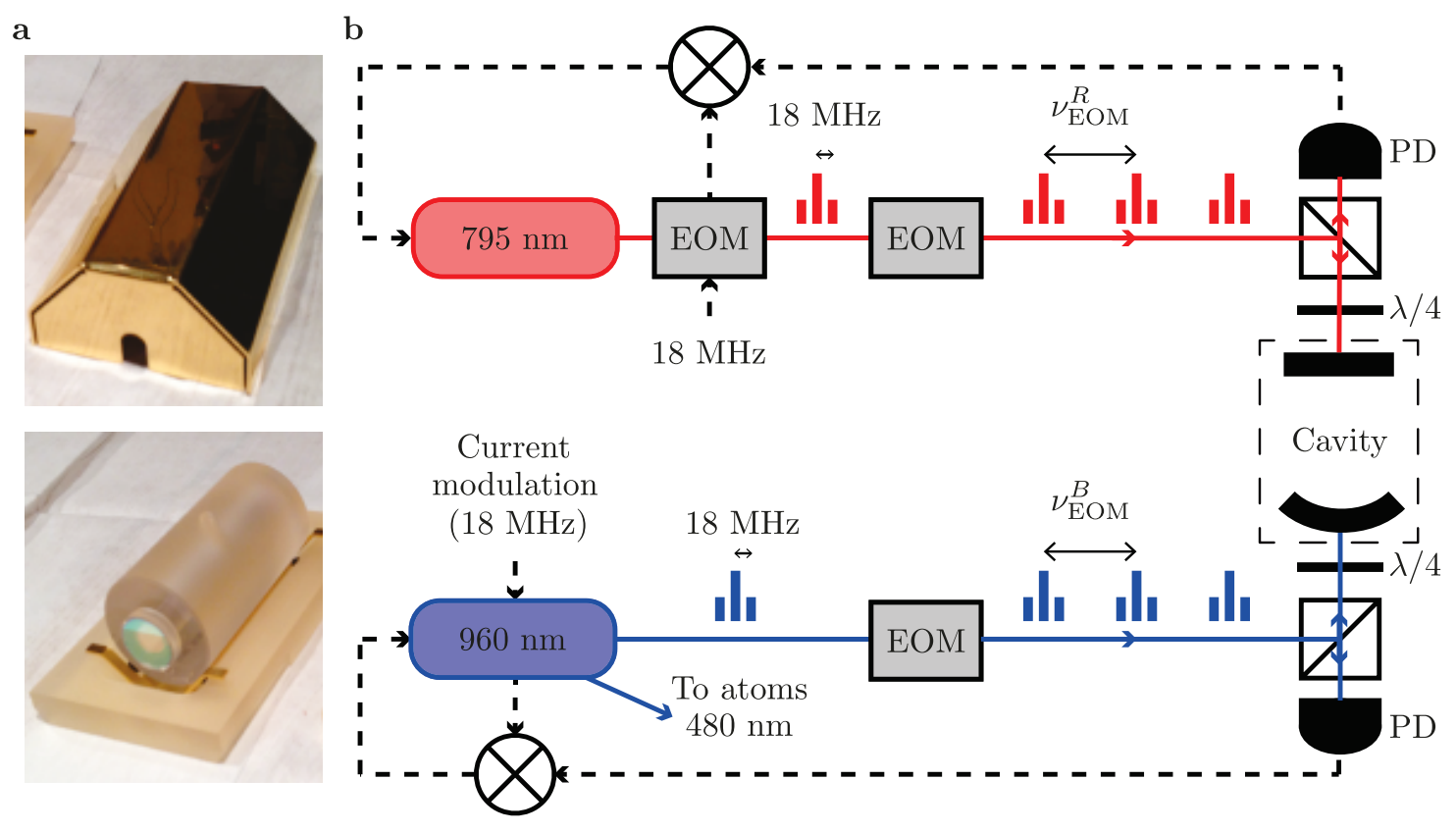

Figure 6.5: Locking the Rydberg lasers. a, Image of our ultra-stable cavity. The mirrors are mounted on a ultra-low expansion (ULE) spacer. The assembly is placed in a thermally insulated vacuum housing. b, Principle of the lock. We send both lasers on the ultra-stable cavity and use a Pound Drever Hall method to narrow down the lasers to linewidths $\leq 10 \mathrm{kHz}$.

a maximal thermal expansion coefficient $\alpha \simeq 2 \times 10^{-9} \mathrm{~K}^{-1}$. As a consequence, the relative cavity length $L$ is passively stable to:

$$
\frac{\Delta L}{L} \leq \alpha \Delta T \simeq 2 \times 10^{-9} \times 2 \times 10^{-3}=4 \times 10^{-12} .
$$

This gives an upper bound on the variations in frequency $\Delta \nu=\nu \Delta L / L$ of light at frequency $\nu=377 \mathrm{THz}$ due to temperature fluctuations of the cavity $\Delta \nu \leq 1.5 \mathrm{kHz}$, showing that temperature fluctuations are not a limiting factor in our case. The cavity has a free spectral range (FSR) of $1.5 \mathrm{GHz}$ and a specified finesse $\geq 1500$ for wavelengths varying between $750 \mathrm{~nm}$ and $1000 \mathrm{~nm}$. At the wavelengths of interest for our locking scheme $(795 \mathrm{~nm}$ and $950 \mathrm{~nm}$ ), the specified finesse is $\geq 20000$, providing reflection peaks with full width at half maximum $\leq 75 \mathrm{kHz}$. The specified value for the finesse is consistent with estimates based on ring-down measurements at a wavelength of $795 \mathrm{~nm}^{7}$.

\footnotetext{
${ }^{7}$ We measure a $\tau \simeq 2.4 \mu$ s lifetime of the photons inside the cavity after switching off the $795 \mathrm{~nm}$ light on a fast timescale using an AOM. This timescale is directly related to the reflection coefficient of the mirrors by $\tau=L /(c(1-R))$, yielding a finesse $F=\pi R /(1-R) \simeq 22500$.
} 


\section{Locking the lasers}

The locking schemes of the blue and the red laser are identical and based on the Pound Drever Hall technique [Black (2001)]. We carefully mode-match the lasers beams to the cavity and we measure the reflection signals from the cavity using two PD100 photodiodes. We send $\simeq 1 \mathrm{~mW}$ of $795 \mathrm{~nm}$ light directly to the cavity. For the blue laser beam, we pick off $\simeq 0.1 \mathrm{~mW}$ of $950 \mathrm{~nm}$ light before the frequency doubling crystal. Both lasers are first modulated at a frequency of $\simeq 18 \mathrm{MHz}$, providing the sidebands necessary for the Pound Drever Hall error signals. We use an electrooptic modulator (from New Focus) for the $795 \mathrm{~nm}$ laser, and we use the built-in current modulation of the $950 \mathrm{~nm}$ laser used to lock the doubling cavity. To reach the resonance condition of the cavity for given frequencies of the red and the blue lasers, we use Lithium Niobate fibered modulators (from EOspace) driven at the frequencies $\nu_{\mathrm{EOM}}^{R}$ and $\nu_{\mathrm{EOM}}^{B}$. This creates replicas of the error signals with variable offset from the carrier, that we can use to lock the laser on the cavity. The choice of the modulation frequency $\nu_{\mathrm{EOM}}^{R}$ is set by the detuning $\Delta / 2 \pi=740 \mathrm{MHz}$, and is fixed. The modulation frequency $\nu_{\mathrm{EOM}}^{B}$ can be scanned and adjusted to reach the resonance condition of the cavity for a given Rydberg transition frequency. The photodetector reflection signals are mixed with the $\simeq 18 \mathrm{MHz}$ local oscillators that are used for the modulation. After phase shifting and filtering, we obtain error signals that we send to reconfigurable laser servos from Vescent Photonics. Those PID controllers correct for the fluctuations of the laser frequencies by direct feedback on the current of the lasers.

We obtain a crude estimate of the linewidths of our lasers by visualizing the error signals:

- The error signal obtained for the $795 \mathrm{~nm}$ laser shows a peak-to-peak voltage of $\simeq 3.1 \mathrm{~V}$ over a spectral range of $\simeq 75 \mathrm{kHz}$. After locking the laser, the root mean square of the error signal is $\simeq 270 \mathrm{mV}$ over a few ms timescale, giving a $\simeq 6.5 \mathrm{kHz}$ estimate for the linewidth of the laser.

- The error signal obtained for the $474 \mathrm{~nm}$ laser shows a peak to peak voltage of $\simeq 156 \mathrm{mV}$ over a spectral range of $\simeq 75 \mathrm{kHz}$. After locking the laser, the root mean square of the error signal is $\simeq 4.5 \mathrm{mV}$ over a few $\mathrm{ms}$ timescale, giving a $\simeq 2 \mathrm{kHz}$ estimate for the linewidth of the laser.

With this new locking scheme, we estimate that we have narrowed the linewidths of the Rydberg lasers to better than $10 \mathrm{kHz}$ over timescales of a few ms, which is 
satisfying for our application. A more accurate method for the measurement of the linewidths of our excitation lasers would consist in doing a self-heterodyne interferometric measurement [Okoshi, Kikuchi, and Nakayama (1980)], but we have not performed this measurement yet.

During our measurements, we also record the wavelength of our lasers using a WSU/10 wavemeter from HighFinesse, which has a $100 \mathrm{kHz}$ resolution and a $10 \mathrm{MHz}$ absolute accuracy. The standard deviation of the laser frequencies measured on the wavemeter over timescales of a few seconds is $100 \mathrm{kHz}$, showing that we are limited by the resolution of the wavemeter, and that the linewidth of the lasers is indeed better than $100 \mathrm{kHz}$. We also observe long-term drifts of a few $\mathrm{MHz}$ over the course of a day, which are most probably due to temperature drifts of the wavemeter itself, as suggested by the experiment in the next section.

\subsubsection{Rydberg detection and coherent excitation to a Rydberg state}

We now suppose that we have one trap, that we are in the single atom regime, and that the lasers are locked at a chosen frequency. We trigger the experiment upon the presence of one atom in the trap. We first apply a vertical magnetic field $B \simeq 3.3 \mathrm{G}$ that defines our quantization axis (see Figure 6.4b) and that allows to optically pump the atoms into the ground state $|g\rangle=\left|5 S_{1 / 2}, F=2, m_{F}=2\right\rangle$. In order to excite the atoms to the chosen Rydberg state, we first turn the trap off so as to avoid any position-dependent light shift induced by the trapping beam during the experiment ${ }^{8}$. We then shine the excitation beams on the atoms for a duration $\tau$. In practice, we use two electro-optic modulators to have switching times on a nanosecond timescale.

\section{Rydberg detection}

Our detection of Rydberg atoms is based on the fact that Rydberg states are not trapped by our trapping beam. Rydberg atoms have long lifetimes $(\sim 100 \mu \mathrm{s})$, and leave the trapping region before decaying back to the ground state. After the excitation of duration $\tau$, we turn the trap on again and measure the fluorescence in the trap by shining some resonant light. We interpret the presence of fluorescence as the fact that the atom has stayed in the ground state, whereas the absence of fluorescence means that the atom has been excited to the Rydberg state. Excitation

\footnotetext{
${ }^{8}$ For that reason, all the Rydberg experiments reported in this manuscript are done in the absence of trapping light.
} 
a

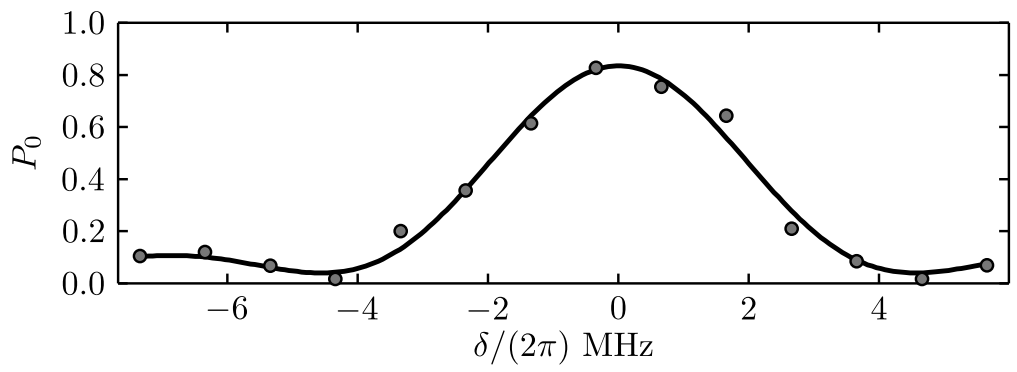

b

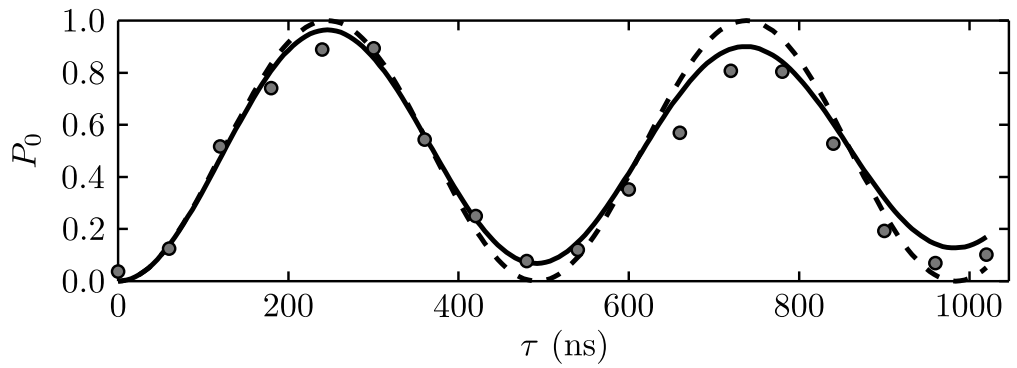

Figure 6.6: Excitation dynamics of a single atom. a, Spectroscopy of the $\left|62 D_{3 / 2}\right\rangle$ state showing the probability of Rydberg excitation as a function of laser detuning $\delta$ from the targeted state (circles). The solid line is a fit by the formula in Equation 6.8, used to measure the center position of the Rydberg line. $\mathbf{b}$, Rabi oscillation on a single atom. The dashed line shows a fit by the Rabi formula, where $\Omega$ is the only fitting parameter (see Equation 6.11). The solid line is a fit by the solution of the optical Bloch equations (see Equation 6.15), where $\Omega$ and $\gamma$ are two fitting parameters. The fits give: $\Omega /(2 \pi)=2.03 \pm 0.02 \mathrm{MHz}$.

of an atom to the Rydberg state is thus inferred from its loss from the corresponding trap at the end of the sequence [Miroshnychenko et al. (2010)]. We repeat each sequence $\simeq 100$ times and reconstruct the populations $P_{i}(i \in\{0 ; 1\})$ where $P_{1}$ is the probability to detect fluorescence at the end of the sequence and $P_{0}=1-P_{1}$ is the probability not to detect fluorescence at the end of the sequence. For 100 repetitions of the sequence, we have a typical $5 \%$ error bar on the measured probabilities.

\section{Spectroscopy}

We first perform the spectroscopy of the Rydberg line: for a given duration of the excitation $\tau$, we measure the loss probability $P_{0}$ as a function of the laser detuning $\delta$. Figure 6.6a shows an example of the spectroscopic line observed experimentally for the $\left|62 D_{3 / 2}\right\rangle$ state using $\simeq 710 \mu \mathrm{W}$ of red power and $\simeq 91 \mathrm{~mW}$ of blue power. Far from resonance, the probability $P_{0}$ of losing the atom is low (most of the atoms stay in the ground state and we recapture them at the end of the sequence). When we approach the resonance, $P_{0}$ increases due to the increase in the Rydberg excitation probability. On resonance, we observe $\mathrm{a} \simeq 86 \%$ excitation peak, showing that we have excited the atom to the Rydberg state with a high efficiency (here, $\tau$ was chosen to 
maximize $P_{0}$ on resonance). The solid line in Figure 6.6a shows a fit of the data to the formula:

$$
P_{0}(\delta)=A+B \frac{\Omega^{2}}{\Omega^{2}+\left(\delta-2 \pi \nu_{B}\right)^{2}} \sin ^{2}\left(\frac{\tau}{2} \sqrt{\Omega^{2}+\left(\delta-2 \pi \nu_{B}\right)^{2}}\right),
$$

where $A, B, \Omega$ and $\nu_{B}$ are fitting parameters. From the fit we obtain the frequency $\nu_{B}$ of the blue laser at resonance:

$$
\nu_{B}=632022092 \pm 10 \mathrm{MHz},
$$

where the uncertainty comes from the absolute accuracy of the wavemeter. The measurement agrees well with the expected value:

$$
\nu_{B, \text { theo }}=632022108 \mathrm{MHz} \text {, }
$$

where we included the light shifts and the Zeeman shifts due to the magnetic field. Over the course of a few weeks, we have noticed that the value $\nu_{\mathrm{EOM}}^{B}$ needed to lock the laser on resonance for a given state is stable to better than $1 \mathrm{MHz}$. Variations observed on the wavemeter are interpreted as temperature drifts of the wavemeter itself. Finally, the fact that the Rabi formula reproduces well our data is an indication that our spectra are essentially power-broadened for this duration of the excitation ( $\tau=200 \mathrm{~ns}$ ), and that other sources of broadening (if any) are negligible. The fit gives a Rabi frequency $\Omega /(2 \pi)=2.0 \pm 0.4 \mathrm{MHz}$.

\section{Rabi oscillation}

In a second experiment, we vary the duration of the excitation while the lasers are locked on resonance. This leads to the Rabi oscillation shown in Figure 6.6b. The oscillation of $P_{0}$ as a function of excitation time $\tau$ shows the coherent character of our excitation scheme from $|g\rangle$ to $|r\rangle$. For a duration of the excitation $\tau=\tau_{\pi}$ ( $\pi$-pulse), the transfer of population to the Rydberg state $|r\rangle$ is maximum $\simeq 90 \%$. The dashed line in Figure $6.6 \mathrm{~b}$ shows a fit of the data by the Rabi formula at resonance:

$$
P_{r}(\tau)=\sin ^{2}\left(\Omega \frac{\tau}{2}\right)
$$

where $\Omega$ is the only fitting parameter (dashed line in Figure 6.6b). The fit gives $\Omega /(2 \pi)=2.03 \pm 0.02 \mathrm{MHz}$. This measurement of $\Omega$ is in agreement with the one obtained from the spectrum, which confirms that our spectra are power-broadened, and that other sources of broadening have a negligible impact for our range of Rabi 
frequencies. Note that an estimation of $\Omega_{\text {eff }}$ using our estimates of $\Omega_{B}$ in Equation 6.6 and $\Omega_{R}$ in Equation 6.5 gives $\Omega_{\text {eff }} \simeq 2 \pi \times 4 \mathrm{MHz}$. The observed deviation might come from systematic errors in the estimate of the amount of excitation light seen by the atoms (due to errors in the measurement of the waists or due to the fact that the atoms are not perfectly centered on the excitation beams for instance).

\subsection{Modeling our system}

Our Rydberg excitation in Figure 6.6b shows some deviations from the theoretical Rabi formula: the measured contrast is smaller than 1, and we observe a slight damping of the oscillation. This indicates that there are some limits to our Rydberg excitation. Some of these limits are well understood (dissipation due to spontaneous emission, dephasing due to fluctuations in the light seen by the atoms) and were presented in detail in [Gaëtan (2009); Béguin (2013)]. We briefly recall them here. During my thesis, we also refined our understanding of the limitations of our detection scheme as detailed in Section 6.4.3. The understanding of these limitations is particularly important since it allows refining our modeling of the system, which plays and important role when increasing the number of atoms.

\subsubsection{Discussion of the limitations on the Rydberg excitation}

In this section, we list the known limitations that lead to a reduction of the efficiency of the Rydberg excitation.

\section{Spontaneous emission}

One first limitation intrinsic to our two-photon excitation scheme itself, comes from the fact that we use the intermediate state $\left|5 P_{1 / 2}, F=2, m_{F}=2\right\rangle$ to excite the Rydberg state. The finite detuning of the red laser $(\Delta /(2 \pi)=740 \mathrm{MHz})$, leads to a residual off-resonant excitation probability of the intermediate state. Population in the intermediate state spontaneously decays to the states $\left|5 S_{1 / 2}, F=1, m_{F}=1\right\rangle$, $\left|5 S_{1 / 2}, F=2, m_{F}=1\right\rangle$ and $\left|5 S_{1 / 2}, F=2, m_{F}=2\right\rangle$ at the rates $\Gamma_{P} / 2, \Gamma_{P} / 6$ and $\Gamma_{P} / 3$, where $\Gamma_{P} /(2 \pi)=5.75 \mathrm{MHz}$ [Steck (2010)]. Spontaneous emission limits our Rydberg excitation since some atoms decay during the excitation, leading to a damping of the oscillations. Atoms that decayed to the states $\left|5 S_{1 / 2}, F=1, m_{F}=1\right\rangle$ and $\left|5 S_{1 / 2}, F=2, m_{F}=1\right\rangle$ are not coupled to $|r\rangle$ anymore, but we keep detecting them as being in $|g\rangle$. As a consequence, for large laser powers, this leads to an asymmetric 
damping [Miroshnychenko et al. (2010)]. In order to limit the effect of spontaneous emission, one has to be sufficiently detuned from the ground state $\left(\Omega_{R}, \Omega_{B} \ll \Delta\right)$. This condition is well verified in our experiment. For our typical experimental parameters, we calculate that the excitation efficiency to the Rydberg state after a time $\tau_{\pi}$ ( $\pi$-pulse) should be better than $95 \%$.

Another source of decay comes from the finite lifetime of Rydberg states. Their typical decay rate $\left(\Gamma_{\text {Ryd }} /(2 \pi) \simeq 10 \mathrm{kHz}\right)$ is small compared to our typical Rabi frequencies $(\Omega /(2 \pi) \simeq 1 \mathrm{MHz})$ so that the damping due to the finite lifetime of Rydberg is limited (less than a few percents) for the typical duration of our sequences (a few $\mu s)$. For our longest sequences, we include the finite lifetime of the Rydberg states in our model (see Chapter 8 for instance, where $\tau$ goes up to $7 \mu \mathrm{s}$ ).

\section{Imperfections in the laser system}

Imperfections in the laser system lead to some dephasing of the oscillations. We have narrowed the laser linewidths down to $\lesssim 10 \mathrm{kHz}$ so that the frequency fluctuations are small. However, fluctuations in the power and polarization of the lasers (estimated to be of the order of a few percents) lead to fluctuations in Rabi frequencies seen by the atoms as well as fluctuating light shifts (see Equation 6.4). The observed Rabi oscillation results from the averaging of several oscillations with different frequencies, which leads to some damping.

\section{Temperature effects}

Another effect is due to the motion of the atoms resulting from their finite temperature $(T \simeq 50 \mu \mathrm{K})$. The thermal velocity of the atoms $v_{\mathrm{th}}=\sqrt{k_{\mathrm{B}} T / m} \simeq 70 \mathrm{~nm} . \mu \mathrm{s}^{-1}$ leads to fluctuations in the laser frequencies seen by the atoms due to the Doppler effect, which varies the detuning of the excitation $\delta$ from shot to shot (with a standard deviation $\left.\sigma_{\delta} /(2 \pi)=\left|\boldsymbol{k}_{R}+\boldsymbol{k}_{B}\right| v_{\mathrm{th}} /(2 \pi) \simeq 150 \mathrm{kHz}\right)$. Variations in the position of the atoms also lead to fluctuations in the quantity of light seen by the atoms, and in $\Omega$. This effect is negligible since the displacements on the timescale of an experimental sequence $\left(v_{\mathrm{th}} \tau \leq 500 \mathrm{~nm}\right)$ are small compared to the size of the excitation beams.

\section{Optical pumping}

Imperfections in the initial optical pumping can also contribute to the reduced efficiency of our Rydberg excitation, since atoms that are not optically pumped in $|g\rangle$ 
can not be excited to $|r\rangle$. We estimate that the quality of our optical pumping is better than $90 \%$, but we have not characterized its efficiency in detail.

\subsubsection{Modeling the system}

We model our Rydberg excitation by solving the master equation for the two-level system $\{|g\rangle ;|r\rangle\}$, where we add a dissipator $\hat{L}[\hat{\rho}]$ to account for the limitations listed previously:

$$
\frac{d \hat{\rho}}{d t}=-\frac{i}{\hbar}[\hat{H} \hat{\rho}-\hat{\rho} \hat{H}]+\hat{L}[\hat{\rho}]
$$

The Hamiltonian of the system is:

$$
\hat{H}=\hbar\left(\begin{array}{cc}
0 & \Omega / 2 \\
\Omega / 2 & -\delta
\end{array}\right)
$$

with $\Omega$ the Rabi frequency and $\delta$ the detuning. The dissipator reads:

$$
\begin{aligned}
\hat{L}[\hat{\rho}] & =\frac{\gamma}{2}\left(2 \hat{\sigma}_{g r} \hat{\rho} \hat{\sigma}_{r g}-\hat{\sigma}_{r r} \hat{\rho}-\hat{\rho} \hat{\sigma}_{r r}\right) \\
& =\gamma\left(\begin{array}{cc}
\rho_{r r} & -\rho_{g r} / 2 \\
-\rho_{r g} / 2 & -\rho_{r r}
\end{array}\right),
\end{aligned}
$$

where $\hat{\sigma}_{i j}=|i\rangle\langle j|(i, j \in\{g ; r\})$ are the transition and projector operators for the atom, and $\gamma$ is a phenomenological damping rate that accounts for the imperfections listed above (here, we assume that all processes are homogeneous). Assuming that all the population is initially in $|g\rangle$ and that $\delta=0$, one obtains [Loudon (2000)]:

$$
P_{r}(t)=\frac{\Omega^{2}}{2 \Omega^{2}+\gamma^{2}}\left[1-\mathrm{e}^{-3 \gamma t / 4}\left(\cos (\widetilde{\Omega} t)+\frac{3 \gamma}{4 \widetilde{\Omega}} \sin (\widetilde{\Omega} t)\right)\right],
$$

where $\widetilde{\Omega}=\sqrt{\Omega^{2}-\gamma^{2} / 16}$, and where we observe the presence of a damping term $\exp (-3 \gamma t / 4)$ that is due to dissipation. We fit the data by the formula of Equation 6.15, where $\gamma$ and $\Omega$ are the only fitting parameters (see solid line in Figure 6.6b), and we obtain the effective damping rate:

$$
\gamma=0.4 \pm 0.1 \mu \mathrm{s}^{-1}
$$

where the error bar comes from the fit. As a consequence, $\gamma^{-1} \simeq 2.5 \mu \mathrm{s}$, which indicates small decoherence during the experimental sequence. In this model, we note that the damping is symmetric, which differs from the type of damping one expects from spontaneous emission from the intermediate $\left|5 P_{1 / 2}\right\rangle$ state. A more sophisticated 
model can be implemented [Gaëtan (2009)], where we include the intermediate state and its three decay channels, leading to a five-level system. Nevertheless, in view of increasing the number of atoms we prefer to work with this simpler model describing the atoms as two-level systems.

\subsubsection{Detection errors}

For $\tau=0$ ns, we observe that we do not recapture $100 \%$ of the atoms: $P_{0}$ is equal to a few percent despite the fact that we have not illuminated the atoms with the excitation beams. These events can not be attributed to excitation of the atom to the Rydberg state, but are attributed to losses that come from two effects:

- Collisions with background gas,

- Escape of the atom from the trap region during the Rydberg excitation sequence, due to the finite temperature of the atoms.

As a consequence, each atom has a small probability $\varepsilon$ to be lost during the sequence, independently of its internal state. When this happens, the event is erroneously counted as a Rydberg excitation. For sequences that are shorter than $\simeq 4 \mu \mathrm{s}$, we have measured that both contributions add up to a few percent detection error. As a consequence, our measured probabilities $P_{1}$ and $P_{0}$, differ from the actual probabilities $P_{g}, P_{r}$ that the atom is in $|g\rangle$ or $|r\rangle$. If we assume that $P_{g}$ is the probability for the atom to be in the ground state at the end of the sequence, then the probability $P_{1}$ to detect some fluorescence at the end of the sequence differs from $P_{g}$ by the quantity $\varepsilon P_{g}$ of atoms that were supposed to be in $P_{g}$ but that were lost during the sequence. Taking into account the fact that $P_{0}+P_{1}=P_{r}+P_{g}=1$, we obtain:

$$
\left\{\begin{array}{l}
P_{1}=(1-\varepsilon) P_{g} \\
P_{0}=(1-\varepsilon) P_{r}+\varepsilon .
\end{array}\right.
$$

These formulae will be generalized later, in the case of 2 and 3 atoms (see Chapter 7 and Chapter 8).

\subsubsection{Summary of the effects}

All the effects discussed above can be summarized in the following expression of $P_{0}(t)$ :

$$
P_{0}(t)=\varepsilon+\eta(1-\varepsilon) \frac{\Omega^{2}}{2 \Omega^{2}+\gamma^{2}}\left[1-\mathrm{e}^{-3 \gamma t / 4}\left(\cos (\widetilde{\Omega} t)+\frac{3 \gamma}{4 \widetilde{\Omega}} \sin (\widetilde{\Omega} t)\right)\right],
$$




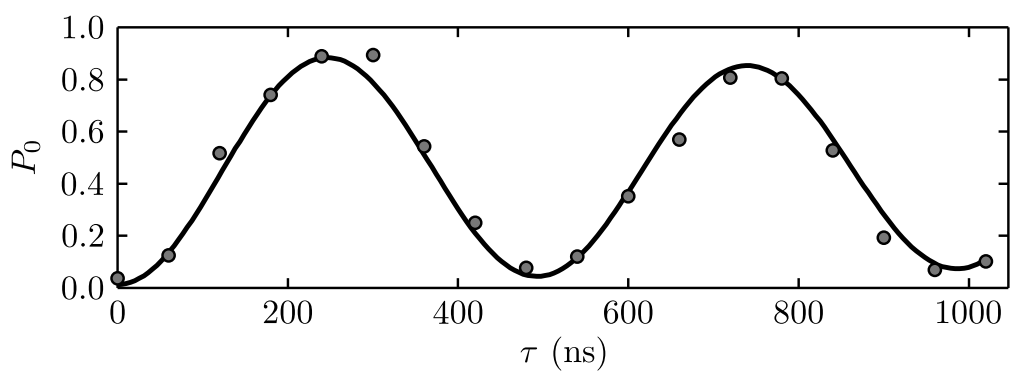

Figure 6.7: Excitation dynamics of a single atom. Fit of the Rabi oscillation shown in Figure 6.6 using the formula Equation 6.18.

where $\varepsilon$ represents the detection errors, and $\eta$ represents the efficiency of our optical pumping and the depumping due to spontaneous emission. The result of the fit is shown in Figure 6.7. We obtain:

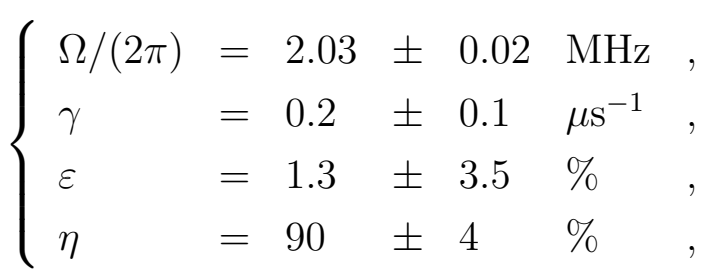

where the error bars come from the fit. The fitted values of $\eta$ and $\epsilon$ are compatible with our estimations of optical pumping $(\gtrsim 90 \%)$ and detection errors $(\simeq$ $5 \%$ ). In general for our experiments, we use single atom measurements as a calibration to obtain an independent measurement of $\Omega$ and $\gamma$ for each individual atom. For this purpose, we fit the data by the simple phenomenological formula $P_{0}(t)=A+B \exp (-3 \gamma t / 4) \sin (\Omega t)$, where we have checked that we obtain similar results for $\gamma$ and $\Omega$ than with Equation 6.18.

\subsection{Control of electric fields}

We have shown our ability to coherently excite single atoms to Rydberg states. Rydberg states are sensitive to electric fields due to their large polarizability (see Chapter 5). L. Béguin has shown during his thesis [Béguin (2013)] that with all electrodes grounded, there exists a residual DC electric field of $\simeq 150 \mathrm{mV} / \mathrm{cm}$ at the position of the atoms. This leads to Stark shifts of a few $\mathrm{MHz}$ as well as state mixing ${ }^{9}$, which degrades the efficiency of the Rydberg excitation. As a consequence, another key feature of our setup is the possibility to cancel stray electric fields. Moreover, a

\footnotetext{
${ }^{9} \mathrm{As}$ an example, an electric field of $150 \mathrm{mV} / \mathrm{cm}$ is enough to shift the $\left|59 D_{3 / 2}, M_{J}=3 / 3\right\rangle$ state by $\approx 4.5 \mathrm{MHz}$, as we will see in Chapter 9 .
} 
good control of the electric field is also required when one wants to reach a Förster resonance for instance as we will see in Chapter 9.

\subsubsection{Compensating residual electric fields}

Our setup was designed to minimize residual electric fields acting on the Rydberg atoms:

- The metallic vacuum chamber is relatively large, so that the glass surfaces of viewports are about $10 \mathrm{~cm}$ away from the atoms, which are further shielded by a large metallic piece holding the aspheric lenses.

- The surfaces of the aspheric lenses facing the atoms are coated with a $\simeq 200 \mathrm{~nm}$ thick Indium-Tin Oxide (ITO) conducting layer and connected to ground.

Moreover, a set of eight independent electrodes in an octopole configuration (shown Figure 6.8a) has been included, allowing us to apply controlled electric fields pointing in any direction at the position of the atoms. For example, when the four electrodes in the half-space $z>0$ are at a potential $V_{z}$ while the four other electrodes are connected to ground, we produce an electric field $\boldsymbol{F}=F_{z} \boldsymbol{u}_{z}$ at the position of the atoms. Similarly, we can apply electrics $\boldsymbol{F}=F_{i} \boldsymbol{u}_{i}$ pointing in the directions $x$ and $y$. We define the effective lengths $L_{i}$ such that:

$$
F_{i}=\frac{\Delta V}{L_{i}}
$$

where $i \in\{x ; y ; z\}$.

\subsubsection{Calibration of the electrodes}

The calibration of the electrodes was described by L Béguin in [Béguin (2013)], and was obtained in the three directions of space by performing the Stark spectroscopy of the state $\left|62 D_{3 / 2}, m_{j}=3 / 2\right\rangle$ as a function of $\Delta V_{i}(i=\{x ; y ; z\})$. The observed shift of the spectroscopic line as a function of the applied electric field was compared to the theoretical expectations from the calculated polarizability of $\left|62 D_{3 / 2}, m_{j}=3 / 2\right\rangle$. This allowed to estimate experimentally the effective lengths $L_{i}$ :

$$
\left\{\begin{array}{l}
L_{x}^{\mathrm{cal}} \simeq 40 \mathrm{~cm} \\
L_{y}^{\mathrm{cal}} \simeq 9 \mathrm{~cm} \\
L_{z}^{\mathrm{cal}} \simeq 5.2 \mathrm{~cm}
\end{array}\right.
$$


a

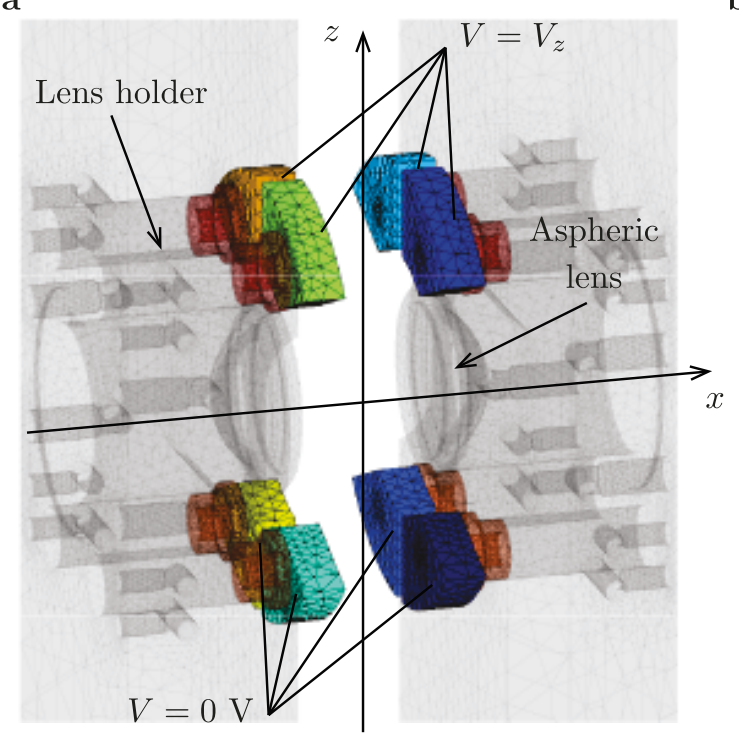

b

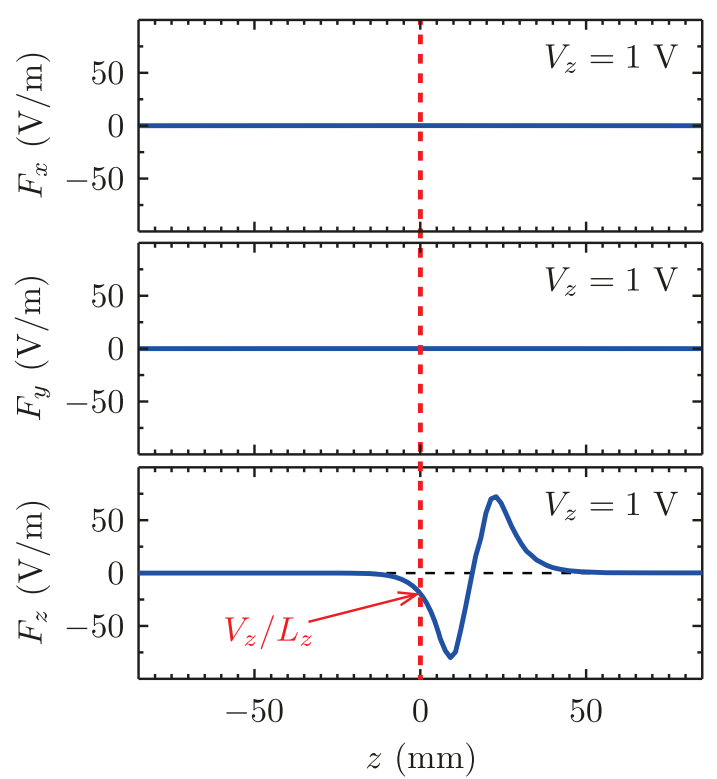

Figure 6.8: Calculation of the electric field profile inside the chamber. a, Our experimental configuration. A set of eight independent electrodes allows us to apply controlled electric fields pointing in any direction. We solve the Laplace equations using the finite element method on the mesh shown here. $\mathbf{b}$, Components of the electric field along the $z$-axis when applying a potential $\Delta V_{z}=1 \mathrm{~V}$. The field points in the $z$-direction.

Compensation is reached when the measured Stark shift is minimum. During my thesis, we reproduced this measurement a couple of times on the $\left|82 D_{3 / 2}\right\rangle$ and $\left|59 D_{3 / 2}\right\rangle$ levels so as to check or adjust the compensation of the electric fields. Over the course of a year, the voltages required to reach compensation did not vary by more than $10 \%$. The Stark spectroscopy of the more sensitive $\left|82 D_{3 / 2}\right\rangle$ state allowed to estimate the residual electric fields after compensation to be well below $\simeq 5 \mathrm{mV} / \mathrm{cm}$.

\subsubsection{Comparison with calculations}

We have also performed a numerical simulation of the expected electric field to get more insight in the electric field profile inside the full chamber. This allowed us to compare the measured values of $L_{i}$ to the expected values. The calculations were performed by M. Besbes, a research engineer at the Institut d'Optique, using a finite element method. Figure 6.8a shows part of the mesh we used to solve the Maxwell equations. All vacuum parts are included in the simulation: the metallic parts are considered as perfect conductors with a specified potential, and the insulating parts are included with their respective dielectric constants. The vacuum chamber is represented by a rectangular box at potential $V=0 \mathrm{~V}$. Figure $6.8 \mathrm{~b}$ shows a result of one 
of these simulations for a potential $\Delta V_{z}=1 \mathrm{~V}$, where we plot the components of the electric field along the $z$ axis. On axis, we observe that the field points along $z$ as expected. At the position of the atoms $(z=0 \mathrm{~mm})$, we obtain a field $F_{z}=19.4 \mathrm{~V} / \mathrm{m}$. Reproducing the same calculations along the axes $x$ and $y$, we obtain the simulated values for the effective lengths $L_{i}$ :

$$
\left\{\begin{array}{l}
L_{x}^{\mathrm{sim}} \simeq 36 \mathrm{~cm} \\
L_{y}^{\mathrm{sim}} \simeq 8.8 \mathrm{~cm} \\
L_{z}^{\mathrm{sim}} \simeq 5.2 \mathrm{~cm}
\end{array}\right.
$$

The results of the simulations are in good agreement with the experiment. In the rest of this thesis, we are interested in small fields along the $z$-direction. In particular, in Chapter 9 we apply vertical electric fields $F_{z} \lesssim 60 \mathrm{mV} / \mathrm{cm}$ at the position $z=0 \mathrm{~V}$. Using the simulation, we evaluate that for an applied electric field $F_{z}=60 \mathrm{mV} / \mathrm{cm}$ at the center of the chamber, the field does not vary by more than $0.3 \mathrm{mV} / \mathrm{cm}$ over a $100 \mu \mathrm{m}$ range around the position of the atoms, the maximal size of the arrays we produce. This indicates that inhomogeneities of the electric field are not a limitation in our experiments.

\subsection{Single-site addressability}

We have demonstrated in Section 6.3.3 Rydberg excitation of a single atom. In view of increasing the number of atoms, we use large excitation beams allowing to simultaneously excite several atoms in an array. However, some proposals for quantum information using Rydberg atoms are based on the possibility to selectively manipulate individual atoms (called "control atoms") in an ensemble [Müller et al. (2009)]. As a consequence, we have implemented during my thesis a way to address some atoms in the array. We achieve selective addressing by shining a red-detuned laser beam on a single ${ }^{87} \mathrm{Rb}$ atom, which induces a light-shift on the ground state of the targeted atom. The Rydberg states stay nearly unaffected so that the targeted atom is off-resonant with the Rydberg excitation, while all the other unperturbed atoms stay in resonance with the Rydberg excitation.

\subsubsection{The setup}

Figure 6.9 shows an illustration of our implementation of single site addressing. We produce the addressing beam using far red-detuned $850 \mathrm{~nm}$ light coming from the 


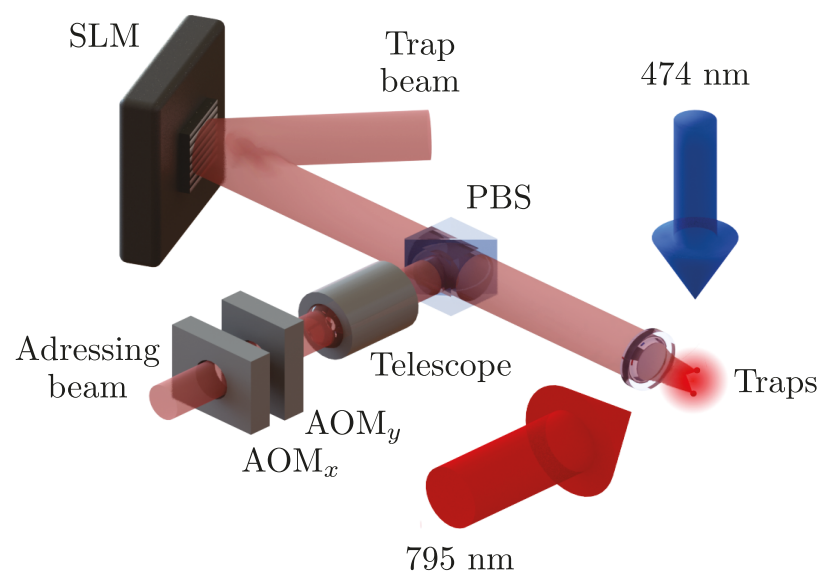

Figure 6.9: Single site addressability in an array of traps. We shine the excitation beams on all the atoms. We induce a light shift on the ground state of a chosen atom using some $850 \mathrm{~nm}$ light so that the atom is no longer resonant with the excitation field.

same laser as the one used for trapping. We superimpose the addressing beam on the phase-manipulated trap beam. We use two crossed AOMs for precise control of the addressing beam position with respect to the targeted trap. The two beams have orthogonal polarizations and are detuned by $\simeq 200 \mathrm{MHz}$ so as to minimize the interference between the beams in the focal plane of the aspheric lens. Before the aspheric lens, the addressing beam has a slightly smaller collimated diameter than the dipole trap beam, which allows obtaining a larger $1 / e^{2}$-radius $(\simeq 1.3 \mu \mathrm{m})$ at the position of the atoms, and ensures that the motional state of the atom is not significantly disturbed when the addressing beam is switched on. Finally, an electrooptic modulator (EOM) allows switching the addressing beam on fast timescales of $\simeq 10 \mathrm{~ns}$.

\subsubsection{Illustration on a single atom}

We illustrate the effect of our addressing beam on a single atom (see Figure 6.10). In the absence of addressing beam, we tune our excitation lasers on resonance with the state $\left|59 D_{3 / 2}\right\rangle$. The blue curve in Figure 6.10 shows the resulting Rabi oscillation, with a Rabi frequency $\Omega \simeq 2 \pi \times 1 \mathrm{MHz}$. In a second experiment, we send $\simeq 4 \mathrm{~mW}$ of addressing light on the atom. The ground state $|g\rangle$ of the atoms experiences an energy shift proportional to the local intensity of the addressing beam at the position of the atom, while the Rydberg state $|r\rangle$ stays nearly unaffected. For induced light shifts 


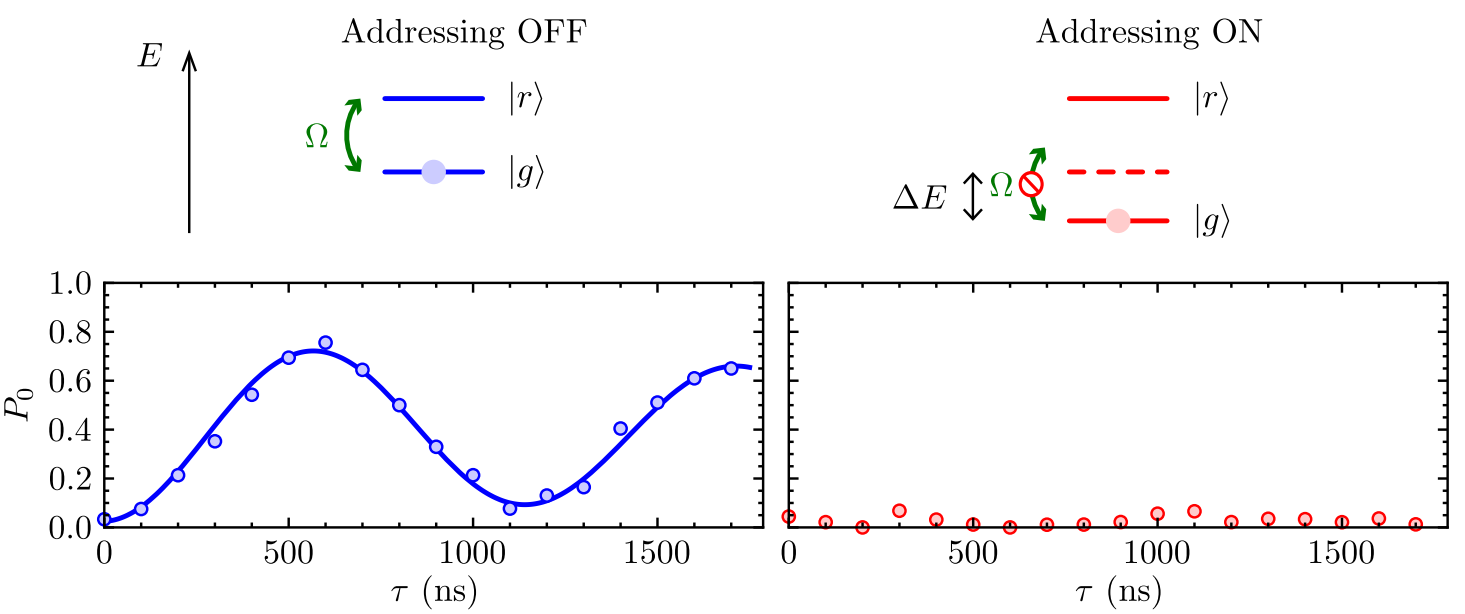

Figure 6.10: Proof of principle of addressability on a single atom. The Rydberg excitation lasers are resonant with the $|r\rangle=\left|59 D_{3 / 2}\right\rangle$ level. When the addressing beam if turned off, we observe a Rabi oscillation between $|g\rangle$ and $|r\rangle$ (where the blue line is a fit by a damped sine). Turning on the addressing beam induces a light shift on the ground state of the atom, while leaving the Rydberg states nearly unaffected. The excitation lasers are not resonant anymore with the transition, which prevents us from exciting the atom to the Rydberg state.

that are larger than the Rydberg excitation linewidth ${ }^{10}$, the lasers are consequently not resonant anymore with the transition $|g\rangle \rightarrow|r\rangle$, which prevents us from exciting the atom to the Rydberg state. The red curve in Figure 6.10 shows the excitation probability of the atom as a function of excitation time in the presence of addressing light. The excitation probability $P_{0}$ stays below $5 \%$, which confirms the inhibition of the Rydberg excitation due to the presence of the addressing beam.

\subsubsection{Addressing in arrays of atoms}

For work with larger ensembles of atoms, we have also checked that the presence of the addressing beam has a negligible impact on other sites of the array. The induced light shift on a neighboring atom as close as $3 \mu \mathrm{m}$ to the targeted atom is estimated to be as low as $\simeq 1 \mathrm{kHz}$, well below the resolution of our experiment. This confirms our ability to selectively address a single atom in an array, with no disturbance on the other atoms ${ }^{11}$. An application of this new feature of our setup is shown in Chapter 8.

\footnotetext{
${ }^{10}$ For $\simeq 2 \mathrm{~mW}$ of total power in the addressing beam, we have measured a maximum light shift of $\Delta E \simeq h \times 5 \mathrm{MHz}$, and for larger addressing beam intensities we have measured light-shifts as large as $\Delta E \simeq h \times 40 \mathrm{MHz}$. Here, we use $\simeq 4 \mathrm{~mW}$ of power, so that $\Delta E / h=10 \mathrm{MHz} \gg \Omega /(2 \pi)$.

${ }^{11}$ More information can be found in [Labuhn et al. (2014)]. The detailed implementation and characterization of single-site addressability on our setup will be given in the thesis of H. Labuhn.
} 


\subsection{Conclusion}

We have demonstrated our ability to trap and manipulate single atoms in optical tweezers. Using a two-photon excitation scheme, we can excite coherently the atoms to Rydberg states. Combined with other tools like the control of electric fields and the possibility to address a particular atom in an array, our system becomes an interesting platform for the study of interactions between Rydberg atoms, which will be the subject of the next chapters. 


\section{Chapter 7}

\section{Van der Waals interactions between two and three Rydberg atoms}

\section{Contents}

7.1 Van der Waals interactions . . . . . . . . . 126

7.1.1 Second-order perturbation . . . . . . . . . . . 127

7.1.2 Expansion in the spherical basis . . . . . . . . . . . 128

7.1 .3 Interaction energy . . . . . . . . . . . . . . . 128

7.1.4 Anisotropy of the interaction . . . . . . . . . . . 133

7.2 Rydberg blockade in three-atom systems . . . . . . 135

7.2.1 Experimental sequence . . . . . . . . . . . . . 136

7.2.2 Demonstration of a robust Rydberg blockade . . . . . . . 137

7.2 .3 Comparison with simulations . . . . . . . . . . . . 139

7.3 Measurement of the van der Waals interaction . . . . . 141

7.3.1 Principle of the experiment . . . . . . . . . . . . . . 142

7.3.2 Excitation dynamics of the two-atom system . . . . . . . 143

7.3.3 Measurement of the interaction energy . . . . . . . . . . . 144

7.4 Angular dependence of the van der Waals interaction . 144

7.4.1 Experimental procedure . . . . . . . . . . . 145

7.4.2 Measurement of the anisotropy of the interaction . . . . . 145

7.4.3 Effects of the anisotropy of the interaction . . . . . . . . 146

7.5 Conclusion................... 151

Chapter 6 described the tools at our disposal for the study of arrays of Rydberg atoms. In the rest of this manuscript, we investigate the effects of interactions between 
Rydberg atoms in small systems of two or three atoms. In most situations, two atoms excited to a given Rydberg state show van der Waals interactions, expressed as $C_{6} / R^{6}$. As a result, most of the early demonstrations of Rydberg blockade were performed in this regime that does not require any active control over the interaction. In this Chapter we concentrate on the van der Waals interactions between Rydberg atoms, leaving the active control over the interaction for Chapter 8 and Chapter 9.

The Rydberg blockade has attracted a lot of interest because of its robustness with respect to fluctuations of experimental parameters. Indeed, the blockade occurs as long as the interatomic interactions in a system are larger than the Rydberg excitation linewidth $(\Delta E \gg \hbar \Omega)$. This criteria can be fulfilled for a large range a parameters, and as a consequence the Rydberg blockade can be observed in disordered systems, where distances or relative angles between the atoms fluctuate. On our setup, L. Béguin demonstrated the blockade between two Rydberg atoms [Béguin (2013)], with significant improvement compared to earlier demonstrations [Urban et al. (2009); Gaëtan et al. (2009)]. In this Chapter, we extend this study to three-atom systems, and illustrate the robustness of the blockade with respect to the geometrical arrangement of the atoms.

With the level of control reached in our system, L. Béguin also showed the possibility to work in the more sensitive regime where $\Delta E \simeq \hbar \Omega$. In this situation, the coherent excitation of the two atoms is not fully blockaded, but is still affected in a characteristic way by the interaction. We can use this feature to directly measure the van der Waals interaction between two Rydberg atoms aligned along the quantization axis as described in [Béguin et al. (2013)]. Here, we use the same method to measure the angular dependence of the van der Waals interaction. We finally observe the influence of anisotropies of the van der Waals interaction on the excitation of a three-atom system.

\subsection{Van der Waals interactions}

When the interatomic distance $R$ is large compared to the size of the atoms ${ }^{1}$, two atoms $A$ and $B$ located at positions $\boldsymbol{R}_{A}$ and $\boldsymbol{R}_{B}$ interact through the dipole-dipole interaction described by the Hamiltonian

$$
\hat{V}_{\mathrm{dip}}=\frac{1}{4 \pi \epsilon_{0}} \frac{\hat{\boldsymbol{d}}_{A} \cdot \hat{\boldsymbol{d}}_{B}-3\left(\hat{\boldsymbol{d}}_{A} \cdot \boldsymbol{n}\right)\left(\hat{\boldsymbol{d}}_{B} \cdot \boldsymbol{n}\right)}{R^{3}},
$$

\footnotetext{
${ }^{1}$ This hypothesis is always verified in this thesis.
} 
where $\hat{\boldsymbol{d}}_{i}$ is the electric dipole moment of atom $i(i=A, B), \boldsymbol{R}=\boldsymbol{R}_{B}-\boldsymbol{R}_{A}$, and $\boldsymbol{n}=\boldsymbol{R} / R$.

\subsubsection{Second-order perturbation}

We consider this Hamiltonian as a weak perturbation to the unperturbed Hamiltonian $\hat{H}_{0}=\hat{H}_{A}+\hat{H}_{B}$ describing the non-interacting two-atom system. In this Chapter, we are interested in the interaction between two atoms excited to the same Rydberg state $|r\rangle=\left|n, L, J, M_{J}\right\rangle$. Because the average value of the dipole moment vanishes in an atomic eigenstate, $\hat{V}_{\text {dip }}$ has no effect to first order. Second-order perturbation theory gives rise to an energy shift of the atom pair, which results in the van der Waals interaction

$$
V_{\mathrm{vdW}}=\frac{C_{6}}{R^{6}}
$$

For a two-atom system excited to $|r r\rangle$, the $C_{6}$ coefficient is given by

$$
C_{6}=\frac{1}{\left(4 \pi \epsilon_{0}\right)^{2}} \sum_{\left|\phi_{A} \phi_{B}\right\rangle} \frac{\left|\left\langle\phi_{A} \phi_{B}\left|\hat{\boldsymbol{d}}_{A} \cdot \hat{\boldsymbol{d}}_{B}\right| r r\right\rangle-3\left\langle\phi_{A} \phi_{B}\left|\left(\hat{\boldsymbol{d}}_{A} \cdot \boldsymbol{n}\right)\left(\hat{\boldsymbol{d}}_{B} \cdot \boldsymbol{n}\right)\right| r r\right\rangle\right|^{2}}{2 E_{r}-\left(E_{\phi_{A}}+E_{\phi_{B}}\right)}
$$

where we sum on all possible states $\left|\phi_{A} \phi_{B}\right\rangle=\left|n_{A}, L_{A}, J_{A}, M_{J, A}\right\rangle \otimes\left|n_{B}, L_{B}, J_{B}, M_{J, B}\right\rangle$ different from $|r r\rangle$. An estimate of the $C_{6}$ coefficient can be obtained using the scaling arguments given in Chapter 5:

- The numerator inside the sum in Equation 7.3 scales as the modulus squared of the product of two dipole matrix elements $\simeq\left(n^{* 2} e a_{0}\right)^{4}$,

- The denominator inside the sum in Equation 7.3 scales as the difference in energy between two Rydberg states $\simeq-2 R_{y} \times n^{*-3}$,

- As a consequence, we obtain an approximate expression for the $C_{6}$ coefficient:

$$
C_{6} \simeq \frac{1}{\left(4 \pi \epsilon_{0}\right)^{2}} \frac{e^{4} a_{0}^{4} n^{* 11}}{2 R_{y}}
$$

Using this scaling, we estimate an interaction energy of $\Delta E / h \simeq 8.5 \mathrm{MHz}$ for two atoms excited to the state $82 D_{3 / 2}$ (the main state of interest in this Chapter), and separated by $\simeq 5 \mu \mathrm{m}$. This energy is almost an order of magnitude larger than our typical Rabi frequencies of $\Omega /(2 \pi) \simeq 1 \mathrm{MHz}$, which is well adapted to the study of Rydberg blockade. 


\subsubsection{Expansion in the spherical basis}

When the internuclear axis defines an angle $\theta$ with the quantization axis $z$, it is convenient to express $\hat{V}_{\text {dip }}$ in the spherical basis using the spherical dipole operators

$$
\left\{\begin{array}{l}
\hat{d}_{0}=\hat{d}_{z} \\
\hat{d}_{+}=-\frac{1}{\sqrt{2}}\left(\hat{d}_{x}+i \hat{d}_{y}\right) \\
\hat{d}_{-}=\frac{1}{\sqrt{2}}\left(\hat{d}_{x}-i \hat{d}_{y}\right)
\end{array}\right.
$$

where the operator $\hat{d}_{0}$ conserves the magnetic quantum number $M_{J}$, the operator $\hat{d}_{+}$ couples states with $\Delta M_{J}=1$, and the operator $\hat{d}_{-}$couples states with $\Delta M_{J}=-1$. In this basis, the dipole-dipole interaction operator can be written as:

$$
\begin{aligned}
\hat{V}_{\text {dip }}= & \frac{1}{4 \pi \epsilon_{0}} \frac{1}{R^{3}}\left[\frac{1-3 \cos ^{2} \theta}{2}\left(\hat{d}_{A,+} \hat{d}_{B,-}+\hat{d}_{A,-} \hat{d}_{B,+}+2 \hat{d}_{A, 0} \hat{d}_{B, 0}\right)\right. \\
& +\frac{3}{\sqrt{2}} \sin \theta \cos \theta\left(\hat{d}_{A,+} \hat{d}_{B, 0}-\hat{d}_{A,-} \hat{d}_{B, 0}+\hat{d}_{A, 0} \hat{d}_{B,+}-\hat{d}_{A, 0} \hat{d}_{B,-}\right) \\
& \left.-\frac{3}{2} \sin ^{2} \theta\left(\hat{d}_{A,+} \hat{d}_{B,+}+\hat{d}_{A,-} \hat{d}_{B,-}\right)\right]
\end{aligned}
$$

where the matrix elements $\left\langle\phi_{A}^{\prime} \phi_{B}^{\prime}\left|\hat{d}_{A, i} \hat{d}_{B, j}\right| \phi_{A} \phi_{B}\right\rangle$ of the Hamiltonian describe the coupling between two oscillating dipoles. In Equation 7.6, we have grouped into three different terms the different operators contributing to the interaction. Each term present the particularity to only contain operators that couple states with a given absolute value of the variation of total magnetic quantum number:

$$
\Delta M_{\mathrm{Tot}}=M_{J, A}+M_{J, B}-2 M_{J}
$$

The first term, with the angular prefactor $\left(1-3 \cos ^{2} \theta\right) / 2$ couples states with $\left|\Delta M_{\text {Tot }}\right|=$ 0 . The second term, with the angular prefactor $3 \sin \theta \cos \theta / \sqrt{2}$ couples states with $\left|\Delta M_{\text {Tot }}\right|=1$. Finally the third term, with the angular prefactor $3 \sin ^{2} \theta / 2$ couples states with $\left|\Delta M_{\text {Tot }}\right|=2$. We observe immediately that for $\theta=0$, only the first term contributes.

\subsubsection{Interaction energy for two atoms aligned along the quantization axis}

When the interatomic axis is aligned with the quantization axis $(\theta=0)$, the interaction Hamiltonian reduces to 


$$
\hat{V}_{\text {dip }}=-\frac{1}{4 \pi \epsilon_{0}} \frac{1}{R^{3}}\left(\hat{d}_{A,+} \hat{d}_{B,-}+\hat{d}_{A,-} \hat{d}_{B,+}+2 \hat{d}_{A, 0} \hat{d}_{B, 0}\right) .
$$

In this case, $V_{\text {dip }}$ only couples pair states with $\left|\Delta M_{\text {Tot }}\right|=0$, which simplifies the study of the interaction since it limits the number of states one has to consider to compute the interaction strength. During his post-doctoral studies in the group, R. Chicireanu developed a Matlab code to calculate the interaction energy in the case $\theta=0^{\circ}$, in a zero magnetic field [Béguin (2013)]. In this Section, we summarize the main steps for the calculation.

\section{Selection rules}

In order to accurately evaluate the interaction energy between two atoms excited to $|r\rangle=\left|n, L, J, M_{J}\right\rangle$, one has to consider all possible couplings between the pair state $|r r\rangle$ and other pair states $\left|\phi_{A} \phi_{B}\right\rangle=\left|n_{A}, L_{A}, J_{A}, M_{J, A}\right\rangle \otimes\left|n_{B}, L_{B}, J_{B}, M_{J, B}\right\rangle$. Selection rules for the electric dipole operator allow to significantly reduce the number of states one has to consider. Indeed, the electric dipole operator only couples states with $\Delta L= \pm 1, \Delta J=0, \pm 1$ and $\Delta M_{J}=0, \pm 1$ [Cohen-Tannoudji, Diu, and Laloë (1977)]. The interaction Hamiltonian contains terms that are products of dipole operators, and we can use these selection rules to restrict the basis to states $\left|\phi_{A} \phi_{B}\right\rangle$ that satisfy $\left|\Delta M_{\text {Tot }}=0\right|$ : for $\theta=0$, we can only keep the Zeeman sub-levels that conserve $M_{\text {Tot }}$. We further limit the number of states by truncating the basis so that:

- $\left|n_{A, B}-n\right| \leq 4$ and $\left|2 E_{r}-\left(E_{\phi_{A}}+E_{\phi_{B}}\right)\right| / h \leq 5 \mathrm{GHz}$, where the energies are obtained from quantum defect theory (see Chapter 5). For the range of interatomic distances considered in this thesis $(R \geq 5 \mu \mathrm{m})$, the interaction energies are smaller than $\simeq 100 \mathrm{MHz}$, which remains small with respect to $5 \mathrm{GHz}$.

- $L_{A, B} \leq 4$ : in this Chapter, we are mainly interested in interactions between atoms that are excited to $D$-states $(L=2)$. Because of the selection rules, $D$ states show non-zero transition dipole moments only with $P$-states $(L=1)$ and $F$-states $(L=3)$. In the calculation of the interaction energy, pair states $\left|\phi_{A} \phi_{B}\right\rangle$ with $L_{A, B} \notin\{1 ; 3\}$ only show indirect couplings to $|r r\rangle$. As a consequence, matrix elements involving states with $L_{A, B}>4$ will have negligible contributions.

We calculate the interaction energy between the atoms in this restricted basis. For the principal quantum numbers considered in this thesis, the basis typically contains a few hundreds to a thousand states. 


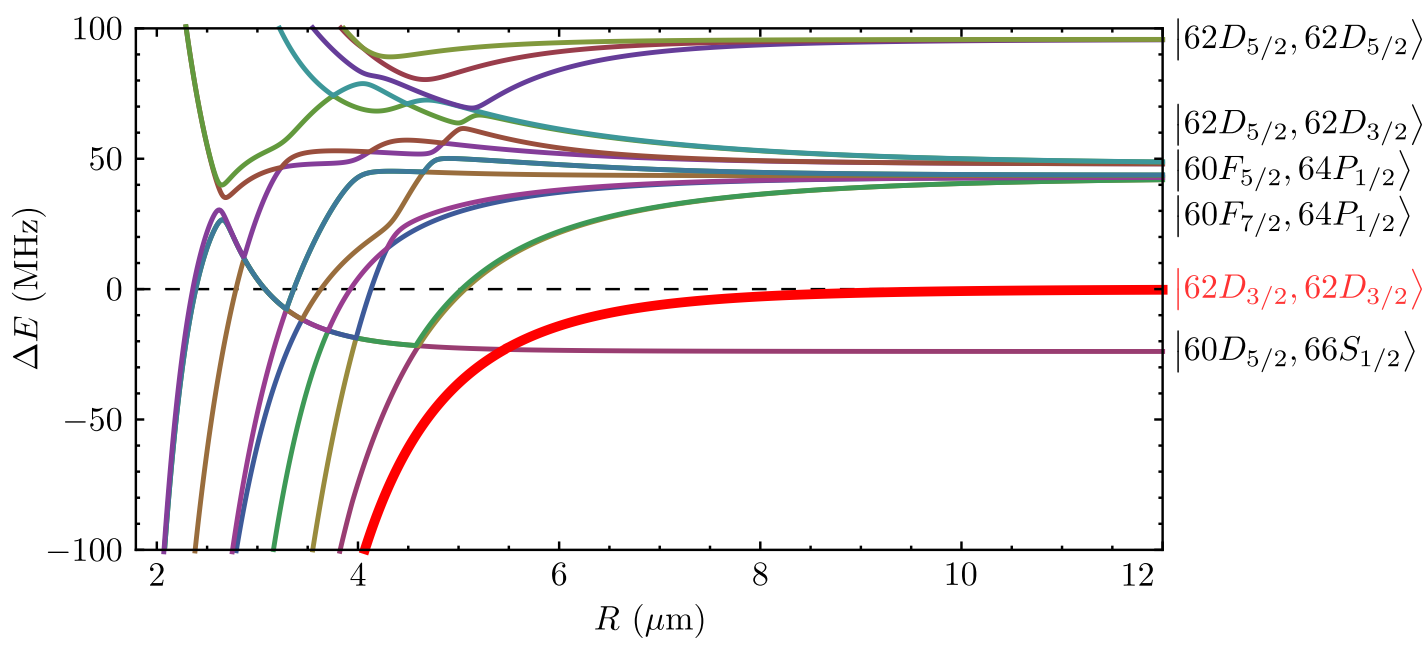

Figure 7.1: Diagonalization of the interaction Hamiltonian. Potential curves corresponding to the interaction energy between two ${ }^{87} \mathrm{Rb}$ atoms around the Rydberg states $\left|62 D_{3 / 2}\right\rangle$. The thick red curve is the potential curve for the state $\left|62 D_{3 / 2}, M_{J}=3 / 2 ; 62 D_{3 / 2}, M_{J}=3 / 2\right\rangle$.

\section{Calculation of the $C_{6}$ coefficient}

We first calculate the interaction energy according to second order perturbation theory. We compute the $C_{6}$ coefficient by calculating the sum of Equation 7.3, in the restricted basis described above, where the radial wave functions are obtained numerically using the Numerov algorithm [Weissbluth (1978); Zimmerman et al. (1979)]. For instance, for the state $\left|82 D_{3 / 2}, m_{J}=3 / 2\right\rangle$ studied in this Chapter, we obtain:

$$
\left|C_{6, \mathrm{th}}\right|=8780 \pm 150 \mathrm{GHz} \cdot \mu \mathrm{m}^{6}
$$

where the error bar comes from an $\simeq 0.5 \%$ uncertainty in the calculation of the dipole matrix elements. Our calculated $C_{6}$ coefficients are in very good agreement with the ones computed in [Reinhard et al. (2007)].

\section{Full diagonalization of the Hamiltonian}

The perturbative approach is not valid for large interaction energy (or equivalently, small interatomic distances). We then diagonalize the interaction Hamiltonian written in the basis described in Section 7.1.3. Figure 7.1 shows the result of the calculation in the case of two interacting atoms in the state $\left|62 D_{3 / 2}, m_{J}=3 / 2\right\rangle$, in the absence of magnetic field (adapted from [Béguin (2013)]). The origin of energies is the energy of the pair state $\left|62 D_{3 / 2}, m_{J}=3 / 2 ; 62 D_{3 / 2}, m_{J}=3 / 2\right\rangle$ in the limit of two infinitely separated atoms. We observe the presence of a large number of potential 


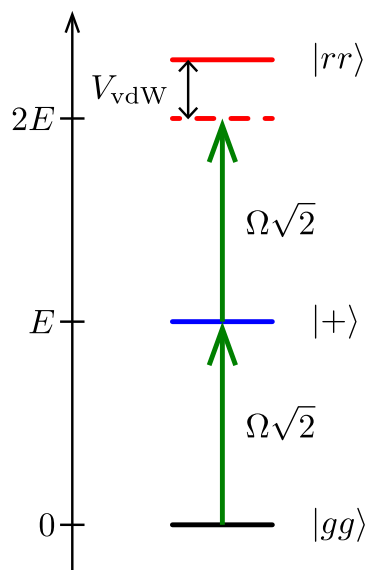

Figure 7.2: Three-level model of the van der Waals interaction. The excitation lasers resonantly couple $|g\rangle$ to $|r\rangle$ with Rabi frequency $\Omega$. Because of the interaction, the energy of $|r r\rangle$ is shifted by a quantity $V_{\mathrm{vdW}}$.

curves due to the multiple couplings allowed by $V_{\text {dip }}$. The thick red curve corresponds to the potential for the state $\left|62 D_{3 / 2}, m_{J}=3 / 2 ; 62 D_{3 / 2}, m_{J}=3 / 2\right\rangle$. At large distances, the interatomic interaction is weak, and we observe almost no shift of the $\left|62 D_{3 / 2}, m_{J}=3 / 2 ; 62 D_{3 / 2}, m_{J}=3 / 2\right\rangle$ state. As we decrease the distance $R$ between the atoms, the interaction energy increases, leading to an increased energy shift of the state. For $\mathrm{a} \simeq 6 \mu \mathrm{m}$ distance, we observe an energy shift corresponding to an interaction energy $\Delta E / h \simeq 15 \mathrm{MHz}$, while a simple $C_{6} / R^{6}$ would give approximately $190 \mathrm{MHz}$.

\section{Description in terms of a three-level system}

We checked that the asymptotic behavior of the potential curve obtained by diagonalizing the Hamiltonian is in good agreement with a $1 / R^{6}$ law. For the states studied in this thesis, and for our typical interatomic distances, we observe that the result of the diagonalization is in very good agreement with the calculation of the $C_{6}$ coefficient obtained from second-order perturbation theory. This shows that in our experiment, the interaction Hamiltonian can be considered as a perturbation. When applying second-order perturbation theory, we neglect the perturbation of the state $|r r\rangle$ due to the interaction, and we simply picture the effect of the interaction between two atoms in the state $|r\rangle$ as an energy shift of the pair state $|r r\rangle$ by a quantity $\Delta E=V_{\mathrm{vdW}}$.

In this regime, the two atoms interacting with each other and with a laser can be modeled by a three-level system containing the pair states $|g g\rangle,|+\rangle=(|g r\rangle+|r g\rangle) / \sqrt{2}$ and $|r r\rangle$. Figure 7.2 shows the three-level diagram and under the rotating wave 

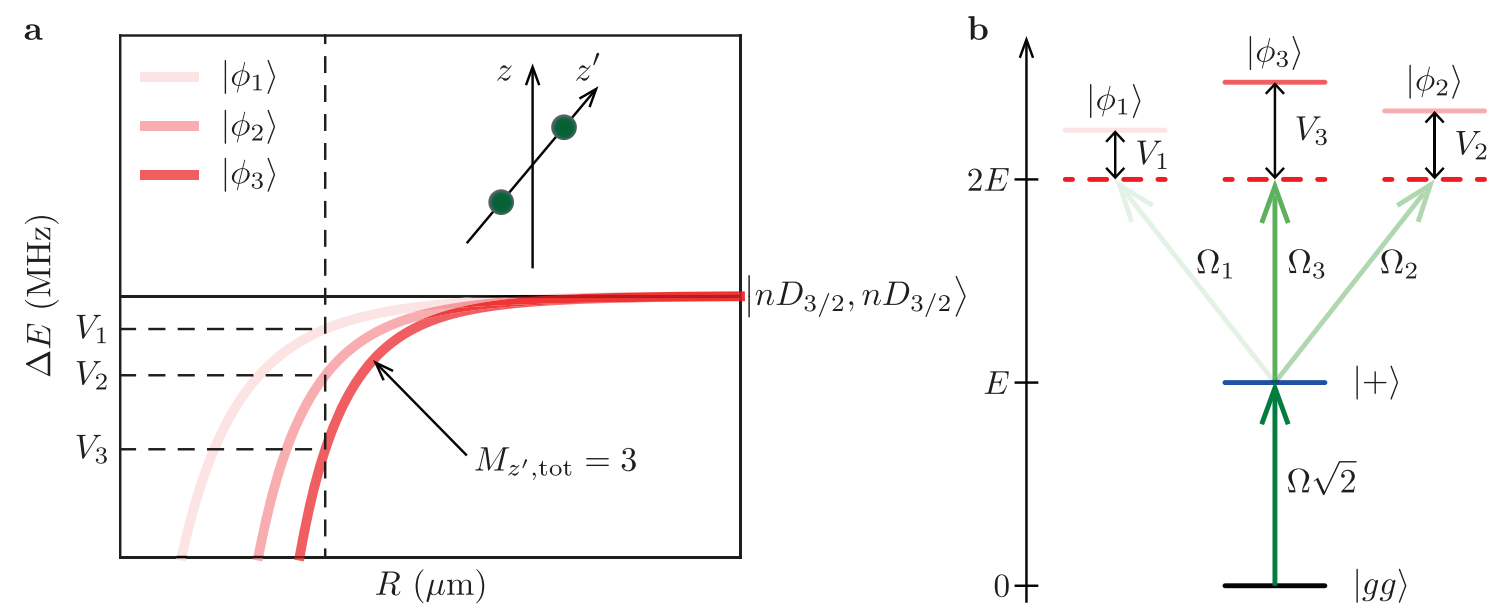

Figure 7.3: Anisotropy of the interaction. a, Potential curves for the different magnetic sublevels of the state $|r r\rangle=\left|n D_{3 / 2}, n D_{3 / 2}\right\rangle$. In the internuclear basis, each curve corresponds to a given value of $M_{z^{\prime}}$,Tot. In the basis defined by the quantization axis, the states are mixed: $|r r\rangle$ contributes to the different potential curves, as represented by the level of red in each curve. b, Diagram of the resulting energy levels, summarizing the different couplings and detunings.

approximation. The Schrödinger equation for this system reads:

$$
i \hbar \frac{\mathrm{d}}{\mathrm{d} t}\left(\begin{array}{c}
c_{g g} \\
c_{+} \\
c_{r r}
\end{array}\right)=\left(\begin{array}{ccc}
0 & \Omega \sqrt{2} / 2 & 0 \\
\Omega \sqrt{2} / 2 & 0 & \Omega \sqrt{2} / 2 \\
0 & \Omega \sqrt{2} / 2 & V_{\mathrm{vdW}} / \hbar
\end{array}\right)\left(\begin{array}{c}
c_{g g} \\
c_{+} \\
c_{r r}
\end{array}\right)
$$

In the low-excitation limit $\hbar \Omega \ll V_{\mathrm{vdW}}$, where the energy shift is large compared to the Rabi frequency (blockaded regime), we adiabatically eliminate the doubly-excited state by setting $\mathrm{d} c_{r r} / \mathrm{d} t=0$ and obtain:

$$
c_{r r}=-\frac{1}{\sqrt{2}} \frac{\hbar \Omega}{V_{\mathrm{vdW}}} c_{+}
$$

The maximal double excitation probability $\left(P_{r r}=\left|c_{r r}\right|^{2}\right)$ is obtained when $\left|c_{+}\right| \simeq 1$ :

$$
P_{r r}^{\mathrm{Max}}=\frac{1}{2} \frac{\hbar^{2} \Omega^{2}}{V_{\mathrm{vdW}}^{2}},
$$

to lowest order in $\Omega / V_{\mathrm{vdW}}$ [Walker and Saffman (2008)]. This number characterizes the blockade in a quantitative way. 


\subsubsection{Anisotropy of the interaction}

The dipole-dipole interaction Hamiltonian in Equation 7.6 shows that the interaction is anisotropic [Carroll et al. (2004); Reinhard et al. (2007)]. When $\theta \neq 0$, all the terms of the dipole-dipole interaction Hamiltonian come into play, and one has to consider couplings to states with $\left|\Delta M_{\text {Tot }}\right|=1$ and $\left|\Delta M_{\text {Tot }}\right|=2$. One of the consequences of these couplings is that the state $|r r\rangle$ is indirectly coupled to all $\left(2 J_{r}+1\right)^{2}$ magnetic substates:

$$
\left|r M ; r M^{\prime}\right\rangle \equiv\left|n_{r}, L_{r}, J_{r}, M\right\rangle \otimes\left|n_{r}, L_{r}, J_{r}, M^{\prime}\right\rangle
$$

where $M$ and $M^{\prime}$ range from $-J$ to $J$ in steps of one. As an example, if we suppose that the interaction Hamiltonian couples the states $|r r\rangle$ and $\left|\phi_{A} \phi_{B}\right\rangle$, then, as soon as $\theta \neq 0,\left|\phi_{A} \phi_{B}\right\rangle$ is necessarily also coupled to the states $\left|r M ; r M^{\prime}\right\rangle$ (with $M$ and $M^{\prime} \in$ $\{ \pm 1 ; \pm 2\})$. The dipole-dipole interaction couples the different magnetic sublevels, and as a consequence, the eigenstates $\left|\phi_{i}\right\rangle$ of the Hamiltonian are superpositions states of the form ${ }^{2}$ :

$$
\left|\phi_{i}\right\rangle=\sum_{M, M^{\prime}} C_{M, M^{\prime}}(R, \theta)\left|r M ; r M^{\prime}\right\rangle
$$

The interaction potentials for the states $\left|\phi_{i}\right\rangle$ are represented in Figure 7.3a as a function of the separation distance $R$ between the atoms. The states $\left|\phi_{i}\right\rangle$ show different interaction strengths, which leads to the different energy shifts $V_{i}(R, \theta)$. When we excite the system, our excitation laser shows couplings to all states $\phi_{i}$ that have a $|r r\rangle$ component $\left(\left\langle r r \mid \phi_{i}\right\rangle \neq 0\right)$. Compared to the $\theta=0^{\circ}$ case, the effect of the interaction can not be reduced to the energy shift of a single level, but the contributions of several potential curves have to be taken into account. We define the couplings $\Omega_{i}$ between $|+\rangle=(|g r\rangle+|r g\rangle) / \sqrt{2}$ and $\left|\phi_{i}\right\rangle$ by:

$$
\Omega_{i}=\sqrt{2} \Omega\left\langle r r \mid \phi_{i}\right\rangle
$$

The resulting situation is represented by the multi-level system in Figure 7.3b, where the energy levels $\left|\phi_{i}\right\rangle$ and $|+\rangle$ are coupled with a Rabi frequency $\Omega_{i}$, and the excitation laser is detuned from those transitions by the energy $V_{i}$. One can write the Schrödinger equations for this system and, in the low-excitation limit, adiabati-

\footnotetext{
${ }^{2}$ Note that in the basis that rotates with the internuclear basis, the Hamiltonian conserves $M_{z^{\prime}}$,Tot . In this basis, the eigenstates of the interaction Hamiltonian are the pure states $\left|r M_{z^{\prime}} ; r M_{z^{\prime}}^{\prime}\right\rangle$. Changing the basis to the laboratory frame using Wigner matrices leads to eigenstates that are superposition states.
} 
cally eliminate the doubly-excited states as in Equation 7.10. We obtain the sets of equations:

$$
c_{i}=-\frac{1}{V_{i}} \frac{\hbar \Omega_{i}}{2} c_{+}
$$

As a consequence, the double-excitation probability $P_{r r}=\sum_{i}\left|c_{i}\right|^{2}$ has an upper limit:

$$
P_{r r}^{\mathrm{Max}}=\frac{1}{4} \sum_{i} \frac{\hbar^{2} \Omega_{i}^{2}}{V_{i}^{2}} .
$$

We notice the similarity with the maximum double-excitation probability calculated in Equation 7.12. Following the procedure in [Walker and Saffman (2008)], we define an effective potential $V_{\text {eff }}$ so that:

$$
P_{r r}^{\mathrm{Max}}=\frac{1}{2} \frac{\hbar^{2} \Omega^{2}}{V_{\mathrm{eff}}^{2}} .
$$

We obtain:

$$
\frac{1}{V_{\mathrm{eff}}^{2}}=\frac{1}{2 \Omega} \sum_{i} \frac{\hbar^{2} \Omega_{i}^{2}}{V_{i}^{2}}=\sum_{i} \frac{\hbar^{2}\left|\left\langle r r \mid \phi_{i}\right\rangle\right|^{2}}{V_{i}^{2}} .
$$

As a conclusion, in the low-excitation limit and for an arbitrary angle $\theta$, we can define a potential $V_{\text {eff }}$ that allows us to effectively place ourselves in a three-level configuration in the low-excitation limit ${ }^{3}$. In this simplified picture, an effective level undergoes a $V_{\text {eff }}$ shift of energy due to the dipole-dipole interaction. The effective potential $V_{\text {eff }}$ is a weighted average of the different potential curves that contribute to the interaction.

This Chapter presents the measurement of the van der Waals interaction in the particular case of the stretched state $\left|82 D_{3 / 2}, 82 D_{3 / 2}\right\rangle$, that shows a maximal $\left|M_{\text {Tot }}\right|=$ 3. For $\theta=0^{\circ}$, the interaction energy is directly obtained from the single potential curve that is labeled by $\left|M_{z, \text { Tot }}\right|=\left|M_{z^{\prime} \text {,Tot }}\right|=3$ in Figure $7.3 \mathrm{a}$. For $\theta \neq 0^{\circ}$, the effective potential $V_{\text {eff }}$ is a weighted average of the different potential curves. We observe in Figure $7.3 \mathrm{a}$ that the potential curve corresponding to the maximal value $\left|M_{z^{\prime}, \text { Tot }}\right|=3$ shows the largest interaction energies. As a consequence, the contributions of the other potential curves in $V_{\text {eff }}$ can only lead to decreased effective interactions when

\footnotetext{
${ }^{3} \mathrm{~A}$ complete theoretical study also requires to consider the effect of the magnetic field, that shifts the levels and adds some couplings to other states. The influence of the magnetic field in our experimental configuration is currently under study by B. Vermersch in the group of P. Zoller in Innsbruck. In the simpler case of the resonant dipole-dipole interaction (see Chapter 9), we will study the influence of the magnetic field in more detail.
} 
$\theta \neq 0^{\circ}$. On our setup, for a given separation distance, pairs of atoms aligned along the $z$-axis will show larger van der Waals interactions than other pairs of atoms ${ }^{4}$. This shows the importance of keeping in mind these anisotropies when working with two-dimensional arrays of traps.

\subsection{Rydberg blockade in three-atoms system with varying geometry}

We have demonstrated in Chapter 6 our ability to produce 2D arrays of traps. As a proof a principle that our 2D arrays are suitable for applications using the Rydberg blockade in large ordered ensembles we first test the robustness of the blockade in elementary 2D systems containing three atoms. In a 2D configuration, some atom pairs necessarily have an internuclear axis not aligned along the quantization axis, which might eventually prevent a perfect blockade [Pohl and Berman (2009); Cano and Fortágh (2012); Qian et al. (2013)]. Here we study the blockade both in a linear and an equilateral triangle configuration where, in the latter case, the anisotropic character of the $D$-state orbital plays a role.

We need large interatomic interactions in order reach a robust blockade. In the van der Waals regime, because of the $1 / R^{6}$ dependence of the interaction, varying $R$ is an efficient way to tune the relative strength of $V_{\mathrm{vdW}}(R)$ with respect to $\hbar \Omega$. Moreover, we chose to work with a large principal quantum number $(n=82)$, so as to increase the interactions. The $|r\rangle=\left|82 D_{3 / 2}, M_{J}=3 / 2\right\rangle$ state has been studied in detail by L. Béguin in the case of two atoms [Béguin et al. (2013)], who measured a $C_{6}$ coefficient of:

$$
\left|C_{6, \mathrm{mes}}(n=82)\right|=8500 \pm 300 \mathrm{GHz} \cdot \mu \mathrm{m}^{6},
$$

in good agreement with the calculated value of $\left|C_{6, \mathrm{th}}(n=82)\right|=8870 \pm 150 \mathrm{GHz} \cdot \mu \mathrm{m}^{6}$ given in Equation 7.9. For a $8 \mu \mathrm{m}$ interatomic distance, we expect an interaction as large as $\simeq 32 \mathrm{MHz}$, placing us in a strong Rydberg blockade regime for our accessible Rabi frequencies $\Omega /(2 \pi) \sim 1 \mathrm{MHz}$. 
a

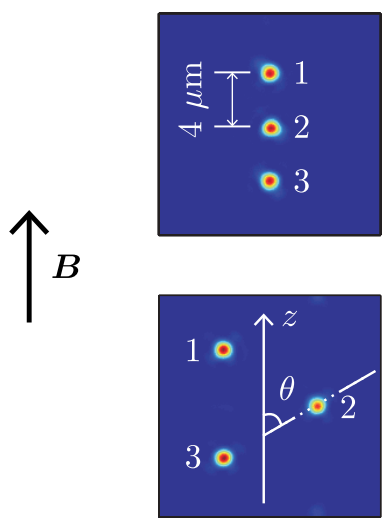

b

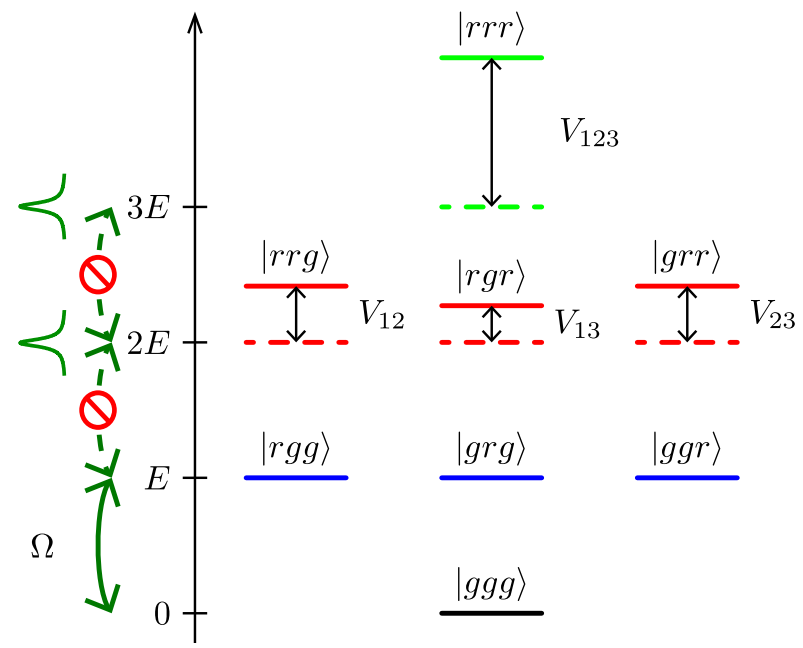

Figure 7.4: Trap arrangements and level configuration in three-atom systems. a, Three single-atoms are trapped in microscopic optical tweezers separated by $R=4 \mu \mathrm{m}$ in a linear arrangement (top), and by $R=8 \mu \mathrm{m}$ in a triangular arrangement (bottom). The quantization axis $\hat{z}$ is set by $\mathbf{a} \simeq 3.3 \mathrm{G}$ external magnetic field. $\mathbf{b}$, Relevant energy levels of a three-atom system with van der Waals interactions $V_{i j}$.

\subsubsection{Experimental sequence}

We consider two different arrangements of three individual atoms. Figure 7.4a shows CCD images of the arrays are shown. The first trap configuration (top panel in Figure 7.4a) is a chain of three atoms separated by $\simeq 4 \mu \mathrm{m}$ and aligned along the quantization axis. In a second experiment, the traps form an equilateral triangle, with $\simeq 8 \mu \mathrm{m}$ sides. We trigger the experimental sequence upon the presence of one atom in each of the three traps. We turn the trap off for $2 \mu \mathrm{s}$, and shine on the atoms an excitation pulse of variable duration $\tau$ that couples $|g\rangle$ to $|r\rangle$. We repeat each measurement about 100 times so as to reconstruct the excitation probabilities $P_{i, j, k}$ (where $i, j$, and $k \in\{0 ; 1\}$ ).

The Rabi frequencies $\Omega_{1}, \Omega_{2}$ and $\Omega_{3}$ of each individual atom are obtained independently from single-atom Rabi oscillation measurements. By careful alignment of the atoms with respect to the center of the excitation beams, we obtain Rabi frequencies $\Omega_{1} \simeq \Omega_{2} \simeq \Omega_{3} \simeq 2 \pi \times 0.8 \mathrm{MHz}$ that are identical ${ }^{5}$ to better than $5 \%$. Figure $7.4 \mathrm{~b}$

\footnotetext{
${ }^{4}$ Note that for spherically symmetric $S$-states, the degree of asymmetry is small, of the order of $\sim 1 \%$ [Walker and Saffman (2008)].

${ }^{5}$ The alignment is particularly sensitive for the triangular configuration, since the $\simeq 7 \mu \mathrm{m}$ height of the triangle has a size which is comparable with the size of the blue beam $\left(w_{B} \simeq 20 \mu \mathrm{m}\right)$, and the quantity of blue light seen by the atom can vary significantly from site to site. As a consequence, we align the center of gravity of the triangle with respect the center of the blue beam so that each atom sees approximately the same quantity of blue light. The size of the red beam is much bigger than the size of the trap pattern $\left(w_{R} \simeq 100 \mu \mathrm{m}\right)$, and variations of the amount of red light seen by
} 
pictures the different energy levels in our three-atom system. The excitation laser resonantly couples the ground state $|g g g\rangle$ to the singly-excited states $|r g g\rangle,|g r g\rangle$ and $|g g r\rangle$. Because of the van der Waals interaction between Rydberg atoms, the doublyexcited states $|r r g\rangle,|r g r\rangle$ and $|g r r\rangle$ and the triply-excited state $|r r r\rangle$ are respectively shifted in energy by the quantities $V_{12}, V_{13}, V_{23}$ and $V_{123}$.

For two $\left|82 D_{3 / 2}, M_{J}=3 / 2\right\rangle$ atoms separated by $\simeq 8 \mu \mathrm{m}$ and aligned along the quantization axis, we have seen that we expect an interaction energy $V_{13} / h \simeq 32 \mathrm{MHz}$. In the linear configuration, the interaction energies $V_{12}$ and $V_{23}$ are 64 times larger than $V_{13}$ due to the $1 / R^{6}$ scaling. As a consequence, the multiply excited states are completely off-resonant with the excitation laser, and we expect the blockade of the excitation of doubly and triply excited states. In the triangular configuration, $V_{12}$ and $V_{23}$ are slightly smaller than $V_{13}$, due to the anisotropy of the interaction. However, the blockade should be robust to these anisotropies of the interaction, since we placed the system in a strong blockade regime, with $V_{13} /(\hbar \Omega) \simeq 40$. A more detailed experimental analysis of the van der Waals interaction angular dependence supporting this statement will be presented in Section 7.3. In the triangular configuration, we should therefore also observe the suppression of the two-atom and three-atom excitation probabilities. When the two-atom and three-atom excitations are blockaded, the excitation laser is only resonant with the three singly-excited state, which leads to a collective excitation of the system (see Chapter 5), and an oscillation at the frequency:

$$
\Omega_{\mathrm{coll}}=\sqrt{\Omega_{1}^{2}+\Omega_{2}^{2}+\Omega_{3}^{2}} \simeq \Omega \sqrt{3}
$$

where $\Omega \equiv \Omega_{1} \simeq \Omega_{2} \simeq \Omega_{3}$.

\subsubsection{Demonstration of a robust Rydberg blockade}

We define the probability $P_{n, 0}$, of losing $n$ atoms at the end of the sequence. In particular, the probability of losing exactly one atom at the end of the sequence is $P_{1,0}=P_{011}+P_{101}+P_{110}$. The probability of losing exactly two atoms at the end of the sequence is $P_{2,0}=P_{001}+P_{010}+P_{001}$. Finally, the probability to lose all the atoms at the end of the sequence is $P_{3,0}=P_{000}$. Figure 7.5b and Figure $7.5 \mathrm{c}$ show the evolution of the three-atom populations as a function of the excitation time. Clear blockade of multiple Rydberg excitations is observed in both configurations, as the

the different atoms are less critical. We nevertheless also align the center of gravity of the triangle with respect to the center of the red beam. 
a

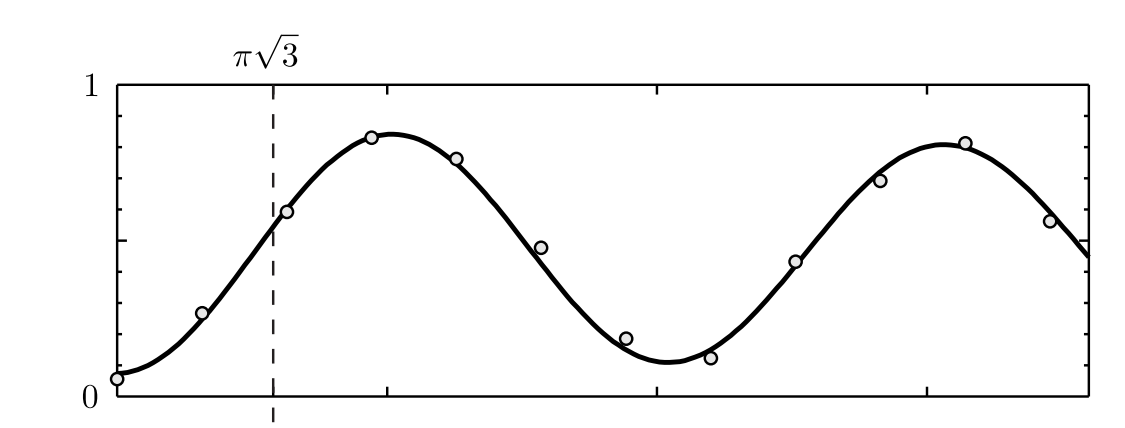

b
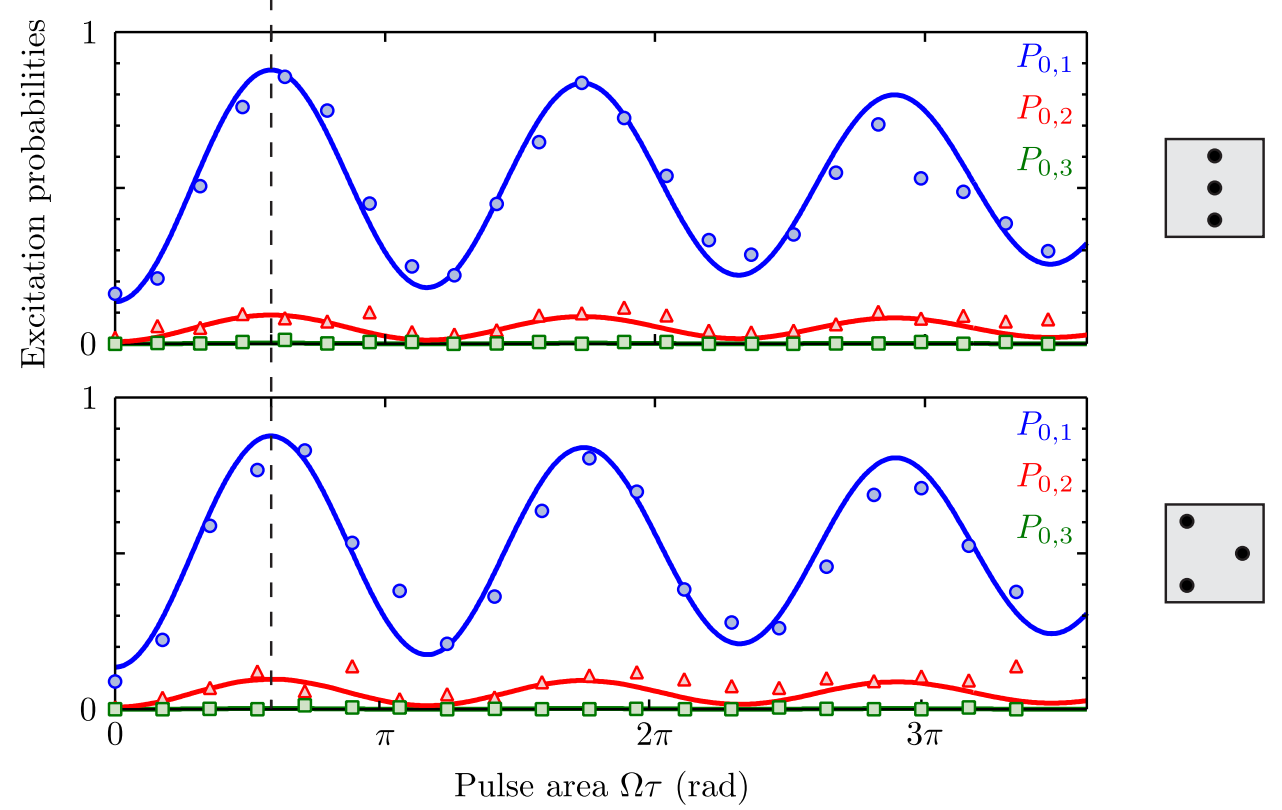

Figure 7.5: Rydberg blockade in three-atom systems. a, Typical single-atom Rabi flopping to the $\left|82 D_{3 / 2}\right\rangle$ state for the central atom in the linear arrangement. Single-atom Rabi frequencies $\Omega \simeq 2 \pi \times 0.8 \mathrm{MHz}$, and damping rates $\gamma \simeq 0.3 \mu \mathrm{s}^{-1}$ for all three atoms are obtained from fits (see solid line) to the solution of the optical Bloch equations for a single two-level atom (see Chapter 6). In this experiment, a $\pi$-pulse corresponds to $\simeq 625 \mathrm{~ns}$. $\mathbf{b}$, Probability of single (blue circles), double (red triangles), and triple (green squares) Rydberg excitation as a function of the excitation pulse area in the linear arrangement. The collective enhancement of the Rabi frequency by $\sqrt{3}$ clearly appears in the data. Solid lines are the result of the model described in the text without any adjustable parameter. $\mathbf{c}$, Same as $\mathbf{b}$ but for the triangular geometry with $R=8 \mu \mathrm{m}$. 
populations $P_{2,0}$ and $P_{3,0}$ are suppressed in the system. We observe that $P_{2,0}$ never exceeds $9 \%$, and $P_{3,0}$ never exceeds $1 \%$. Moreover, the single excitation probability $P_{1,0}$ shows oscillations with a frequency $(1.72 \pm 0.02) \Omega$, compatible with the expected $\sqrt{3} \Omega$, which confirms the collective character of the excitation. In particular, after an excitation time $\tau=\pi /(\sqrt{3} \Omega)$, the system has evolved to the collective state:

$$
|\Psi\rangle=\frac{1}{\sqrt{3}}\left(\mathrm{e}^{i \boldsymbol{k} \cdot \boldsymbol{R}_{1}}|r g g\rangle+e^{i \boldsymbol{k} \cdot \boldsymbol{R}_{2}}|g r g\rangle+e^{i \boldsymbol{k} \cdot \boldsymbol{R}_{3}}|g g r\rangle\right),
$$

where $\boldsymbol{k}$ is the wave vector of the excitation and $\boldsymbol{R}_{i}(i \in\{1,2,3\})$ are the positions of the atoms (see Chapter 5).

The strong similarities between the two sets of data demonstrates that we are in a strong Rydberg blockade regime that is robust to fluctuations of the interactions due to variations of relative distances or angles between the atoms. Despite the presence of anisotropies in the systems, both experiments show the Rydberg blockade, which is promising for applications of Rydberg blockade in larger arrays of atoms. Nevertheless, we observe that the probability $P_{2,0}$ can be as high as $9 \%$, and oscillates in phase with $P_{1,0}$. In the next Section, we demonstrate that this effect is mostly due to detection errors, and that the blockade is actually even better than suggested by these observations.

\subsubsection{Comparison with simulations}

We now simulate the dynamics of the three-atom system, to gain more insight into the quality of the blockade for our experimental parameters.

\section{Hamiltonian of the system}

An atom at site $i$ has its ground state $\left|g_{i}\right\rangle$ coupled to the Rydberg state $\left|r_{i}\right\rangle$ with a Rabi frequency $\Omega_{i}$. The atoms interact via pairwise interactions, with interaction energies $V_{i j}$. In the van der Waals regime, the effect of the interaction is to shift the energy of the doubly excited states by the quantity $V_{i j}$, and the system is described by the Hamiltonian ${ }^{6}$ [Lesanovsky (2011)]:

\footnotetext{
${ }^{6}$ Interestingly, this Hamiltonian has the form of an Ising Hamiltonian, showing that our setup can be used for the simulation of spin systems. We note that all parameters of the Hamiltonian can be tuned by a proper choice of the experimental settings, which shows the versatility of our system. In particular, choosing $|r\rangle=\left|n D_{3 / 2}\right\rangle$ gives a degree of freedom to tune $V_{i j}$ due to the anisotropy of the interaction.
} 


$$
\hat{H}=\sum_{i=1}^{3} \frac{\hbar \Omega_{i}}{2}\left(\hat{\sigma}_{r g}^{(i)}+\hat{\sigma}_{g r}^{(i)}\right)+\sum_{i<j} V_{i j} \hat{\sigma}_{r r}^{(i)} \hat{\sigma}_{r r}^{(j)},
$$

where $\hat{\sigma}_{r g}^{(i)}=\left|r_{i}\right\rangle\left\langle g_{i}\left|, \hat{\sigma}_{g r}^{(i)}=\right| g_{i}\right\rangle\left\langle r_{i}\right|$, and $\hat{\sigma}_{r r}^{(i)}=\left|r_{i}\right\rangle\left\langle r_{i}\right|$ are transition and projector operators for the atom at site $i$. In the simulation with no adjustable parameters, we use the $1 / R^{6}$ scaling of the interaction to extrapolate the coefficients $V_{i j}$ from independent measurements of the interaction energy we performed on two $\left|82 D_{3 / 2}, M_{J}=3 / 2\right\rangle$ atoms separated by $\simeq 12 \mu \mathrm{m}$ at angles $\theta=0$ and $\theta=60^{\circ}$, as we will discuss in Section 7.3.

\section{Optical Bloch equations}

We solve the optical Bloch equations for the three-atom system to include dissipation in our model (see Chapter 6). We use the Hamiltonian of Equation 7.23, and we add a sum of independent single-atom dissipators to account for the small experimental damping $\gamma_{i}$ observed experimentally on single-atom Rabi oscillations:

$$
L[\rho]=\sum_{i} \frac{\gamma_{i}}{2}\left(2 \hat{\sigma}_{g r}^{(i)} \rho \hat{\sigma}_{r g}^{(i)}-\hat{\sigma}_{r r}^{(i)} \rho-\rho \hat{\sigma}_{r r}^{(i)}\right)
$$

The coefficients $\Omega_{i}$ and $\gamma_{i}$ are obtained from fits of Rabi oscillations taken on each atom individually (see Chapter 6 ). We obtain $\gamma_{i} \simeq 0.3 \mu \mathrm{s}^{-1}$, and we observe that all the $\gamma_{i}$ are equal within 10\%. Figure 7.5 (top panel), shows a typical single-atom Rabi oscillation.

\section{Detection errors}

We also include in the simulation the detection errors (see Chapter 6), to account for the small probability to lose atoms from the trapping region independently of their actual state. Generalized to the case of three atoms, the relation between the observed probabilities $P_{i j k}(i, j, k \in\{0 ; 1\})$ and the actual excitation probabilities $P_{l m n}(l, m, n \in\{g ; r\})$ reads: 


$$
\left\{\begin{array}{l}
P_{111}=(1-\varepsilon)^{3} P_{g g g} \\
P_{011}=(1-\varepsilon)^{2}\left(P_{r g g}+\varepsilon P_{g g g}\right) \\
P_{101}=(1-\varepsilon)^{2}\left(P_{g r g}+\varepsilon P_{g g g}\right) \\
P_{110}=(1-\varepsilon)^{2}\left(P_{g g r}+\varepsilon P_{g g g}\right) \\
P_{001}=(1-\varepsilon)\left(P_{r r g}+\varepsilon\left(P_{r g g}+P_{g r g}\right)+\varepsilon^{2} P_{g g g}\right) \\
P_{010}=(1-\varepsilon)\left(P_{r g r}+\varepsilon\left(P_{g g r}+P_{r g g}\right)+\varepsilon^{2} P_{g g g}\right) \\
P_{100}=(1-\varepsilon)\left(P_{g r r}+\varepsilon\left(P_{g r g}+P_{g g r}\right)+\varepsilon^{2} P_{g g g}\right) \\
P_{000}=P_{r r r}+\varepsilon\left(P_{g r r}+P_{r g r}+P_{r r g}\right)+\varepsilon^{2}\left(P_{g g r}+P_{r g g}+P_{g r g}\right)+\varepsilon^{3} P_{g g g}
\end{array}\right.
$$

A release and recapture measurement realized on a single atom allows to estimate $\varepsilon$. Turning the trap off for a total duration $\tau=2 \mu$ s leads to a $95 \pm 1 \%$ recapture probability. In the simulation, we use $\varepsilon=5 \pm 1 \%$. Because of the error detection $\varepsilon$, the measured probability $P_{2,0}$ differs from the double excitation probability $P_{2, r}$. In the case of a perfect blockade, one has $P_{2, r} \simeq 0$ and, using Equation 7.25, we obtain $P_{2,0} \sim 2 \varepsilon P_{1, r}$ to first order in $\varepsilon$. Despite the fact that the blockade is perfect, the probability $P_{2,0}$ oscillates between 0 and $2 \varepsilon \simeq 10 \%$, in phase with $P_{1, r}$, as can be seen on the experimental data (see Figure 7.5).

\section{Quality of the blockade}

The results of the simulation, with no adjustable parameters, are plotted in Figure 7.5 (solid lines in Figure 7.5b and Figure 7.5c). The very good agreement with the experimental data further supports the quality of the blockade. In particular, due to detection errors included in the model, we observe that the predicted probability $P_{2,0}$ that two atoms are lost at the end of the sequence agrees well with the measured probability, which can reach up to $\simeq 10 \%$, despite the fact that the model predicts a maximal double excitation probability $P_{2, r}$ of $\sim 0.1 \%$. For the population $P_{2,0}$ in the line configuration, we measure an average residual of $1.5 \%$ with a residual standard deviation of $2.1 \%$. We can estimate that in our experiment, the actual double excitation probability is lower than $2 \%$, showing an efficient blockade.

\subsection{Measurement of the van der Waals interaction between two atoms}

The Rydberg blockade demonstrated above is a manifestation of strong interactions between Rydberg atoms that occurs above a certain threshold of the interaction 
a

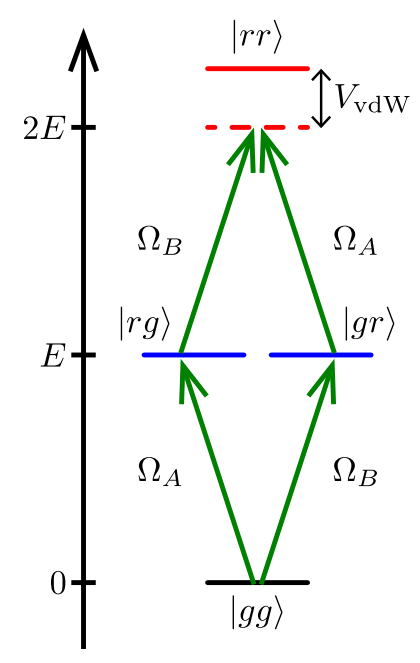

b
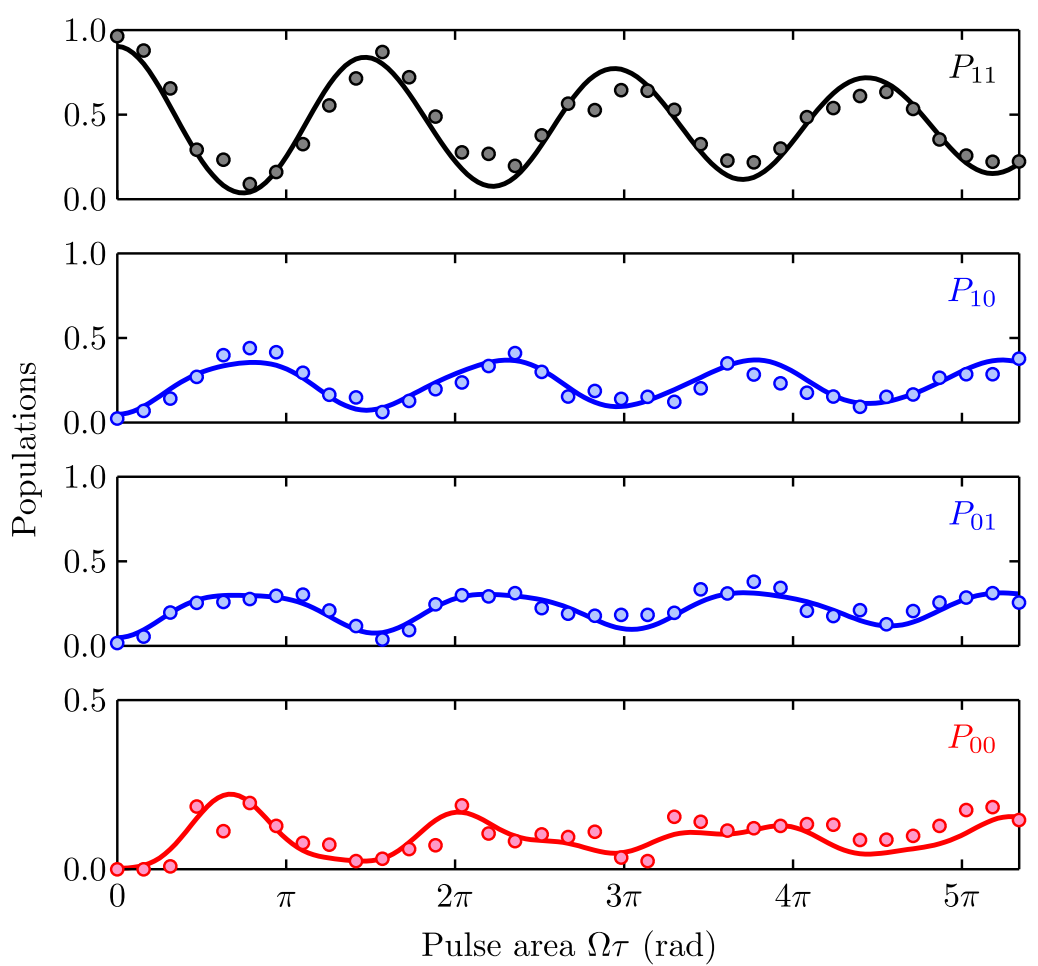

Figure 7.6: Partial blockade dynamics for two atoms. a, Configuration of the levels. The excitation lasers resonantly couple $|g g\rangle$ to $|g r\rangle$ and $|r g\rangle$. Because of the interaction, the energy of $|r r\rangle$ is shifted by a quantity $V_{\mathrm{vdW}}$. $\mathbf{b}$, Measuring the excitation dynamics of a two-atom system. Here, $|r\rangle=\left|82 D_{3 / 2}, m_{J}=3 / 2\right\rangle$, the two atoms are aligned along the quantization axis and separated by $R=12.4 \mu \mathrm{m}$. The Rabi frequencies are $\Omega_{A}=\Omega_{B}=$ $2 \pi \times 1.57 \mathrm{MHz}$. The solid lines are the fits used to extract $V_{\mathrm{vdW}}$.

energy $(\Delta E \gg \hbar \Omega)$, which explains its robustness. With the level of control we have on our setup, we can also consistently study the excitation of the system in the more sensitive regime where $\hbar \Omega \simeq \Delta E$. In this regime, L. Béguin measured the van der Waals interaction between two atoms aligned along the quantization axis [Béguin (2013)]. Here, we recall the method used, and measure the interaction energy for two atoms excited to the state $\left|82 D_{3 / 2}\right\rangle$, with $\theta=0$. In Section 7.4 , we apply the same method to measure the anisotropy of the van der Waals interaction.

\subsubsection{Principle of the experiment}

We consider two atoms aligned along the quantization axis $z$. For each atom $A$ and $B$, the excitation lasers resonantly couple $|g\rangle$ to $|r\rangle$, with couplings $\Omega_{A}$ and $\Omega_{B}$. Figure 7.6a shows the relevant energy levels for the two-atom system. The four pairstates $\{|g g\rangle,|r g\rangle,|g r\rangle,|r r\rangle\}$ are represented, where the doubly-excited state $|r r\rangle$ is 
shifted by an energy $V_{\mathrm{vdW}}$ due to the van der Waals interaction. Our goal is to measure the excitation dynamics of the system with $V_{\mathrm{vdW}} \simeq \hbar \Omega_{A, B}$. This particular regime is intermediate between the situation where the atoms behave totally independently, allowing the coherent excitation of $|r r\rangle$, and the situation where the double excitation is blockaded. The excitation laser is not exactly resonant with the doubly excited state due to the dipole-dipole interaction, but has a sufficiently large Rabi frequency to partially populate $|r r\rangle$. As a consequence, we refer to this situation as partial blockade. In this case, a characteristic beating between the two frequency components $V_{\mathrm{vdW}} / \hbar$ and $\Omega$ appears in the excitation dynamics of the system, showing the influence of the interaction [Béguin et al. (2013)].

\subsubsection{Excitation dynamics of the two-atom system in the partial blockade regime}

We now perform the excitation of two atoms in the partial blockade regime. In this experiment, the excitation lasers are resonant with the state $|r\rangle=\left|82 D_{3 / 2}, M_{J}=3 / 2\right\rangle$. In order to reach the partial blockade regime, we need an interaction energy comparable to the Rabi frequency. With an interatomic distance of $R \simeq 12.4 \mu \mathrm{m}$, we expect an interaction energy of:

$$
\frac{1}{h} V_{\mathrm{vdW}}=\frac{1}{h} \frac{C_{6, \mathrm{th}}}{R^{6}} \simeq 2.4 \mathrm{MHz},
$$

which is of the order of our typical Rabi frequency. By careful alignment of the system, we achieve couplings $\Omega \equiv \Omega_{A} \simeq \Omega_{B} \simeq 2 \pi \times 1.57 \mathrm{MHz}$ that are equal within $5 \%$. This places us in the regime where $V_{\mathrm{vdW}} \simeq \hbar \Omega_{A, B}$. We turn the trap off for $2 \mu \mathrm{s}$, and we shine an excitation laser pulse of duration $\tau$ on the atoms. We then measure the evolution of the populations $P_{i j}(i, j \in\{0 ; 1\})$ as a function of the excitation time.

Figure 7.6b shows the result of the experiment. We observe the coherent evolution of the populations as a function of the excitation pulse area $\Omega \tau$. The excitation of the doubly-excited state is not fully blockaded, but is limited by the dipole-dipole interaction since $P_{00}$ only goes up to $\simeq 20 \%$. In the partial blockade regime, the excitation dynamics are influenced by the two frequency components $\Omega$ and $V_{\mathrm{vdW}}$. Our goal is to extract from this measurement a value for the interaction. 


\subsubsection{Measurement of the interaction energy}

We fit the data using $V_{\mathrm{vdW}}$ as the only fitting parameter. Our fitting functions are obtained by solving the optical Bloch equations using similar Hamiltonian and dissipator as in Equation 7.23 and Equation 7.24, written in the two-atom case. All input parameters (Rabi frequencies $\Omega_{i}$ and damping rates $\gamma_{i}$ ) were independently measured for each single atoms taken individually. We also include the detection error $\varepsilon$ so as to compute the observed values $P_{11}, P_{01}, P_{10}$ and $P_{11}$ :

$$
\left\{\begin{array}{l}
P_{11}=(1-\varepsilon)^{2} P_{g g} \\
P_{01}=(1-\varepsilon)\left(P_{r g}+\varepsilon P_{g g}\right) \\
P_{10}=(1-\varepsilon)\left(P_{g r}+\varepsilon P_{g g}\right) \\
P_{00}=P_{r r}+\varepsilon\left(P_{r g}+P_{g r}\right)+\varepsilon^{2} P_{g g}
\end{array}\right.
$$

The only remaining free parameter in the model is the coefficient $V_{\mathrm{vdW}}$. For different values of $V_{\mathrm{vdW}}$, we compare the calculated populations $P_{i j}(i, j \in\{0 ; 1\})$ with the data. We apply the minimum chi-square method [Bevington and Robinson (2003)] to obtain the interaction energy:

$$
\frac{V_{\mathrm{vdW}}}{h}=2.5 \pm 0.5 \mathrm{MHz}
$$

where the error represents one standard deviation confidence interval in the fit. The solid lines in Figure $7.6 \mathrm{~b}$ show the result of the best fit superimposed with the experimental data. The result is in good agreement with the theoretical value $V_{\mathrm{vdW}, \mathrm{th}} / h=2.4 \mathrm{MHz}$.

\subsection{Angular dependence of the van der Waals interaction}

We now vary the angle $\theta$ between the internuclear axis and the quantization axis $z$. For $\theta \neq 0^{\circ}$, we have seen in Section 7.1 that extra terms appear in the dipoledipole Hamiltonian, which leads to a more complex situation than in the case $\theta=0^{\circ}$. However, in the low excitation limit, we can define an effective potential $V_{\text {eff }}(\theta)$, which allows to describe the effect of the interaction by a shift of the doubly excited state by a quantity $V_{\text {eff }}(\theta)$, as it is the case for $\theta=0^{\circ}$. Here, we measure this effective potential as a function of $\theta$. 


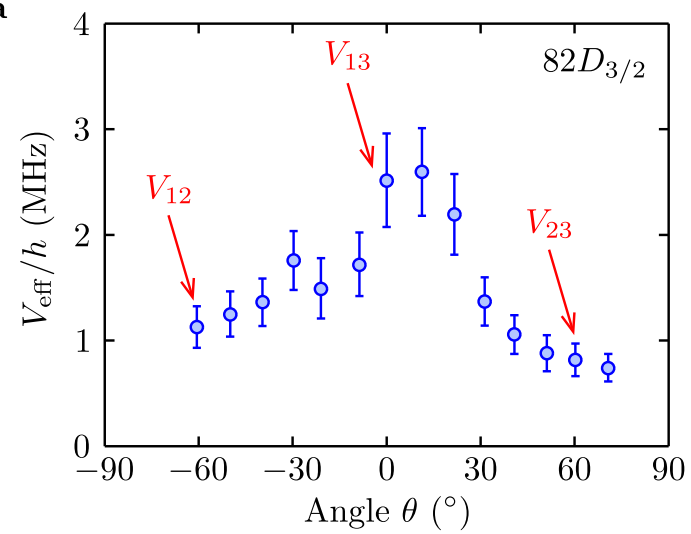

b

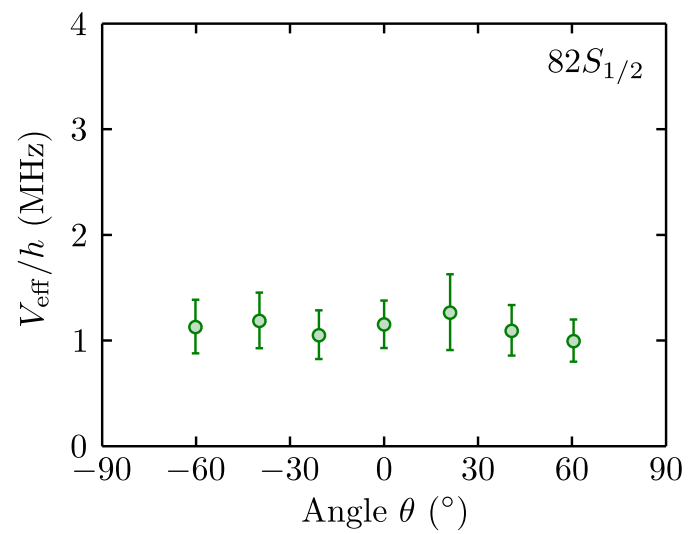

Figure 7.7: Angular dependence of the effective interaction energy $V_{\text {eff }}$ for two atoms. The interatomic distance is $R=12.4 \mu \mathrm{m}$, and $\theta$ is the angle between the internuclear axis and the quantization axis $z$. a, Angular dependence of the interaction for the $\left|82 D_{3 / 2}, m_{J}=3 / 2\right\rangle$ state. $V_{13}, V_{23}$, and $V_{12}$ indicate the measured energy shifts used for the simulation of the three-atom system. $\mathbf{b}$, The angular dependence of the interaction for the spherically symmetric $\left|82 S_{1 / 2}, M_{J}=1 / 2\right\rangle$ state is shown for comparison. Error bars represent one standard deviation confidence intervals in the fits.

\subsubsection{Experimental procedure}

The experimental procedure we use is exactly the same as the one described in Section 7.3, where we replace $V_{\mathrm{vdW}}$ by $V_{\mathrm{eff}}(\theta)$ in the level configuration in Figure 7.6a. The interatomic distance is fixed to $R=12.4 \mu \mathrm{m}$, and we very the angle $\theta$ by changing the phase displayed on the SLM. In the partial blockade regime, we measure the excitation dynamics of the two-atom system and fit them with the solution of the optical Bloch equations with $V_{\text {eff }}(\theta)$ as the only fitting parameter.

\subsubsection{Measurement of the anisotropy of the interaction}

The result of this approach is shown in Figure 7.7 a for the $\left|82 D_{3 / 2}, M_{J}=3 / 2\right\rangle$ state. The measured interaction energies show a clear anisotropy as a function of the angle. As expected, the energy shift is maximum around $\theta=0$ and decreases for larger angles: we measure a relative change of interaction strength ${ }^{7}$ by a factor $\simeq 3$ when $\theta$ varies from $\theta=0^{\circ}$ to $\theta=60^{\circ}$.

We observe an unexpected, slight asymmetry in the angular dependence of $V_{\text {eff }}$, probably due to small systematic effects. We have ruled out several possible systematic effects, such as imperfections in the generation of the traps or longitudinal shifts

\footnotetext{
${ }^{7} V_{13}, V_{23}$, and $V_{12}$ indicate the measured energy shifts used to extrapolate the interaction energies at a distance $R=8 \mu \mathrm{m}$ in the simulation of the three-atom system Section 7.2.
} 
between the two trap foci. One possible reason for this asymmetry comes from the fact that the theoretical derivation of the effective potential is only justified in a limit where the double-excitation probability $P_{r r}$ vanishes (see Section 7.1). However, our measurement method requires $P_{r r} \neq 0$. For the negative angles in Figure 7.7a, we adapted the Rabi frequency $\Omega$ to keep the double excitation lower than $\simeq 30 \%$ everywhere. However, for the positive angles, we generally used larger Rabi frequencies, leading to double excitation probabilities as high as $\simeq 60 \%$, which could be a source of asymmetry. Those effects, along with a detailed study of the angular dependence of the van der Waals interaction including all 16 Zeeman sublevels with their van der Waals couplings, are under investigation by B. Vermersch, a postdoctoral researcher in the group of P. Zoller in Innsbruck. Nonetheless, the observed degree of asymmetry has a negligible impact on what follows.

We have finally measured the effective interaction for the spherically symmetric $\left|82 S_{1 / 2}, M_{J}=1 / 2\right\rangle$ state $^{8}$. For comparison, the result is shown in Figure $7.7 \mathrm{~b}$ and shows, as expected, almost no anisotropy of the interaction energy.

\subsubsection{Effects of the anisotropy of the interaction on a three-atom system in the partial blockade regime}

The angular dependence of $V_{\text {eff }}$ manifests itself in our system as soon as we use 2D arrays of atoms. In Section 7.2, we have demonstrated that the Rydberg blockade is robust to anisotropies of the interaction. However, in the partial blockade regime, one should directly observe anisotropic effects in the excitation dynamics of the threeatom system. Now that we have characterized the interactions between pairs of atoms in all directions, one natural thing to do is to use the additivity of van der Waals interactions to describe interacting systems containing more atoms. However, even in the case of $N=3$, some situations have been identified where many body effects in the presence of resonant dipole-dipole interactions lead to the non-additivity of interaction potentials [Pohl and Berman (2009); Cano and Fortágh (2012)]. In the particular situation of the van der Waals regime investigated here, we will check on a three-atom system that the physics can be understood in terms of pairwise interactions. 

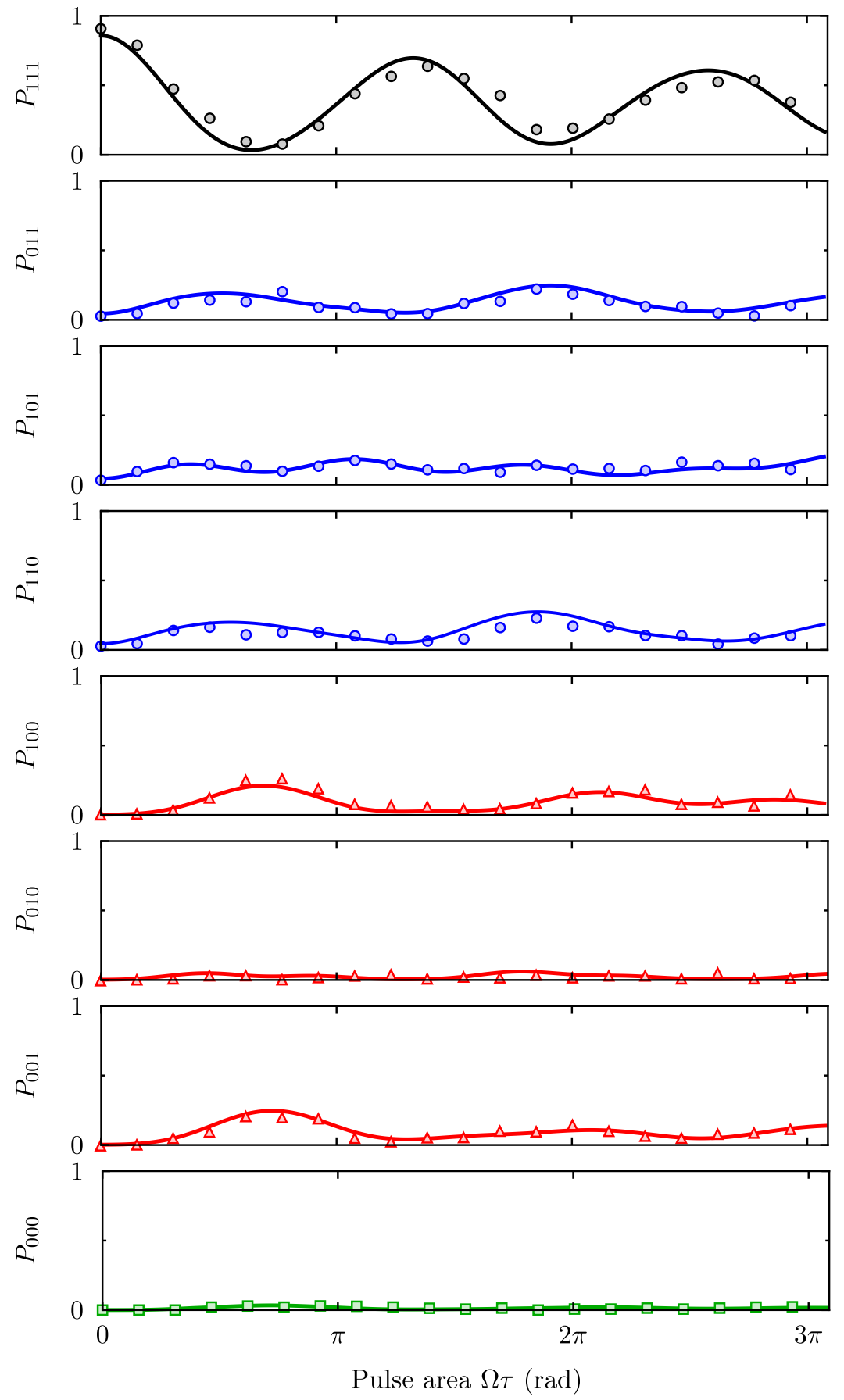

Figure 7.8: Partial blockade dynamics of three interacting atoms for $\Omega /(2 \pi) \simeq 0.8 \mathrm{MHz}$. Populations $P_{i j k}$ of the three-atom system versus excitation pulse area $\Omega \tau$ for driving Rabi frequencies $\Omega_{i} /(2 \pi) \simeq 0.8 \mathrm{MHz}$. The distance between the traps is $R=12.4 \mu \mathrm{m}$. The ratio between the effective pairwise interaction energies is $V_{13} / V_{12} \simeq V_{13} / V_{23} \simeq 3$ for $\theta=60^{\circ}$. Solid lines are the solution of the OBEs for the three-atom system without any adjustable parameter. 

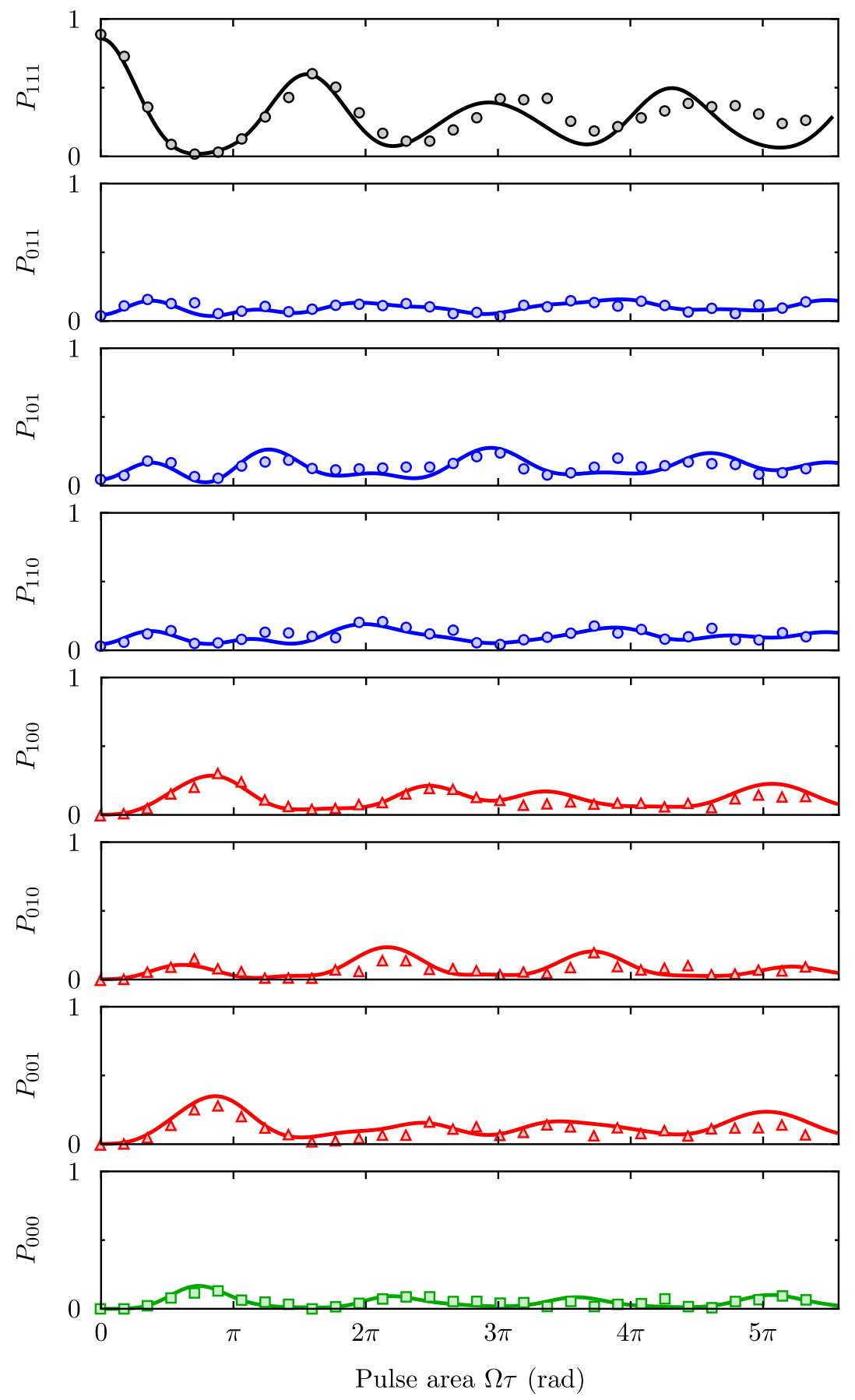

Figure 7.9: Partial blockade dynamics of three interacting atoms for $\boldsymbol{\Omega} /(\mathbf{2} \boldsymbol{\pi}) \simeq \mathbf{1 . 6} \mathrm{MHz}$. Same as Figure 7.8 , where we increase the Rabi frequency to $\Omega_{i} /(2 \pi) \simeq 1.6 \mathrm{MHz}$, allowing to partially excite the state $|r r r\rangle$. 


\section{Experimental observations}

We thus come back to an equilateral triangle configuration as in Section 7.2. The excitation lasers are resonant with the state $|r\rangle=\left|82 D_{3 / 2}, M_{J}=3 / 2\right\rangle$. Compared to the blockaded situation, we reach the partial blockade regime by increasing the sides of the triangle to $R \simeq 12.4 \mu \mathrm{m}$. The effective interaction energies present in the system are directly obtained from Figure 7.7 , and read $\left(V_{12}, V_{23}, V_{13}\right) \simeq h \times(0.9,1.1,2.6) \mathrm{MHz}$. For two different Rabi frequencies $\Omega$, we shine an excitation pulse of duration $\tau$ on the atoms, and we measure the eight recapture probabilities $P_{i j k}(i, j, k \in\{0 ; 1\})$ as a function of $\tau$.

- Figure 7.8 shows a first dataset with:

$$
\Omega=2 \pi \times 0.8 \mathrm{MHz} \simeq V_{12} / \hbar \simeq V_{23} / \hbar<V_{13} / \hbar
$$

We observe that $\hbar \Omega$ is of the order of the interactions $V_{12}$ and $V_{23}$ for the atom pairs that form an angle $\theta= \pm 60^{\circ}$ with the quantization axis. However, the Rabi frequency is more than three times smaller than the interaction between the two atoms that are aligned along the quantization axis. Due to the anisotropy of the interaction, one pair of atoms is consequently in the blockade regime, whereas the two other pairs are in the partial blockade regime. This effect is directly observed on the excitation dynamics of the system shown in Figure 7.8, in particular when looking at the probabilities $P_{100}, P_{010}$ and $P_{001}$ to lose two atoms. The probability $P_{010}$ remains below $5 \%$, due to the blockade of the double excitation for the two atoms aligned along the quantization axis, whereas the probabilities $P_{001}$ and $P_{100}$ show up to $30 \%$ excitation of double excitation for the other pairs of atoms. Note that the anisotropy of the interaction also affects the single excitation probabilities, as we can observe on the probabilities $P_{011}$, $P_{101}$ and $P_{110}$ measured experimentally: $P_{011}$ and $P_{110}$ show similar behavior, whereas $P_{101}$ shows faster dynamics.

- Figure 7.9 shows a second dataset with:

$$
V_{12} / \hbar \simeq V_{23} / \hbar \lesssim \Omega=2 \pi \times 1.6 \mathrm{MHz} \lesssim V_{13} / \hbar
$$

We observe that the Rabi frequency is intermediate between the interactions $V_{12} \simeq V_{23}$ and $V_{13}$, allowing all the pairs to be in the partial blockade regime,

\footnotetext{
${ }^{8}$ For excitation to the $S$-state, we changed the polarization of the $474 \mathrm{~nm}$ laser to $\sigma^{-}$.
} 
but with slightly different interactions. In Section 7.3, we have seen that the excitation dynamics of a system in the partial blockade regime strongly depend on the interaction strength, a property which allowed to measure the van der Waals interaction between pairs of atoms. As a consequence we expect again to observe different dynamics between the different pairs of atoms. Indeed, we observe in Figure 7.9 that $P_{100}$ and $P_{001}$ (resp. $P_{110}$ and $P_{011}$ ) show a similar behavior, which differs from $P_{010}\left(\operatorname{resp} P_{101}\right)$. Compared to Figure 7.8 , we observe that the double excitation of the atoms aligned along the quantization axis and therefore the triple excitation are not blockaded anymore, as $P_{010}$ and $P_{000}$ can be as high as $\simeq 15 \%$.

Note that the fact that the populations $P_{100}$ and $P_{001}$ show a similar behavior in Figure 7.8 and Figure 7.9 supports that the observed asymmetry in our measurement of the angular dependence of the van der Waals interaction (see Figure 7.7) comes from a bias of our measurement.

\section{Comparison with the simulations}

To refine our understanding of the evolution of the populations during the excitation and to investigate the validity of the description of the system from pairwise interactions, we perform a simulation using the optical Bloch equations for the three-atom system, as described in Section 7.2. We use the Hamiltonian of Equation 7.23 and the dissipator of Equation 7.24. In this model with no adjustable parameters, the singleatom Rabi frequencies $\Omega_{i}$ and damping coefficients $\gamma_{i}$ are obtained from single-atom Rabi oscillations. A fit of the data gives the same Rabi frequencies $\Omega_{i} \equiv \Omega$ for the three atoms within $5 \%$ and same $\gamma_{i}$ for the three atoms within $10 \%$. The interaction energies measured at $\theta=0^{\circ}$ and $\theta= \pm 60^{\circ}$ in Figure $7.7 \mathrm{a}$ are introduced. We finally include in the simulation the $\varepsilon=5 \%$ probability of detection errors.

The result of the simulation is shown by the solid lines in Figure 7.8 and Figure 7.9. Using the effective energy shifts measured for each pair of atoms, it is possible to reproduce the three-atom dynamics with high accuracy. In particular, the fact that the simulation can accurately describe the evolution of the triply excited state $P_{000}$ in Figure 7.9 suggests that, for our choice of parameters, the pairwise addition of van der Waals level shifts $V_{123}=V_{12}+V_{13}+V_{23}$ is valid to a good approximation (we have not tried to put an upper limit on a possible non-additive shift of the $|r r r\rangle$ state). This result demonstrates our good understanding of the excitation of our system in the van der Waals regime, and suggests that the anisotropy of the interaction could 
be used to tailor the interactions in systems containing a larger number of atoms.

\subsection{Conclusion}

In summary, we have investigated the excitation dynamics of systems of two and three Rydberg atoms in the van der Waals regime [Walker and Saffman (2008)]. Using systems containing three atoms, we have checked that, for a proper choice of parameters, the Rydberg blockade is robust with respect to anisotropies of the interaction, which is a prerequisite for the scalability of quantum information processing proposals using 2D arrays of dipole traps [Saffman, Walker, and Mølmer (2010)]. The level of control reached in our system on the relative distances and angles between atoms enabled us to investigate the effects of the van der Waals interaction in the more sensitive regime where the blockade is only partial, allowing the measurement of the angular dependence of the interaction.

We concentrated on the van der Waals interaction between atoms excited to the same Rydberg state in this Chapter. This does not require any control of the interaction. In this case, we have shown that we can describe accurately the system from the pairwise addition of van der Waals level shifts. However, this additivity of the potential may not hold in the case of resonant dipole-dipole interactions [Cano and Fortágh (2012)], where interesting effects like the "breaking" of the Rydberg blockade [Pohl and Berman (2009)] or the formation of trimers bound by the dipole-dipole interaction [Kiffner, Li, and Jaksch (2013)] have been predicted recently. Moreover, from the point of view of quantum engineering in large arrays of atoms, resonant dipole-dipole interactions are interesting since they show a larger strength and a longer range than in the van der Waals regime. This motivated the study, in our system, of this other regime of dipole-dipole interactions, as we will discuss in Chapter 8 and Chapter 9 . 


\section{Chapter 8}

\section{Coherent resonant excitation transfer in a chain of three Rydberg atoms}

\section{Contents}

8.1 Resonant interaction between two Rydberg atoms . . . . 157

8.1 .1 Finding a resonance . . . . . . . . . . . . . . 157

8.1.2 Resonant interaction between two Rydberg atoms . . . . . . 158

8.1.3 Resonant interaction for the simulation of spin systems . . . 159

8.2 Choice of the Rydberg states . . . . . . . . . 160

8.2 .1 Microwave transition . . . . . . . . . . . . . 160

8.2 .2 Two-level system . . . . . . . . . . . . . . . . . 162

8.2.3 Estimating the needed microwave power . . . . . . . . . 163

8.3 Coherent manipulation of Rydberg states . . . . . . . . 164

8.3 .1 Setup . . . . . . . . . . . . . . . . 164

8.3.2 Experimental sequence . . . . . . . . . . . . . . 165

8.3.3 Microwave spectroscopy . . . . . . . . . . . . . . . . 165

8.3.4 Excitation dynamics of a single atom . . . . . . . . . 166

8.4 Coherent resonant energy transfer between two Rydberg atoms . . . . . . . . . . . . . . 167

8.4.1 Preparation of the system . . . . . . . . . . . 168

8.4.2 Interaction Dynamics of a two-atom system at resonance . . 170

8.4.3 Simulation of the dynamics . . . . . . . . . . . . 174

8.4.4 Origin of the reduced contrast . . . . . . . . . . 175

8.4.5 Influence of the interaction during the preparation $\ldots . .176$

8.4.6 Creation of entanglement . . . . . . . . . . . 179 
8.5 Coherent resonant energy transfer in a chain of Rydberg atoms . . . . . . . . . . . . . . . 179

8.5.1 Linear chain of three Rydberg atoms . . . . . . . . . . . 179

8.5.2 Experimental implementation . . . . . . . . . . . . . 183

8.6 Full numerical simulation of the three-atom system . . . 184

8.6.1 Hamiltonian of the system . . . . . . . . . . . . . 184

8.6.2 Imperfection of the state preparation . . . . . . . . . . 186

8.6 .3 Optical Bloch equations . . . . . . . . . . . . . . 186

8.6 .4 Finite temperature of the atoms . . . . . . . . . 188

8.6.5 Detection errors . . . . . . . . . . . . . . . . . . 190

8.6.6 Comparison with the data . . . . . . . . . . . . . . 194

8.7 Conclusion . . . . . . . . . . . . . . . . . . 194

We have used, in the previous Chapter, two atoms excited to the same Rydberg state $|r\rangle$ to investigate the van der Waals interaction between two particles. In this case, the interaction is a consequence of the off-resonant coupling of the two-atom state $|r r\rangle$ to many other pair states $\left|\phi_{A} \phi_{B}\right\rangle$. This leads to an energy shift of the state $|r r\rangle$ by a quantity $V_{\mathrm{vdW}}$ arising from non-degenerate second-order perturbation theory (see Chapter 7):

$$
V_{\mathrm{vdW}}=\frac{C_{6}}{R^{6}}
$$

We investigate, in this Chapter, the situation where the two atoms are excited to two different Rydberg states $|r\rangle$ and $\left|r^{\prime}\right\rangle$ that show a non-zero transition dipole moment. As a consequence, the two degenerate pair states $\left|r r^{\prime}\right\rangle$ and $\left|r^{\prime} r\right\rangle$ are coupled by the interaction, leading to a non-zero average energy $\left\langle r^{\prime} r\left|\hat{V}_{\text {dip }}\right| r r^{\prime}\right\rangle$. In this case, the interaction energy is $\propto 1 / R^{3}$, and is referred to as "resonant dipole-dipole interaction".

This type of resonant dipole-dipole interaction naturally occurs in chemical or biological photosystems for instance. For example, photosynthesis in plants relies on the resonant transfer of excitations from light-gathering molecules (chromophores) to the photosynthetic reaction centers (see Figure 8.1). The particularly high efficiency of light-harvesting in plants triggered some interest for the study of resonant dipole interactions in the early days of quantum mechanics ${ }^{1}$ [Oppenheimer (1941); Förster (1948); Arnold and Oppenheimer (1950)]. Today, resonant energy transfers have found applications in microscopy and biophysics [Lakowicz (2007)], where their use as a "spectroscopic ruler" [Stryer and Haugland (1967)] allows the measurement of

\footnotetext{
${ }^{1}$ For a historical review, see [Clegg (2006)].
} 


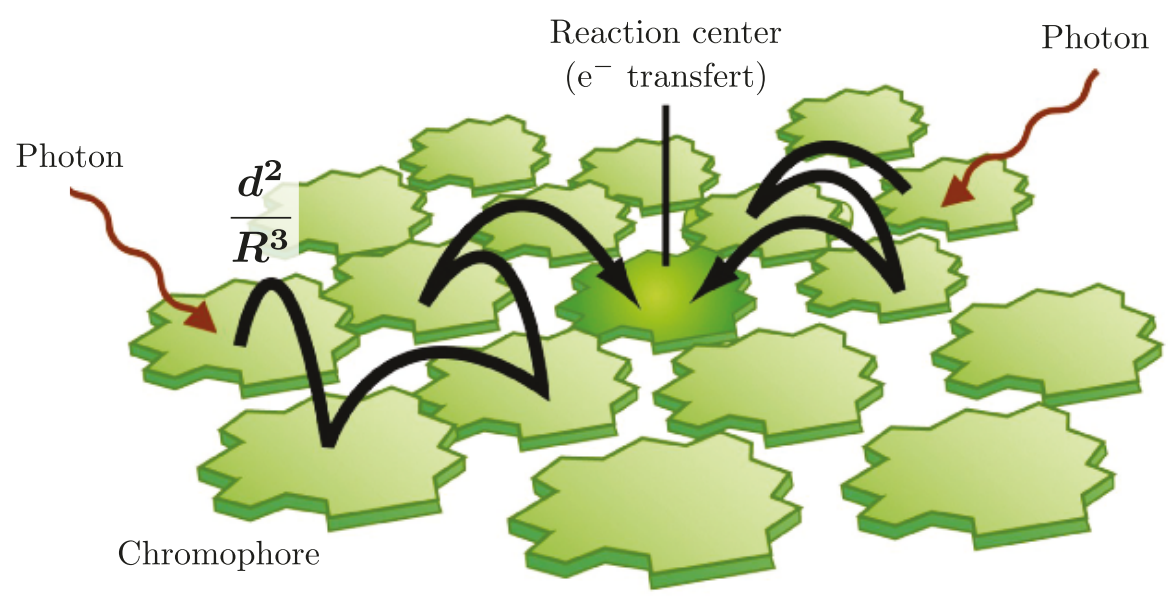

Figure 8.1: Role of resonant energy transfers in photosynthesis (adapted from [Freeman (2005)]). One chromophore receives a light excitation. The excitation is then resonantly transferred from chromophore to chromophore to reach the reaction center, where the chemical reactions of photosynthesis takes place.

nanoscale distances or binding energies in macromolecules like DNA. Still, the deep understanding of energy transport in photosynthetic systems remains an active field of research [Engel et al. (2007); Panitchayangkoon et al. (2010); Collini et al. (2010)], also motivated by the technological challenge of manufacturing efficient artificial light harvesting complexes like solar cells [Hardin et al. (2009); Huang et al. (2013)]. In particular, the origin of the coherence observed is still an open question [Briggs and Eisfeld (2011); Miller (2012)], showing that the potential role of quantum coherence [Olaya-Castro et al. (2008); Wilde, McCracken, and Mizel (2010)] or entanglement [Sarovar et al. (2010); Fassioli and Olaya-Castro (2010); Whaley, Sarovar, and Ishizaki (2011)] in natural light harvesting complexes is still not well understood.

In the early days of quantum mechanics, F. Perrin [Perrin (1933)] gave a first theoretical explanation for the resonant energy transfer between two single particles with the same resonance frequency. In this model, each particle is viewed as an oscillating dipole, so that the two-particle system is the quantum analog of two coupled classical pendula. An oscillating particle creates a field with a $1 / R^{3}$ contribution in the near field and a $1 / R$ radiation part. When a second particle is located in the near field ( $1 / R^{3}$ contribution), an excitation can be transferred from the first particle to the other particle through the exchange of virtual photons ("non-radiative energy transfer"). According to F. Perrin's theoretical predictions, the transfer should occur at distances as large as $R_{0} \sim 100 \mathrm{~nm}$. However, this prediction did not in agree with observations made by experimentalists [Cario and Franck (1922); Beutler and Josephy (1929)], who measured energy transfers between two chemical species in mixtures 
a

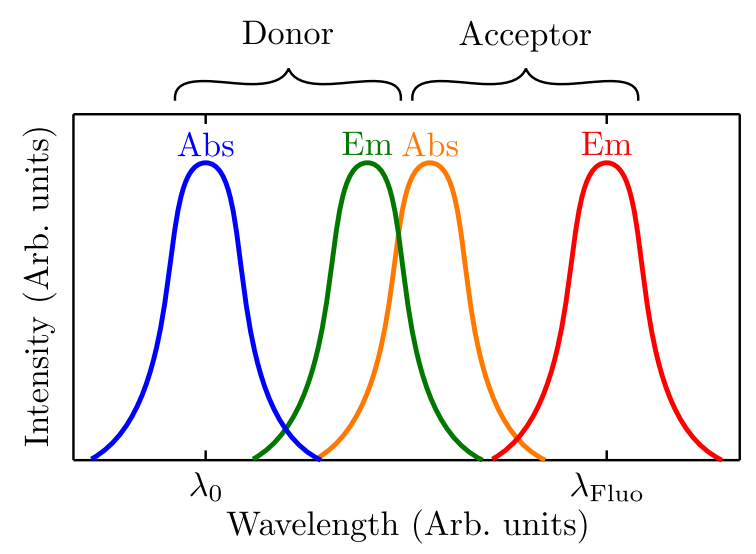

b

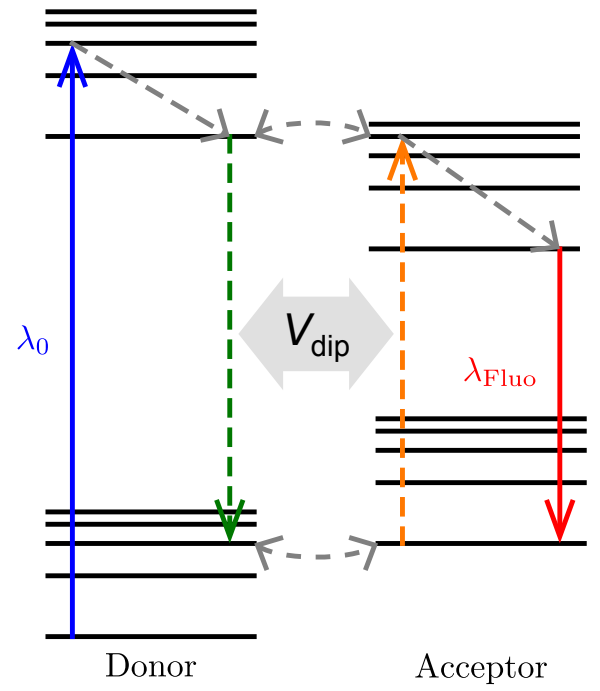

Figure 8.2: Förster model of resonant energy transfers. a, In most systems found in nature, the two particles are not exactly resonant. However the spectral broadening of each particle can result in an overlap between the emission (Em) spectrum of the first one (donor) and the absorption (Abs) spectrum of the second one (acceptor). b, A resonant energy transfer is therefore a consequence of the interaction between a state and a continuum of energy. One can obtain the transfer rate by applying a Fermi golden rule.

of vapors over much shorter distances $\left(R_{0} \sim 10 \mathrm{~nm}\right)$. Förster later refined the model to a more general situation [Förster (1948)] that did not require an exact match of the energy levels of the two particles, but only an overlap between broad emission and absorption spectra (see Figure 8.2a). In this case, the resonant energy transfer results from the contribution of a continuum of states (see Figure 8.2b). Applying Fermi's golden rule allowed Förster to obtain the rate of energy transfer:

$$
k_{E T}=\frac{1}{\tau_{e}}\left(\frac{R_{0}}{R}\right)^{6},
$$

where $\tau_{e}$ is the lifetime of the donor in its excited state and $R_{0}$ is the distance where the rate of energy transfer is equal to the rate of fluorescence emission. This formula gave excellent agreement with the experimental observations [Stryer and Haugland (1967)].

Even if the final energy transfer rate is $\propto 1 / R^{6}$, the underlying mechanism at play here is indeed the one described by Perrin, arising from dipole-dipole interaction between the two particles. In this Chapter, we use two Rydberg atoms to implement the Perrin model in its most pristine form. After describing the theoretical background of the resonant interactions between two Rydberg atoms, we study the resonant energy transfer between two Rydberg atoms, and we use the coherence properties of 
the system to measure the interaction energy between two atoms separated by up to 50 microns. We then extend the system to a linear chain of three atoms and measure the resonant dynamics of the system. We finally show that simulations with no adjustable parameters agree well with our measurements.

\subsection{Resonant interaction between two Rydberg atoms}

We show in this Section how our system can be used to implement Perrin's model of the resonant dipole-dipole interaction.

\subsubsection{Finding a resonance}

One way to obtain resonant interactions between two Rydberg atoms is to prepare them in two distinct states $|n L\rangle$ and $\left|n^{\prime} L^{\prime}\right\rangle$ that show a non-zero transition dipole moment $\left(\left\langle n L|\hat{\boldsymbol{d}}| n^{\prime} L^{\prime}\right\rangle \neq 0\right)$. The degenerate two-atom states $\left|n L ; n^{\prime} L^{\prime}\right\rangle$ and $\left|n^{\prime} L^{\prime} ; n L\right\rangle$ are then coupled by the dipole-dipole interaction Hamiltonian ${ }^{2}$. As a consequence, the two atoms interact to first order through the channel (see Figure 8.3)

$$
n L+n^{\prime} L^{\prime} \longleftrightarrow n^{\prime} L^{\prime}+n L
$$

After preparation in the non-stationary state $\left|n L, n^{\prime} L^{\prime}\right\rangle$ for example, the system oscillates back and forth between its two degenerate configurations at a frequency given by the interaction energy. This can be seen as the hopping of one $n^{\prime} L^{\prime}$ excitation from one site to the other as represented in Figure 8.3.

Transitions between Rydberg states typically lie in the microwave domain, with wavelengths $\lambda \sim 1 \mathrm{~cm}$ much longer than the typical separation distance $R$ between the atoms in our experiment $(R \sim 10 \mu \mathrm{m})$. This ensures that the atoms are located in the near field of the transition $n L \rightarrow n^{\prime} L^{\prime}$. This places us exactly in the situation described by Perrin, where energy is transferred non-radiatively between the atoms (through the exchange of virtual photons).

\footnotetext{
${ }^{2}$ Another way to obtain resonant interactions between two Rydberg atoms is to apply an electric field so as to reach a Förster resonance, as we will demonstrate in Chapter 9.
} 
Atom 1

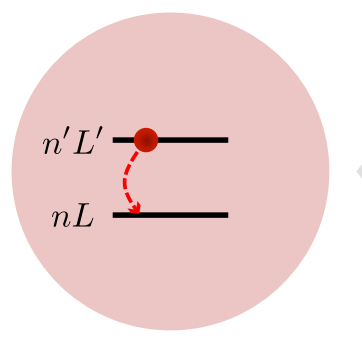

Atom 2

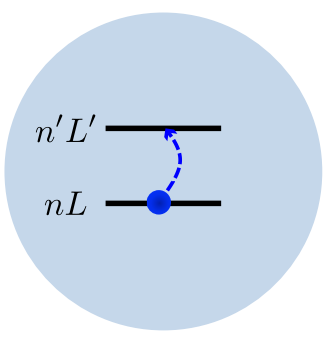

Figure 8.3: Principle of the experiment. When we excite the system to the pair state $\left|n L, n^{\prime} L^{\prime}\right\rangle$, it is exactly on resonance with $\left|n^{\prime} L^{\prime}, n L\right\rangle$ independently of external fields. The system starts oscillating under the influence of the dipole-dipole coupling $V_{\text {dip }}$.

\subsubsection{Resonant interaction between two Rydberg atoms aligned along the quantization axis}

We suppose in this Section that two Rydberg atoms are excited to the states $|n L\rangle$ and $\left|n^{\prime} L^{\prime}\right\rangle$ and that the degenerate two-atom states $\left|n L, n^{\prime} L^{\prime}\right\rangle$ and $\left|n^{\prime} L^{\prime}, n L\right\rangle$ are coupled by the dipole-dipole Hamiltonian ${ }^{3}$ with a dipolar coupling given by

$$
\left\langle n L, n^{\prime} L^{\prime}\left|\hat{V}_{\text {dip }}\right| n^{\prime} L^{\prime}, n L\right\rangle=\frac{C_{3}}{R^{3}} .
$$

The total Hamiltonian of the system is, in the basis $\left\{\left|n L, n^{\prime} L^{\prime}\right\rangle ;\left|n^{\prime} L^{\prime}, n L\right\rangle\right\}$,

$$
\hat{H}=\left(\begin{array}{cc}
0 & C_{3} / R^{3} \\
C_{3} / R^{3} & 0
\end{array}\right) .
$$

The diagonalization of this Hamiltonian gives the eigenenergies $E_{ \pm}$of the system :

$$
E_{ \pm}= \pm \frac{\left|C_{3}\right|}{R^{3}}
$$

The interaction mixes the states, as shown in Figure 8.4, and the eigenstates of the interacting system are :

$$
\left\{\begin{aligned}
|+\rangle & =\frac{1}{\sqrt{2}}\left(\left|n L, n^{\prime} L^{\prime}\right\rangle+\left|n^{\prime} L^{\prime}, n L\right\rangle\right) \\
|-\rangle & =\frac{1}{\sqrt{2}}\left(\left|n L, n^{\prime} L^{\prime}\right\rangle-\left|n^{\prime} L^{\prime}, n L\right\rangle\right) .
\end{aligned}\right.
$$

\footnotetext{
${ }^{3}$ Note that the two pair states $|n L, n L\rangle$ and $\left|n^{\prime} L^{\prime}, n^{\prime} L^{\prime}\right\rangle$ are also coupled by the dipole-dipole interaction Hamiltonian. However, those pair states are separated in energy by a few tens of $\mathrm{GHz}$, and thus the effect of the dipolar coupling is negligible. We therefore restrict the two-atom basis to $\left\{\left|n L, n^{\prime} L^{\prime}\right\rangle ;\left|n^{\prime} L^{\prime}, n L\right\rangle\right\}$.
} 

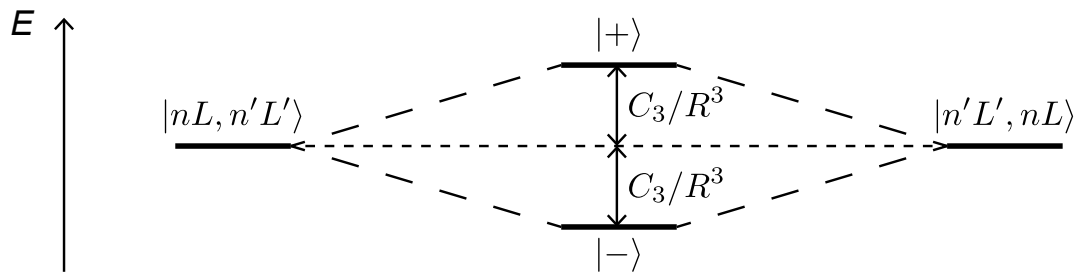

Figure 8.4: Eigenvectors of the two-atom interacting system. The pair states $\left|n L, n^{\prime} L^{\prime}\right\rangle$ and $\left|n^{\prime} L^{\prime}, n L\right\rangle$ are degenerate. Because of the dipole-dipole coupling, those pair states are not eigenstates of the two-atom interacting system. The eigenstates of the interacting system are the superposition states $|+\rangle$ and $|-\rangle$, shifted in energy by the quantity $C_{3} / R^{3}$.

We first observe that the eigenvalues are shifted in energy by a quantity $\propto 1 / R^{3}$. We recover the first-order interaction characteristic to the case of resonant dipoledipole interactions. The two degenerate states $\left|n L, n^{\prime} L^{\prime}\right\rangle$ and $\left|n^{\prime} L^{\prime}, n L\right\rangle$ are not eigenstates of the interacting system. The stationary states are the symmetric and antisymmetric combinations $|+\rangle$ and $|-\rangle$. If we prepare the pair of atoms in the state $\left|n L, n^{\prime} L^{\prime}\right\rangle$ at an instant $t=0$, the evolution of the system as a function of time is non-stationary. The system oscillates between the two configurations $\left|n L, n^{\prime} L^{\prime}\right\rangle$ and $\left|n^{\prime} L^{\prime}, n L\right\rangle$ at a frequency given by the difference in energy between the two eigenstates, namely $2 C_{3} / R^{3}$. After a time $t$, the probability to find the system in the state $\left|n L, n^{\prime} L\right\rangle$ is

$$
P_{\left|n L, n^{\prime} L^{\prime}\right\rangle}(t)=\cos ^{2}\left(2 \pi \frac{C_{3}}{R^{3}} t\right)=\frac{1}{2}\left[1+\cos \left(2 \pi \frac{2 C_{3}}{R^{3}} t\right)\right]
$$

\subsubsection{Resonant interaction for the simulation of spin systems}

Interestingly, the hopping of one excitation from site to site is a mechanism taking part in the dynamics of interacting, localized spin systems. In the presence of resonant interactions, our system naturally implements a spin-exchange Hamiltonian. In the basis $\left\{\left|n L, n^{\prime} L^{\prime}\right\rangle ;\left|n^{\prime} L^{\prime}, n L\right\rangle\right\}$, the resonant dipole-dipole interaction Hamiltonian can be written as:

$$
\hat{H}_{\mathrm{int}}=\frac{C_{3}}{R^{3}}\left(\left|n^{\prime} L^{\prime}, n L\right\rangle\left\langle n L, n^{\prime} L^{\prime}|+| n L, n^{\prime} L^{\prime}\right\rangle\left\langle n^{\prime} L^{\prime}, n L\right|\right) .
$$

If we encode two spin states in $|\uparrow\rangle=|n L\rangle$ and $|\downarrow\rangle=\left|n^{\prime} L^{\prime}\right\rangle$, the dipole-dipole interaction between the atoms is given by the Hamiltonian

$$
H=\frac{1}{2} \sum_{i, j} V_{i j}\left(\sigma_{i}^{+} \sigma_{j}^{-}+\sigma_{i}^{-} \sigma_{j}^{+}\right)
$$


where $\sigma^{ \pm}=\sigma^{x} \pm i \sigma^{y}$ are spin-flip operators and $V_{i j} \sim\left|\boldsymbol{r}_{i}-\boldsymbol{r}_{j}\right|^{-3}$. We thus realize a spin-exchange, or XY spin-Hamiltonian [Bettelli et al. (2013); Günter et al. (2013)]. Note that this situation is different from the one discussed in the case of van der Waals interactions (see Chapter 7), for which we have shown that our system can be described by an Ising-type Hamiltonian [Weimer et al. (2008); Lesanovsky (2011)]:

$$
H=\frac{1}{2} \sum_{i, j} V_{i j} \sigma_{i}^{z} \sigma_{j}^{z},
$$

where $\sigma^{z}$ is the $z$-Pauli matrix and $V_{i j} \sim\left|\boldsymbol{r}_{i}-\boldsymbol{r}_{j}\right|^{-6}$. Working with resonant interactions offers the possibility to implement another type of spin Hamiltonian, with long-range interactions $\propto 1 / R^{3}$. In the rest of this Chapter, our experiments can be seen as the implementation of XY Hamiltonians in elementary spin systems of 2 and 3 spins. We will show in particular that the long-range character of the interaction in our system plays a crucial role in the interacting dynamics of the atoms, which are not only influenced by the interactions between nearest neighbors. An accurate description of the system requires to take into account the interactions between next-nearest neighbors.

\subsection{Choice of the Rydberg states}

We want to excite two neighboring atoms to two different Rydberg states in order to have them interact resonantly. We realized this situation experimentally by combining single-site addressability (see Chapter 6) and microwave coupling between Rydberg states, as we will demonstrate in Section 8.3. In this Section, we detail our choice of Rydberg states.

\subsubsection{Microwave transition}

Using an optical excitation, we can excite one atom from the ground state to a Rydberg state (see Chapter 6). This state can then be coupled to other Rydberg states, that satisfy $\Delta L= \pm 1$ and $\Delta M_{J}=0, \pm 1$. Starting from an optically excited $D$ state, we can access to $P$ or $F$ states. $P$ states present the advantage of having a finestructure splitting of the order of a few hundreds of $\mathrm{MHz}$ for the typical principal quantum numbers used in our experiments. This value is large compared to our typical excitation linewidths, which ensures that the $n P_{1 / 2}$ and $n P_{3 / 2}$ states are separated 
$\mathbf{a}$

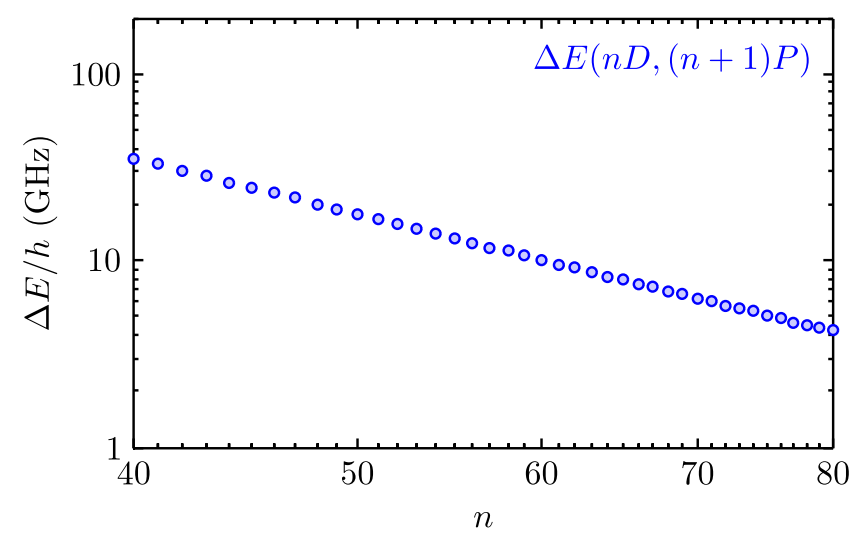

b

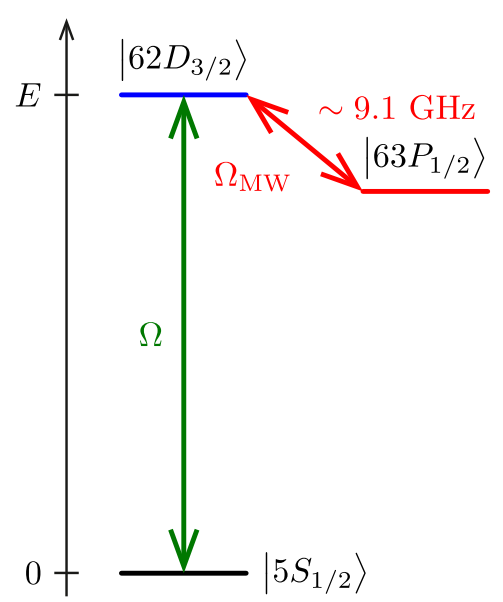

Figure 8.5: Choice of the Rydberg states. a, Energy separation (in $\mathrm{GHz}$ ) between the states $n D_{3 / 2}$ and $(n+1) P_{1 / 2}$ as a function of $n$. We note that the scaling law $1 / n^{3}$ is well verified. b, Excitation level scheme. The optical excitation couples the ground state $\left|5 S_{1 / 2}\right\rangle$ and the Rydberg state $\left|62 D_{3 / 2}\right\rangle$ with an effective Rabi frequency $\Omega$. A microwave field of Rabi frequency $\Omega_{\mathrm{MW}}$ couples the $\left|62 D_{3 / 2}\right\rangle$ and the $\left|63 P_{1 / 2}\right\rangle$ states.

enough from each other ${ }^{4}$. We can work either with $n P_{3 / 2}$ or with $n P_{1 / 2}$. We decided to use $n P_{1 / 2}$ states because they only have two Zeeman sub-levels, which eases the isolation of a two-level system. Transitions between Rydberg states typically lie in the microwave domain and can be addressed experimentally using a microwave (MW) synthesizer.

We calculate the position of the Rydberg states using the quantum defects (see Chapter 5). The closest state from $n D_{3 / 2}$ is the level $(n+1) P_{1 / 2}$. Figure 8.5 a shows the difference in energy $\Delta E / h$ between those two states, expressed as a function of $n$. For principal quantum numbers smaller than 56, the transition frequency is larger than $12.75 \mathrm{GHz}$, the highest frequency that can be produced by our MW synthesizer. For $n \simeq 60$, the transition frequency $\simeq 10 \mathrm{GHz}$ corresponds perfectly to what we can do experimentally. We chose to work with the states $\left|62 D_{3 / 2}\right\rangle$ and $\left|63 P_{1 / 2}\right\rangle$. Figure $8.5 \mathrm{~b}$ shows the chosen energy levels. In the absence of magnetic field, the calculated separation in energy between $\left|62 D_{3 / 2}\right\rangle$ and $\left|63 P_{1 / 2}\right\rangle$ is $9.1235 \mathrm{GHz}$. 


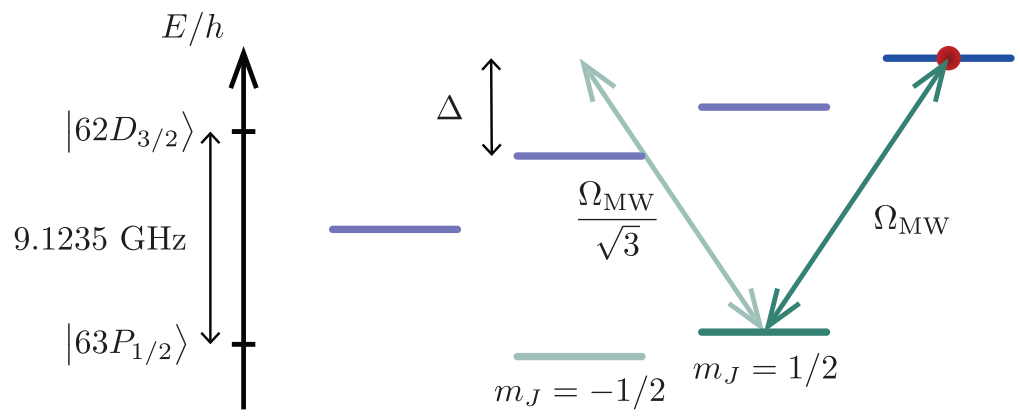

Figure 8.6: Isolating a two-level system. The full sub-Zeeman structure of the chosen states contains 6 levels. Applying a $6.6 \mathrm{G}$ magnetic field, we shift the different Zeeman sub-levels. Because of the differential Zeeman effect, only two levels are resonant with the microwave field.

\subsubsection{Two-level system}

Both $62 D_{3 / 2}$ and $63 P_{1 / 2}$ levels present a Zeeman sub-structure, and care has to be taken in order to select only two levels out of the six levels in Figure 8.6. We do not control well the polarization of the microwave field: its polarization at the position of the atoms is mainly a combination of $\sigma_{+}, \sigma_{-}$, and to a lesser extent $\pi$ polarizations, as we will see in Section 8.3. As a result, several transitions are addressed by the microwave field. In the absence of magnetic field to lift the degeneracy, the states $\left|62 D_{3 / 2}, m_{J}=3 / 2\right\rangle$ and $\left|63 P_{1 / 2}, m_{J}=1 / 2\right\rangle$ as well as the states $\left|63 P_{1 / 2}, m_{J}=1 / 2\right\rangle$ and $\left|62 D_{3 / 2}, m_{J}=-1 / 2\right\rangle$ are coupled by the microwave. This would place us outside the framework of the model of F. Perrin since at least three levels have to be considered.

We therefore apply a magnetic field to shift some of the levels out of resonance from the microwave field. Because of a differential Zeeman effect ${ }^{5}$, the transition frequency between the states $\left|62 D_{3 / 2}, m_{J}=-1 / 2\right\rangle$ and $\left|63 P_{1 / 2}, m_{J}=1 / 2\right\rangle$ shifts by a quantity $\Delta$ from the transition frequency between the states $\left|62 D_{3 / 2}, m_{J}=3 / 2\right\rangle$ and $\left|63 P_{1 / 2}, m_{J}=1 / 2\right\rangle$. In order to be resonant with only one transition, the linewidth of the microwave excitation has to be smaller than the energy separation between the two states $\left(\Omega_{\mathrm{MW}} /(2 \pi) \ll \Delta\right)$. Similarly to our optical transitions, our microwave transitions have a typical strength of $\Omega_{\mathrm{MW}}=2 \pi \times 5 \mathrm{MHz}$. With a $6.6 \mathrm{G}$ magnetic field, we obtain $\Delta \simeq 14.8 \mathrm{MHz}>\Omega_{\mathrm{MW}} /(2 \pi)$. Using the Rabi formula, we obtain an upper bound on the probability to populate the state $\left|62 D_{3 / 2}, m_{J}=-1 / 2\right\rangle$ due to of

\footnotetext{
${ }^{4} F$-states have fine-structure splittings of a few $\mathrm{MHz}$ or less, which is of the same order as our typical excitation linewidths. This makes the situation more complex, since in this case we would off-resonantly couple to several levels.

${ }^{5}$ The Landé factor for the $|d\rangle$ (resp. $\left.|p\rangle\right)$ state is $g_{J}=4 / 5$ (resp. $g_{J}=2 / 3$ ).
} 
off-resonant coupling after a microwave $\pi$-pulse:

$$
P \leq \frac{\left(\Omega_{\mathrm{MW}} / \sqrt{3}\right)^{2}}{\left(\Omega_{\mathrm{MW}} / \sqrt{3}\right)^{2}+\Delta^{2}}=3.7 \%,
$$

where $\Omega / \sqrt{3}$ is the Rabi frequency corresponding to the transition between the states $\left|62 D_{3 / 2}, m_{J}=3 / 2\right\rangle$ and $\left|63 P_{1 / 2}, m_{J}=1 / 2\right\rangle$ (see Figure 8.6). We can ignore the effect of the other Zeeman sub-levels. In the rest of this Chapter, we are interested in the transition between the stretched states $|d\rangle \equiv\left|62 D_{3 / 2}, m_{J}=3 / 2\right\rangle$ and $|p\rangle \equiv\left|63 P_{1 / 2}, m_{J}=1 / 2\right\rangle$. Taking into account the $6.6 \mathrm{G}$ magnetic field, we calculate that the transition frequency between $|d\rangle$ and $|p\rangle$ is $9.1315 \mathrm{GHz}$. This will be verified experimentally in Section 8.3.

\subsubsection{Estimating the needed microwave power}

We now concentrate on the transition between the states $|d\rangle$ and $|p\rangle$ that are separated by $9.1315 \mathrm{GHz}$. The dipole coupling between $|d\rangle$ and $|p\rangle$ is given by the dipole matrix element (calculated numerically)

$$
\left\langle 62 D_{3 / 2}, m_{j}=3 / 2\left|\hat{d}_{+}\right| 63 P_{1 / 2}, m_{j}=1 / 2\right\rangle \simeq 2858 e a_{0} .
$$

This dipole element is large, meaning that the amount of MW power needed to transfer one atom from $|d\rangle$ to $|p\rangle$ is low. We quantify this by calculating an order of magnitude of the power needed to obtain a microwave Rabi frequency $\Omega_{\mathrm{MW}}=$ $2 \pi \times 5 \mathrm{MHz}$. We make the approximation that the radiation pattern emitted from the antenna is isotropic and that the atoms are located at a distance $z \sim 20 \mathrm{~cm}$ away from the antenna. We suppose that only half of the emitted power is in the polarization $\sigma+$, and that we have $3 \mathrm{~dB}$ losses in the one meter RG58 cable. In the far field, the amplitude of the microwave field is approximately

$$
E_{\mathrm{MW}} \simeq \frac{1}{2} \sqrt{\frac{P_{\mathrm{MW}}}{2 \pi z^{2} \mathrm{c} \varepsilon_{0}}},
$$

and the Rabi frequency is given by

$$
\hbar \Omega_{\mathrm{MW}}=-\left\langle d\left|\hat{d}_{+}\right| p\right\rangle E_{\mathrm{MW}}
$$

In order to obtain the typical Rabi frequency $\Omega_{\mathrm{MW}}=2 \pi \times 5 \mathrm{MHz}$, we estimate that we need $\sim 40 \mu \mathrm{W}$ of MW power $(-14 \mathrm{dBm})$. This number will be confirmed experimentally in Section 8.3.4. 


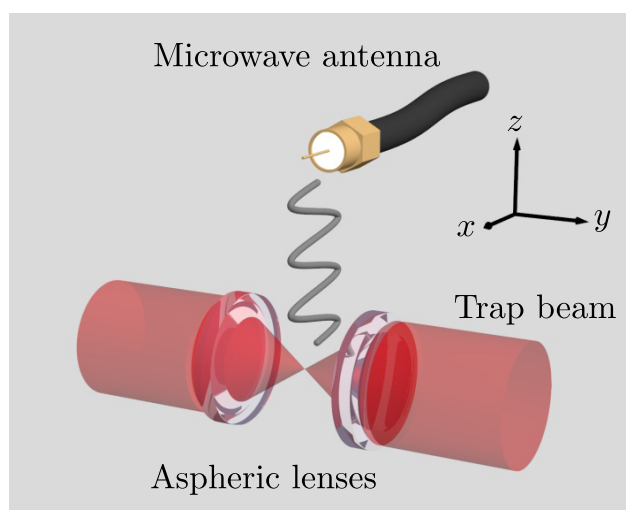

Figure 8.7: Experimental setup. A RG58 cable facing the atoms outside the vacuum chamber is connected to a MW synthesizer. We apply pulses of microwave field on the atoms to couple $|d\rangle$ to $|p\rangle$.

\subsection{Coherent manipulation of Rydberg states using microwave fields}

We have demonstrated in Chapter 6 that we are able to coherently excite a single atom from $|g\rangle$ to a Rydberg state $|d\rangle$ by sending resonant optical photons on the atoms. We now demonstrate our ability to perform transitions between Rydberg states, lying in the microwave domain.

\subsubsection{Setup}

We perform transitions between Rydberg states using a microwave field produced by a MW synthesizer SMB 100A from Rohde\&Schwartz that we connect to a RG58 cable. One end of the cable has been stripped over a $\sim 5 \mathrm{~mm}$ range, producing an electric dipole antenna that we place horizontally $\sim 20 \mathrm{~cm}$ away from the atoms (see Figure 8.7). The antenna is placed outside the vacuum chamber, above a big viewport. After free-space propagation, we would expect the polarization of the microwave field to be an equal combination of $\sigma^{+}$and $\sigma^{-}$polarizations at the position of the atoms. Due to the presence of the metallic parts of the vacuum system, a standing wave pattern probably forms inside the chamber and the polarization at the position of the atoms is more complicated. 
a

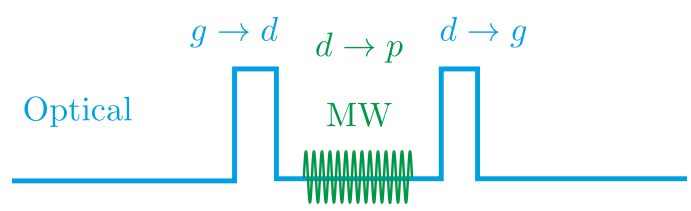

b

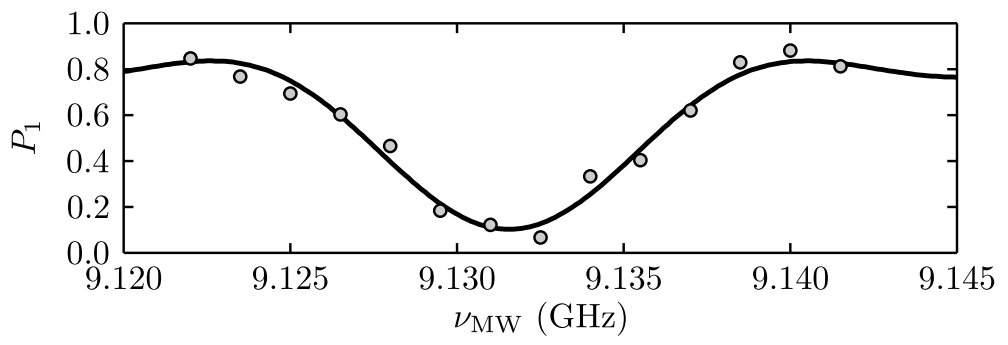

Figure 8.8: Microwave spectroscopy of $|p\rangle$. a, We optically excite the atom from $|g\rangle$ to $|d\rangle$. After optical excitation we turn on the microwave for a duration $\tau_{\mathrm{MW}}$. The microwave field couples $|d\rangle$ to $|p\rangle$. We read out the state of the system by applying a de-excitation pulse that couples back $|d\rangle$ to $|g\rangle$ and leaves $|p\rangle$ unchanged. b. Evolution of $P_{1}$ (circles) as a function of $\nu_{\mathrm{MW}}$. The solid line is a fit by the formula used in Chapter 6 to fit our single-atom optical excitation spectra (see Equation 6.8).

\subsubsection{Experimental sequence}

We first perform a spectroscopic measurement on a single atom to find the transition frequency to the $|p\rangle$ state. Figure 8.8a shows the experimental sequence. Our excitation lasers couple $|g\rangle$ to $|d\rangle$, with an excitation pulse of duration $t_{\pi}$ to prepare the system in $|d\rangle$. We then send a pulse of microwave field (of fixed duration $\tau_{\mathrm{MW}}$ ) that couples $|d\rangle$ to $|p\rangle$. Our detection scheme described in Chapter 6 is based on the loss of Rydberg atoms, and does not allow to discriminate between an atom in $|p\rangle$ and an atom in $|d\rangle$. In order to read out the state of the system at the end of the sequence, we apply a de-excitation laser $\pi$-pulse identical to the ones used during the preparation for Rydberg excitation to $|d\rangle$. The read-out pulse couples $|d\rangle$ down to $|g\rangle$ while leaving $|p\rangle$ unchanged. We then reconstruct the probability $P_{1}$ to detect fluorescence at the end of the sequence. If we make the assumption that our excitation and detection are perfect, the probability $P_{1}$ thus coincides with the probability $P_{d}$ for the atom to be in the $|d\rangle$ state.

\subsubsection{Microwave spectroscopy}

Figure $8.8 \mathrm{~b}$ shows the evolution of $P_{1}$ as a function the absolute MW frequency $\nu_{\mathrm{MW}}$. We observe the excitation spectrum of $|p\rangle$ : when $\nu_{\mathrm{MW}}$ is far from resonance, the atoms are not affected by the microwave field, and there is a high probability of recapturing the atoms at the end of the sequence. When the microwave field is resonant with the 


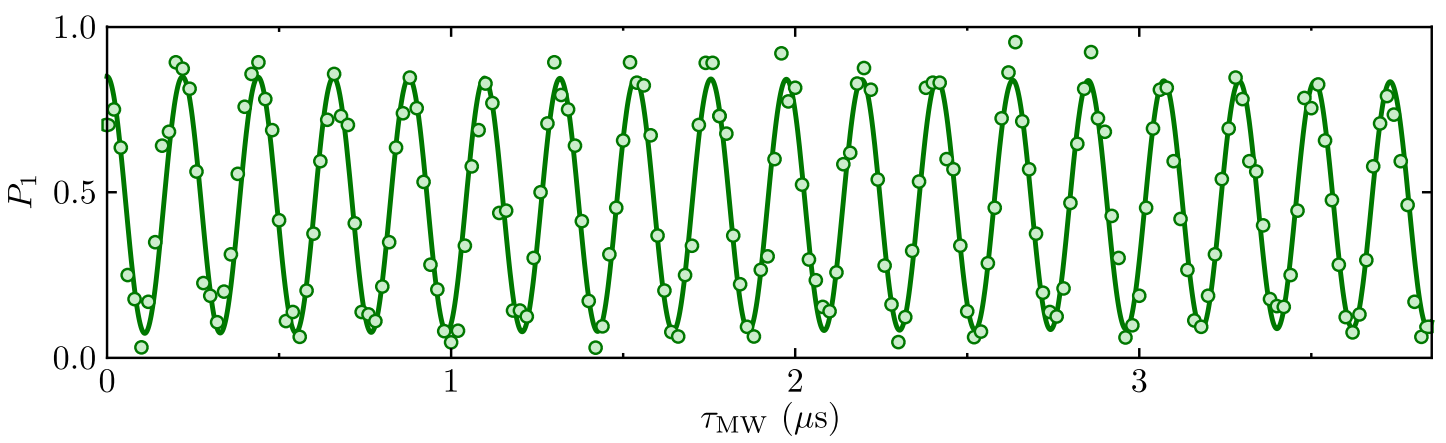

Figure 8.9: Single atom Rabi oscillation between $|d\rangle$ and $|p\rangle$. The data points (circles) show the evolution of $P_{1}$ as a function of $\tau_{\mathrm{MW}}$. The solid line is a fit by a sine.

transition $|d\rangle \leftrightarrow|p\rangle$, population moves to $|p\rangle$, leading to atom losses at the end of the sequence. When $P_{1}$ is minimum, all the population has been transferred to $|p\rangle$. To extract the position of the resonance we fit the spectrum by the Rabi formula given in Equation 6.8 (see Chapter 6). We obtain a line centered on

$$
\nu_{\mathrm{MW}}=9.1316 \pm 0.0002 \mathrm{GHz}
$$

in agreement with our theoretical expectations. The fact that the Rabi formula reproduces well our data is an indication that, for this duration of excitation $\left(\tau_{\mathrm{MW}}=\right.$ $100 \mathrm{~ns}$ ), the linewidth of the spectrum is essentially set by the Rabi frequency, and that other sources of broadening (if any) are negligible. From the fit, we obtain a Rabi frequency of:

$$
\Omega_{\mathrm{MW}} /(2 \pi)=4.5 \pm 0.4 \mathrm{MHz}
$$

where the error bar is the one-sigma confidence interval from the fit.

\subsubsection{Excitation dynamics of a single atom}

We then measure the excitation dynamics of a single atom. The sequence is identical to the one in Figure 8.8a. The frequency $\nu_{\mathrm{MW}}$ is fixed at the position of the resonance, and we vary the duration $\tau_{\mathrm{MW}}$ of the microwave pulse. We measure the probability $P_{i}(i \in\{0 ; 1\})$ to recapture or lose the atom at the end of the sequence. We observe in Figure 8.9 the Rabi oscillation of the system, with $P_{1}$ oscillating as a function of the pulse duration $\tau_{\mathrm{MW}}$. For a duration of the excitation $\tau=\tau_{\pi, \mathrm{MW}}$, the transfer of population to the Rydberg state $|p\rangle$ is maximum. We measure up to 17 Rabi cycles with no appreciable damping, showing the coherent character of our excitation scheme from $|d\rangle$ to $|p\rangle$. The solid line in Figure 8.9 shows a fit of the data by a 
damped sine. We make the two following observations:

- For $\simeq 31.6 \mu \mathrm{W}$ of MW power, we measure a Rabi frequency of:

$$
\Omega_{\mathrm{MW}} /(2 \pi)=4.560 \pm 0.001 \mathrm{MHz}
$$

which is in agreement with our estimate from Equation 8.15. This value is in agreement with the one obtained by fitting the spectrum in Figure 8.8b, which confirms that our spectra are power-broadened, and that other sources of broadening (e.g. fluctuating electric fields) have a negligible impact for our range of Rabi frequencies.

- The data also shows almost no damping, contrary to what we observed in the case of the optical excitation. Moreover, the measured contrast of the oscillation is $\simeq 80 \%$, which approximately corresponds to the efficiency of our optical excitation to the $|d\rangle$ state. Indeed, transitions in the Rydberg manifold are done using a one-photon excitation process between two long-lived states, which frees ourselves from spontaneous emission during the process. Our microwave source is also stable to better than a $\mathrm{Hz}$, leading to less dephasing than for the laser excitation. As a consequence, in the rest of this Chapter, we will consider the microwave excitation ideal.

These results therefore show our ability to coherently control the state of a single Rydberg atom using a microwave field. We use this new feature of our setup to study resonant dipole-dipole interaction in systems of two and three atoms.

\subsection{Coherent resonant energy transfer between two Rydberg atoms}

We demonstrate experimentally the excitation of a pair of atoms to the two-atom state $|d p\rangle$ in this Section. This state is exactly resonant with $|p d\rangle$, and the two atoms interact resonantly. For the transition studied here, we have $\Delta M_{\text {tot }}=0$. The dipole-dipole Hamiltonian reads

$$
\hat{V}_{\text {dip }}=\frac{1}{4 \pi \epsilon_{0}} \frac{1}{R^{3}}\left(\hat{d}_{A,+} \hat{d}_{B,-}+\hat{d}_{A,-} \hat{d}_{B,+}+2 \hat{d}_{A, 0} \hat{d}_{B, 0}\right)
$$

and the two atoms show a non-zero interaction at $\theta=0$. We concentrate on the case where the atoms are aligned along the quantization axis. The calculated interaction 


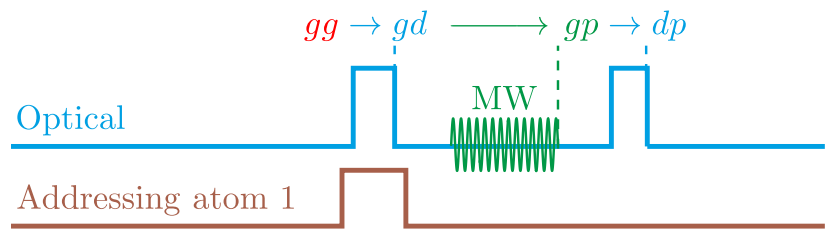

Figure 8.10: Experimental sequence for the preparation of the pair state $|d p\rangle$. By combining single site addressing demonstrated in Section 6.6 and microwave excitation form $|d\rangle$ to $|p\rangle$, we are able to prepare the state $|d p\rangle$ under the condition that $\hbar \Omega_{\mathrm{L}} \gg C_{3} / R^{3}$.

energy is

$$
C_{3}=\frac{\left|\left\langle d\left|\hat{d}_{+}\right| p\right\rangle\right|^{2}}{4 \pi \varepsilon_{0}} \simeq 7965 \mathrm{MHz} . \mu \mathrm{m}^{3} .
$$

We measure experimentally interaction energies between two atoms that are separated by distances as large as $50 \mu \mathrm{m}$, which illustrates the long-range character of the resonant dipole-dipole interaction.

\subsubsection{Preparation of the system}

We excite the system to the two-atom state $|d p\rangle$, in order to observe resonant interactions between two atoms.

\section{Excitation of $|d p\rangle$}

Experimentally, we prepare the system by combining the microwave excitation from $|d\rangle$ to $|p\rangle$ with single site addressing demonstrated in Chapter 6. Figure 8.10 shows our excitation scheme. The two-atom system is initially in the state $|g g\rangle$. After turning off the trap, we shine the addressing beam on the first atom while optically exciting the second atom ( $\pi$-pulse). The presence of the addressing beam prevents the excitation of the first atom, allowing us to reach the state $|g d\rangle$. A microwave $\pi$-pulse then transfers the system to the state $|g p\rangle$. We finally send a second optical excitation pulse that couples $|g p\rangle$ to $|d p\rangle$. One can immediately notice that some care has to be taken during this last optical pulse since $|d p\rangle$ and $|p d\rangle$ are not eigenstates of the interacting system, as discussed in Section 8.1.2. Due to the resonant interaction between the atoms, the eigenstates are the superposition states $|+\rangle$ and $|-\rangle$ that are detuned from the excitation laser by a quantity $C_{3} / R^{3}$. We intuitively understand that the dipole-dipole interaction between the atoms influences the preparation of the system. 
a

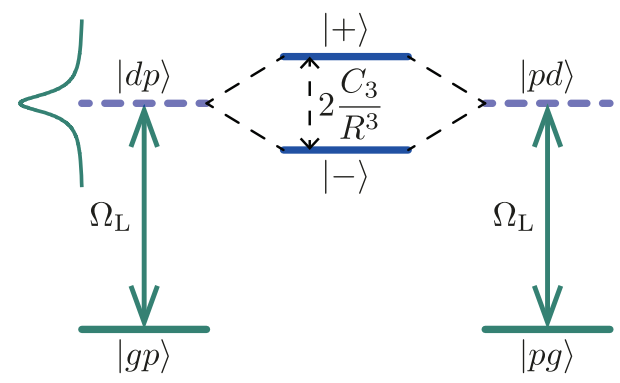

b

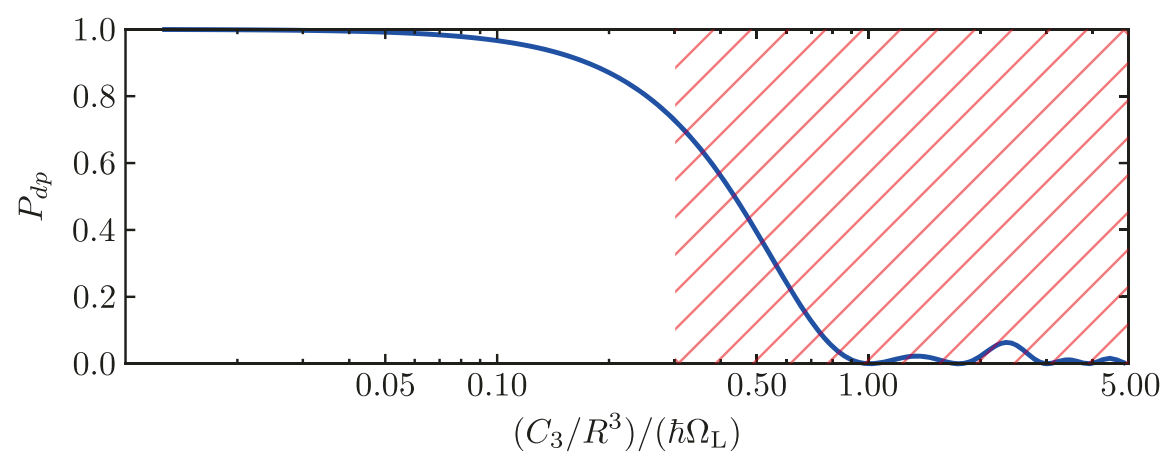

Figure 8.11: Influence of the dipole-dipole interaction on the preparation of the state $|\boldsymbol{d} \boldsymbol{p}\rangle$. a, Schematic of the energy levels that enter in the preparation and how the dipole-dipole interaction affects them (see text for details). b, Excitation probability $P_{d p}$ at the end of the preparation sequence as a function of $\left(C_{3} / R_{3}\right) /\left(\hbar \Omega_{\mathrm{L}}\right)$, showing that for $C_{3} / R^{3} \ll \hbar \Omega_{\mathrm{L}}$, the preparation of the state $|d p\rangle$ is efficient (note that the horizontal axis is logarithmic). In the experiments that follow, we have $\left(C_{3} / R_{3}\right) /\left(\hbar \Omega_{\mathrm{L}}\right) \leq 0.3$.

\section{Influence of the interaction during the preparation}

Figure 8.11a shows a diagram of the energy levels and couplings during the last preparation step. We assume that all the population has been placed in the state $|g p\rangle$ thanks to the first two steps of the preparation. The excitation lasers then couple $|g p\rangle$ to $|d p\rangle$ (and $|p g\rangle$ to $|p d\rangle$ ) with a Rabi frequency $\Omega_{\mathrm{L}}$. As we are trying to excite the system, the states $|d p\rangle$ and $|p d\rangle$ are also coupled by the dipole-dipole interaction, of strength $C_{3} / R^{3}$. As a consequence, the interaction affects the preparation of the state $|d p\rangle$. Three different regimes can be identified:

- When $C_{3} / R^{3} \gg \hbar \Omega_{\mathrm{L}}$, the linewidth of the excitation laser is smaller than the frequency shifts of the states $|+\rangle$ and $|-\rangle$ that are due to the resonant dipoledipole interaction. As a consequence, the excitation lasers can not couple to $|+\rangle$ or $|-\rangle$, and the preparation of the system is blockaded.

- When $C_{3} / R^{3} \ll \hbar \Omega_{\mathrm{L}}$, the laser linewidth is bigger than the separation between the states $|+\rangle$ and $|-\rangle$ allowing to couple efficiently to the $|d p\rangle$ component of the states $|+\rangle$ and $|-\rangle$. The interaction dynamics can be considered frozen 
during the optical excitation, which allows us to prepare the state $|d p\rangle$ with a good fidelity.

- When $C_{3} / R^{3} \simeq \hbar \Omega_{\mathrm{L}}$, the duration of the optical excitation $\left(t_{\pi}=\pi / \Omega_{\mathrm{L}}\right)$ is comparable to the interaction time needed by the system to evolve from $|d p\rangle$ to $|p d\rangle$ under the influence of the resonant dipole-dipole interaction. As a result, we excite a superposition of the states $|d p\rangle$ and $|p d\rangle$.

As a consequence, we will try to prepare the system in the regime where $C_{3} / R^{3} \ll \hbar \Omega_{\mathrm{L}}$.

\section{Simulation of the preparation}

We estimate the influence of the dipole-dipole interaction on the fidelity of our preparation scheme, by solving the Schrödinger equation for the four-level system in Figure 8.11a. In the basis $\{|g p\rangle ;|p g\rangle ;|d p\rangle ;|p d\rangle\}$, the total Hamiltonian reads

$$
\hat{H}=\hbar\left(\begin{array}{cccc}
0 & 0 & \Omega_{\mathrm{L}} / 2 & 0 \\
0 & 0 & 0 & \Omega_{\mathrm{L}} / 2 \\
\Omega_{\mathrm{L}} / 2 & 0 & 0 & C_{3} / R^{3} \\
0 & \Omega_{\mathrm{L}} / 2 & C_{3} / R^{3} & 0
\end{array}\right) .
$$

We assume that the first two steps of the preparation (optical pulse and microwave pulse) are perfect since their limitations have nothing to do with the dipole-dipole interaction, meaning that initially $P_{g p}=1$. We use an optical Rabi frequency $\Omega_{\mathrm{L}}=$ $2 \pi \times 5 \mathrm{MHz}$ that corresponds to our typical experimental parameters. Figure $8.11 \mathrm{~b}$ shows the probability $P_{d p}$ of having excited $|d p\rangle$ at the end of the preparation sequence as a function of $\left(C_{3} / R^{3}\right) /\left(\hbar \Omega_{\mathrm{L}}\right)$. When $\left(C_{3} / R^{3}\right) /\left(\hbar \Omega_{\mathrm{L}}\right) \leq 0.1$, we prepare $|d p\rangle$ with an efficiency $>95 \%$. For $\left(C_{3} / R^{3}\right) /\left(\hbar \Omega_{\mathrm{L}}\right) \geq 1$ we prepare a superposition of the four states $\{|g p\rangle,|p g\rangle,|d p\rangle,|p d\rangle\}$, leading to a more complicated situation.

\subsubsection{Interaction Dynamics of a two-atom system at resonance}

Once in the state $|d p\rangle$, the two-atom system evolves freely under the influence of the dipole-dipole interaction. Because the system is at resonance, we expect it to evolve back and forth between the states $|d p\rangle$ and $|p d\rangle$ at the frequency $f_{\text {osc }}=2 C_{3} /\left(h R^{3}\right)$ (see Equation 8.8). 


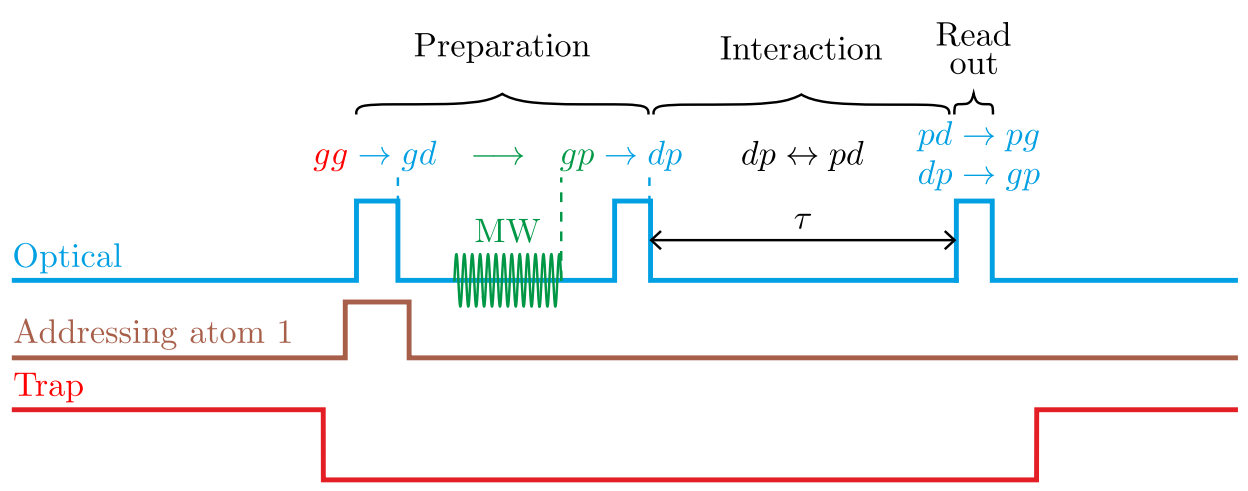

Figure 8.12: Experimental sequence for measuring the interaction dynamics. After preparing the system as described in Section 8.4.1, we let the system interact for a duration $\tau$. We finally read out the state of the system.

\section{Full experimental sequence}

We use the experimental sequence shown in Figure 8.12, in order to observe these dynamics experimentally. After preparing the system as described in the previous paragraph, we let the system evolve for a duration $\tau$. At the end this interaction time, we read out the state of the system. We apply a de-excitation laser $\pi$-pulse identical to the ones used during the preparation for Rydberg excitation to $|d\rangle$. The read-out pulse couples $|d p\rangle$ to $|g p\rangle(|p d\rangle$ to $|p g\rangle)$. Note that identically to what happens during the preparation, the system still interacts during the read-out pulse. However, as long as we are in the situation $\hbar \Omega_{\mathrm{L}} \gg C_{3} / R^{3}$, we can assume that the dipole-dipole interaction dynamics are frozen during the read out pulse (see Figure 8.11). We repeat the sequence $\simeq 100$ times to reconstruct the probabilities $P_{i j}(i, j \in\{0 ; 1\})$ after the read out. Assuming perfect Rydberg excitation, the probabilities $P_{10}$ and $P_{01}$ measured at the end of the sequence would coincide with the populations in $P_{d p}$ and $P_{p d}$.

\section{Experimental results}

Figure 8.13a shows the measured evolution of $P_{10}$ and $P_{01}$ as a function of $\tau$ for different distances $R$ ranging from $17.5 \mu \mathrm{m}$ up to $50.1 \mu \mathrm{m}$. We observe the oscillations of $P_{10}$ and $P_{01}$, a consequence of the excitation hopping between the pair states $|p d\rangle$ and $|d p\rangle$. The oscillation frequency depends on the distance $R$ between the atoms, which confirms that we are witnessing the interaction-induced energy transfer between two particles. The presence of only two-atoms at a controlled distance is fundamental to the observation of the coherence of the energy transfer. In other systems, only 




Figure 8.13: Coherent excitation transfer between two atoms. a, Experimental data for $P_{10}$ (blue) and $P_{10}$ (red) for two Rydberg atoms separated by a distance $R$ as a function of interaction time $\tau$. The solid lines are fits to sines. $\mathbf{b}$, Simulation of the interaction dynamics solving the Schrödinger equation, where the first two steps of the preparation are supposed to be perfect (see Section 8.4.3). 


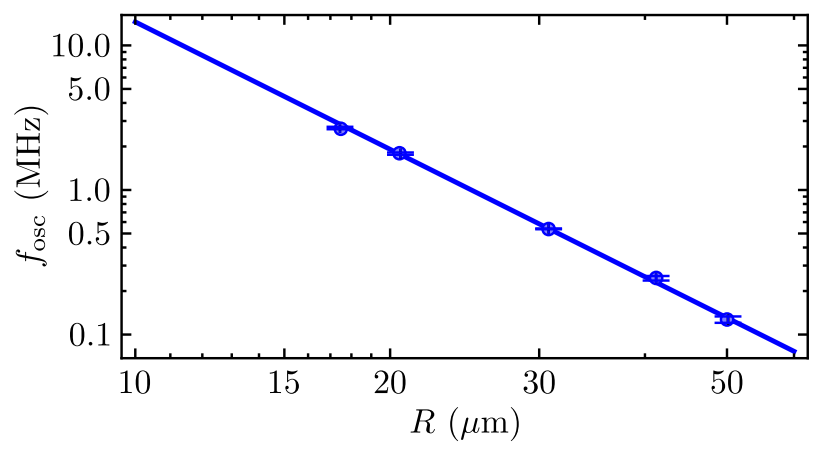

Figure 8.14: Experimental determination of $C_{3}$. Double logarithmic plot of the fitted oscillation frequency $f_{\text {osc }}$ as a function of the distance $R$. To obtain the oscillation frequency, we fit $P_{10}$ and $P_{10}$ by damped sines of the form $P(\tau)=A+B \mathrm{e}^{-\gamma \tau} \cos \left(2 \pi f_{\text {osc }}\left(\tau-\tau_{0}\right)\right)$. The error bars represent the $1 \sigma$ confidence interval on the fit. The solid line is a fit by a power law, which gives a fitted exponent of $-2.93 \pm 0.16$.

indirect effects of this coherence could be observed [Nipper et al. (2012a,b)], due to the distribution of interatomic distances that scrambles the interaction dynamics ${ }^{6}$.

For a distance as large as $R=50.1 \mu \mathrm{m}$, we observe that the $p$-excitation has hopped from one site to the other after $\simeq 3.95 \pm 0.20 \mu \mathrm{s}$. This corresponds to an oscillation at the frequency $f_{\text {osc }}=126 \pm 6 \mathrm{kHz}$, in excellent agreement with the expected oscillation at the frequency $2 C_{3} / R^{3}=126.4 \mathrm{kHz}$. The possibility to measure a $\simeq 100 \mathrm{kHz}$ interatomic interaction for distances as large as $\simeq 50 \mu \mathrm{m}$ illustrates well the strength of the resonant dipole-dipole interaction. It contrasts with the case of the van der Waals interaction studied in Chapter 7 for two atoms in the state $82 D_{3 / 2}$. Despite the fact that an atom in the state $\left|82 D_{3 / 2}\right\rangle$ has an electric dipole nearly twice as large as an atom in the state $62 D_{3 / 2}$, a $50 \mu \mathrm{m}$ separation distance in Chapter 7 would have led to an interaction energy smaller than $1 \mathrm{kHz}$ that we cannot resolve experimentally. In the present situation, the interaction is two orders of magnitude larger. In principle, one could observe coherent energy transfer for even larger distances. Currently, our limitations come from the finite size of our excitation laser beams which prevents efficient laser excitation for larger interatomic distances, and temperature effects that lead to large atom losses for long interrogation times $(\tau \geq 8 \mu \mathrm{s})$, as we will discuss in Section 8.6. 


\section{Measurement of the $C_{3}$ coefficient}

When we decrease $R$, we observe that the oscillation frequency increases. Figure 8.14 shows a double logarithmic plot of the fitted oscillation frequency as a function of the distance $R$. The data features a power-law behavior of exponent $-2.93 \pm 0.16$, in excellent agreement with the expected $R^{-3}$ behavior. Fixing the exponent to -3 , we determine $C_{3}$ experimentally:

$$
C_{3}=7.95 \pm 0.11 \mathrm{GHz} \cdot \mu \mathrm{m}^{3},
$$

where the error represents one standard deviation confidence interval in the fit. Note that we also have a systematic $5 \%$ uncertainty on the determination of $R$. This value is also in very good agreement with the expected value $C_{3, \mathrm{th}}=7.97 \mathrm{GHz} \cdot \mu \mathrm{m}^{3}$.

\subsubsection{Simulation of the dynamics}

Experimentally, we measure oscillations with a contrast of about $60 \%$. If the preparation and detection were perfect, we would expect a $100 \%$ contrast. To get more insight into what actually limits us, we simulate the interaction dynamics of the system. For this simulation, we assume that the first optical and microwave transfers to Rydberg states during the preparation are perfect. This allows us to restrict ourselves to the four-level system $\{|g p\rangle,|p g\rangle,|d p\rangle,|p d\rangle\}$ in Figure 8.11, with $P_{g p}=1$ for initial condition. The system is described by the Hamiltonian of Equation 8.21, where we include in the simulation the laser excitation pulse from $|g p\rangle$ to $|d p\rangle$ and the read-out pulse that are separated by a varying interaction time $\tau$.

Figure $8.13 \mathrm{~b}$ shows the result of simulations obtained by solving numerically the Schrödinger equation for the four levels:

- For large separation distances between the atoms $(R \geq 30 \mu \mathrm{m})$, we observe that the simulation shows an almost $100 \%$ transfer from $|d p\rangle$ to $|p d\rangle$. This is expected since we have considered the case of ideal optical and microwave excitations (see Equation 8.8). For large distances, the observed reduction of the contrast in the measured oscillations is thus mainly due to technical limitations during the preparation and the read out, as we will detail in Section 8.4.4.

\footnotetext{
${ }^{6}$ In studies performed with disordered systems, the presence of an ensemble of atoms with disordered positions washes out the back and forth transfer of the excitation, leading to the diffusion of the excitation [Anderson, Veale, and Gallagher (1998); Mourachko et al. (1998); Günter et al. (2013)]. Those studies, in connection with Förster resonances will be discussed in Chapter 9.
} 
- For smaller separation distances between the atoms $(T \leq 30 \mu \mathrm{m})$, we observe in Figure 8.13b that the contrast of the simulated oscillations is lower than $100 \%$. We also observe the presence of a phase shift and some beating in the simulated oscillations as we decrease the interatomic distance. Since we have considered our optical and microwave excitations perfect, these effects can only be related to the presence of the dipole-dipole interaction during the preparation and read out. Indeed, for the smallest distances used here we are close to the regime $\hbar \Omega_{\mathrm{L}} \sim C_{3} / R^{3}$ where the dipole-dipole interaction affects the preparation and read out as explained in Section 8.4.1. We will detail those limitations in Section 8.4.5.

As a consequence, we observe that several effects contribute to the reduction of the contrast. In what follows, we first discuss the technical limitations that affect the contrast independently of the interatomic distance. We then discuss the effects that are specific to small interaction distances and that are due to the dipole-dipole interaction.

\subsubsection{Origin of the reduced contrast}

We measure that, for large distances, the contrast of the measured oscillations is of the order of $\simeq 60 \%$, which is smaller than the expected $100 \%$ contrast. This shows that the system has been placed in states that do not take part in the interaction dynamics, with $\mathrm{a} \simeq 40 \%$ probability. Those imperfections in the preparation of $|d p\rangle$ are mainly due to dissipation, that reduces the optical excitation of an atom from $|g\rangle$ to $|d\rangle$ as discussed in Chapter 6.

\section{Influence of dissipation}

Our optical Rydberg excitation from $|g\rangle$ to $|d\rangle$ is limited by dissipation due to spontaneous emission, and by some dephasing due to fluctuations in our laser system (see Chapter 6). On the other hand, we have shown in Section 8.3.4 that the microwave excitation could be considered ideal, due to the fact that we only couple long-lived states and also due to the stability of our microwave source. An independent measurement of the microwave single-atom Rabi oscillation from $|d\rangle$ to $|p\rangle$ shows a $\simeq 80 \%$ contrast, which indicates that we have optically excited the $|d\rangle$ state with a $\simeq 0.8$ efficiency. As a consequence, our preparation of the state $|d p\rangle$ is limited. At the end of the preparation, we estimate the population in $|d p\rangle$ by calculating the product of the different transfer efficiencies: 


$$
P_{d p}=P_{g g \rightarrow g d} P_{g d \rightarrow g p} P_{g p \rightarrow d g} \simeq 0.8 \times 1.0 \times 0.8=0.64 .
$$

This number shows good agreement with our experimental observations.

\section{Offset between the two curves}

Experimentally, we also observe that the curve $P_{10}$ shows an offset with respect to the curve $P_{01}$. As mentioned earlier, our read out procedure does not allow to measure $P_{d p}$ and $P_{p d}$ directly. During the read out pulse, different transitions between pair states can contribute to $P_{10}$ and $P_{01}$. The measured probabilities are the sum of the transitions probabilities:

$$
\begin{aligned}
& P_{10}=P_{d p \rightarrow g p}+P_{g p \rightarrow g p}+P_{g d \rightarrow g d}+P_{d g \rightarrow g d}+P_{g g \rightarrow g d}+P_{d d \rightarrow g d}, \\
& P_{01}=P_{p d \rightarrow p g}+P_{p g \rightarrow p g}+P_{d g \rightarrow d g}+P_{g d \rightarrow d g}+P_{g g \rightarrow d g}+P_{d d \rightarrow d g} .
\end{aligned}
$$

The first term (in red) in each sum of Equation $8.24\left(P_{d p \rightarrow g p}\right.$ and $\left.P_{p d \rightarrow p g}\right)$ is the oscillating term that corresponds to the interaction dynamics we want to measure. All the other terms are small contributions to $P_{01}$ and $P_{10}$, that are due to imperfections in our optical excitation. The contributions in black are of the same order of magnitude, of the order of a few percent. However, due to the way we prepare the system, the terms in blue $\left(P_{g p \rightarrow g p}\right.$ and $\left.P_{p g \rightarrow p g}\right)$ are not of the same order. Indeed, since we specifically excite the second atom to $|p\rangle$ using our addressing beam, some population can remain in $|g p\rangle$, due to spontaneous emission during the last optical excitation pulse of the preparation. However, exciting the system in $|p g\rangle$ is extremely unlikely. As a consequence, $P_{g p \rightarrow g p}>P_{p g \rightarrow p g}$ and the $P_{10}$ is shifted with respect to $P_{01}$.

\subsubsection{Influence of the interaction during the preparation for smaller distances}

We observe experimentally, for our smallest values of $R(R=20.6 \mu \mathrm{m}$ and $R=$ $17.5 \mu \mathrm{m})$, that the contrast of the oscillations is further reduced and that the oscillations start with a non-zero phase. These features can also be observed on the simulated oscillations and are due to the dipole-dipole interaction itself as discussed earlier (see Figure 8.13b). In order to deconvolve those effects from the more technical limitations discussed in Section 8.4.4, we consider here that the optical and microwave excitations are perfect, and that we can place $100 \%$ of the population in $|g p\rangle$ after the microwave excitation. 


\section{Phase shift of the oscillations}

For $R \leq 30 \mu \mathrm{m}$, we enter in the regime $\Omega_{\mathrm{L}} \sim C_{3} / R^{3}$ where the interaction dynamics between the atoms cannot be considered frozen during the preparation of the system (see Figure 8.11). Some transfer of population from $|d p\rangle$ to $|p d\rangle$ occurs during the preparation itself, which translates to a phase shift in our measurement since the dynamics have already started at $\tau=0 \mu$ s.

\section{Further reduced contrast}

Another effect related to the interaction during the preparation and readout affects the dynamics of the system. During those steps, the excitation light is indeed detuned from the states $|+\rangle$ and $|-\rangle$ by a quantity $C_{3} / R^{3}$ as already mentioned earlier in Section 8.4.1. Our excitation scheme leads to the excitation of a superposition of the states $\{|d p\rangle ;|g p\rangle ;|p d\rangle ;|p g\rangle\}$. The fact that we do not place all the population in $|d p\rangle$ leads to the reduction of the contrast of the oscillations. Since we keep the Rabi frequency constant in those experiments, the effect is more noticeable for small interatomic distances, where the interaction is larger.

\section{Beating in the oscillations}

Another consequence of the off-resonant excitation to $|d p\rangle$ can be seen in Figure 8.13b. For $R=20.6 \mu \mathrm{m}$ and $R=17.5 \mu \mathrm{m}$, we observe some beating in the interaction dynamics of the two-atom system, which reveals the presence of an extra frequency component in the oscillations. This is an interference effect occurring between the last preparation pulse and the read out pulse due to the fact that the excitation laser is detuned with respect to the transition between the states $|d p\rangle$ and $|+\rangle$ (and similarly $|d p\rangle$ and $|-\rangle)$ by a frequency $C_{3} /\left(h R^{3}\right)$. As a result, during the interaction time $\tau$, the two states accumulate a relative phase $\Delta \phi$ (in the frame rotating at the frequency of the laser):

$$
\Delta \phi=\frac{C_{3}}{R^{3}} \frac{\tau}{\hbar}
$$

As a result, upon reading out the state of the system, an interference effect similar to the one observed in a Ramsey sequence occurs ${ }^{7}$. This leads to a modulation of the measured populations at a frequency $C_{3} /\left(h R^{3}\right)$, on top of the expected oscillation at frequency $C_{3} /\left(2 h R^{3}\right)$.

\footnotetext{
${ }^{7}$ The difference with a Ramsey sequence is that our preparation and read-out pulses are $\pi$-pulses and not $\frac{\pi}{2}$-pulses. As a consequence, the amplitude of the modulation is not maximal.
} 
Note that this interference effect is a "one-atom" effect that differs fundamentally from the interaction dynamics we are interested in. We illustrate this point by showing that the same effect arises when exciting a single atom from $|g\rangle$ to $|d\rangle$ with the excitation laser detuned from the transition by a frequency $C_{3} / R^{3}$. At resonance, one can theoretically excite the atom to $|d\rangle$ with a $100 \%$ probability by shining an excitation pulse of duration $t_{\pi}$ on the atom. Here, we shine an excitation pulse of duration $t_{\pi}$ on the atoms, but the laser excitation is detuned from the transition, and we excite a superposition:

$$
\Psi_{1 \mathrm{at}}\left(t_{\pi}\right)=\alpha|g\rangle+\beta|r\rangle
$$

where $\alpha$ and $\beta$ are coefficients given by the Rabi formula, that depend on the Rabi frequency and the detuning. We then wait for a variable duration $\tau$, during which the atom and the laser accumulate a phase $\Delta \phi$ :

$$
\Psi_{1 \mathrm{at}}\left(t_{\pi}+\tau\right)=\alpha|g\rangle+\beta \mathrm{e}^{i \Delta \phi}|r\rangle
$$

The state becomes, after shining the second laser pulse:

$$
\begin{aligned}
\Psi_{1 \mathrm{at}}\left(2 t_{\pi}+\tau\right) & =\alpha(\alpha|g\rangle+\beta|r\rangle)+\beta \mathrm{e}^{i \Delta \phi}(\alpha|r\rangle-\beta|g\rangle) \\
& =\left(\alpha^{2}-\beta^{2} \mathrm{e}^{i \Delta \phi}\right)|g\rangle+\alpha \beta\left(1+\mathrm{e}^{i \Delta \phi}\right)|r\rangle .
\end{aligned}
$$

The final probability $P_{g}$ to find the atom in $|g\rangle$ depends on $\Delta \phi$, and on $\tau$ :

$$
P_{g}=\left|\alpha^{2}-\beta^{2} \mathrm{e}^{i \Delta \phi}\right|^{2}=\alpha^{4}+\beta^{4}-2 \alpha^{2} \beta^{2} \cos (\Delta \phi)
$$

Finally, in our situation of two interacting atoms, this interference occurs between the last excitation pulse and the read out pulse. As a consequence, a small modulation at the frequency $C_{3} /\left(h R^{3}\right)$ adds up on top of the interaction dynamics, which results in some beating. Fortunately, in the regime $\Omega_{\mathrm{L}} \gg C_{3} / R^{3}$, we theoretically excite $|d p\rangle$ with a fidelity $>95 \%$ (see Section 8.4.1) the amplitude of this extra modulation remains small compared to the amplitude of the interaction dynamics we are interested in.

\section{Working with smaller distances}

We have seen here that working with distances smaller than $20 \mu \mathrm{m}$ exposes us to parasitic effects that perturb the interaction dynamics of the two-atom system. In the limit $\Omega_{\mathrm{L}} \ll C_{3} / R^{3}$, the excitation of the system is even blockaded, preventing the preparation of the system. In principle, working with smaller distances would 
be possible as long as we have enough available laser power to increase the Rabi frequency $\Omega_{\mathrm{L}}$. However, more laser power leads to more spontaneous emission during the preparation, and a decreased contrast. For the states $\left|62 D_{3 / 2}\right\rangle$ and $\left|61 P_{1 / 2}\right\rangle$ studied in this Chapter, we can hardly work with smaller interatomic distances. In order to extend this kind of study to large arrays of atoms (which necessarily means smaller interatomic distances), a better option would be to chose states that show a smaller dipole-dipole coupling (a smaller $C_{3}$ coefficient).

\subsubsection{Creation of entanglement}

Let us call $|\Psi(t)\rangle$ the state of the interacting two-atom system. Ideally, during the interaction time, the state of the two-atom system $|\Psi(t)\rangle$ evolves under the influence of the dipole-dipole interaction Hamiltonian, so that:

$$
\begin{aligned}
|\Psi(\tau)\rangle & =\frac{1}{\sqrt{2}}\left(\exp \left(i E_{+} \tau\right)|+\rangle+\exp \left(i E_{-} \tau\right)|-\rangle\right) \\
& =\cos \left(\frac{1}{\hbar} \frac{C_{3}}{R^{3}} \tau\right)|d p\rangle+i \sin \left(\frac{1}{\hbar} \frac{C_{3}}{R^{3}} \tau\right)|p d\rangle
\end{aligned}
$$

After a time $\tau_{\text {Ent }}=\pi \hbar R^{3} /\left(4 C_{3}\right)$, the system has evolved to the entangled state ${ }^{8}$ $\Psi\left(\tau_{\text {Ent }}\right)=(|d p\rangle+i|p d\rangle) / \sqrt{2}$. This offers a way to produce entanglement in our system. The full characterization of this entangled state requires to measure the fidelity of this entanglement procedure [Wilk et al. (2010)] by applying rotations on the state. Such a measurement was not possible to perform in the current status of the experiment.

\subsection{Coherent resonant energy transfer in a chain of Rydberg atoms}

We have observed the coherent energy transfer of an excitation between two single atoms. In this Section, we study the propagation of an excitation along a three-atom chain.

\subsubsection{Linear chain of three Rydberg atoms}

To get some insight into the propagation of an excitation in a three-atom spin chain, we first generalize the simple approach of Section 8.4 to the case of three atoms

\footnotetext{
${ }^{8}$ Note that one needs to stop the evolution after $\tau_{\text {Ent }}$. One possibility is to apply a read out pulse so as to map this state down to the state $\left(\mathrm{e}^{i \boldsymbol{k} \cdot \boldsymbol{R}_{A}}|g p\rangle+i \mathrm{e}^{i \boldsymbol{k} \cdot \boldsymbol{R}_{B}}|p g\rangle\right) / \sqrt{2}$.
} 

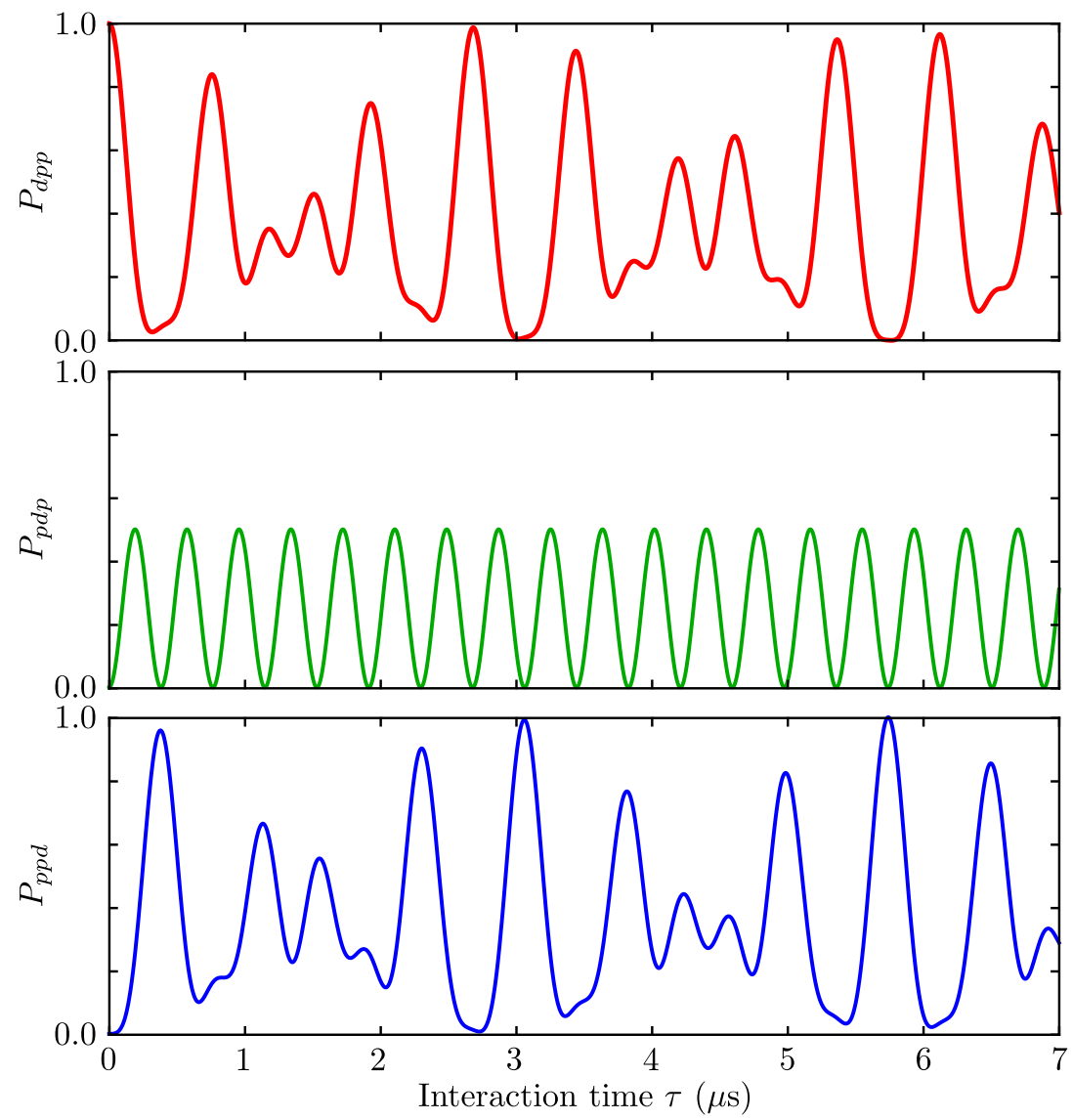

Figure 8.15: Propagation of an excitation along a chain of three Rydberg atoms. The system is initially prepared in the state $|d p p\rangle$. A calculation of the evolution of the system under the influence of $\hat{H}_{\text {int }}$ is shown. We observe the oscillations of the probabilities $P_{d p p}, P_{p d p}$ and $P_{p p d}$, as a consequence of the hopping of the $d$-excitation from site to site. The observed beating is due to the presence of several frequency components, resulting from the interplay of nearest and next-nearest neighbor interaction.

in a line. The separation distance between two neighboring atoms is chosen to be $R=20.5 \mu \mathrm{m}$. The distance between the two outermost atoms is $2 R=41 \mu \mathrm{m}$ for which we have also demonstrated the coherent oscillation in the case of two atoms in Fig 8.13a.

\section{Hamiltonian of the system}

We consider one $d$-excitation propagating in a chain of atoms in the state $|p\rangle$. Conservation of the excitation number allows to restrict the study to the three states $|d p p\rangle,|p d p\rangle$ and $|p p d\rangle$ which are degenerate in energy. Those levels are resonantly coupled by the dipole-dipole interaction, and the interaction Hamiltonian reads 


$$
\begin{aligned}
\hat{H}_{\mathrm{int}} & =h \frac{C_{3}}{R^{3}}(|d p p\rangle\langle p d p|+| p d p\rangle\langle d p p|)+h \frac{C_{3}}{R^{3}}(|p d p\rangle\langle p p d|+| p p d\rangle\langle p d p|) \\
& +h \frac{C_{3}}{(2 R)^{3}}(|d p p\rangle\langle p p d|+| p p d\rangle\langle d p p|) .
\end{aligned}
$$

The factors $C_{3} / R^{3}$ in front of the first two terms correspond to the nearest-neighbor interaction. The factor $C_{3} /(2 R)^{3}$ in front of the last term is the next-nearest-neighbor term. The interaction between the two outermost atoms is eight times smaller than the interaction between two neighboring atoms:

$$
\hat{H}_{\mathrm{int}}=h \frac{C_{3}}{R^{3}}\left(\begin{array}{ccc}
0 & 1 & 1 / 8 \\
1 & 0 & 1 \\
1 / 8 & 1 & 0
\end{array}\right)
$$

The diagonalization of $\hat{H}_{\text {int }}$ gives the eigenvalues $\lambda_{1}, \lambda_{2}$ and $\lambda_{3}$ :

$$
\left\{\begin{array}{l}
\lambda_{1}=h \frac{C_{3}}{R^{3}} \frac{1}{16}(1+3 \sqrt{57}) \\
\lambda_{2}=h \frac{C_{3}}{R^{3}} \frac{1}{16}(1-3 \sqrt{57}) \\
\lambda_{3}=-h \frac{C_{3}}{R^{3}} \frac{1}{8} .
\end{array}\right.
$$

The dynamics of the three-atom system is governed by the three different frequency components $\lambda_{1}-\lambda_{2}, \lambda_{2}-\lambda_{3}$ and $\lambda_{1}-\lambda_{3}$, which are incommensurate. As a consequence, the evolution of the populations is aperiodic and results from the beating of those frequency components. For an interatomic separation $R=20.5 \mu \mathrm{m}$ in the experiment, we expect the following frequency components:

$$
\left\{\begin{array}{l}
\nu_{1}=2.62 \mathrm{MHz} \\
\nu_{2}=1.48 \mathrm{MHz} \\
\nu_{3}=1.14 \mathrm{MHz}
\end{array}\right.
$$

\section{Simulation of the three-atom system}

Figure 8.15 shows the result of the numerical simulation of this system. We solve the Schrödinger equation for the Hamiltonian $\hat{H}_{\text {int }}$, where we suppose that all the population is initially in $|d p p\rangle$. The curves $P_{d p p}, P_{p d p}$ and $P_{p p d}$ exhibit an oscillatory behavior as the $d$-excitation is transferred from site to site. After $\simeq 0.2 \mu \mathrm{s}$, the system has evolved from the state $|d p p\rangle$ to a state very close to $|p p d\rangle$, showing the 

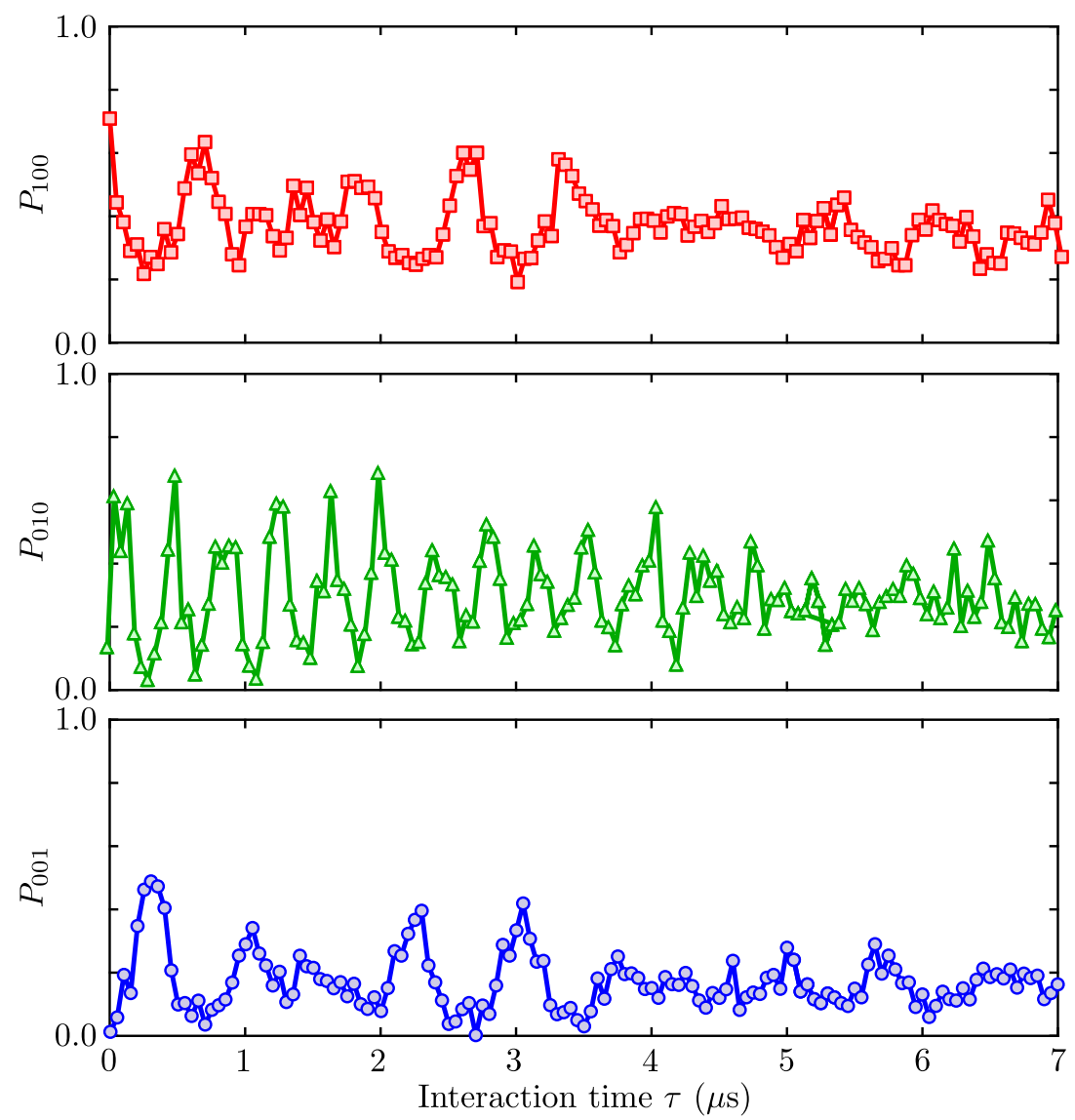

Figure 8.16: Energy transport along a chain of three Rydberg atoms. We prepare the system in the state $|d p p\rangle$. The evolution of the system after excitation is shown. We observe the oscillations of the populations $P_{100}, P_{010}$ and $P_{001}$, a consequence of the hopping of the $d$-excitation.

transfer of the $d$-excitation between the two outermost sites located $41 \mu \mathrm{m}$ away. Note that this transfer time is much faster than the one measured in the case of only two atoms separated by $41 \mu \mathrm{m}(\simeq 2 \mu$ s in Figure 8.13), as the middle atom mediates the energy transfer, with $P_{p d p}$ showing fast oscillations between 0 and $1 / 2$. We also observe that the probabilities $P_{d p p}$ and $P_{p p d}$ are aperiodic and show the presence of multiple frequency components resulting from the modulation of the energy transfer by a slowly varying envelope. This beating is an other clear illustration of the long-range character of the resonant dipole-dipole interaction, where the interplay between the couplings $C_{3} / R^{3}$ and $C_{3} /(2 R)^{3}$ makes the eigenvalues of the Hamiltonian incommensurate, leading to the observed aperiodic behavior. 


\subsubsection{Experimental implementation}

The experimental sequence used for the implementation of the three-atom spin chain is similar to the one presented in Figure 8.12 for two atoms.

\section{Experimental sequence}

To prepare the system in $|d p p\rangle$ we start by addressing the first atom in order to optically excite the state $|g d d\rangle$. The large separation distance between two neighboring atoms $(R=20.5 \mu \mathrm{m})$ ensures that the excitation to $|g d d\rangle$ is not blockaded by the van der Waals interaction between the two atoms in $|d\rangle$ (their van der Waals interaction is $\simeq 10 \mathrm{kHz}$, much smaller than the optical Rabi frequency). We then transfer the system to the state $|g p p\rangle$ using a microwave pulse and we finally optically excite the first atom to reach the state $|d p p\rangle$. After an interaction time $\tau$, we read out the state of the system by applying an optical pulse that couples $|d\rangle$ back to $|g\rangle$. We repeat the sequence $\simeq 100$ times to reconstruct the probabilities $P_{i j k}(i, j, k \in\{0 ; 1\})$. Assuming perfect excitation and read out, the probabilities $P_{100}, P_{010}$ and $P_{001}$ coincide with $P_{d p p}, P_{p d p}$ and $P_{p p d}$.

\section{Experimental results}

Figure 8.16 shows the experimental measurement of $P_{100}, P_{010}$ and $P_{001}$. We observe a qualitative agreement with the behavior observed in Figure 8.15, in particular the transfer of the $D$-excitation as a function of time, with $P_{100}$ and $P_{001}$ reaching successive maxima and minima. We also observe the beating that is the signature of the long-range interaction. A sum of the Fourier transforms of $P_{100}, P_{010}$ and $P_{001}$ gives the spectrum shown Figure 8.17. As expected, we clearly see the presence of three frequency components in the dynamics of the system. The measured frequencies agree to better than $6 \%$ with the ones expected from the diagonalization of $\hat{H}_{\text {int }}$. Discrepancies probably come from a systematic error on the calibration of the interatomic distance.

\section{Limitations}

We finally observe that the contrast of the measured oscillations is smaller than the theoretical one. This is expected since we are subject to the same limitations as discussed in the two-atom case. Dissipation during the optical excitation due to spontaneous emission limits our excitation efficiency. Moreover, the preparation is also affected by the resonant dipole-dipole interaction since $C_{3} / R^{3} \sim \hbar \Omega_{\mathrm{L}}$ (see Section 8.4.1), 


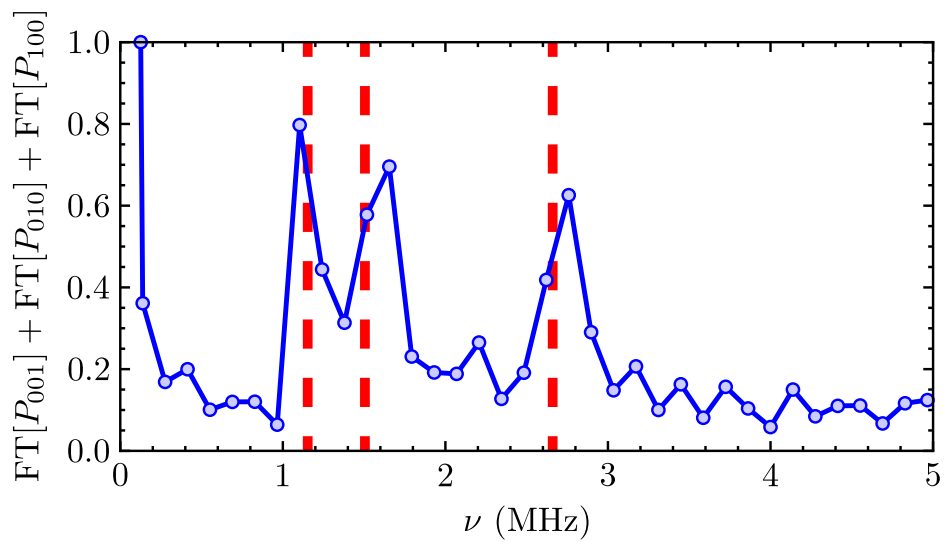

Figure 8.17: Sum of the Fourier transforms of $P_{100}, P_{010}$ and $P_{001}$. The Fourier transform shows the presence of three frequency components in the dynamics of the system. Those frequencies agree well with the expected ones, marked with red dashed lines.

which leads to some significant population in $|p d p\rangle$ as soon as the oscillation starts. We also observe the presence of some damping, which becomes significant beyond an interaction time of $\sim 4 \mu \mathrm{s}$. This effect essentially comes from the finite temperature of the atoms, which leads to changes in the distances between the atoms, and thus in the couplings from run to run. To go beyond this qualitative understanding of our limitations, we study the system in detail in the next Section, by incorporating all the known imperfections in the simulation.

\subsection{Full numerical simulation of the three-atom system}

We investigate the different contributions of the effects leading to the reduced contrast and the damping of the oscillations, by performing a simulation including all the known imperfections of the experiment. For each atom, we consider the three states $\{|g\rangle,|d\rangle,|p\rangle\}$ and we include all the steps of the experimental sequence, from the preparation to the final read out.

\subsubsection{Hamiltonian of the system}

In the presence of both the laser and the microwave fields, and in the rotating wave approximation, the one-atom Hamiltonian for atom $i$ is: 


$$
\hat{H}_{i}=\hbar\left(\begin{array}{ccc}
0 & \Omega_{\mathrm{L}}^{(i)} / 2 & \Omega_{\mathrm{MW}}^{(i)} / 2 \\
\Omega_{\mathrm{L}}^{(i)} / 2 & \delta_{\mathrm{L}}^{(i)} & 0 \\
\Omega_{\mathrm{MW}}^{(i)} / 2 & 0 & \delta_{\mathrm{L}}^{(i)}+\delta_{\mathrm{MW}}^{(i)}
\end{array}\right)
$$

where $\Omega_{\mathrm{L}}^{(i)}$ is the optical Rabi frequency and $\Omega_{\mathrm{MW}}^{(i)}$ is the microwave Rabi frequency for atom $i . \quad \delta_{\mathrm{L}}^{(i)}$ is the detuning of the laser frequency from the optical transition $|g\rangle \rightarrow|d\rangle$, and $\delta_{\mathrm{MW}}^{(i)}$ is the detuning of the microwave frequency from the transition $|d\rangle \rightarrow|p\rangle$. Slight variations of Rabi frequencies $\Omega$ and detunings $\delta$ can occur from site to site due to inhomogeneities in the excitation fields. In practice, we measure all these parameters experimentally:

- Independent measurements of $\delta_{\mathrm{MW}}^{(i)}$ and $\Omega_{\mathrm{MW}}^{(i)}$ performed on each atom taken individually gave results that are identical to better than a percent, showing that microwave field inhomogeneities can be neglected. In the rest of this Chapter, we consequently consider $\Omega_{\mathrm{MW}}^{(i)}=\Omega_{\mathrm{MW}}$ and $\delta_{\mathrm{MW}}^{(i)}=0$.

- As for the laser excitation, the finite extension of the laser Gaussian beams leads to non-negligible inhomogeneities in $\Omega_{\mathrm{L}}^{(i)}$ and $\delta_{\mathrm{L}}^{(i)}$ (which takes into account the light shifts), especially when working with large distances. We measure independently the laser detunings $\delta_{\mathrm{L}}^{(i)}$ and the single-atom Rabi frequencies $\Omega_{\mathrm{L}}^{(i)}$ by performing a spectroscopic measurement of the Rydberg line and the Rabi oscillation on each atom taken individually (see Section 6.3).

The Hamiltonian describing the coupling of the light and microwave field to the atoms reads, in the $N$-atom case:

$$
\hat{H}_{\mathrm{L}}+\hat{H}_{\mathrm{MW}}=\sum_{i} \frac{\hbar \Omega_{\mathrm{L}}^{(i)}}{2}\left(\hat{\sigma}_{d g}^{(i)}+\hat{\sigma}_{g d}^{(i)}\right)+\frac{\hbar \Omega_{\mathrm{MW}}}{2}\left(\hat{\sigma}_{p d}^{(i)}+\hat{\sigma}_{d p}^{(i)}\right)+\hbar \delta_{\mathrm{L}}^{(i)}\left(\hat{\sigma}_{d d}^{(i)}+\hat{\sigma}_{p p}^{(i)}\right)
$$

and the interaction Hamiltonian is given by:

$$
\hat{H}_{\mathrm{int}}=\frac{h}{2} \sum_{i \neq j} \frac{C_{3}}{R_{i, j}^{3}}\left(\hat{\sigma}_{p d}^{(i)} \hat{\sigma}_{d p}^{(j)}+\hat{\sigma}_{d p}^{(i)} \hat{\sigma}_{p d}^{(j)}\right)
$$

where $\hat{\sigma}_{l l^{\prime}}^{(i)}=\left|l^{(i)}\right\rangle\left\langle l^{\prime(i)}\right|$ are transition and projector operators for atom at site $i$, where $l$ and $l^{\prime}$ can take the values $g, d$ and $p$. The total Hamiltonian of the system is:

$$
\hat{H}_{\text {tot }}=\hat{H}_{\mathrm{L}}+\hat{H}_{\mathrm{MW}}+\hat{H}_{\text {int }} \text {. }
$$




\subsubsection{Imperfection of the state preparation}

Similarly to what we described in Section 8.4.1, the presence of the interaction during the last preparation step and the read out affects the measured interaction dynamics. In this experiment, we work with an optical Rabi frequency $\Omega_{L} /(2 \pi) \simeq 5 \mathrm{MHz}$ providing short optical pulses $\left(t_{\pi} \simeq 100 \mathrm{~ns}\right)$, while keeping a reasonable level of spontaneous emission. For the interatomic distance used here $(R=20.5 \mu \mathrm{m})$, we have $C_{3} / R^{3} \simeq \hbar \Omega_{\mathrm{L}} /(10 \pi)$, which places us in the regime where the interaction dynamics due to the dipole-dipole interaction has already started during the last excitation and read out pulses. This shows up in the data as a phase in the oscillations, since, at $\tau=0$, some population has already been transferred from $|d p p\rangle$ to $|p d p\rangle^{9}$. To model this effect, we include in the simulation the full experimental sequence, from the beginning of the preparation to the final read out pulse.

\subsubsection{Optical Bloch equations}

Another source of imperfections in our system is dissipation. As discussed in Chapter 6 , dissipation mainly comes from spontaneous emission through the intermediate state $\left|5 P_{1 / 2}\right\rangle$ during the optical pulses and fluctuations in the laser system. Figure 8.16 shows interaction times as long as $\tau=7 \mu \mathrm{s}$, and therefore we also take the finite lifetime of Rydberg states into account. We include those effects in the simulation as a single decay channel to the state $|g\rangle$, by solving the optical Bloch equations (see in Section 6.4.2).

\section{Dissipator}

To account for experimental damping $\gamma^{(i)}$ of the single-atom oscillations mainly due to off-resonant spontaneous emission through the intermediate state $\left|5 P_{1 / 2}\right\rangle$ (see Chapter 6), we add a sum of independent single atom dissipators:

$$
L[\rho]=\frac{1}{2} \sum_{i} \gamma^{(i)}\left(2 \hat{\sigma}_{g d}^{(i)} \rho \hat{\sigma}_{d g}^{(i)}-\hat{\sigma}_{d d}^{(i)} \rho-\rho \hat{\sigma}_{d d}^{(i)}\right),
$$

where the parameters $\gamma^{(i)}$ and similarly are obtained independently from single-atom measurements. Typical values are $1 / \gamma^{(i)} \sim 1 \mu$ s. To account for the finite lifetime of the Rydberg states, we add the sum of dissipators

\footnotetext{
${ }^{9}$ Our optical pulse is short enough to avoid any transfer to the third resonant state $|p p d\rangle$ at $\tau=0$.
} 


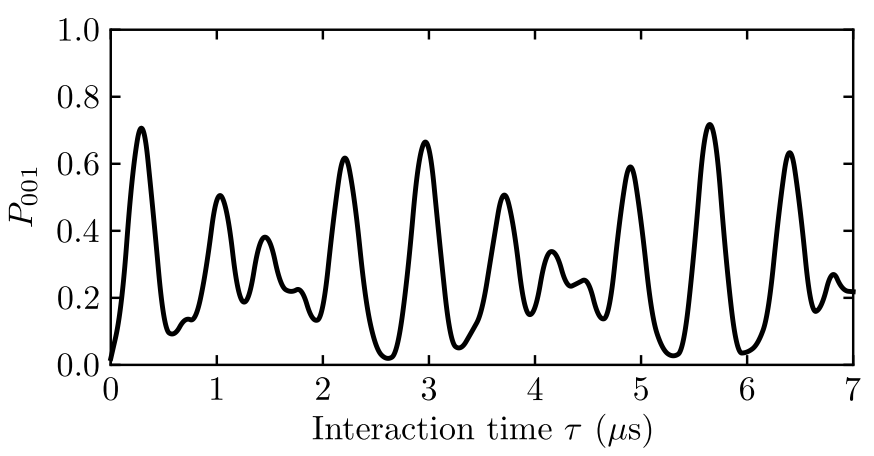

Figure 8.18: Solution of the Bloch equations for a three-atom chain. The curve shows the evolution of the population $P_{001}$ as a function of the interaction time. The limited preparation efficiency and the dissipation lead to a reduction of the amplitude of the oscillations.

$$
L[\rho]=\frac{1}{2} \sum_{i} \gamma_{d}\left(2 \hat{\sigma}_{g d}^{(i)} \rho \hat{\sigma}_{d g}^{(i)}-\hat{\sigma}_{d d}^{(i)} \rho-\rho \hat{\sigma}_{d d}^{(i)}\right)+\gamma_{p}\left(2 \hat{\sigma}_{g p}^{(i)} \rho \hat{\sigma}_{p g}^{(i)}-\hat{\sigma}_{p p}^{(i)} \rho-\rho \hat{\sigma}_{p p}^{(i)}\right) .
$$

where we use the effective values $1 / \gamma_{d} \sim 101 \mu \mathrm{s}$ and $1 / \gamma_{p} \sim 135 \mu$ s given in [Beterov et al. (2009) $]^{10}$.

\section{Results of the simulation}

Figure 8.18 shows the result of the simulation for three atoms with a $20.5 \mu \mathrm{m}$ separation between each atom (see Section 8.5). The only input of the simulation are the parameters $\Omega_{\mathrm{L}}^{(i)}, \delta_{\mathrm{L}}^{(i)}, \gamma^{(i)}$ and $\Omega_{\mathrm{MW}}$ obtained independently from single-atom measurements. The probability $P_{001}$ shows the expected oscillating behavior, with successive collapses and revivals in the dynamics. Compared to Figure 8.15 where no dissipation was included, the effect of the dissipation is to reduce the contrast of the oscillation. Indeed, dissipation during the preparation limits the efficiency of the preparation in $|d p p\rangle$, which thus reduces the contrast of the oscillations. For short interaction times, the simulation fits reasonably well the data. However, for larger interaction times, the simulation does not reproduce the damping of the oscillation observed experimentally.

\footnotetext{
${ }^{10}$ Note that those values are large compared to the maximal duration of the sequence $\leq 8 \mu \mathrm{s}$. As a consequence, for the number of atoms used here and on the timescale of this experiment, the effect of the Rydberg states lifetimes is small.
} 


\subsubsection{Finite temperature of the atoms}

The damping observed experimentally essentially arises from this dephasing due to the finite temperature of the atoms of $\simeq 50 \mu \mathrm{K}$. Indeed, every time we repeat the experiment, the atoms are randomly distributed inside the traps. The interatomic distance and the coupling between the atoms changes slightly. This effect alters the interaction strength between the different states during the experiment, and an effective damping of the observed oscillation thus arises from the averaging of oscillations with different frequencies.

\section{Fluctuating interatomic distances}

Under the assumption of thermal equilibrium, the distribution of positions of an atom of mass $m$ in an harmonic trap of radial trapping frequency $\omega$ is a Gaussian of standard deviation $\sqrt{k_{\mathrm{B}} T /\left(m \omega^{2}\right)}$. With a radial trap frequency of $\omega_{r} /(2 \pi) \simeq 86.5 \mathrm{kHz}$ and a temperature of $50 \mu \mathrm{K}$ (see Chapter 6), the spreads of the initial positions of an atom are approximately, in the radial directions, $\sigma_{z}=\sigma_{y} \simeq 120 \mathrm{~nm}$. In the simulation, we only consider the displacements along the z-axis, since they contribute the most to the variation of the interatomic distance $R$. Small displacements along the other axes only contribute to second order to the variation of $R$, as long as $R$ is large compared to the standard deviations $\sigma_{x}$ and $\sigma_{y}$. This is verified experimentally since $\sigma_{y} \simeq 120 \mathrm{~nm} \ll \mathrm{R}$ and $\sigma_{x}=\sqrt{k_{\mathrm{B}} T /\left(m \omega_{x}^{2}\right)} \sim 1 \mu \mathrm{m} \ll R$. As a consequence, the distribution of distances between two atoms is a Gaussian centered on $R$, of standard deviation

$$
\sigma_{R}=\sqrt{2} \sqrt{\frac{k_{\mathrm{B}} T}{m \omega_{r}^{2}}} .
$$

\section{Thermal velocity of the atoms}

The thermal velocity of an atom is, at a $50 \mu \mathrm{K}$ temperature:

$$
v_{\mathrm{th}}=\sqrt{k_{B} T / m} \simeq 70 \mathrm{~nm} \cdot \mu \mathrm{s}^{-1}
$$

The traps are turned off during the interaction time, and therefore the position of the atom varies during the sequence. During its free flight, the atom undergoes a displacement $v_{z} t$ along the z-axis, where the distribution of velocities $v_{z}$ is a Gaussian centered on 0 , of standard deviation $v_{\text {th }}$. 


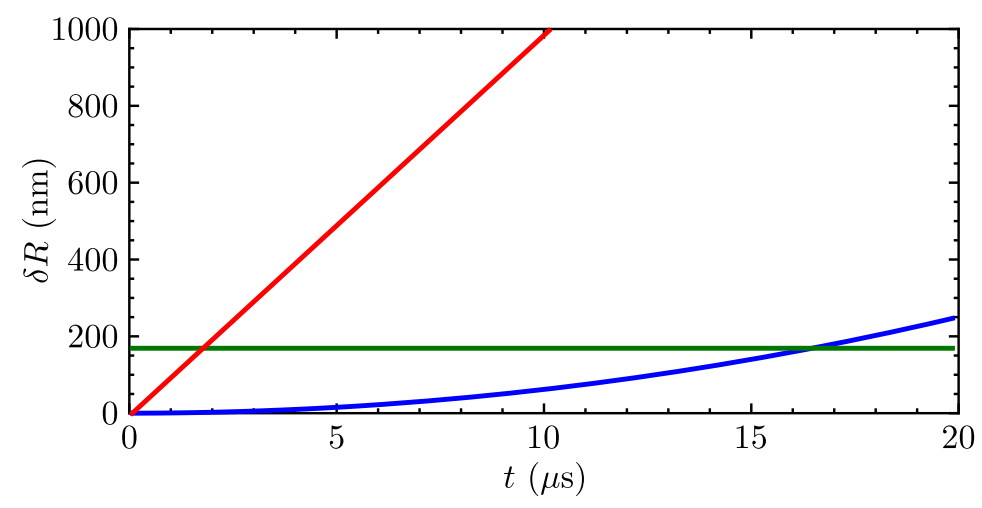

Figure 8.19: Evolution of the interatomic distance during the sequence. We plot the influence of the finite temperature of the atoms due to shot-to-shot fluctuations of the initial positions (green line) and due to the thermal velocity of the atoms (red curve). The effect of interatomic forces is shown in blue, for a separation distance $R=20.5 \mu \mathrm{m}$. For the durations $\tau$ we used experimentally $(\tau<7 \mu \mathrm{s})$, the forces between the atoms can be neglected.

\section{Forces between the atoms}

Because of their finite temperature, the atoms move when the trap is turned off during the experimental sequence. In order to follow their trajectories, one needs to consider interatomic forces that are due to the interaction. Here, we use a classical model to estimate the effect of the force. A full quantum treatment is complicated since the forces are only present when the atoms are in the Rydberg state, which leads in general to entanglement between external and internal degrees of freedom of the atoms. The force between the atoms derives from the interaction energy:

$$
F_{\text {dip }}=-\frac{\mathrm{d}}{\mathrm{d} R}\left(h \frac{C_{3}}{R^{3}}\right)=\frac{3 h}{R} \frac{C_{3}}{R^{3}} .
$$

The equation of motion reads:

$$
\mu \frac{d^{2} R}{d t^{2}}=3 h \frac{C_{3}}{R^{4}}
$$

where the reduced mass $\mu$ is equal to half the mass of ${ }^{87} \mathrm{Rb}$. The blue curve in Figure 8.19 shows the solution of Equation 8.44 for two atoms separated by $20.5 \mu \mathrm{m}$ and with a zero initial velocity. The variation of the interatomic distance $\delta R$ is represented as a function of the time $t$. For comparison, we also represented the typical fluctuations of the initial positions $\sigma_{R}$ (green line) and the typical displacement $\sqrt{2} v_{\text {th }} t$ due to the thermal velocity of the atoms during the free flight (red line). The graph shows that for our sequence durations the effect of the forces can be neglected compared to the thermal effects. For $\tau=7 \mu \mathrm{s}$, the maximal duration used 


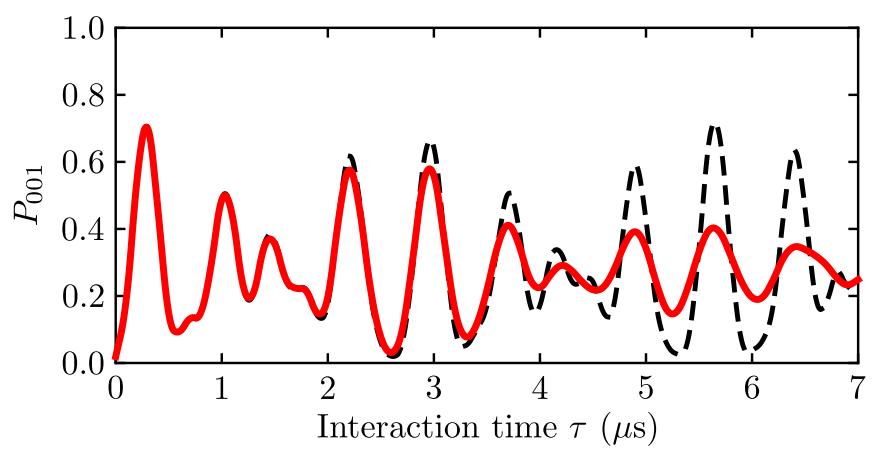

Figure 8.20: Effect of the finite temperature of the atoms. Simulated probability $P_{001}$ for traps that are separated by $20.5 \mu \mathrm{m}$. The black dashed line corresponds to $T=0$. The solid red line corresponds to $T=50 \mu \mathrm{K}$, giving a dispersion on the initial positions of the atoms $\sim 120 \mathrm{~nm}$ and a rms velocity of $\sim 7 \mathrm{~cm} / \mathrm{s}$.

experimentally, forces between the atoms induce a $\delta R$ as low as $30 \mathrm{~nm}$, whereas the thermal effects induce variations of a few hundreds of $\mathrm{nm}$ in the interatomic distance. In what follows we neglect the effects due to forces between the atoms.

\section{Model}

A more accurate description of the system requires to take into account in the simulation the variations of interatomic distances between the atoms between and during each shot:

$$
R(t)=R+\Delta z_{0}+\Delta v_{z} t
$$

where $\Delta z_{0}$ is a random variable of Gaussian distribution centered around 0 with standard deviation $\sigma_{R}$, and $\Delta v_{z}$ is a random variable of Gaussian distribution centered around 0 with standard deviation $\sqrt{2} v_{t h}$. We draw initial positions and initial velocities and solve the optical Bloch equations as in Figure 8.18. We average the calculated populations over 100 realizations of the simulation. We observe in Figure 8.20 that the effect of the temperature shows as a damping in the oscillations of $P_{001}$. The effect becomes important after an interaction time of $\simeq 4 \mu s$, which agrees well with the experimental observations.

\subsubsection{Detection errors}

Finally, the detection errors need to be taken into account (see Section 6.4.3). Some atoms leave the trapping region during the experiment and are detected as a lost independently of their actual state. Because of our loss-based detection scheme, 


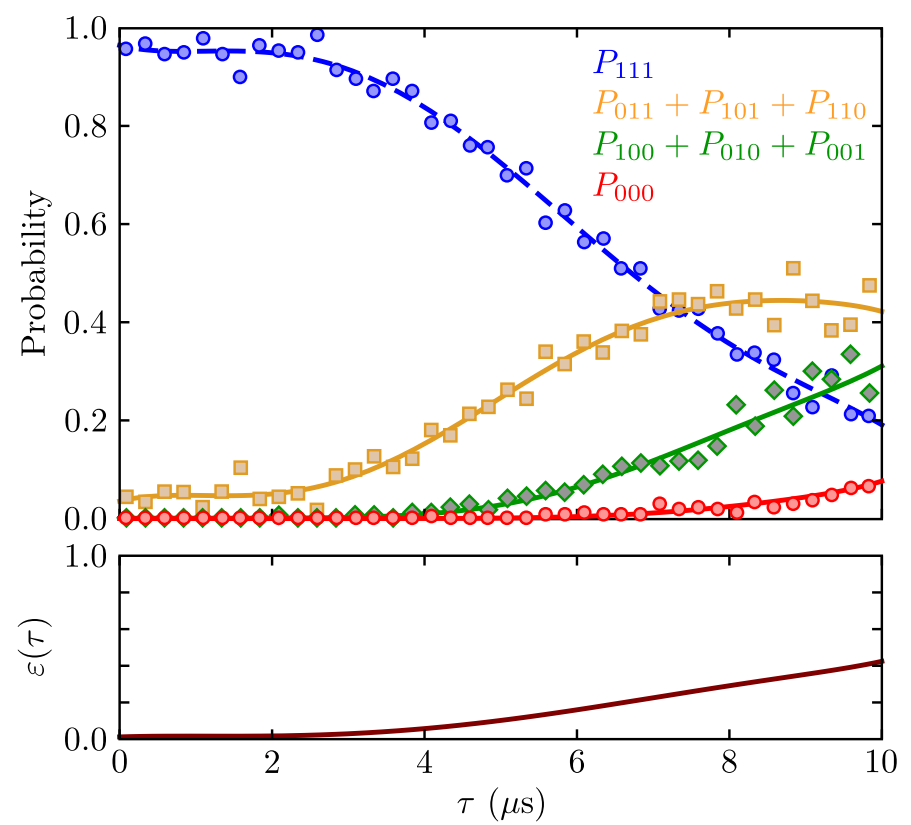

Figure 8.21: Measurement of the detection error $\varepsilon(\tau)$. a, The data points show the evolution of the populations $P_{111}, P_{011}+P_{101}+P_{110}, P_{001}+P_{010}+P_{100}$ and $P_{000}$ when we switch of the trap for a variable duration of time. The dashed line is a polynomial fit of $P_{111}$, from which we extract $\varepsilon(\tau)$. $\mathbf{b}$, Extracted $\varepsilon(\tau)$ from the measured probabilities.

there exists a probability to falsely detect an atom to be in Rydberg state state. As discussed earlier, this effect arises from the finite temperature of the atoms and also from collisions with background gas. As a consequence, detection errors increase with the length of the sequence. In Chapter 6 , we measured an $\varepsilon \simeq 5 \%$ detection error for experimental sequences that are shorter than $\simeq 4 \mu \mathrm{s}$. Here, we switch off the trap for durations which are as large as $\tau=7 \mu \mathrm{s}$, and it is thus necessary to take into account the variation of $\varepsilon$ as we increase the duration of the sequence.

\section{Measurement of $\varepsilon(\tau)$}

We perform a release and recapture experiment to measure $\varepsilon(\tau)$. We trap three ground state atoms in a line, in the same configuration as in Section $8.5(20.5 \mu \mathrm{m}$ separation distance between the atoms). We switch the trap off for a variable duration $\tau$ and we measure the recapture probabilities at the end of the sequence (see Figure 8.21). We extract $\varepsilon(\tau)$ from a polynomial fit of the measured $P_{111}$ (see dashed line in Figure 8.21a), where

$$
P_{111}=(1-\varepsilon(\tau))^{3}
$$




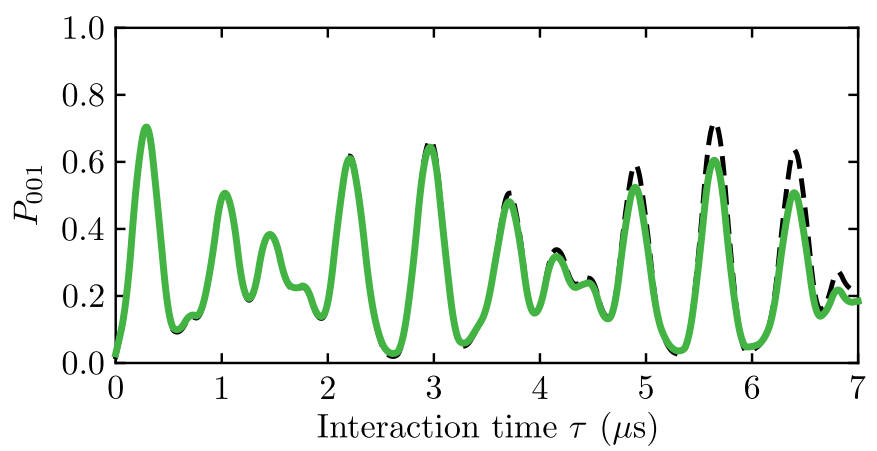

Figure 8.22: Effect of the detection errors on the simulation for the three atom chain. The solid curve shows the solution of the Bloch equations for the population $P_{001}$ as a function of the interaction time $\tau$, the detection error $\varepsilon(\tau)$ is taken in to account. For comparison, the dashed line shows $P_{001}$ obtained from the optical Bloch equations only.

Using the fitted $\varepsilon(\tau)$, we evaluate the evolution of the rest of the recapture probabilities using the relations between the measured probabilities and the actual ones (see Chapter 7):

$$
\left\{\begin{array}{l}
P_{011}+P_{101}+P_{110}=3 \varepsilon(\tau)[1-\varepsilon(\tau)]^{2} \\
P_{100}+P_{010}+P_{001}=3 \varepsilon(\tau)^{2}[1-\varepsilon(\tau)] \\
P_{000}=\varepsilon(\tau)^{3}
\end{array}\right.
$$

The comparison of the calculated recapture probabilities (see solid lines in Figure 8.21a) with the experimentally measured populations supports the consistency of our loss model. For $\tau=7 \mu \mathrm{s}, \varepsilon$ can be as large as $20 \%$, showing the importance of this calibration measurement for our simulation.

\section{Consequences in the simulation}

We now include the detection errors in the simulation of the interaction dynamics for the three-atoms chain. As an example, we compute the measured probability $P_{001}$ using the formula:

$$
\begin{aligned}
P_{001} & =(1-\varepsilon(\tau))\left[P_{d d g}+P_{d p g}+P_{p d g}+P_{p p g}\right. \\
& \left.+\varepsilon(\tau)\left(P_{d g g}+P_{p g g}+P_{g d g}+P_{g p g}\right)+\varepsilon(\tau)^{2} P_{g g g}\right] .
\end{aligned}
$$

Figure 8.22 shows the solution of the Bloch equations for $P_{001}$, when taking into account the detection errors (solid curve) compared to the solution of the Bloch equation only (dashed curve). Significant effects start to be visible for interaction times $\geq 4 \mu \mathrm{s}$, and contributes to the explanation of the damping observed experimentally. 

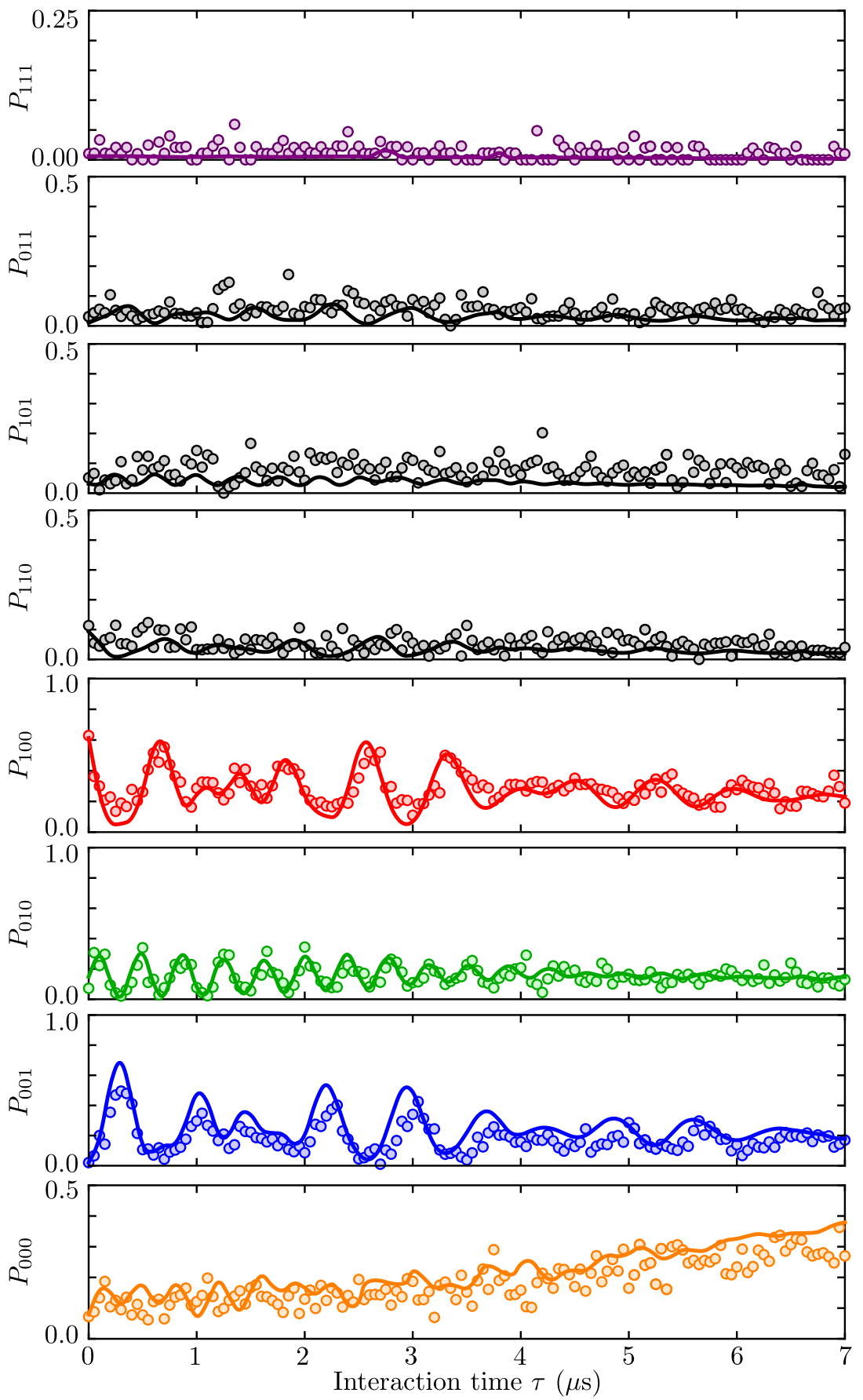

Figure 8.23: Energy transport along a chain of three Rydberg atoms: comparison with the simulation. All the probabilities $P_{i j k}(i, j, k \in\{0 ; 1\})$ are represented as a function of the interaction time. The points show the experimental data, and the solid lines show the results of the simulation with no adjustable parameters. 


\subsubsection{Comparison with the data}

We finally simulate the three-atom dynamics using the optical Bloch equations and including all the effects described above (temperature effects, dissipation and detection errors). The results of the simulation are shown as solid lines in Figure 8.23, without any adjustable parameter. The simulation compares well with the experimental observations. We clearly see the coherent evolution of the system for interaction times as large as $\tau=7 \mu \mathrm{s}$, and the simulation reproduces well the limited contrast (mainly due to dissipation) and the observed damping (mainly due to temperature effects and detection errors). Small remaining discrepancies, in particular in the contrast of $P_{001}$ could be due to other effects that we ignored here, like imperfections in our optical pumping or addressing for instance, or to a $5 \%$ uncertainty in our calibration of the interatomic distances.

\subsection{Conclusion}

We have demonstrated fast coherent manipulation of a single Rydberg atom with a microwave field. Using this tool, we measured the coupling between two atoms, separated by distances as large as $50 \mu \mathrm{m}$. The coupling strength follows the $C_{3} / R^{3}$ dependence of the resonant dipole-dipole interaction, and the measured values agree well with the calculated value of the $C_{3}$ coefficient. Adding a third atom, we simulate the evolution dynamics of an elementary chain of three interacting spins. The results agree well with our simulation, showing that we have a good understanding of our system.

One of the main technical limitation in this experiment is the finite temperature of the atoms. Reducing the temperature [Kaufman, Lester, and Regal (2012); Thompson et al. (2013)] could allow us to probe the coherence of the system for even longer timescales, which might be useful to extend this kind of study to larger and more complex ensembles. In an attempt to investigate the limitations of the finite temperature, we simulate the evolution of the system at $T=10 \mu \mathrm{K}$. To estimate $\varepsilon(\tau)$ at $T=10 \mu \mathrm{K}$, we perform Monte-Carlo simulations of the trajectories of the single atoms, taking into account the expected energy distribution of an atom in the trap [Tuchendler et al. (2008)]. The result is shown in Figure 8.24, where we compare the simulation for $T=10 \mu \mathrm{K}$ (solid lines) with the curves obtained at $T=50 \mu \mathrm{K}$ (dashed lines). In this case, the damping of the oscillations are drastically reduced for interaction times longer than $\sim 4 \mu$ s. This suggests that a reduction of the temperature of 

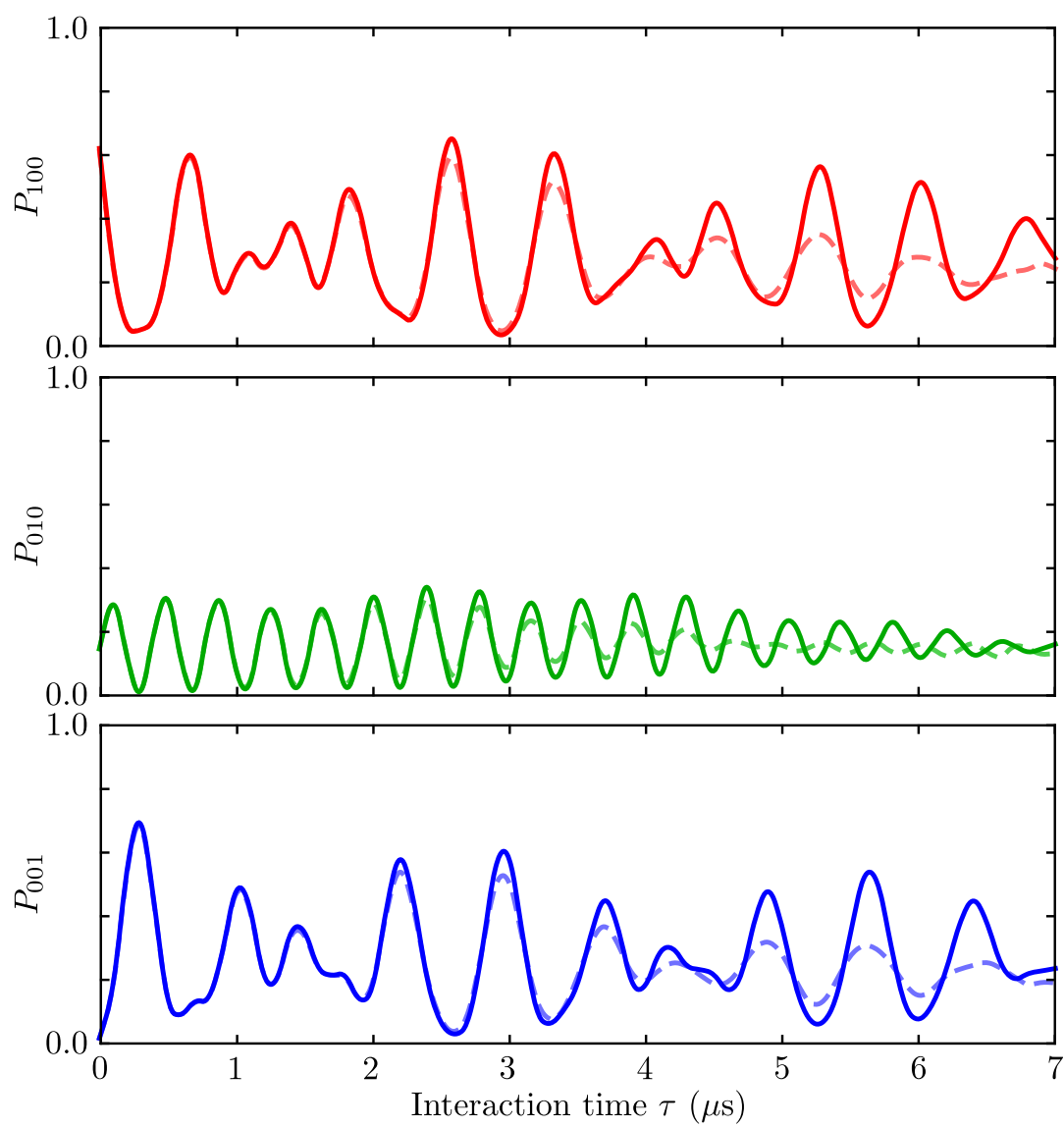

Figure 8.24: Decreasing the temperature of the atoms. Simulation of the probabilities $P_{100}, P_{010}$ and $P_{001}$ for a $T=10 \mu \mathrm{K}$ temperature of the atoms (solid line). For comparison, the dashed line shows the dynamics obtained for a temperature $T=50 \mu \mathrm{K}$. Decreasing the temperature by a factor five could already improve significantly the quality of the data.

the atoms by only one order of magnitude would be enough to make motional effects negligible on the timescales used in the experiment, so as to enable the nearly ideal quantum simulation of larger spin systems.

Another more fundamental imperfection comes from the fact that we can not switch the interaction off during the sequence. This perturbs our preparation and read out steps, especially at small distances. In the extreme limit of small separation distances such that $C_{3} / R^{3} \gg \hbar \Omega_{\mathrm{MW}}$, multiple excitations would even blockaded by the resonant interaction, preventing the excitation of the system. In the next Chapter, we demonstrate a way to overcome this problem by controlling the interactions in the system using electric fields so as to switch the system on and off from resonance. 


\section{Chapter 9}

\section{Coherent dipole-dipole coupling between two single atoms at a Förster resonance}

\section{Contents}

9.1 Finding a Förster resonance between two Rydberg atoms 200

9.1.1 Accidental degeneracy . . . . . . . . . . . . . . . . . . 201

9.1 .2 Interacting pair states . . . . . . . . . . . . . 202

9.1.3 Tuning the system to resonance . . . . . . . . . . . . 202

9.2 Interaction between two atoms at a Förster resonance . 203

9.2.1 Single interaction channel model . . . . . . . . . . . . . . 204

9.2 .2 Förster interaction limit . . . . . . . . . . . . . . 205

9.2.3 Van der Waals interaction limit . . . . . . . . . . . . 206

9.2.4 Four-level system . . . . . . . . . . . . . . . . 206

9.3 Spectroscopy of two interacting atoms at a Förster resonance ....................... 207

9.3.1 Experimental sequence . . . . . . . . . . . . . . . . 207

9.3.2 Observation of the avoided crossing . . . . . . . . . . 207

9.3.3 Measurement of the $C_{3}$ coefficient . . . . . . . . . . . 210

9.3.4 Switch of the interaction . . . . . . . . . . . . . 213

9.3.5 Simulation using the Schrödinger equation . . . . . . . . . 214

9.4 Observation of Förster oscillations . . . . . . . . 217

9.4.1 Experimental sequence . . . . . . . . . . . . . . 217

9.4.2 Experimental results . . . . . . . . . . . . . . . . 218 
9.4.3 Measurement of the $C_{3}$ coefficient . . . . . . . . . . 218

9.4.4 Discussion of the limitations . . . . . . . . . . . . . 220

9.5 Angular dependence of the interaction . . . . . . . 225

9.5.1 Several Förster resonances . . . . . . . . . . . . . 226

9.5.2 Measurement of the angular dependence . . . . . . . . . . 227

9.5.3 Spectroscopy of the system for $\theta=30^{\circ} \ldots \ldots$. . . . . . 231

9.6 Förster resonance in a magnetic field . . . . . . . . 233

9.6.1 Zeeman Hamiltonian . . . . . . . . . . . . . . . . . . 233

9.6 .2 Stark Hamiltonian . . . . . . . . . . . . . . . . . 234

9.6 .3 Total Hamiltonian . . . . . . . . . . . . . . . . 235

9.6.4 Including the magnetic field in the simulation . . . . . . . 236

9.7 Conclusion . . . . . . . . . . . . . . . 236

We have studied in Chapter 8 the resonant interactions between two atoms excited to two different Rydberg states that are coupled by the dipole-dipole interaction. Our system allows preparing in a controllable fashion the atoms to two different Rydberg states by combining three tools: the single-site addressability, the optical excitation to Rydberg states and the microwave transfer in the Rydberg manifold. Because it only requires the excitation of the atoms to a single Rydberg state, a preferred way to initiate resonant energy transfers in most experimental systems is to use a so-called "Förster resonance" [Gallagher (2005); Walker and Saffman (2005)]. For two atoms excited to the same Rydberg state $|r\rangle$, we have seen in Chapter 7 that the most general interaction is the van der Waals interaction, arising from second-order perturbation theory:

$$
V_{\mathrm{vdW}}=\frac{1}{R^{6}} \sum_{\left|\phi_{A} \phi_{B}\right\rangle} \frac{\left|\left\langle\phi_{A} \phi_{B}\left|\hat{\boldsymbol{d}}_{A} \cdot \hat{\boldsymbol{d}}_{B}\right| r r\right\rangle-3\left\langle\phi_{A} \phi_{B}\left|\left(\hat{\boldsymbol{d}}_{A} \cdot \boldsymbol{n}\right)\left(\hat{\boldsymbol{d}}_{B} \cdot \boldsymbol{n}\right)\right| r r\right\rangle\right|^{2}}{\left(4 \pi \epsilon_{0}\right)^{2}\left(2 E_{r}-E_{\phi_{A}}-E_{\phi_{B}}\right)}
$$

It is apparent from Equation 9.1 that a singularity occurs when there exists a degeneracy in energy between two pair states $\left(2 E_{r}=E_{\phi_{A}}+E_{\phi_{B}}\right)$, thus canceling the denominator. Indeed, if there exists a situation where two coupled pair states are exactly resonant, the system is then subject to resonant dipole-dipole interactions as described in Chapter8. Figure 9.1 shows the example of a state $|r\rangle$, which energy is located midway between the energies of two other states $\left|\phi_{A}\right\rangle$ and $\left|\phi_{B}\right\rangle$. The pair states $|r r\rangle$ and $\left|\phi_{A} \phi_{B}\right\rangle$ (which are coupled by the dipole-dipole interaction Hamiltonian $\hat{V}_{\text {dip }}$ ) are degenerate, leading to a resonant interaction: one atom undergoes a transition from $|r\rangle$ to $\left|\phi_{A}\right\rangle$ while the other one undergoes a transition from $|r\rangle$ to 


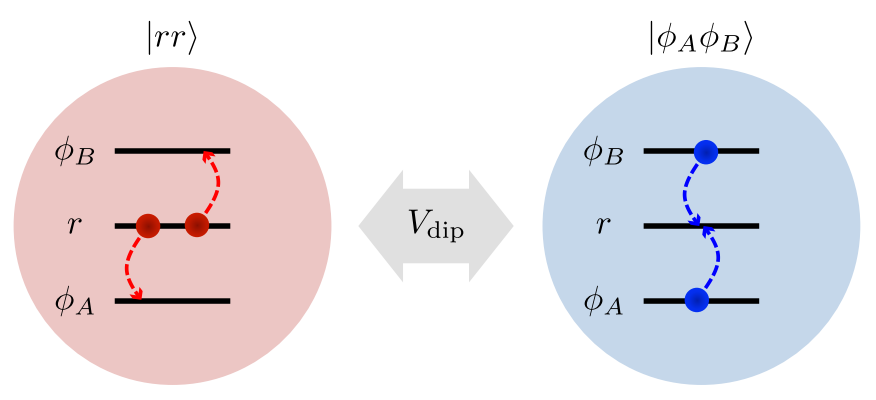

Figure 9.1: Principle of the experiment. The pair states $|r r\rangle$ and $\left|\phi_{A} \phi_{B}\right\rangle$, which are coupled by the dipole-dipole interaction Hamiltonian, are degenerate. As a consequence, they are subject to a resonant dipole-dipole interaction. This type of resonance is called "Förster resonance".

$\left|\phi_{B}\right\rangle$. In practice, for a given atom, rare accidental degeneracies exist, but more importantly, two atoms can be Stark-tuned to exact resonance by applying small electric fields [Gallagher (2005); Safinya et al. (1981); Anderson, Veale, and Gallagher (1998); Mourachko et al. (1998)]. This mechanism has been named Förster resonance [Walker and Saffman (2005)] by analogy with resonant energy transfers observed in biology or photochemistry [Förster (1948); Andrews and Demidov (1999); Clegg (2006)].

First studies were performed in effusive thermal beams of Sodium [Safinya et al. (1981)], where the atoms resonantly interacted through the channel

$$
n S+n S \longrightarrow n P+(n-1) P
$$

which was viewed as a resonant collision between internal degrees of freedom of two Rydberg atoms. Due to the long range character of the resonant interaction, the collision showed large interaction times $(\sim 1 \mathrm{~ns})$ and cross sections $\sim 10^{3}$ larger than in the absence of resonance. Generalizations of those studies to cold atom systems [Anderson, Veale, and Gallagher (1998); Mourachko et al. (1998)] led to the observation of resonant dipole-dipole interactions in frozen gases where the atoms could be considered as motionless, far from the original picture of collisions. For example, Mourachko et al. in [Mourachko et al. (1998)] studied the Förster resonance

$$
n P_{3 / 2}+n P_{3 / 2} \longrightarrow n S+(n+1) S
$$

between Cesium atoms. In the frozen gas limit, they noticed that correlations between particles could build up on timescales that are faster than the motion of the atoms, leading to the observation of many-body effects. As a matter of fact, once created using the Förster resonance, the $n S$ and $(n+1) S$ excitations could diffuse in the medium through resonant energy transfers governed by the interaction channels 


$$
\begin{aligned}
& n P_{3 / 2}+n S \quad \longrightarrow \quad n S+n P_{3 / 2} \\
& n P_{3 / 2}+(n+1) S \longrightarrow(n+1) S+n P_{3 / 2} \text {, }
\end{aligned}
$$

as described in Chapter 8. After those pioneering studies, the long-range character of the interaction at a Förster resonance led to the observation of a strong, electricallytuned Rydberg blockade in cold atom ensembles [Vogt et al. (2006, 2007); Reinhard, Younge, and Raithel (2008)]. The incoherent transfer of excitations between two spatially resolved locations was also observed in 2008 [van Ditzhuijzen et al. (2008)]. However, in all those experiments the coherent nature of the interaction could not be observed due to the presence of a distribution of interatomic distances in disordered systems, the motion of the atoms induced by the interaction and the diffusion of the excitations through the interaction channels in Equation 9.4 [Anderson et al. (2002); Mudrich et al. (2005); Westermann et al. (2006); Ryabtsev et al. (2010a,b); Nipper et al. (2012a,b); Vogt et al. (2007); Reinhard, Younge, and Raithel (2008); Ryabtsev et al. (2010a); Nipper et al. (2012a,b)].

We study in this chapter the interactions between two Rydberg atoms separated by a controlled distance and tuned to a Förster resonance similar to the one in Equation 9.3. The presence of only two atoms allows concentrating on the Förster interaction process itself, deconvolved from any other interaction channels that lead to diffusion, as in Equation 9.4. We study, both spectroscopically and in the time domain, the coherent, dipolar induced exchange of electronic excitations between the two-atoms, and verify that the coherent oscillation occurs at a frequency that scales as the inverse third power of the interatomic distance [Walker and Saffman (2005)]. The system can be tuned to resonance using our control of electric fields, which allows switching off the interaction during the preparation and read out of the system, and shows our ability to actively switch on and off strong interactions in the system. Finally, we measure the angular dependence of resonant dipole-dipole interactions.

\subsection{Finding a Förster resonance between two Rydberg atoms}

We have demonstrated in Chapter 6 that we can optically excite $\left|n D_{3 / 2}\right\rangle$ Rydberg states. Starting from this state, selection rules (see Chapter 7) show that direct dipolar couplings are allowed between the pair state $\left|n D_{3 / 2} ; n D_{3 / 2}\right\rangle$ and all the pair states that combine the one-atom states $\left|n P_{1 / 2}\right\rangle,\left|n P_{3 / 2}\right\rangle$ and $\left|n F_{5 / 2}\right\rangle$. In view of 
a

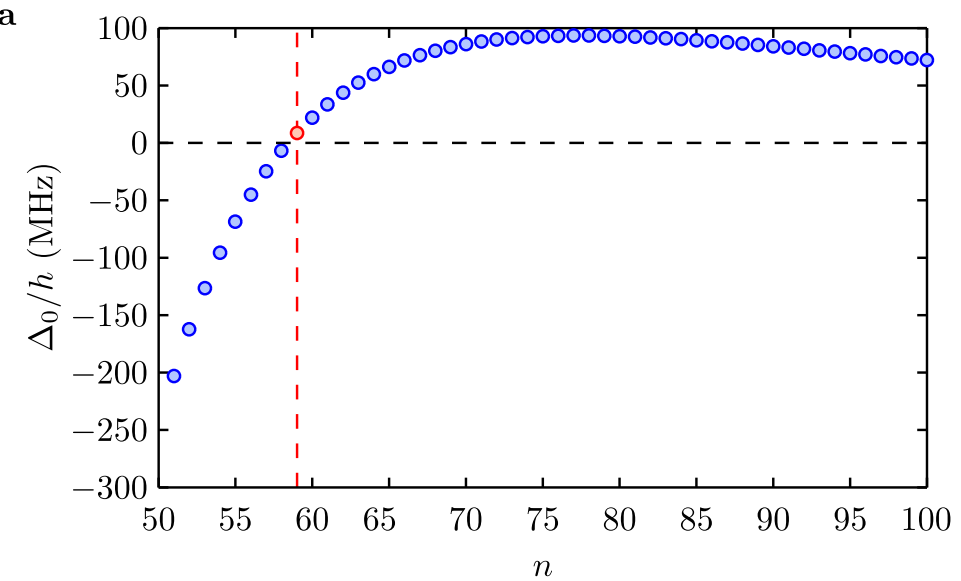

b

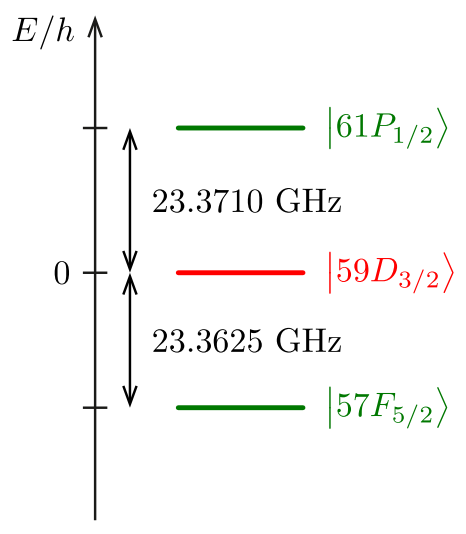

Figure 9.2: Choice of the Rydberg states. a, Förster defects for the interaction channel in Equation 9.5 as a function of the principal quantum number $n$ in the absence of electric field. Despite the large energy separations $\sim 10 \mathrm{GHz}$ between Rydberg states, the pair states of interest are separated by less than $200 \mathrm{MHz}$ for $n \geq 50$. For $n=59$, the Förster defect is $8.5 \mathrm{MHz}$. We will use those pair states to benefit from this accidental quasi-degeneracy. $\mathbf{b}$, Levels of interest in this Chapter. In the absence of electric field, the state $\left|59 D_{3 / 2}\right\rangle$ is located almost midway between the states $\left|61 P_{1 / 2}\right\rangle$ and $\left|57 F_{5 / 2}\right\rangle$.

working at a Förster resonance with rubidium atoms, one interesting interaction channel that has been studied before theoretically [Walker and Saffman (2005)] and experimentally [Gaëtan (2009)] is:

$$
n D_{3 / 2}+n D_{3 / 2} \longleftrightarrow(n+2) P_{3 / 2}+(n-2) F_{5 / 2} .
$$

\subsubsection{Accidental degeneracy}

We need to tune the two pair states $\left|n D_{3 / 2} ; n D_{3 / 2}\right\rangle$ and $\left|(n+2) P_{3 / 2} ;(n-2) F_{5 / 2}\right\rangle$ to exact resonance in order to observe the Förster resonance described by Equation 9.5. An important parameter is the energy difference between those two pair states. This energy difference $\Delta_{0}$ is called "Förster defect":

$$
\Delta_{0}=E\left[(n+2) P_{3 / 2}\right]+E\left[(n-2) F_{5 / 2}\right]-2 E\left[n D_{3 / 2}\right] .
$$

We have seen in Chapter 8 that the typical energy separation between Rydberg states is as large as a few $\mathrm{GHz}$ (in frequency units). On the other hand, by combining one-atom states with different energies, one can create pair states which are much closer in energy. Figure 9.2a shows the "Förster defect" (see Equation 9.6) between the pair states $\left|n D_{3 / 2} ; n D_{3 / 2}\right\rangle$ and $\left|(n+2) P_{3 / 2} ;(n-2) F_{5 / 2}\right\rangle$ as a function of the principal quantum number $n$ in the absence of electric field. One interest of the 
interaction channel in Equation 9.5 is that it involves pair states $\left|n D_{3 / 2} ; n D_{3 / 2}\right\rangle$ and $\left|(n+2) P_{3 / 2} ;(n-2) F_{5 / 2}\right\rangle$, that are separated by less than $200 \mathrm{MHz}$ for principal quantum number that are larger than $n=50$. In particular for $n=59$, the Förster defect is as low as $\Delta_{0} / h=8.5 \mathrm{MHz}$. Indeed, in the absence of electric field, the one-atom state $\left|59 D_{3 / 2}\right\rangle$ is almost exactly midway between the states $\left|61 P_{1 / 2}\right\rangle$ and $\left|57 F_{5 / 2}\right\rangle$ (see Figure 9.2b), leading to the observed accidental quasi-degeneracy. In the rest of this Chapter we use these Rydberg states to study the Förster resonance between two atoms ${ }^{1}$.

\subsubsection{Interacting pair states}

The excitation lasers couple $|g\rangle$ to the stretched state $\left|59 D_{3 / 2}, M_{J}=3 / 2\right\rangle$. We are initially interested in the Förster interaction between two atoms aligned along the quantization axis. In this case, the Zeeman sub-levels taking part in the interaction are the ones fulfilling the condition $\Delta M_{\text {Tot }}=0$, as explained in Section 7.1. When $\theta=0^{\circ}$, the Zeeman sub-levels that are directly coupled by the dipole-dipole interaction satisfy $M_{\mathrm{Tot}}=3$ :

$$
\begin{aligned}
& |d d\rangle \equiv\left|59 D_{3 / 2}, M_{J}=3 / 2 ; 59 D_{3 / 2}, M_{J}=3 / 2\right\rangle \\
& |p f\rangle \equiv\left|61 P_{1 / 2}, M_{J}=1 / 2 ; 57 F_{5 / 2}, M_{J}=5 / 2\right\rangle \\
& |f p\rangle \equiv\left|57 F_{5 / 2}, M_{J}=5 / 2 ; 61 P_{1 / 2}, M_{J}=1 / 2\right\rangle
\end{aligned}
$$

Note that $|d d\rangle$ couples to the two degenerate states $|p f\rangle$ and $|f p\rangle$ that consist of a permutation of the same pair states, and show the same properties.

\subsubsection{Tuning the system to resonance}

We have seen that in the absence of electric field, the Förster defect between the states $|d d\rangle$ and $|p f\rangle$ (or $|f p\rangle$ ) is $\Delta_{0} /(2 \pi)=8.5 \mathrm{MHz}$. One way to tune the system to exact resonance is to use electric fields. Table 9.1 shows the polarizabilities of those pair-states. Because $P$-states, $D$-states and $F$-states have different sensitivities to the electric field, the pair states have different polarizabilities. As a consequence, when increasing the vertical electric field $F_{z}$, the states are subject to a differential Stark effect. The state $|d d\rangle$ shifts as $\alpha_{d d} F_{z}^{2} / 2$, whereas the state $|p f\rangle$ shifts as $\alpha_{p f} F_{z}^{2} / 2$. As a consequence, the two states are degenerate for an electric field of:

\footnotetext{
${ }^{1}$ Note that the Förster defect for the state $\left|58 D_{3 / 2}\right\rangle$ is even smaller. However, it is negative which does not allow tuning the system to exact resonance using electric fields as we will demonstrate in Section 9.1.3.
} 
Table 9.1: Polarizabilities for the pair states $|d d\rangle,|p f\rangle$ and $|f p\rangle$. The polarizabilities were calculated using second-order perturbation theory as described in Chapter 5. They are given in $\left(\mathrm{kHz} .(\mathrm{mV} / \mathrm{cm})^{-2}\right)$.

\begin{tabular}{ll}
\hline State & $\boldsymbol{\alpha}$ \\
\hline$|d d\rangle$ & 1.1 \\
$|p f\rangle$ & 16.45 \\
$|f p\rangle$ & 16.45 \\
\hline
\end{tabular}

$$
F_{z}=F_{\text {res }} \simeq 33 \mathrm{mV} / \mathrm{cm}
$$

which corresponds to the position of the resonance. Note that the choice $n=59$ allows working with a small vertical electric field for which the states can be considered unperturbed. At resonance, the states $|d d\rangle,|p f\rangle$ and $|f p\rangle$ are degenerate, and the system interacts resonantly through the exchange of virtual photons as shown in Equation 9.5:

$$
59 D_{3 / 2}+59 D_{3 / 2} \longleftrightarrow 61 P_{1 / 2}+57 F_{5 / 2}
$$

\subsection{Interaction between two Rydberg atoms at a Förster resonance}

We first consider the case of two atoms aligned along the quantization axis. The dipolar coupling between the state $|d d\rangle$ and the states $|p f\rangle$ and $|f p\rangle$ is given by

$$
\left\langle d d\left|\hat{V}_{\text {dip }}\right| p f\right\rangle=\left\langle d d\left|\hat{V}_{\text {dip }}\right| f p\right\rangle=\frac{C_{3}}{R^{3}},
$$

where $\hat{V}_{\text {dip }}$ is the interaction Hamiltonian in Equation 7.8. Numerical calculations give a theoretical $C_{3}$ coefficient of:

$$
\frac{C_{3, \mathrm{th}}}{h} \simeq 2.54 \mathrm{GHz} \cdot \mu \mathrm{m}^{3} .
$$

Since the state $|d d\rangle$ can couple to $|p f\rangle$ or $|f p\rangle$ with equal strength, it is convenient to work in the basis $\{|d d\rangle ;|\widetilde{p f}\rangle ;|\widetilde{f p}\rangle\}$ containing the symmetric and antisymmetric 
combinations of $|p f\rangle$ and $|f p\rangle$ :

$$
\begin{aligned}
& \widetilde{p f}\rangle \equiv \frac{|p f\rangle+|f p\rangle}{\sqrt{2}}, \\
& |\widetilde{f p}\rangle \equiv \frac{|p f\rangle-|f p\rangle}{\sqrt{2}}
\end{aligned}
$$

The dipolar coupling between $|d d\rangle$ and the symmetric state $|\widetilde{p f}\rangle$ is:

$$
\left\langle d d\left|\hat{V}_{\text {dip }}\right| \widetilde{p f}\right\rangle=\sqrt{2} \frac{C_{3}}{R^{3}},
$$

whereas the states $|d d\rangle$ and $|\widetilde{f p}\rangle$ do not show any dipolar coupling:

$$
\left\langle d d\left|\hat{V}_{\text {dip }}\right| \widetilde{f p}\right\rangle=0
$$

For two atoms that are aligned along the quantization axis, this allows to restrict the basis to $\{|d d\rangle ;|\widetilde{p f}\rangle\}$. Couplings of $|d d\rangle$ to other non-resonant states also contribute to second-order to the interaction. However, here we only consider the resonant interaction channel of Equation 9.9 and ignore other couplings.

\subsubsection{Single interaction channel model}

In the basis $\{|d d\rangle ;|\widetilde{p f}\rangle\}$, the total Hamiltonian of the system reads :

$$
\hat{H}=\left(\begin{array}{cc}
0 & \sqrt{2} C_{3} / R^{3} \\
\sqrt{2} C_{3} / R^{3} & \Delta
\end{array}\right) .
$$

The diagonalization of this Hamiltonian gives the eigenenergies $E_{ \pm}$of the system :

$$
E_{ \pm}=\frac{\Delta}{2} \pm \sqrt{\left(\frac{\Delta}{2}\right)^{2}+\left(\frac{\sqrt{2} C_{3}}{R^{3}}\right)^{2}}
$$

The interaction mixes the states and the eigenstates are :

$$
\left\{\begin{array}{l}
|+\rangle=\sin \theta|d d\rangle+\cos \theta|\widetilde{p f}\rangle \\
|-\rangle=\cos \theta|d d\rangle-\sin \theta|\widetilde{p f}\rangle
\end{array}\right.
$$

where $\tan 2 \theta=2\left(\sqrt{2} C_{3} / R^{3}\right) / \Delta$. The Förster interaction corresponds to the limit where $|\Delta| \ll\left|C_{3}\right| / R^{3}$. 


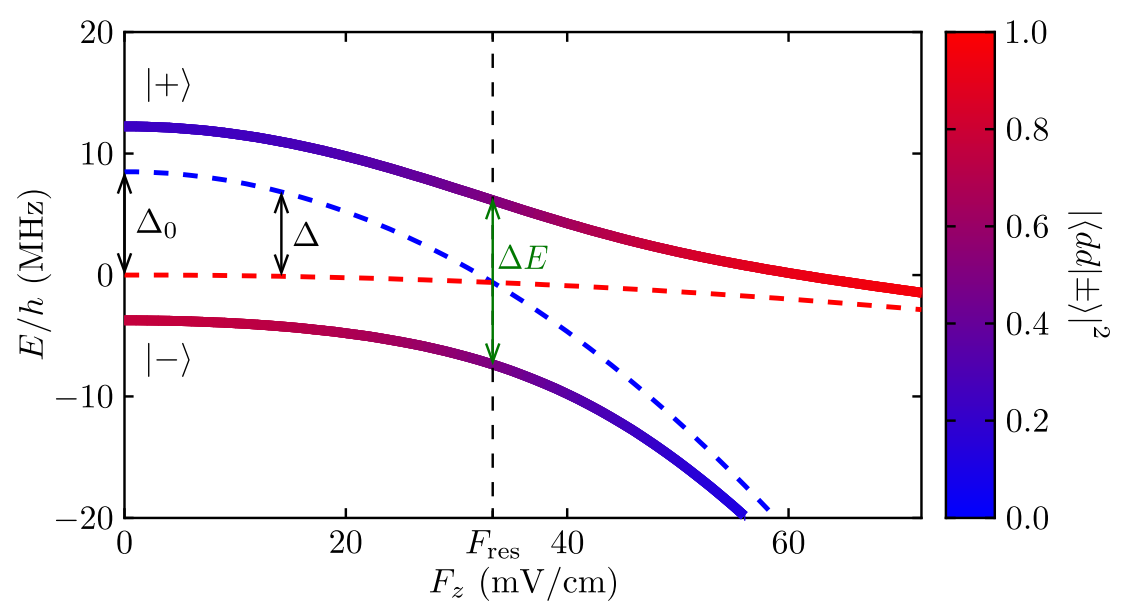

Figure 9.3: Eigenenergies of the system as a function of the electric field. In the absence of coupling, the two pair-state energy levels (dotted lines) vary when an external electric field $F_{z}$ is applied, and cross each other for $F_{z}=F_{\text {res }}$. The coupled system (solid lines) undergoes an avoided crossing at the resonance indicated by a vertical dashed line.

\subsubsection{Förster interaction limit}

We first focus on the limiting case of the Förster interaction, when $|\Delta| \ll\left|C_{3}\right| / R^{3}$. In this case, the eigenenergies of the system are:

$$
E_{ \pm}= \pm \sqrt{2} \frac{\left|C_{3}\right|}{R^{3}}
$$

and the eigenstates of the system are the superposition states:

$$
| \pm\rangle=\frac{|d d\rangle \pm|\widetilde{p f}\rangle}{\sqrt{2}}
$$

As observed in Section 8.1.2, the eigenvalues of the system are shifted in energy by a quantity $\propto 1 / R^{3}$, which is characteristic of resonant dipole-dipole interactions. Moreover, the interaction mixes the states $|d d\rangle$ and $|\widetilde{p f}\rangle$. At resonance, the stationary states are the symmetric and antisymmetric combinations $|+\rangle$ and $|-\rangle$. Figure 9.3 shows the evolution of the eigenstates of the system when scanning $\Delta$ across the resonance. The dashed lines in Figure 9.3 show $E_{d d}$ and $E_{p f}$ in the absence of dipoledipole interaction. The system reaches the resonance at the position where the two curves cross, for a value $F_{\text {res }}$ of the vertical electric field. The solid lines show the evolution of the eigenenergies $E_{ \pm}$of the coupled system in the presence of interactions, where the colorscale represents the $|d d\rangle$ contribution to the eigenstates. We observe that the system undergoes an avoided crossing. At resonance, if we prepare initially the system in the non-stationary state $|d d\rangle$, it starts oscillating between the two 
configurations $|d d\rangle$ and $|\widetilde{p f}\rangle$ at a frequency given by the difference in energy between the two eigenstates $2 \sqrt{2} C_{3} / R^{3}$. After a time $t$, the probability to find the system in the state $|d d\rangle$ is

$$
P_{d d}(t)=\cos ^{2}\left(2 \pi \frac{\sqrt{2} C_{3}}{h R^{3}} t\right)=\frac{1}{2}\left[1+\cos \left(2 \pi \frac{2 \sqrt{2} C_{3}}{h R^{3}} t\right)\right] .
$$

We therefore expect similar dynamics as in Chapter 8.

\subsubsection{Van der Waals interaction limit}

For the sake of completeness, we next recall the other limit, where the Förster defect is large compared to the interaction $\left(2 \sqrt{2} C_{3} / R^{3} \ll|\Delta|\right)$. In this case the eigenenergies of the system are

$$
\frac{1}{\hbar} E_{ \pm}=\frac{\Delta}{2} \pm \frac{|\Delta|}{2}\left(1+\frac{4 C_{3}^{2}}{\Delta^{2} R^{6}}\right)
$$

and the eigenstates remain almost unperturbed $(|-\rangle \simeq|d d\rangle$ and $|+\rangle \simeq|\widetilde{p f}\rangle$ to first order). We observe that the eigenenergies show a $1 / R^{6}$ behavior. This regime corresponds to the van der Waals interaction, where the shift in energy of the state $|d d\rangle$ is given by $V_{\mathrm{vdW}}=C_{6} / R^{6}$, with :

$$
C_{6}=-\frac{\left(\sqrt{2} C_{3}\right)^{2}}{\Delta}
$$

\subsubsection{Four-level system}

We concentrate, in the rest of this Chapter, on the case of the Förster interaction close to resonance. Figure 9.4 shows the relevant energy levels for our experimental configuration at resonance. Our excitation laser couples $|g\rangle$ to $|d\rangle$ with a Rabi frequency $\Omega$, and therefore couples $|g g\rangle$ to $|\widetilde{d g}\rangle \equiv(|d g\rangle+|g d\rangle) / \sqrt{2}$ with a Rabi frequency $\sqrt{2} \Omega$. Finally, the excitation laser couples $|\widetilde{d g}\rangle$ to $|d d\rangle$ with a Rabi frequency $\sqrt{2} \Omega$, and thus partially couples $|\widetilde{d g}\rangle$ to $|+\rangle$ and $|-\rangle$. 


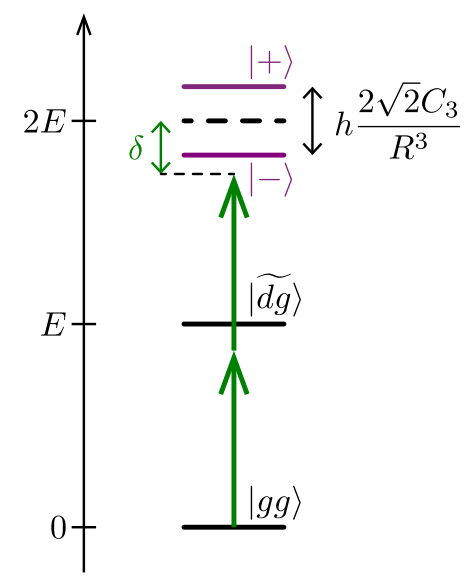

Figure 9.4: Structure of the coupled levels at resonance. We scan the excitation laser (green arrow) to perform a two-atom excitation from $|g g\rangle$ to $|d d\rangle$ via the intermediate state $|\widetilde{d g}\rangle$. The detuning between the laser frequency and the position of $|d d\rangle$ in the absence of interactions (black horizontal dashed line) is called $\delta$.

\subsection{Spectroscopy of two interacting atoms at a Förster resonance}

We first find the position of the Förster resonance by performing a spectroscopic measurement of the two interacting atoms.

\subsubsection{Experimental sequence}

For different values of the vertical electric $F_{z}$, we scan the laser detuning $\delta$ (defined as in Figure 9.4) and perform a spectroscopic measurement of the interacting system. We turn the trap off for $\simeq 2 \mu$ s and shine the excitation lasers on the two atoms for a fixed duration $\tau$. We repeat the sequence $\simeq 100$ times to reconstruct the probabilities $P_{i j}(i, j \in\{0 ; 1\})$. If our excitation and detection were perfect, the probability $P_{00}$ would coincide with the double excitation probability $P_{r r}$.

\subsubsection{Varying the electric field: observation of the avoided crossing}

As a calibration for this experiment, we first measure the position of the one-atom Rydberg line and the one-atom Rabi frequency $\Omega$ independently on a single-atom. The position of the one-atom line corresponds to $\delta=0$ in our spectra (see Figure 9.4). We then fix the interatomic distance to $R=8.1 \mu \mathrm{m}$. Due to the dipole-dipole inter- 
action, the position of the doubly excited state shifts. Scanning the laser allows us to tune the excitation laser to resonance for the two-atom state. Far from the Förster resonance, we expect to observe one line shifted by the van der Waals interaction energy between the two atoms. At the Förster resonance, we expect to observe two lines separated by the energy $\Delta E=\hbar \times 2 \sqrt{2} C_{3} / R^{3}$. Having a fixed set of parameters to probe those different regimes requires a proper choice of the one-atom Rabi frequency $\Omega$ and excitation time $\tau$, as detailed below.

\section{Choice of the Rabi frequency}

At the position of the Förster resonance, we expect to observe two excitation peaks that are separated by the interaction energy $\Delta E / h \simeq 13.7 \mathrm{MHz}$, where we used the calculated $C_{3, \text { th }}$ coefficient (see Equation 9.11). In order to resolve the two peaks we use an excitation linewidth that is smaller than the interaction energy $(\hbar \Omega \leq \Delta E)$. For Rabi frequencies that are too small $(\hbar \Omega \ll \Delta E)$, we have noticed experimentally that the double-excitation probability is smaller than $\lesssim 20 \%$. Instead, we prefer working in a regime with $\hbar \Omega \lesssim \Delta E$, where the double excitation through the intermediate state $|\widetilde{d g}\rangle$ experimentally showed efficiencies that are larger than $60 \%$. For a separation distance $R=8.1 \mu \mathrm{m}$, we chose a Rabi frequency $\Omega /(2 \pi)=2.9 \mathrm{MHz}$, as $\Delta E / \hbar \simeq 13.7 \mathrm{MHz}$.

\section{Choice of the excitation time}

In the absence of interaction, we would maximize the two-atom excitation probability for a duration of the excitation that corresponds to the one-atom $\pi$-pulse $\left(\tau_{\pi}=\pi / \Omega \simeq 172 \mathrm{~ns}\right)$. In the presence of interactions, we have seen that the excitation laser is detuned from the intermediate state $|\widetilde{d g}\rangle$. At the Förster resonance, the oscillation between $|d d\rangle$ and $|p f\rangle$ starts occurring while optically exciting the system. Experimentally, we optimized the duration of the excitation pulse to observe comparable maximized double excitation probabilities at all fields. In this experiment, we used $\tau \simeq 210$ ns at all $F_{z}$.

\section{Experimental results}

Figure 9.5a shows a plot of the excitation spectra obtained for electric fields that vary between $0 \mathrm{mV} / \mathrm{cm}$ and $70 \mathrm{mV} / \mathrm{cm}$. When scanning the electric field, we observe the expected avoided crossing between the states $|d d\rangle$ and $|\widetilde{p f}\rangle$ that is due to the Förster resonance. For $F_{z}=0$, we observe a single line centered on $\delta /(2 \pi) \approx-5 \mathrm{MHz}$. This 
a

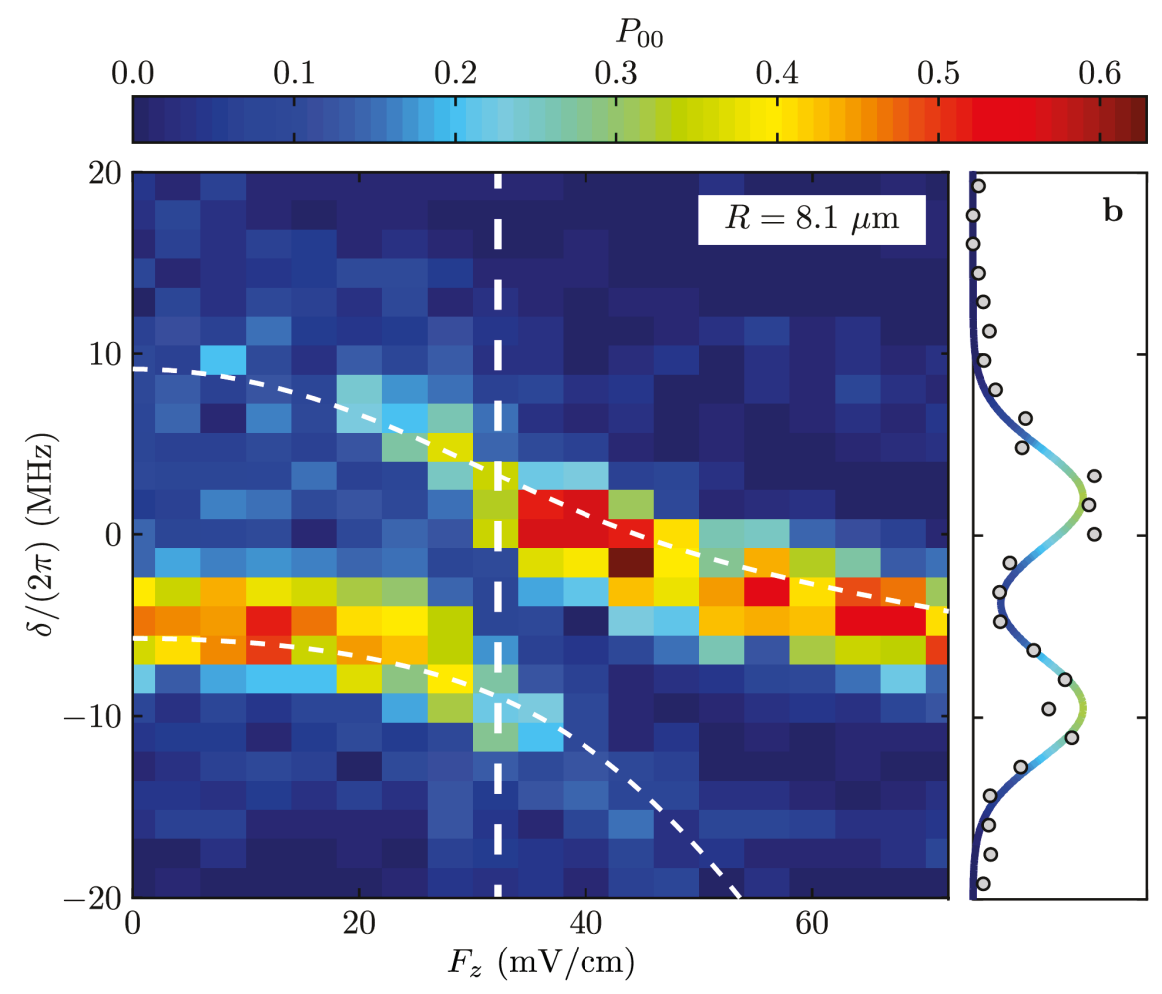

c
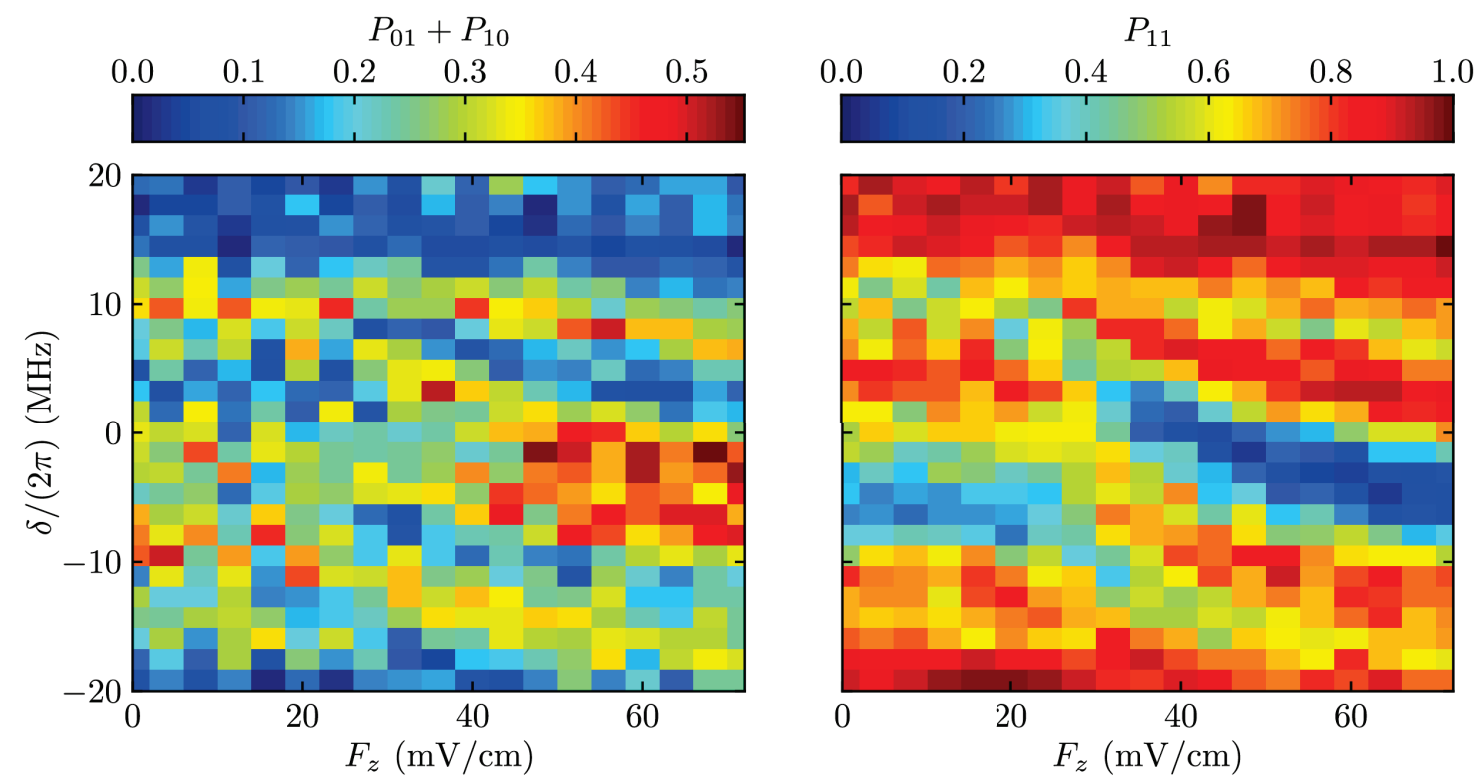

Figure 9.5: Spectroscopy at the Förster resonance. a, Two-atom excitation spectrum where the population of double loss $P_{00}$ is plotted as a function of $\delta$ and $F_{z}$. We observe the avoided crossing between the resonant pair states. The white vertical dashed line indicates the position of the Förster resonance. The white curved dashed lines represents the Stark map calculated in the presence of interactions with $C_{3} /(2 \pi)=2.26 \mathrm{GHz} \cdot \mu \mathrm{m}^{3}$ (see Section 9.3.3) b, Spectrum $P_{00}(\delta)$ at resonance for $R=8.1 \mu \mathrm{m}$. The solid line is a fit by a a sum of two Gaussian functions. c, The populations $P_{01}+P_{10}$ and $P_{11}$ are given for completeness. 
number gives the strength of the van der Waals attraction between the two atoms in a zero electric field. For $F_{z} \approx 20 \mathrm{mV} / \mathrm{cm}$ we observe on the spectrum the apparition of a repulsive potential branch. Increasing the field even further allows scanning across the avoided crossing until only one peak is visible again. The system is at the Förster resonance when we observe two symmetric peaks that are separated by a minimal distance. We measure:

$$
F_{\text {res }}=32 \pm 4 \mathrm{mV} \cdot \mathrm{cm}^{-1}
$$

which is in good agreement with the expected value (see Equation 9.8). The spectrum at resonance is shown in Figure 9.5b. A fit of this spectrum by a sum of two Gaussians gives a separation distance of $\Delta E_{\mathrm{fit}} / h \simeq 10 \pm 0.4 \mathrm{MHz}$. The discrepancy with the expected value $(13.7 \mathrm{MHz})$ comes from a bias in the measurement introduced when fitting the spectrum by a sum of two Gaussians (see Section 9.3.5), and from a possible systematic error on $R$ due to a $\simeq 5 \%$ uncertainty on the calibration of the distance.

\subsubsection{Varying the distance: measurement of the $C_{3}$ coefficient}

We then measure the evolution of the spectra at resonance when varying the interatomic distance $R$.

\section{Measurement of the $C_{3}$ coefficient}

For each distance, we adapt the one-atom Rabi frequency $\Omega$ and the excitation time $\tau$, so as to obtain large excitation probabilities while still being able to resolve the two excitation peaks. We observe in Figure 9.6 the expected trend of the splitting between the two double-excitation peaks with the distance $R$. When increasing $R$, the interaction between the atoms decreases and thus the distance between the peaks decreases. We fit each spectrum by a sum of two Gaussian functions in order to extract the splitting in energy $\Delta E_{\text {fit }}$. Figure 9.7 shows a double-logarithmic plot of $\Delta E_{\mathrm{fit}}$ versus $R$. The data shows a power-law behavior of exponent $-3.2 \pm 0.2$, consistent with the expected $C_{3} / R^{3}$ law. Fixing the exponent to -3 , we obtain:

$$
C_{3, \mathrm{fit}}=2.1 \pm 0.1 \mathrm{GHz} \cdot \mu \mathrm{m}^{3}
$$

The mismatch between the measured value and the expected one comes from a bias in the measurement introduced by fitting the spectra by two Gaussians (see simulation in Section 9.3.5), and a possible systematic error on $R$ due to a $\simeq 5 \%$ uncertainty on 


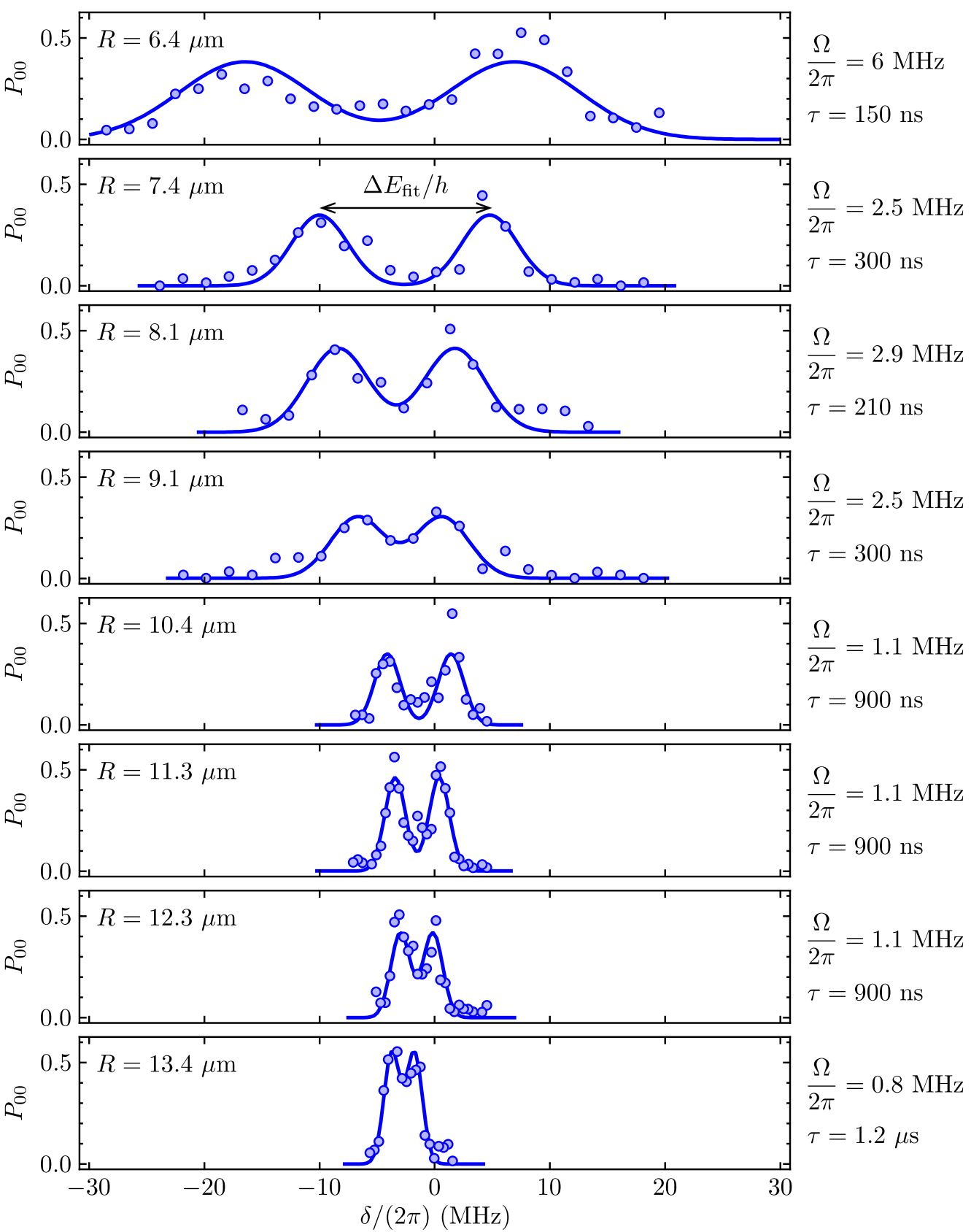

Figure 9.6: Spectra at resonance for different interatomic distances. Blue circles show the measurement of $P_{00}$ for different interatomic distances $R$. As expected, we observe that the distance between the two double-excitation peaks increases when we increase the interaction (or decrease $R$ ). Blue lines are fits by a sum of two Gaussian functions, used to measure the frequency splitting $\Delta E_{\text {fit }}$ between the two peaks. The Rabi frequency and excitation duration used for each measurement are indicated on the right. 


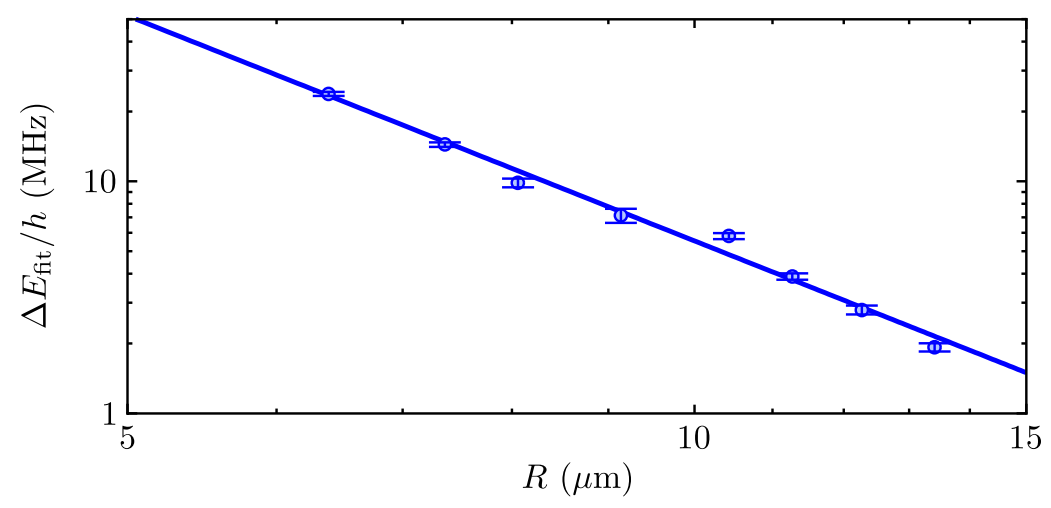

Figure 9.7: Experimental determination of $C_{3}$. Double logarithmic plot of the splitting $\Delta E$ between the peaks at resonance, as a function of $R$. The solid line is a fit by a power law, which gives an exponent of $-3.2 \pm 0.2$. Fixing the exponent to -3 gives the fitted coefficient $C_{3, \mathrm{fit}}=2.1 \pm 0.1 \mathrm{GHz} \cdot \mu \mathrm{m}^{3}$.

the calibration of the camera.

\section{Discussion of the observed shifts}

We observe in Figure 9.6 that the spectra at resonance are not centered exactly around the same frequency. We would expect to observe a symmetric evolution of the spectra around one central position as we vary the interatomic distance ${ }^{2}$. The measurements in Figure 9.6 were performed with different Rabi frequencies $\Omega$, leading to differential light shifts between the measurements. To rescale the frequency axis, we have calibrated the one-atom light shift by measuring the position of the oneatom line for a few values of $\Omega$. However, we have not systematically measured the position of the one-atom line for each scan. The observed residual shifts between the spectra come from calibration errors, most probably due to long-term drifts in the laser power seen by the atoms over the entirety of the measurement, leading to errors in our estimation of $\Omega$. However, our measurement of the $C_{3}$ coefficient is not affected by these global shifts since we are only interested in the frequency difference between the two peaks.

\footnotetext{
${ }^{2}$ As a reminder, the frequency $\delta /(2 \pi)=0$ in Figure 9.6 corresponds to the position of the oneatom line in a zero electric field. As a consequence, we do not expect the central position to be $\delta /(2 \pi)=0$, due to the presence of Stark shifts for the electric field $F_{z}=F_{\text {res }}$ used at resonance. Moreover, dipole couplings to other, non-resonant states also results in a van der Waals shift of the lines. Finally, possible residual light shifts in the two-photon spectroscopy might also play a role.
} 


\subsubsection{Switch of the interaction}

An interesting feature of the Förster resonance is that it allows us to use the electric field $F_{z}$ as a switch for the interatomic interaction. This can be seen directly in Figure 9.5, where we observe the transition from the Förster regime for $F_{z}=F_{\text {res }}$ to the van der Waals regime for larger values of $F_{z}$. In the Förster regime, the system shows strong interactions that scale as $1 / R^{3}$, whereas in the van der Waals regime the system shows weaker interactions with a $1 / R^{6}$ dependency. To illustrate this fact, we measure the one-atom and two-atom excitation spectra for an electric field $F_{z} \simeq 64 \mathrm{mV} / \mathrm{cm}$. For this value of the electric field, we expect a Förster defect of $\Delta /(2 \pi) \simeq-31 \mathrm{MHz}$, placing the atoms far from the Förster resonance. We first measure the position of the one-atom line by performing a spectroscopy on a single atom. This measurement allows us to calibrate the frequency axis, where $\delta=0$ now corresponds to the position one-atom Rydberg line ${ }^{3}$ for $F_{z}=64 \mathrm{mV} / \mathrm{cm}$. Under the same experimental conditions, we then perform the two-atom spectroscopy for two atoms that are separated by $R=9.1 \mu \mathrm{m}$. Figure 9.8 plots the probability of doubleloss $P_{00}$ as a function of the laser detuning. We observe the presence of a single excitation peak, which confirms that we are far from the Förster resonance. To extract the position of the center of the line in a simple way, we fit the spectrum by a Gaussian. We observe that the line is shifted by an energy $\Delta E$, with:

$$
\frac{\Delta E}{h}=882 \pm 222 \mathrm{kHz}
$$

We have seen in Chapter 7 that the shift of the double-excitation line corresponds to the van der Waals interaction energy between the two atoms. The measured value is compatible with the expected van der Waals shift obtained using Equation 9.22 for a Förster defect of $\Delta /(2 \pi) \simeq-31 \mathrm{MHz}$ :

$$
\frac{V_{\mathrm{vdW}}}{h}=-\frac{\left(\sqrt{2} C_{3}\right)^{2}}{\Delta} \frac{1}{R^{6}}=733 \mathrm{kHz} .
$$

This contrasts with the 7.1 MHz interaction energy we have measured at the Förster resonance (see Figure 9.7). Changing the field by $\simeq 30 \mathrm{mV} / \mathrm{cm}$ thus allows to change to interaction by a factor of 10 . We will use this ability to tune the interactions in Section 9.4 .

\footnotetext{
${ }^{3}$ Note that this convention is different from the one used in the rest of this Chapter where $\delta=0$ corresponds to the position of the one-atom line for $F_{z}=0 \mathrm{mV} / \mathrm{cm}$. We exceptionally change the convention in this paragraph to simplify the interpretation of the two-atom spectrum.
} 


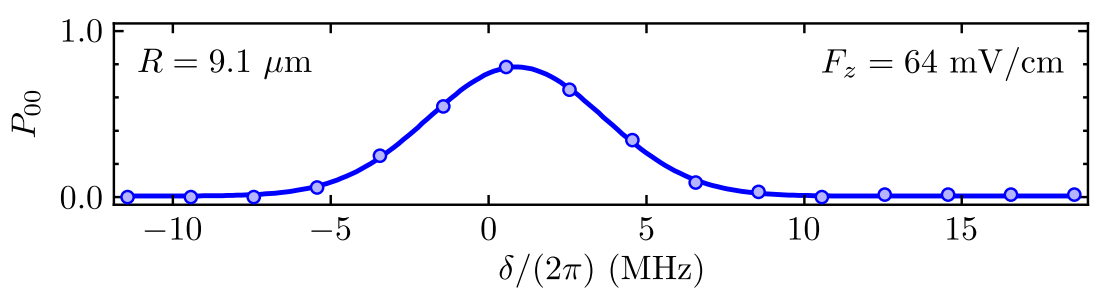

Figure 9.8: Switching the interaction. Two-atom spectrum obtained for an electric field $F_{z}=64 \mathrm{mV} / \mathrm{cm}$. The probability $P_{00}$ shows only one peak, which confirms that we have placed the system in the van der Waals regime. The interaction energy between the two atoms is given by the energy shift of the two-atom line compared to the one-atom line. The frequency $\delta /(2 \pi)=0$ corresponds to the position one-atom line. The solid line is a fit of the spectrum by a Gaussian [Pérez Galván, Zhao, and Orozco (2008)], which allows us to measure that the two-atom line is centered at the position $\delta /(2 \pi)=882 \pm 222 \mathrm{kHz}$.

\subsubsection{Simulation using the Schrödinger equation}

A difficulty of the spectroscopic measurement resides in the fact that we measure double excitation probabilities for different interaction strengths, both in the van der Waals and in the Förster regime. Out of resonance, our excitation lasers directly couple $|g g\rangle$ to $|d d\rangle$, whereas on resonance the lasers only partially couple $|g g\rangle$ to $|+\rangle$ and $|-\rangle$. Moreover, we have seen that the excitations lasers are detuned from the intermediate level $|\widetilde{d g}\rangle$ by an energy which is of the same order as $\hbar \Omega$, so that the state $|\widetilde{d g}\rangle$ also has to be considered in the excitation dynamics of the system. Finally, the atoms interact during the excitation, which complicates even more the excitation of the system. To get a better understanding of our two atom spectra, we simulate them numerically.

\section{Hamiltonian of the system}

The two-atom system is described, in the basis $\{|g g\rangle,|\widetilde{d g}\rangle,|d d\rangle,|\widetilde{p f}\rangle\}$, by the Hamiltonian:

$$
\hat{H}=\hbar\left(\begin{array}{cccc}
\Delta E_{g g} & \sqrt{2} \Omega / 2 & 0 & 0 \\
\sqrt{2} \Omega / 2 & \Delta E_{d g}-\delta / 2 & \sqrt{2} \Omega / 2 & 0 \\
0 & \sqrt{2} \Omega / 2 & \Delta E_{d d}-\delta & 2 \sqrt{2} C_{3, \mathrm{th}} / R^{3} \\
0 & 0 & 2 \sqrt{2} C_{3, \mathrm{th}} / R^{3} & \Delta_{0}+\Delta E_{p f}-\delta
\end{array}\right)
$$

where $\Delta E_{i j}=-\alpha_{i j} F_{z}^{2} / 2$ is the Stark shift for the pair state $|i j\rangle, \Delta_{0}$ is the Förster defect in a zero electric field and $\delta$ is the laser detuning. For different parameters $R$, $\Omega, \tau$ that we used experimentally (see Figure 9.6), we simply solve numerically the Schrödinger equation without taking into account dissipation or detection efficiency. 
a

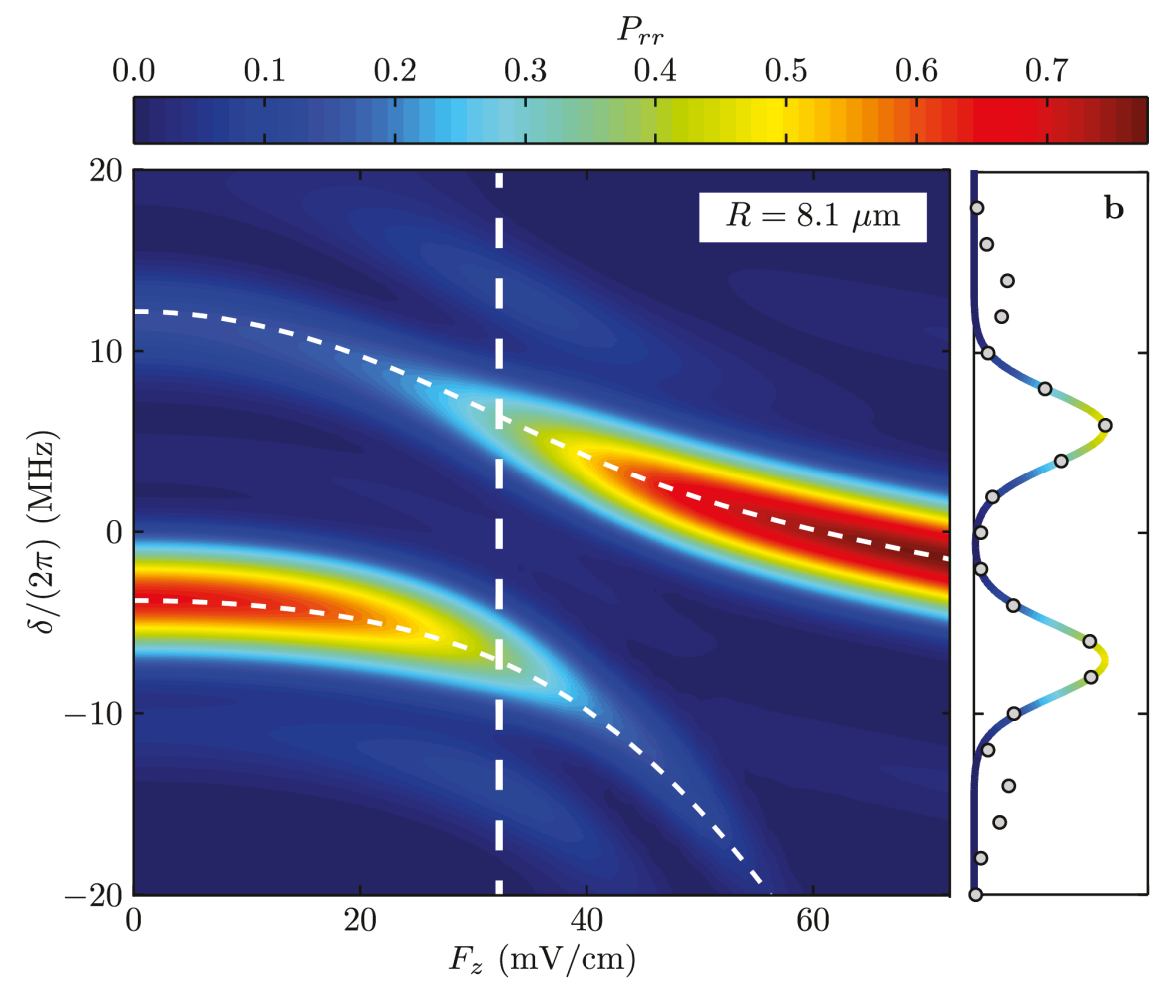

c

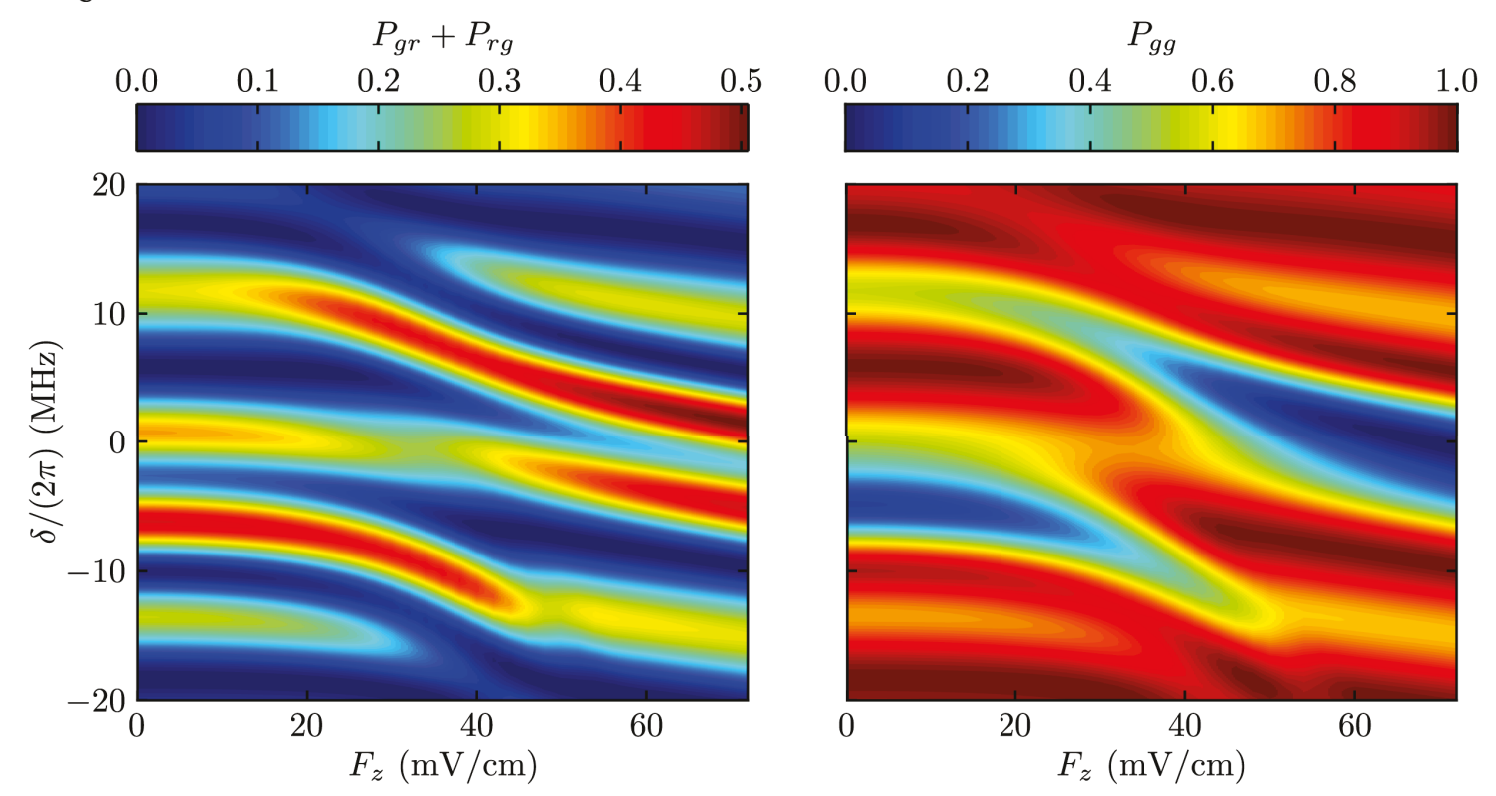

Figure 9.9: Numerical simulation of the spectroscopy at the Förster resonance. a, Two-atom excitation spectrum where the population in the doubly-excited state $P_{r r}$ is plotted as a function of $\delta$ and $F_{z}$. We observe the avoided crossing between the resonant pair states. The white vertical dashed line indicates the position of the Förster resonance. The white curved dashed lines represents the Stark map calculated in the presence of interactions with $C_{3}=C_{3, \text { th }}$ b, Spectrum $P_{r r}(\delta)$ at resonance for $R=8.1 \mu \mathrm{m}$. The solid line is a fit by a sum of two Gaussian functions. c, The populations $P_{g g}$ and $P_{g r}+P_{r g}$ are given for completeness. They show qualitative agreement with the experimental observations (see Figure 9.5). 


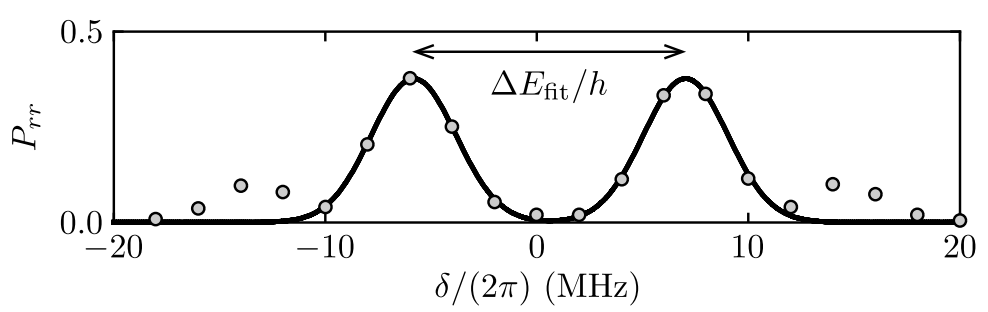

Figure 9.10: Bias introduced by the fitting procedure. For the interatomic distance $R=8.1 \mu \mathrm{m}$ and for the parameters $\Omega$ and $\tau$ used experimentally, we simulate the double excitation spectrum (black disks). To extract the distance between the two peaks in a simple way, we fit the spectrum by a sum of two Gaussian functions. The fit gives $\Delta E_{\text {fit }} / h=$ $12.8 \pm 0.1 \mathrm{MHz}$. We use the simulation to evaluate the bias introduced by this fitting procedure on the measurement of $\Delta E$.

\section{Results of the simulation}

The results of the simulation of the spectroscopy are shown in Figure 9.9 and qualitatively reproduce the observations made experimentally (see Figure 9.5). As expected, the double excitation spectrum $P_{r r}$ shows the avoided crossing between the two resonant states $|d d\rangle$ and $|\widetilde{p f}\rangle$. We also observe qualitative agreement for the populations $P_{g g}$ and $P_{g r}+P_{r g}$ (see Figure 9.9c). Finally, Figure 9.9b plots the spectrum at resonance (black disks), where the solid line is a fit by a sum of two Gaussian functions, used to measure the distance between the two peaks. As expected, this phenomenological fit does not reproduce completely the structure of the spectrum at resonance. In particular, the simulated spectra show secondary side lobes next to the two main peaks. As a consequence, we use the simulation to test the validity of our fitting procedure.

\section{Bias introduced by the fitting procedure}

Figure 9.10 shows the simulated spectrum at the Förster resonance using the same experimental parameters, $\Omega$ and $\tau$ as in Figure 9.5 and for a separation distance $R \simeq$ $8.1 \mu \mathrm{m}$. From the fit by a sum of two Gaussian functions (solid line in Figure 9.10), we extract a splitting $\Delta E_{\mathrm{fit}} / h=12.8 \pm 0.1 \mathrm{MHz}$ between the two peaks. At a distance $R=8.1 \mu \mathrm{m}$, we expect the states $|+\rangle$ and $|-\rangle$ to be separated by:

$$
\frac{\Delta E}{h}=\frac{1}{2 \pi} \frac{2 \sqrt{2} C_{3, \text { th }}}{R^{3}}=13.7 \mathrm{MHz} .
$$

As a consequence, the fit of $\Delta E_{\text {fit }}$ gives a slight underestimate of the energy splitting $\Delta E$. Reproducing the same simulation for all the different values of $R, \Omega$ and $\tau$ used experimentally in Figure 9.6 gives similar results. In the end we observe that: 




Figure 9.11: Experimental sequence for observing the Förster oscillation between two atoms. After exciting the atoms to $|d d\rangle$ (first $\pi$ pulse), we switch on the resonant interaction for a variable amount of time $\tau$ by tuning the electric field to $F_{\text {res }}$. The system oscillates back and forth between the two pair states $|d d\rangle$ and $|\widetilde{p f}\rangle$ for the duration $\tau$. A second optical $\pi$-pulse allows reading out the state of the system.

$$
\Delta E=(1.08 \pm 0.02) \times \Delta E_{\mathrm{fit}} .
$$

We thus take this correction factor into account in the spectroscopic measurement of the $C_{3}$ coefficient presented in Section 9.3.3:

$$
C_{3, \text { Spectro }}=1.08 \times C_{3, \mathrm{fit}}=2.26 \pm 0.15 \mathrm{GHz} \cdot \mu \mathrm{m}^{3} .
$$

The remaining discrepancy with the expected value $C_{3, \mathrm{th}} \simeq 2.54 \mathrm{GHz} \cdot \mu \mathrm{m}^{3}$ probably comes from a systematic error on our measurement of $R$ due to a $\simeq 5 \%$ uncertainty on the calibration of the camera.

\subsection{Observation of Förster oscillations}

In this Section, we study the coherence properties of the system at the Förster resonance.

\subsubsection{Experimental sequence}

Figure 9.11 shows the experimental sequence. We first turn the traps off for $\simeq 2 \mu \mathrm{s}$. We shine a $100 \mathrm{~ns}$ optical $\pi$-pulse on the atoms to prepare the system in the state $|d d\rangle$. We perform the optical excitation in a $F \simeq 64 \mathrm{mV} / \mathrm{cm}$ electric field, thus placing the atoms out of resonance, where they interact in the van der Waals regime as observed in Section 9.3.4. During the preparation, the small interatomic interactions 
between the atoms of $\sim 100 \mathrm{kHz}$ allow us to easily overcome the Rydberg blockade, and to place the two atoms in a pure $|d d\rangle$ state. We then switch rapidly (risetime below $10 \mathrm{~ns}$ ) the electric field to $F_{z}=F_{\text {res }}$ for a variable duration $\tau$ to turn on the resonant interaction in the system. Switching the electric field back to its initial value $F_{z} \simeq 64 \mathrm{mV} / \mathrm{cm}$ then allows us to freeze the interaction. We finally read out the state of the system by applying a second optical $\pi$-pulse that couples $|d d\rangle$ back to $|g g\rangle$, while leaving $|\widetilde{p f}\rangle$ unchanged. We repeat so sequence $\sim 100$ times and reconstruct the recapture probabilities $P_{i j}(i, j \in\{0 ; 1\})$. Assuming perfect excitation and read out, the probability $P_{00}$ (resp. $\left.P_{11}\right)$ coincides with $P_{p f}\left(\right.$ resp. $\left.P_{d d}\right)$.

\subsubsection{Experimental results}

Figure. 9.12 shows the measured evolution of $P_{11}$ as a function of $\tau$ for different interatomic distances $R$ ranging from $7.4 \mu \mathrm{m}$ to $13.4 \mu \mathrm{m}$. We observe the expected oscillations of $P_{11}$, which is a direct consequence of the resonant interactions between the two atoms (see Equation 8.8). The oscillation frequencies $\nu_{\mathrm{osc}}$ depend on the distance $R$, which further confirms that we are observing the interaction-induced coherent oscillation between $|d d\rangle$ and $|\widetilde{p f}\rangle$ at the Förster resonance. A fit of the data for $R=8.07 \mu \mathrm{m}$ gives the oscillation frequency:

$$
\nu_{\mathrm{osc}}=12.3 \pm 0.8 \mathrm{MHz} \text {, }
$$

where the error bar comes from the fit and a $150 \mathrm{~nm}$ statistical error in the determination of $R$. This value is close to the expected oscillation frequency $2 \sqrt{2} C 3 /\left(2 \pi R^{3}\right)=$ 13.7 MHz. We attribute the observed deviation to a systematic error on the measurement of $R$. As mentioned in Chapter 8, the presence of only two atoms separated by a controlled distance is fundamental to this observation. In disordered systems of atoms, oscillations with different frequencies average out due to the presence of a distribution of interatomic distances, which prevents this direct observation of the coherence of the interaction.

\subsubsection{Measurement of the $C_{3}$ coefficient}

To extract the oscillation frequency $\nu_{\mathrm{osc}}$, we fit the dynamics by damped $\operatorname{sines}^{4}$ (see Figure 9.12). Figure 9.13 shows a double-logarithmic plot of the obtained values of $\nu_{\text {osc }}$ for the different separation distances $R$. The data shows a power-law behavior of

\footnotetext{
${ }^{4}$ Simulations of the dynamics using the total Hamiltonian in Equation 9.27 show that this fitting procedure allows extracting the correct value for $C_{3}$, with no need for a correction factor.
} 

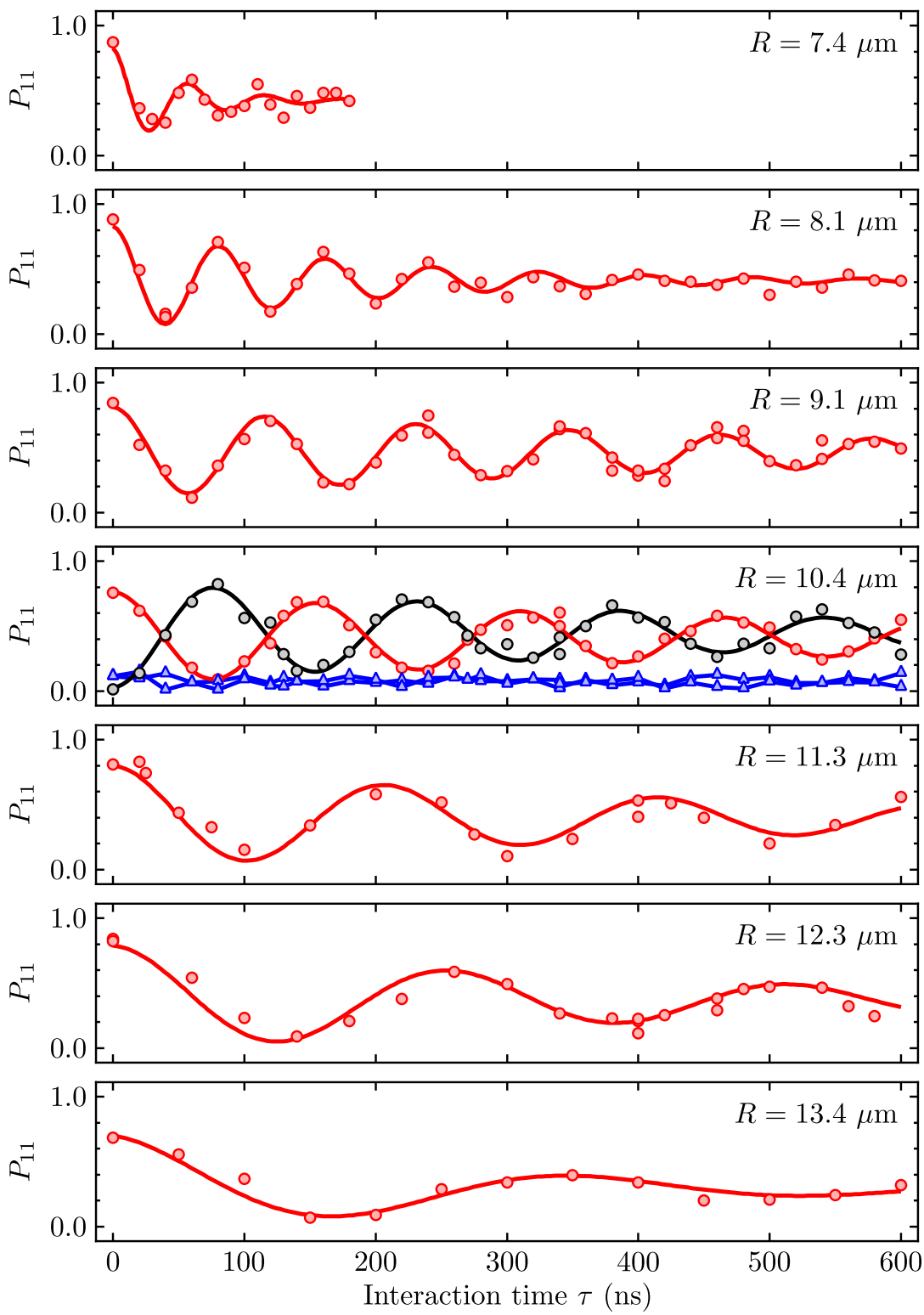

Figure 9.12: Observation of Förster oscillations between two atoms. Evolution of $P_{11}$ as a function of the interaction time for different interatomic distances. $P_{11}$ oscillates at a frequency $\nu_{\text {osc }}$ that depends on $R$. For information, the populations $P_{00}$ (black), $P_{01}$ and $P_{10}$ (blue) are also given for the $R=10.4 \mu \mathrm{m}$ distance. The solid lines are fits by damped sines. 


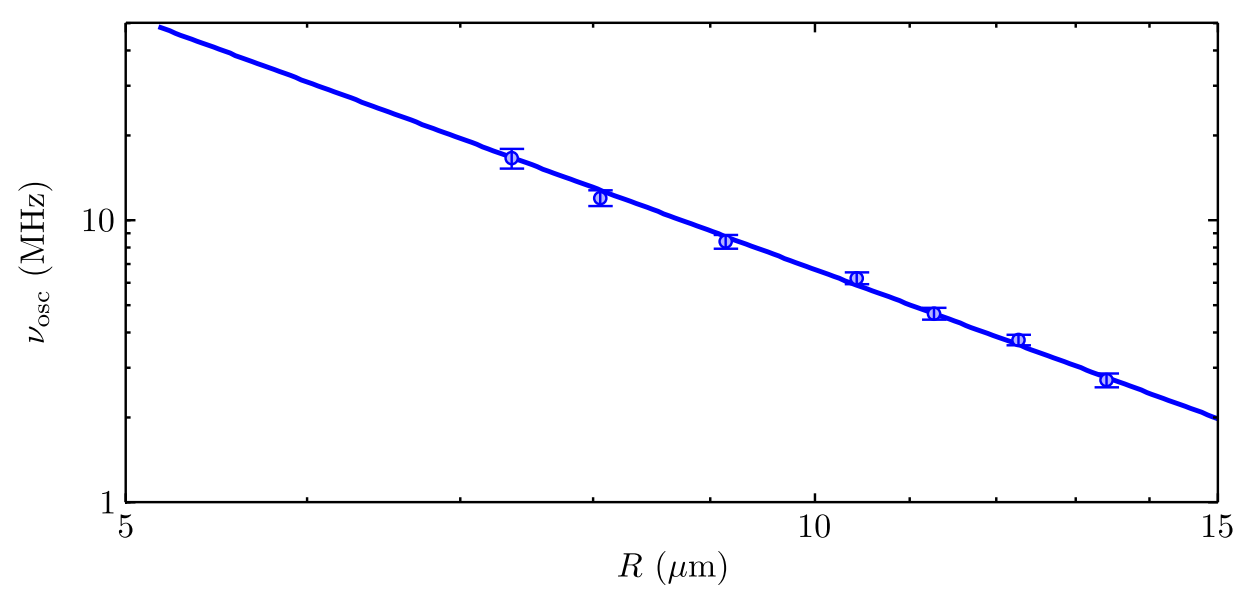

Figure 9.13: Experimental determination of $C_{3}$. Double logarithmic plot of the oscillation frequency $\nu_{\mathrm{osc}}$ as a function of $R$. The error bars come from the fit of the oscillation frequencies as well as from the initial dispersion in interatomic distances due to the temperature of the atoms $\left(\sigma_{R}=170 \mathrm{~nm}\right)$. A fit by a power law gives an exponent $-2.9 \pm 0.1$. This value of the exponent is in good agreement with the expected $1 / R^{3}$ behavior. Fixing the exponent to -3 , the fit gives $C_{3}=2.44 \pm 0.06 \mathrm{GHz} \cdot \mu \mathrm{m}^{3}$ (solid line).

exponent $-2.9 \pm 0.1$, again in excellent agreement with the expected $R^{-3}$ behavior. Fixing the exponent to -3 , we obtain:

$$
C_{3, \mathrm{osc}}=2.44 \pm 0.06 \mathrm{GHz} \cdot \mu \mathrm{m}^{3} .
$$

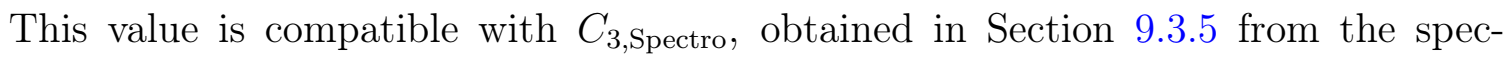
troscopic measurement. This value is also close to the theoretical value, where the observed mismatch is explained by $\mathrm{a} \simeq 5 \%$ systematic error on $R$ due to calibration errors.

\subsubsection{Discussion of the limitations}

The measured oscillations show a contrast that is smaller than $100 \%$, as well as some damping. Most of the limitations discussed in Chapter 8 are also present in this experiment. In particular, dissipation during the optical pulses reduces the efficiency of the preparation and read-out steps, leading to a reduction of the contrast of the oscillations. Temperature effects lead to some dephasing which results in damping. Other limitations discussed in Chapter 8 related to the presence of resonant interactions during the preparation and read out of the system are not present here since we have the possibility to switch off the resonant interactions by pulsing the electric field. However, fluctuations in the applied electric field are another source of dephasing in the system. We discuss those limitations in this Section. 


\section{Temperature effects}

We have seen in Section 8.6.4 that the finite temperature leads to fluctuations in the interatomic distance that are summarized in Equation 8.45:

$$
R(t)=R_{0}+\Delta z_{0}+\Delta v_{z} t
$$

The random variable $\Delta z_{0}$ accounts for shot-to-shot fluctuations of $R$ with a standard deviation $\sigma_{R} \simeq 170 \mathrm{~nm}$. The random variable $\Delta v_{z}$ accounts for the thermal motion $v_{\mathrm{th}} \simeq 70 \mathrm{~nm} . \mu \mathrm{s}^{-1}$ of the atoms during the sequence. Note that the maximal interaction time in Figure 9.12 is $\tau=600$ ns. At the end of this interaction time, the thermal motion of the atoms has led to a variation $\Delta R$ of the separation distance between the atoms, that we estimate to be of the order of:

$$
\Delta R \sim \sqrt{2} v_{\mathrm{th}} \tau \simeq 60 \mathrm{~nm}
$$

For $\tau=600 \mathrm{~ns}$, we also estimate that the variation in $R$ due to forces between the atoms is (see Section 8.6.4):

$$
\Delta R \sim \frac{3}{2} \frac{\hbar C_{3}}{m_{\mathrm{eff}} R^{4}} \tau^{2} \simeq 4 \mathrm{~nm}
$$

where we have taken $R=7.4 \mu \mathrm{m}$ (the shortest interactomic distance in Figure 9.12). As a consequence, we first consider only shot to shot fluctuations $\left(R=R_{0}+\Delta z_{0}\right)$. Those fluctuations in $R$ lead to fluctuations in the interaction energy between the two atoms so that $P_{d d}$ is the average of oscillations with different frequencies:

$$
\begin{aligned}
P_{d d} & =\left\langle\cos ^{2}\left(2 \pi \frac{\sqrt{2} C_{3}}{R^{3}} \tau\right)\right\rangle_{R} \\
& =\frac{1}{2}+\frac{1}{2}\left\langle\cos \left(2 \pi \frac{2 \sqrt{2} C_{3}}{R^{3}} \tau\right)\right\rangle_{R} \\
& =\frac{1}{2}+\frac{1}{2} \frac{1}{\sqrt{2 \pi} \sigma_{R}} \int_{-\infty}^{+\infty} d R \cos \left(2 \pi \frac{2 \sqrt{2} C_{3}}{R^{3}} \tau\right) \exp \left(-\frac{\left(R-R_{0}\right)^{2}}{2 \sigma_{R}^{2}}\right) .
\end{aligned}
$$

For our range of experimental parameters, $\Delta z_{0} \ll R_{0}$. In this limit, we obtain an approximate value for the integral in Equation 9.36 by expanding around $R_{0}$ the term $\propto 1 / R^{3}$, and by using the properties of Fourier transform for Gaussian distributions:

$$
P_{d d}=\frac{1}{2}+\frac{1}{2} \exp \left[-\frac{1}{2}\left(2 \pi \frac{3 \sigma_{R}}{R_{0}} \frac{2 \sqrt{2} C_{3}}{R_{0}^{3}} \tau\right)^{2}\right] \cos \left(2 \pi \frac{2 \sqrt{2} C_{3}}{R_{0}^{3}} \tau\right)
$$



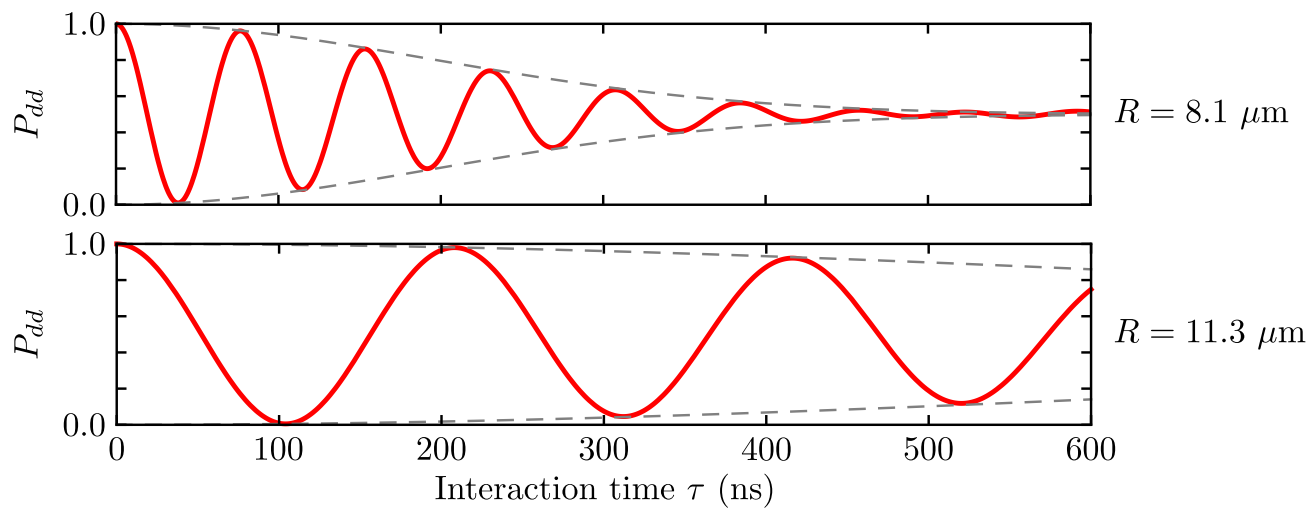

Figure 9.14: Effect of the finite temperature of the atoms. Fluctuations in the distance $R$ lead to some dephasing. The solid lines show the solution of the Schrödinger equations averaged over 1000 realizations of the experiment for $R=8.1 \mu \mathrm{m}$ and $R=11.3 \mu \mathrm{m}$. The grey dashed lines plot the decaying envelopes calculated in Equation 9.37.

We observe that the shot-to-shot fluctuations in the $R$ lead to a Gaussian damping of the oscillations. The decaying envelopes for the Förster oscillation obtained in Equation 9.37 are plotted in Figure 9.14 for different distances (grey dashed lines).

\section{Solutions of the Schrödinger equations}

We now compare the result of the previous calculation to a numerical simulation of the system. We simulate the two-atom system by solving numerically the Schrödinger equations. We consider that we have prepared the system in $|d d\rangle$ with a $100 \%$ efficiency. At the Förster resonance, the Hamiltonian of the interacting system written in the basis $\{|d d\rangle ;|\widetilde{p f}\rangle\}$ is:

$$
\hat{H}=\hbar\left(\begin{array}{cc}
0 & \frac{\sqrt{2} C_{3}}{\left(R_{0}+\Delta z_{0}+\Delta v_{z} t\right)^{3}} \\
\frac{\sqrt{2} C_{3}}{\left(R_{0}+\Delta z_{0}+\Delta v_{z} t\right)^{3}} & 0
\end{array}\right),
$$

where $\Delta z_{0}$ and $v_{z}$ are random variables with Gaussian distributions and standard deviations $\sigma_{R}$ and $\sqrt{2} v_{\text {th }}$, as described in Section 8.6.4. We average the solutions of the Schrödinger equations over 1000 realizations of the experiment. The results are shown in Figure 9.14 for $R=8.1 \mu \mathrm{m}$ and for $R=11.3 \mu \mathrm{m}$ (solid lines). As expected, we observe that shot-to-shot fluctuations in the positions of the atoms lead to a damping in the Förster oscillations. The decaying envelopes of the oscillations agree well with the ones predicted in Equation 9.37 (grey dashed lines), which confirms that the effect of the thermal velocity of the atoms on the damping is small for the short interaction times we are probing in this experiment $(\tau \leq 600 \mathrm{~ns})$. Finally, we observe that due 


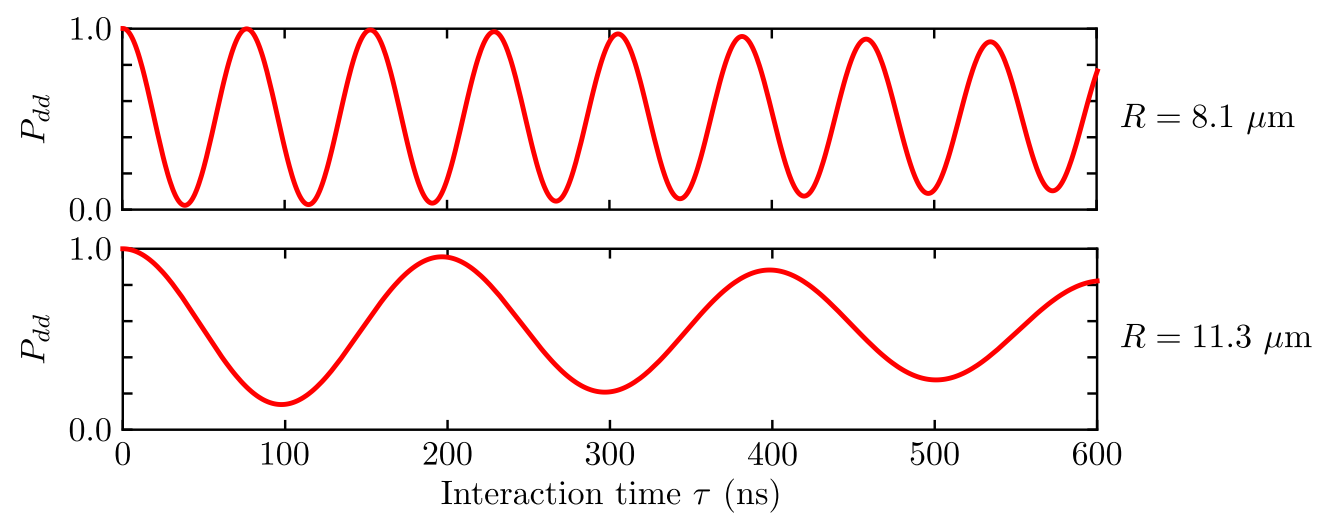

Figure 9.15: Effect of fluctuations of the electric field on the oscillations. Fluctuations of the electric field leads to more dephasing. The solid lines show the solution of the Schrödinger equations averaged over 1000 realizations of the experiment for $R=8.1 \mu \mathrm{m}$ and $R=11.3 \mu \mathrm{m}$. We observe that the effect dominates for the largest interatomic distances.

to the $1 / R^{3}$ dependence of the interaction energy, the temperature effects dominate at small distances. Indeed, we observe a significant damping for the $R=8.1 \mu \mathrm{m}$ distance. However, for the $R=11.3 \mu \mathrm{m}$ distance, we qualitatively observe that the calculation does not reproduce the damping observed experimentally in Figure 9.12. Indeed, another effect contributes to the damping at larger distances.

\section{Fluctuations of the electric field}

Electric noise in the voltage sources we use to pulse the electric field to resonance leads to fluctuations of $F_{z}$, with a standard deviation that we estimate to be of the order of a few $\mathrm{mV} . \mathrm{cm}^{-1}\left(\sigma_{F} \simeq 4 \mathrm{mV} . \mathrm{cm}^{-1}\right)$. To take those fluctuations into account in our model, we introduce the Förster defect $\Delta$ in the Hamiltonian describing the system. We first simulate the effect of the fluctuations of the electric field in the absence of temperature effects. In this case, the interatomic distance is fixed to $R=R_{0}$, and the Hamiltonian of the system reads:

$$
\hat{H}=\hbar\left(\begin{array}{cc}
0 & \frac{\sqrt{2} C_{3}}{R_{0}^{3}} \\
\frac{\sqrt{2} C_{3}}{R_{0}^{3}} & \Delta
\end{array}\right)
$$

where $\Delta$ is a random variable centered around $0 \mathrm{MHz}$, with a standard deviation:

$$
\sigma_{\Delta}=\left(\alpha_{p f}-\alpha_{d d}\right) F_{\text {res }} \sigma_{F} \simeq 2 \mathrm{MHz}
$$


As a consequence, the system is not tuned to exact resonance at all times. The interaction energy between the two atoms fluctuates from shot-to-shot, which is a source of dephasing since the interaction dynamics of the system result from the averaging of Förster oscillations with slightly different frequencies ${ }^{5}$. Figure 9.15 shows the solutions of the Schrödinger equations averaged over 1000 realizations of the experiment for $R=8.1 \mu \mathrm{m}$ and for $R=11.3 \mu \mathrm{m}$ (solid lines). In this case, we note that the effect dominates at large distances:

- For $R=8.1 \mu \mathrm{m}$, the interaction energy between the atoms $(\sim 13 \mathrm{MHz})$ is large compared to the fluctuations of the Förster defect $\left(2 \sqrt{2} C_{3} / R^{3} \gg \Delta\right)$, and we observe only a slight damping of the oscillations in Figure 9.15.

- For larger interatomic distances, the interaction energy between the atoms decreases, and the system is more sensitive to fluctuations of $\Delta$. In the case $R=11.3 \mu \mathrm{m}$ for example, the interaction energy between the atoms $(\sim 5 \mathrm{MHz})$ is of the same order as $\sigma_{\Delta}$. As a consequence, we observe in Figure 9.15 a more significant damping of the oscillations.

Fluctuations of the electric field thus contribute to the damping of the oscillations observed for large interatomic distances.

\section{Qualitative comparison to the data}

We have seen that dephasing in the system mainly comes from the thermal motion of the atoms at small distances, and from fluctuations of the electric field at larger distances. Note that in principle, both sources of damping can be strongly decreased by technical improvements in the setup, using colder atoms [Kaufman, Lester, and Regal (2012); Thompson et al. (2013)] and more stable voltage sources. We now perform a simulation that includes those two effects by averaging the solutions of the Schrödinger equation over 1000 realizations of the experiment, for the system described by the total Hamiltonian:

$$
\hat{H}=\hbar\left(\begin{array}{cc}
0 & \frac{\sqrt{2} C_{3}}{\left(R_{0}+\Delta z_{0}+\Delta v_{z} t\right)^{3}} \\
\frac{\sqrt{2} C_{3}}{\left(R_{0}+\Delta z_{0}+\Delta v_{z} t\right)^{3}} & \Delta
\end{array}\right) .
$$

\footnotetext{
${ }^{5}$ When $\Delta \neq 0$, the system is out of resonance. Because the noise on the electrodes has a frequency $\ll 1 \mathrm{MHz}$, this leads to shot-to-shot fluctuations in the frequency of the Förster oscillation. Out of resonance, the contrast of the oscillation is also reduced, and thus the transfer of population from $|d d\rangle$ to $|\widetilde{p f}\rangle$ is not maximal. As the consequence, the damping of the oscillations due to fluctuations of the electric field is asymmetric.
} 


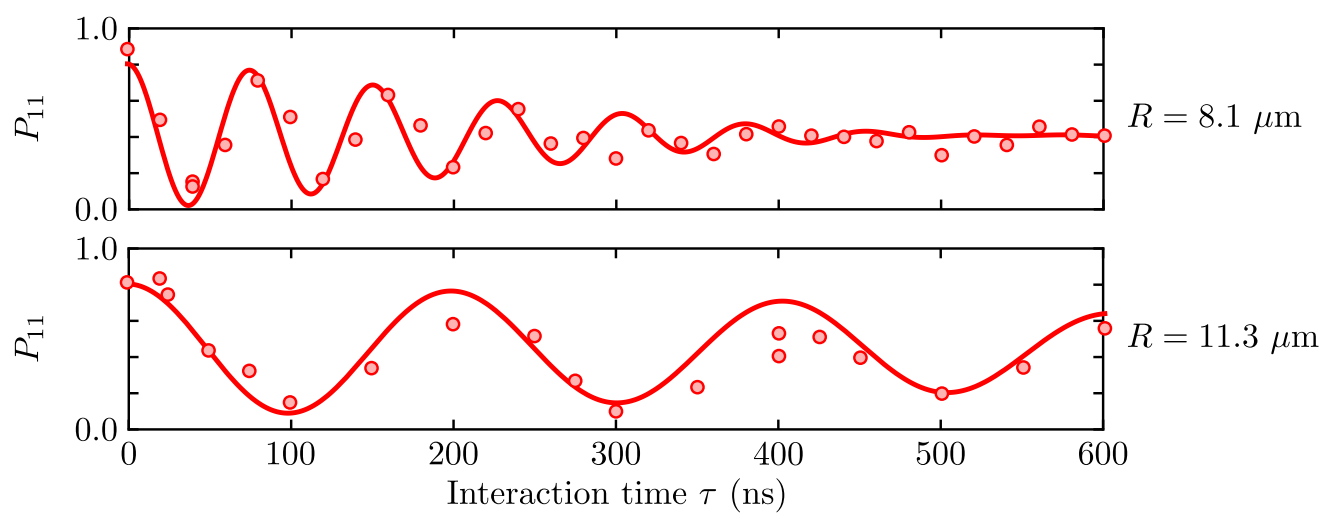

Figure 9.16: Qualitative comparison to the data. The solid lines show the average of 1000 realizations of the simulation including temperature effects and fluctuations in the electric field. The curves are multiplied by the phenomenological factor 0.8 to take into account limitations in the preparation of the system. The simulation qualitatively reproduces the data (red circles).

We include in this model the effect of the dissipation during the preparation in a phenomenological way by adapting the initial conditions in the Schrödinger equation. Due to the presence of dissipation during our optical excitation (see Chapter 6), we have measured that we prepare the system in $|d d\rangle$ with a $80 \%$ probability (see the maximal double excitation in the independent measurement of the two-atom spectrum in Figure 9.8). We use for initial conditions $P_{d d}=0.8$ and $P_{p f}=0$. We observe in Figure 9.16 that the simulation qualitatively reproduces the observed damping, which suggests that our sources of damping are well understood. However, a more quantitative study would require to solve the optical Bloch equations for the system in order to take into account dissipation during the preparation as well as during the read out. Other systematic effects like a constant residual Förster defect (detuning the electric field from the Förster resonance by $2 \mathrm{mV} . \mathrm{cm}^{-1}$ leads to a Förster defect of $\simeq 1 \mathrm{MHz}$ ) or small gradients in the electric field might also contribute to the reduction of the contrast of the Förster oscillations. Finally, we have not taken into account the presence of the magnetic field or the coupling to other non-resonant states that also contribute to the interaction, as we will see in Section 9.5.

\subsection{Angular dependence of the interaction}

We now extend the study of the resonance between $|d d\rangle$ and $|p f\rangle$ to the case where the internuclear axis is not aligned with the quantization axis, and measure the angular dependence of the interaction [Reinhard et al. (2007); Carroll et al. (2004)], in view of tailoring even further the interactions between two particles. In the most general 
Table 9.2: Polarizabilities of the pair states of interest in this Chapter. The polarizabilities are obtained in the weak field limit as described in Chapter 5. They are given in $\mathrm{kHz} .(\mathrm{mV} / \mathrm{cm})^{-2}$.

\begin{tabular}{ll}
\hline State & $\boldsymbol{\alpha}$ \\
\hline$|d d\rangle$ & 1.1 \\
$\left|\widetilde{p f_{1}}\right\rangle$ & 16.45 \\
$\left|\widetilde{p f}_{2}\right\rangle$ & 26.7 \\
$\left|\widetilde{p f}_{3}\right\rangle$ & 32.5 \\
\hline
\end{tabular}

case, the interaction Hamiltonian reads (see Chapter 7):

$$
\begin{aligned}
\hat{V}_{\text {dip }}= & \frac{1}{4 \pi \epsilon_{0}} \frac{1}{R^{3}}\left[\frac{1-3 \cos ^{2} \theta}{2}\left(\hat{d}_{A,+} \hat{d}_{B,-}+\hat{d}_{A,-} \hat{d}_{B,+}+2 \hat{d}_{A, 0} \hat{d}_{B, 0}\right)\right. \\
& +\frac{3}{\sqrt{2}} \sin \theta \cos \theta\left(\hat{d}_{A,+} \hat{d}_{B, 0}-\hat{d}_{A,-} \hat{d}_{B, 0}+\hat{d}_{A, 0} \hat{d}_{B,+}-\hat{d}_{A, 0} \hat{d}_{B,-}\right) \\
& \left.-\frac{3}{2} \sin ^{2} \theta\left(\hat{d}_{A,+} \hat{d}_{B,+}+\hat{d}_{A,-} \hat{d}_{B,-}\right)\right]
\end{aligned}
$$

where $\theta$ is the angle between the quantization axis and the internuclear axis. For $\theta \neq 0^{\circ}, \hat{V}_{\text {dip }}$ induces some couplings to other Zeeman sub-levels $\left(\Delta M_{\text {tot }}=0, \pm 1, \pm 2\right)$, which influence the interaction.

\subsubsection{Several Förster resonances}

For $\theta=0^{\circ}$, we have seen that there exists a Förster resonance between the states $|d d\rangle$ and $|\widetilde{p f}\rangle$, which corresponds to $\Delta M_{\text {Tot }}=0$. For $\theta \neq 0^{\circ}$, the interaction Hamiltonian $\hat{V}_{\text {dip }}$ couples $|d d\rangle$ to the states $\left|p f_{i}\right\rangle$ and $\left|f p_{i}\right\rangle$, where:

$$
\left\{\begin{aligned}
\left|p f_{1}\right\rangle & \equiv\left|61 P_{1 / 2}, M_{J}=1 / 2 ; 57 F_{5 / 2}, M_{J}=5 / 2\right\rangle \quad\left(\Delta M_{\mathrm{Tot}}=0\right) \\
\left|p f_{2}\right\rangle & \equiv\left|61 P_{1 / 2}, M_{J}=1 / 2 ; 57 F_{5 / 2}, M_{J}=3 / 2\right\rangle \quad\left(\Delta M_{\mathrm{Tot}}=1\right) \\
\left|p f_{3}\right\rangle & \equiv\left|61 P_{1 / 2}, M_{J}=1 / 2 ; 57 F_{5 / 2}, M_{J}=1 / 2\right\rangle \quad\left(\Delta M_{\mathrm{Tot}}=2\right)
\end{aligned}\right.
$$

We define the symmetrized states $\left|\widetilde{p f}_{i}\right\rangle$ : 


$$
\left|\widetilde{p f}_{i}\right\rangle \equiv \frac{\left|p f_{i}\right\rangle+\left|f p_{i}\right\rangle}{\sqrt{2}}
$$

The polarizabilities of the pair states $\left\{|d d\rangle ;\left|\widetilde{p f} f_{i}\right\rangle\right\}$ are given in Table 9.2. They are obtained by summing the one-atom polarizabilities calculated in the weak field limit as described in Chapter 5 .

We notice that the polarizability $\alpha$ depends on $M_{J}$. Figure 9.17 shows the evolution of the energies of the different states as a function of the electric field, in the absence of dipole-dipole interactions. We observe that the pair state $|d d\rangle$ can be brought in resonance with the three pair states $\left|\widetilde{p f}_{i}\right\rangle(i \in\{1 ; 2 ; 3\})$ for three different values of the electric field. In the absence of magnetic field, we thus expect three Förster resonances for the system. We note that each resonance corresponds to a different value of $\Delta M_{\text {Tot }}$ and is thus associated with one particular angular prefactor in the dipole-dipole interaction Hamiltonian in Equation 9.42. Here, we are interested in the resonant dipole-dipole interaction between $|d d\rangle$ and $\left|\widetilde{p f}_{1}\right\rangle$, as studied in Section 9.3 and Section 9.4. If we tune the field to this particular resonance, the other states $\left|\widetilde{p f}_{2}\right\rangle$ and $\left|\widetilde{p f}_{3}\right\rangle$ are slightly off-resonant. In a first approximation, the resonant dipole-dipole interaction Hamiltonian is given by:

$$
\hat{V}_{\text {dip }}=\frac{1}{4 \pi \epsilon_{0}} \frac{1}{R^{3}} \frac{1-3 \cos ^{2} \theta}{2}\left(\hat{d}_{A,+} \hat{d}_{B,-}+\hat{d}_{A,-} \hat{d}_{B,+}+2 \hat{d}_{A, 0} \hat{d}_{B, 0}\right),
$$

where we neglected the terms in the Hamiltonian that corresponds to states that are not exactly resonant. At the Förster resonance between $|d d\rangle$ and $\left|\widetilde{p f}_{1}\right\rangle$, we expect the interaction energy to vary as $\left(1-3 \cos ^{2} \theta\right) / 2$.

\subsubsection{Measurement of the angular dependence}

We now measure the angular dependence of the resonant interaction between the atoms for an electric field $F_{z} \simeq 32 \mathrm{mV} / \mathrm{cm}$, which corresponds to the position of the resonance between $|d d\rangle$ and $\left|\widetilde{p f}{ }_{1}\right\rangle$.

\section{Experimental procedure}

We reproduce the experimental sequence presented in Figure 9.11, and measure the coherent evolution of the two-atom system. The interatomic distance is fixed to $R=9.14 \mu \mathrm{m}$, and we vary the angle between $-90^{\circ}$ and $90^{\circ}$. For every angle, the strength of the interaction is given by the frequency $\nu_{\text {osc }}$ of the oscillation between $|d d\rangle$ and $\left|\widetilde{p f}{ }_{1}\right\rangle$. 


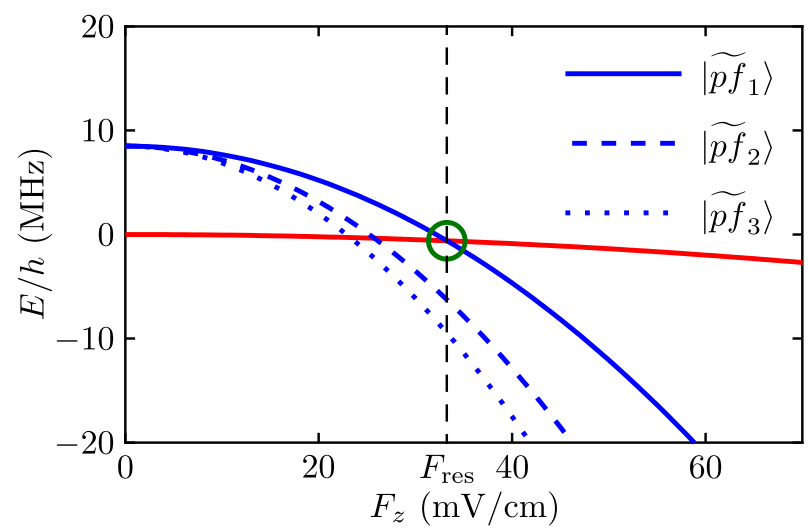

Figure 9.17: Stark effect of the pair states $\left|\widetilde{p f}_{i}\right\rangle$. The Förster resonances occur at the crossing between the blue curves and the red curve. Because the polarizability $\alpha$ depends on $M_{J}$, the different crossings occur for different electric fields $F_{z}$. We thus obtain three Förster resonances, each having its own angular dependence. The green circle indicates the position of the Förster resonance under study in this Chapter.

\section{Experimental results}

Figure 9.18 shows the coherent oscillations obtained for a few different angles. The anisotropy of the interaction is evident on the data, as the frequency of the Förster oscillation depends on $\theta$. For $\theta=0^{\circ}$ we observe the coherent oscillation at resonance, as described in Section 9.4. As $\theta$ increases, the frequency of the oscillation decreases. Around $\theta=55^{\circ}$, we do not observe any oscillation, but we only observe the decrease in the population $P_{d d}$ due to dephasing in the system (see Section 9.4.4). This angle corresponds to the so-called "magic angle" $\left(\theta=54.7^{\circ}\right)$, where the angular prefactor $\left(1-3 \cos ^{2} \theta\right) / 2$ (and thus the interaction) cancels. Increasing the angle even further allows us to observe again a coherent interaction with increasing frequencies. We measure the oscillation frequency $\nu_{\mathrm{osc}}$ as a function of $\theta$ by fitting the data by a damped sine. Figure 9.19a shows the result of this approach, where the sign of the interaction has not been determined experimentally, but has been inferred so as to match the the theoretical expectations. A polar representation of the same results is shown in Figure 9.19b, where we recognize the main features of the function $\left(1-3 \cos ^{2} \theta\right) / 2$, and in particular the cancellation for the angle $54.7^{\circ}$. We next analyze the measured angular dependence in more detail.

\section{Analysis of the results}

We fit the measured angular dependence shown in Figure 9.19 using the function:

$$
f(\theta)=K \frac{1-3 \cos ^{2} \theta}{2}
$$




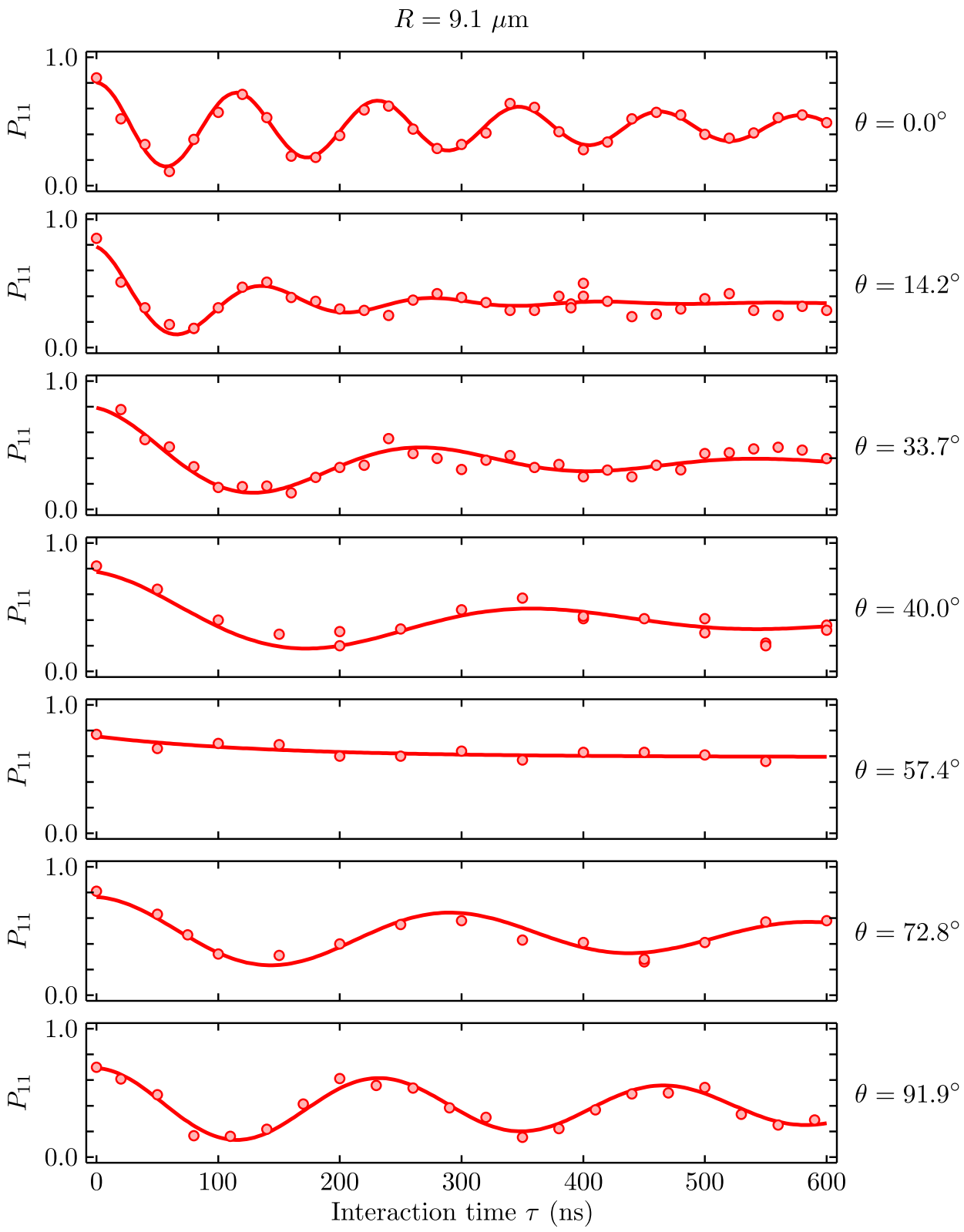

Figure 9.18: Förster oscillations for different angles $\boldsymbol{\theta}$. In this experiment the interatomic distance is fixed to $R=9.1 \mu \mathrm{m}$. The Figure shows the Förster oscillations obtained for various angle $\theta$. We observe that the frequency of the oscillation depends on $\theta$, which shows the anisotropy of the interaction. In particular the interaction cancels around the "magic angle" $\theta=54.7^{\circ}$. 
a

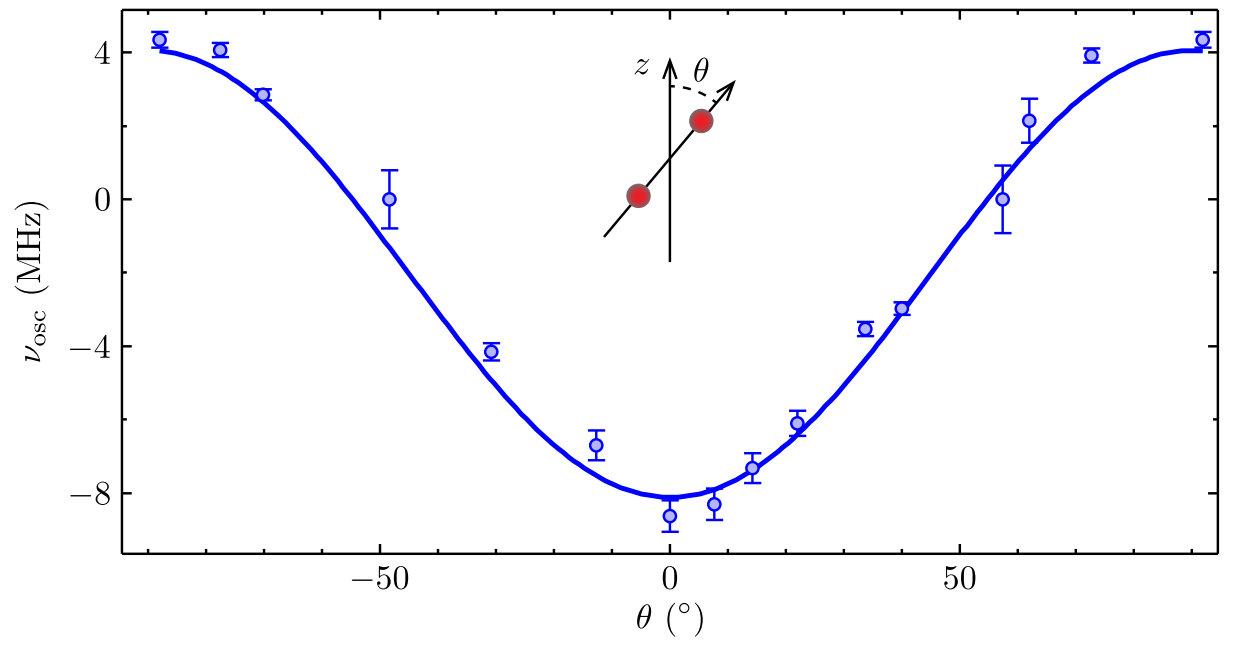

b

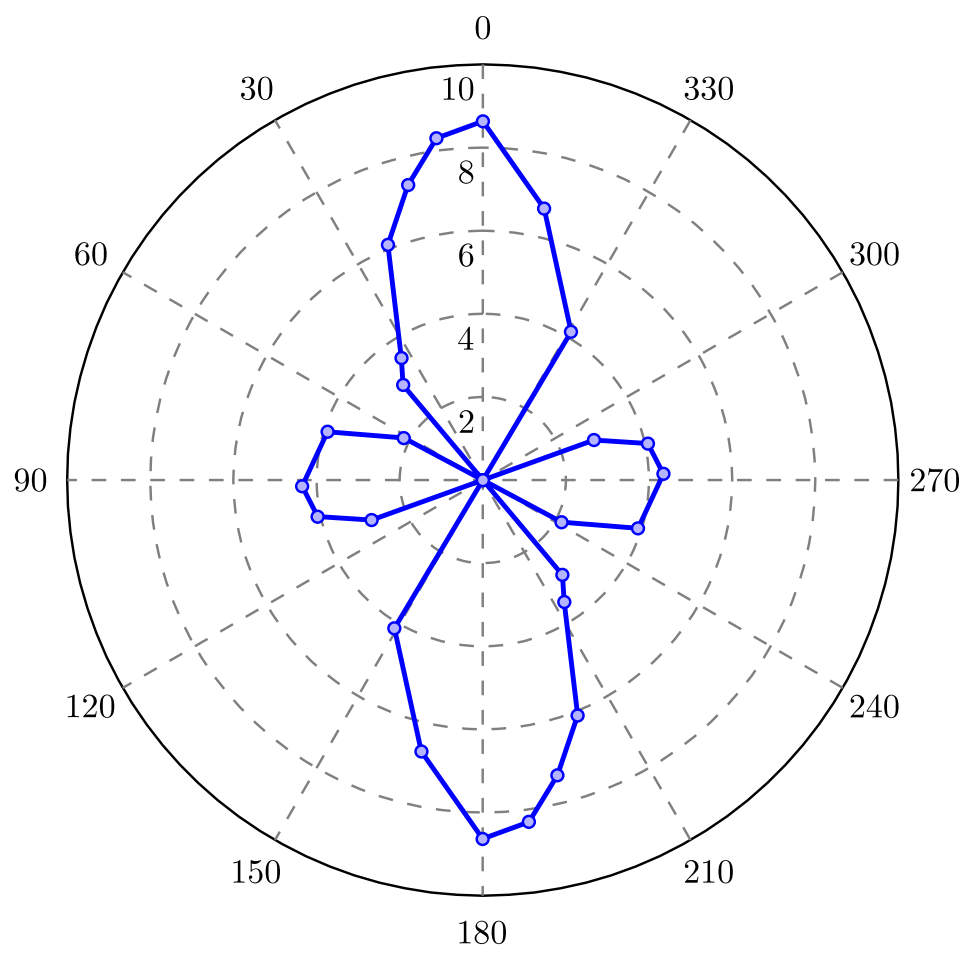

Figure 9.19: Angular dependence of the interaction. a, Fits of the Förster oscillations by damped sines allow measuring the evolution of the strength of the interaction. The graph plots $\nu_{\text {osc }}$ as a function of $\theta$. The solid line is a fit by function $K\left(1-3 \cos ^{2} \theta\right) / 2$. The fit gives $K=8.1 \pm 0.1 \mathrm{MHz}$, with a reduced chi-squared $\chi_{\text {red }} \simeq 5$. b, Representation in polar coordinates of the same set of data. The points for the angles $\theta$ and $\theta+180^{\circ}$ are identical. 
we obtain:

$$
K=8.1 \pm 0.1 \mathrm{MHz},
$$

with a reduced chi-squared $\chi_{\text {red }} \simeq 5$ (solid line in Figure 9.19). The fit shows a significant deviation from $\chi_{\text {red }}=1$, showing that the chosen fitting function does not fully agree with the measured angular dependence. This might be an indication that the other neighboring Förster resonances also contribute to the interaction, so that the system can not be described by only two levels at resonance, as we considered in a first approximation. Moreover, for the angles $\theta=14.2^{\circ}$ and $\theta=33.7^{\circ}$, we observe in Figure 9.18 that the Förster oscillations show a more pronounced damping, which could also indicate the presence of a beatnote between several frequencies. As a consequence, we perform the spectroscopy of the system for $\theta \neq 0^{\circ}$.

\subsubsection{Spectroscopy of the system for $\theta=30^{\circ}$}

We now fix the angle to $\theta=30^{\circ}$, and perform a spectroscopic measurement of the system as described in Section 9.3.

\section{Experimental procedure}

In this experiment, we fix the interatomic distance to $R=9.1 \mu \mathrm{m}$. For electric fields that vary between $4 \mathrm{mV} / \mathrm{cm}$ and $44 \mathrm{mV} / \mathrm{cm}$, we scan the laser detuning $\delta$ and measure the probability $P_{00}$ of loosing both atoms. In order to obtain a better spectral resolution than in Section 9.3, we narrow down our excitation linewidth and increase the interrogation time. Here, $\Omega / 2 \pi=0.76 \mathrm{MHz}$ and $\tau=1000 \mathrm{~ns}$.

\section{Experimental results}

Figure 9.20a shows the result of this spectroscopic measurement. The experimental spectrum shows several avoided crossings, which confirms the presence of other Förster resonances for the system, between $|d d\rangle$ and the different $\left|\widetilde{p f}_{i}\right\rangle$ Zeeman sublevels. We resolve the avoided crossing at $F \simeq 32 \mathrm{mV} / \mathrm{cm}$ corresponding to the resonance between $|d d\rangle$ and $\left|\widetilde{p f}_{1}\right\rangle$ studied earlier for $\theta=0^{\circ}$ (see Section 9.1). Compared to the situation at $\theta=0^{\circ}$, the separation between the two peaks at resonance (and the strength of the interaction) is reduced, which is another piece of evidence of the fact that the interatomic interaction is smaller for $\theta=30^{\circ}$ than for $\theta=0^{\circ}$. However, the proximity of the different Förster resonances suggest that for a given field, several 
a

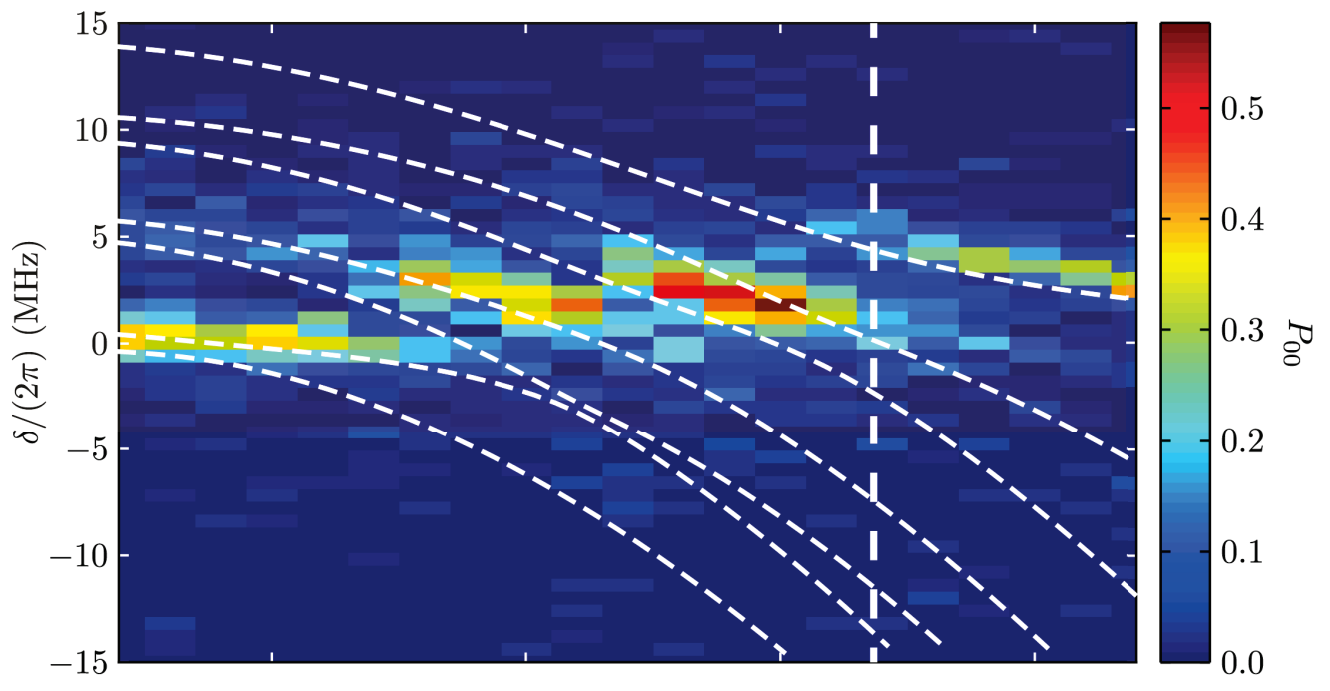

b

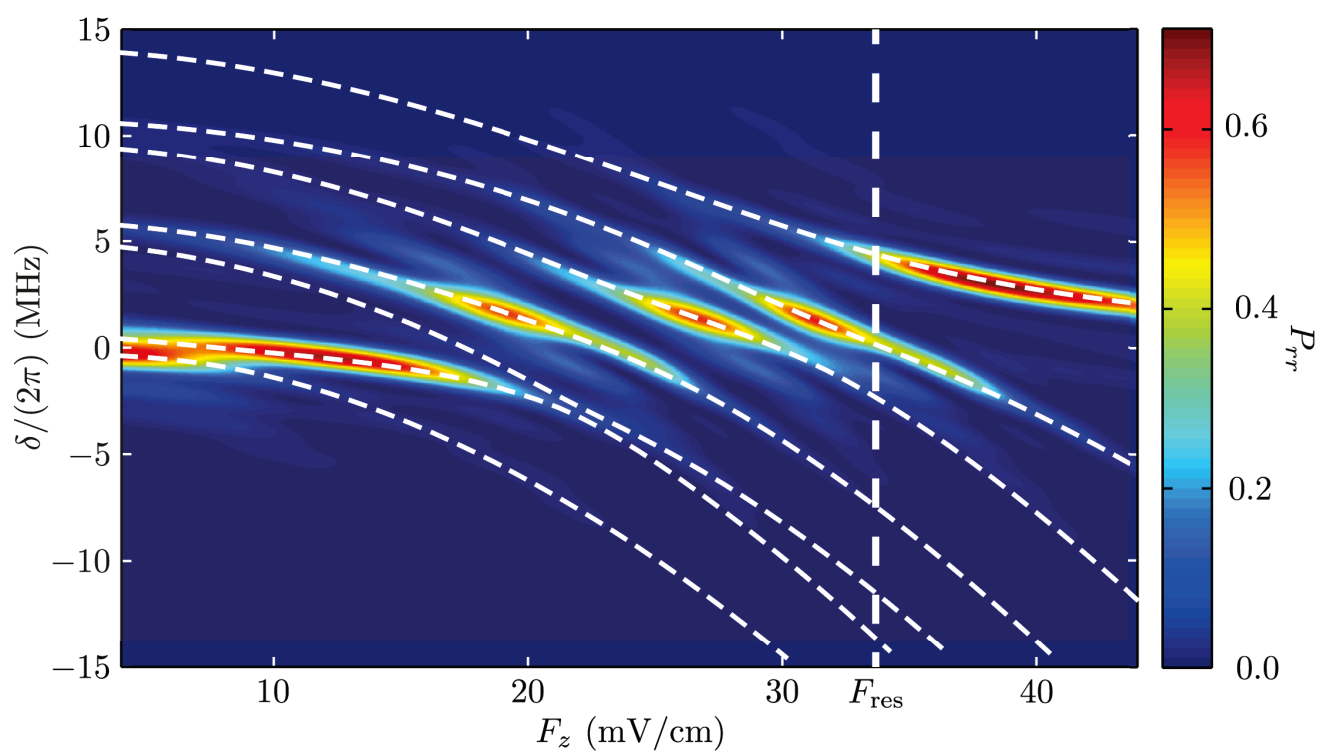

Figure 9.20: Spectroscopy at the Förster resonance for $\boldsymbol{\theta}=30^{\circ}$. a, Experimental excitation spectrum for two atoms separated by $R=9.1 \mu \mathrm{m}$. The population of double loss $P_{00}$ is plotted as a function of $\delta$ and $F_{z}$. We observe several avoided crossings between the different resonant pair states. The white vertical dashed line indicates the position of the Förster resonance we studied. The white curved dashed lines represent the Stark map of the pair states, calculated in the presence of interactions using the measured $C_{3 \text {,osc }}$ coefficient (see Section 9.4). b, Simulated two-atom excitation spectrum where the population in the doubly-excited state $P_{r r}$ is plotted as a function of $\delta$ and $F_{z}$. We include the $3.3 \mathrm{G}$ magnetic field in the simulation, as we will describe in Section 9.6.1. 
levels can contribute to the interaction energy, leading to complicated angular dependence of the interaction. Note that the relative position between the different Zeeman sub-levels $\left|\widetilde{p f}_{i}\right\rangle$, and between the different resonances can be varied by changing the strength of the magnetic field we apply on the atoms (see Chapter 5). Using the Zeeman effect, we have the possibility to tailor even further the angular dependence of the dipole-dipole interaction. In the next Section, we simulate the system by taking into account the magnetic field.

\subsection{Förster resonance in a magnetic field}

So far, we have not considered the effect of the magnetic field in our simulations. However, when $\theta \neq 0^{\circ}$, several Zeeman sublevels contribute to the interaction. The relative position of the different states depends on the value of the magnetic field. The magnetic field offers a degree of freedom in the system to tailor the angular dependence of the interaction. In this Section, we include the Zeeman effect in our simulations.

\subsubsection{Zeeman Hamiltonian}

The Landé factors for the different states of interest in this Chapter are given in Table 5.2. For $S$-states, $P$-states and $D$-states, we have seen in Section 5.3.1 that the perturbative approach is valid, due to their large fine-structure splittings. For twoatom states that are combinations of $|g\rangle,|p\rangle$ or $|d\rangle$, the effect of the magnetic field is to shift the eigenenergies of the system by a quantity $\Delta E_{B}$ without disturbing the eigenstates. However, the situation is different for $F$-states, where we have to consider the couplings to states of different $J$ introduced by the Zeeman Hamiltonian. Indeed, $\hat{H}_{B}$ couples the states $\left|57 F_{5 / 2}, M_{J}\right\rangle$ with the states $\left|57 F_{7 / 2}, M_{J}\right\rangle$, that are split by a fine structure splitting of less than $1 \mathrm{MHz}$, which is of the same order or smaller as our typical Zeeman shifts. In total, we have to consider in our simulation the nine pair states $\left\{|g g\rangle,|\widetilde{g d}\rangle,|d d\rangle,\left|\widetilde{p f} f_{i}\right\rangle\right.$, where $i$ ranges from 1 to 6 , and where we have defined:

$$
\left\{\begin{aligned}
\left|p f_{4}\right\rangle & \equiv\left|61 P_{1 / 2}, M_{J}=1 / 2 ; 57 F_{7 / 2}, M_{J}=5 / 2\right\rangle \\
\left|p f_{5}\right\rangle & \equiv\left|61 P_{1 / 2}, M_{J}=1 / 2 ; 57 F_{7 / 2}, M_{J}=3 / 2\right\rangle \\
\left|p f_{6}\right\rangle & \equiv\left|61 P_{1 / 2}, M_{J}=1 / 2 ; 57 F_{7 / 2}, M_{J}=1 / 2\right\rangle
\end{aligned}\right.
$$


Table 9.3: Polarizabilities of the pair states $\left|\widetilde{p f}_{i}\right\rangle$ with $i \in\{4 ; 5 ; 6\}$. The polarizabilities are obtained in the weak field limit as describe in Section 5.3.2. They are given in $\mathrm{kHz} .(\mathrm{mV} / \mathrm{cm})^{-2}$.

\begin{tabular}{ll}
\hline State & $\boldsymbol{\alpha}$ \\
\hline$\left.\widetilde{p f}_{4}\right\rangle$ & 25.4 \\
$\left.\widetilde{p f}_{5}\right\rangle$ & 31 \\
$\left|\widetilde{p f}_{6}\right\rangle$ & 33.1 \\
\hline
\end{tabular}

The diagonal matrix elements for $\hat{H}_{B}$ are the different Zeeman shifts for every pair state. We calculate them by summing the one-atom Zeeman shifts obtained using Equation 5.9. The non-diagonal matrix elements are combinations of Clebsch-Gordan coefficients, calculated using Equation 5.11. The Hamiltonian $\hat{H}_{B}$ couples states of same $M_{J}$, and we obtain the following coupling coefficients between the states $\left|\widetilde{p f}_{i}\right\rangle$ :

$$
\left\{\begin{array}{l}
\left\langle\widetilde{p f}_{1}\left|\hat{H}_{B}\right| \widetilde{p f}_{4}\right\rangle=\left\langle f_{1}\left|\hat{H}_{B}\right| f_{4}\right\rangle=-\sqrt{6} / 7 \mu_{B} B \\
\left\langle\widetilde{p f}_{2}\left|\hat{H}_{B}\right| \widetilde{p f}_{5}\right\rangle=\left\langle f_{2}\left|\hat{H}_{B}\right| f_{5}\right\rangle=-\sqrt{10} / 7 \mu_{B} B \\
\left\langle\widetilde{p f}_{3}\left|\hat{H}_{B}\right| \widetilde{p f}_{6}\right\rangle=\left\langle f_{3}\left|\hat{H}_{B}\right| f_{6}\right\rangle=-2 \sqrt{3} / 7 \mu_{B} B .
\end{array}\right.
$$

\subsubsection{Stark Hamiltonian}

We have seen in Section 5.3.2 that for the small fields used in this thesis, the Stark effect can be treated perturbatively using second-order perturbation theory. To second order the electric field shifts the eigenenergies of the system while the eigenstates stay unperturbed. As a consequence, the Stark Hamiltonian $\hat{H}_{F}$ is diagonal, and its diagonal elements are given by $-\alpha F_{z}^{2} / 2$, where $\alpha$ are the polarizabilities of the different pair states. The calculated polarizabilitites for the states $\left\{\left|\widetilde{p f}_{i}\right\rangle(i \in\{4 ; 5 ; 6\})\right.$ are given in Table 9.3, giving us all the information to compute the Stark Hamiltonian ${ }^{6}$ (see Section 5.3.2).

\footnotetext{
${ }^{6}$ The polarizabilities for the other states were given in Table 9.2. The polarizability for the $|g g\rangle$ is $\simeq 0$, and the polarizability for the state $|\widetilde{g d}\rangle$ is half the polarizability given for the pair state $|d d\rangle$.
} 


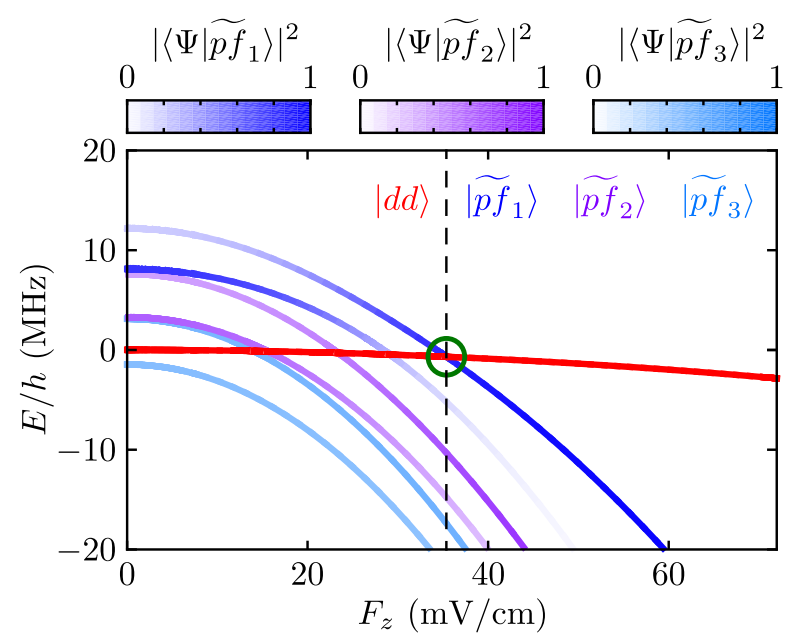

Figure 9.21: Effect of combined electric and magnetic field. When adding a $3.3 \mathrm{G}$ magnetic field, the states $\left|\widetilde{p f}_{i}\right\rangle(i \in\{1 ; 2 ; 3\})$ respectively mix with the states $\left|\widetilde{p f}_{j}\right\rangle(j \in$ $\{4 ; 5 ; 6\})$. We obtain five distinct resonances of various strengths, given by the degree of admixture of the states. The state $|d d\rangle$ is shown in red. For every eigenstate $|\Psi\rangle$ of the hamiltonian $\hat{H}_{\text {tot }}$, the weights of the states $\left|\widetilde{p f}_{1}\right\rangle,\left|\widetilde{p f}_{2}\right\rangle$ and $\left|\widetilde{p f}_{3}\right\rangle$ are given by the color scales. The green circle indicates the position of the resonance we chose to study in this Chapter.

\subsubsection{Total Hamiltonian}

The system is described, in the presence of an electric and a magnetic field, by the total Hamiltonian

$$
\hat{H}_{\mathrm{Tot}}=\hat{H}_{0}+\hat{H}_{F}+\hat{H}_{B}
$$

where $\hat{H}_{0}$ is the unperturbed Hamiltonian, and where we have not considered dipoledipole interactions. The result of the diagonalization of $\hat{H}_{\text {Tot }}$ is shown in Figure 9.21. Because all the states have different polarizabilities, the system exhibits a discrete set of Förster resonances. The magnetic field shifts the energies of the different states, which gives the possibility to tune the positions of the different resonances. The magnetic field also adds some couplings to other states, thus adding extra resonances. In total, we observe the presence of five resonances, the strength of which depends on the degree of admixture of the states at the position of the crossing, and can thus also be tuned by varying the magnetic field. In this Chapter, we studied in detail the resonance occurring between the states $|d d\rangle$ and $\left|\widetilde{p f}_{1}\right\rangle$ that is indicated by a green circle in Figure 9.21. 


\subsubsection{Including the magnetic field in the simulation}

We simulate the system by generalizing the approach of Section 9.3 in the nine-state basis $\left\{|g g\rangle, \widetilde{|d g\rangle}, \widetilde{|d d\rangle},\left|\widetilde{p f}{ }_{i}\right\rangle\right\}$ that includes all relevant Zeeman sub-levels. For $\theta=0^{\circ}$, the only state that contributes directly to the dipole-dipole interaction is $\left|\widetilde{p f}_{1}\right\rangle$. The state $\left|\widetilde{p f}_{4}\right\rangle$ also contributes indirectly to the dipole-dipole interaction via its Zeeman coupling to $\left|\widetilde{p f}_{1}\right\rangle$ that mixes the two states. At the position of the resonance, the mixing between $\left|\widetilde{p f}_{1}\right\rangle$ and $\left|\widetilde{p f}_{4}\right\rangle$ is small enough, and does not change significantly the conclusions of the simulations discussed earlier. This stays true as long as the dipole coupling between $|d d\rangle$ and $\left|\widetilde{p f}_{1}\right\rangle$ is larger than the magnetic coupling between $\left|\widetilde{p f}_{1}\right\rangle$ and $\left|\widetilde{p f}_{4}\right\rangle$ (i.e. for $R<16 \mu \mathrm{m}$, which is verified in our experiments).

We then fix the angle to $\theta=30^{\circ}$. In this case, $\hat{V}_{\text {dip }}$ induces couplings between $|d d\rangle$ and the states $\left|\widetilde{p f}_{1}\right\rangle,\left|\widetilde{p f}_{2}\right\rangle$ and $\left|\widetilde{p f}_{3}\right\rangle$ verifying respectively $\Delta M_{\text {tot }}=0,1$ and 2 (see Equation 9.42). There exist multiple resonances in the system, each with a particular angular dependence directly related to $\Delta M_{\text {tot }}$. Because of the presence a $3.3 \mathrm{G}$ magnetic field that mixes $\left|\widetilde{p f}_{i}\right\rangle(i \in\{1 ; 2 ; 3\})$ with $\left|\widetilde{p f}_{j}\right\rangle(j \in\{4 ; 5 ; 6\}$ respectively) there exist five different crossings with $|d d\rangle$ (see Figure 9.21). We simulate the spectrum of the system for an interatomic distance $R=9.14 \mu \mathrm{m}$, with $\Omega=2 \pi \times 0.76 \mathrm{MHz}$ and $\tau=1000 \mathrm{~ns}$ (parameters used experimentally in Section 9.5.3). The result of the simulation is presented in Figure 9.20b. The spectrum shows four avoided crossings, corresponding to different Förster resonances. Note that the number of resonances and their positions qualitatively agree with the spectrum observed experimentally in Figure 9.20a. In particular, the simulation confirms that we do not resolve the first two resonances shown in Figure 9.21. We finally notice that for our parameters, the resonance between $\left|\widetilde{p f}_{1}\right\rangle$ and $\widetilde{|d d\rangle}$ occurs at the highest electric field and is well separated from the other resonances. The proximity of the other resonances only slightly influences the angular dependence of the interaction, which explains the small deviations from $\left(1-3 \cos ^{2} \theta\right) / 2$ observed in Section 9.5.

\subsection{Conclusion}

We directly observed the oscillation between the two degenerate pair states of two single Rydberg atoms at a Förster resonance, demonstrating the coherent nature of the mechanism underlying resonant energy transfer. The presence of only two atoms at a controlled distance, with well defined internal states, was fundamental to this study. Förster resonances offer the possibility to work with strong and switchable 
interactions, which will prove useful for applications in larger arrays of atoms. We finally measured the angular dependence for one particular resonance. Controlling the relative position between several resonances using the Zeeman effect would finally allow to tailor the angular dependence of the interaction, therefore reaching a full level of control on the interaction between two atoms. One interesting line of research for the future will be to extend the system to a few atoms, so as to observe three-body or four-body interactions [Gurian et al. (2012)] (see Outlook). In larger ensembles of atoms, Förster resonances will also allow to study the propagation of entanglement in disordered systems of atoms [Günter et al. (2013)]. 
238 Chapter 9: Coherent dipole-dipole coupling at a "Förster" resonance 


\section{Outlook}

This thesis describes my work on two different experimental setups developed for quantum engineering. I have first described the fabrication and characterization of tapered optical nanofibers that are promising to interface cold ${ }^{87} \mathrm{Rb}$ atoms with a superconducting qubit. I have then characterized the interactions between two and three Rydberg atoms in different regimes, ranging from van der Waals to resonant dipole-dipole interactions. Here, I briefly describe the latest developments and future goals for both setups.

\section{JQI setup}

Since I left JQI, significant progress has been achieved towards the realization of the hybrid quantum system. Atom trapping has been demonstrated around the nanofiber, a new resonator has been fabricated and tested, and the design of the full setup has evolved to take into account what has been learned from the preliminary studies presented in this first part:

- The team at JQI have now demonstrated atom trapping using our nanofibers in a vacuum chamber. Figure 1a shows preliminary data concerning the transmission of a resonant $(780 \mathrm{~nm})$ probe through the fiber, when $\sim 500$ atoms are loaded in the optical dipole traps around the nanofiber. We observe a strong absorption line due to the trapped atoms. The shift, broadening and asymmetry of the line come from state dependent light shift of the transition frequency frequency induced by the trapping light.

- Our estimates of losses due to Rayleigh scattering in the nanofiber (see Chapter 3) showed that the amount of light impinging on the resonator in the initial design would be detrimental for its quality factor. This has led to a new design of the resonator, where the position of the fiber minimizes the amount of stray light. The new resonator is shown in Figure 1b. The superconducting circuit is 
a

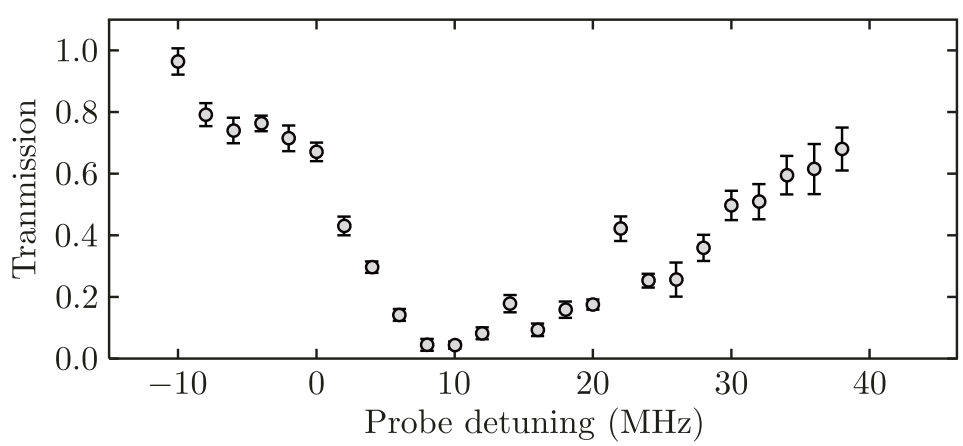

b

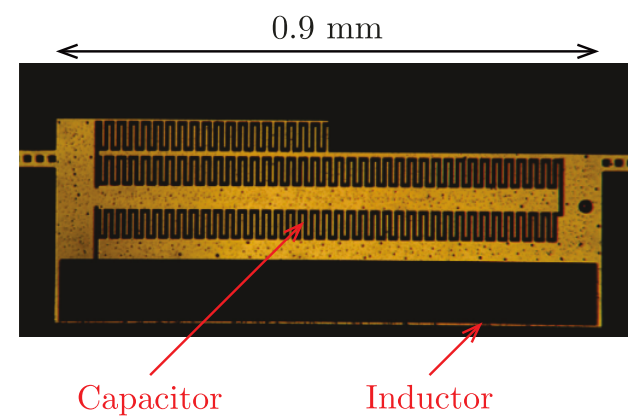

c

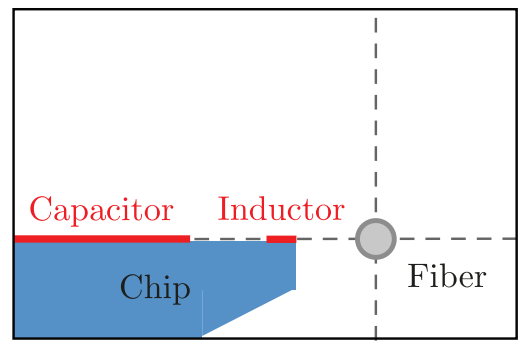

d

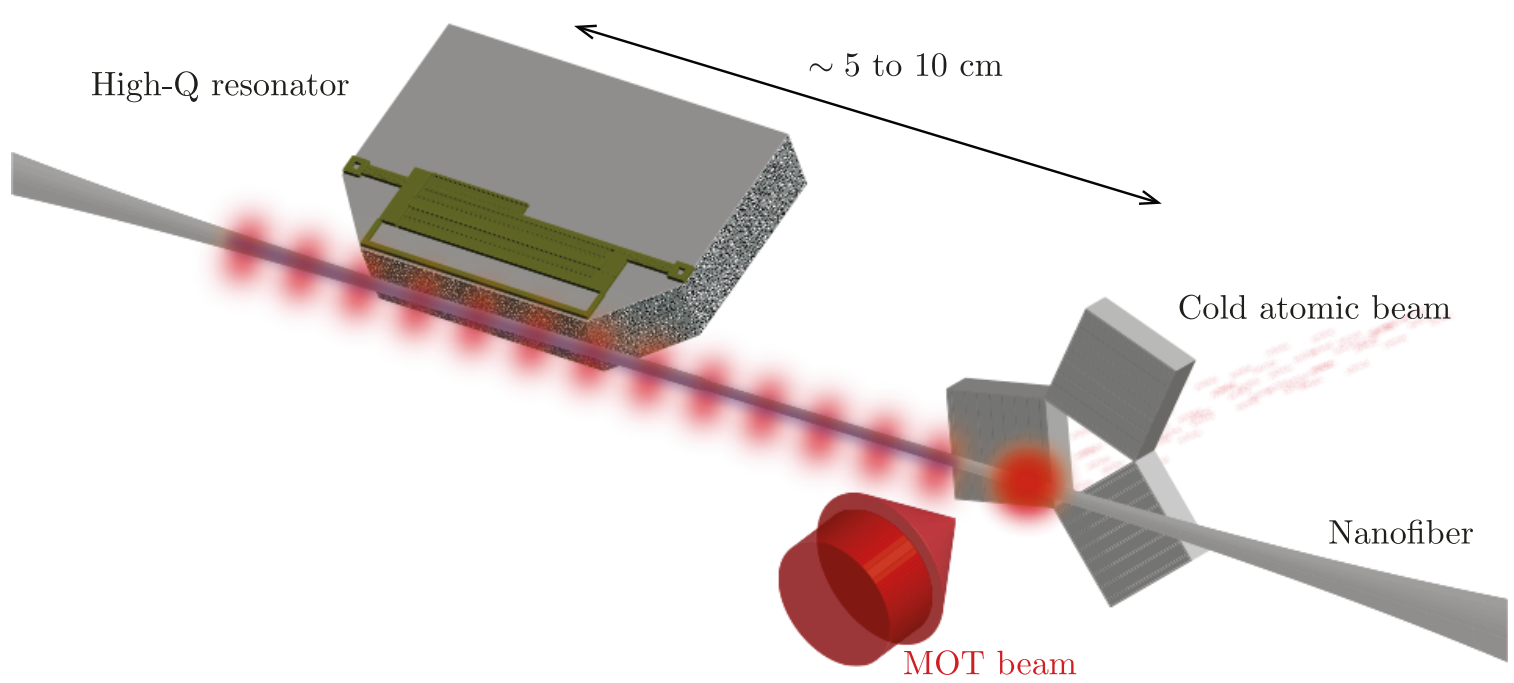

Figure 1: Latest results and future directions for the JQI setup. a, Absorption spectrum of a probe beam by atoms trapped around the nanofiber. $\mathbf{b}$, New design of the resonator. c, Position of the nanofiber with respect to the resonator. d, Current envisioning of the full setup. 
made of aluminum, and its quality factor has been measured to be greater than $10^{5}$. As before, it is composed of an inductor and an interdigitated capacitor. The latter has a larger surface area, making it more prone to the detrimental effects of stray light. However, the atoms only need to be close to the inductor, in order to maximize their magnetic coupling to the resonator. The adopted design has the fiber and the resonator in the same plane, the atoms being close to the inductor (see Figure 1c). Preliminary tests have shown that in this configuration, the quantity of light absorbed by the resonator is indeed greatly decreased, by about two orders of magnitude.

- Finally, the next steps will be to integrate the various elements in the cryogenic environment: the MOT for producing cold atoms in a $3.5 \mathrm{~K}$ stage, the superconducting resonator in the $10 \mathrm{mK}$ stage, and the nanofiber for transporting the atoms from the MOT to the resonator. Figure 1d shows the current design. A 2D MOT, under construction, will serve as a source of slow atoms to load a pyramid MOT, which has the advantage of being compact and of using only one beam [Lee et al. (2013)]. The MOT mirrors will be thermally anchored to the $3.5 \mathrm{~K}$ stage of the cryostat, that has a cooling power of the order of a few watts, at a distance of about 5 to $10 \mathrm{~cm}$ from the superconducting chip. Moreover, with such a design, the MOT coils are far from the chip, and the MOT magnetic field should not affect the resonator. The final step will be to transport the trapped atoms from the MOT to the chip using the nanofiber as an optical conveyer belt over $\sim 10 \mathrm{~cm}$. Recently, such a transport was demonstrated over millimeter distances [Schneeweiss et al. (2013)]. The challenge will consist in extending the transport distance by one order of magnitude.

\section{IOGS setup}

During my thesis, we have studied in great detail systems of two and three interacting Rydberg atoms, in both the van der Waals and the resonant dipole-dipole regimes. This has allowed us to characterize the building blocks of a future Rydberg quantum simulator. Our long term goal is to extend this approach to larger arrays containing a few tens of atoms, for instance in order to simulate spin systems. Two important steps along those lines have already been achieved in the laboratory, and will be detailed in the thesis of H. Labuhn: 
a
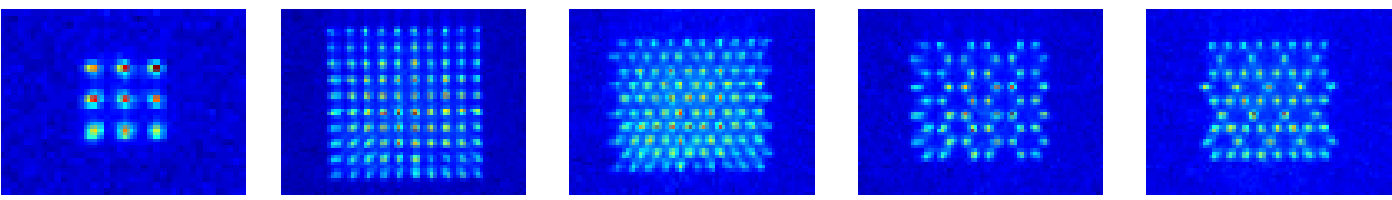

b

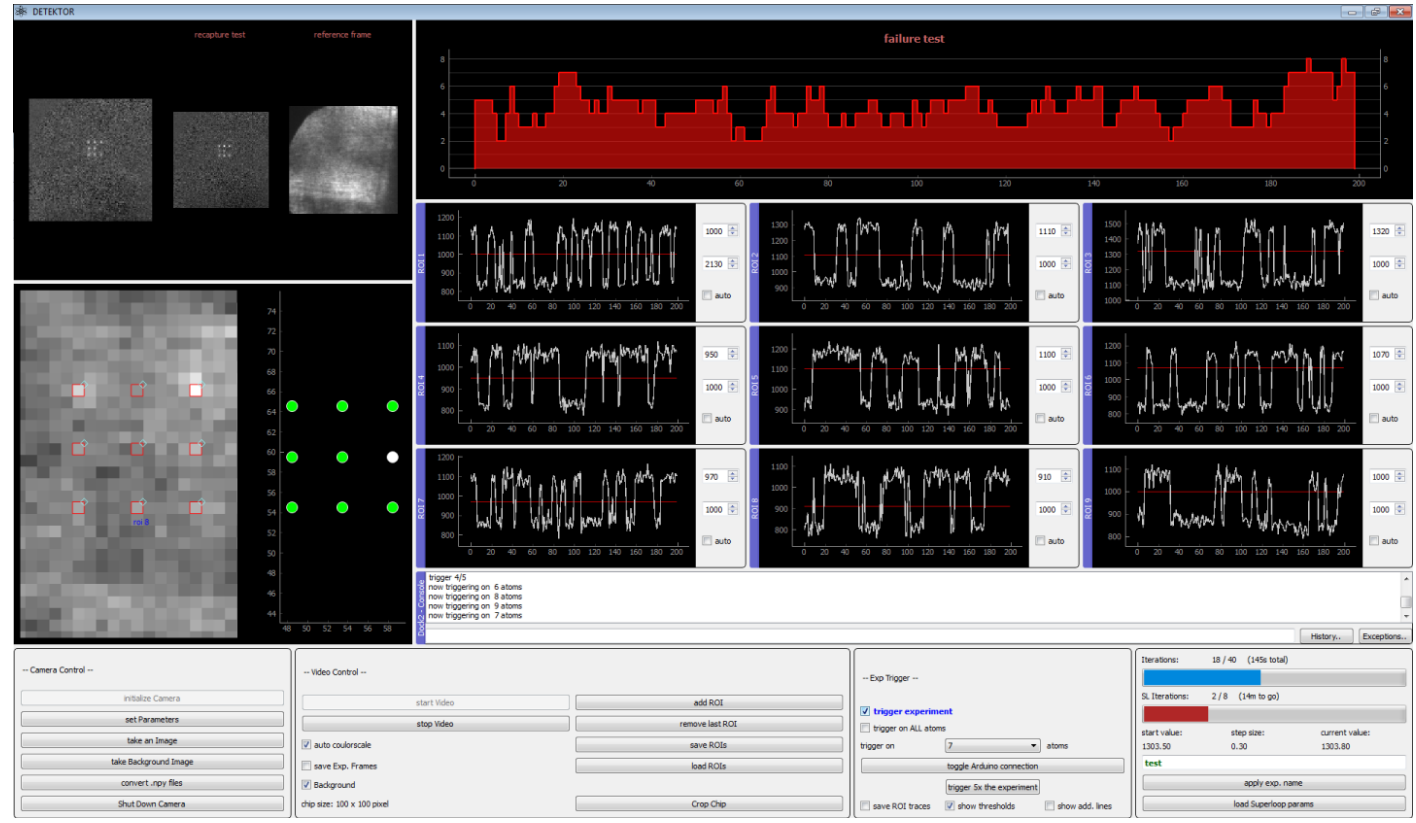

c
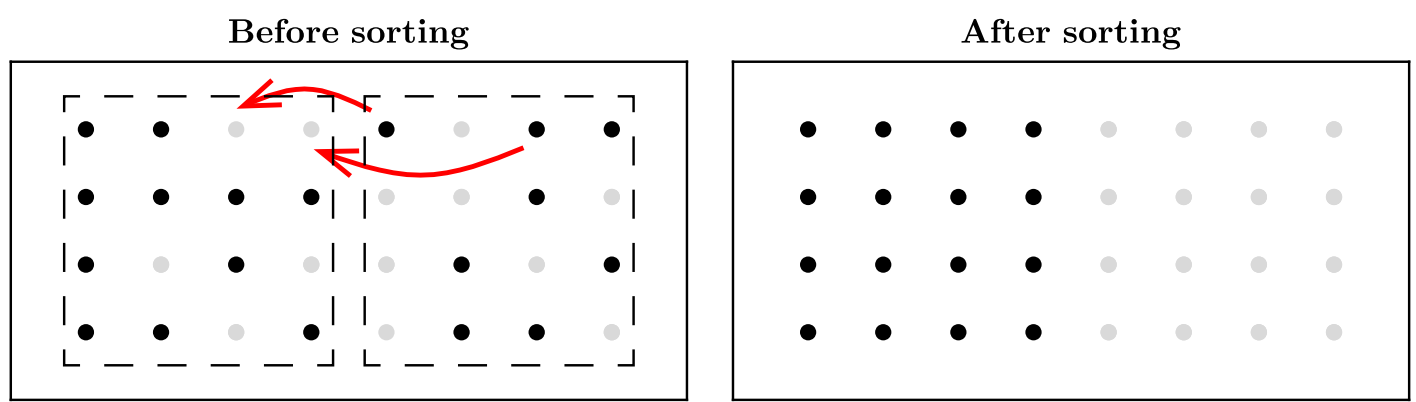

"Target array"

"Reservoir"

Figure 2: Towards larger number of atoms on the IOGS setup. a, Example of arrays of microtraps with geometries that are relevant for quantum simulation [Nogrette et al. (2014)]. b, Screen capture of the new control software used to acquire the fluorescence of atoms using the EMCCD camera. Fluorescence images of atoms in a $3 \times 3$ square matrix are shown on the left. The white traces plot the atom signals for each of the nine sites. We observe in red the evolution of the number of loaded sites as a function of time. c, Cartoon of a possible way to obtain a fully-loaded $4 \times 4$ square matrix ("target array"). We juxtapose two versions of the array we want to load. Using our current loading scheme, we obtain a sparsely-loaded array. The idea is to use our movable optical tweezer to rearrange the configuration of the atoms. 
- We have demonstrated [Nogrette et al. (2014)] that the SLM allows creating arrays containing up to 100 microtraps with arbitrary two-dimensional geometries, as exemplified in Figure 2a.

- More recently, we have changed the fluorescence detection system, by replacing the three fiberized avalanche photodiodes by an EMCCD camera, where each pixel can be considered as a single photon detector with millisecond time resolution. A new software (see screen capture in Figure 2b) has been developed to acquire and process rapidly $(\sim 50 \mathrm{~ms})$ the EMCCD images and trigger the experimental sequences.

With these tools, some interesting applications can already be implemented with low number of deterministically positioned atoms. Indeed, for small arrays containing less than five or six atoms, it is still reasonable to wait for all traps to be loaded at the same time (for five traps the duty cycle of the experiment is $\sim 10 \mathrm{~s}$ ). As a consequence, natural extensions of the work presented in this thesis can be carried out:

- Production of W-states with up to six atoms using the Rydberg blockade, a natural extension of the work performed with two atoms [Wilk et al. (2010)].

- Study of the propagation of spin excitations along a small spin chain or around plaquettes. In particular, for the case of several excitations, the equivalence between spin excitations and hard-core bosons should lead to correlations in the dynamics of the excitations.

- Study of few-body Förster resonances as observed recently in group of P. Pillet in Orsay [Gurian et al. (2012); Cheinet (2014)] for the three-body and four-body cases.

For some experiments with much larger arrays, the probabilistic loading will introduce randomness in the filling of the array with a filling factor $\sim 1 / 2$, as can be seen in Figure 2b. This intrinsic randomness can nevertheless be seen as an asset. For example, microwave spectroscopy of Rydberg atoms randomly loaded in a large array could shed light on the anomalous diffusion of Rydberg excitations observed in frozen Rydberg gases [Anderson, Veale, and Gallagher (1998); Mourachko et al. (1998); Günter et al. (2013)] or allow for the study of dipole-coupled spins in a sparsely loaded array as done recently with polar molecules in optical lattices [Yan et al. (2013)]. 
Finally, for some applications in quantum information processing and quantum simulation, one would need to work with deterministically loaded arrays of microtraps. We will explore possible ways to achieve more deterministic loading. Recent proof-of-principle experiments using either the Rydberg blockade [Saffman and Walker (2002); Ebert et al. (2014)] or modified light-assisted collisions [Grunzweig et al. (2010); Carpentier et al. (2013)] have shown increased loading probabilities for a single trap, of $60 \%$ and $90 \%$ respectively. Another approach that we might also try would consist in sparsely loading a larger array, and dynamically rearranging the configuration of the atoms by using the movable optical tweezer described in Chapter 6 to move the atoms from filled sites to empty sites, as represented in Figure 2c, in the spirit of the pioneering experiments described in [Dumke et al. (2002); Miroshnychenko et al. (2006a,b); Kruse et al. (2010); Schlosser et al. (2011)].

\section{Coupling Rydberg atoms to superconducting circuits}

As a final concluding remark, it would be interesting to explore the coupling between Rydberg atoms and a superconducting circuit, by the way of an on-chip resonant microwave guide. This route would thus combine the tools developed in the two parts of this thesis. In this approach, one would use the electric dipole coupling between the field above the waveguide and the strong dipole of the transition between two Rydberg states. As the wavelength of the Rydberg transitions around $n=50$ lies in the 10-100 GHz range, the wavelength is on the order of the typical size of a microwave resonator circuit $(\sim 1 \mathrm{~mm})$ and this architecture seems natural. This route is actually followed by several groups, in particular at ETH Zurich [Hogan et al. (2012)] and at the University of Tübingen [Cano et al. (2011)]. Among the challenges one has to face is the presence of surfaces close to the Rydberg atoms, which usually leads to shifts of the Rydberg lines due to the fluctuating patch charges on the surfaces [Hattermann et al. (2012)]. Recently, the team of M. Brune has demonstrated a solution to this problem by saturating the surface by a layer of rubidium [Celistrino Teixeira (2014)]. Another problem if one wants to couple a few, well-defined number of Rydberg atoms to an on-chip waveguide is to position them close to the surface carrying the guide. This is where the optical nano-fibered could prove useful. Despite the atoms being close to the fiber ( $\sim 100 \mathrm{~nm})$, the small size of the fiber ( $\sim 100 \mathrm{~nm}$ also) could minimize the amount of dielectric seen by the atoms and therefore the detrimental effect of the patch charges when they are excited in a Rydberg state. Other problems would have to be considered, such as the forces between the atoms and the surface, but their 
effect could be minimized by placing the atoms in the Rydberg states only for short amount of times. Finally, one could use the control over the interactions between Rydberg atoms demonstrated in this thesis to engineer quantum states of a few-atom ensembles that could be largely immune to the effect of fluctuating electric fields. A lot remains to be explored in this direction. 


\section{Bibliography}

Afrousheh, K., Bohlouli-Zanjani, P., Vagale, D., Mugford, A., Fedorov, M., and Martin, J. D. D., "Spectroscopic Observation of Resonant Electric Dipole-Dipole Interactions between Cold Rydberg Atoms," Phys. Rev. Lett. 93, 233001 (2004).

Anderson, W. R., Robinson, M. P., Martin, J. D. D., and Gallagher, T. F., "Dephasing of resonant energy transfer in a cold Rydberg gas," Phys. Rev. A 65, 063404 (2002).

Anderson, W. R., Veale, J. R., and Gallagher, T. F., "Resonant Dipole-Dipole Energy Transfer in a Nearly Frozen Rydberg Gas," Phys. Rev. Lett. 80, 249-252 (1998).

Andre, A., DeMille, D., Doyle, J. M., Lukin, M. D., Maxwell, S. E., Rabl, P., Schoelkopf, R. J., and Zoller, P., "A coherent all-electrical interface between polar molecules and mesoscopic superconducting resonators," Nat. Phys. 2, 636-642 (2006).

Andrews, D. L. and Demidov, A. A., Resonance Energy Transfer (Wiley, 1999).

Arnold, W. and Oppenheimer, J. R., "Internal conversion in the photosynthetic mechanism of blue-green algae," J. Gen. Physiol. 33, 423-435 (1950).

Ashkin, A., "Acceleration and Trapping of Particles by Radiation Pressure," Phys. Rev. Lett. 24, 156-159 (1970).

Aspuru-Guzik, A. and Walther, P., "Photonic quantum simulators," Nat. Phys. 8, 285-291 (2012).

Balewski, J. B., Krupp, A. T., Gaj, A., Peter, D., Buchler, H. P., Low, R., Hofferberth, S., and Pfau, T., "Coupling a single electron to a Bose-Einstein condensate," Nature 502, 664-667 (2013). 
Balykin, V. I., Hakuta, K., Le Kien, F., Liang, J. Q., and Morinaga, M., "Atom trapping and guiding with a subwavelength-diameter optical fiber," Phys. Rev. A 70, 011401 (2004).

Barends, R., Wenner, J., Lenander, M., Chen, Y., Bialczak, R. C., Kelly, J., Lucero, E., O’Malley, P., Mariantoni, M., Sank, D., Wang, H., White, T. C., Yin, Y., Zhao, J., Cleland, A. N., Martinis, J. M., and Baselmans, J. J. A., "Minimizing quasiparticle generation from stray infrared light in superconducting quantum circuits," Appl. Phys. Lett. 99, 113507 (2011).

Béguin, L., Measurement of the van der Waals interaction between two Rydberg atoms, Theses, Institut d'Optique Graduate School (2013).

Béguin, L., Vernier, A., Chicireanu, R., Lahaye, T., and Browaeys, A., "Direct Measurement of the van der Waals Interaction between Two Rydberg Atoms," Phys. Rev. Lett. 110, 263201 (2013).

Bergamini, S., Darquié, B., Jones, M., Jacubowiez, L., Browaeys, A., and Grangier, P., "Holographic generation of microtrap arrays for single atoms by use of a programmable phase modulator," J. Opt. Soc. Am. B 21, 1889-1894 (2004).

Bernon, S., Hattermann, H., Bothner, D., Knufinke, M., Weiss, P., Jessen, F., Cano, D., Kemmler, M., Kleiner, R., Koelle, D., and Fortágh, J., "Manipulation and coherence of ultra-cold atoms on a superconducting atom chip," Nat. Commun. 4 (2013).

Beterov, I. I., Ryabtsev, I. I., Tretyakov, D. B., and Entin, V. M., "Quasiclassical calculations of blackbody-radiation-induced depopulation rates and effective lifetimes of Rydberg $n S$, $n P$, and $n D$ alkali-metal atoms with $n \leq 80$," Phys. Rev. A 79, 052504 (2009).

Beterov, I. I., Tretyakov, D. B., Entin, V. M., Yakshina, E. A., Ryabtsev, I. I., MacCormick, C., and Bergamini, S., "Deterministic single-atom excitation via adiabatic passage and Rydberg blockade," Phys. Rev. A 84, 023413 (2011).

Bettelli, S., Maxwell, D., Fernholz, T., Adams, C. S., Lesanovsky, I., and Ates, C., "Exciton dynamics in emergent Rydberg lattices," Phys. Rev. A 88, 043436 (2013).

Beugnon, J., Single trapped atom internal state control and two-photon interference experiments: towards quantum computing with neutral atoms, Theses, Université Paris Sud - Paris XI (2007). 
Beugnon, J., Jones, M. P. A., Dingjan, J., Darquié, B., Messin, G., Browaeys, A., and Grangier, P., "Quantum interference between two single photons emitted by independently trapped atoms," Nature 440, 779-782 (2006).

Beutler, H. and Josephy, B., "Resonanz bei Stößen in der Fluoreszenz und Chemilumineszenz," Z. Physik 53, 747-765 (1929).

Bevington, P. R. and Robinson, D. K., Data reduction and error analysis for the physical sciences, McGraw-Hill Higher Education (McGraw-Hill, 2003).

Bilodeau, F., Hill, K. O., Johnson, D. C., and Faucher, S., "Compact, low-loss, fused biconical taper couplers: overcoupled operation and antisymmetric supermode cutoff," Opt. Lett. 12, 634-636 (1987).

Birks, T. and Li, Y., "The shape of fiber tapers," J. Lightwave Technol. 10, 432-438 (1992).

Black, E. D., "An introduction to Pound-Drever-Hall laser frequency stabilization," Am. J. Phys. 69, 79-87 (2001).

Blatt, R. and Roos, C. F., "Quantum simulations with trapped ions," Nat. Phys. 8, 277-284 (2012).

Bloch, I., "Exploring quantum matter with ultracold atoms in optical lattices," J. Phys. B 38, S629 (2005).

Bloch, I., Dalibard, J., and Nascimbene, S., "Quantum simulations with ultracold quantum gases,” Nat. Phys. 8, 267-276 (2012).

Bohr, N., "On the constitution of atoms and molecules," Philosophical Magazine Series 6, Philos. Mag. 26, 1-25 (1913).

Boyer, V., Chandrashekar, C. M., Foot, C. J., and Laczik, Z. J., "Dynamic optical trap generation using FLC SLMs for the manipulation of cold atoms," Journal of Modern Optics, J. Mod. Opt. 51, 2235-2240 (2004).

Brambilla, G., "Optical fibre nanowires and microwires: a review," J. Opt. 12, 043001 (2010).

Brambilla, G., Finazzi, V., and Richardson, D., "Ultra-low-loss optical fiber nanotapers," Opt. Express 12, 2258-2263 (2004). 
Briggs, J. S. and Eisfeld, A., "Equivalence of quantum and classical coherence in electronic energy transfer," Phys. Rev. E 83, 051911 (2011).

Bures, J. and Ghosh, R., "Power density of the evanescent field in the vicinity of a tapered fiber,” J. Opt. Soc. Am. A 16, 1992-1996 (1999).

Cano, D. and Fortágh, J., "Nonadditive potentials between three Rydberg atoms," Phys. Rev. A 86, 043422 (2012).

Cano, D., Hattermann, H., Kasch, B., Zimmermann, C., Kleiner, R., Koelle, D., and Fortágh, J., "Experimental system for research on ultracold atomic gases near superconducting microstructures," Eur. Phys. J. D 63, 17-23 (2011).

Cario, G. and Franck, J., "Über Zerlegung von Wasserstoffmolekülen durch angeregte Quecksilberatome," Z. Physik 11, 161-166 (1922).

Carpentier, A. V., Fung, Y. H., Sompet, P., Hilliard, A. J., Walker, T. G., and Andersen, M. F., "Preparation of a single atom in an optical microtrap," Laser Phys. Lett. 10, 125501 (2013).

Carroll, T. J., Claringbould, K., Goodsell, A., Lim, M. J., and Noel, M. W., "Angular Dependence of the Dipole-Dipole Interaction in a Nearly One-Dimensional Sample of Rydberg Atoms," Phys. Rev. Lett. 93, 153001 (2004).

Celistrino Teixeira, R., Mechanical effects of dipolar interaction of Rydberg atoms probed by microwave spectroscopy, Theses, Université Pierre et Marie Curie (2014).

Cheinet, P., "Private communication," (2014).

Chen, G. Y., Ding, M., Newson, T. P., and Brambilla, G., "A review of microfiber and nanofiber based optical sensors," Open Optic J. 7, 32-57 (2013).

Cirac, J. I. and Zoller, P., "Goals and opportunities in quantum simulation," Nat. Phys. 8, 264-266 (2012).

Clegg, R. M., "The History of Fret," in Reviews in Fluorescence 2006, Reviews in Fluorescence, Vol. 2006, edited by C. D. Geddes and J. R. Lakowicz (Springer US, 2006) pp. 1-45.

Cohen-Tannoudji, C., Diu, B., and Laloë, F., Quantum mechanics (Wiley, 1977).

Cohen-Tannoudji, C., Dupont-Roc, J., and Grynberg, G., Processus d'interaction entre photons et atomes (InterÉd, 1988). 
Collini, E., Wong, C. Y., Wilk, K. E., Curmi, P. M. G., Brumer, P., and Scholes, G. D., "Coherently wired light-harvesting in photosynthetic marine algae at ambient temperature," Nature 463, 644-647 (2010).

Comparat, D. and Pillet, P., "Dipole blockade in a cold Rydberg atomic sample [Invited],” J. Opt. Soc. Am. B 27, A208-A232 (2010).

Córcoles, A. D., Chow, J. M., Gambetta, J. M., Rigetti, C., Rozen, J. R., Keefe, G. A., Beth Rothwell, M., Ketchen, M. B., and Steffen, M., "Protecting superconducting qubits from radiation," Appl. Phys. Lett. 99, 181906-181906-3 (2011).

Courtois, J.-Y., Courty, J.-M., and Mertz, J. C., "Internal dynamics of multilevel atoms near a vacuum-dielectric interface," Phys. Rev. A 53, 1862-1878 (1996).

Darquié, B., Manipulation d'atomes dans des pièges dipolaires microscopiques et émission contrôlée de photons par un atome unique, Theses, Université Paris Sud - Paris XI (2005).

Dehmelt, H., "Experiments with an isolated subatomic particle at rest," Rev. Mod. Phys. 62, 525-530 (1990).

Devoret, M. H. and Martinis, J. M., "Implementing Qubits with Superconducting Integrated Circuits," Quantum Inf. Process. 3, 163-203 (2004).

Dudin, Y. O. and Kuzmich, A., "Strongly Interacting Rydberg Excitations of a Cold Atomic Gas,” Science 336, 887-889 (2012).

Dumke, R., Volk, M., Müther, T., Buchkremer, F. B. J., Birkl, G., and Ertmer, W., "Micro-optical Realization of Arrays of Selectively Addressable Dipole Traps: A Scalable Configuration for Quantum Computation with Atomic Qubits," Phys. Rev. Lett. 89, 097903 (2002).

Ebert, M., Gill, A., Gibbons, M., Zhang, X., Saffman, M., and Walker, T. G., "Atomic Fock State Preparation Using Rydberg Blockade," Phys. Rev. Lett. 112, 043602 (2014).

Eisaman, M. D., Fan, J., Migdall, A., and Polyakov, S. V., "Invited Review Article: Single-photon sources and detectors," Rev. Sci. Instrum. 82, 071101 (2011). 
Engel, G. S., Calhoun, T. R., Read, E. L., Ahn, T.-K., Mancal, T., Cheng, Y.-C., Blankenship, R. E., and Fleming, G. R., "Evidence for wavelike energy transfer through quantum coherence in photosynthetic systems," Nature 446, 782-786 (2007).

Engler, H., Weber, T., Mudrich, M., Grimm, R., and Weidemüller, M., "Very long storage times and evaporative cooling of cesium atoms in a quasielectrostatic dipole trap,” Phys. Rev. A 62, 031402 (2000).

Farley, J. W. and Wing, W. H., "Accurate calculation of dynamic Stark shifts and depopulation rates of Rydberg energy levels induced by blackbody radiation. Hydrogen, helium, and alkali-metal atoms," Phys. Rev. A 23, 2397-2424 (1981).

Fassioli, F. and Olaya-Castro, A., "Distribution of entanglement in light-harvesting complexes and their quantum efficiency," New J. Phys. 12, 085006 (2010).

Fatemi, F. K., "Cylindrical vector beams for rapid polarization-dependent measurements in atomic systems," Opt. Express 19, 25143-25150 (2011).

Feynman, R. P., "Simulating physics with computers," Int. J. Theor. Phys. 21, 467488 (1982).

Förster, T., "Zwischenmolekulare Energiewanderung und Fluoreszenz," Ann. Phys. (Berlin) 437, 55-75 (1948).

Frawley, M. C., Petcu-Colan, A., Truong, V. G., and Nic Chormaic, S., "Higher order mode propagation in an optical nanofiber," Opt. Commun. 285, 4648 - 4654 (2012).

Freeman, S., Biological Science (Pearson Prentice Hall, 2005).

Fuhrmanek, A., Bourgain, R., Sortais, Y. R. P., and Browaeys, A., "Light-assisted collisions between a few cold atoms in a microscopic dipole trap," Phys. Rev. A 85 , 062708 (2012).

Gaëtan, A., Entanglement of two atoms using the Rydberg blockade, Theses, Université Paris Sud - Paris XI Paris Sud - Paris XI (2009).

Gaëtan, A., Miroshnychenko, Y., Wilk, T., Chotia, A., Viteau, M., Comparat, D., Pillet, P., Browaeys, A., and Grangier, P., "Observation of collective excitation of two individual atoms in the Rydberg blockade regime," Nat. Phys. 5, 115-118 (2009). 
Gallagher, T. F., Rydberg Atoms, Cambridge Monographs on Atomic, Molecular and Chemical Physics (Cambridge University Press, 2005).

Gaunt, A. L. and Hadzibabic, Z., "Robust Digital Holography For Ultracold Atom Trapping," Sci. Rep. 2 (2012).

Gaunt, A. L., Schmidutz, T. F., Gotlibovych, I., Smith, R. P., and Hadzibabic, Z., "Bose-Einstein Condensation of Atoms in a Uniform Potential," Phys. Rev. Lett. 110, 200406 (2013).

Georgescu, I. M., Ashhab, S., and Nori, F., "Quantum simulation," Rev. Mod. Phys. 86, 153-185 (2014).

Gerchberg, R. W. and Saxton, W. O., "A practical algorithm for the determination of the phase from image and diffraction plane pictures," Optik 35, 237-246 (1972).

Goban, A., Choi, K. S., Alton, D. J., Ding, D., Lacroûte, C., Pototschnig, M., Thiele, T., Stern, N. P., and Kimble, H. J., "Demonstration of a State-Insensitive, Compensated Nanofiber Trap," Phys. Rev. Lett. 109, 033603 (2012).

Gounand, F., Fournier, P. R., Cuvellier, J., and Berlande, J., "Determination of natural radiative lifetimes for highly excited $P$ states in rubidium," Phys. Lett. A 59, $23-24$ (1976).

Grimm, R., Weidemüller, M., and Ovchinnikov, Y. B., "Optical dipole traps for neutral atoms," Adv. At. Mol. Opt. Phys. 42, 95-170 (2000).

Grunzweig, T., Hilliard, A., McGovern, M., and Andersen, M. F., "Neardeterministic preparation of a single atom in an optical microtrap," Nat. Phys. 6, 951-954 (2010).

Günter, G., Schempp, H., Robert-de-Saint-Vincent, M., Gavryusev, V., Helmrich, S., Hofmann, C. S., Whitlock, S., and Weidemüller, M., "Observing the Dynamics of Dipole-Mediated Energy Transport by Interaction-Enhanced Imaging," Science 342, 954-956 (2013).

Gurian, J. H., Cheinet, P., Huillery, P., Fioretti, A., Zhao, J., Gould, P. L., Comparat, D., and Pillet, P., "Observation of a Resonant Four-Body Interaction in Cold Cesium Rydberg Atoms," Phys. Rev. Lett. 108, 023005 (2012). 
Han, J., Jamil, Y., Norum, D. V. L., Tanner, P. J., and Gallagher, T. F., "Rb nf quantum defects from millimeter-wave spectroscopy of cold ${ }^{85}$ Rb Rydberg atoms," Phys. Rev. A 74, 054502 (2006).

Hankin, A. M., Jau, Y.-Y., Parazzoli, L. P., Chou, C. W., Armstrong, D. J., Landahl, A. J., and Biedermann, G. W., "Two-atom Rydberg blockade using direct $6 S$ to nP excitation," Phys. Rev. A 89, 033416 (2014).

Hardin, B. E., Hoke, E. T., Armstrong, P. B., Yum, J.-H., Comte, P., Torres, T., Frechet, J. M. J., Nazeeruddin, M. K., Gratzel, M., and McGehee, M. D., "Increased light harvesting in dye-sensitized solar cells with energy relay dyes," Nat. Photon. 3, 406-411 (2009).

Haroche, S., "Nobel Lecture: Controlling photons in a box and exploring the quantum to classical boundary," Rev. Mod. Phys. 85, 1083-1102 (2013).

Haroche, S. and Raimond, J., Exploring the Quantum: Atoms, Cavities, and Photons, Oxford Graduate Texts (OUP Oxford, 2006).

Hattermann, H., Mack, M., Karlewski, F., Jessen, F., Cano, D., and Fortágh, J., "Detrimental adsorbate fields in experiments with cold Rydberg gases near surfaces," Phys. Rev. A 86, 022511 (2012).

Heidemann, R., Raitzsch, U., Bendkowsky, V., Butscher, B., Löw, R., and Pfau, T., "Rydberg Excitation of Bose-Einstein Condensates," Phys. Rev. Lett. 100, 033601 (2008).

Hoffman, J. E., Atoms coupled to superconducting qubits: optical nanofiber fabrication and analysis, Ph.D. thesis, University of Maryland (2014).

Hoffman, J. E., Grover, J. A., Kim, Z., Wood, A. K., Anderson, J. R., Dragt, A. J., Hafezi, M., Lobb, C. J., Orozco, L. A., Rolston, S. L., Taylor, J. M., Vlahacos, C. P., and Wellstood, F. C., "Atoms talking to SQUIDs," Rev. Mex. Fis. S 57, $1-5(2011)$.

Hoffman, J. E., Ravets, S., Grover, J. A., Solano, P., Kordell, P. R., WongCampos, J. D., Orozco, L. A., and Rolston, S. L., "Ultrahigh transmission optical nanofibers," AIP Advances 4, 067124 (2014).

Hogan, S. D., Agner, J. A., Merkt, F., Thiele, T., Filipp, S., and Wallraff, A., "Driving Rydberg-Rydberg Transitions from a Coplanar Microwave Waveguide," Phys. Rev. Lett. 108, 063004 (2012). 
Houck, A. A., Tureci, H. E., and Koch, J., "On-chip quantum simulation with superconducting circuits," Nat. Phys. 8, 292-299 (2012).

Huang, J.-S., Goh, T., Li, X., Sfeir, M. Y., Bielinski, E. A., Tomasulo, S., Lee, M. L., Hazari, N., and Taylor, A. D., "Polymer bulk heterojunction solar cells employing Förster resonance energy transfer," Nat. Photon. 7, 479-485 (2013).

Imamoğlu, A., "Cavity QED Based on Collective Magnetic Dipole Coupling: Spin Ensembles as Hybrid Two-Level Systems," Phys. Rev. Lett. 102, 083602 (2009).

Isenhower, L., Urban, E., Zhang, X. L., Gill, A. T., Henage, T., Johnson, T. A., Walker, T. G., and Saffman, M., "Demonstration of a Neutral Atom ControlledNOT Quantum Gate," Phys. Rev. Lett. 104, 010503 (2010).

Jaksch, D., Briegel, H.-J., Cirac, J. I., Gardiner, C. W., and Zoller, P., "Entanglement of Atoms via Cold Controlled Collisions," Phys. Rev. Lett. 82, 1975-1978 (1999).

Jaksch, D., Cirac, J. I., Zoller, P., Rolston, S. L., Côté, R., and Lukin, M. D., "Fast Quantum Gates for Neutral Atoms,” Phys. Rev. Lett. 85, 2208-2211 (2000).

Jessen, F., Knufinke, M., Bell, S., Vergien, P., Hattermann, H., Weiss, P., Rudolph, M., Reinschmidt, M., Meyer, K., Gaber, T., Cano, D., Günther, A., Bernon, S., Koelle, D., Kleiner, R., and Fortágh, J., "Trapping of ultracold atoms in a 3He/4He dilution refrigerator," Appl. Phys. B 116, 665-671 (2014).

Johnson, T. A., Urban, E., Henage, T., Isenhower, L., Yavuz, D. D., Walker, T. G., and Saffman, M., "Rabi Oscillations between Ground and Rydberg States with Dipole-Dipole Atomic Interactions," Phys. Rev. Lett. 100, 113003 (2008).

Jurcevic, P., Lanyon, B. P., Hauke, P., Hempel, C., Zoller, P., Blatt, R., and Roos, C. F., "Quasiparticle engineering and entanglement propagation in a quantum many-body system," Nature 511, 202-205 (2014).

Kaufman, A. M., Lester, B. J., and Regal, C. A., "Cooling a Single Atom in an Optical Tweezer to its Quantum Ground State," Phys. Rev. X 2, 041014 (2012).

Kien, F. L., Liang, J., Hakuta, K., and Balykin, V., "Field intensity distributions and polarization orientations in a vacuum-clad subwavelength-diameter optical fiber," Opt. Commun. 242, $445-455$ (2004).

Kiffner, M., Li, W., and Jaksch, D., "Three-Body Bound States in Dipole-Dipole Interacting Rydberg Atoms,” Phys. Rev. Lett. 111, 233003 (2013). 
Kim, Z., Vlahacos, C. P., Hoffman, J. E., Grover, J. A., Voigt, K. D., Cooper, B. K., Ballard, C. J., Palmer, B. S., Hafezi, M., Taylor, J. M., Anderson, J. R., Dragt, A. J., Lobb, C. J., Orozco, L. A., Rolston, S. L., and Wellstood, F. C., "Thin-film superconducting resonator tunable to the ground-state hyperfine splitting of ${ }^{87} \mathrm{Rb}$," AIP Advances 1, 042107 (2011).

Kimble, H. J., "The quantum internet," Nature 453, 1023-1030 (2008).

Kruse, J., Gierl, C., Schlosser, M., and Birkl, G., "Reconfigurable site-selective manipulation of atomic quantum systems in two-dimensional arrays of dipole traps," Phys. Rev. A 81, 060308 (2010).

Labuhn, H., Ravets, S., Barredo, D., Béguin, L., Nogrette, F., Lahaye, T., and Browaeys, A., "Single-atom addressing in microtraps for quantum-state engineering using Rydberg atoms," Phys. Rev. A 90, 023415 (2014).

Ladd, T. D., Jelezko, F., Laflamme, R., Nakamura, Y., Monroe, C., and O'Brien, J. L., "Quantum computers," Nature 464, 45-53 (2010).

Lakowicz, J., Principles of Fluorescence Spectroscopy (Springer, 2007).

Landau, L. and Lifshitz, E., Mechanics, Butterworth Heinemann (ButterworthHeinemann, 1976).

Landragin, A., Courtois, J.-Y., Labeyrie, G., Vansteenkiste, N., Westbrook, C. I., and Aspect, A., "Measurement of the van der Waals Force in an Atomic Mirror," Phys. Rev. Lett. 77, 1464-1467 (1996).

Le Kien, F., Balykin, V. I., and Hakuta, K., "Atom trap and waveguide using a twocolor evanescent light field around a subwavelength-diameter optical fiber," Phys. Rev. A 70, 063403 (2004).

Lee, J., Grover, J. A., Orozco, L. A., and Rolston, S. L., "Sub-Doppler cooling of neutral atoms in a grating magneto-optical trap," J. Opt. Soc. Am. B 30, 2869-2874 (2013).

Leon-Saval, S., Birks, T., Wadsworth, W., Russell, P. S. J., and Mason, M., "Supercontinuum generation in submicron fibre waveguides," Opt. Express 12, 2864-2869 (2004).

Lesanovsky, I., "Many-Body Spin Interactions and the Ground State of a Dense Rydberg Lattice Gas,” Phys. Rev. Lett. 106, 025301 (2011). 
Li, W., Mourachko, I., Noel, M. W., and Gallagher, T. F., "Millimeter-wave spectroscopy of cold Rb Rydberg atoms in a magneto-optical trap: Quantum defects of the ns, np, and nd series," Phys. Rev. A 67, 052502 (2003).

Liebisch, T. C., Reinhard, A., Berman, P. R., and Raithel, G., "Atom Counting Statistics in Ensembles of Interacting Rydberg Atoms," Phys. Rev. Lett. 95, 253002 (2005).

López-Quesada, C., Andilla, J., and Martín-Badosa, E., "Correction of aberration in holographic optical tweezers using a Shack-Hartmann sensor," Appl. Opt. 48, 1084-1090 (2009).

Loudon, R., The Quantum Theory of Light (OUP Oxford, 2000).

Löw, R., Weimer, H., Krohn, U., Heidemann, R., Bendkowsky, V., Butscher, B., Büchler, H. P., and Pfau, T., "Universal scaling in a strongly interacting rydberg gas," Phys. Rev. A 80, 033422 (2009).

Lukin, M. D., Fleischhauer, M., Cote, R., Duan, L. M., Jaksch, D., Cirac, J. I., and Zoller, P., "Dipole Blockade and Quantum Information Processing in Mesoscopic Atomic Ensembles," Phys. Rev. Lett. 87, 037901 (2001).

Marcos, D., Wubs, M., Taylor, J. M., Aguado, R., Lukin, M. D., and Sørensen, A. S., "Coupling Nitrogen-Vacancy Centers in Diamond to Superconducting Flux Qubits," Phys. Rev. Lett. 105, 210501 (2010).

Maxwell, D., Szwer, D. J., Paredes-Barato, D., Busche, H., Pritchard, J. D., Gauguet, A., Weatherill, K. J., Jones, M. P. A., and Adams, C. S., "Storage and Control of Optical Photons Using Rydberg Polaritons," Phys. Rev. Lett. 110, 103001 (2013).

Miller, W. H., "Perspective: Quantum or classical coherence?" J. Chem. Phys. 136, 210901 (2012).

Miroshnychenko, Y., Alt, W., Dotsenko, I., Förster, L., Khudaverdyan, M., Meschede, D., Schrader, D., and Rauschenbeutel, A., "Quantum engineering: An atomsorting machine," Nature 442, 151-151 (2006a).

Miroshnychenko, Y., Alt, W., Dotsenko, I., Förster, L., Khudaverdyan, M., Rauschenbeutel, A., and Meschede, D., "Precision preparation of strings of trapped neutral atoms," New J. Phys. 8, 191 (2006b). 
Miroshnychenko, Y., Gaëtan, A., Evellin, C., Grangier, P., Comparat, D., Pillet, P., Wilk, T., and Browaeys, A., "Coherent excitation of a single atom to a Rydberg state," Phys. Rev. A 82, 013405 (2010).

Monz, T., Schindler, P., Barreiro, J. T., Chwalla, M., Nigg, D., Coish, W. A., Harlander, M., Hänsel, W., Hennrich, M., and Blatt, R., "14-Qubit Entanglement: Creation and Coherence," Phys. Rev. Lett. 106, 130506 (2011).

Morrissey, M. J., Deasy, K., Frawley, M., Kumar, R., Prel, E., Russell, L., Truong, V. G., and Nic Chormaic, S., "Spectroscopy, Manipulation and Trapping of Neutral Atoms, Molecules, and Other Particles Using Optical Nanofibers: A Review," Sensors 13, 10449-10481 (2013).

Mourachko, I., Comparat, D., de Tomasi, F., Fioretti, A., Nosbaum, P., Akulin, V. M., and Pillet, P., "Many-Body Effects in a Frozen Rydberg Gas," Phys. Rev. Lett. 80, 253-256 (1998).

Mudrich, M., Zahzam, N., Vogt, T., Comparat, D., and Pillet, P., "Back and Forth Transfer and Coherent Coupling in a Cold Rydberg Dipole Gas," Phys. Rev. Lett. 95, 233002 (2005).

Müller, M., Lesanovsky, I., Weimer, H., Büchler, H. P., and Zoller, P., "Mesoscopic Rydberg Gate Based on Electromagnetically Induced Transparency," Phys. Rev. Lett. 102, 170502 (2009).

Nelson, K. D., Li, X., and Weiss, D. S., "Imaging single atoms in a three-dimensional array," Nat. Phys. 3, 556-560 (2007).

Nielsen, M. and Chuang, I., Quantum Computation and Quantum Information, Cambridge Series on Information and the Natural Sciences (Cambridge University Press, 2000).

Nipper, J., Balewski, J. B., Krupp, A. T., Butscher, B., Löw, R., and Pfau, T., "Highly Resolved Measurements of Stark-Tuned Förster Resonances between Rydberg Atoms," Phys. Rev. Lett. 108, 113001 (2012a).

Nipper, J., Balewski, J. B., Krupp, A. T., Hofferberth, S., Löw, R., and Pfau, T., "Atomic Pair-State Interferometer: Controlling and Measuring an InteractionInduced Phase Shift in Rydberg-Atom Pairs," Phys. Rev. X 2, 031011 (2012b). 
Nogrette, F., Labuhn, H., Ravets, S., Barredo, D., Béguin, L., Vernier, A., Lahaye, T., and Browaeys, A., "Single-Atom Trapping in Holographic 2D Arrays of Microtraps with Arbitrary Geometries," Phys. Rev. X 4, 021034 (2014).

Okoshi, T., Kikuchi, K., and Nakayama, A., "Novel method for high resolution measurement of laser output spectrum," Electron. Lett. 16, 630-631 (1980).

Olaya-Castro, A., Lee, C. F., Olsen, F. F., and Johnson, N. F., "Efficiency of energy transfer in a light-harvesting system under quantum coherence," Phys. Rev. B 78, 085115 (2008).

Oppenheimer, J. R., "Minutes of the Pasadena, California, Meeting," Phys. Rev. 60, 158-165 (1941).

Orucevic, F., Lefèvre-Seguin, V., and Hare, J., "Transmittance and near-field characterization of sub-wavelength tapered optical fibers," Opt. Express 15, 13624-13629 (2007).

Panitchayangkoon, G., Hayes, D., Fransted, K. A., Caram, J. R., Harel, E., Wen, J., Blankenship, R. E., and Engel, G. S., "Long-lived quantum coherence in photosynthetic complexes at physiological temperature," Proc. Natl. Acad. Sci. U.S.A. 107, 12766-12770 (2010).

Pechkis, J. A. and Fatemi, F. K., "Cold atom guidance in a capillary using bluedetuned, hollow optical modes," Opt. Express 20, 13409-13418 (2012).

Pérez Galván, A., Zhao, Y., and Orozco, L. A., "Measurement of the hyperfine splitting of the $6 S_{1 / 2}$ level in rubidium," Phys. Rev. A 78, 012502 (2008).

Perrin, F., "Interaction entre atomes normal et active. Transferts d'activation. Formation d'une molécule activée." Ann. Inst. Henri Poincaré 3, 279-318 (1933).

Peyronel, T., Firstenberg, O., Liang, Q.-Y., Hofferberth, S., Gorshkov, A. V., Pohl, T., Lukin, M. D., and Vuletic, V., "Quantum nonlinear optics with single photons enabled by strongly interacting atoms," Nature 488, 57-60 (2012).

Pohl, T. and Berman, P. R., "Breaking the Dipole Blockade: Nearly Resonant Dipole Interactions in Few-Atom Systems,” Phys. Rev. Lett. 102, 013004 (2009).

Qian, J., Zhao, X.-D., Zhou, L., and Zhang, W., "Anisotropic deformation of the Rydberg-blockade sphere in few-atom systems," Phys. Rev. A 88, 033422 (2013). 
Rabl, P., DeMille, D., Doyle, J. M., Lukin, M. D., Schoelkopf, R. J., and Zoller, P., "Hybrid Quantum Processors: Molecular Ensembles as Quantum Memory for Solid State Circuits," Phys. Rev. Lett. 97, 033003 (2006).

Ravets, S., Hoffman, J. E., Kordell, P. R., Wong-Campos, J. D., Rolston, S. L., and Orozco, L. A., "Intermodal energy transfer in a tapered optical fiber: optimizing transmission," J. Opt. Soc. Am. A 30, 2361-2371 (2013a).

Ravets, S., Hoffman, J. E., Orozco, L. A., Rolston, S. L., Beadie, G., and Fatemi, F. K., "A low-loss photonic silica nanofiber for higher-order modes," Opt. Express 21, 18325-18335 (2013b).

Reif, F., Fundamentals of statistical and thermal physics, McGraw-Hill series in fundamentals of physics (McGraw-Hill, 1965).

Reinhard, A., Liebisch, T. C., Knuffman, B., and Raithel, G., "Publisher's Note: Level shifts of rubidium Rydberg states due to binary interactions," Phys. Rev. A 75, 039902 (2007).

Reinhard, A., Younge, K. C., and Raithel, G., "Effect of Förster resonances on the excitation statistics of many-body Rydberg systems," Phys. Rev. A 78, 060702 (2008).

Reitzenstein, S. and Forchel, A., "Quantum dot micropillars," J. Phys. D: Appl. Phys. 43, 033001 (2010).

Richerme, P., Gong, Z.-X., Lee, A., Senko, C., Smith, J., Foss-Feig, M., Michalakis, S., Gorshkov, A. V., and Monroe, C., "Non-local propagation of correlations in quantum systems with long-range interactions," Nature 511, 198-201 (2014).

Ryabtsev, I. I., Tretyakov, D. B., Beterov, I. I., and Entin, V. M., "Observation of the Stark-Tuned Förster Resonance between Two Rydberg Atoms," Phys. Rev. Lett. 104, 073003 (2010a).

Ryabtsev, I. I., Tretyakov, D. B., Beterov, I. I., Entin, V. M., and Yakshina, E. A., "Stark-tuned Förster resonance and dipole blockade for two to five cold Rydberg atoms: Monte Carlo simulations for various spatial configurations," Phys. Rev. A 82, 053409 (2010b).

Saffman, M. and Walker, T. G., "Creating single-atom and single-photon sources from entangled atomic ensembles," Phys. Rev. A 66, 065403 (2002). 
Saffman, M., Walker, T. G., and Mølmer, K., "Quantum information with Rydberg atoms," Rev. Mod. Phys. 82, 2313-2363 (2010).

Safinya, K. A., Delpech, J. F., Gounand, F., Sandner, W., and Gallagher, T. F., "Resonant Rydberg-Atom-Rydberg-Atom Collisions," Phys. Rev. Lett. 47, 405-408 (1981).

Sagué, G., Baade, A., and Rauschenbeutel, A., "Blue-detuned evanescent field surface traps for neutral atoms based on mode interference in ultrathin optical fibres," New J. Phys. 10, 113008 (2008).

Sarovar, M., Ishizaki, A., Fleming, G. R., and Whaley, K. B., "Quantum entanglement in photosynthetic light-harvesting complexes," Nat. Phys. 6, 462-467 (2010).

Schauß, P., Cheneau, M., Endres, M., Fukuhara, T., Hild, S., Omran, A., Pohl, T., Gross, C., Kuhr, S., and Bloch, I., "Observation of spatially ordered structures in a two-dimensional Rydberg gas," Nature 491, 87-91 (2012).

Schlosser, M., Tichelmann, S., Kruse, J., and Birkl, G., "Scalable architecture for quantum information processing with atoms in optical micro-structures," Quantum Inf. Process. 10, 907-924 (2011).

Schlosser, N., Reymond, G., and Grangier, P., "Collisional Blockade in Microscopic Optical Dipole Traps," Phys. Rev. Lett. 89, 023005 (2002).

Schlosser, N., Reymond, G., Protsenko, I., and Grangier, P., "Sub-poissonian loading of single atoms in a microscopic dipole trap," Nature 411, 1024-1027 (2001).

Schneeweiss, P., Dawkins, S. T., Mitsch, R., Reitz, D., Vetsch, E., and Rauschenbeutel, A., "A nanofiber-based optical conveyor belt for cold atoms," Appl. Phys. B 110, 279-283 (2013).

Shih, C.-Y. and Chapman, M. S., "Nondestructive light-shift measurements of single atoms in optical dipole traps," Phys. Rev. A 87, 063408 (2013).

Singer, K., Reetz-Lamour, M., Amthor, T., Marcassa, L. G., and Weidemüller, M., "Suppression of Excitation and Spectral Broadening Induced by Interactions in a Cold Gas of Rydberg Atoms," Phys. Rev. Lett. 93, 163001 (2004).

Snyder, A. and Love, J., Optical Waveguide Theory, Science paperbacks (Springer, 1983). 
Sørensen, A. S., van der Wal, C. H., Childress, L. I., and Lukin, M. D., "Capacitive Coupling of Atomic Systems to Mesoscopic Conductors," Phys. Rev. Lett. 92, 063601 (2004).

Sortais, Y. R. P., Marion, H., Tuchendler, C., Lance, A. M., Lamare, M., Fournet, P., Armellin, C., Mercier, R., Messin, G., Browaeys, A., and Grangier, P., "Diffractionlimited optics for single-atom manipulation," Phys. Rev. A 75, 013406 (2007).

Steck, D. A., "Rubidium 87 D Line Data," available online at http://steck.us/alkalidata (revision2.1.4, 23 December 2010).

Stryer, L. and Haugland, R. P., "Energy transfer: a spectroscopic ruler," Proc. Natl. Acad. Sci. U.S.A. 58, 719-726 (1967).

Tey, M. K., Chen, Z., Aljunid, S. A., Chng, B., Huber, F., Maslennikov, G., and Kurtsiefer, C., "Strong interaction between light and a single trapped atom without the need for a cavity," Nat. Phys. 4, 924-927 (2008).

Thompson, J. D., Tiecke, T. G., Zibrov, A. S., Vuletić, V., and Lukin, M. D., "Coherence and Raman Sideband Cooling of a Single Atom in an Optical Tweezer," Phys. Rev. Lett. 110, 133001 (2013).

Tian, L., Rabl, P., Blatt, R., and Zoller, P., "Interfacing Quantum-Optical and Solid-State Qubits," Phys. Rev. Lett. 92, 247902 (2004).

Tong, D., Farooqi, S. M., Stanojevic, J., Krishnan, S., Zhang, Y. P., Côté, R., Eyler, E. E., and Gould, P. L., "Local Blockade of Rydberg Excitation in an Ultracold Gas," Phys. Rev. Lett. 93, 063001 (2004).

Tong, L., Gattass, R. R., Ashcom, J. B., He, S., Lou, J., Shen, M., Maxwell, I., and Mazur, E., "Subwavelength-diameter silica wires for low-loss optical wave guiding," Nature 426, 816-819 (2003).

Tuchendler, C., Lance, A. M., Browaeys, A., Sortais, Y. R. P., and Grangier, P., "Energy distribution and cooling of a single atom in an optical tweezer," Phys. Rev. A 78, 033425 (2008).

Urban, E., Johnson, T. A., Henage, T., Isenhower, L., Yavuz, D. D., Walker, T. G., and Saffman, M., "Observation of Rydberg blockade between two atoms," Nat. Phys. 5, 110-114 (2009). 
van Ditzhuijzen, C. S. E., Koenderink, A. F., Hernández, J. V., Robicheaux, F., Noordam, L. D., and van Linden van den Heuvell, H. B., "Spatially Resolved Observation of Dipole-Dipole Interaction between Rydberg Atoms," Phys. Rev. Lett. 100, 243201 (2008).

van Ditzhuijzen, C. S. E., Koenderink, A. F., Noordam, L. D., and van Linden van den Heuvell, H. B., "Simultaneous position and state measurement of Rydberg atoms," Eur. Phys. J. D 40, 13-17 (2006).

Verdú, J., Zoubi, H., Koller, C., Majer, J., Ritsch, H., and Schmiedmayer, J., "Strong Magnetic Coupling of an Ultracold Gas to a Superconducting Waveguide Cavity," Phys. Rev. Lett. 103, 043603 (2009).

Vetsch, E., Reitz, D., Sagué, G., Schmidt, R., Dawkins, S. T., and Rauschenbeutel, A., "Optical Interface Created by Laser-Cooled Atoms Trapped in the Evanescent Field Surrounding an Optical Nanofiber," Phys. Rev. Lett. 104, 203603 (2010).

Viteau, M., Bason, M. G., Radogostowicz, J., Malossi, N., Ciampini, D., Morsch, O., and Arimondo, E., "Rydberg Excitations in Bose-Einstein Condensates in QuasiOne-Dimensional Potentials and Optical Lattices," Phys. Rev. Lett. 107, 060402 (2011).

Vogt, T., Viteau, M., Chotia, A., Zhao, J., Comparat, D., and Pillet, P., "ElectricField Induced Dipole Blockade with Rydberg Atoms," Phys. Rev. Lett. 99, 073002 (2007).

Vogt, T., Viteau, M., Zhao, J., Chotia, A., Comparat, D., and Pillet, P., "Dipole Blockade at Förster Resonances in High Resolution Laser Excitation of Rydberg States of Cesium Atoms," Phys. Rev. Lett. 97, 083003 (2006).

Walker, T. G. and Saffman, M., "Zeros of Rydberg-Rydberg Föster interactions," J. Phys. B: At. Mol. Opt. Phys. 38, S309 (2005).

Walker, T. G. and Saffman, M., "Consequences of Zeeman degeneracy for the van der Waals blockade between Rydberg atoms," Phys. Rev. A 77, 032723 (2008).

Wallquist, M., Hammerer, K., Rabl, P., Lukin, M., and Zoller, P., "Hybrid quantum devices and quantum engineering," Phys. Scr. 2009, 014001 (2009).

Warken, F., Ultra thin glass fibers as a tool for coupling light and matter, Ph.D. thesis, Rheinische Friedrich-Wilhelms-Universität Bonn (2007). 
Weimer, H., Löw, R., Pfau, T., and Büchler, H. P., "Quantum Critical Behavior in Strongly Interacting Rydberg Gases," Phys. Rev. Lett. 101, 250601 (2008).

Weissbluth, M., Atoms and molecules (Academic Press, 1978).

Westermann, S., Amthor, T., de Oliveira, A. L., Deiglmayr, J., Reetz-Lamour, M., and Weidemüller, M., "Dynamics of resonant energy transfer in a cold rydberg gas," Eur. Phys. J. D 40, 37-43 (2006).

Whaley, K. B., Sarovar, M., and Ishizaki, A., "Quantum entanglement phenomena in photosynthetic light harvesting complexes," Procedia Chem. 3, 152 - 164 (2011).

Wilde, M. M., McCracken, J. M., and Mizel, A., "Could light harvesting complexes exhibit non-classical effects at room temperature?" Proc. R. Soc. A 466, 1347-1363 (2010).

Wilk, T., Gaëtan, A., Evellin, C., Wolters, J., Miroshnychenko, Y., Grangier, P., and Browaeys, A., "Entanglement of Two Individual Neutral Atoms Using Rydberg Blockade," Phys. Rev. Lett. 104, 010502 (2010).

Wineland, D. J., "Nobel lecture: Superposition, entanglement, and raising Schrödinger's cat," Rev. Mod. Phys. 85, 1103-1114 (2013).

Wineland, D. J., Bollinger, J. J., Itano, W. M., Moore, F. L., and Heinzen, D. J., "Spin squeezing and reduced quantum noise in spectroscopy," Phys. Rev. A 46, R6797-R6800 (1992).

Xiang, Z.-L., Ashhab, S., You, J. Q., and Nori, F., "Hybrid quantum circuits: Superconducting circuits interacting with other quantum systems," Rev. Mod. Phys. 85, 623-653 (2013).

Yan, B., Moses, S. A., Gadway, B., Covey, J. P., Hazzard, K. R. A., Rey, A. M., Jin, D. S., and Ye, J., "Observation of dipolar spin-exchange interactions with lattice-confined polar molecules," Nature 501, 521-525 (2013).

Yariv, A., Optical Electronics, The Oxford Series in Electrical and Computer Engineering (Oxford University Press, 1990).

Zimmerman, M. L., Littman, M. G., Kash, M. M., and Kleppner, D., "Stark structure of the Rydberg states of alkali-metal atoms," Phys. Rev. A 20, 2251-2275 (1979). 
